\title{
Aggregatenbildung der Rußteilchen in Kohlenwasserstoff/Luftflammen in einem Druckbereich von ein bis fünf bar
}

\author{
Dissertation \\ zur Erlangung des Doktorgrades \\ der Mathematisch-Naturwissenschaftlichen Fakultäten \\ der Georg-August-Universität zu Göttingen
}

vorgelegt von

Wilhelm Stahlberg

aus Bremen

Göttingen, den 16.03.2004 
Auf die Rückseite:

D 7

Referentin/Referent: Prof. Dr. Dr. h.c.mult. H.-Gg. Wagner

Korreferentin/Korreferent: Prof. Dr. Lutz Fitjer

Tag der mündlichen Prüfung: 28.04.2004 
Meinem verehrten Lehrer, Herrn Prof. Dr. H. Gg. Wagner, danke ich herzlich für die Anregung und Förderung dieser Arbeit.

Mein ganz besonderer Dank gilt Frau Dr. H. Jander, für die Anleitung dieser Arbeit, die fachlichen Gespräche und ganz speziell für die ständige Hilfsbereitschaft.

Nicht zuletzt gilt mein Dank den Angehörigen der Werkstätten des Instituts unter der Leitung von Herrn V. Meyer und A. Knorr sowie der Feinmechanikwerkstatt des Max-Planck-Instituts für Strömungsforschung unter der Leitung von R. Schrader.

Abschließend möchte ich mich besonders Herrn K. Pape für seine entscheidende Hilfe unter anderem bei der Konstruktion der Brennerhaube bedanken. 


\section{Inhaltsverzeichnis}

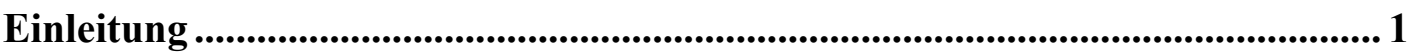

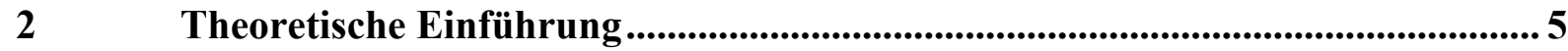

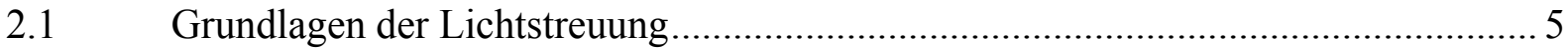

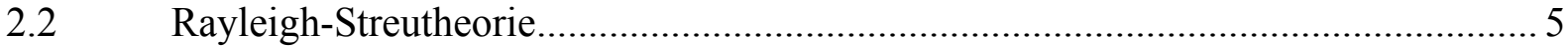

2.3 Die Streutheorie von Mie in der Rayleigh-Näherung ........................................ 8

2.4 Anwendung auf Streulichtmessungen in rußenden Flammen ................................. 10

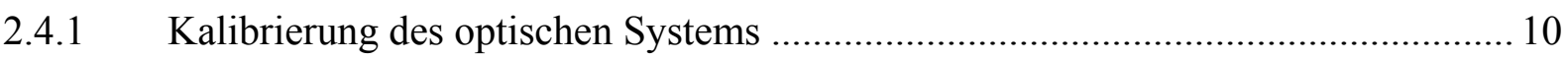

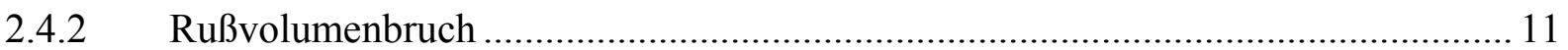

2.4.3 Teilchendurchmesser und Teilchenzahldichte .................................................... 12

2.5 Streutheorie für polydisperse Aggregate RDG/FA-Theorie .................................. 13

2.6 Untersuchung der Rußaggregate und deren Morphologie nach der RDG/FA-Theorie

3 Experimentelle Aufbauten und Durchführung der Messungen ........................ 20

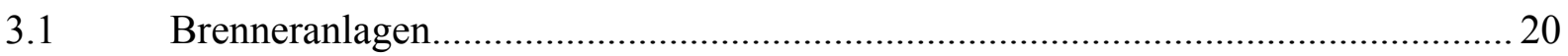

3.1.1 Röhrchenbrenner für die Normaldruckmessungen............................................. 20

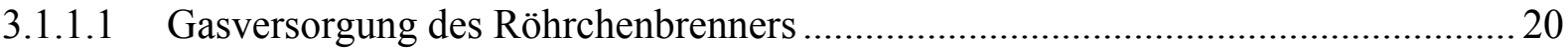

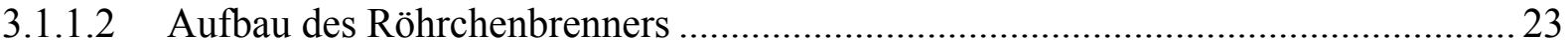

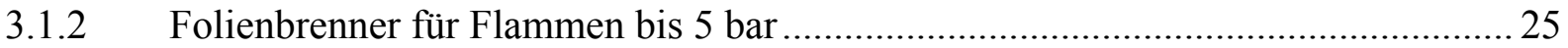

3.1.2.1 Gasdosierung der 15 bar Brenneranlage .................................................................. 25

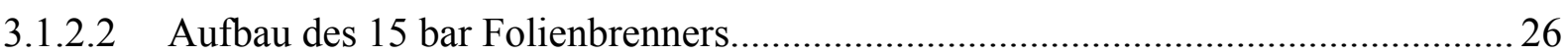

3.1.2.3 Aufbau und Betrieb der Hochdruckbrenneranlage.................................................. 28

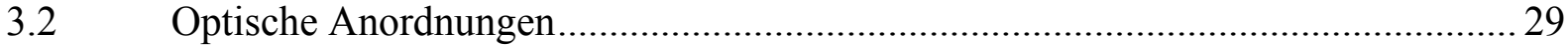

3.2.1 Optische Anordnung der 1 bar Brenneranlage ....................................................29

3.2.2 Optische Messungen mit dem Hochdruckbrenner ............................................... 31

3.2.2.1 Winkelabhängiges optisches Messen mit Lichtleitern .......................................... 31

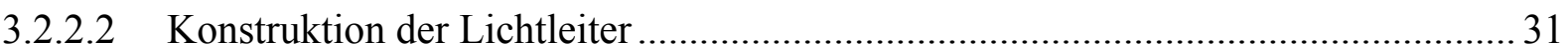

3.2.2.3 Eichung der Lichtleiter für die Messungen ........................................................... 33

3.2.2.4 Optische Anordnung der Hochdruckbrenneranlage ............................................. 35 
$4 \quad$ Auswertung der Meßdaten ....................................................................................3 37

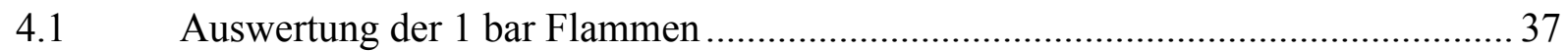

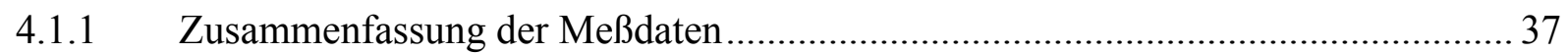

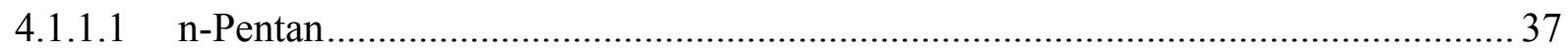

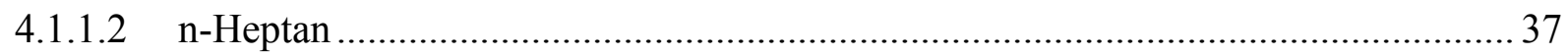

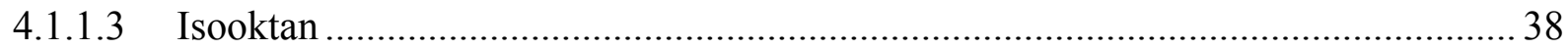

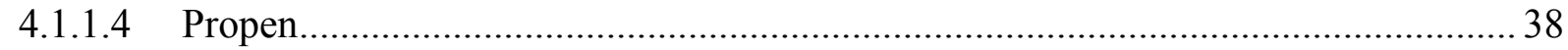

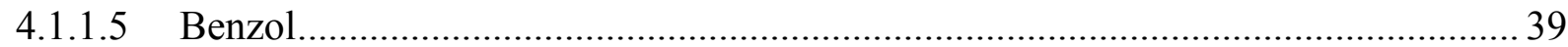

4.1.2 Detaillierte Darstellung der 1 bar Flammen am Beispiel der Benzolflamme .......... 40

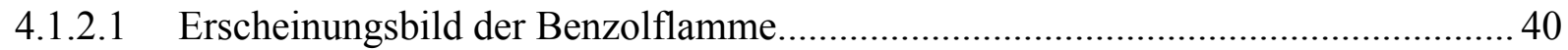

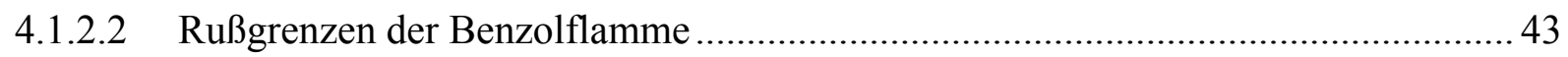

4.1.2.3 Flammentemperaturen der Benzolflammen nach Kurlbaum .................................. 44

4.1.2.4 Winkelabhängige Streukurven der rußenden Benzol-Luft-Flammen ...................... 47

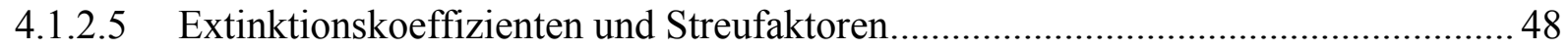

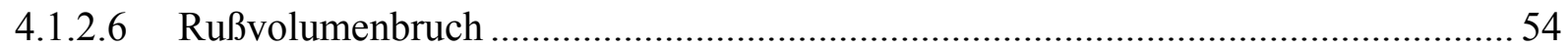

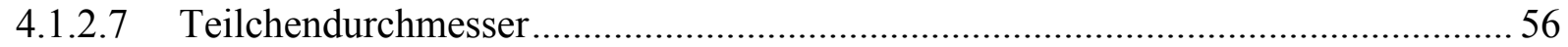

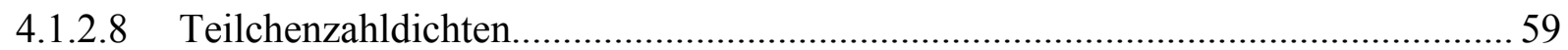

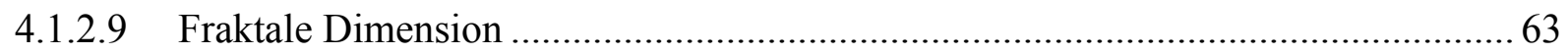

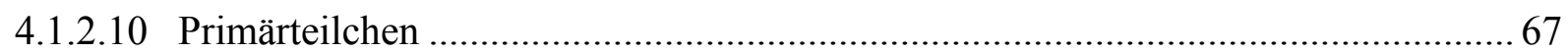

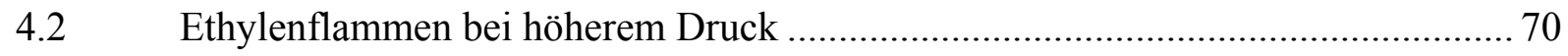

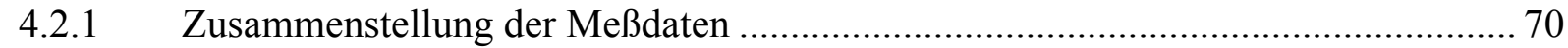

4.2.2 Erscheinungsbild der Ethylen-Luft-Flammen im Druckbereich von 2 bis 5 bar ..... 71

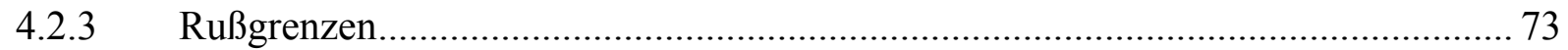

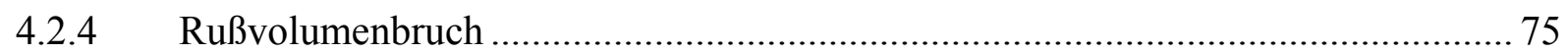

4.2.5 Teilchendurchmesser und Teilchenzahldichten .................................................... 77

4.2.5.1 Teilchendurchmesser, volumenäquivalenter Durchmesser und scheinbarer Radius 77

4.2.5.2 Teilchenzahldichte und Aggregatenzahldichte .................................................. 79

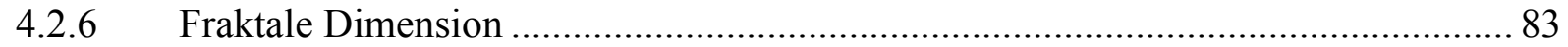

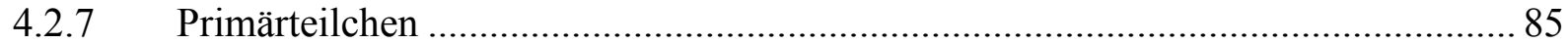

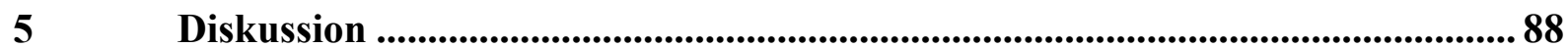

5.1 Vergleich der Meßergebnisse bei Normaldruck.................................................. 88

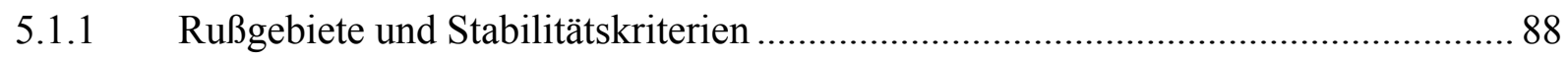

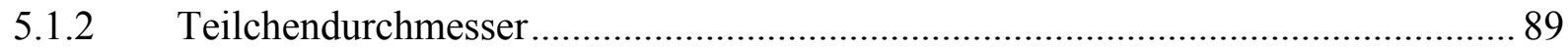




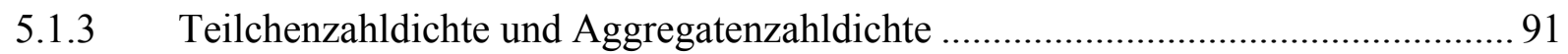

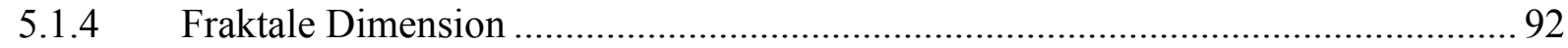

5.2 Vergleich der Meßerbegnisse mit der Mie-Theorie ............................................ 95

$6 \quad$ Zusammenfassung ................................................................................................. 97

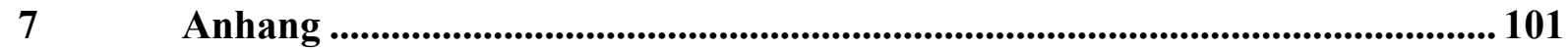

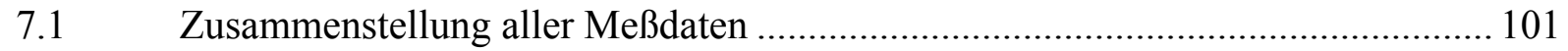

7.1.1 Flammen mit dem 1 bar Röhrchenbrenner.......................................................... 101

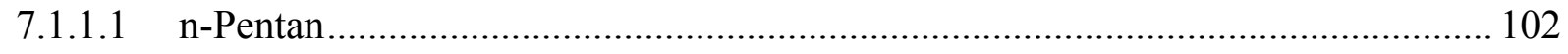

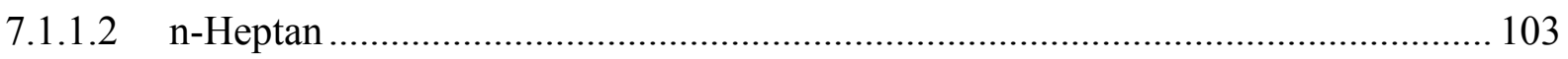

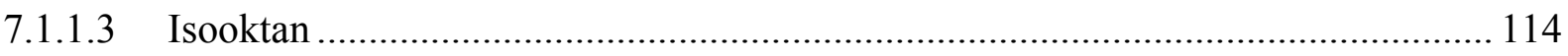

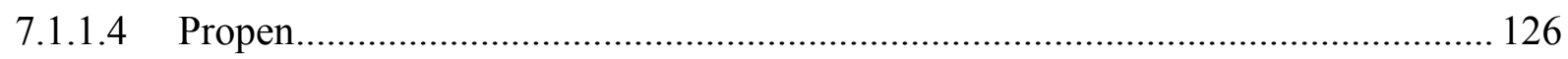

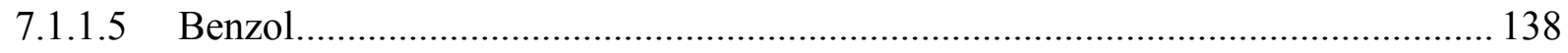

7.1.2 Zusätzliche Meßdaten der Ethylen-Luft-Flammen im Hochdruck ........................ 139 


\section{Verzeichnis der benutzten Symbole}

a

$\Delta \mathrm{A}$

C

$\mathrm{C}_{\text {sca }}$

$\mathrm{C}_{\mathrm{VV}}$

$\mathrm{D}_{30}$

$\mathrm{D}_{30}^{3}$

$\mathrm{D}_{60}^{6}$

d

$\mathrm{D}_{\mathrm{f}}$

$\mathrm{d}_{\mathrm{g}}$

$\mathrm{d}_{\mathrm{M}}$

$\mathrm{d}_{\mathrm{p}}$

$\mathrm{d}_{\mathrm{R}}$

$E(\widetilde{m})$

$\overrightarrow{\mathrm{E}}_{0}$

$\mathrm{E}_{0, \text { int }}$

$\mathrm{F}(\widetilde{\mathrm{m}})$

$f_{n}$

$f_{V}$

$f\left(\mathrm{q}_{\mathrm{i}}^{2} \overline{\mathrm{R}_{\mathrm{g}}^{2}}\right)$

$\mathrm{h}$

$\overrightarrow{\mathrm{H}}_{0}$

I

$\mathrm{I}_{\mathrm{ext}}$

$\mathrm{I}_{\mathrm{HV}}$

$\mathrm{I}_{\mathrm{VV}}$

Abstand

vom Streulicht bestrahlte Fläche

differentieller Streuquerschnitt pro Teilchen

Streuquerschnitt pro Teilchen

differentieller Streuquerschnitt pro Teilchen mit vertikalen

einfallenden/gestreuten Licht

volumenäquivalenter Durchmesser $\mathrm{D}_{30}=\mathrm{n}^{1 / 3} \mathrm{~d}_{\mathrm{p}}[\mathrm{nm}]$

drittes Moment der Aggregatenverteilungsfunktion

sechstes Moment der Aggregatenverteilungsfunktion

Teilchendurchmesser

fraktale Dimension (engl.: fractal Dimension)

geometrischer Mittelwert des Primärteilchendurchmessers [nm]

Modalwert des Primärteilchendurchmessers [nm]

Primärteilchendurchmesser [nm]

Teilchendurchmesser nach Rayleigh [nm]

$\operatorname{Im}\left[\left(\mathrm{m}^{2}-1\right) /\left(\mathrm{m}^{2}+2\right)\right]$

elektrischer Feldvektor des einfallenden Lichtes

elektrische Feldkonstante $\left[\mathrm{A} \mathrm{s} \mathrm{V}^{-1} \mathrm{~m}^{-1}\right]$ im Vakuum eines Objekts

$\left|\left(\mathrm{m}^{2}-1\right) /\left(\mathrm{m}^{2}+2\right)\right|^{2}$

Momentenverhältnis $\overline{\mathrm{n}^{2}} /\left(\overline{\mathrm{n}^{1}}\right)^{2}$

Rußvolumenbruch (particle volume fraction)

Strukturfunktion eines Aggregats

Höhe über der Brennerkante [mm]

magnetischer Feldvektor des einfallenden Lichtes

gestreute Lichtintensität eines Teilchens

Lichtintensität bei einer Extinktionsmessung

horizontal polarisierte Streulichtintensität bei vertikal polarisierten

einfallendem Licht

vertikal polarisierte Streulichtintensität bei vertikal polarisierten einfallendem

Licht 


\begin{tabular}{|c|c|}
\hline $\mathrm{I}_{\mathrm{sca}}$ & Intensität des Streulichts \\
\hline $\mathrm{I}_{0}$ & Intensität des einfallenden Lichtes \\
\hline $\operatorname{Im}$ & Imaginärteil \\
\hline $\mathrm{k}$ & Wellenzahl $2 \pi / \lambda\left[\mathrm{cm}^{-1}\right]$ \\
\hline $\mathrm{k}_{\mathrm{f}}$ & fraktaler Vorfaktor \\
\hline$k_{\text {abs }}$ & Absorptionskoeffizient $\left[\mathrm{cm}^{-1}\right]$ \\
\hline $\mathrm{k}_{\mathrm{ext}}$ & Extinktionskoeffizient $\left[\mathrm{cm}^{-1}\right]$ \\
\hline $\mathrm{L}$ & optische Weglänge \\
\hline $\mathrm{m}$ & Realteil des komplexen Brechungsindex \\
\hline$\widetilde{\mathrm{m}}$ & komplexer Brechungsindex \\
\hline $\mathrm{m}^{\prime}$ & Imaginärteil des komplexen Brechungsindex \\
\hline $\mathrm{n}$ & reeller Brechungsindex (nur Kap. 2.2) \\
\hline $\mathrm{n}$ & Anzahl der Primärteilchen pro Aggregat (Kap. 2.5 und 2.6) \\
\hline$\overline{\mathrm{n}^{1}}$ & 1. Moment (Mittelwert) der Größenverteilungsfunktion der Aggregate \\
\hline$\overline{\mathrm{n}^{2}}$ & 2. Moment (rel. Breite) der Größenverteilungsfunktion der Aggregate \\
\hline $\mathrm{N}$ & Teilchenzahldichte allgemein \\
\hline $\mathrm{N}_{\mathrm{R}}$ & Teilchenzahldichte nach Rayleigh $\left[\mathrm{cm}^{-3}\right]$ \\
\hline $\mathrm{N}_{\mathrm{R}, \infty}$ & Teilchenzahldichte $\mathrm{N}_{\mathrm{R}}$ am Ende des Verbrennungsprozesses $\left[\mathrm{cm}^{-3}\right]$ \\
\hline $\mathrm{N}_{\text {Agg }}$ & Aggregatenzahldichte nach der Aggregaten-Theorie $\left[\mathrm{cm}^{-3}\right]$ \\
\hline $\mathrm{N}_{\mathrm{Agg}, \infty}$ & Aggregatenzahldichte $\mathrm{N}_{\mathrm{Agg}}$ am Ende des Verbrennungsprozesses $\left[\mathrm{cm}^{-3}\right]$ \\
\hline $\mathrm{n}_{\mathrm{g}}$ & geometrischer Mittelwert der Primärpartikel pro Aggregat \\
\hline $\mathrm{n}_{\mathrm{p}}$ & Primärteilchenzahldichte $\left[\mathrm{cm}^{-3}\right]$ \\
\hline $\mathrm{n}^{\mathrm{q}}$ & q-tes Moment des n-ten Aggregats \\
\hline $\mathrm{p}$ & Dipolmoment \\
\hline $\operatorname{pdf}(\mathrm{N})$ & Aggregatgrößenverteilung (aggregate size distribution) \\
\hline $\mathrm{q}_{\mathrm{i}}$ & Modulus des Streuvektors $q_{i}=\frac{4 \pi}{\lambda} \sin \frac{\Theta}{2}$ \\
\hline$\left(\mathrm{q}_{\mathrm{i}}^{2} \overline{\mathrm{R}_{\mathrm{g}}^{2}}\right)$ & Formfaktor \\
\hline $\mathrm{Q}_{\text {sca }}$ & Streukoeffizient $\left[\mathrm{cm}^{2}\right.$ sterad $\left.^{-1}\right]$ \\
\hline $\mathrm{Q}_{\mathrm{VV}}$ & Streufaktor (Streulicht und einfallendes Licht vertikal polarisiert) $\left[\mathrm{cm}^{-1}\right.$ sterad $\left.^{-1}\right]$ \\
\hline $\mathrm{r}$ & Teilchenradius; Kugelradius \\
\hline $\mathrm{R}_{\mathrm{g}}$ & scheinbarer Radius, auch Gyrationsradius (ㄹadius of Gyration) [nm] \\
\hline $\mathrm{R}_{\mathrm{VV}}$ & Dissymmetrieverhältnis, auch z \\
\hline $\mathrm{T}$ & Flammentemperatur $[\mathrm{K}]$ \\
\hline
\end{tabular}


V

$\Delta \mathrm{V}$

$\mathrm{X}_{\mathrm{a}}$

$\mathrm{X}_{\mathrm{p}}$

Z

$\varepsilon_{0}$

$\varepsilon_{1,2}$

$\vartheta$

$\lambda$

$\rho$

$\rho_{\mathrm{V}}$

$\sigma$

$\sigma_{\mathrm{g}}$

$\varphi$

$\chi$

$\psi$

$\omega$

$\Delta \Omega$

Strömungsgeschwindigkeit $[\mathrm{cm} / \mathrm{s}]$

Streuvolumen; Volumen

optischer Größenparameter eines Aggregats mit $2 \pi \mathrm{R}_{\mathrm{g}} / \lambda$

optischer Größenparameter eines Primärteilchens mit $\pi \mathrm{d}_{\mathrm{p}} / \lambda=\mathrm{x}_{\mathrm{p}}$

Dissymmetrieverhältnis, auch $\mathrm{R}_{\mathrm{VV}}$

elektrische Feldkonstante

Permittivitätszahl (früher: Dielektrizitätszahl) des Objekts, der Umgebung

Beobachtungswinkel

Wellenlänge [nm]

Dichte

Depolarisationsverhältnis $\left(\mathrm{I}_{\mathrm{HV}} / \mathrm{I}_{\mathrm{VV}}\right)$ für vertikal polarisiertes einfallendes Licht Standardabweichung

Standardabweichung der Primärpartikelzahl pro Aggregat

Winkel zwischen der auf die xy-Ebene projizierten Streurichtung und dem Dipol

Azimutwinkel

Winkel zwischen Streurichtung und Dipol

Verhältnis des Stoßparameters geladener zu dem ungeladener Teilchen

Raumwinkel 
Technische Verbrennungsprozesse liefern einen großen Teil der insgesamt industriell erzeugten Primärenergie. Auch am Anfang des dritten Jahrtausends spielen regenerative Energiequellen mit 3\% Anteil eine untergeordnete Rolle [48]. Andere moderne Energieträger, wie die Kernenergie mit einem Primärenergieanteil von 13\%, stehen zwar mit einem breiten Anwendungsspektrum zur Verfügung, werden aber aufgrund öffentlichen Drucks nicht ausgeweitet, sondern vielmehr eingeschränkt. So wurden im Jahr 2001 in Deutschland 84\% des Primärenergiebedarfs über technische Verbrennungsprozesse fossiler Brennstoffe gedeckt. Als Brennstoffe werden hauptsächlich Erdöl (38\%), Erdgas (und andere fossil vorkommende Gase wie Grubengas und Klärgas, 22\%), Steinkohle (13\%) und Braunkohle (11\%) herangezogen [48]. Drei Sekundärenergiearten werden dabei hauptsächlich produziert: Mechanische Energie, z.B. Antrieb einer Kurbelwelle, elektrischen Energie, z.B. Betrieb eines Generators und Wärmeenergie, die z.B. zum Heizen benötigt wird. Durch die Verbrennung fossiler Brennstoffe können neben Ruß und Kohlendioxid Schadstoffe entstehen, wie Schwefeldioxid $\mathrm{SO}_{2}$, Stickoxide $\mathrm{NO}_{\mathrm{x}}$, Kohlenmonoxid $\mathrm{CO}$ und Kohlenwasserstoffe $\mathrm{CH}_{\mathrm{x}}$. Neben den Gefahren dieser Schadstoffe für Mensch und Umwelt an sich, kann durch ihr Auftreten die Energieeffizienz der Verbrennung verschlechtert werden, da bei der Entstehung von Kohlenmonoxid, Ruß und Kohlenwasserstoffen nicht die maximal mögliche Verbrennungswärme freigesetzt wird. Allerdings wird gerade der Ruß für die Wärmeabstrahlung in Feuerungsanlagen zumindest als Zwischenprodukt benötigt. Deshalb werden technische Verbrennungsprozesse oft unter brennstoffreichen Flammenbedingungen durchgeführt, in denen sich dann Rußteilchen bilden können. Größe und Beschaffenheit der Rußteilchen hängen dabei weitgehend von Flammenbedingungen, wie Brennstoffart, Gemischzusammensetzung, Flammentemperatur, Brennkammerdruck sowie der Aufenthaltszeit der Rußteilchen in der Flamme, ab ([1], [3], [4], [5], [6], [7], [11], [14],[15], [17], [27], [28], [29] und [32]). Sowohl ökonomische als auch ökologische Gesichtspunkte sind Motivation für eine Optimierung der Verbrennungsprozesse. Die Kenntnis der Rußbildungsmechanismen steht dabei neben der Erforschung von Strömungs- und Transportvorgängen im Mittelpunkt der Grundlagenforschung. Die Rußbildung wird wissenschaftlich mit Hilfe von Modellflammen, Strömungsreaktoren und Pyrolysen in Stoßrohren experimentell untersucht. In dieser Arbeit wird die Rußentstehung in Flammen behandelt. 
In der ersten Rußbildungsphase wachsen die entstehenden Rußpartikel zunächst zu kleinen pseudosphärischen Teilchen an [37]. Koagulation und Massenwachstum lassen größere Rußteilchen mit veränderter Morphologie entstehen. Je nach Flammenbedingung können aus zunächst kugelförmig entstandenen Rußteilchen größere Rußpartikel mit sehr differenzierter Struktur entstehen, diese werden dann als Rußaggregate bezeichnet. Die Rußaggregate können sowohl in kompakter Struktur wie auch als stark verzweigte Ketten vorliegen.

In den letzten Jahren sind eine große Zahl an experimentellen ([1], [3], [4], [6], [7], [11], [27], [29] und [32]) als auch an theoretische Arbeiten ([24], [25], [50] und [51]) über die Wachstumsprozesse der Rußteilchen in Kohlenwasserstoff-Flammen veröffentlicht worden.

Für die experimentellen Arbeiten über die Rußteilchen und ihre Eigenschaften wurden meistens die Lichtstreuung sowie die Extinktion als Untersuchungsmethoden eingesetzt. Die Ermittlung der Teilchenparameter wie Teilchendurchmesser und Teilchenzahldichte erfolgte im allgemeinen nach der Mie-Streutheorie (oft in ihrer Rayleigh-Näherung) sowie der Rayleigh-Streutheorie. Diese „klassischen“ Methoden machen die Annahme einer kugelförmigen Gestalt der Rußteilchen ([3], [4], [7], [13], [14], [27], [29] und [46]) und liefern volumenäquivalente Durchmesser.

Für sehr brennstoffreiche Flammen reichen die Rayleigh- und die Mie-Theorie hingegen nicht aus, da zum einen die Rußteilchen von der sphärischen Form abweichen und zu anderen weil sie als polydisperse Systeme mit hohen Teilchenzahldichten vorliegen, die zu einer Mehrfachstreuung führen. Eine Theorie die sowohl die von einer Kugel abweichende Gestalt einzelner Rußpartikel als auch deren Polydispersität berücksichtigt ist die sogenannte „Aggregatentheorie“, die auch genauer als RDG/FA-Theorie (Rayleigh-Debye-Gans/Fractal Aggregate Theorie)[33] bezeichnet wird. Die RDG/FA-Theorie beruht auf den Grundlagen der Streutheorie von Rayleigh, Debye und Gans [34]. Zusätzlich wird in der RDG/FA-Theorie die Bildung von Massenfraktalen [33], die ihrerseits aus stets gleichen Primärteilchen aufgebaut sind, angenommen. Diese Annahme eines fraktalen Wachstums eröffnete theoretische und experimentell bestätigte Lösungsmodelle für die Berechnung des optischen Streuquerschnitts sehr großer Rußteilchen, und ermöglichte damit, die Morphologie von Aggregaten aus Streulicht- und Extinktionsmessungen experimentell zu ermitteln. 
Die Anwendung der RDG/FA-Theorie auf Rußteilchen wurde in mehreren Arbeitsgruppen untersucht: Neben Charalampopoulos [12] (1992), Felske et.al. [23] (1986) und Massoli et.al. [18] (1995) haben zwei amerikanische Autorengruppen um Dobbins und Megaridis ([19] und [20]) sowie um Köylü und Faeth ([35] und [36]) die RDG/FA-Theorie auf Rußteilchen angewandt.

Die Diplomarbeit des Verfassers [47] beschäftigte sich mit der Aggregatenbildung in EthylenLuft-Flammen bei Normaldruck. Die Ausweitung der zu untersuchenden Flammen sowohl in Hinblick auf höhere Brennstoffe als auch unter erhöhten Druck andererseits ist Gegenstand dieser Arbeit.

Zielsetzung dieser Arbeit

Die Bestimmung der Morphologie der Rußteilchen aus winkelabhängigen Streulichtmessungen sowie Extinktionsmessungen mit Hilfe der RDG/FA-Theorie ist eine Zielsetzung dieser Arbeit.

Die in dieser Arbeit zu untersuchenden Brennstoffe wurden nach Gesichtspunkten der Vergleichbarkeit untereinander und mit Ethylen einerseits als auch unter dem Aspekt der industriellen Relevanz andererseits, ausgewählt. Im Einzelnen sind es Propen, ein höheres Alken, n-Pentan, n-Heptan und Isooktan, höhere Alkane, die in raffinierten Erdölfraktionen und damit in Kraftstoffen vorkommen und Benzol, ein Aromat, der ebenfalls in Kraftstoffen enthalten ist. Außerdem wurde für den Brennstoff Ethylen der untersuchte Druckbereich bis auf 5 bar erweitert. Für die jeweiligen Flammen wurden Durchsatz und Gemischzusammensetzung variiert. Einige Flammen wurden außerdem in mehreren Höhen über der Brenneroberfläche untersucht. Es sollen dabei die Ergebnisse der nach der RayleighTheorie ausgewerteten Meßergebnisse den RDG/FA-Resultaten gegenübergestellt werden.

Aus den Extinktionsmessungen wird der Rußvolumenbruch $f_{v}$ erhalten. Die RayleighAuswertung liefert Resultate für den Teilchenradius $d_{R}$ sowie der Teilchenzahldichte $\mathrm{N}_{\mathrm{R}}$. Die RDG/FA-Theorie macht deren unmittelbare Vergleichsgrößen den volumenäquivalenten Durchmesser $\mathrm{D}_{30}$ und die dazu korrespondierende Aggregatenzahldichte $\mathrm{N}_{\text {Agg }}$ zugänglich. Ferner läßt sich nach der Aggregatentheorie aus einer doppeltlogarithmischen Auftragung die 
fraktale Dimension $\mathrm{D}_{\mathrm{f}}$ ermitteln, die Aussagen über die Gestalt der Rußteilchen macht. Die fraktale Dimension macht ihrerseits den Primärteilchendurchmesser $d_{p}$ und die Primärteilchenzahldichte $n_{p}$ zugänglich. Über das Dissymmetrieverhältnis $z$ kann der scheinbare Radius $R_{g}$ berechnet werden. Der scheinbare Radius ist eine massenbezogene Größe, die aber anders als der Name vermuten läßt eher mit den anderen Teilchendurchmessern zu vergleichen ist.

Die Abhängigkeit aller aufgeführten Parameter in bezug auf Brennstoffart, Brennkammerdruck, Durchsatz, Gemischzusammensetzung und Höhe über dem Brenner ist Gegenstand der vorliegenden Arbeit. 
2.1 Grundlagen der Lichtstreuung

Die Theorie der Lichtstreuung und deren Messung ist in den Lehrbüchern von van de Hulst [49] und Kerker [34] ausführlich beschrieben.

Wenn eine ebene elektromagnetische Welle ein Dielektrikum (z.B. Rußteilchen) mit einer im Vergleich zum Dielektrikum (z.B. Teilchengröße) kleineren Wellenlänge $\lambda_{0}$ trifft und dabei nicht absorbiert wird, kommt es zu anderen Wechselwirkungen wie Brechung, Beugung und Reflexion. Ist die Wellenlänge $\lambda_{0}$ der eingestrahlten Welle hingegen gleich groß wie das Dielektrikum oder größer, kann zwischen Brechung, Beugung und Reflektion nicht mehr eindeutig unterschieden werden. Diese undifferenzierte Wechselwirkung wird als Streuung bezeichnet. Falls die elektromagnetische Welle eine Lichtwelle ist spricht man dann auch von Lichtstreuung. Es werden zwei Arten der Streuung unterschieden: Ist die Wellenlänge des gestreuten Lichts $\lambda_{\mathrm{SCA}}$ gleich der Wellenlänge des eingestrahlten Lichts $\left(\lambda_{\mathrm{SCA}}=\lambda_{0}\right)$ handelt es sich um elastische (z.B. Rayleigh-Streuung) Streuung, ändert sich die Wellenlänge des gestreuten Lichts im Vergleich zur Wellenlänge des eingestrahlten Lichts $\left(\lambda_{\mathrm{SCA}} \neq \lambda_{0}\right)$ liegt inelastische Streuung vor (z.B. Raman Effekt). Außerdem wird zwischen kohärenter und inkohärenter Streuung unterschieden, d.h. Streuung unter Phasenbeibehaltung oder Verschiebung der Phase des eingestrahlten Lichts. Die Meßergebnisse dieser Arbeit resultieren ausschließlich aus elastischer kohärenter Streuung bei einer Wellenlänge $\lambda=488$ nm.

\subsection{Rayleigh-Streutheorie}

Von Mie [42] wurde 1908 eine Streutheorie formuliert, die exakt für die Einfachstreuung an isotropen sphärischen Teilchen gültig ist. Rayleigh vereinfachte diese Theorie (RayleighTheorie) mit der zusätzlichen Einschränkung die Wellenlänge des eingestrahlten Lichts sei deutlich größer als die als Streuzentren dienenden Teilchen $(\lambda \geq 10 \mathrm{~d})$. Zweckmäßig ist die Einführung des Größenparameter $\alpha$, der das Verhältnis zwischen Teilchenradius und Wellenlänge angibt: 


$$
\alpha=\frac{\pi \cdot \mathrm{d}}{\lambda}, \text { mit } \alpha \leq 0.3
$$

Im folgenden soll die Lichtstreuung an einer dielektrischen Kugel erläutert werden. Ein Rußteilchen, welches als eine dielektrische Kugel mit dem Radius $\mathrm{r}$ zu betrachten ist, befindet sich in einem homogenen elektrischen Feld und wird durch dieses polarisiert. Der so induzierte Dipolmoment ist von 4 Parametern abhängig: 1. Dem Kugelradius, 2. der elektrischen Feldstärke des äußeren Feldes 3. der Permittivitätszahl des Mediums $\varepsilon_{1}$ und 4. der Permittivitätszahl der Umgebung $\varepsilon_{2}$. Periodische Schwingungen des äußeren Feldes, ziehen entsprechende Schwingungen des Dipols in Form von nach außen in alle Raumrichtungen tretender elektromagnetischer Sekundärstrahlung, der Streustrahlung, nach sich.

Die Intensität des gestreuten Lichts (I) im Beobachtungsabstand a läßt sich für aus z-Richtung senkrecht polarisiert einfallendes Licht $\left(\mathrm{I}_{0}\right)$ berechnen:

$$
\mathrm{I}=\mathrm{I}_{0} \cdot \frac{16 \pi^{4} \cdot \mathrm{r}^{6}}{\mathrm{a}^{2} \cdot \lambda^{4}} \cdot\left(\frac{\varepsilon_{1}-\varepsilon_{2}}{\varepsilon_{1}+2 \varepsilon_{2}}\right)^{2} \cdot \sin ^{2} \psi
$$

Mit $\Psi$ als dem von Streurichtung und Dipolachse eingeschlossenen Winkel.

Zur Vereinfachung soll in dieser Arbeit nur das senkrecht zur Schwingungsebene der Lichtquelle gestreute Licht betrachtet werden. In diesem Fall ist $\Psi=90^{\circ}$ und folglich $\sin ^{2} \Psi=1$. Außerdem werden die Permittivitätszahlen in einer Näherung $\varepsilon_{1} / \varepsilon_{2}=\varepsilon=\mathrm{n}^{2}$ durch den Brechungsindex n ersetzt. Damit erhält man:

$$
I=I_{0} \cdot \frac{16 \pi^{4} \cdot r^{6}}{a^{2} \cdot \lambda^{4}} \cdot\left(\frac{n^{2}-1}{n^{2}+2}\right)^{2}
$$

In Abb. 2.1 ist die so vereinfachte zugrundeliegende Geometrie der Lichtstreuung am Beispiel der Kugel dargestellt: 


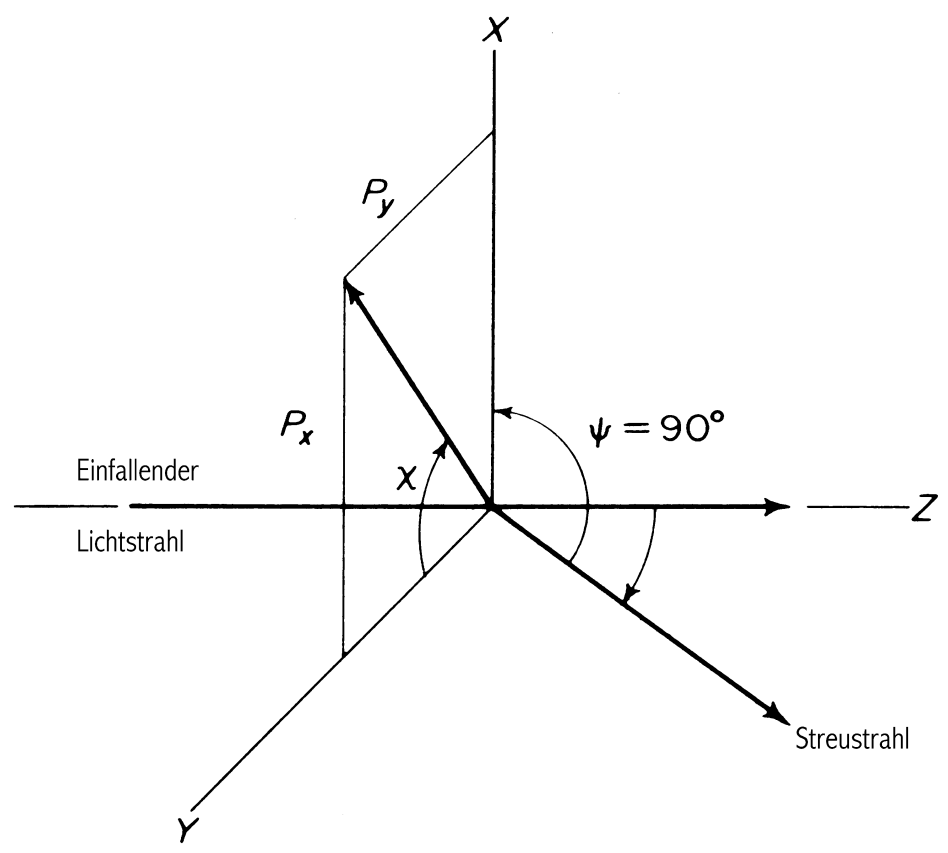

Abb. 2.1: Sphärisches Streuzentrum mit Lichtstreuung in der yz-Ebene und einem induzierten Dipolmoment in der xy-Ebene

Die Achse des Dipols schließt zusammen mit $\mathrm{y}$-Achse einen Azimutwinkel $\chi$ ein und hat deshalb zusätzlich zur y- eine x-Komponente. Für die vertikale polarisiert gestreutes Licht gilt dann:

$$
I_{V}(\chi)=I_{0} \cdot \frac{16 \pi^{4} \cdot r^{6}}{a^{2} \cdot \lambda^{4}} \cdot\left(\frac{n^{2}-1}{n^{2}+2}\right)^{2} \cdot \sin ^{2} \chi
$$

Durch einen Polarisationsfilter (s. Kap. 3) wird nur der senkrecht zur Streuebenen polarisierte Anteil des Streustrahls detektiert. Damit beträgt $\chi=90^{\circ}$ oder $\pi / 2$. Unter Beachtung der Konvention, daß der erste Index für die Polarisationsebene der gestreuten Welle und zweite Index für die Polarisationsebene der einfallenden Welle steht, erhält man:

$$
\mathrm{I}_{\mathrm{V}}\left(\chi=90^{\circ}\right)=\mathrm{I}_{\mathrm{VV}}, \quad \mathrm{I}_{\mathrm{H}}\left(\chi=90^{\circ}\right)=\mathrm{I}_{\mathrm{HV}}
$$

Daraus folgt, daß die Horizontalkomponente des Streulichts für senkrecht polarisiertes einfallendes Licht gleich null ist $\left(\mathrm{I}_{\mathrm{HV}}=0\right)$. Damit vereinfacht sich die Gleichung (2.4) zu:

$$
I_{\mathrm{VV}}=\mathrm{I}_{0} \cdot \frac{16 \pi^{4} \cdot \mathrm{r}^{6}}{\mathrm{a}^{2} \cdot \lambda^{4}} \cdot\left(\frac{\mathrm{n}^{2}-1}{\mathrm{n}^{2}+2}\right)^{2}
$$

Ein einzelnes Teilchen hat einen Streuquerschnitt (integrierte Form) von: 


$$
\mathrm{C}_{\text {sca }}=\int \frac{\mathrm{I}}{\mathrm{I}_{0}} \cdot \mathrm{a}^{2} \cdot \mathrm{d} \Omega, \quad \operatorname{mit} \mathrm{d} \Omega=\sin \Psi \cdot \mathrm{d} \Psi \cdot \mathrm{d} \varphi
$$

$\Omega$ ist der Öffnungswinkel oder Raumwinkel aus dem das Streusignal detektiert werden kann. Um den Raumwinkel aus der Gleichung zu eliminieren wird der differentielle Streuquerschnitt eingeführt:

$$
\mathrm{C} \equiv \frac{\mathrm{dC}_{\mathrm{sca}}}{\mathrm{d} \Omega}=\frac{\mathrm{I}}{\mathrm{I}_{0}} \cdot \mathrm{a}^{2}
$$

Gleichung (2.3) in Gl. (2.8) eingesetzt ergibt:

$$
\mathrm{C}_{\mathrm{vV}}=\frac{16 \pi^{4} \mathrm{r}^{6}}{\lambda^{4}} \cdot\left(\frac{\mathrm{n}^{2}-1}{\mathrm{n}^{2}+2}\right)^{2}\left[\mathrm{~cm}^{2} \cdot \mathrm{sterad}^{-1}\right]
$$

Bisher wurde von einer absorptionsfreien Streuung ausgegangen. Dies ist bei Ruß aber offensichtlich nicht der Fall. Deshalb muß der (relative) Brechungsindex $n$ durch einen komplexen Brechungsindex $\tilde{\mathrm{m}}$ bzw. m ersetzt werden, für den gilt:

$$
\tilde{\mathrm{m}}=\mathrm{m}-i \cdot \mathrm{m}^{\prime} \text {, oft auch } \mathrm{m}=\mathrm{n}-i \cdot \mathrm{k}
$$

Das $m$ rechts vom Gleichheitszeichen gibt wie auch $\mathrm{n}$ den Realteil des komplexen Brechungsindex an. Der Imaginärteil im' bzw. ik berücksichtigt den absorbierten Anteil des eingestrahlten Lichts. Der Streuquerschnitt eines Teilchens wird dann zu:

$$
\mathrm{C}_{\mathrm{VV}}=\frac{16 \pi^{4} \mathrm{r}^{6}}{\lambda^{4}} \cdot\left|\frac{\tilde{\mathrm{m}}^{2}-1}{\tilde{\mathrm{m}}^{2}+2}\right|^{2}\left[\mathrm{~cm}^{2} \cdot \mathrm{sterad}^{-1}\right]
$$

Multipliziert man den Streuquerschnitt eines einzelnen Teilchens $\mathrm{C}_{\mathrm{VV}}$ mit der Teilchenzahldichte N, so erhält man den Streufaktor $Q_{\mathrm{Vv}}$ :

$$
\mathrm{Q}_{\mathrm{VV}}=\mathrm{N} \cdot \mathrm{C}_{\mathrm{vV}}=\left(\frac{4 \pi}{\lambda}\right)^{4} \cdot\left|\frac{\mathrm{m}^{2}-1}{\mathrm{~m}^{2}+2}\right|^{2} \cdot \mathrm{N} \cdot \mathrm{r}^{6} \quad\left[\mathrm{~cm}^{-1} \cdot \mathrm{sterad}^{-1}\right]
$$

2.3 Die Streutheorie von Mie in der Rayleigh-Näherung

Für Kugeln mit einem Radius bis zu $r \leq 0.05 \lambda$ ist die Rayleigh-Streutheorie uneingeschränkt gültig. Das Streuverhalten größerer Teilchen weicht mehr und mehr von den nach der Rayleigh-Theorie zu erwartenden Ergebnissen ab [34]. Die Elektronen der sphärischen Teilchen mit einem Durchmesser in der Größenordnung der Wellenlänge des eingestrahlten Lichts interferieren mit den Photonen der Lichtquelle, dies führt zu einer winkelabhängigen teilweisen Auslöschung der Streuintensität. Die tatsächliche Streuintensität größerer Teilchen 
wird damit in Abhängigkeit des Streuwinkels gegenüber der Rayleigh-Streuung kleiner. Außerdem sind Extinktion und Absorption sowohl von kleinen als auch von größeren Teilchen in der ursprünglichen Rayleigh-Theorie nicht berücksichtigt.

Das Lambert'sche Gesetz gibt die beim Durchtreten von Licht durch ein homogenes Objekt einhergehende Extinktion an:

$$
\begin{aligned}
& \mathrm{dI}=-\mathrm{I} \cdot \mathrm{k}_{\text {ext }} \cdot \mathrm{dL} \text { durch Integration erhält man : } \\
& \frac{\mathrm{I}}{\mathrm{I}_{0}}=\mathrm{e}^{-\mathrm{k}_{\text {ext }} \cdot \mathrm{L}}
\end{aligned}
$$

Mit L als optische Weglänge, $\mathrm{I}_{0}$ als Intensität des eingestrahlten Lichts und I als Intensität des transmittierten Lichts. Der Extinktionskoeffizient $k_{\text {ext }}$ ist die Summe der Koeffizienten der Absorption $\mathrm{k}_{\mathrm{abs}}$ und der Streuung $\mathrm{k}_{\mathrm{sca}}$ :

$$
\mathrm{k}_{\mathrm{ext}}=\mathrm{k}_{\mathrm{abs}}+\mathrm{k}_{\mathrm{sca}}
$$

Für eine Kugel, die sich im Ursprung eines sphärischen Koordinatensystems befindet, können die Maxwell'schen Gleichungen unter der Annahme, daß die Bessel- und Hankelfunktionen einzelner Feldkomponenten in einem ausreichend großem Abstand vom Koordinatenursprung verschwinden, gelöst werden. Unter Berücksichtigung der elektrischen Dipol- und Quadrupolmomente sowie des magnetischen Dipolmoments kann eine Reihenentwicklung im Falle hinreichend kleiner Teilchen nach dem ersten Glied abgebrochen werden, da die $(r / \lambda)^{2}-$ Terme für kleine Teilchenradien gegen Null konvergieren (Mie-Theorie in der RayleighNäherung)[34].

Für den Absorptionskoeffizienten erhält man dann:

$$
\mathrm{k}_{\mathrm{abs}}=-\frac{8 \pi^{2}}{\lambda} \operatorname{Im}\left(\frac{\widetilde{\mathrm{m}}^{2}-1}{\widetilde{\mathrm{m}}^{2}+2}\right) \cdot \mathrm{Nr}^{3}
$$

Für den Streukoeffizienten ergibt sich:

$$
\mathrm{k}_{\text {sca }}=\frac{128 \pi^{5}}{3 \lambda^{4}}\left|\frac{\widetilde{\mathrm{m}}^{2}-1}{\widetilde{\mathrm{m}}^{2}+2}\right|^{2} \cdot \mathrm{Nr}^{6}
$$

Für kleine Durchmesser ist außerdem der Absorptionskoeffizient in etwa gleich dem Extinktionskoeffizienten, daher folgt aus Gleichung (2.13) und (2.15):

$$
-\frac{1}{\mathrm{~L}} \cdot \ln \frac{\mathrm{I}}{\mathrm{I}_{0}}=\mathrm{k}_{\mathrm{ext}} \approx \mathrm{k}_{\mathrm{abs}} \sim \mathrm{Nr}^{3}
$$


Als Streuintensität erhält man aus Gl.(2.16) einen in bezug auf N und $r$ zur Gleichung (2.12) analogen Zusammenhang:

$$
\mathrm{I}_{\mathrm{VV}} \sim \mathrm{N} \cdot \mathrm{C}_{\mathrm{VV}} \sim \mathrm{Nr}^{6}
$$

\subsection{Anwendung auf Streulichtmessungen in rußenden Flammen}

\subsubsection{Kalibrierung des optischen Systems}

Streulichtmessungen können sowohl mit einer Absolutmethode als auch einer Relativmethode durchgeführt werden. Die Absolutmethode hat den Nachteil, daß an das Material (Linsen, Filter und SEV) und die optische Justierung höchste Anforderungen gestellt werden. Relative Streulichtmessungen, das heißt Messungen im Vergleich zu einer Eichmessung, sind mit vergleichsweise geringen Anforderungen an den optischen Aufbau zu realisieren. Für die Eichmessungen dieser Arbeit wurde Luft bei Zimmertemperatur und Normaldruck als Eichgas eingesetzt. Der Streuquerschnitt von Gasen wird wie folgt berechnet:

$$
\mathrm{C}_{\mathrm{vV}}=\frac{4 \pi^{2}}{\lambda^{4} \cdot \mathrm{N}_{0}^{2}} \cdot\left(\mathrm{n}_{0}-1\right)^{2} \cdot \frac{3}{3-4 \rho_{\mathrm{V}}}
$$

Mit $\mathrm{N}_{0}$ als Teilchenzahldichte des Eichgases, $\mathrm{n}_{0}$ als seinen Brechungsindex und $\rho_{\mathrm{V}}$ als das Depolarisationsverhältnis $\left(\rho_{\mathrm{V}}=\mathrm{I}_{\mathrm{HV}} / \mathrm{I}_{\mathrm{VV}}\right)$ für vertikal polarisiertes einfallendes Licht.

Der Streuquerschnitt von Ruß wird über das Verhältnis der Streuintensitäten von Ruß und Luft (Eichgas) erhalten:

$$
\frac{\mathrm{I}_{\mathrm{VV}}(\mathrm{Ru} \beta)}{\mathrm{I}_{\mathrm{VV}}(\mathrm{Luft})}=\frac{\mathrm{N} \cdot \mathrm{C}_{\mathrm{VV}}(\mathrm{Ru} \beta)}{\mathrm{N}_{0} \cdot \mathrm{C}_{\mathrm{VV}}(\mathrm{Luft})}=\frac{\mathrm{Q}_{\mathrm{VV}}(\mathrm{Ru} \beta)}{\mathrm{Q}_{\mathrm{VV}}(\mathrm{Luft})}
$$

Mit dem idealen Gasgesetz kann $\mathrm{N}_{0}$ berechnet werden. $\mathrm{C}_{\mathrm{Vv}}$ (Luft) wird über die Gleichung (2.19 erhalten. Die Depolarisationsverhältnisse von Stickstoff und Sauerstoff wurden von Bridge und Buckingham [9] übernommen.

Da der Laserstrahl beim Durchtreten der Flamme, aber auch der Streustrahl vom Streuzentrum aus zum SEV innerhalb dieser Flamme eine Abschwächung erfahren, muß die Extinktion zur Korrektur der Streufaktoren mit berücksichtigt werden. Bei einem runden Brenner und einer entsprechenden Justierung auf sein Zentrum entspricht die Länge dieser 
Abschwächung genau der optischen Weglänge L. Für einen rechteckigen Brenner muß die Geometrie entsprechend berücksichtigt werden. Man erhält für den Streufaktor $\mathrm{Q}_{\mathrm{Vv}}$ dann:

$$
Q_{V V}(\operatorname{Ru} \beta)=N \cdot C_{V V}(R u B)=\left|\frac{\frac{I_{V V}(\text { Ru } \beta)}{I_{V V}(\text { Luft })}}{1-\frac{\Delta I_{\text {ext }}}{I_{0}}}\right| \cdot N_{0} \cdot C_{V V}(\text { Luft })
$$

Eine weitere Korrektur ist nur bei sehr schwach rußenden Flammen erforderlich und berücksichtigt die gasförmigen Verbrennungsprodukte durch Messung der Streuintensität in der Hauptoxidationszone. Die Hauptoxidationszone ist durch ein Minimum der höhenabhängigen Streuintensität charakterisiert. Von der durch die Extinktion korrigierten Streuintensität müßte also noch der Quotient $\mathrm{I}_{\mathrm{Vv}}(\mathrm{Ox}) / \mathrm{I}_{\mathrm{Vv}}(\mathrm{Luft})$ subtrahiert werden. Gegenstand dieser Arbeit ist aber gerade die Untersuchung stark rußender Flammen, deshalb wurde auf diese Korrektur verzichtet.

Die Extinktionsintensität einer rußenden Flamme wird aus der Differenz der Extinktionssignale von rußender und nichtrußender Flamme erhalten. Der Extinktionskoeffizient wird über das umgeformte Lambert'schen Gesetz, Gl. (2.17), aus den Extinktionsmessungen gewonnen.

Der komplexe Brechungsindex des Rußes von $\widetilde{\mathrm{m}}=1.57-0.56 \mathrm{i}$ wurde einer Veröffentlichung von Dalzell und Sarofim [16] entnommen.

\subsubsection{Rußvolumenbruch}

Der Rußvolumenbruch $f_{v}$ gibt das Verhältnis des Volumenanteils des Rußes und dem gesamten Flammenvolumen an. Der Rußvolumenbruch ergibt sich aus einer geometrischen Kugel $\frac{4 \pi}{3} \cdot r^{3}$ multipliziert mit der Teilchenzahldichte $N$ unter der Annahme eines mittleren Radius. Dann entspricht die geometrische Kugel einer Teilchenkugel mit einem mittleren Teilchenvolumen $\overline{\mathrm{V}_{\mathrm{K}}}$.

$$
\mathrm{f}_{\mathrm{v}}=\frac{4 \pi}{3} \cdot \mathrm{N} \cdot \mathrm{rr}^{-3}=\overline{\mathrm{V}_{\mathrm{K}}} \cdot \mathrm{N}
$$

Diese Gleichung impliziert damit ebenfalls die sphärische Gestalt der Rußteilchen. Der Rußvolumenbruch kann mit Kenntnis des komplexen Brechungsindex der Rußteilchen aus dem Extinktionskoeffizienten berechnet werden. Dazu wird die Gleichung (2.22) nach $\mathrm{Nr}^{3}$ aufgelöst und in Gleichung (2.15)) unter der Annahme von Gleichung (2.17) eingesetzt: 


$$
\mathrm{f}_{\mathrm{v}}=-\frac{\lambda}{6 \pi} \cdot \frac{1}{\mathrm{E}(\widetilde{\mathrm{m}})} \cdot \mathrm{k}_{\text {ext }}, \quad \text { mit } \mathrm{E}(\widetilde{\mathrm{m}})=\operatorname{Im}\left(\frac{\widetilde{\mathrm{m}}^{2}-1}{\widetilde{\mathrm{m}}^{2}+2}\right)
$$

Für die konkrete Berechnung muß zusätzlich die Wellenlänge bekannt sein. Die Extinktionsmessungen für diese Arbeit wurden ausnahmslos bei einer Wellenlänge von $\lambda=$ $488 \mathrm{~nm}$ durchgeführt. Damit reduziert sich die Gleichung (2.23) zu:

$$
\mathrm{f}_{\mathrm{v}} \approx \mathrm{k}_{\mathrm{ext}} \cdot 9.9766 \cdot 10^{-6} \sim \mathrm{k}_{\mathrm{ext}} \cdot 10^{-5}, \text { für } \lambda=488 \mathrm{~nm}
$$

\subsubsection{Teilchendurchmesser und Teilchenzahldichte}

Aus Streufaktor und Extinktionskoeffizient lassen sich der Teilchendurchmesser und die Teilchenzahldichte berechnen (Gln. (2.25)-(2.28)). Für den Teilchendurchmesser wird der Quotient von Streufaktor und Extinktionskoeffizient gebildet:

$$
\frac{\mathrm{Q}_{\mathrm{VV}}}{\mathrm{k}_{\mathrm{ext}}}=\frac{2 \pi^{2} \cdot \mathrm{F}(\tilde{\mathrm{m}}) \cdot \mathrm{r}^{3}}{\lambda^{3} \cdot \mathrm{E}(\tilde{\mathrm{m}})}=\frac{2 \pi^{2} \cdot \mathrm{F}(\tilde{\mathrm{m}}) \cdot\left(\frac{\mathrm{d}_{\mathrm{R}}}{2}\right)^{3}}{\lambda^{3} \cdot \mathrm{E}(\tilde{\mathrm{m}})}
$$

Den Teilchendurchmesser erhält man dann durch Auflösung der Gleichung (2.25) nach dem Teilchendurchmesser:

$$
\mathrm{d}_{\mathrm{R}}=2 \mathrm{r}=2 \sqrt[3]{\frac{\mathrm{Q}_{\mathrm{VV}}}{\mathrm{k}_{\mathrm{ext}}} \cdot \frac{\mathrm{E}(\tilde{\mathrm{m}})}{\mathrm{F}(\tilde{\mathrm{m}})} \cdot \frac{\lambda^{3}}{2 \pi^{2}}}
$$

Der Quotient des Quadrates des Extinktionskoeffizienten und des Streufaktors ermöglicht die Ermittlung der Teilchenzahldichte:

$$
\frac{\mathrm{k}_{\mathrm{ext}}^{2}}{\mathrm{Q}_{\mathrm{VV}}}=\frac{4 \lambda^{2} \cdot \mathrm{E}^{2}(\tilde{\mathrm{m}})}{\mathrm{F}(\tilde{\mathrm{m}})} \cdot \mathrm{N} ; \quad \text { mit } \mathrm{F}(\tilde{\mathrm{m}})=\left|\frac{\tilde{\mathrm{m}}^{2}-1}{\tilde{\mathrm{m}}^{2}+2}\right|^{2}
$$

Die Teilchenzahldichte wird durch Auflösung von Gl. (2.27) erhalten:

$$
\mathrm{N}_{\mathrm{R}}=\mathrm{N}=\frac{\mathrm{k}_{\mathrm{ext}}^{2}}{\mathrm{Q}_{\mathrm{VV}}} \cdot \frac{\mathrm{F}(\tilde{\mathrm{m}})}{\mathrm{E}^{2}(\tilde{\mathrm{m}})} \cdot \frac{1}{4 \lambda^{2}}\left[\mathrm{~cm}^{-3}\right]
$$

Der Teilchendurchmesser, die Teilchenzahldichte und der Rußvolumenbruch setzen mehrere Annahmen voraus:

1. Die Rußteilchen sind klein genug, damit die Rayleigh-Theorie gültig ist, bzw. die Reihenentwicklung der Mie-Streufunktion nach dem ersten Glied abgebrochen 
werden kann. Bei einer Wellenlänge von $\lambda=488 \mathrm{~nm}$ ist die Teilchengröße dann auf $\mathrm{d}_{\mathrm{R}} \sim 50 \mathrm{~nm}$ beschränkt.

2. Die Teilchen werden sowohl nach Rayleigh als auch nach Mie als kugelförmig angenommen. Die Bildung von langen Ketten und Aggregaten wird nicht berücksichtigt.

3. Alle Rußteilchen sind einheitlich gleich groß, d.h. monodispers verteilt. In Wirklichkeit liegen aber Größenverteilungen, die einer logarithmischen Normalverteilung entsprechen, vor.

4. Der verwendete komplexe Brechungsindex nach Dalzell und Sarofim gilt bei der eingestellten Wellenlänge von $\lambda=488 \mathrm{~nm}$ universell für alle Flammenregionen und alle untersuchten Flammen.

5. Eine Mehrfachstreuung wird ausgeschlossen.

Die ersten drei Einschränkungen sollen in folgendem Abschnitt relativiert werden.

\subsection{Streutheorie für polydisperse Aggregate RDG/FA-Theorie}

Da Rußteilchen nicht als kugelförmige und monodisperse Teilchen vorliegen, wurden in den letzten Jahren Untersuchungen über die Aggregatenbildung von Rußteilchen durchgeführt ([12], [18], [19], [20], [23], [35] und [36]), die sich unter anderem mit der Morphologie der Rußteilchen beschäftigen. In diesen Arbeiten wurde die Bildung der fraktalen polydispersen Aggregate theoretisch behandelt.

Besonders in brennstoffreichen Flammen können die Rußteilchen als Aggregate, d.h. als polydisperses System von unterschiedlich gestalteten Rußteilchen mit unterschiedlicher Masse, vorliegen. Die Teilchenzahldichte ist oft sehr hoch und Mehrfachstreuungen treten auf. Als polydispers bezeichnet man eine Verteilung an Aggregaten dann, wenn die einzelnen Aggregate aus unterschiedlichen Anzahlen sog. Primärteilchen bestehen. Bei monodispersen Aggregaten ist die Anzahl der Primärteilchen hingegen in jedem einzelnen Aggregat stets

gleich, es gilt $\mathrm{n}_{0}=\mathrm{n}_{\mathrm{g}}=\mathrm{n}_{\infty}$. Dabei ist $\mathrm{n}_{\mathrm{g}}$ der geometrische Mittelwert der Primärteilchenzahl pro Aggregat.

Im folgenden soll die Aggregaten-Theorie und ihre Anwendung auf rußende Flammen nach Dobbins und Megaridis ([19], [20] und [41]) sowie Köylü und Faeth ([36] und [35]) kurz eingeführt werden. 
Die Grundlage der Aggregaten-Theorie ist die Streutheorie von Rayleigh, Debye und Gans [34] mit der zusätzlichen Annahme, daß die Aggregate als Massenfraktale vorliegen. Dabei können sich eine beliebige Anzahl an Primärteilchen zu Aggregaten mit unterschiedlicher Größe und Gestalt zusammenfügen. Die Primärteilchen selbst sind kleine kugelförmige Teilchen mit einem einheitlichen Durchmesser und Brechungsindex, die sich im Aggregat berühren. Für den optischen Größenparameter der Primärteilchen gilt:

$$
\mathrm{x}_{\mathrm{p}} \cdot|\widetilde{\mathrm{m}}-1|<<1, \text { mit } \mathrm{x}_{\mathrm{p}}=\pi \mathrm{d}_{\mathrm{p}} / \lambda
$$

Die Anzahl an Primärteilchen pro Aggregat $n$ läßt sich aus der Annahme, daß Aggregate Massenfraktale mit eine scheinbaren Radius $R_{g}$ (auch Gyrationsradius genannt) sind [33], berechnen:

$$
\mathrm{n}=\mathrm{k}_{\mathrm{f}}\left(\frac{\mathrm{R}_{\mathrm{g}}}{\mathrm{d}_{\mathrm{p}}}\right)^{\mathrm{D}_{\mathrm{f}}}
$$

Dabei ist der scheinbare Radius $\mathrm{R}_{\mathrm{g}}$ für ein Aggregat definiert nach:

$$
\mathrm{R}_{\mathrm{g}}^{2}=\frac{1}{\mathrm{n}} \cdot \sum_{\mathrm{i}=1}^{\mathrm{n}} \mathrm{r}_{\mathrm{i}}^{2}
$$

Mit $n$ als Anzahl der Primärteilchen pro Aggregat und $r_{i}$ als Abstand des i-ten Primärteilchens vom Massenschwerpunkt des Aggregats. Der fraktale Vorfaktor (präexponentieller Faktor) $\mathrm{k}_{\mathrm{f}}$ scheint nach vorangegangenen Experimenten von Rußteilchen in Flammen ([20], [35]), unter anderem mit TEM (Transmissions-Elektronenmikoskopie), einen verhältnismäßig einheitlichen Wert von $\mathrm{k}_{\mathrm{f}}=8.5$ anzunehmen, was auf eine universelle Eigenschaft der Rußaggregate hindeutet. Die Primärteilchen sollen hinreichend klein sein, damit sie in den Bereich der Rayleigh-Streuung fallen, für den optischen Größenparameter $x_{p}$ muß dann $x_{p} \leq 0.3$ gelten.

Der differentielle Streufaktor $\mathrm{Q}_{\mathrm{VV}}^{\mathrm{A}}(\Theta)$ für vertikal polarisiertes Streulicht bei vertikal polarisiert einfallenden Licht ist das Produkt aus Aggregatenzahldichte $\mathrm{N}_{\mathrm{Agg}}\left[\mathrm{cm}^{-3}\right]$ und dem differentiellen Streuquerschnitt $\mathrm{C}_{\mathrm{vV}}^{\mathrm{A}}(\Theta)$ für ein Aggregat:

$$
\mathrm{Q}_{\mathrm{VV}}^{\mathrm{A}}(\Theta)=\mathrm{N}_{\mathrm{Agg}} \cdot \mathrm{C}_{\mathrm{VV}}^{\mathrm{A}}(\Theta)\left[\mathrm{cm}^{-1} \cdot \mathrm{sterad}^{-1}\right]
$$

Für polydisperse Aggregate erhält man nach Dobbins und Megaridis [20] einen allgemeinen Ausdruck für den differentiellen Streufaktor $\mathrm{Q}_{\mathrm{VV}}^{\mathrm{A}}(\Theta)$ von:

$$
\mathrm{Q}_{\mathrm{VV}}^{\mathrm{A}}(\Theta)=\frac{\overline{\mathrm{n}^{2}} \cdot \mathrm{N}_{\mathrm{Agg}} \cdot \mathrm{x}_{\mathrm{p}}^{6} \cdot \mathrm{F}(\tilde{\mathrm{m}})}{\mathrm{k}^{2}} \cdot f\left(\mathrm{q}_{\mathrm{i}}^{2} \cdot \overline{\mathrm{R}_{\mathrm{g}}^{2}}\right) ; \text { mit: } \overline{\mathrm{n}^{\mathrm{q}}}=\sum_{\mathrm{n}} \mathrm{n}^{\mathrm{q}} \cdot \mathrm{p}(\mathrm{n})
$$


Dabei ist $\mathrm{n}^{\mathrm{q}}$ das q-te Moment des $\mathrm{n}$-ten Aggregats, $\mathrm{p}(\mathrm{n})$ ist die Verteilungsfunktion der Wahrscheinlichkeiten für die relative Anzahl an Aggregaten mit n Primärteilchen und $\mathrm{k}$ ist eine Wellenzahl, für die $\mathrm{k}=2 \pi / \lambda$ gilt.

Die Strukturfunktion $f\left(\mathrm{q}_{\mathrm{i}}^{2} \cdot \overline{\mathrm{R}_{\mathrm{g}}^{2}}\right)$ hat zwei Gültigkeitsbereiche, die jeweils von Größe und Masse der Aggregate abhängen und vom Streuwinkel abhängig sind ([19], [20]). Der Modulus des Streuwinkels $\mathrm{q}_{\mathrm{i}}$ ist definiert als:

$$
\mathrm{q}_{\mathrm{i}}=\frac{4 \pi}{\lambda} \sin \frac{\Theta}{2}
$$

Der Absorptionskoeffizient nach der RDG/FA-Theorie beträgt:

$$
\mathrm{k}_{\mathrm{abs}}^{\mathrm{A}}=\frac{4 \pi \cdot \overline{\mathrm{n}^{1}} \cdot \mathrm{N}_{\mathrm{Agg}} \cdot \mathrm{x}_{\mathrm{p}}^{3} \cdot \mathrm{E}(\tilde{\mathrm{m}})}{\mathrm{k}^{2}} ; \text { mit: } \overline{\mathrm{n}^{1}} \cdot \mathrm{N}_{\mathrm{Agg}}=\mathrm{n}_{\mathrm{p}}\left[\mathrm{cm}^{-3}\right]
$$

$\overline{\mathrm{n}^{1}}$ ist das 1. Moment (Mittelwert) einer Größenverteilungsfunktion der Aggregate, $\mathrm{N}_{\text {Agg }}$ ist die Aggregatenzahldichte pro $\mathrm{cm}^{3}$ und $\mathrm{n}_{\mathrm{p}}$ ist die Primärteilchendichte pro $\mathrm{cm}^{3}$.

2.6 Untersuchung der Rußaggregate und deren Morphologie nach der RDG/FA-Theorie

Die Kombination der Gleichungen (2.33) und (2.35) ermöglicht die Berechnung der Rußaggregate, deren Morphologie sowie auch ihrer Primärteilchen. Es können anhand der Gleichungen (2.33), (2.35) und (2.37) Werte für den scheinbaren Radius $\mathrm{R}_{\mathrm{g}}$, den volumenäquivalenten Durchmesser $\mathrm{D}_{30}$ und der Aggregatenanzahldichte $\mathrm{N}_{\text {Agg }}$ erhalten werden. Für die Primärteilchen werden die Parameter, Primärteilchendurchmesser $d_{p}$ und Primärteilchenzahldichte $n_{p}$, bestimmt. Außerdem sollen Ausdrücke für den Rußvolumenbruch $\mathrm{f}_{\mathrm{v} . \mathrm{A}}$, der fraktalen Dimension $\mathrm{D}_{\mathrm{f}}$, des Dissymmetrieverhältnisses $\mathrm{z}$, des Momentenverhältnisses $\mathrm{f}_{\mathrm{n}}$ und der Strukturfunktion $f\left(\mathrm{q}_{\mathrm{i}}^{2} \cdot \overline{\mathrm{R}_{\mathrm{g}}^{2}}\right)$ ermittelt werden.

Die Berechnung des scheinbaren Radius $\mathrm{R}_{\mathrm{g}}$ erfolgt in dieser Arbeit über das Dissymmetrieverhältnis z, welches der Quotient zweier Streufaktoren für die Winkel von Vorwärts- und Rückwärtsstreuung in gleichem Abstand vom 90-Winkel ist.

$$
\mathrm{z}=\mathrm{R}_{\mathrm{VV}}=\frac{\mathrm{Q}_{\mathrm{VV}}\left(\Theta_{\mathrm{i}}\right)}{\mathrm{Q}_{\mathrm{VV}}\left(\Theta_{\mathrm{j}}\right)}=\frac{\mathrm{Q}_{\mathrm{VV}}\left(45^{\circ}\right)}{\mathrm{Q}_{\mathrm{VV}}\left(135^{\circ}\right)} ; \text { mit: } \Theta_{i}<\Theta_{j}
$$


Der scheinbare Radius kleiner Aggregate kann im sogenannten Guinier-Bereich, s. Gl. (2.45), aus dem Dissymmetrieverhältnis z berechnet werden:

$$
\mathrm{R}_{\mathrm{g}}^{2}=\frac{3 \ln z}{\mathrm{q}_{\mathrm{i}}^{2}-\mathrm{q}_{\mathrm{j}}^{2}}=\frac{3 \ln \left[\frac{\mathrm{Q}_{\mathrm{vv}}^{\mathrm{A}}\left(\Theta_{\mathrm{i}}\right)}{\mathrm{Q}_{\mathrm{vv}}^{\mathrm{A}}\left(\Theta_{\mathrm{j}}\right)}\right]}{\mathrm{q}_{\mathrm{i}}^{2}-\mathrm{q}_{\mathrm{j}}^{2}}=\frac{3 \ln \left[\frac{\mathrm{Q}_{\mathrm{vv}}^{\mathrm{A}}\left(\Theta_{\mathrm{i}}\right)}{\mathrm{Q}_{\mathrm{vv}}^{\mathrm{A}}\left(\Theta_{\mathrm{j}}\right)}\right]}{\left[\frac{4 \pi}{\lambda} \sin \left(\frac{\Theta_{\mathrm{i}}}{2}\right)\right]^{2}-\left[\frac{4 \pi}{\lambda} \sin \left(\frac{\Theta_{\mathrm{j}}}{2}\right)\right]^{2}}
$$

Für größere Aggregate, die als Massenfraktale beschrieben werden können und in den Power Law Bereich, s. Gl. (2.45), fallen, muß zur empirischen Berechnung des scheinbaren Radius zusätzlich deren fraktale Dimension bekannt sein.

$$
\mathrm{n}=\mathrm{k}_{\mathrm{f}}\left(\frac{\mathrm{R}_{\mathrm{g}}}{\mathrm{d}_{\mathrm{p}}}\right)^{\mathrm{D}_{\mathrm{f}}}
$$

Mit dem Quadrat des scheinbaren Radius wird die Summe der quadratischen Abstände von den Primärteilchen in einem Aggregat vom Massenschwerpunkt dieses Aggregats dividiert durch die Primärteilchenzahl bezeichnet, es handelt sich also um das mittlere Abstandsquadrat der Primärteilchen in einem Aggregat: (Wiederholung der Gl. (2.31))

$$
\mathrm{R}_{\mathrm{g}}^{2}=\frac{1}{\mathrm{n}} \sum_{\mathrm{i}=1}^{\mathrm{n}} \mathrm{r}_{\mathrm{i}}^{2}
$$

Der volumenäquivalente Teilchendurchmesser $D_{30}$ bezieht sich im Gegensatz zum scheinbaren Radius auf das Volumen der Aggregate. $\mathrm{D}_{30}$ und wird über den Quotienten des Streufaktors $\mathrm{Q}_{\mathrm{VV}}^{\mathrm{A}}(\Theta)$, Gl. (2.33), und des Absorptionskoeffizienten $\mathrm{k}_{\mathrm{abs}}^{\mathrm{A}}$, Gl. (2.35), ermittelt:

$$
\frac{\mathrm{Q}_{\mathrm{VV}}^{\mathrm{A}}(\Theta)}{\mathrm{k}_{\mathrm{abs}}^{\mathrm{A}}}=\frac{\overline{\mathrm{n}^{2}} \cdot \pi^{3} \cdot \mathrm{d}_{\mathrm{p}}^{3} \cdot \mathrm{F}(\widetilde{\mathrm{m}})}{\overline{\mathrm{n}^{1}} \cdot 4 \pi \cdot \lambda^{3} \cdot \mathrm{E}(\widetilde{\mathrm{m}}) \cdot f\left(\mathrm{q}_{\mathrm{i}}^{2} \cdot \overline{\mathrm{R}_{\mathrm{g}}^{2}}\right)}
$$

$\overline{\mathrm{n}^{1}}$ gibt das 1. Moment, den Mittelwert, der Größenverteilungsfunktion an. $\overline{\mathrm{n}^{2}}$ gibt das 2. Moment, ein Maß der relativen Breite, der Größenverteilungsfunktion an. Im Falle monodisperser Aggregate gehen die Momente in die Primärteilchenzahl pro Aggregat bzw. das Quadrat der Primärteilchenzahl pro Aggregat über: $\overline{n^{1}} \rightarrow n, \overline{n^{2}} \rightarrow n^{2}$. Für polydisperse Aggregate wird der optische Größenparameter für Momente eines Aggregats $x_{30}^{3}$, für den $\mathrm{x}_{30}^{3}=\left(\pi \mathrm{D}_{30}^{3} / \lambda\right)^{3}=\overline{\mathrm{n}^{1}} \mathrm{x}_{\mathrm{p}}^{3}$ bzw. $\mathrm{x}_{60}^{6}=\left(\pi \mathrm{D}_{60}^{6} / \lambda\right)^{6}=\overline{\mathrm{n}^{2}} \mathrm{x}_{\mathrm{p}}^{6} \quad$ gilt, analog zum optischen Größenparameters eines Primärpartikels $\mathrm{x}_{\mathrm{p}}$ definiert.

Für die Berechnung des volumenäquivalenten Durchmessers $\mathrm{D}_{30} \mathrm{mu}$ das 2 . Moment zuerst durch die zu Hilfenahme des Momentenverhältnisses $f_{n}$ der Verteilungsfunktion vorher 
eliminiert werden. Dazu wird das Momentenverhältnis $f_{n}$, eingeführt und zum Einsetzen in die Gleichung (2.40) umgeformt:

$$
f_{n}=\frac{D_{60}^{6}}{\left(D_{30}^{3}\right)^{2}}=\frac{\overline{n^{2}}}{\left(\overline{n^{1}}\right)^{2}} \quad \text { bzw. } \frac{\overline{n^{2}}}{\overline{n^{1}}}=\overline{n^{1}} \cdot f_{n}
$$

Das Momentenverhältnis $f_{n}$ beträgt für monodisperse Aggregate $f_{n}=1$ und erreicht im Falle polydisperser Aggregate mit durchgehender selbsterhaltender Verteilung Werte bis unterhalb von zwei. In den experimentellen Untersuchungen der Rußaggregate von Dobbins ([19] und [20]) wurde ein konstanter Wert für das Momentenverhältnis von $f_{n}=1.77$ gefunden. Dies ermöglicht dann die Vereinfachung der Gleichung (2.40) zu:

$$
\frac{\mathrm{Q}_{\mathrm{VV}}^{\mathrm{A}}(\Theta)}{\mathrm{k}_{\mathrm{abs}}^{\mathrm{A}}}=\frac{\mathrm{f}_{\mathrm{n}} \cdot \pi^{3} \cdot \overline{\mathrm{n}^{1}} \cdot \mathrm{d}_{\mathrm{p}}^{3} \cdot \mathrm{F}(\widetilde{\mathrm{m}})}{4 \pi \cdot \lambda^{3} \cdot \mathrm{E}(\widetilde{\mathrm{m}}) \cdot f\left(\mathrm{q}_{\mathrm{i}}^{2} \cdot \overline{\mathrm{R}_{\mathrm{g}}^{2}}\right)}
$$

Den volumenäquivalenten Durchmesser $\mathrm{D}_{30}$ erhält man als Produkt aus der 3. Wurzel des 1 . Moments der Verteilungsfunktion der Aggregate und dem Primärteilchendurchmesser $d_{p}$ [19].

$$
\mathrm{D}_{30}=\sqrt[3]{\mathrm{n}^{1}} \cdot \mathrm{d}_{\mathrm{p}}
$$

Die Primärpartikel nehmen dabei einen einheitlichen Primärteilchendurchmesser $d_{p}$ an.

Wird die Gleichung (2.43) in die Gleichung (2.42) eingesetzt, erhält man durch Auflösung nach dem volumenäquivalenten Durchmesser $\mathrm{D}_{30}$ den folgenden Ausdruck:

$$
\mathrm{D}_{30}=\frac{\lambda}{\pi} \cdot\left[\frac{4 \pi \cdot \mathrm{E}(\widetilde{\mathrm{m}}) \cdot \mathrm{Q}_{\mathrm{Vv}}^{\mathrm{A}}\left(\Theta_{\mathrm{i}}\right)}{\mathrm{f}_{\mathrm{n}} \cdot \mathrm{F}(\widetilde{\mathrm{m}}) \cdot \mathrm{k}_{\mathrm{abs}}^{\mathrm{A}} \cdot f\left(\mathrm{q}_{\mathrm{i}}^{2} \cdot \overline{\mathrm{R}_{\mathrm{g}}^{2}}\right)}\right]^{1 / 3}
$$

Experimentelle Untersuchungen über die Strukturfunktion $f\left(\mathrm{q}_{\mathrm{i}}^{2} \cdot \overline{\mathrm{R}_{\mathrm{g}}^{2}}\right)$ haben ergeben, daß es zwei verschiedene Lösungen für die Strukturfunktion, eine für den Guinier Bereich und eine für den Power Law Bereich des Formfaktors $\mathrm{q}_{\mathrm{i}}^{2} \cdot \overline{\mathrm{R}_{\mathrm{g}}^{2}}$, gibt [19], [20]. Die Bereiche sind über die relative Größe des Formfaktors zur fraktalen Dimension $D_{\mathrm{f}}$ definiert, damit erhält man für die Strukturfunktion folgende Beziehung:

$$
f\left(\mathrm{q}_{\mathrm{i}}^{2} \cdot \overline{\mathrm{R}_{\mathrm{g}}^{2}}\right)=\left\{\begin{array}{l}
\exp \left[-\frac{\mathrm{q}_{\mathrm{i}}^{2} \cdot \overline{\mathrm{R}_{\mathrm{g}}^{2}}}{3}\right] ; \mathrm{mit}_{\mathrm{i}}^{2} \cdot \overline{\mathrm{R}_{\mathrm{g}}^{2}} \leq 1.5 \cdot \mathrm{D}_{\mathrm{f}}: \text { Guinier Bereich } \\
\left(\frac{3 \mathrm{D}_{\mathrm{f}}}{2 e \cdot \mathrm{q}_{\mathrm{i}}^{2} \cdot \overline{\mathrm{R}_{\mathrm{g}}^{2}}}\right)^{\mathrm{D}_{\mathrm{f}} / 2} ; \mathrm{mit}_{\mathrm{i}}^{2} \cdot \overline{\mathrm{R}_{\mathrm{g}}^{2}}>1.5 \cdot \mathrm{D}_{\mathrm{f}}: \text { Power Law B. }
\end{array}\right.
$$




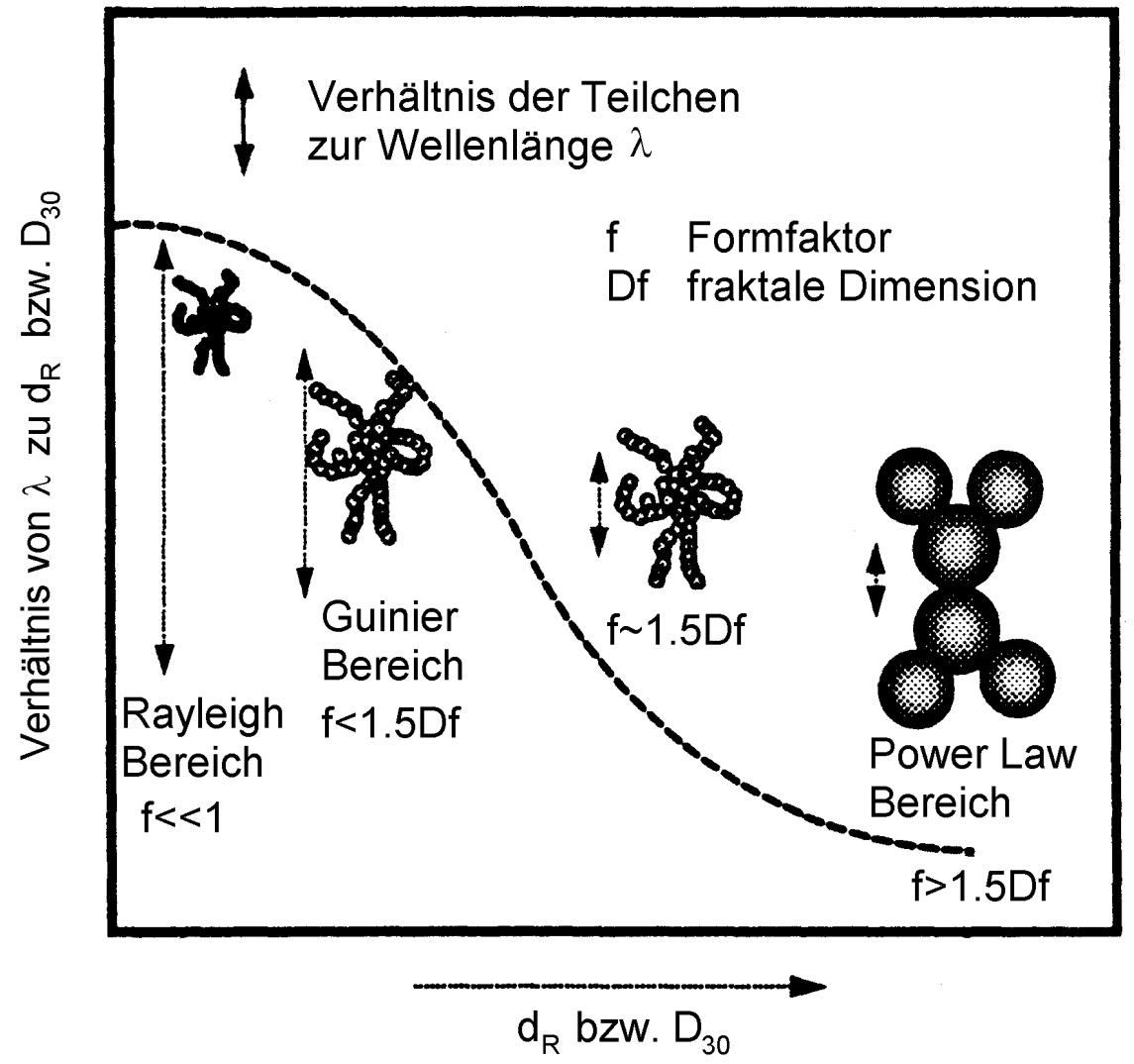

Abb. 2.2: Die verschiedenen Bereiche in der RDG/FA-Theorie im Zusammenhang mit dem Formfaktor $\mathrm{q}_{\mathrm{i}}^{2} \cdot \overline{\mathrm{R}_{\mathrm{g}}^{2}}[31]$.

Aus der Power Law Bedingung der Gleichung (2.45) wird eine empirische von der fraktalen Dimension abhängige Größe $C_{p}$ formuliert, die für $\mathrm{D}_{\mathrm{f}}=1.80$ etwa den Wert eins annimmt:

$$
\mathrm{C}_{\mathrm{p}}=\left(1.5 \cdot \mathrm{D}_{\mathrm{f}} / e\right)^{\mathrm{D}_{\mathrm{f}} / 2}
$$

Die Aggregatenzahldichte pro Volumen $\mathrm{N}_{\mathrm{Agg}}$ kann aus dem volumenäquivalenten Durchmesser $\mathrm{D}_{30}$ und dem Rußvolumenbruch $\mathrm{f}_{\mathrm{v}, \mathrm{A}}$ wie folgt ermittelt werden:

$$
\mathrm{N}_{\mathrm{Agg}}=\frac{6 \cdot \mathrm{f}_{\mathrm{v}, \mathrm{A}}}{\pi \cdot \mathrm{D}_{30}^{3}}
$$

Der Rußvolumenbruch nach der RDG/FA-Theorie $\mathrm{f}_{\mathrm{v}, \mathrm{A}}$ wird dabei berechnet nach:

$$
\mathrm{f}_{\mathrm{v}, \mathrm{A}}=\frac{\lambda}{6 \pi \cdot \mathrm{E}(\tilde{\mathrm{m}})} \cdot \mathrm{k}_{\mathrm{abs}}^{\mathrm{A}}
$$

Einsetzen der Gleichung (2.48) in die Gleichung (2.47) ergibt dann: 


$$
\mathrm{N}_{\mathrm{Agg}}=\frac{\lambda \cdot \mathrm{k}_{\mathrm{abs}}^{\mathrm{A}}}{\pi^{2} \cdot \mathrm{D}_{30}^{3} \cdot \mathrm{E}(\tilde{\mathrm{m}})}
$$

Die fraktale Dimension $\mathrm{D}_{\mathrm{f}}$, die eine quantitative Größe für die Anordnung der Aggregate im dreidimensionalen Raum darstellt, kann aus den Gleichungen (2.33) und (2.35) und der Umformung der Gleichung (2.38) hergeleitet werden:

$$
\mathrm{R}_{\mathrm{g}}^{\mathrm{D}_{\mathrm{f}}}=\frac{\mathrm{n} \cdot \mathrm{d}_{\mathrm{p}}^{\mathrm{D}_{\mathrm{f}}}}{\mathrm{k}_{\mathrm{f}}}
$$

Außerdem muß der Formfaktor für den Power Law Bereich gelten, d.h. $\mathrm{q}_{\mathrm{i}}^{2} \cdot \overline{\mathrm{R}_{\mathrm{g}}^{2}}>1.5 \cdot \mathrm{D}_{\mathrm{f}}$ sein. Der fraktale Vorfaktor $\mathrm{k}_{\mathrm{f}}$ hat nach Köylü [35] den festen Wert von $\mathrm{k}_{\mathrm{f}}=8.5$.

Für den Quotienten aus Streufaktor und Absorptionskoeffizienten ergibt sich dann:

$$
\frac{Q_{V V}^{A}(\Theta)}{k_{a b s}^{A}}=\frac{k_{f} \cdot F(\widetilde{m}) \cdot f_{n} \cdot x_{p}^{3-D_{f}} \cdot C_{p}}{4 \pi \cdot E(\widetilde{m}) \cdot[4 \sin (\Theta / 2)]^{D_{f}}}
$$

Die Steigung einer Ausgleichgeraden des linearen, dem Power Law Bereich entsprechenden, Teils einer Auftragung von $\log \left[\frac{\mathrm{Q}_{\mathrm{VV}}^{\mathrm{A}}}{\mathrm{k}_{\mathrm{abs}}^{\mathrm{A}}}\right]$ gegen $\log \left[\frac{1}{4 \sin (\Theta / 2)}\right]$ liefert den Zahlenwert für die fraktalen Dimension $\mathrm{D}_{\mathrm{f}}$.

Für die Berechnung der Primärteilchenparameter wird die Gleichung (2.51) nach $\mathrm{x}_{\mathrm{p}}$ aufgelöst:

$$
\mathrm{x}_{\mathrm{p}}=\left\{4 \pi \frac{\mathrm{E}(\tilde{\mathrm{m}})}{\mathrm{F}(\widetilde{\mathrm{m}})} \frac{\left.\mathrm{Q}_{\mathrm{VV}}^{\mathrm{A}}\right)}{\mathrm{k}_{\mathrm{abs}}^{\mathrm{A}}} \frac{[4 \sin (\Theta / 2)]^{\mathrm{D}_{\mathrm{f}}}}{\mathrm{k}_{\mathrm{f}}}\right\}^{1 /\left(3-\mathrm{D}_{\mathrm{f}}\right)}
$$

Aus dem optischen Größenparameter der Primärteilchen $\mathrm{x}_{\mathrm{p}}$ kann über die Beziehung

$$
\mathrm{d}_{\mathrm{p}}=\frac{\mathrm{x}_{\mathrm{p}} \cdot \lambda}{2 \pi}
$$

der Primärteilchendurchmesser $d_{p}$ berechnet werden. Analog zur Aggregatenzahldichte wird die Primärteilchenzahldichte $\mathrm{n}_{\mathrm{p}}$ aus dem Primärteilchenradius berechnet:

$$
n_{p}=\frac{6 f_{v}}{\pi d_{p}^{3}}
$$




\subsection{Brenneranlagen}

Für die Messungen wurden zwei verschiedene Brenner, ein Röhrchenbrenner für die Normaldruckmessungen und ein Folienbrenner für die Messungen bei höherem Druck, verwendet. Beide Brenner sind bereits in verschiedenen Arbeiten ausführlich beschrieben [30] (Röhrchenbrenner), bzw. [22], [45] (Folienbrenner).

\subsubsection{Röhrchenbrenner für die Normaldruckmessungen}

\subsubsection{Gasversorgung des Röhrchenbrenners}

Die Versorgung der 1-bar-Brenneranlage mit gasförmigen Brennstoffen erfolgte weitgehend der im Arbeitskreis üblichen Anordnung [47]. Die Gasversorgung des Röhrchenbrenners erfolgte jeweils für den Innenbrenner bzw. für den Außenbrenner unabhängig voneinander. Die Leitungen wurden zu diesem Zweck vor den Nadelventilen im Vordruckbereich verzweigt. Der Innenbrenner konnte sowohl mit gasförmigen als auch mit flüssigen Brennstoffen versorgt werden.

\section{$\underline{\text { Außenbrenner }}$}

Der Außenbrenner wurde ausschließlich mit einem Ethylen-Luft-Gemisch versorgt. Die Luft wurde mit Hilfe eines Preßluftgenerators auf 40-100 bar komprimiert, so daß ein genügender Vordruck von 3-5 bar stabil aufrechterhalten werden konnte. Zur Trocknung wurde die Luft durch einen Trockenzylinder mit Silicagel (orange) geleitet. Um Staubpartikel der Luft und des Silicagels vom Brenner fernzuhalten wurde die trockene Luft anschließend durch ein Membranfilter geführt. Das Ethylen wurde aus Druckgasflaschen mit Ethylen technischer Reinheit (Firma Messer Griesheim GmbH), hier 2.7, das einspricht einem Reinheitsgrad von 99.7\%, entnommen. Die Dosierung der Luft und des Ethylens erfolgte mit Nadelventilen. Zur Messung der Massenflüsse wurden, hier teilweise nur als Flowmeter eingesetzte, Flowcontroller (Fa. Tylan) verwendet: (sl bedeutet Standard-Liter) 
Luft für den Außenbrenner:

Ethylen für den Außenbrenner
200 sl/min Luft, Tylan Typ FC 262

10 sl/min Ethylen, Tylan Typ FC261

Die Flowcontroller, waren vor den Messungen wiederholt mit den entsprechenden Gasen, mit Hilfe von ausgeliterten Glaszylindern, geeicht worden.

\section{Innenbrenner, gasförmige Brennstoffe}

Mit dem Innenbrenner wurden Ethylen oder Propylen als gasförmige Brennstoffe verbrannt. Das Propylen (Ethylen s.o.) entsprach technischer Reinheit (Firma Messer Griesheim GmbH, Propylen 2.5). Die Gasdosierung erfolgte wie beim Außenbrenner mit Nadelventilen, als Meßinstrumente kamen folgende geeichte Flowcontroller der Firma Tylan zum Einsatz:

Luft für den Innenbrenner: 100 sl/min Luft, Tylan Typ FC262

Ethlyen, Propylen für den Innenbrenner:

10 sl/min Ethylen, Tylan Typ FC261

Die Eichung des $10 \mathrm{sl} / \mathrm{min}$ Ethylenmassenflußreglers für die Propylen-Messungen erfolgte zusätzlich mit Propylen, dabei wurde die zu vermutende Differenz der Anzeige infolge unterschiedlicher Molmassen bestätigt. Die Linearität blieb ebenfalls erhalten, deshalb konnte dieser Massenflußregler unter Zuhilfenahme der jeweiligen Eichgeraden für beide Kohlenwasserstoffe verwendet werden.

\section{Innenbrenner mit Flüssigbrennstoffen}

Für die Messungen der Flüssigbrennstoffe wurde ein zweistufiger Sättiger (Abb. 3.1) verwendet. Für die Brennstoffsättigung wurde die Luft dem Innenbrenner in zwei unterschiedlich großen Teilen zugeführt. Der kleinere Teil (etwa 1/3) der Luft wurde aus einer separaten Druckflasche (Preßluft aus dem Kompressor) entnommen und mit einem zusätzlichen Nadelventil dosiert. Der genaue Teil der Sättiger-Luft hängt einerseits von den Vorgaben der Flamme (C/O-Verhältnis, Frischgasgeschwindigkeit) ab. Andererseits soll die Temperatur im Sättiger deutlich unter dem Siedepunkt des eingefüllten Kohlenwasserstoffs liegen. Außerdem kann der Sättiger nur einen begrenzten Maximalfluß verkraften. 


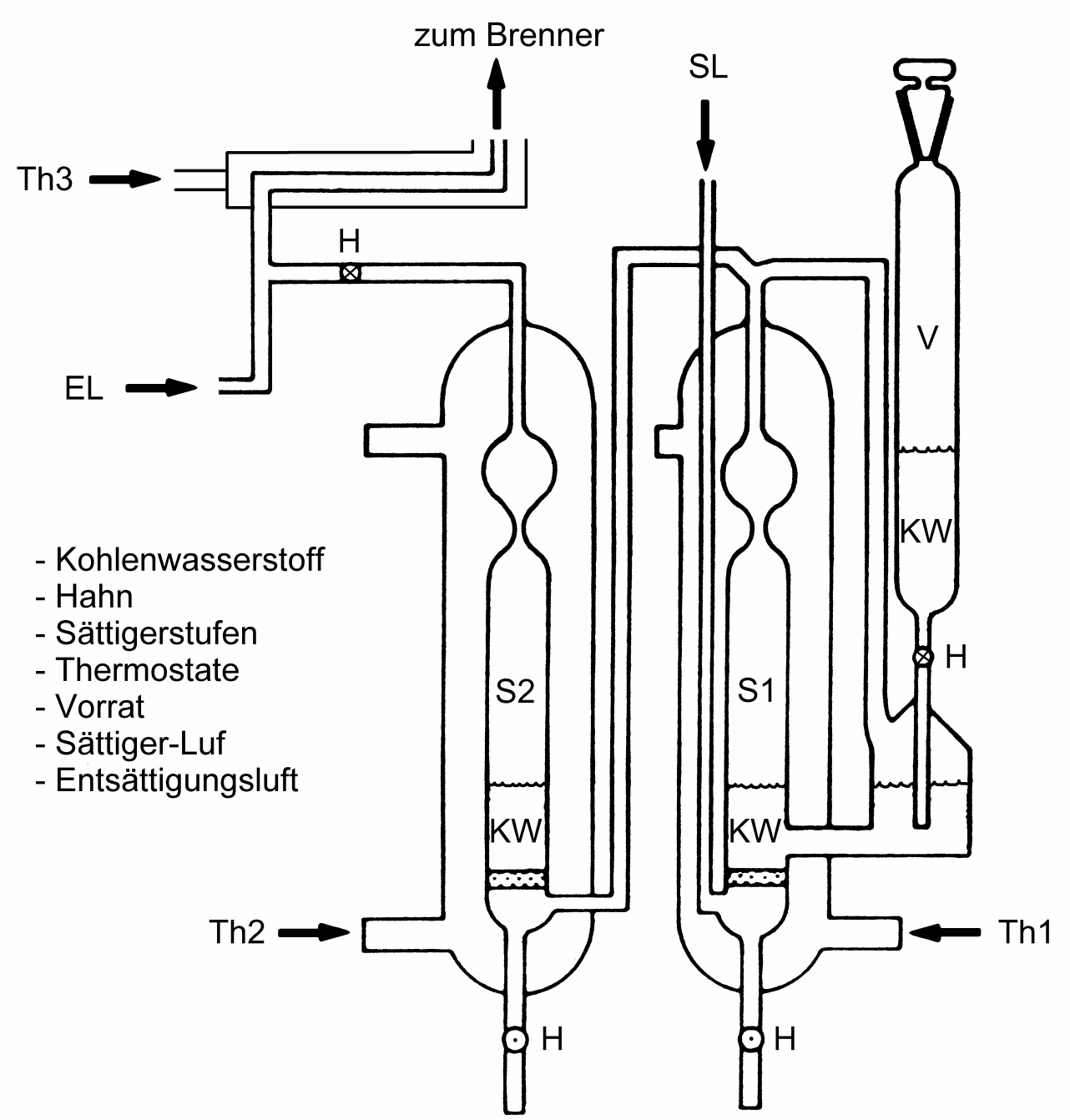

Abb. 3.1: $\quad$ Schematischer Aufbau des Sättigers

Zur Messung des Massenflusses der Sätiger-Luft diente ein weiterer Flowcontroller der Firma Tylan:

Sättiger Luft:

10 s1/min Wasserstoff, Tylan Typ FC261

Die sog. Sättigerluft (SL) wurde durch den zweistufigen Sättiger geleitet. Dabei reicherte sich die Luft im Sättiger mit dem jeweils eingesetzten Kohlenwasserstoff (KW) an.

$$
\phi_{K W}=\phi_{S L} \frac{p_{K W}}{p_{\text {Luft }}}=\frac{p_{K W}}{p_{\text {Labor }}-p_{K W}}
$$

Dazu müssen die Temperaturen der Flüssigkeiten in den beiden Sättigerstufen und der Luftdruck gemessen werden. Die Temperatur der ersten Stufe des Sättigers wurde um ungefähr $0.2 \mathrm{~K}$ höher als die Temperatur der zweiten Stufe eingestellt, um eine möglichst 
vollständige Sättigung zu erreichen. Die Temperaturregelung erfolgte mit zwei Thermostaten. Die anzureichernde Luft passiert dabei zuerst die erste um etwa $0.2 \mathrm{~K}$ wärmere Stufe, reichert sich dementsprechend mit Brennstoff an, um dann in der zweiten Stufe den noch fehlenden Teil an Kohlenwasserstoff aufzunehmen oder einen Überschuß abzugeben. Der größere Teil der Luft, die sogenannte Entsättigungsluft (EL) wurde, um eine Kondensation der Kohlenwasserstoffe zu verhindern, unmittelbar hinter dem Sättiger mit dem Gasgemisch aus dem Sättiger vereinigt. Der Versuch einer Regelung mit den Flowcontrollern führte zu Schwankungen der Sättigungsluft im Sättiger und der Entsättigungsluft. Die rußende Flamme begann zu oszillieren, d.h. zwischen leuchtender und nicht oder geringer leuchtender Flamme zu pendeln. Aus diesem Grund wurden die Flowcontroller lediglich als Flowmeter benutzt. Um ein Auskondensieren der Kohlenwasserstoffe zu vermeiden, war die Leitung zum Brenner mit Heizbändern (Fa. Horst) umwickelt. Der kontinuierliche Betrieb des Sättigers wurde über den Vorrat aufrechterhalten.

Für Flammen mit Flüssigbrennstoff wurde zunächst eine nichtrußende Ethylen/Luft-Flamme gezündet. Danach wurde ein kleiner Teil der Sättiger-Luft in den Sättiger gegeben. Die mit Kohlenwasserstoff gesättigte Luft wurde mit dem Ethylen/Luft-Gemisch vereinigt. Es wurde abwechselnd Ethylen reduziert und der Flüssigbrennstoff über die Sättiger-Luft erhöht. Dies wurde so lange wiederholt bis die gewünschte Flamme eingestellt war.

Als flüssige Kohlenwasserstoffe wurden n-Pentan (Fa. Merck., per Analysis, >99\% Reinheit), n-Heptan (Fa. Merck., per Analysis, >99.5\%), Isooktan (2,2,4-Trimethylpentan) (Fa. Merck., per Analysis, $>99.5 \%$ ) und Benzol (Fa. Fluka., puriss., $>99.5 \%$ Reinheit) eingesetzt.

\subsubsection{Aufbau des Röhrchenbrenners}

Der für die 1-bar-Flammen verwendete Röhrchenbrenner bestand aus einem Innenbrenner, dem Zentralbrenner, und einem Außenbrenner. Der Außenbrenner umgab den Innenbrenner vollständig und diente zur Stabilisierung der Innenbrennerflamme. Mit dem Innenbrenner konnte damit eine flache nicht gestörte rußende Flamme realisiert werden.

Der Innenbrenner bestand aus einem Edelstahlrohr von $56 \mathrm{~mm}$ Durchmesser und einer Höhe von $100 \mathrm{~mm}$. Dieses Rohr wurde in den eigentlichen Brenner und eine Mischkammer 
unterteilt. Im Brennerteil befanden sich in etwa 1200 Edelstahlröhrchen von $60 \mathrm{~mm}$ Länge und $1.5 \mathrm{~mm}$ Außendurchmesser bei einer Wandstärke von $0.25 \mathrm{~mm}$. Die Edelstahlröhrchen standen dicht gepackt auf einer Sinterplatte. Diese ermöglichte eine gleichmäßige und planare Anordnung der Röhrchen. Die Mischkammer war mit Glasraschigringen gefüllt. Der Innenbrenner wurde nicht gekühlt. Auf dem Innenbrenner brannten Flammen mit Frischgasgeschwindigkeiten von $3.5 \mathrm{~cm} / \mathrm{s}$ bis zu knapp $8.0 \mathrm{~cm} / \mathrm{s}$.

Der Außenbrenner hatte einen Außendurchmesser von $80 \mathrm{~mm}$ und damit eine Dicke von knapp $24 \mathrm{~mm}$. Im Außenbrenner standen ebenfalls dichtest gepackte Edelstahlröhrchen von 24.5 bis $25.0 \mathrm{~mm}$ Länge und $2.0 \mathrm{~mm}$ Durchmesser bei einer Wandstärke von $0.25 \mathrm{~mm}$. Der Brenner wurde mit etwa $55{ }^{\circ} \mathrm{C}$ warmen Wasser gekühlt. Auf dem Außenbrenner brannten ausschließlich nichtrußende Flammen mit Frischgasgeschwindigkeiten um die $8 \mathrm{~cm} / \mathrm{s}$.

In etwa $50 \mathrm{~mm}$ über dem Brenner befand sich ein Drahtnetz, mit einer Maschenweite von etwa $2.5 \mathrm{~mm}$ oder $4.0 \mathrm{~mm}$ und einem Durchmesser der Drähte von $0.8 \mathrm{~mm}$

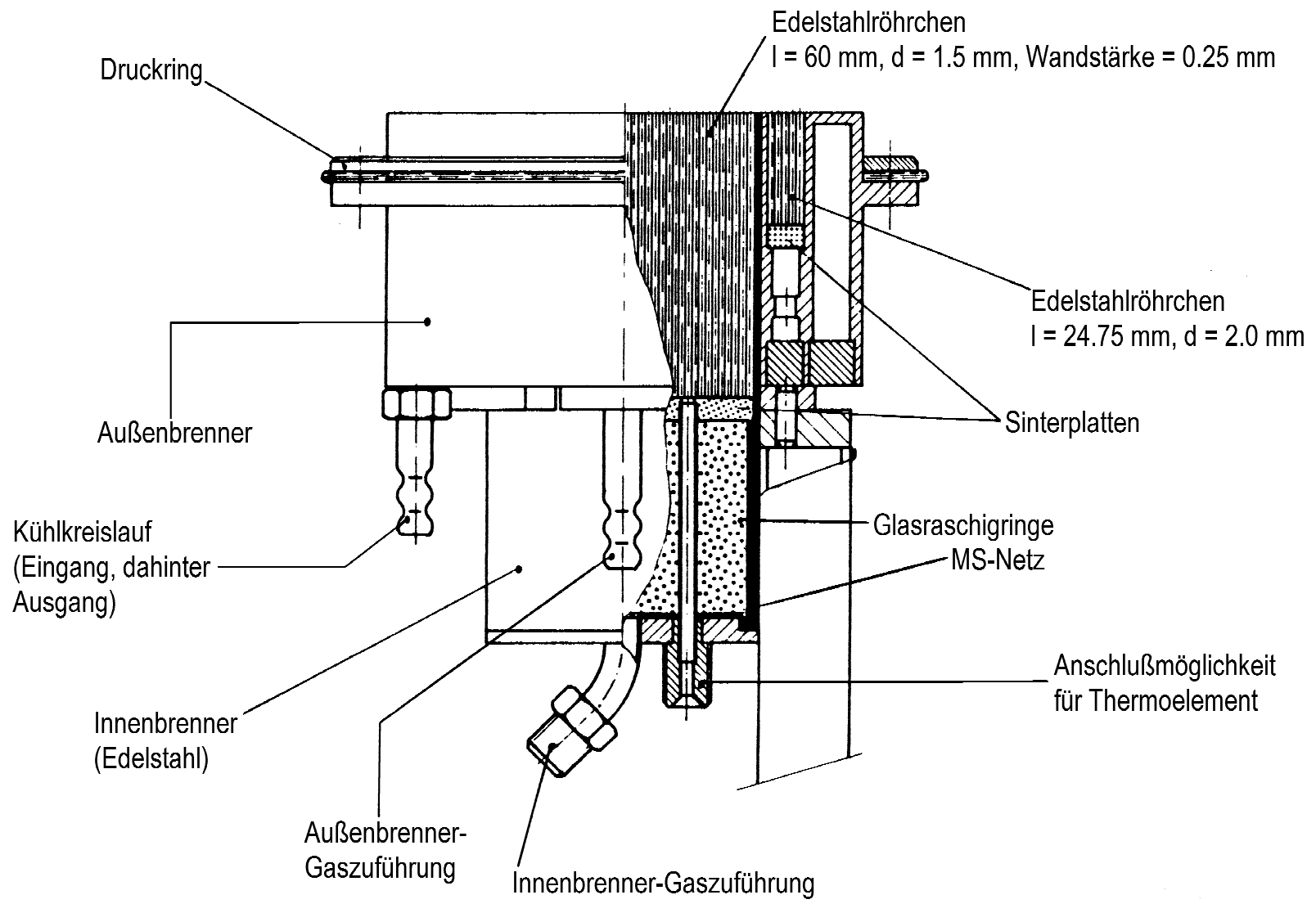

Abb. 3.2: Röhrchenbrenner 


\subsubsection{Folienbrenner für Flammen bis 5 bar}

\subsubsection{Gasdosierung der 15 bar Brenneranlage}

Für die Flammen bis 5 bar wurde Luft mit dem Kompressor (s. Kap. 3.1.1.1) komprimiert und anschließend gereinigt und getrocknet. Der Brennstoff Ethylen stammte aus Gasflaschen (Fa. Messer Griesheim, Reinheit 2.7). Der Vordruck wurde auf 10 bar eingestellt, um auch bei Messungen mit einem höheren Druck bis 5 bar eine konstante Gasversorgung zu gewährleisten. Da Ethylen in Druckflaschen teilweise kondensiert vorliegt, wurde Ethylen zu diesem Zweck in eine zweite Flasche überführt, damit es vollständig gasförmig vorlag. Innenbrenner und Außenbrenner des 15 bar Brenners wurden weitgehend unabhängig voneinander mit einem Ethylen-Luft-Gemisch versorgt.

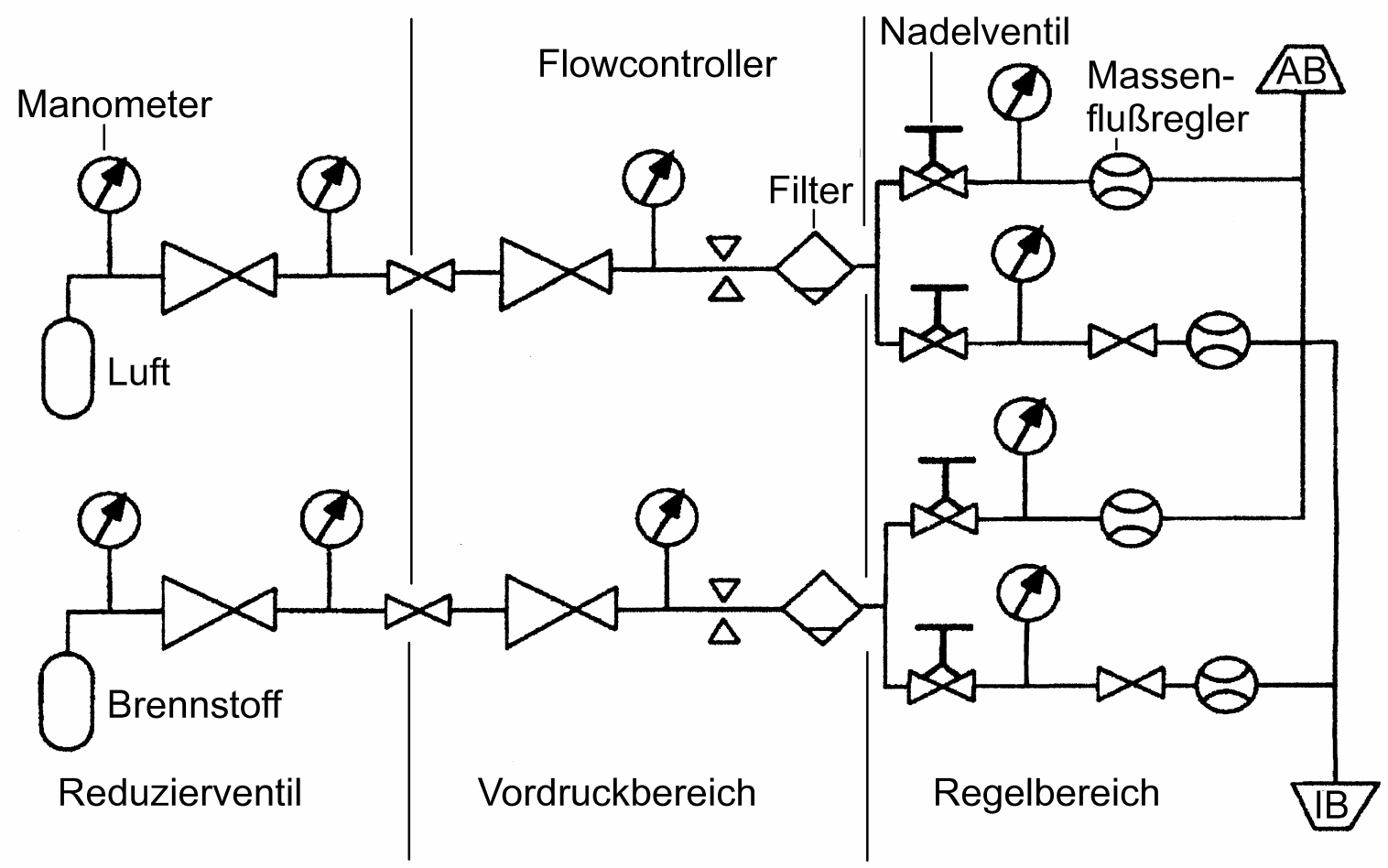

Abb. 3.3: Schaltplan der Gasdosierung des 15 bar Brenner

Die Einstellung erfolgte durch Nadelventile (Fa. Nupro). Zur Messung der eingesetzten Gasströme dienten Massenflußanzeigen (Flowmeter, Fa. Tylan):

Luft Innenbrenner, 100 sl/min Luft, $\pm 1 \%$; Luft Außenbrenner, 500 sl/min Luft, $\pm 1 \%$

Ethylen Innenbrenner, $15 \mathrm{sl} / \mathrm{min}$ Ethylen, $\pm 1 \%$; Ethylen Außenbrenner, $50 \mathrm{sl} / \mathrm{min}$ Ethylen, $\pm 1 \%$ 
Die Massenflußanzeigen wurden entweder mit der Seifenblasenmethode (bis v = 40 1/min) oder mit einer Durchfluß-Meßturbine ( $\mathrm{v}=20 \mathrm{1} / \mathrm{min} \leq \phi \leq 400 \mathrm{l} / \mathrm{min})$ geeicht. Als Auslesegerät diente eine Vierkanalanzeige (Eigenbau Elektronikwerkstatt IPC). Die EthylenVentile wurden mit Heizbändern (Fa. Horst) warm gehalten, um einer durch die Expansion von Gasen mit großem Durchsatz entstehenden Abkühlung (Joule-Thomson-Effekt) entgegenzuwirken. Brennstoff und Luft wurden erst kurz vor dem Brenner in einer Mischkammer zusammengeführt und gemischt. Das Beobachtungsfenster mußte vom anfallenden Kondenswasser frei gehalten werden. Dies wurde mit Helium (Fa. Messer Griesheim, Reinheit 4.6) erreicht, das von einem Nadelventil geregelt von unten durch eine Düse gegen das Fenster geblasen wurde.

\subsubsection{Aufbau des 15 bar Folienbrenners}

Für die Messungen bei höherem Druck von 1.3 bar bis 5 bar wurde ein bis 15 bar ausgelegter rechteckiger Folienbrenner verwendet, der im vollen Umfang von einem runden Sinterplattenbrenner als Außenbrenner umgeben war. Dieser Brenner wurde bereits in mehreren Arbeiten ausführlich beschrieben [21], [44] und [45].

Als Flammenhalter des Innenbrenners zur Stabilisierung der zu untersuchenden Ethylen/LuftFlammen diente eine mit Aluminiumfolien gepackte Kassette aus Kupfer von $37.5 \mathrm{~mm}$ Länge, 20.5 mm Breite und 18 mm Höhe (s. Abb.3.4).

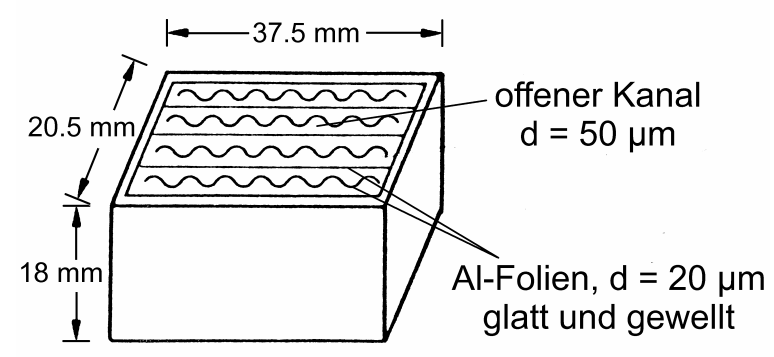

Abb. 3.4: Kassette des 15 bar Folienbrenners

Die Aluminiumfolien selbst hatten eine Dicke von $20 \mu \mathrm{m}$ und waren abwechselnd im glatten und gewellten Zustand in der Kassette angeordnet worden. Dabei entstanden Kanäle von etwa $50 \mu \mathrm{m}$ Durchmesser, durch die die Gase strömen konnten. Der Durchmesser der Gaskanäle von nur $50 \mu \mathrm{m}$ unterschreitet dabei den Löschabstand aller möglichen zur Untersuchung 
kommenden Flammen deutlich. Das günstige Verhältnis von offenen Kanälen zur Oberfläche der Folienkanten stellte außerdem eine homogene streifenfreie Flamme bei den meisten Flammenbedingungen sicher. Durch Erodierung der Schnittkanten der Aluminiumfolien wurde außerdem eine homogene Brenneroberfläche geschaffen, die entsprechend flache, laminare und homogen Flammen ermöglicht.

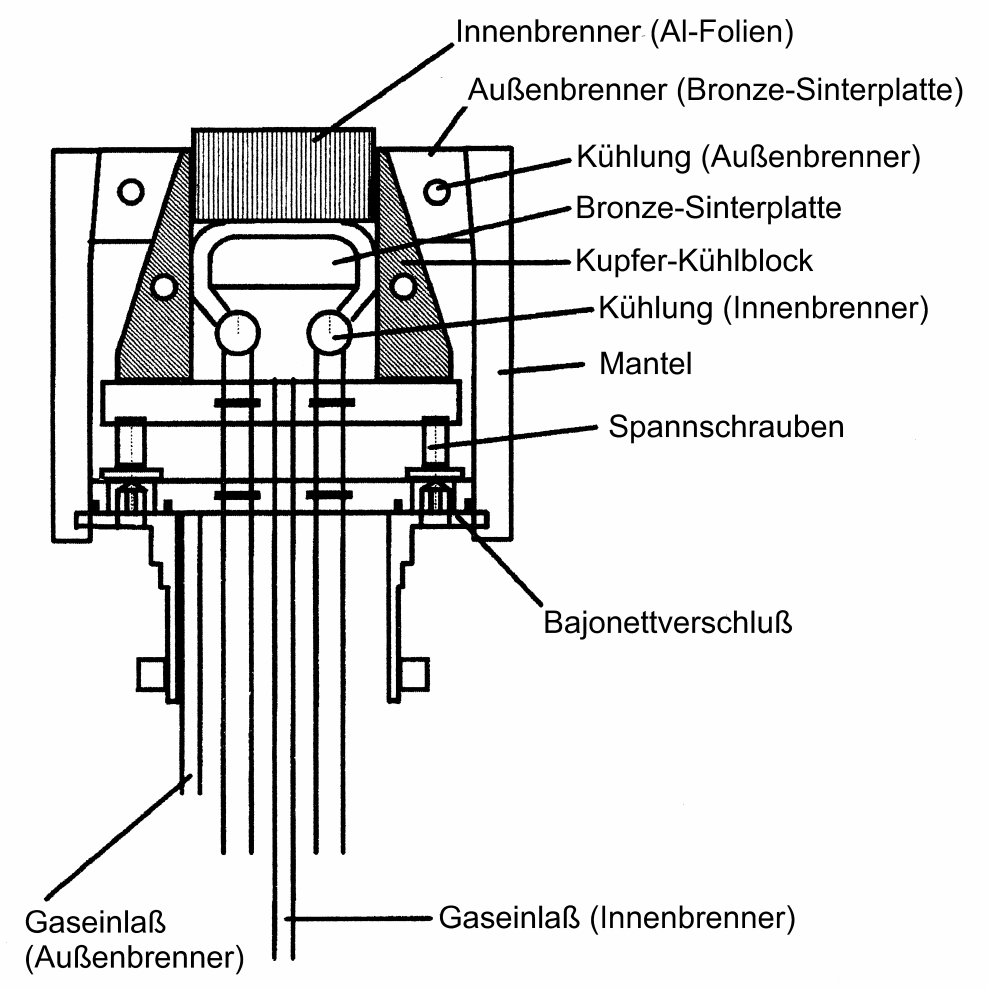

Abb. 3.5: $\quad$ Skizze des 15 bar Folienbrenners

Der Außenbrenner (s. Abb. 3.5) bestand aus einer $22 \mathrm{~mm}$ dicken Sinterplatte aus Bronze (Fa. Thyssen, Siperm B40) und hatte einen Außendurchmesser von $81 \mathrm{~mm}$. In die Sinterplatte wurde ein Kupferrohr von $6 \mathrm{~mm}$ Durchmesser als Kühlmittelleitung einmontiert. Der Außenbrenner soll eine homogene Flammentemperatur und ein ausgeglichenes Strömungsprofil der zu untersuchenden Flamme des Innenbrenners sicherstellen.

Der Brenner war zur Stabilisierung der Flammen von einem Keramikrohr (Fa. Schiedel) umgeben. In einer Höhe von etwa $3.5 \mathrm{~cm}$ über dem Brenner befand sich ein Drahtnetz, welches ebenfalls der Stabilisierung der Flammen diente. Ein Ölkühlkreislauf stellte eine ausreichende Wärmeabfuhr für beide Brennerbestandteile bereit. Der für die Kühlung erforderliche Durchfluß an Öl wurde von einer Zahnradpumpe (Fa. Verder Liquiflo) angetrieben. Zum besseren Wärmeaustausch wurden die Pumpen ihrerseits mit Luft gekühlt (Ventilator) und die Ölthermostaten mit Wasser (Thermostate). 


\subsubsection{Aufbau und Betrieb der Hochdruckbrenneranlage}

Der Brenner wurde von einer druckdichten Brennerhaube aus Edelstahl umgeben (Abb. 3.6).

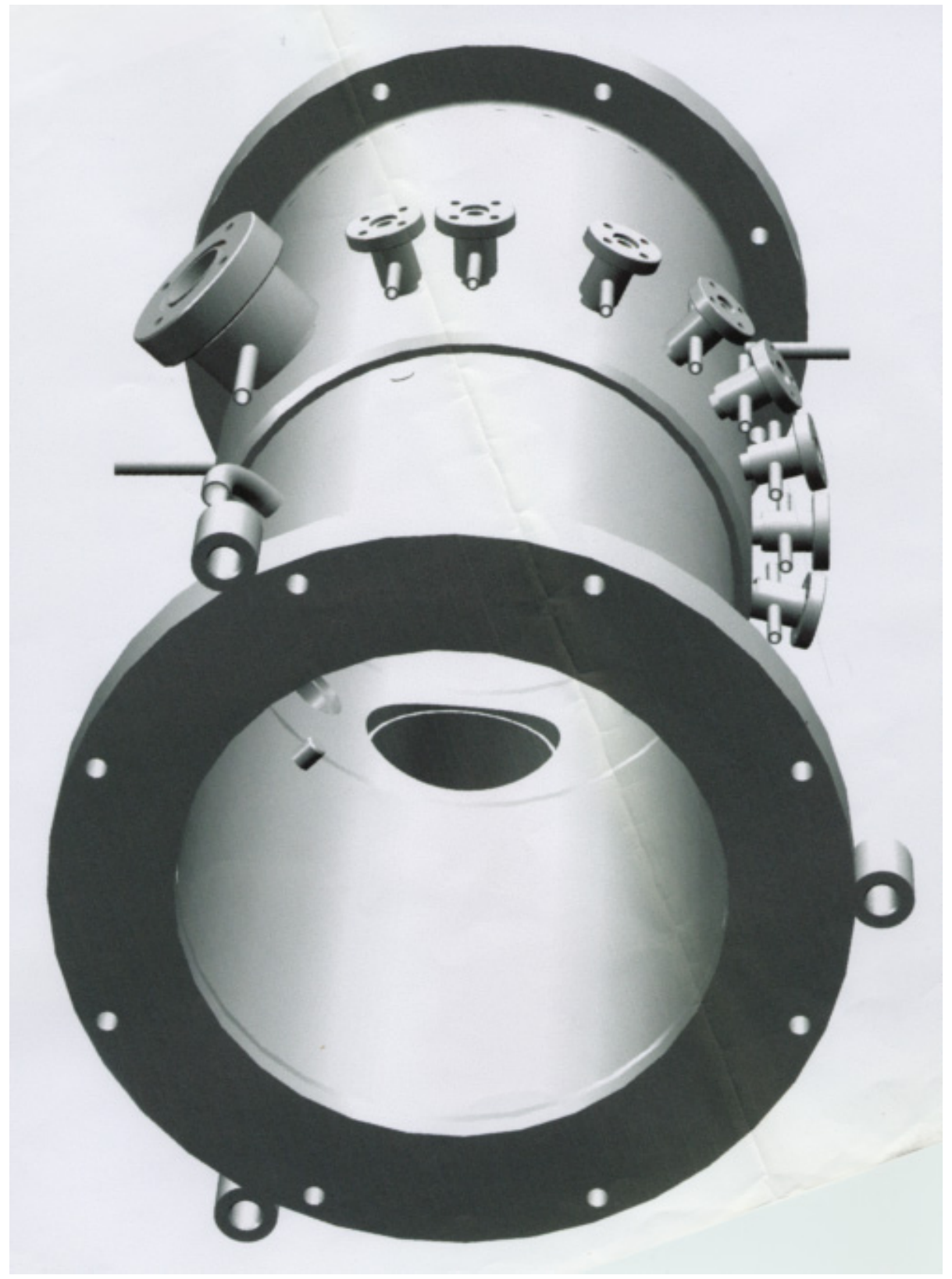

Abb. 3.6: Brennerhaube mit Einschüben für die Lichtleiter

Die Haube besaß mehrere Flanschansätze:

- $\quad$ Das Zündloch zum Zünden des Brenners unter Atmosphärendruck

- $\quad$ Ein größeres Fenster (Fa. Auersieg) zur Beobachtung der Flammen.

- $\quad 7$ Flanschen für Lichtleiter.

- $\quad 2$ Öffnungen für die Einsätze von im Brewster-Winkel stehenden Quarzscheiben.

Sie ermöglichen den Strahleneintritt bzw. Austritt des Laserstrahls für die optischen Messungen. 
Alle Einsätze wurden mit einem auf $35{ }^{\circ} \mathrm{C}$ thermostatisierten Wasserkreislauf (Schlauchpumpe) gekühlt. Der Brenner konnte mit Hilfe eines Spindeltriebes (Genauigkeit \pm $0.25 \mathrm{~mm}$ ) in der Höhe verstellt werden. Der Zwischenraum von Brenner und Außenwand war mit einer Schamotteauskleidung von $10 \mathrm{~cm}$ Innendurchmesser ausgefüllt. Das Abgas wurde über ein wassergekühltes Rohr in eine stählerne Kühlfalle (Abgastonne) geleitet. Die Kühlfalle war mit Steinwolle und Metallspänen gefüllt und diente zur Abscheidung von Ruß und Reaktionswasser aus dem Abgas, um ein Verstopfen der Druckventile zu vermeiden. Das auskondensierte Wasser konnte unterhalb der Kühlfalle über einen Hahn abgelassen werden.

Der Druck innerhalb der gesamten Apparatur wurde über ein der Abgastonne nachgeschaltetes Blendensystem und mit Hilfe von zwei Nadelventilen geregelt. Der Druck wurde an einem Manometer $(\mathrm{Kl} .=0.6)$ abgelesen. Durch ein den Blenden und Ventilen nachgeschaltetes Rohr wurde das expandierte Abgas ins Freie abgeleitet.

\subsection{Optische Anordnungen}

\subsubsection{Optische Anordnung der 1 bar Brenneranlage}

Ein 4 Watt Argon-Ionen-Laser (Fa. Coherent Modell Innova 90) wurde als Lichtquelle verwendet. Der Laser erzeugte polarisiertes Licht bei einer gewählten Wellenlänge von 488 $\mathrm{nm}$ und einer Ausgangsleistung von etwa 1.4 Watt für die Streulichtmessungen und $60 \mathrm{~mW}$ für die Extinktionsmessungen. Der Laserstrahl wurde von einem Chopper auf eine Frequenz von $150 \mathrm{~Hz}$ moduliert. Im Strahlengang schloß sich ein Achromat $\mathrm{L}_{1}$ mit der Brennweite $\mathrm{f}=$ $300 \mathrm{~mm}$ (Fa. Spindler \& Hoyer) an, um den Laserstrahl auf den Mittelpunkt des Brenners zu fokussieren. Um den Lichtstrahl möglichst weitgehend abzuschirmen, wurden zwei Edelstahlrohre von $6 \mathrm{~mm}$ Durchmesser parallel in den Strahlengang eingebracht. Die Rohre wurden während der Messungen mit Stickstoff durchflutet, um ein Hineinschlagen der Flamme zu verhindern.

Im Detektionsteil der optischen Anordnung wurde der divergierende Streustrahl von einer Linse $\mathrm{L}_{2}(\mathrm{f}=100 \mathrm{~mm})(\mathrm{Fa}$. Spindler \& Hoyer) auf die Eintrittsöffnung des SEV fokussiert. Hinter der zweiten Linse $\mathrm{L}_{2}$ befand sich während der Durchführung der Streulichtmessungen ein Polarisationsfilter (Spindler \& Hoyer Typ 10K Durchlässigkeit $2 * 10^{-3} \%$ ), um zusätzlich 
zum Chopper Störstrahlungen vom SEV fernzuhalten. Außerdem sollte nur der Anteil des, die Polarisationsebene beibehaltene Streulicht detektiert werden.

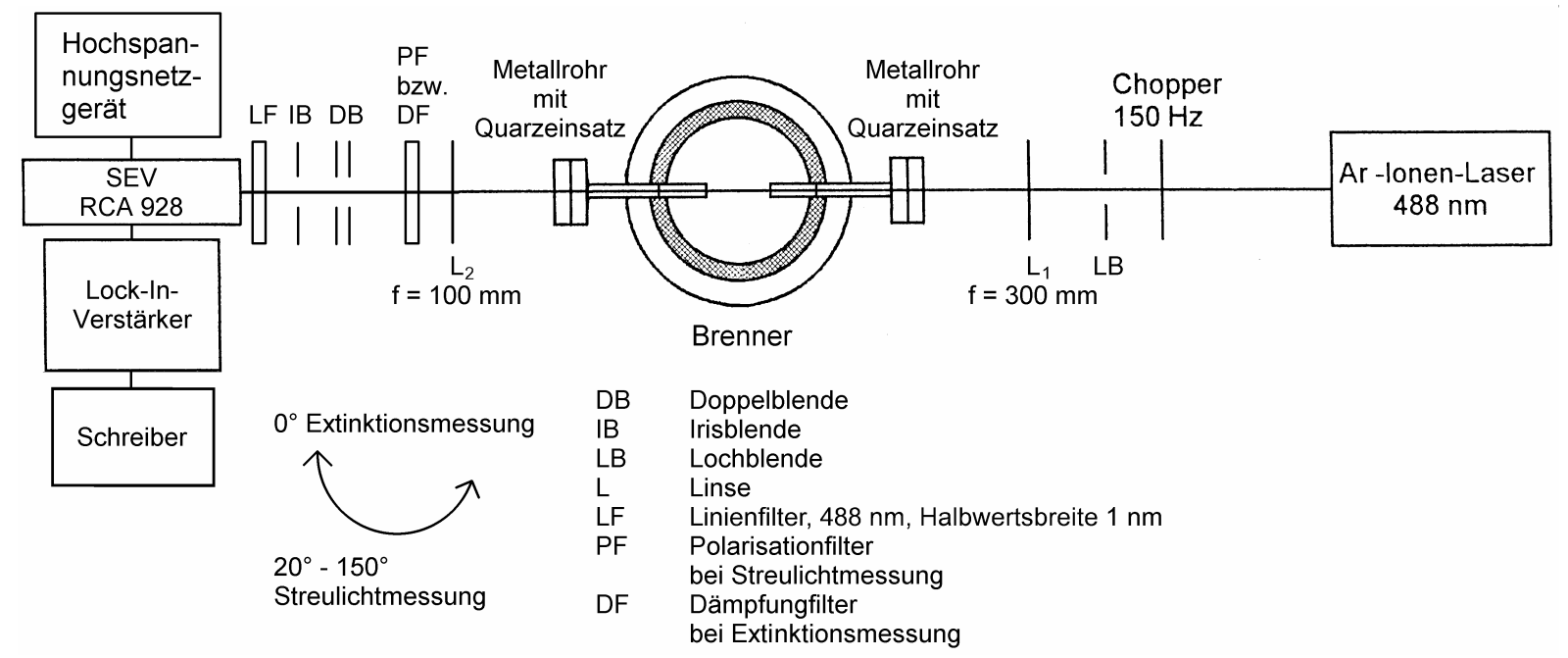

Abb. 3 7: $\quad$ Skizze der optischen Anordnung.

Für die Extinktionsmessungen befand sich auf der Detektorseite ein Dämpfungsfilter (Firma Spindler \& Hoyer Typ D1), um das für den Empfindlichkeitsbereich des SEV zu intensive Signal zusätzlich abzuschwächen.

An die jeweils eingesetzten Filter schloß sich eine Doppelblende an, mit welcher der Meßstrahl horizontal und vertikal ausgeblendet werden konnte. Hinter der Doppelblende befand sich eine Irisblende, mit welcher der Meßstrahl noch weiter begrenzt werden konnte. Für die Streulicht- und Absorptionsmessungen wurde ein Laserline-Filter (Linienfilter) mit einer Halbwertsbreite bei $\lambda=488 \mathrm{~nm}$ von $1 \mathrm{~nm}$ (Fa. Spindler \& Hoyer) im Anschluß an die Irisblende in den Strahlengang eingebracht. Die Detektion der Streulicht bzw. Extinktionssignale erfolgte durch einen Sekundärelektronenvervielfacher (SEV; Fa. Hamamatsu, Typ R928), auf dessen Eintrittsöffnung der Laserstrahl genau justiert wurde. An den Hochspannungseingang des SEV wurde mittels eines Netzgerätes (Fa. Nucletron, Hochspannungseinschub Typ NU1250B) je nach den Erfordernissen der untersuchten Flammen Spannungen zwischen 700 und 1100 Watt angelegt.

Der gesamte Detektionsteil der Meßapparatur ist auf einer zwischen den Winkeln von $0^{\circ}$ bis $150^{\circ}$ schwenkbaren optischen Schiene montiert worden. Zusätzlich wurde der gesamte Strahlengang so weit wie möglich vor Einflüssen von Hintergrundstrahlungen abgeschirmt. Die Meßsignale des SEV wurden mit einem doppelt abgeschirmten Kabel in einen Lock-in- 
Verstärker (Keithley, Modell 840) geleitet. Das verstärkte Signale wurden von einem Schreiber (Fa. Philips, Modell PM222 two line recorder) für die weitere Auswertung aufgezeichnet.

\subsubsection{Optische Messungen mit dem Hochdruckbrenner}

\subsubsection{Winkelabhängiges optisches Messen mit Lichtleitern}

Vorangegangene winkelabhängige optische Messungen hatten ergeben, daß für aussagekräftige Ergebnisse mindestens 5 Winkeleinstellungen erforderlich sind. Um eine maximale Anzahl an Winkelmeßstellen zu erhalten, wurden wegen ihres geringen Durchmessers Lichtleiter gewählt. Außerdem gewähren Lichtleiter eine hohe Lichtdurchlässigkeit, so daß sie für die vorliegende Aufgabenstellung geeignet erschienen. Da die Lichtleiter auf der flammenzugewandten Seite erheblichen Temperaturen von etwa $\mathrm{T}$ $\approx 600^{\circ} \mathrm{C}$ ausgesetzt sind, mußten sie in Eigenproduktion aus Meterware hergestellt werden (Lichtleiter im Handel halten Temperaturen nur bis $\mathrm{zu} 125^{\circ} \mathrm{C}$ aus). Es wurde eine Silica/Silica-multimode Faser mit einem Kerndurchmesser von $\mathrm{r}_{1}=365 \mu \mathrm{m}$ und einem Manteldurchmesser von $\mathrm{r}_{2}=400 \mu \mathrm{m}$ mit einer numerischen Apertur von 0.22 gewählt (Fa. Thorlabs, Grünstadt; Typ CF01493-42, für UV/VIS). Für eine höhere Lichtausbeute wurden 2-adrige Lichtleiter gebaut.

\subsubsection{Konstruktion der Lichtleiter}

Von jeweils zwei Fasern wurden am flammenseitigen Ende die Kunststoffummantelungen entfernt. Die zwei entisolierten Glasfasern wurden anschließend in ein Degussitrohr $\left(\mathrm{d}_{\mathrm{A}}=4\right.$ $\mathrm{mm})$ mit zwei Bohrungen $\left(\mathrm{d}_{\text {Bohrung }}=1.2 \mathrm{~mm}\right)$ geschoben. Die Glasfasern wurden an der brennerabgewandten Seite mit einem hitzebeständigen Spezialklebstoff (Fa. Thorlabs; Epoxyd-Kleber (Hochtemperaturfest) AB9123) mit dem Degussitrohr verklebt. Die beiden anderen Enden der Glasfasern wurden zusammen in die Spitze eines optischen Steckers (Fa. Thorlabs; Connector 10440A SMA, stainless steel multimode) geführt. Damit beide Lichtleiter in der Spitze des Steckers Platz finden, mußte dieser auf einen Durchmesser von $0.9 \mathrm{~mm}$ aufgebohrt werden. 
Über den freien Teil der Lichtleiter zwischen Degussitrohr und Stecker wurde ein Schutzmantel gezogen. Das Degussitrohr wurde in ein Edelstahlrohr mit Flansch geschoben und mit Epoxyd-Kleber und einem Schrumpfschlauch befestigt.

Insgesamt wurden 7 Lichtleiter hergestellt und in den Winkeln von $25^{\circ}, 45^{\circ}, 70^{\circ}, 90^{\circ}, 110^{\circ}$, $130^{\circ}$ und $150^{\circ}$ in die dafür vorgesehenen Öffnungen in die Brennerhaube geschoben. Die Stecker wurden auf einen drehbaren optischen Umschalter (s. Abb. 3.10) aufgesteckt. Der optische Umschalter war fest am SEV montiert und gewährte die Weiterleitung des Lichts zum SEV für jeweils einen Lichtleiter. Direkt vor der Eintrittsöffnung des SEV (Fa. Hamamatsu; Typ R 928, spektrale Empfindlichkeit $\lambda_{\max }=500$ nm, 9 Verstärkerstufen) befand sich ein Laserline-Filter (Fa. Spindler \& Hoyer). Der optische Umschalter ermöglichte durch Drehen und Einrasten einen präzisen, für die Lichtleiter torsionsfreien, Wechsel der Lichtleiter. So wurde eine effiziente Lichtausbeute, die für die Messungen eine genügende optische Genauigkeit bereitstellte, gewährleistet. In den folgenden Abbildungen sind ein Lichtleiter im Metallrohr mit Flansch sowie sein Stecker abgebildet:

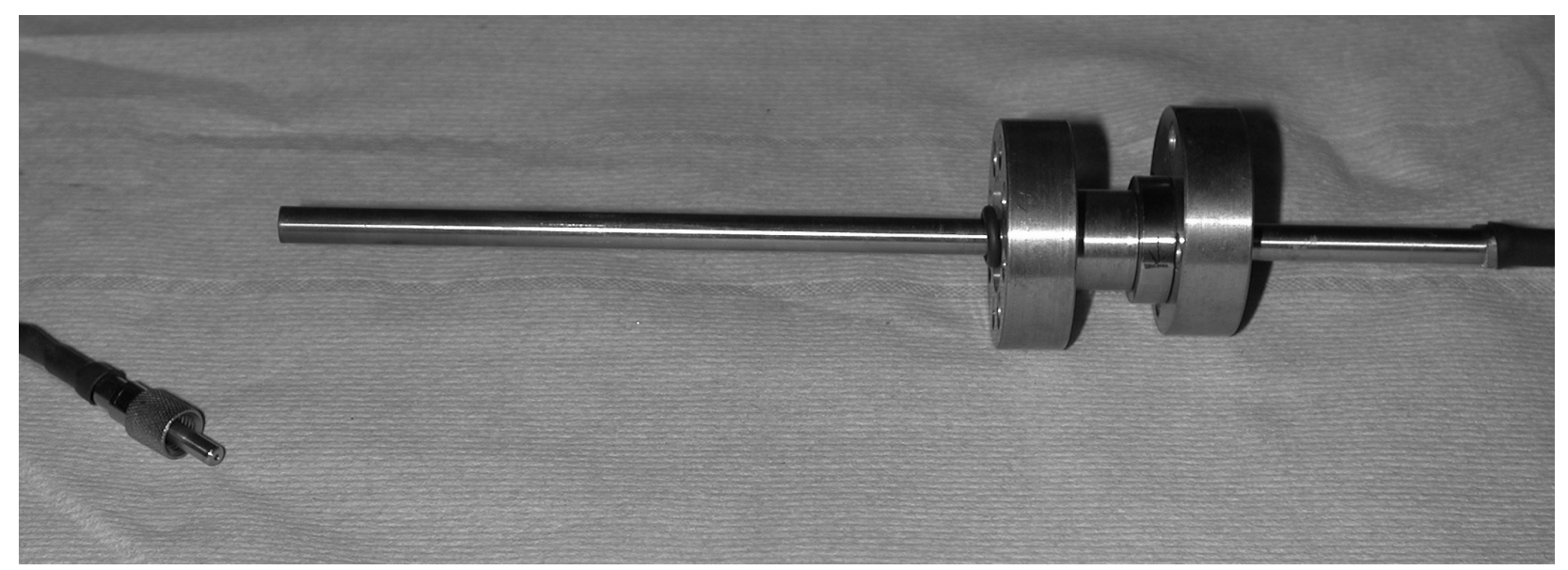

Abb. 3.8: Lichtleiter in Längsrichtung aufgenommen

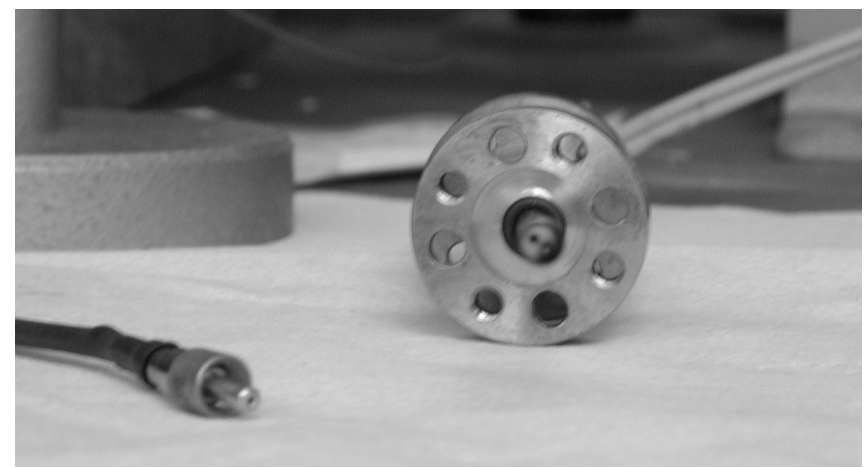

Abb. 3.9: Lichtleiter von der Flammenseite aufgenommen 
In der Abb. 3.9 kann man das Degussitrohr innerhalb des Metallrohres erkennen. In den beiden kleineren Löchern innerhalb des Degussitrohrs sind die Lichtleiterfasern untergebracht.

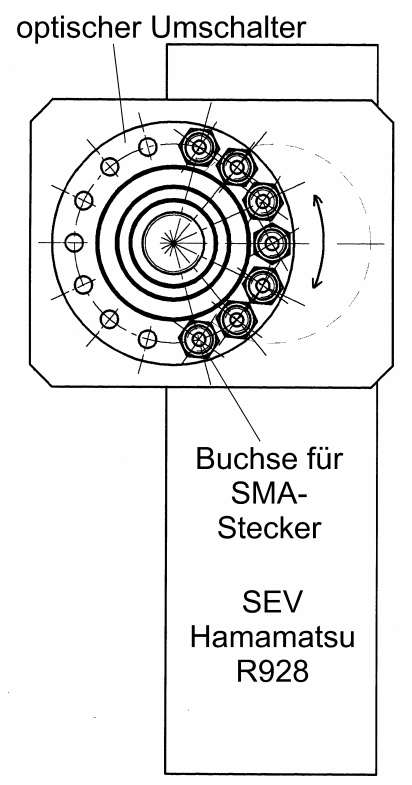

Abb. 3.10: $\quad$ Skizze des optischen Umschalters

\subsubsection{Eichung der Lichtleiter für die Messungen}

Die Lichtleiter wurden auf die Einsatzfähigkeit, quantitative Streulichtmessungen an rußenden Hochdruckflammen ausführen zu können, einem sorgfältigen Testprogramm unterzogen. Folgende Ergebnisse wurden für die einzelnen Lichtleiter erhalten:

- Räumliche Auflösung: a) horizontal: $8 \mathrm{~mm}$ (die Lichtleiterfasern liegen horizontal nebeneinander)

b) vertikal: $6 \mathrm{~mm}$

- Nachweisempfindlichkeit: Streuquerschnitt $\mathrm{Qvv}_{\mathrm{vv}}= \pm 2 * 10^{-9} \mathrm{~cm}^{-1} \mathrm{sterad}^{-1}$, dies entspricht einem Teilchenradius von $\mathrm{d}=6 \mathrm{~nm}$

- Reproduzierbarkeit: $\quad \sim 2 \%$

- Linearität des Signals, s. Abb. 3.11.

Die erhaltenen Resultate zeigen, daß jeder einzelne Lichtleiter für eine quantitativer Untersuchung pro festem Winkel einzusetzen ist, daß die Lichtleiter untereinander aber nicht die Bedingung $I_{n}\left(\Theta_{n}\right)=I_{n+1}\left(\Theta_{n+1}\right)$ mit $n=1$ bis 7 erfüllen. Die gefertigten Lichtleiter besitzen 
hinsichtlich der Lichtübertragung eine eigene Charakteristik. Mit Hilfe eines Eichverfahrens, das im folgendem kurz beschrieben werden soll, konnten die Lichtleiter aufeinander eingeeicht werden.

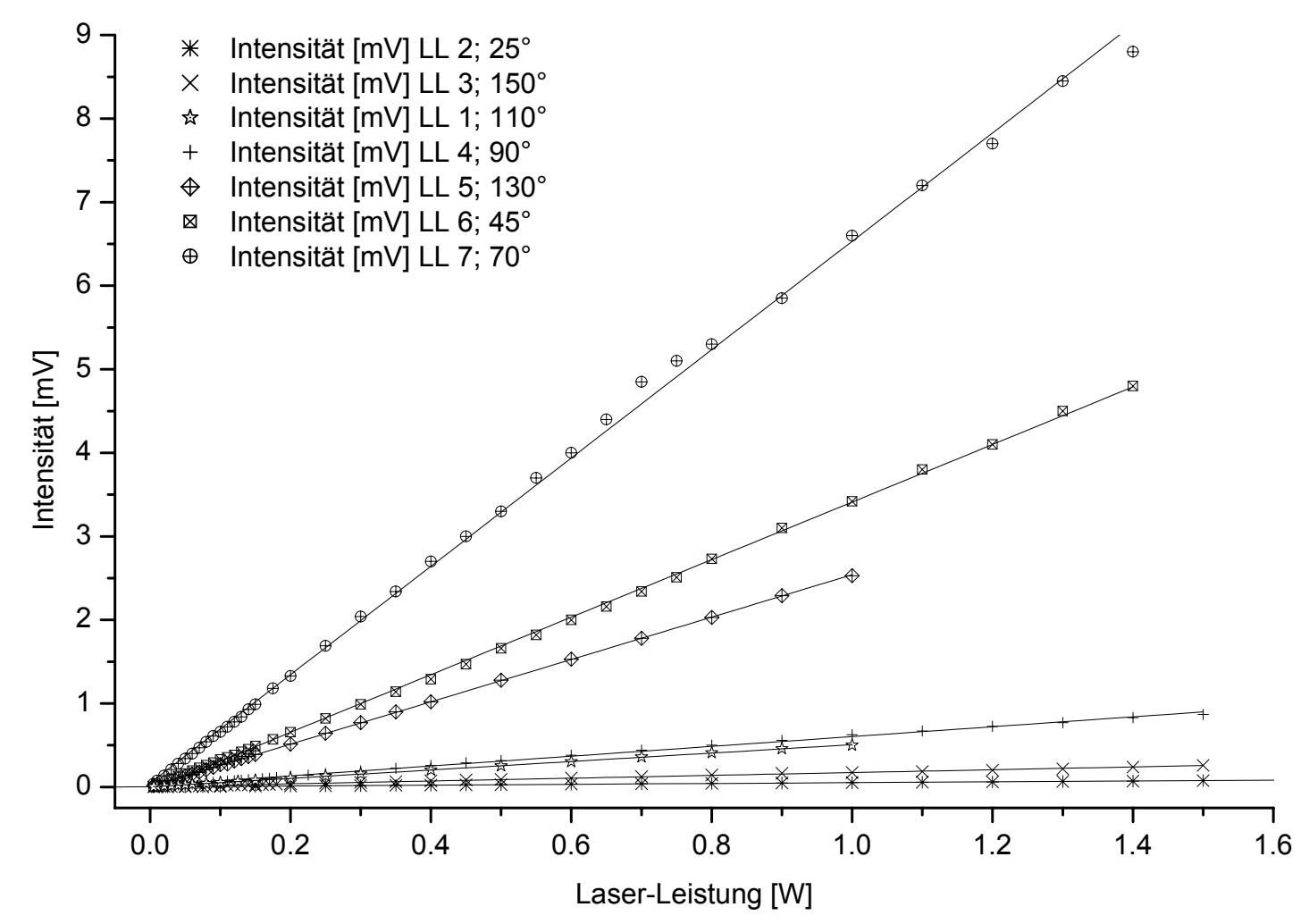

Abb. 3.11: Auftragung der Linearitätsmessung

Es wurde das Streusignal von Luft bei einem festen Winkel, hier $90^{\circ}$, von allen Lichtleitern aufgenommen. Derjenige Lichtleiter $\left(\operatorname{LL90^{\circ }}\right)$, der für die späteren Messungen bei $\Theta=90^{\circ}$ eingesetzt werden sollte war der Bezugslichtleiter und bekam einen Korrekturfaktor von 1. Das Verhältnis der Signalintensitäten aus den verschiedenen Lichtleitern I(LL $\Theta) /$ I(LL90 $\left.{ }^{\circ}\right)$ (dabei ist $\Theta$ der spätere Meßwinkel der jeweiligen Lichtleiter und die Verschraubung am optischen Umschalter blieb erhalten) war dann der Korrekturfaktor der einzelnen Lichtleiter.

Eine weitere Schwierigkeit, die bei der Durchführung der optischen Messungen auftrat, war die Hintergrundstrahlung im Brennerraum, hervorgerufen durch Streuung und Reflektionen des Laserstrahls. Sorgfältige Justierung und Änderungen im optischen Aufbau konnten diese Störstrahlung nicht restlos beseitigen. Der Anteil des Störstrahls konnte auf folgende Weise ermittelt werden: 
Vergleichende Messung an der 1 bar Anlage, bei der diese Art der Störstrahlung nicht auftrat (offenes System) ergaben, daß die Hintergrundsstrahlung bei $\Theta=90^{\circ}$ auch am Hochdruckbrenner vernachlässigbar war. Dies bedeutet, daß das Meßsignal bei $\Theta=90^{\circ}$ einem echten Streusignal ohne überlagerter Störstrahlung entspricht. Nach der RayleighTheorie ist der Streuwert an Gasen unabhängig vom Streuwinkel $\Theta$ und nur abhängig von der Sinusfunktion des Streuvolumens. Das hat zur Folge, daß die Streukurve mit Hilfe des bei $\Theta$ $=90^{\circ}$ gemessenen Wertes berechnet werden kann. Der Anteil der Störstrahlung ergibt sich dann aus der Differenz des tatsächlich gemessenen Signals und des berechneten Wertes.

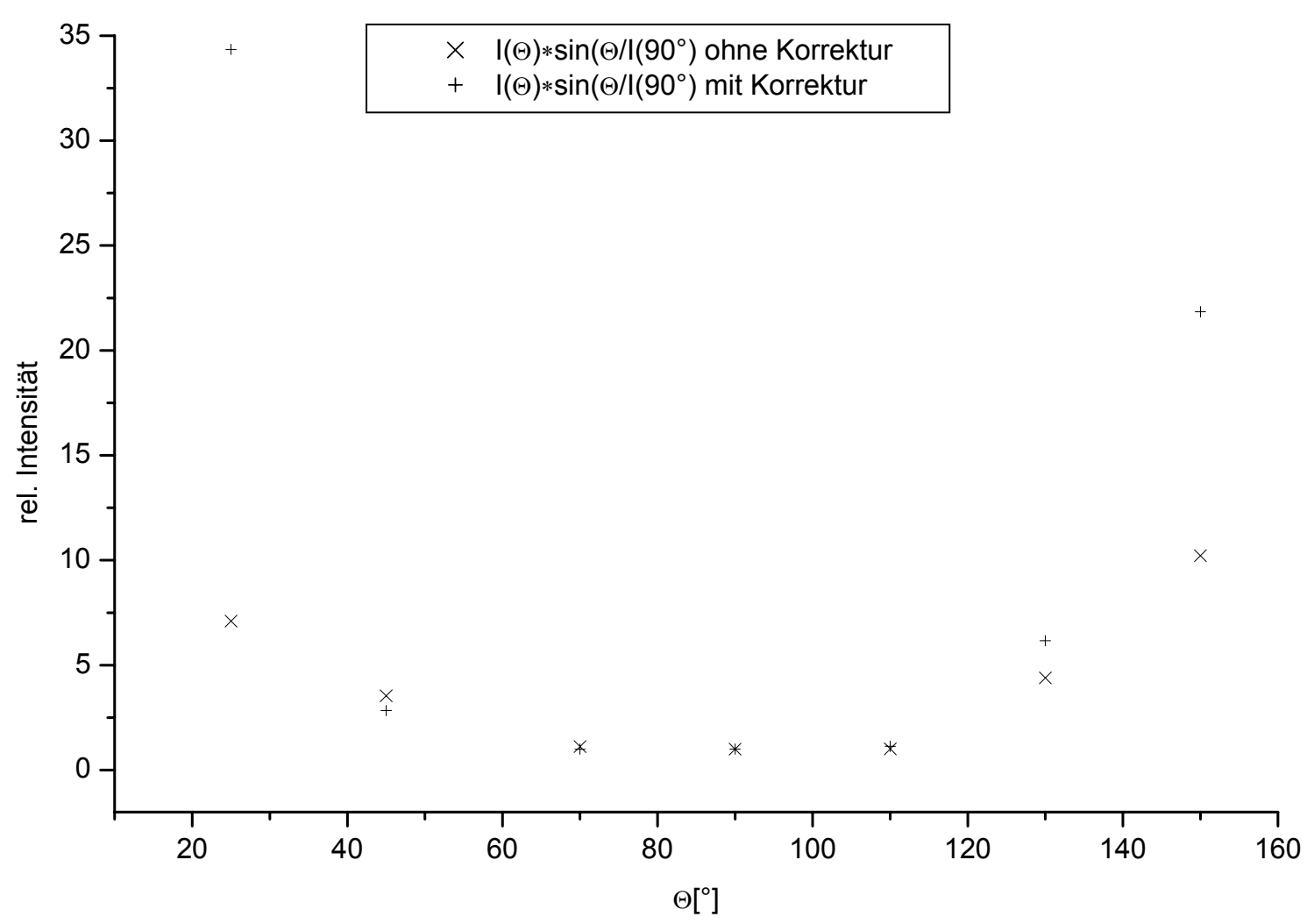

Abb. 3.12: Vergleich der normierten Streukurven mit und ohne Lichtleiterkorrektur

\subsubsection{Optische Anordnung der Hochdruckbrenneranlage}

Als Lichtquelle diente ein 4 Watt Argon-Ionen-Laser (Fa. Coherent Modell Innova 90). Der Laser erzeugte polarisiertes Licht bei einer gewählten Wellenlänge von $488 \mathrm{~nm}$ und einer Ausgangsleistung von 1 Watt. Der Laserstrahl wurde von einer rotierenden unterbrochenen Scheibe (Chopper) auf eine Frequenz von $150 \mathrm{~Hz}$ moduliert. Das Licht des Lasers wurde mit einer Linse (Achromat) mit einer Brennweite von $\mathrm{f}=300 \mathrm{~mm}$ (Fa. Spindler \& Hoyer) 
gebündelt. Eine Lochblende begrenzte den Lichtstrahl, damit der Eintritt durch das BrewsterFenster in die Brennkammer möglichst frei von Störstrahlen in den Randbereichen war. Hinter dem Brewster-Fenster befand sich ein Edelstahlrohr mit einem Durchmesser von $d=5$ mm, das Reflexionen des Lasertrahls in der Brennerkammer unterbinden sollte.

Für die Absorptionsmessung konnte der Strahl durch ein weiteres Brewster-Fenster mit einem davor angebrachten Edelstahlrohr aus der Brennerhaube austreten. Der Lichtstrahl wurde mit einer Kombination zweier Density-Filter und einer Lochblende abgeschwächt. Das transmittierte Laserlicht wurde hinter einem Laserlinefilter (Linienfilter, mit einer Halbwertsbreite bei $\lambda=488 \mathrm{~nm}$ von $1 \mathrm{~nm}$, Fa. Spindler \& Hoyer) mit einem SEV (Fa. Hamamatsu, Typ R928) detektiert. Ein Netzgerät (Fa. Nucletron, Hochspannungseinschub Typ NU1250B) lieferte die benötigten Hochspannungen von 700-800 Volt. Das Signal wurde von einem Lock-In-Verstärker (s. o.) verstärkt und von einem Schreiber (s. o.) festgehalten.

Winkelabhängige Streulichtmessungen konnten mit Hilfe der Lichtleiter in den Winkeln von $25^{\circ}, 45^{\circ}, 70^{\circ}, 90^{\circ}, 110^{\circ}, 130^{\circ}$ und $150^{\circ}$ ausgeführt werden. Die Signale der Lichtleiter wurden mit einem SEV mit optischem Umschalter nacheinander detektiert. Der SEV wurde mit Spannungen zwischen 800 und 1100 Volt betrieben. Das Signal wurde wie bei der Absorption beschrieben weitergeleitet.

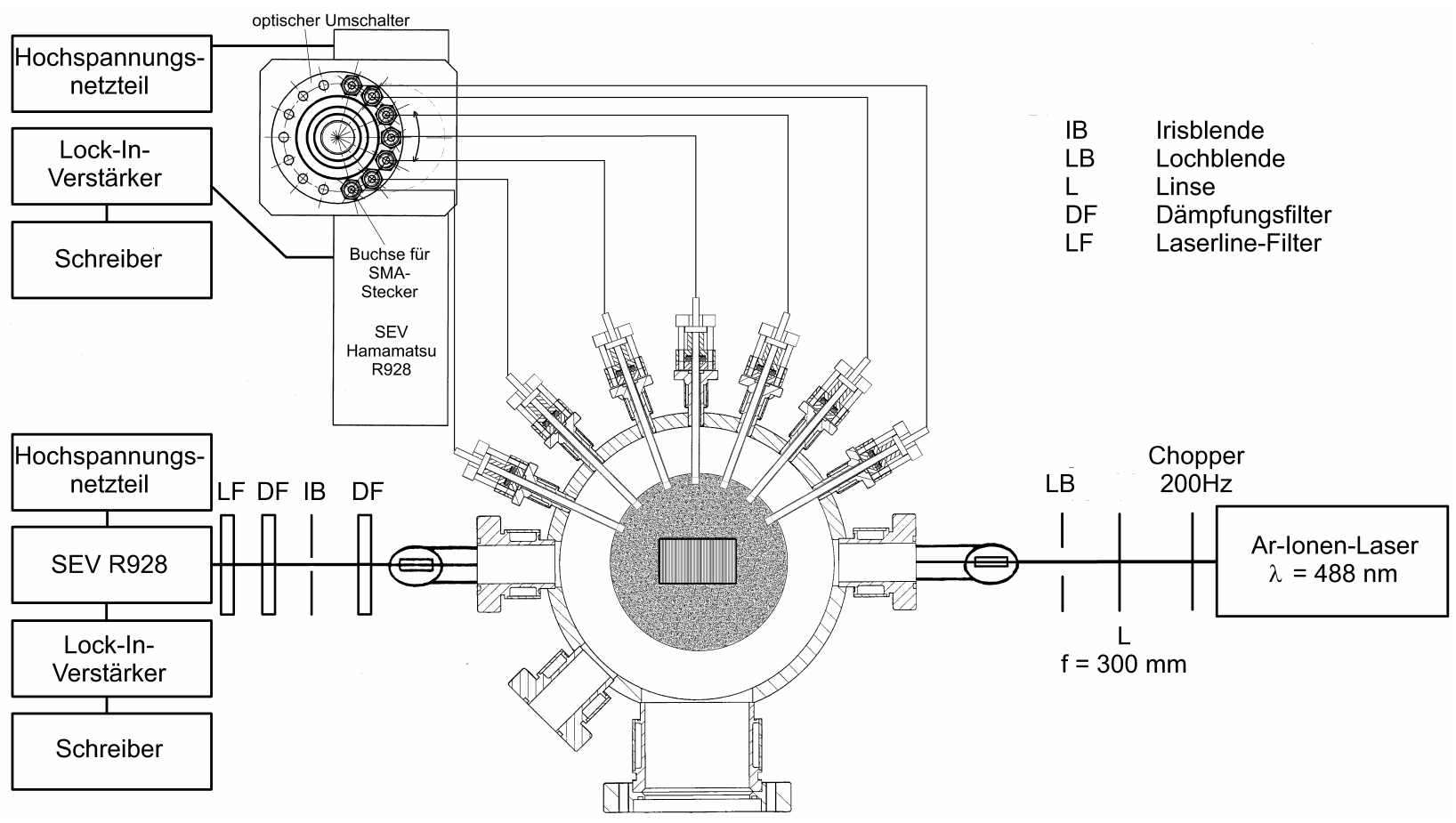

Abb. 3.13: $\quad$ Skizze der optischen Anordnung am Hochdruckbrenner 
4.1 Auswertung der 1 bar Flammen

\subsubsection{Zusammenfassung der Meßdaten}

In den folgenden Tabellen soll eine Übersicht über die mit dem Röhrchenbrenner (s. Kap. 3.1.1, S. 20ff) bei 1 bar untersuchten Flammen gegeben werden. Die vollständigen Übersichtstabellen mit allen ausgewerteten und in der Diskussion verwendeten Parametern (Rußvolumenbruch $f_{v}$, Teilchendurchmesser $d_{R}$, Teilchenzahldichte $N_{R}$, volumenäquivalenter Durchmesser $\mathrm{D}_{30}$, Aggregatenzahldichte $\mathrm{N}_{\mathrm{Agg}}$, scheinbarer Radius $\mathrm{R}_{\mathrm{g}}$, fraktale Dimension $\mathrm{D}_{\mathrm{f}}$, Primärteilchendurchmesser $d_{p}$ und Primärteilchenzahldichte $n_{p}$ ) sind im Anhang (Kap. 7.1.1) beigefügt. Die Sortierung erfolgt nach steigender Frischgasgeschwindigkeit.

\subsubsection{1 n-Pentan}

\begin{tabular}{|l|l|l|l|}
\hline $\begin{array}{l}\text { Frischgasgeschwindigkeit } \\
{[\mathrm{cm} / \mathrm{s}]}\end{array}$ & C/O-Verhältnis & $\begin{array}{l}\text { Höhe über dem } \\
\text { Brenner }[\mathrm{mm}]\end{array}$ & Messung-Nr. \\
\hline 4.0 & 0.66 & $14.2 ; 16.3 ; 19.2$ & 0003141 \\
\hline 4.4 & 0.68 & $10.2 ; 15.2 ; 19.2 ; 22.2$ & 0003081 \\
\hline 4.6 & 0.60 & $11.3 ; 14.3 ; 17.2$ & 0003101 \\
\hline 4.6 & 0.60 & $11.9 ; 15.0 ; 18.1$ & 0003131 \\
\hline 5.4 & 0.68 & $9.4 ; 12.4 ; 16.4 ; 19.1$ & 0003061 \\
\hline 5.4 & 0.68 & $8.1 ; 11.2 ; 14.2 ; 18.2$ & 0003062 \\
\hline
\end{tabular}

Tab. 4.1: Röhrchenbrenner, n-Pentan-Messungen

Die vollständige Tabelle befindet sich im Anhang 7.1.1.1.

\subsubsection{2 n-Heptan}

\begin{tabular}{|l|l|l|l|}
\hline $\begin{array}{l}\text { Frischgasgeschwindigkeit } \\
{[\mathrm{cm} / \mathrm{s}]}\end{array}$ & C/O-Verhältnis & $\begin{array}{l}\text { Höhe über dem } \\
\text { Brenner }[\mathrm{mm}]\end{array}$ & Messung-Nr. \\
\hline 3.8 & 0.54 & $13.8 ; 17.6$ & 0003172 \\
\hline 3.8 & 0.65 & $12.0 ; 15.8 ; 19.2$ & 0003171 \\
\hline 4.9 & 0.63 & $11.2 ; 15.2 ; 18.2 ; 21.4$ & 0003161 \\
\hline 6.1 & 0.59 & $16.8 ; 21.9$ & 0003173 \\
\hline
\end{tabular}

Tab. 4 .2: $\quad$ Röhrchenbrenner, n-Heptan-Messungen 
Die vollständige Tabelle sowie einige Auftragungen befinden sich im Anhang 7.1.1.2.

\subsubsection{Isooktan}

\begin{tabular}{|l|l|l|l|}
\hline $\begin{array}{l}\text { Frischgasgeschwindigkeit } \\
{[\mathrm{cm} / \mathrm{s}]}\end{array}$ & $\mathrm{C} / \mathrm{O}-$ Verhältnis & Höhen $[\mathrm{mm}]$ & Messung-Nr. \\
\hline 3.5 & 0.62 & $13.8 ; 16.8 ; 19.8 ; 22.6$ & 0003221 \\
\hline 3.9 & 0.55 & $14.9 ; 18.9$ & 0003232 \\
\hline 4.7 & 0.62 & $14.3 ; 18.7 ; 21.7 ; 24.7$ & 0003231 \\
\hline 4.8 & 0.67 & $22.9 ; 26.9$ & 0003233 \\
\hline
\end{tabular}

Tab. 4.3: $\quad$ Röhrchenbrenner, Messungen mit Isooktan

Die vollständige Tabelle sowie einige Auftragungen befinden sich im Anhang 7.1.1.3.

\subsubsection{Propen}

Die Messungen mit Propen erfolgten zu Vergleichszwecken mit den Brennstoffen Benzol und Ethylen im Hochdruck sowie den Daten in der Diplomarbeit.

\begin{tabular}{|l|l|l|l|}
\hline $\begin{array}{l}\text { Frischgasgeschwindigkeit } \\
{[\mathrm{cm} / \mathrm{s}]}\end{array}$ & C/O-Verhältnis & Höhe $[\mathrm{mm}]$ & Messung-Nr. \\
\hline 6.2 & 0.71 & 16.7 & prop001 \\
\hline 5.2 & 0.71 & 16.7 & prop002 \\
\hline 4.3 & 0.71 & 16.7 & prop003 \\
\hline 3.7 & 0.71 & 16.7 & prop000 \\
\hline 8.4 & 0.69 & 16.7 & prop005 \\
\hline 8.3 & 0.67 & 16.7 & prop005a \\
\hline 7.3 & 0.72 & 16.7 & prop004 \\
\hline 7.4 & 0.70 & 16.7 & prop004a \\
\hline 7.4 & 0.70 & 16.7 & prop004b \\
\hline 6.3 & 0.65 & 16.7 & prop006 \\
\hline 6.3 & 0.75 & 16.7 & prop007 \\
\hline 4.3 & 0.71 & 16.7 & prop008 \\
\hline 4.3 & 0.71 & 24.4 & prop008a \\
\hline 4.3 & 0.71 & 24.4 & prop008a \\
\hline 4.3 & 0.71 & 16.7 & prop009 \\
\hline 4.3 & 0.71 & 11.5 & prop009a \\
\hline 5.2 & 0.71 & 16.7 & prop010_1 \\
\hline 5.2 & 0.71 & 16.7 & prop010_2 \\
\hline 5.2 & 0.71 & 16.7 & prop010_3 \\
\hline 5.2 & 0.71 & 24.3 & prop010a \\
\hline 6.2 & 0.71 & 16.7 & prop011_1 \\
\hline & & & \\
\hline
\end{tabular}




\begin{tabular}{|l|l|l|l|}
\hline 6.2 & 0.71 & 16.7 & prop011_2 \\
\hline 7.4 & 0.70 & 16.7 & prop012 \\
\hline 4.8 & 0.71 & 16.7 & prop013 \\
\hline 5.7 & 0.71 & 16.7 & prop014_1 \\
\hline 5.7 & 0.71 & 16.7 & prop014_2 \\
\hline
\end{tabular}

Tab. 4.4: Röhrchenbrenner, Messungen mit Propen

Die vollständige Tabelle sowie einige Auftragungen befinden sich im Anhang

\subsubsection{Benzol}

\begin{tabular}{|l|l|l|l|}
\hline $\begin{array}{l}\text { Frischgasgeschwindigkeit } \\
{[\mathrm{cm} / \mathrm{s}]}\end{array}$ & C/O-Verhältnis & Höhe $[\mathrm{mm}]$ & Messung-Nr. \\
\hline 6.2 & 0.71 & & 011130 \\
\hline 6.6 & 0.68 & 13.1 & 011214 \\
\hline 6.6 & 0.68 & 17.7 & 011217 \\
\hline 5.6 & 0.68 & 17.7 & 011221 \\
\hline 7.6 & 0.68 & 17.7 & 020103 \\
\hline 7.6 & 0.68 & 17.7 & $020103 \_2$ \\
\hline 7.1 & 0.68 & 17.7 & $020103 \mathrm{a}$ \\
\hline 7.1 & 0.68 & 17.7 & $020103 \mathrm{a} 22$ \\
\hline 4.6 & 0.68 & 17.7 & 020108 \\
\hline 4.6 & 0.68 & 17.7 & $020108 \_2$ \\
\hline 3.8 & 0.68 & 17.7 & $020108 \mathrm{a}$ \\
\hline 3.8 & 0.68 & 17.7 & $020108 \mathrm{a} 22$ \\
\hline 5.1 & 0.68 & 17.7 & 020115 \\
\hline 5.1 & 0.68 & 17.7 & $020115 \mathrm{a}$ \\
\hline 6.1 & 0.68 & 17.7 & $020115 \_2$ \\
\hline 6.1 & 0.68 & 17.7 & $020115 \_2 \mathrm{a}$ \\
\hline 6.1 & 0.68 & 8.0 & $020115 \_3$ \\
\hline 5.1 & 0.75 & 17.7 & 020118 \\
\hline 5.1 & 0.75 & 17.7 & $020118 \mathrm{a}$ \\
\hline 5.1 & 0.72 & 17.7 & $020118 \_2$ \\
\hline 5.1 & 0.72 & 17.7 & $020118 \_2 \mathrm{a}$ \\
\hline 5.1 & 0.63 & 17.7 & $020118 \_3$ \\
\hline 5.1 & 0.63 & 17.7 & $020118 \_3 \mathrm{a}$ \\
\hline 5.1 & 0.72 & 9.1 & $020118 \_4$ \\
\hline 5.1 & 0.72 & 9.1 & $020118 \_4 \mathrm{a}$ \\
\hline 5.1 & 0.72 & 13.3 & $020118 \_5$ \\
\hline & & \\
\hline
\end{tabular}

Tab. 4.5: Röhrchenbrenner, Messungen mit Benzol

Die vollständige Tabelle befindet sich im Anhang. Die Beschreibung dieser Flammen erfolgt im nächsten Abschnitt (Kap. 4.1.2). 


\subsubsection{Detaillierte Darstellung der 1 bar Flammen am Beispiel der Benzolflamme}

\subsubsection{Erscheinungsbild der Benzolflamme}

Untersucht wurden vorgemischte laminare Benzol-Luft-Flammen mit Frischgasgeschwindigkeiten von $3.75 \mathrm{~cm} / \mathrm{s}$ und $7.6 \mathrm{~cm} / \mathrm{s}$. Es wurde ein C/O-Verhältnis von 0.68 gewählt. Bei einer Frischgasgeschwindigkeit von $5.1 \mathrm{~cm} / \mathrm{s}$ wurde das $\mathrm{C} / \mathrm{O}-$ Verhältnis variiert. Alle Flammen wurden mit einer nichtrußenden Ethylen-Luft-Flamme des Außenbrenners mit einer Frischgasgeschwindigkeit von etwa $10 \mathrm{~cm} / \mathrm{s}$ stabilisiert, um eine möglichst homogene Benzolflamme zu erhalten. Eine weitere Stabilisierung wurde durch ein Drahtnetz in $7 \mathrm{~cm}$ Höhe über dem Brenner erreicht. Die langsamste Flamme weicht wie in Abb. 4.1 dargestellt schon deutlich von einer zylindrischen Form ab und ist kegelförmig. Die leuchtend gelbe Rußzone beginnt in $2.9 \mathrm{~mm}$ Höhe.

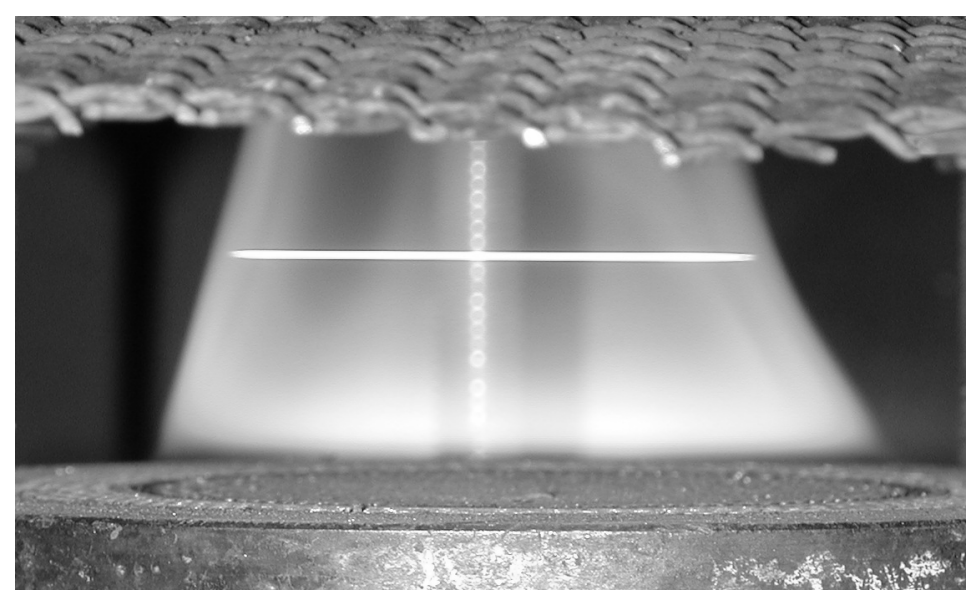

Abb. 4.1: $\quad$ Benzol-Luft-Flamme mit $\mathrm{v}=3.75 \mathrm{~cm} / \mathrm{s}$ und $\mathrm{C} / \mathrm{O}=0.68$; (Der horizontale weiße Strich ist der Laserstrahl; der vertikale Strich wird durch Reflektion an einer Halterungsschraube verursacht)

Ab einer Frischgasgeschwindigkeit von $5.1 \mathrm{~cm} / \mathrm{s}$ ist die Form fast zylindrisch, wie in der Abb. 4.2 dargestellt. Die Rußzone beginnt hier in $2.7 \mathrm{~mm}$ Höhe 


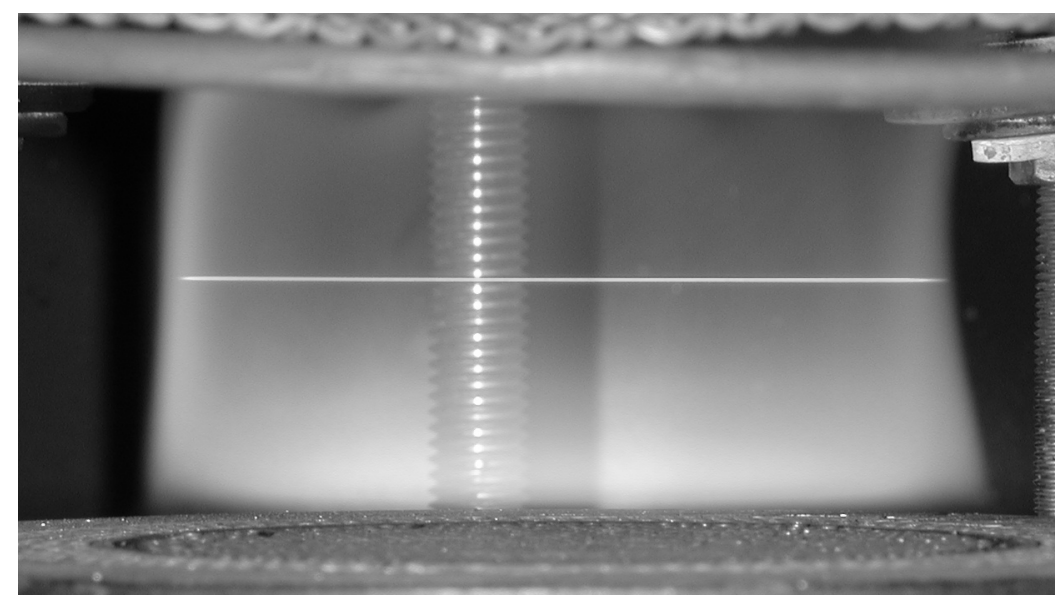

Abb. 4.2: $\quad$ Benzol-Luft-Flamme mit $\mathrm{v}=5.1 \mathrm{~cm} / \mathrm{s}$ und $\mathrm{C} / \mathrm{O}=0.68$

Mit einer Frischgasgeschwindigkeit von $6.1 \mathrm{~cm} / \mathrm{s}$ brannte die Benzolflamme in einer zylindrischen Form, wie in Abb. 4.3 zu sehen ist.

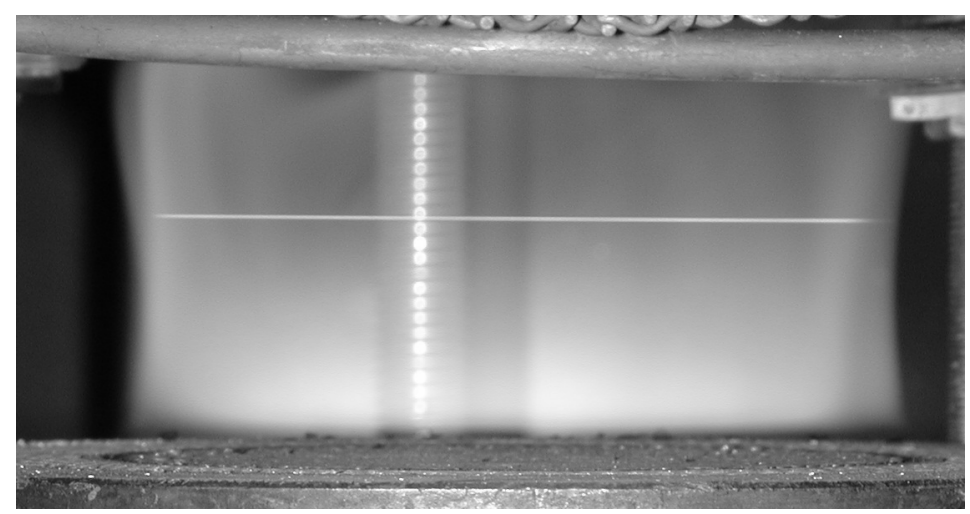

Abb. 4.3: $\quad$ Benzol-Luft-Flamme mit $\mathrm{v}=6.1 \mathrm{~cm} / \mathrm{s}$ und $\mathrm{C} / \mathrm{O}=0.68$

Die Rußzone ist ab einer Höhe von $2.2 \mathrm{~mm}$ zu erkennen.

Mit noch höheren Durchsätzen zieht sich die leuchtende Zone der Flammen mit der Höhe leicht kegelförmig auseinander, da das Drahtnetz für diese Flammen einen zu großen Widerstand darstellt und die Flammen deshalb etwas gestaucht werden. Die Rußzone liegt mit steigender Geschwindigkeit immer dichter an der Brenneroberfläche. Ein höheres C/OVerhältnis hat auf die untere Grenze des Rußgebiets nur einen geringen Effekt, wie die Abb. 4.4 zeigt. 


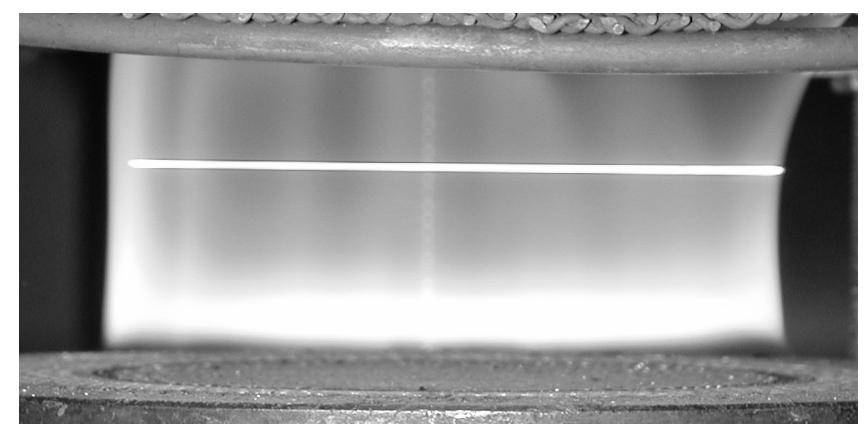

Abb. 4.4: Benzol-Luft-Flamme mit $\mathrm{v}=5.1 \mathrm{~cm} / \mathrm{s}$ und einem $\mathrm{C} / \mathrm{O}-$ Verhältnis von 0.75

In der Abb. 4.4 ist das deutlich stärkere Leuchten der fetteren Flamme mit einem C/OVerhältnis von $0.75 \mathrm{im}$ Vergleich mit der Flamme mit einem C/O-Verhältnis von 0.68 (Abb. 4.2) zu erkennen.

Geringere C/O-Verhältnisse bewirken aber ein Absinken der Rußzone in Richtung Brenneroberfläche hin, da die Flammen heißer werden. In der Abb. 4.5 ist eine Benzolflamme mit einem C/O-Verhältnis von 0.63 dargestellt. Der Beginn der Rußzone liegt hier bei 1.7 $\mathrm{mm}$.

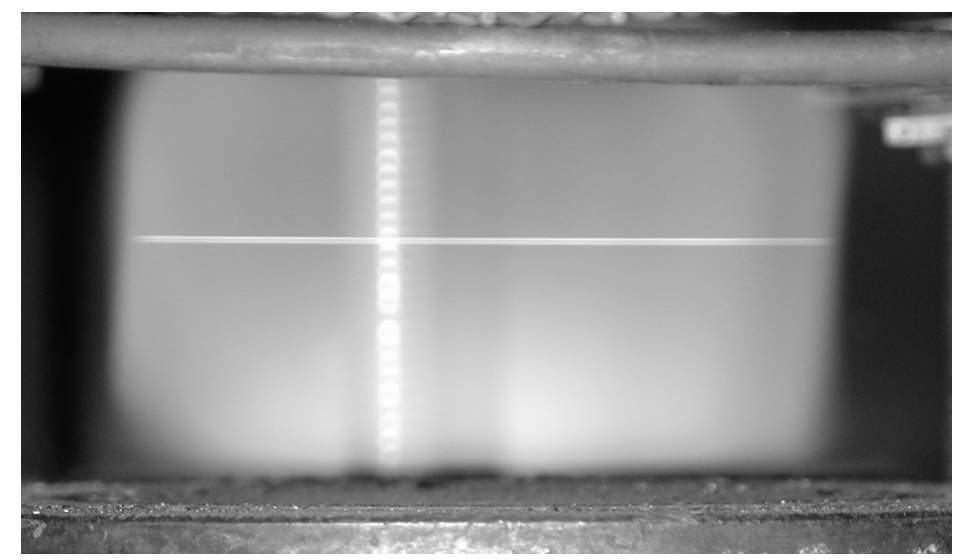

Abb. 4.5: Benzol-Luft-Flamme mit $\mathrm{v}=5.1 \mathrm{~cm} / \mathrm{s}$ und einem $\mathrm{C} / \mathrm{O}-$ Verhältnis von 0.63

Bei den Flammen bis $\mathrm{C} / \mathrm{O}=0.68$ sind mit bloßem Auge die bläulichen Hauptoxidationszonen $\mathrm{zu}$ erkennen. Auf den Fotografien ist dies aber wegen der Blendwirkung der Rußzonen meistens nicht mehr zu erkennen. Die Hauptoxidationszone liegt bei einem C/O-Verhältnis von 0.68 etwa $1 \mathrm{~mm}$ unterhalb der Rußzone und ist nur ein sehr schmaler Streifen mit einer vertikalen Ausdehnung von $1 \mathrm{~mm}$. Mit steigendem $\mathrm{C} / \mathrm{O}-\mathrm{Verhältnis}$ ist die Hauptoxidationszone nicht mehr zu erkennen. 
Stabile Benzol-Luft-Flammen konnten mit dem verwendeten Brenner nur in einem Bereich (s. auch Kap. 4.1.2.2; Abb. 4.6) von Frischgasgeschwindigkeiten $\left(3.5 \mathrm{~cm} / \mathrm{s} \leq \mathrm{v}_{\mathrm{FG}} \leq 8 \mathrm{~cm} / \mathrm{s}\right)$ und $\mathrm{C} / \mathrm{O}-$ Verhältnissen $(0.58 \leq \mathrm{C} / \mathrm{O} \leq 0.83)$ realisiert werden. Von einer stabilen Flamme spricht man, wenn diese keine Strukturen wie Wellen oder Dächer aufweist, außerdem darf eine stabile Flamme nicht von der Brenneroberfläche abheben und nur noch über das Drahtnetz in Richtung Brenner gedrückt sein. Die Grenze der stabilen Flammen zu höheren Geschwindigkeiten und $\mathrm{C} / \mathrm{O}-$ Verhältnissen wird als Stabilitätsgrenze bezeichnet. Die Stabilitätsgrenze von Benzol-Luft-Flammen ist in Kap. 4.1.2.2 in der Abb. 4.6 aufgetragen. Zum Vergleich wurden Werte für gerade instabil werdende Flammen, die aus einer anderen Arbeit [30] unter Verwendung desselben Brenners stammen, dargestellt. Beide Messungen zeigen übereinstimmend die Begrenzung des Bereichs stabiler Flammen bei $\mathrm{C} / \mathrm{O}-$ Verhältnissen zwischen 0.74 und 0.84. Eine stabile Flamme mit dem höchsten C/O-Verhältnis von etwa 0.84 kann bei einer Frischgasgeschwindigkeit von $5 \mathrm{~cm} / \mathrm{s}$ erreicht werden.

\subsubsection{Rußgrenzen der Benzolflamme}

Rußteilchen zeigen in vorgemischten Flammen eine breitbandige Lichtemission mit einer für das menschliche Auge gelb bis leicht rötlich erscheinenden Farbe. Die brennstoffärmste Gemischzusammensetzung, bei der die Rußbildung einsetzt, wird Rußgrenze genannt. Das dazugehörige C/O-Verhältnis wird als kritisches C/O-Verhältnis bezeichnet. Die Rußgrenzen wurden mit bloßem Auge bei vollständig abgedunkelter Umgebung ermittelt. Das menschliche Auge kann das Rußleuchten ab einem Rußvolumenbruch von $10^{-9}\left[\mathrm{~cm}^{3} / \mathrm{cm}^{3}\right]$ wahrnehmen. Diese Grenze hängt aber stark vom individuellen Sehvermögen des Beobachters ab. Die Rußgrenze ist außerdem stark temperaturabhängig und deshalb für jeden Brenner verschieden.

In der Abb. 4.6 ist die Rußgrenze in Abhängigkeit von der Frischgasgeschwindigkeit und vom $\mathrm{C} / \mathrm{O}-$ Verhältnis aufgetragen. 


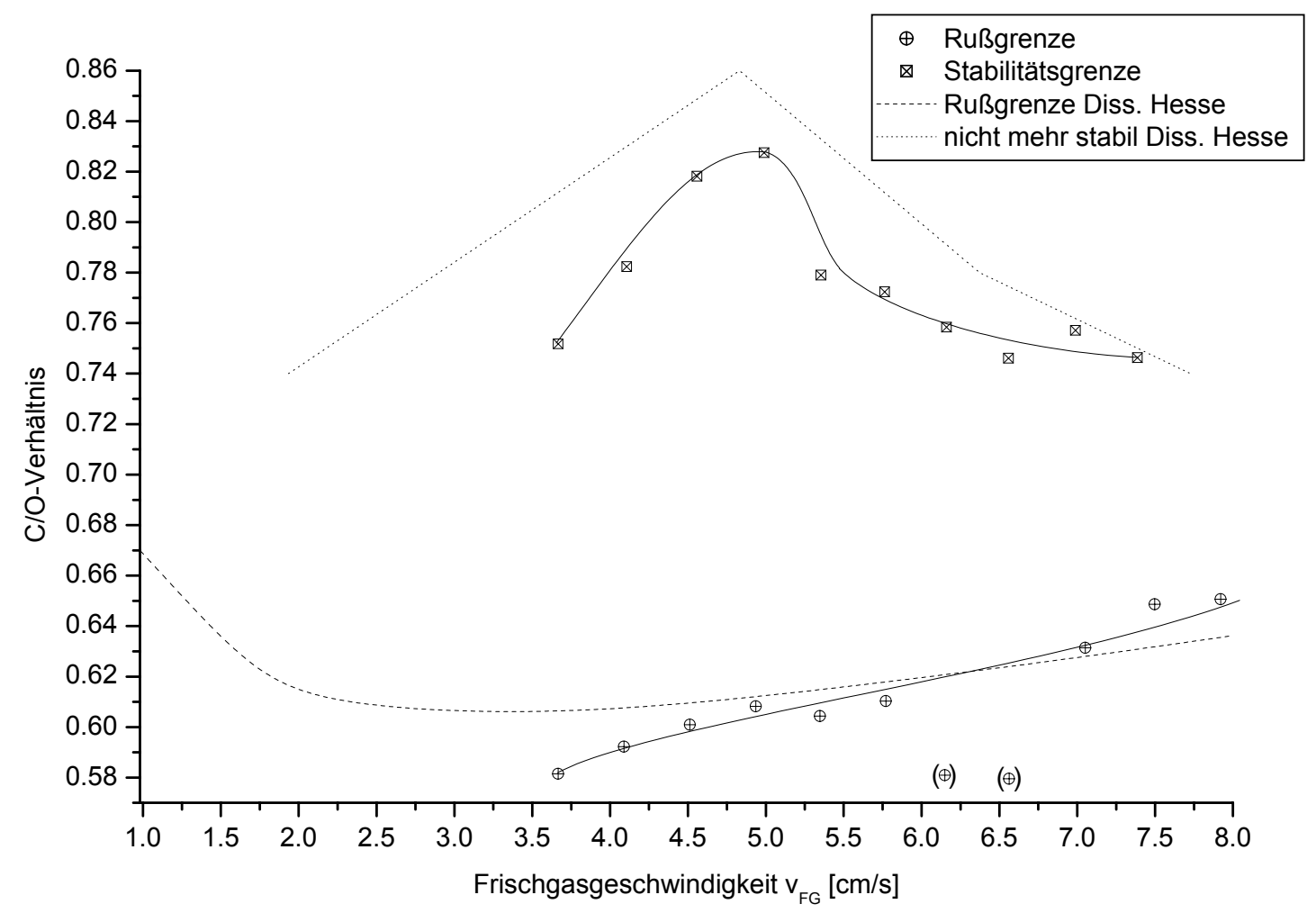

Abb. 4.6: Rußgrenze und Stabilitätsgrenze von Benzol-Luft-Flammen bei 1 bar

Die durchgezogene Linie gibt eine Vergleichsmessung aus einer anderen Arbeit [30] wieder. Da beide Messungen mit demselben Brenner durchgeführt wurden, liegen die ermittelten Rußgrenzen dicht zusammen. Die Rußgrenze fällt mit steigendem Durchsatz zunächst annähernd linear $a b$, durchläuft bei einer Frischgasgeschwindigkeit von $3.25 \mathrm{~cm} / \mathrm{s}$ ein Minimum, um anschließend wieder linear, aber etwas flacher, anzusteigen. Der Ast mit den geringen Geschwindigkeiten wurde in dieser Arbeit nicht erneut ermittelt, da die Vergleichswerte vorlagen. Ein C/O-Verhältnis von etwa 0.59 ist das geringste kritische C/OVerhältnis für das Auftreten von Ruß. Benzol-Luft-Flammen mit einem C/O-Verhältnis von 0.68 und höher rußen im untersuchten Bereich bei 1 bar immer.

\subsubsection{Flammentemperaturen der Benzolflammen nach Kurlbaum}

Die Flammentemperaturen der Benzol-Luft-Flammen wurden nach der Kurlbaum-Methode ermittelt [26]. Mit dieser Methode war es möglich, für fast alle optisch untersuchten BenzolLuft-Flammen die Flammentemperatur zu bestimmen. Nur die magerste eingestellte Flamme mit einem $\mathrm{C} / \mathrm{O}-$ Verhältnis von $\mathrm{C} / \mathrm{O}=0.63$ sowie die schnellste untersuchte Flamme mit einer 
Frischgasgeschwindigkeit von $7.6 \mathrm{~cm} / \mathrm{s}$ ließen die Temperaturbestimmung nach Kurlbaum nicht zu. Diese Werte können aber anhand der homologen Reihen von Flammen mit unterschiedlichem C/O-Verhältnissen bzw. Frischgasgeschwindigkeiten sehr gut extrapoliert werden. Außerdem lieferte die langsamste untersuchte Flamme mit einer Frischgasgeschwindigkeit von $\mathrm{v}=3.75 \mathrm{~cm} / \mathrm{s}$ nur ungenaue Resultate, da diese Flamme nicht stabil genug war. Die Kurlbaum-Methode beruht auf dem Prinzip der Teilchenstrahlung und kann daher die Flammentemperaturen nur im rußenden Abgas erfassen. Damit fehlen die maximalen Flammentemperaturen der Hauptoxidationszone.

\section{Höhenabhängigkeit der Flammentemperatur}

Die Flammentemperaturen aller untersuchten Benzol-Luft-Flammen nehmen in der Rußzone etwa linear mit der Höhe ab. Die Flammentemperaturprofile verlaufen in geringen Höhen mit zunehmender Frischgasgeschwindigkeit immer flacher (Abb. 4.8). So fällt die Flammentemperatur einer Benzol-Luft-Flamme mit zunehmender Höhe bei einer Frischgasgeschwindigkeit $\mathrm{v}_{\mathrm{FG}}=7.1 \mathrm{~cm} / \mathrm{s}$ mit $12 \mathrm{~K} / \mathrm{mm}$. Die Flammentemperatur einer Benzol-Luft-Flamme mit einer Frischgasgeschwindigkeit von $\mathrm{v}_{\mathrm{FG}}=4.6 \mathrm{~cm} / \mathrm{s}$ fällt mit 45 $\mathrm{K} / \mathrm{mm}$ fast viermal so stark. Mit niedrigeren $\mathrm{C} / \mathrm{O}$-Verhältnissen hingegen ist die Steigung etwas flacher als bei fetteren Flammen, wie in der Abb. $4.7 \mathrm{zu}$ erkennen ist, da bei rußenden Flammen zusätzlich zur Wärmestrahlung auch die Teilchenstrahlung proportional mit dem $\mathrm{C} / \mathrm{O}-$ Verhältnis an Bedeutung gewinnt. Liegen die Flammentemperaturen zweier Flammen mit unterschiedlichen $\mathrm{C} / \mathrm{O}-$ Verhältnissen von $\mathrm{C} / \mathrm{O}=0.68 \mathrm{bzw} . \mathrm{C} / \mathrm{O}=0.75$ in $4.5 \mathrm{~cm}$ Höhe nur um $50 \mathrm{~K}$ auseinander so sind es in $18 \mathrm{~cm}$ Höhe bereits etwa $200 \mathrm{~K}$. Dies ist mit der erhöhten Teilchenstrahlung in fetten Flammen $\mathrm{zu}$ erklären. Bei hohen Reaktionszeiten und kohlenstoffreicheren Gemischzusammensetzungen flachen die linearen Kurvenverläufe aber etwas ab. Dies kann in den hohen Rußkonzentrationen begründet sein, die zu einer größeren Wärmekapazität durch die große Zahl an Rußteilchen führt und deshalb das Verhältnis von abgestrahlter und im System verbleibender Wärme verschiebt.

\section{$\underline{\text { Abhängigkeit der Flammentemperatur vom C/O-Verhältnis }}$}

Mit steigendem C/O-Verhältnis nimmt die Flammentemperatur im untersuchten Bereich von $\mathrm{C} / \mathrm{O}=0.68$ bis $\mathrm{C} / \mathrm{O}=0.75$ ab. Die Gemischzusammensetzung mit dem höchsten Temperaturprofil liegt damit außerhalb der in dieser Arbeit mit der Kurlbaum-Methode untersuchten Flammen. In einer anderen Arbeit [30] wird für die größte maximale Flammentemperatur einer rußenden Benzol-Luft-Flamme ein $\mathrm{C} / \mathrm{O}-$ Verhältnis von $\mathrm{C} / \mathrm{O}_{\max }=$ 
0.66 angegeben. Damit nehmen die Flammentemperaturen für eine Höhe $(\mathrm{h}=$ const.) mit steigendem C/O-Verhältnis im gesamten Rußgebiet ab. Von einer für Benzol-Luft-Flammen stöchiometrischen Gemischzusammensetzung von $\mathrm{C} / \mathrm{O}_{\text {stöch }}=0.40$ nehmen die Flammentemperaturen zunächst im nichtrußenden Teil und dann noch etwas im leicht rußenden Teil bis zu einem C/O-Verhältnis von 0.66 zu. Dieser Verlauf ist nach [30] auch bei Ethylen-Luft-Flammen zu beobachten $\left(\mathrm{C} / \mathrm{O}_{\text {stöch }}=0.33 ; \mathrm{C} / \mathrm{O}_{\text {krit }}=0.60\right.$ und $\left.\mathrm{C} / \mathrm{O}_{\max }=0.65\right)$.

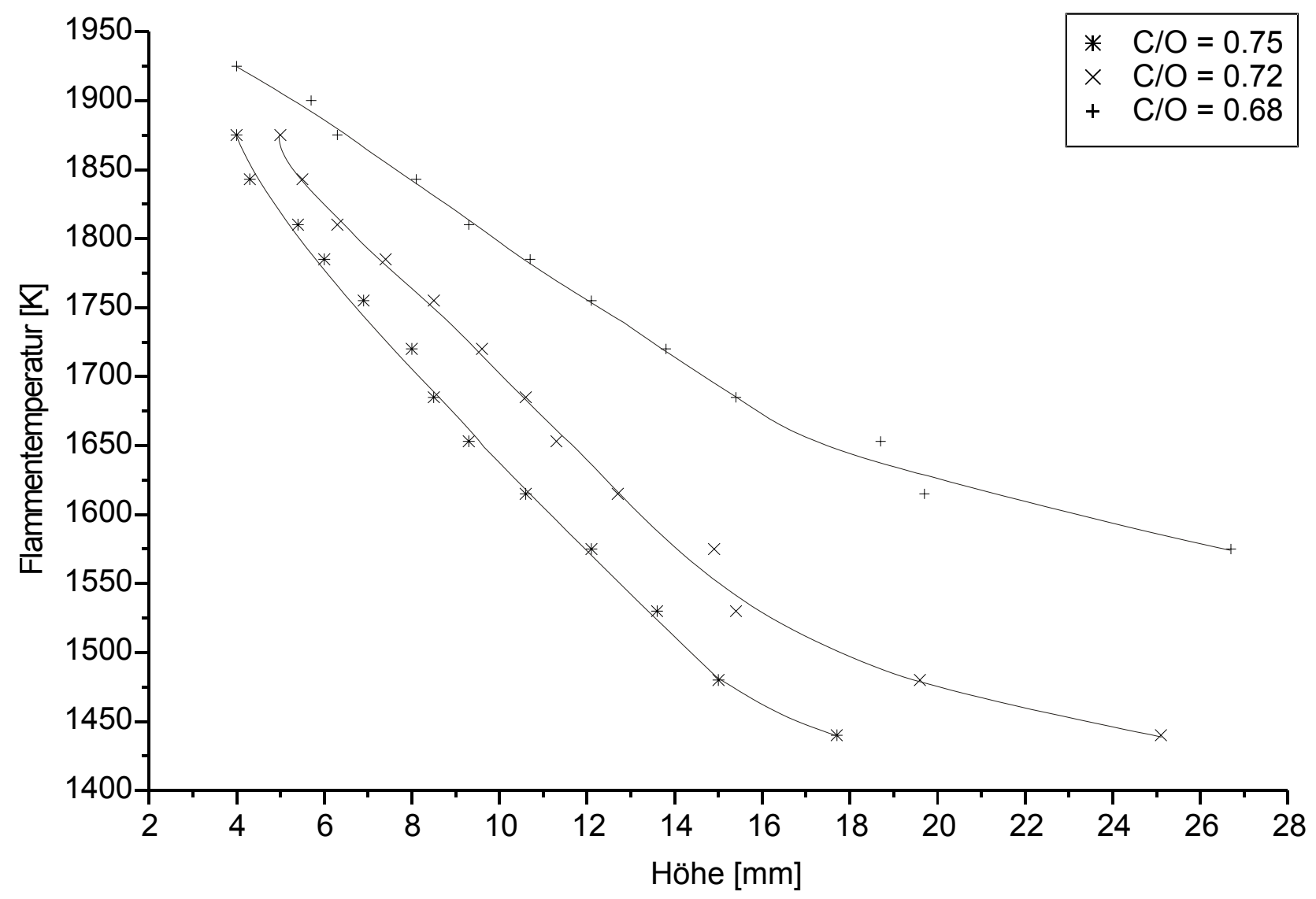

Abb. 4.7: Flammentemperaturprofile von Benzol-Luft-Flammen unterschiedlicher C/OVerhältnisse bei einer Frischgasgeschwindigkeit von $5.1 \mathrm{~cm} / \mathrm{s}$

Durchsatzabhängigkeit der Flammentemperaturen

In der Abb. 4.8 sind die Flammentemperaturprofile für eine Reihe von Frischgasgeschwindigkeiten von $\mathrm{v}=4.6 \mathrm{~cm} / \mathrm{s}$ bis $\mathrm{zu} \mathrm{v}=7.1 \mathrm{~cm}$ im Abstand von $0.5 \mathrm{~cm} / \mathrm{s}$ aufgetragen. Wie bereits beschrieben sind die Kurvenverläufe ungefähr linear. Besonders bei geringeren Frischgasgeschwindigkeiten fällt die Flammentemperatur mit zunehmender Höhe immer geringer. Die Temperaturen der einzelnen Temperaturprofile streben unterhalb des untersuchten Höhenintervalls der maximalen Flammentemperatur (Kurlbaum) zu, die hier in 
einem kleinen Bereich zwischen $\mathrm{T}=2000 \mathrm{~K}$ und $\mathrm{T}=2040 \mathrm{~K}$ bei einer Höhe über dem Brenner von $\mathrm{h}=2 \mathrm{~mm}$ liegt (die adiabatische Flammentemperatur liegt bei $\mathrm{T}=2375 \mathrm{~K}$ [38]). Die Abstände zwischen den einzelnen Temperaturprofilen mit $\Delta \mathrm{v}_{\mathrm{FG}}=0.5 \mathrm{~cm} / \mathrm{s}$ betragen fast im gesamten untersuchten Höhenbereich etwa 75 K. So läßt sich z.B. das Temperaturprofil einer Benzol-Luft-Flamme mit Frischgasgeschwindigkeit von $v=7.6 \mathrm{~cm} / \mathrm{s}$ ziemlich gut extrapolieren.

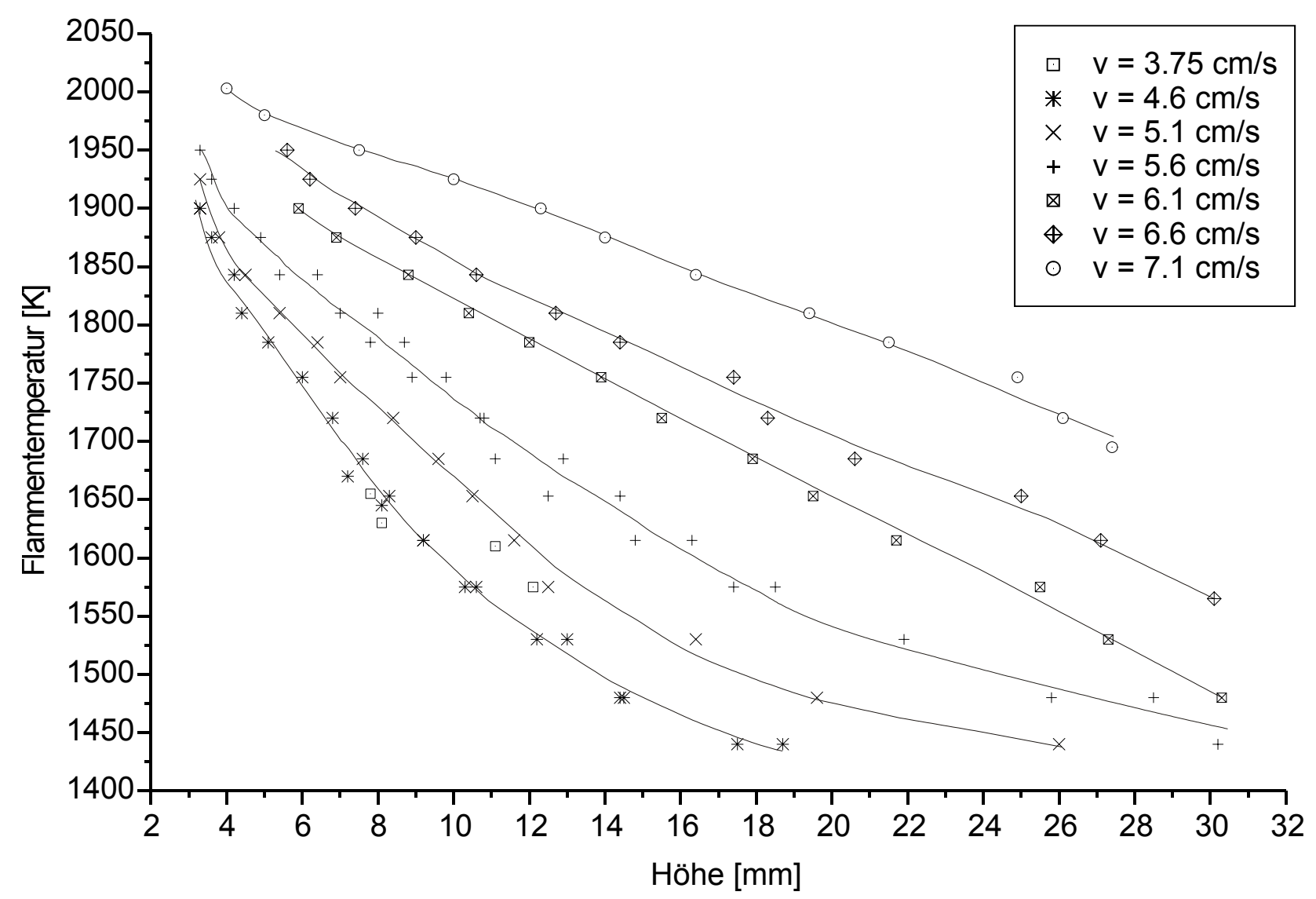

Abb. 4.8: Flammentemperaturprofile von Benzol-Luft-Flammen mit unterschiedlichen Frischgasgeschwindigkeiten bei einem C/O-Verhältnis von 0.68

\subsubsection{Winkelabhängige Streukurven der rußenden Benzol-Luft-Flammen}

Jeder winkelabhängigen Streulichtmessung einer Benzol-Luft-Flamme gehen eine oder mehrere Lufteichungen voraus. Die Lufteichung dient einerseits dazu, die Genauigkeit der optischen Justierung einzuschätzen und andererseits als Schnittstelle zwischen relativen gemessenen Streuintensitäten und deren absoluten Werte über den bekannten Streuquerschnitt 
von Gasen, in diesem Fall Luft. In der Abb. 4.9 ist der normierte winkelabhängige Verlauf der Streuintensität $\mathrm{I}_{\mathrm{Vv}}$ von Luft und von einer rußenden Benzol-Luft-Flamme mit einem C/OVerhältnis von $\mathrm{C} / \mathrm{O}=0.68$, einer Frischgasgeschwindigkeit von $\mathrm{v}=6.64 \mathrm{~cm} / \mathrm{s}$ und einer Meßhöhe von $\mathrm{h}=13.1 \mathrm{~mm}$ über der Brenneroberfläche dargestellt.

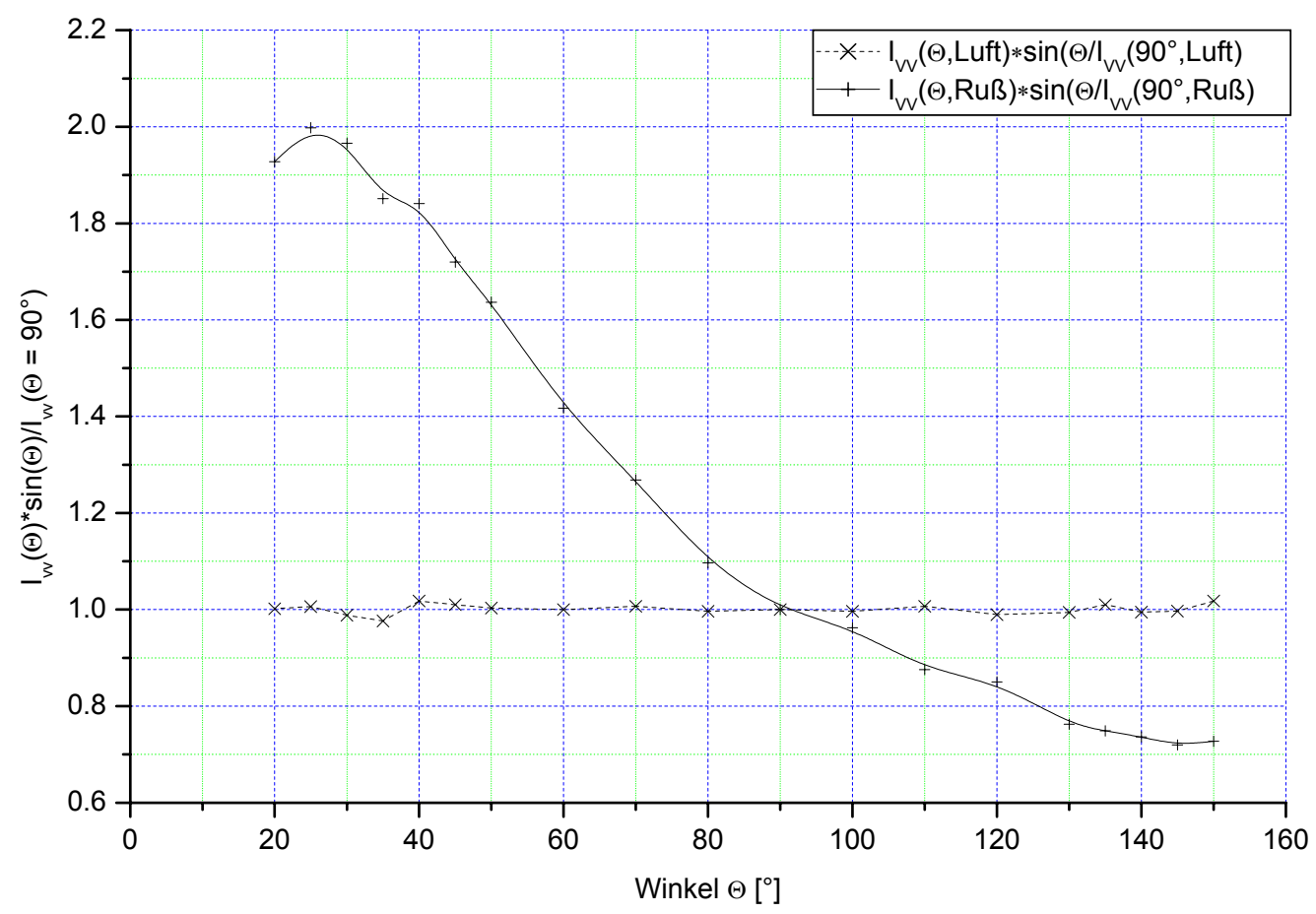

Abb. 4.9: Winkelabhängige normierte Streukurven von Luft bei NTP und Ruß einer Benzol-Luft-Flamme

\subsubsection{Extinktionskoeffizienten und Streufaktoren}

\section{$\underline{\text { Extinktionskoeffizienten }}$}

Die Extinktionskoeffizienten (s. Kap. 2.3) erhält man aus Extinktionsmessungen (Absorptionsmessungen) der unterschiedlichen Flammen. Im folgenden soll die Abhängigkeit des Extinktionskoeffizienten von der Höhe, dem C/O-Verhältnis und der Frischgasgeschwindigkeit diskutiert werden, weitere Ergebnisse für alle Flammen befinden sich im Anhang 7.1.1.5.

Die Abb. 4.10 zeigt den Verlauf des Extinktionskoeffizienten bei einer Wellenlänge von $\lambda=$ $488 \mathrm{~nm}$ mit der Höhe für eine Benzol-Luft-Flamme mit einem $\mathrm{C} / \mathrm{O}-$ Verhältnis von $\mathrm{C} / \mathrm{O}=$ 
0.72 und einer Frischgasgeschwindigkeit von $\mathrm{v}=5.1 \mathrm{~cm} / \mathrm{s}$. Die Kreuze geben die Meßpunkte wieder, die Kurve ist der angenommene Verlauf. Mit Beginn des Rußgebiets bei etwa $5 \mathrm{~mm}$ nimmt der Extinktionskoeffizient zunächst deutlich zu. In einer Höhe von etwa $7 \mathrm{~mm}$ durchläuft die Kurve ihren Wendepunk und flacht anschließend mit weiterer Höhenzunahme mehr und mehr ab.

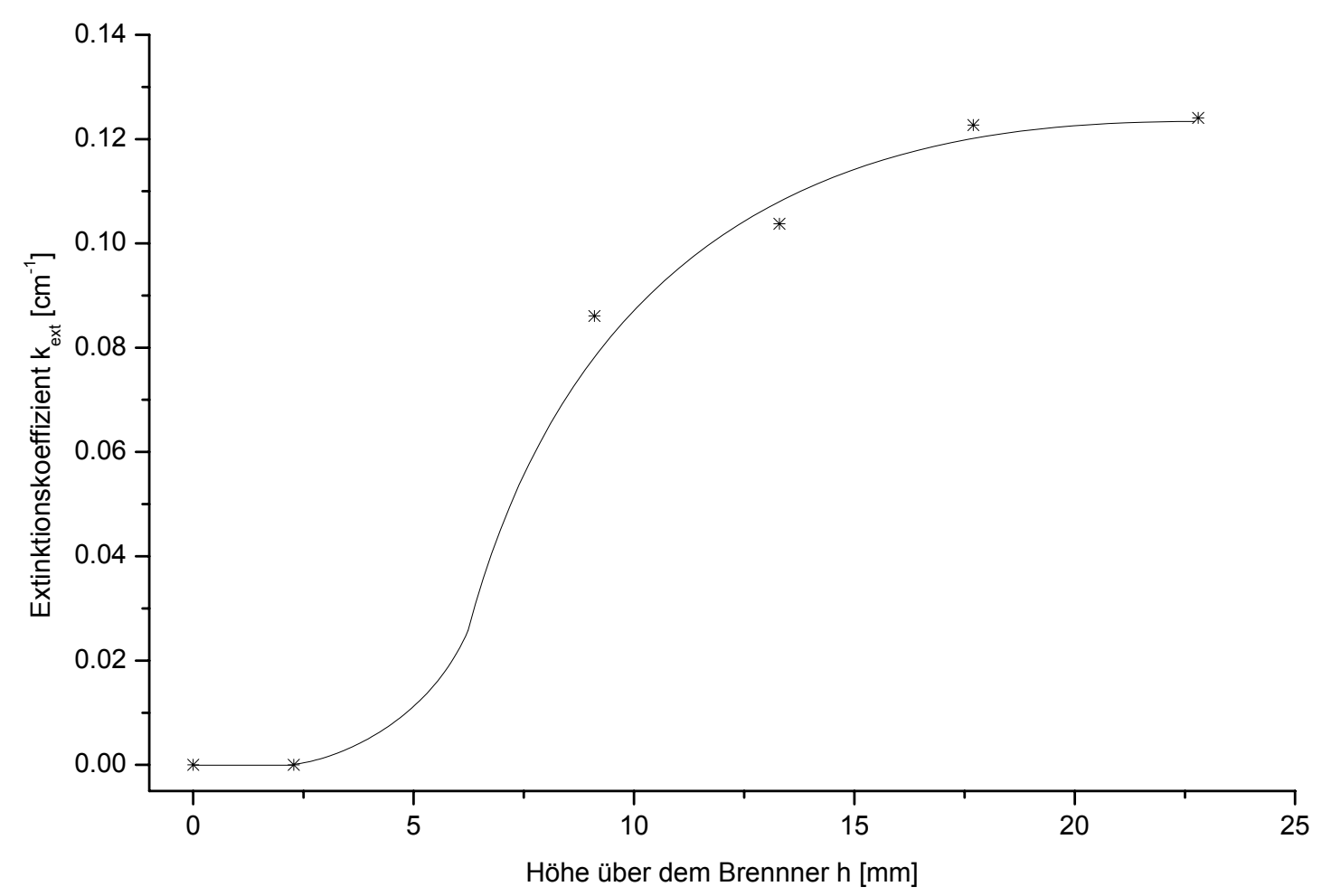

Abb. 4.10: Höhenabhängigkeit des Extinktionskoeffizienten einer Benzol-Luft-Flamme mit einem $\mathrm{C} / \mathrm{O}-$ Verhältnis von $\mathrm{C} / \mathrm{O}=0.72$ und einer Frischgasgeschwindigkeit von $\mathrm{v}=5.1 \mathrm{~cm} / \mathrm{s}$

In der Abb. 4.11 ist die Abhängigkeit des Extinktionskoeffizienten vom C/O-Verhältnis in einer Höhe von $\mathrm{h}=17.7 \mathrm{~mm}$ und einer Frischgasgeschwindigkeit von $\mathrm{v}=5.1 \mathrm{~cm} / \mathrm{s}$ dargestellt. Im untersuchten Bereich nimmt der Extinktionskoeffizient mit steigendem C/O-Verhältnis überproportional zu. Sehr brennstoffreiche Flammen ließen die Bestimmung des Extinktionskoeffizienten nicht zu, da diese oberhalb der Stabilitätsgrenze liegen. Frühere Messungen [30] haben aber gezeigt, daß der Anstieg der Extinktionskoeffizienten mit dem C/O-Verhältnis wieder abflacht und auf einen konstanten Wert zuläuft. 


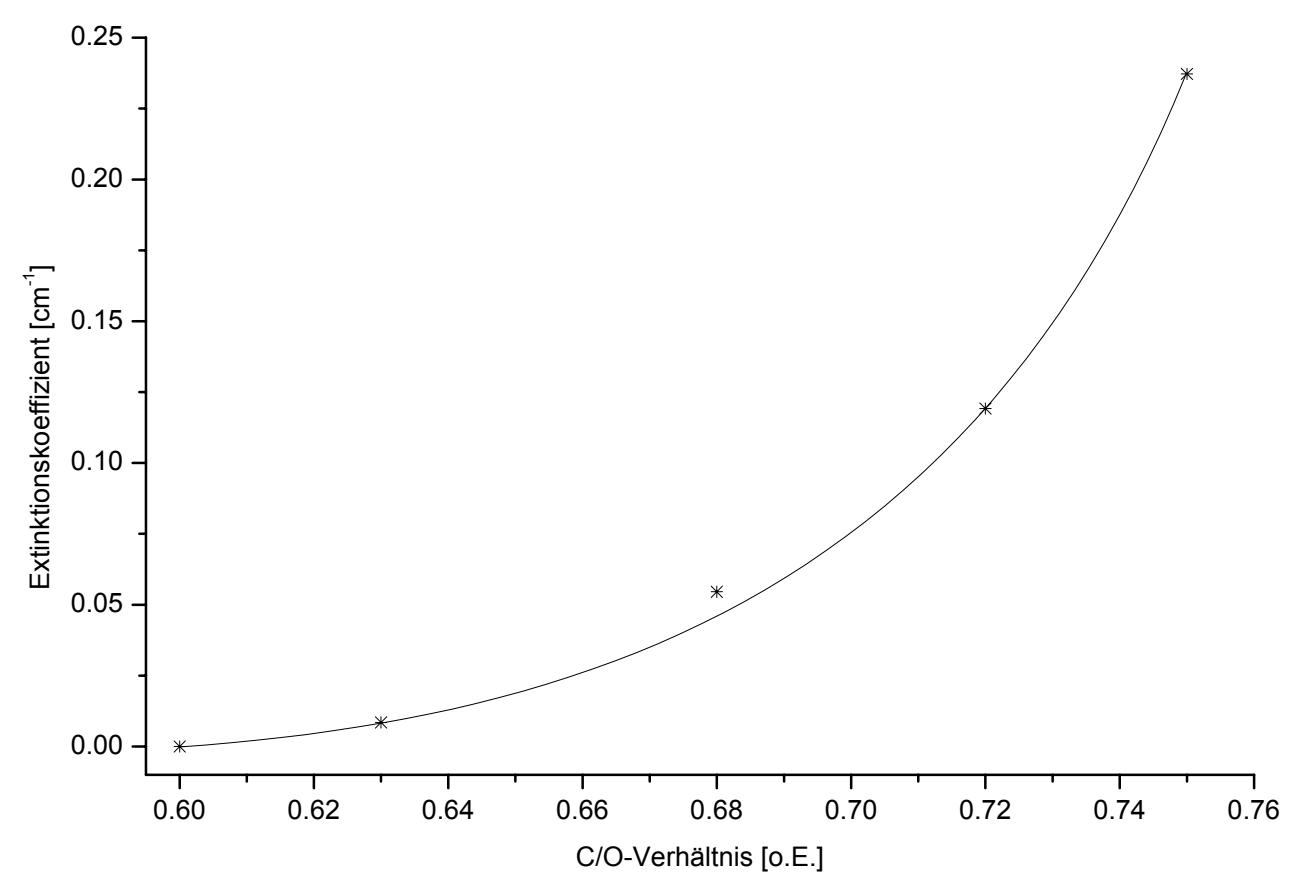

Abb. 4.11: Abhängigkeit des Extinktionskoeffizienten vom C/O-Verhältnis bei einer Frischgasgeschwindigkeit von $\mathrm{v}=5.1 \mathrm{~cm} / \mathrm{s}$ und einer Höhe von $17.7 \mathrm{~mm}$

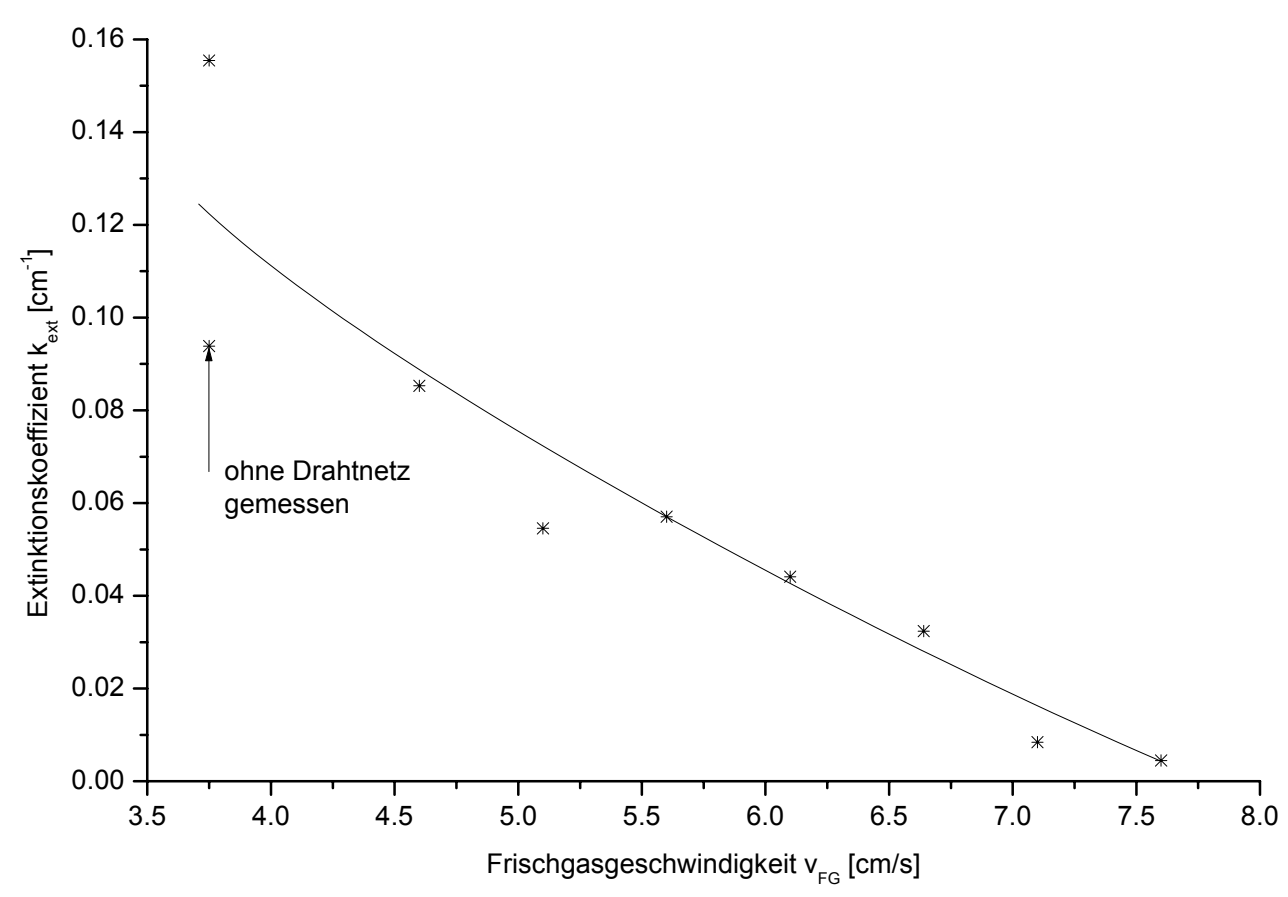

Abb. 4.12: Abhängigkeit des Extinktionskoeffizienten von der Frischgasgeschwindigkeit bei einem $\mathrm{C} / \mathrm{O}-$ Verhältnis von $\mathrm{C} / \mathrm{O}=0.68 \mathrm{~cm} / \mathrm{s}$ und einer Höhe von $17.7 \mathrm{~mm}$ 
Der Extinktionskoeffizient fällt innerhalb des Meßbereichs, wie in der Abb. 4.12 dargestellt, linear mit zunehmender Frischgasgeschwindigkeit ab. Eine Benzol-Luft-Flamme mit einer Frischgasgeschwindigkeit von $\mathrm{v}=7.6 \mathrm{~cm} / \mathrm{s}$ rußt nur noch sehr schwach, so daß oberhalb von $\mathrm{v}=8 \mathrm{~cm} / \mathrm{s}$ bei Benzol-Luft-Flammen mit dem verwendeten optischen Aufbau keine Extinktion mehr gemessen werden kann. Ein Maximum, wie bei Ethylen beobachtet, liegt beim Benzol außerhalb oder genau um Rand des untersuchten Bereich bei oder unter $\mathrm{v}=3.5$ $\mathrm{cm} / \mathrm{s}$.

\section{$\underline{\text { Streufaktoren }}$}

Aus den Streulicht- und den Extinktionsmessungen lassen sich die Streufaktoren Qvv berechnen (s. Kap. 2.4.1). Wie der Extinktionskoeffizient hängt der Streufaktor einer rußenden Flamme stark von der Höhe, der Gemischzusammensetzung und dem Durchsatz ab. Zusätzlich ist der Streufaktor vom Beobachtungswinkel abhängig. Im Anschluß soll der winkelabhängige Verlauf des Streufaktors in seiner Abhängigkeit von den 3 anderen Parametern (Höhe, C/O-Verhältnis und Frischgasgeschwindigkeit) für Benzol-Luft-Flammen gezeigt werden.

Die Abb. 4.13 zeigt den winkelabhängigen Verlauf des Streufaktors für mehrere Höhen. Man kann einen exponentiellen Abfall des Streufaktors mit zunehmendem Streuwinkel erkennen. Dabei gleicht der Kurvenverlauf sehr dem des Ethylens [47].

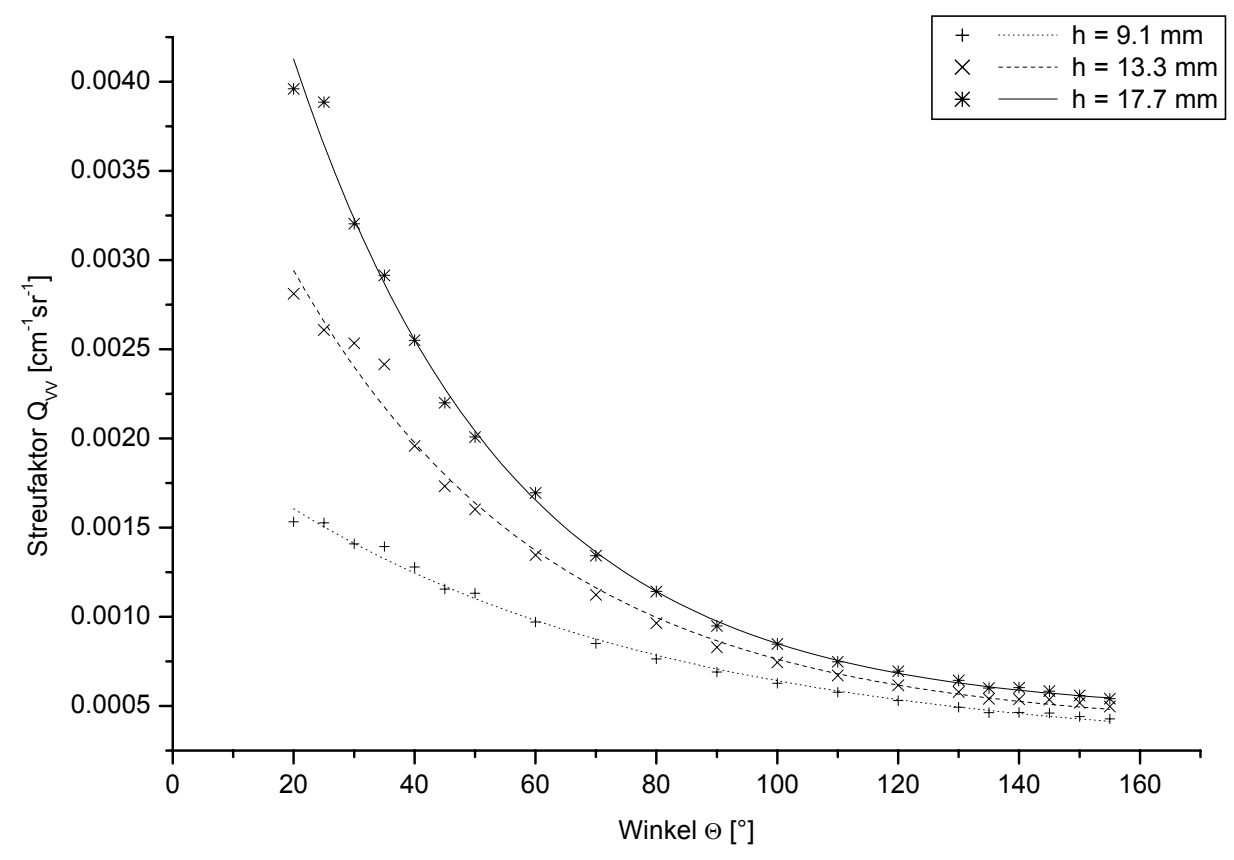

Abb. 4.13: Höhen- und Winkelabhängigkeit des Streufaktors für ein C/O-Verhältnis von $\mathrm{C} / \mathrm{O}=0.72$ und einer Frischgasgeschwindigkeit von $\mathrm{v}=5.1 \mathrm{~cm} / \mathrm{s}$ 
In größeren Höhen aufgenommene Streukurven haben einen stärkeren exponentiellen Abfall als Streukurven, die in geringerer Höhe bestimmt wurden. Somit ist die Höheabhängigkeit bei kleineren Streuwinkeln größer als bei großen Streuwinkeln. Diesem Phänomen liegen die großen Teilchendurchmesser der Rußpartikel zugrunde, die damit nicht mehr eine reine Dipolstrahlung ausüben. Außerdem sind die Rußteilchen einer Benzol-Luft-Flamme mit einem $\mathrm{C} / \mathrm{O}-$ Verhältnis von $\mathrm{C} / \mathrm{O}=0.72$ nicht mehr streng kugelförmig, sondern weichen davon ab wie z.B. durch Aggregatbildung.

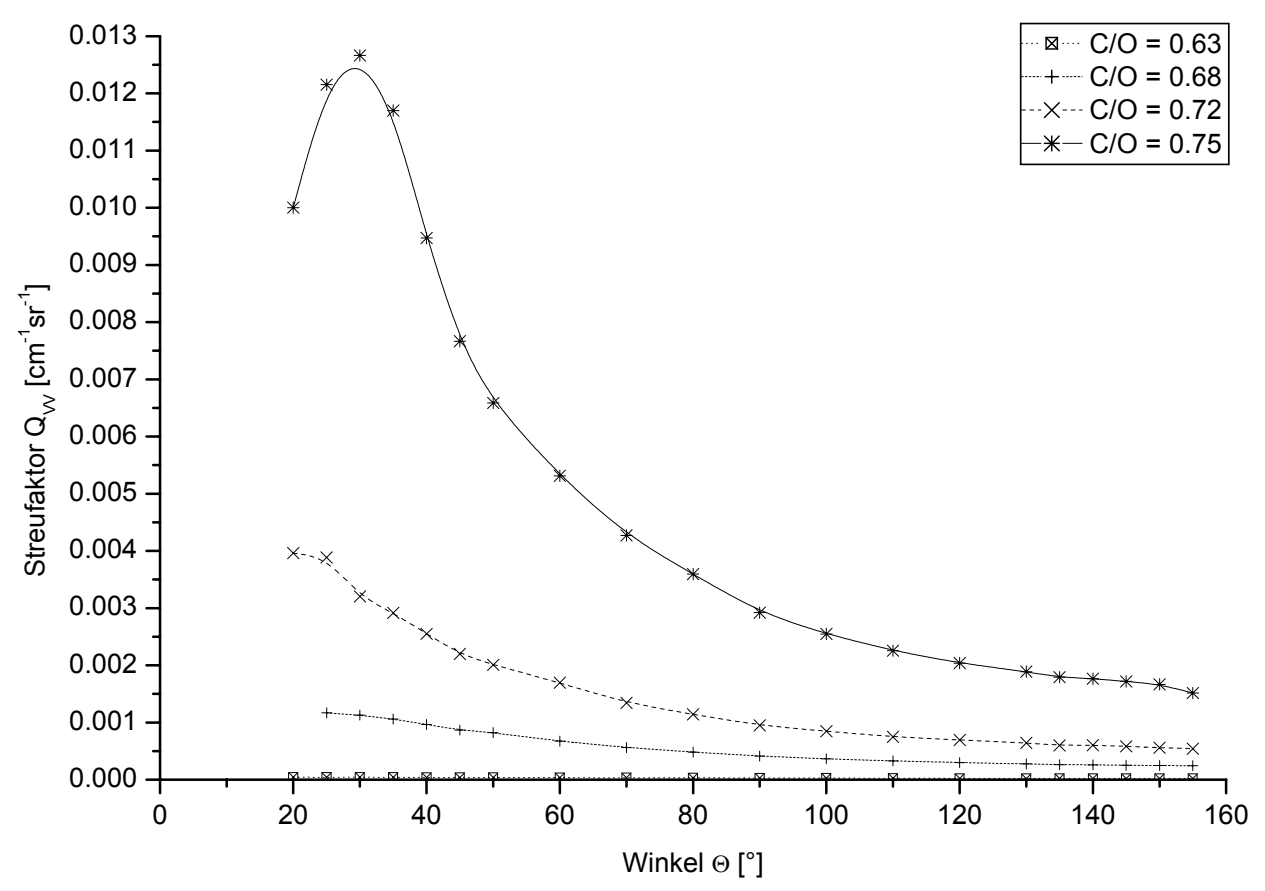

Abb. 4.14: Abhängigkeit des Streufaktors vom C/O-Verhältnis für eine Höhe von $\mathrm{h}=17.7$ $\mathrm{mm}$ und einer Frischgasgeschwindigkeit von $\mathrm{v}=5.1 \mathrm{~cm} / \mathrm{s}$ in einer Auswahl

Die Abhängigkeit des winkelabhängigen Verlaufs der Streufaktoren vom C/O-Verhältnis ist der Höhenabhängigkeit ähnlich (Abb. 4.14). Allerdings geht der Streufaktor bei sehr fetten Gemischzusammensetzungen und sehr spitzen Winkeln wieder etwas zurück, da hier noch ausgeprägter als in großen Höhen für eine reine Dipolstrahlung zu große und nicht mehr kugelförmige Teilchen vorliegen. Zusätzlich spielt hier die Qualität der Lufteichung hinein. Bei spitzen Winkeln wird bei einer Lufteichung ein Teil des einfallenden Laserlichts mitdetektiert, besonders in stark rußenden Flammen wird dieser Effekt ganz oder teilweise aufgehoben. Bei einem $\mathrm{C} / \mathrm{O}-\mathrm{Verhältnis} \mathrm{von} \mathrm{C} / \mathrm{O}=0.63$ ist der Streufaktor auf einer linearen 
Skala im Vergleich mit höheren $\mathrm{C} / \mathrm{O}-$ Verhältnissen fast gegen null zurückgegangen (er liegt im Bereich $10^{-5}$ ). Mit brennstoffreicheren Flammen werden analog zur Höheabhängigkeit mit dem C/O-Verhältnis exponentiell anwachsende Streufaktoren erhalten. Dies ist wiederum um so ausgeprägter, desto kleiner der Streuwinkel ist. Im Gegensatz zum Ethylen ist eine Abschwächung dieser Tendenz bis zu einer Sättigung bei besonders hohen C/O-Verhältnissen außerhalb des untersuchten Bereichs und damit nicht festzustellen aber $\mathrm{zu}$ vermuten. Abweichend zur Höhenabhängigkeit laufen die Streufaktoren unterschiedlicher C/OVerhältnisse nicht mit großen Winkeln vollständig zusammen.

Die beiden folgenden Abbildungen (Abb. 4.15 und Abb. 4.16) zeigen die Durchsatzabhängigkeit des Streufaktors. Mit zunehmender Frischgasgeschwindigkeit nimmt der Streufaktor $a b$, da die Teilchen bei höheren Flammentemperaturen nicht mehr so groß werden. Dieses Erscheinungsbild entsprich den Beobachtungen beim Ethylen, dabei sind die Frischgasgeschwindigkeiten aber geringer als die beim Ethylen untersuchten. Die Geschwindigkeitsabhängigkeit wird von leicht veränderten $\mathrm{C} / \mathrm{O}-$ Verhältnissen deutlich überlagert. So passen die Messungen mit den Frischgasgeschwindigkeiten v $=5.1 \mathrm{~cm} / \mathrm{s}$ und v $=6.1 \mathrm{~cm} / \mathrm{s}$ nicht in die homologe Reihe, da daß C/O-Verhältnis etwas höher war.

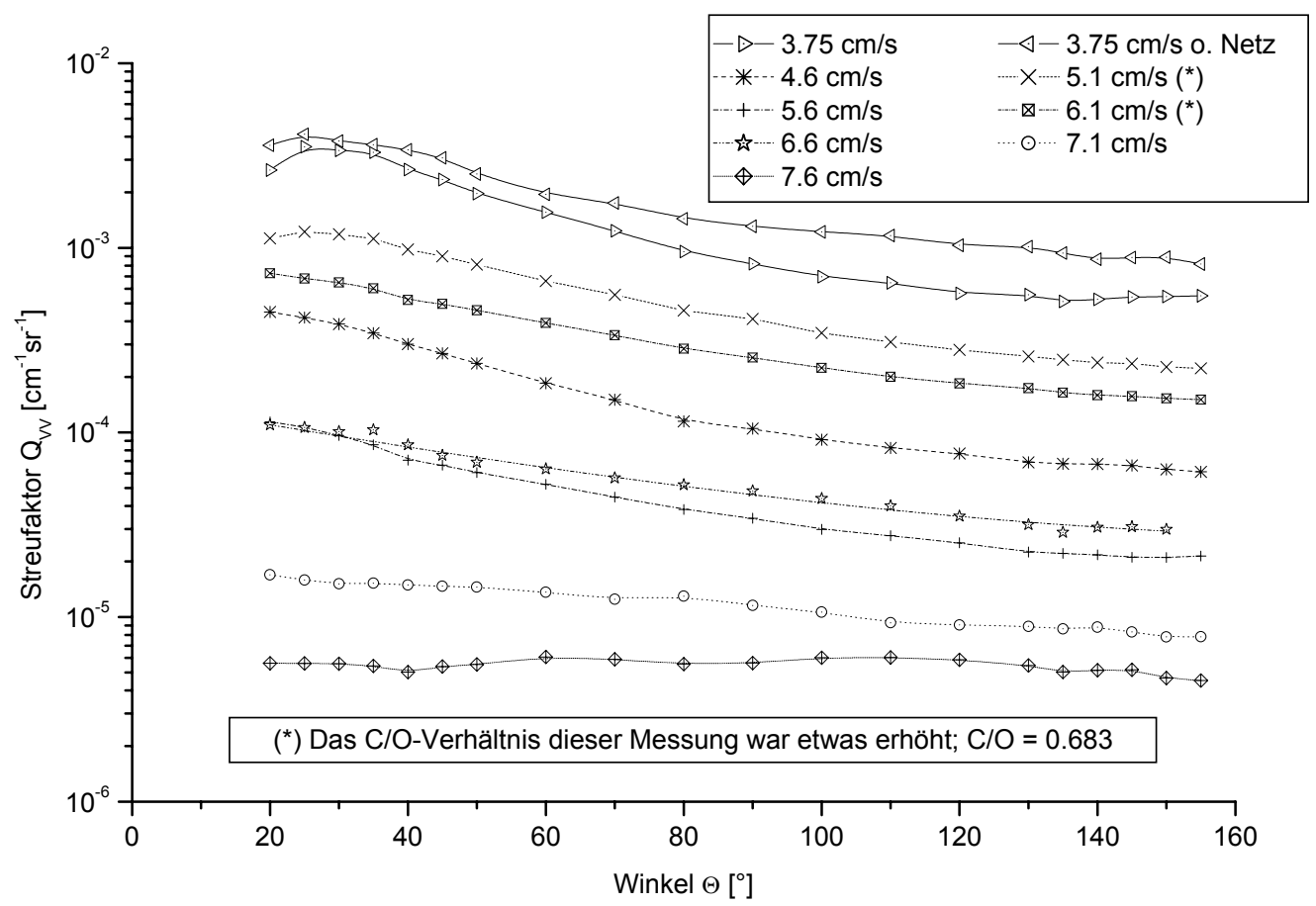

Abb. 4.15: Abhängigkeit des Streufaktors von der Frischgasgeschwindigkeit bei einem $\mathrm{C} / \mathrm{O}-$ Verhältnis von $\mathrm{C} / \mathrm{O}=0.68$ und einer Höhe von $\mathrm{h}=17.7 \mathrm{~mm}$ in logarithmischer Auftragung 


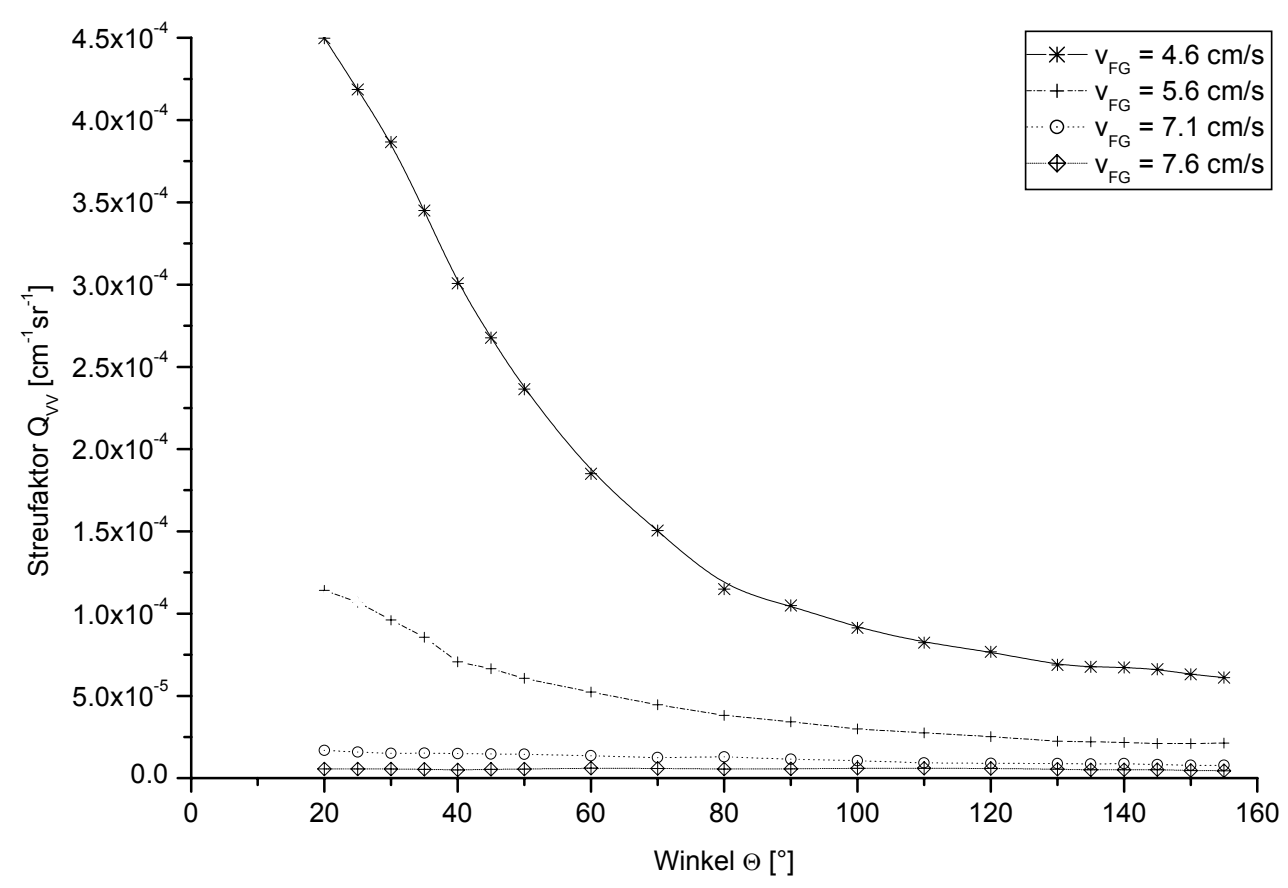

Abb. 4.16: Abhängigkeit des Streufaktors von der Frischgasgeschwindigkeit bei einem $\mathrm{C} / \mathrm{O}-$ Verhältnis von $\mathrm{C} / \mathrm{O}=0.68$ und einer Höhe von $\mathrm{h}=17.7 \mathrm{~mm}$. Auswahl an Frischgasgeschwindigkeiten in linearer Auftragung.

\subsubsection{Rußvolumenbruch}

Aus den Extinktionskoeffizienten lassen sich über den komplexen Brechungsindex die Rußvolumenbrüche berechnen. Da der komplexe Brechungsindex für alle Messungen als konstant angesehen wurde, gelten alle Aussagen für die Kurvenverläufe der Extinktionskoeffizienten analog auch für die so definierten Rußvolumenbrüche (Definition s. Kap. 2.4.2). Die Änderung des Rußvolumenbruchs mit der Höhe in Abb. 4.17 stellt einen Ausschnitt des Verlaufs der Abb. 4.10 dar. In diesem Meßbereich der Streulichtmessungen ist der Kurvenverlauf nur geringfügig von einem linearen Verlauf abweichend.

Die weiteren Abbildungen (Abb. 4.18, Abb. 4.19) reproduzieren exakt die Kurvenverläufe des Extinktionskoeffizienten der Abb. 4.11 und Abb. 4.12, nur die Ordinateskala hat eine andere Dimension $\left(\mathrm{cm}^{3} \mathrm{Ru} / \mathrm{cm}^{3}\right.$ Flammenvolumen) und Größenordnung. Für die verwendete Wellenlänge von $\lambda=488 \mathrm{~nm}$ und dem komplexen Brechungsindex von $\mathrm{m}=1.57-0.56 \mathrm{i}$ gilt: $\mathrm{k}_{\mathrm{ext}} * 9.975 * 10^{-6}=\mathrm{f}_{\mathrm{v}}$. 


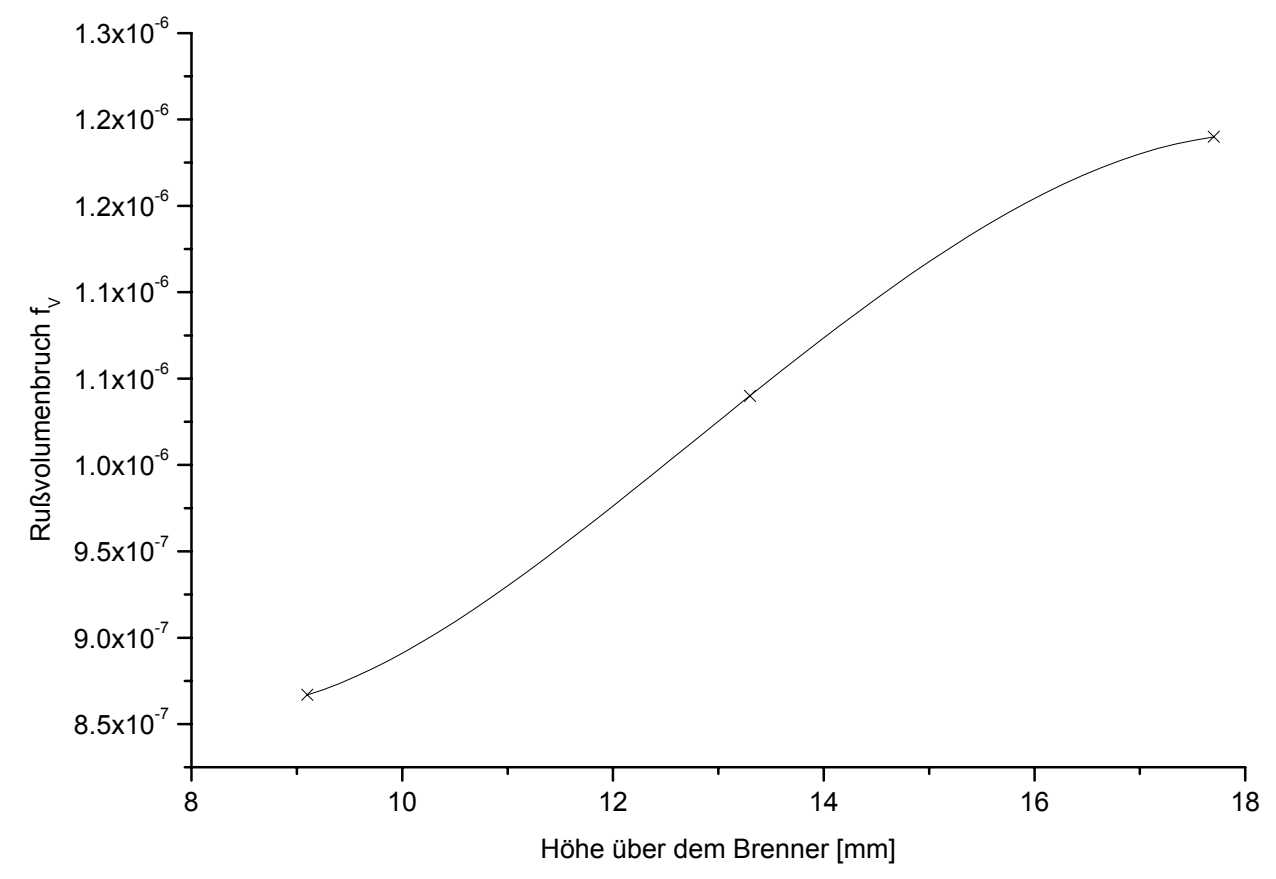

Abb. 4.17: Höhenabhängigkeit des Rußvolumenbruchs bei einem $\mathrm{C} / \mathrm{O}-$ Verhältnis von $\mathrm{C} / \mathrm{O}$ $=0.72$ und einer Frischgasgeschwindigkeit von $\mathrm{v}=5.1 \mathrm{~cm} / \mathrm{s}$

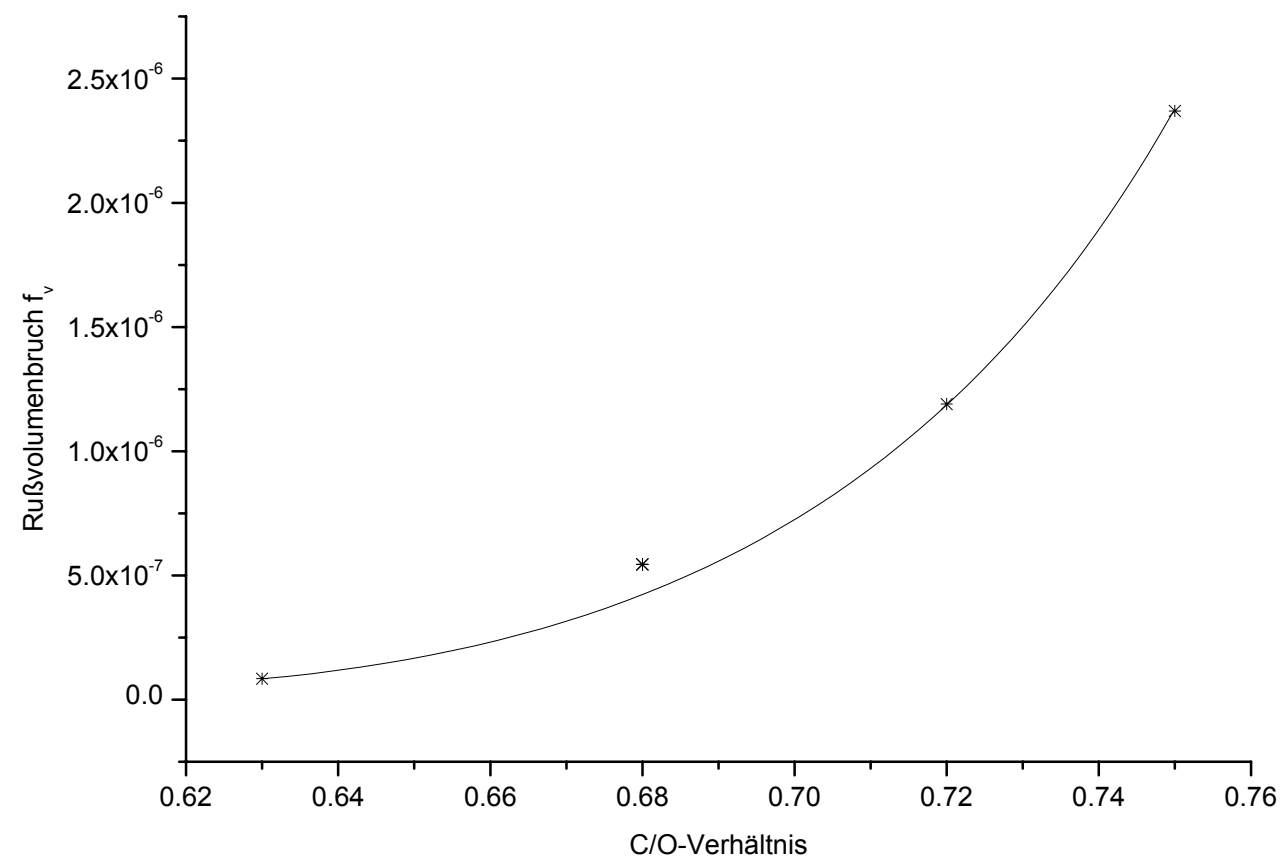

Abb. 4.18: Abhängigkeit des Rußvolumenbruchs vom C/O-Verhältnis für eine Höhe von $\mathrm{h}$ $=17.7 \mathrm{~mm}$ und eine Frischgasgeschwindigkeit von $\mathrm{v}=5.1 \mathrm{~cm} / \mathrm{s}$ 


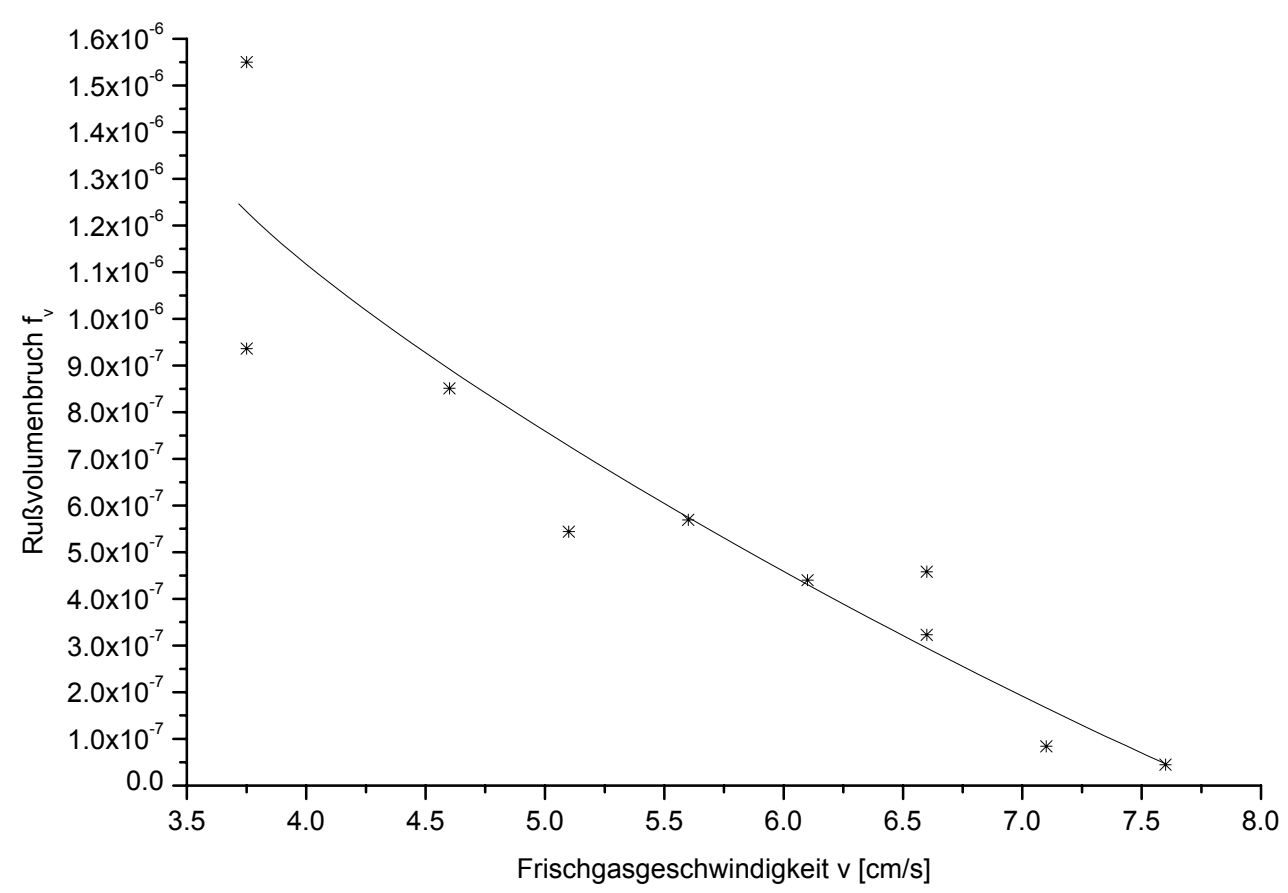

Abb. 4.19: Durchsatzabhängigkeit des Rußvolumenbruchs für eine Höhe von $\mathrm{h}=17.7 \mathrm{~mm}$ und einem $\mathrm{C} / \mathrm{O}-$ Verhältnis von $\mathrm{C} / \mathrm{O}=0.68$

\subsubsection{Teilchendurchmesser}

Teilchenradien der Rußpartikel lassen sich nach Rayleigh $\left(\mathrm{d}_{\mathrm{R}}\right.$; s. Kap. 2.4.3) und mit der RDG/FA-Methode (scheinbarer Radius, $\mathrm{R}_{\mathrm{g}}$ und volumenäquivalenter Durchmesser, $\mathrm{D}_{30}$; $\mathrm{s}$. Kap. 2.6 ) ermitteln. Die verschieden Teilchendurchmesser sollen hier gemeinsam gezeigt und diskutiert werden. Es gilt allerdings die unterschiedlichen Definitionen zu beachten:

Dem Rayleigh-Durchmesser $\left(d_{R}\right)$ liegt die Mie-Streutheorie in ihrer Rayleigh-Näherung zugrunde. Annahmen sind kugelförmige Teilchen mit einem Durchmesser, der deutlich unter der Wellenlänge der Lichtquelle für die Streuung liegt. Rußteilchen entfernen sich aber mit zunehmendem $\mathrm{C} / \mathrm{O}-$ Verhältnis und zunehmender Höhe von einer Kugelgestalt und bilden fraktale Körper, die Aggregate.

Der volumenäquivalente Durchmesser $\left(\mathrm{D}_{30}\right)$ ist über den Mittelwert relativer Häufigkeiten an Aggregaten mit einer unterschiedlichen Anzahl von Primärteilchen definiert. Die Primärteilchen mit dem Radius $d_{p}$ sind dabei stets einheitlich groß

Der scheinbare Radius $\left(\mathrm{R}_{\mathrm{g}}\right)$ ist im Gegensatz zu den vorangegangenen Durchmessern keine volumenbezogene Größe, sondern eine über die Masse definierte Größe. Das Quadrat des 
scheinbaren Radius eines Aggregats ist das mittlere Abstandsquadrat seiner Teilchen vom Massenschwerpunkt. Der scheinbare Radius ist eigentlich ein Durchmesser und kann deshalb direkt mit den anderen Durchmesser verglichen werden. Der scheinbare Radius sagt etwas über die Massenverteilung in den Teilchen aus.

Der Rayleigh-Durchmesser $d_{R}$ zeigt keine Höhenabhängigkeit (Abb. 4.20), sondern liegt im gesamten untersuchten Höhenintervall bei etwa $76 \mathrm{~nm}$. Der volumenäquivalente Durchmesser $\mathrm{D}_{30}$ sowie der scheinbare Radius $\mathrm{R}_{\mathrm{g}}$ steigen zunächst etwas mit der Höhe an, um dann in ihrem Anstieg abzuflachen. Dabei erreichen $\mathrm{D}_{30}$ und $\mathrm{R}_{\mathrm{g}}$ in größeren Höhen Werte von $95 \mathrm{~nm}$ bzw. $92 \mathrm{~nm}$ und liegen damit deutlich höher als $d_{R}$. Erwartungsgemäß wäre der scheinbare Radius eigentlich etwas größer als der volumenäquivalenter Durchmesser. Damit läßt sich feststellen, daß die Höhenabhängigkeit der unterschiedlichen Teilchendurchmesser in BenzolLuft-Flammen ausschließlich am Ende des Teilchenbildungsprozesses untersucht wurde. Das Teilchenwachstum ist schon in der untersten Meßhöhe von $\mathrm{h}=9 \mathrm{~mm}$ nahezu abgeschlossen.

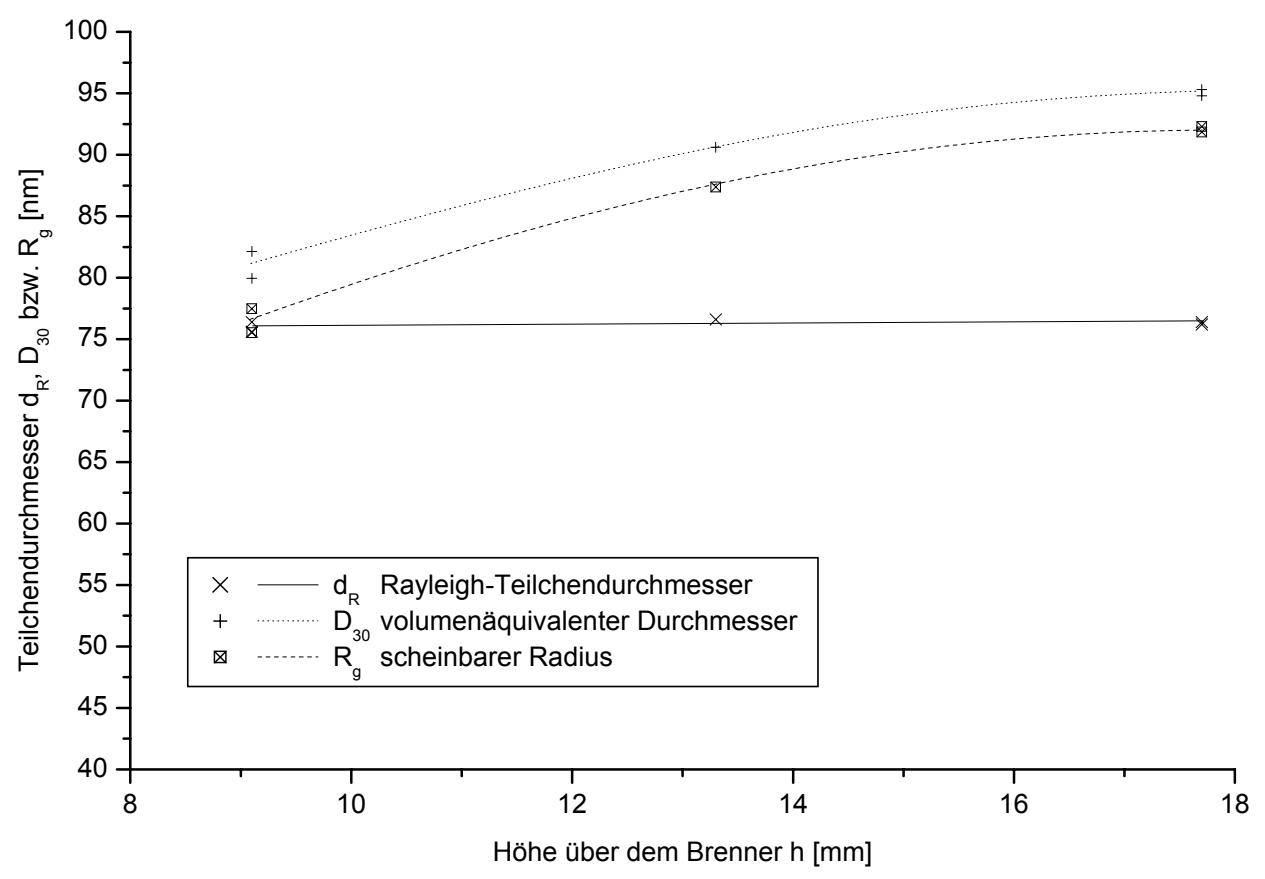

Abb. 4.20: Höhenabhängigkeit der Teilchendurchmesser für ein $\mathrm{C} / \mathrm{O}-$ Verhältnis von $\mathrm{C} / \mathrm{O}$ $=0.72$ und einer Frischgasgeschwindigkeit von $5.1 \mathrm{~cm} / \mathrm{s}$

Diese Kurvenverläufe bestätigen außerdem, daß in größere Höhe keine rein kugelförmigen Teilchen mehr vorliegen und daß die maximale Größe der Rußteilchen ab einer bestimmten Höhe nicht mehr zunimmt. Der Kurvenverläufe entsprechen im Falle des 
volumenäquivalenten Durchmessers $\mathrm{D}_{30}$ und des scheinbaren Radius $\mathrm{R}_{\mathrm{g}}$ dem Verhalten beim Ethylen. Dies trifft auch für den Rayleigh-Teilchenradius $d_{R}$ für die oberen beiden Meßpunkte $\mathrm{zu}$.

Alle Durchmesser nehmen in Flammen mit brennstoffreicheren Gemischzusammensetzungen zu (Abb. 4.21). Der Rayleigh-Durchmesser $d_{R}$ wächst dabei auch hier am geringsten in Übereinstimmung mit den Ethylenflammen. Die Kurvenverläufe des scheinbaren Radius $\mathrm{R}_{\mathrm{g}}$ sowie des volumenäquivalenten Durchmessers $\mathrm{D}_{30}$ zeigen ebenfalls ein mit steigendem $\mathrm{C} / \mathrm{O}-$ Verhältnis abflachendes Steigungsverhalten (wie beim Ethylen). Die Werte des volumenäquivalenten Durchmessers für $\mathrm{C} / \mathrm{O}=0.75$ liegen etwa $20 \mathrm{~nm}$ zu hoch und sollten wie bei den Messungen der Ethylen-Luft-Flammen leicht unterhalb oder auf gleicher Höhe der scheinbaren Radien $\mathrm{R}_{\mathrm{g}}$ liegen.

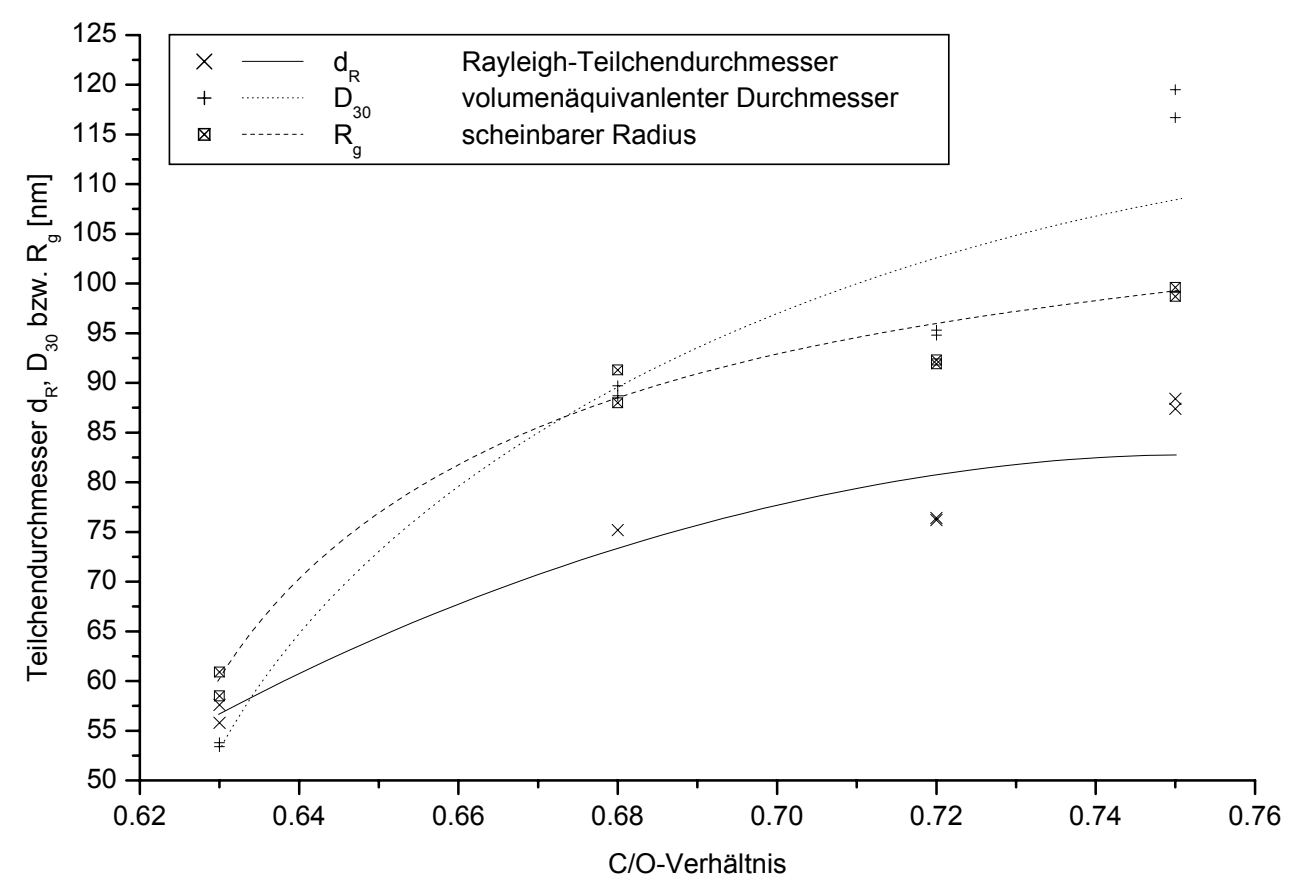

Abb. 4.21: Abhängigkeit der verschiedenen Teilchenradien vom $\mathrm{C} / \mathrm{O}-$ Verhältnis für eine Höhe von $\mathrm{h}=17.7 \mathrm{~mm}$ und eine Frischgasgeschwindigkeit von $\mathrm{v}=5.1 \mathrm{~cm} / \mathrm{s}$

Die Abhängigkeit von der Frischgasgeschwindigkeit ist in Abb. 4.22 dargestellt. Alle drei Teilchendurchmesser $\left(\mathrm{d}_{\mathrm{R}}, \mathrm{D}_{30}\right.$ und $\left.\mathrm{R}_{\mathrm{g}}\right)$ nehmen einen ähnlichen Verlauf mit der Frischgasgeschwindigkeit. Man findet in Übereinstimmung mit der Rußgrenze (s. Abb. 4.6), daß Benzol-Luft-Flammen (mit dem verwendeten Brenner) mit Frischgasgeschwindigkeiten 
von über $8 \mathrm{~cm} / \mathrm{s}$ nicht mehr rußen. Die Kurvenverläufe für alle Durchmesser sind dem Verhalten in Ethylen-Luft-Flammen sehr ähnlich.

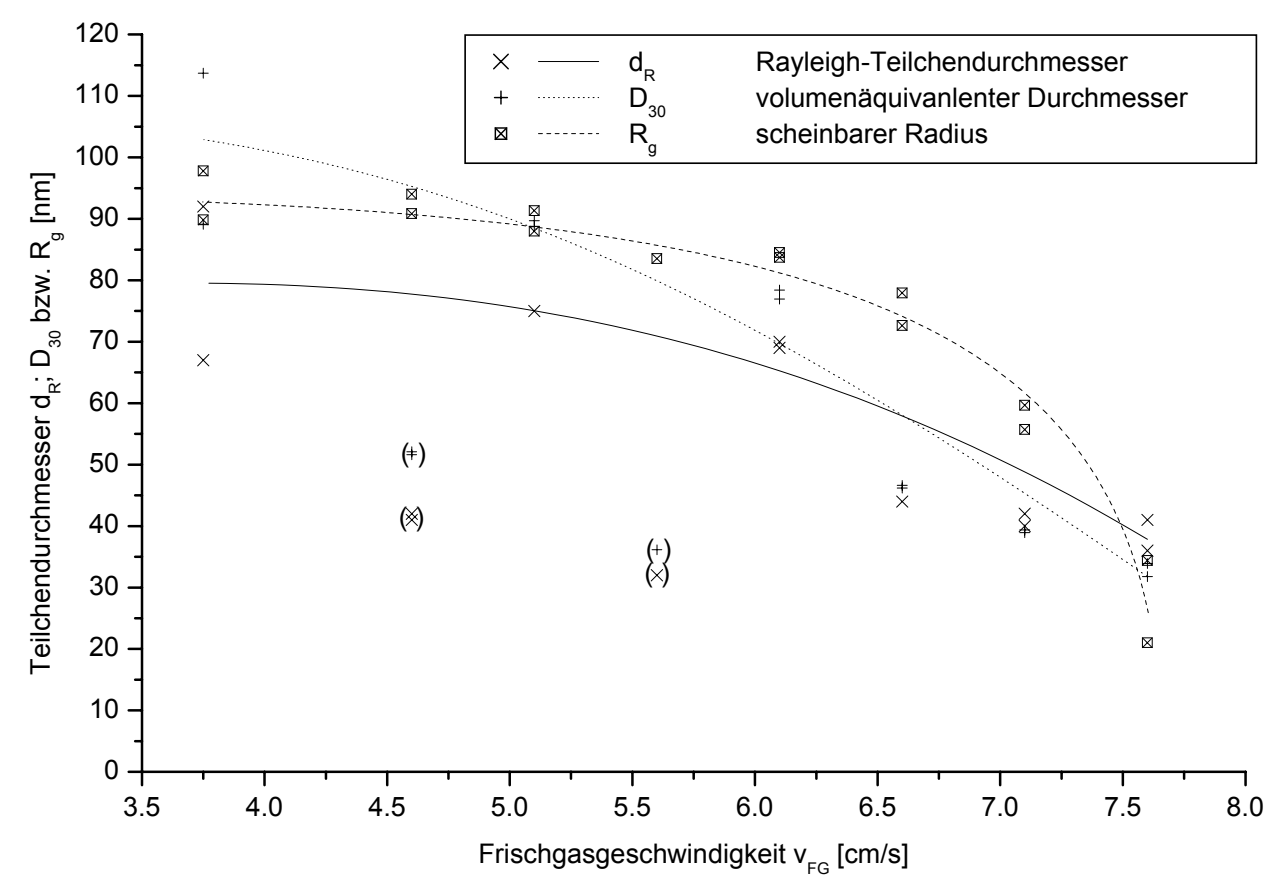

Abb. 4.22: Durchsatzabhängigkeit der verschiedenen Teilchenradien für eine Höhe von $\mathrm{h}$ $=17.7 \mathrm{~mm}$ und einem $\mathrm{C} / \mathrm{O}-$ Verhältnis von $\mathrm{C} / \mathrm{O}=0.68$

\subsubsection{Teilchenzahldichten}

Die beiden Teilchenzahldichten, Rayleigh-Teilchenzahldichte $\left(\mathrm{N}_{\mathrm{R}} ;\right.$ s. Kap. 2.4.3) bzw. Aggregatenzahldichte $\left(\mathrm{N}_{\mathrm{Agg}} ;\right.$ s. Kap. 2.6), sind korrespondierende Größen zu den Teilchendurchmessern, dem Rayleigh-Teilchendurchmesser $\left(d_{R},\right)$ bzw. dem volumenäquivalenten Durchmesser $\left(\mathrm{D}_{30}\right)$. Die Teilchendurchmesser und die Teilchenzahldichten sind über die Beziehung $N_{X}=\frac{6 f_{v}}{\pi D_{X}^{3}}$ verknüpft. Dies gilt sowohl für die Rayleigh-Parameter $(X=R)$ als auch für die Aggregate $(X=30$ bzw. Agg). Damit nimmt die Teilchenzahldichte umgekehrt proportional mit der dritten Potenz des Teilchendurchmessers ab. Mit Eintritt in die Rußzone entstehen zunächst sehr kleine Rußpartikel, die mit steigender Höhe durch Massenwachstum und Koagulation immer größer werden. Die Teilchenzahldichte nimmt deshalb mit zunehmender Höhe ab. 
Die Abb. 4.23 zeigt die Teilchenzahldichte $\mathrm{N}_{\mathrm{R}}$ sowie die Aggregatenzahldichte $\mathrm{N}_{\text {Agg }}$ in ihrer Höhenabhängigkeit. Die Meßwerte der Teilchenzahldichte $\mathrm{N}_{\mathrm{R}}$ liefern einen, den Erwartungen in bezug auf die Höhenabhängigkeit genau widersprechenden und liegen im Bereich von $4 *$ $10^{-9}\left[\mathrm{~cm}^{-3}\right]$, Verlauf. Die Aggregatenzahldichte $\mathrm{N}_{\text {Agg }}$ konvergiert hingegen im untersuchten Höhenintervall $(\mathrm{h}=9$ bis $18 \mathrm{~mm})$ gegen $2.6 * 10^{9}\left[\mathrm{~cm}^{-3}\right]$. Damit ist die Aggregatenzahldichte $\mathrm{N}_{\text {Agg }}$ im untersuchten Höhenintervall wie nach der RDG/FA-Theorie zu erwarten war, etwas kleiner als die Teilchenzahldichte $\mathrm{N}_{\mathrm{R}}$. Diese Werte entsprechen ungefähr dem Verlauf bei Ethylen-Luft-Flammen. Die in dieser Arbeit untersuchten Höhen liegen alle am oberen Ende der Teilchenbildungszone, die Teilchenbildung ist fast abgeschlossen und die Teilchenzahldichte bzw. Aggregatenzahldichte nehmen konstante Werte an $\left(\mathrm{N}_{\mathrm{R}, \infty}=4 * 10^{-9}\right.$ $\left[\mathrm{cm}^{-3}\right]$ bzw. $\mathrm{N}_{\mathrm{Agg}, \infty}=2.6 * 10^{-9}\left[\mathrm{~cm}^{-3}\right]$. Der Verlauf der Teilchenzahldichte $\mathrm{N}_{\mathrm{R}}$ in kleineren Höhen wurde mit Hilfe von Ergebnissen einer anderen Arbeit [2] ergänzt (Abb. 4.23).

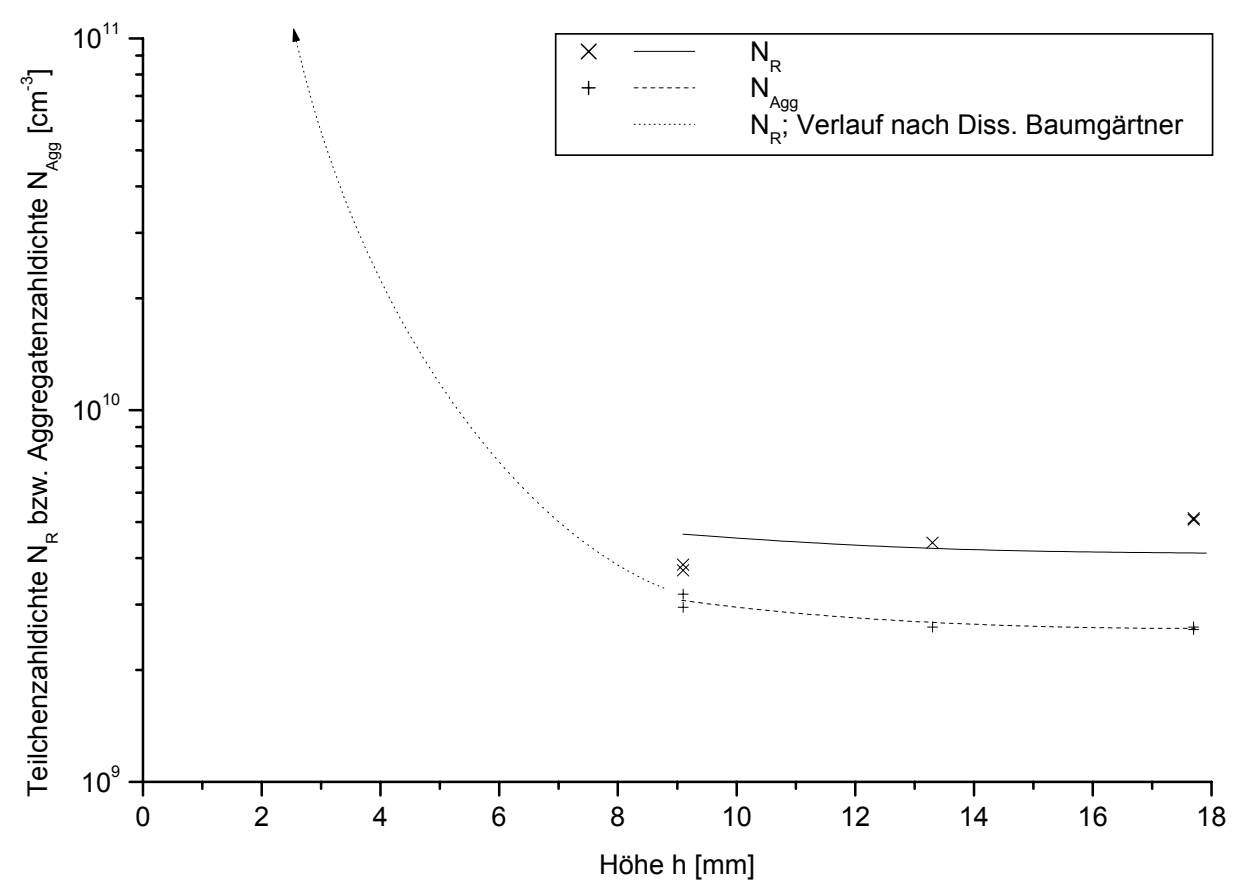

Abb. 4.23: Höhenabhängigkeit der Teilchenzahldichte einer Benzol-Luft-Flamme für ein $\mathrm{C} / \mathrm{O}-$ Verhältnis von $\mathrm{C} / \mathrm{O}=0.72$ und einer Frischgasgeschwindigkeit von 5.1 $\mathrm{cm} / \mathrm{s}$

Am oberen Ende der Teilchenbildungszone in einer Höhe von $\mathrm{h}=17.7 \mathrm{~mm}$ steigen die Teilchenzahldichte $\mathrm{N}_{\mathrm{R}, \infty}$ (Teilchenzahldichte am Ende des Verbrennungsprozesses) und die Aggregatenzahldichte $\mathrm{N}_{\mathrm{Agg}, \infty}$ (Aggregatenzahldichte am Ende des Verbrennungsprozesses) 
linear mit zunehmendem C/O-Verhältnis an (Abb. 4.24). Die Steigung der Aggregatenzahldichte $\left(\mathrm{s} \sim 4.9 * 10^{10} \mathrm{~cm}^{-3}\right)$ sollte dabei eine geringere Steigung haben als die der Teilchenzahldichte nach Rayleigh $\left(\mathrm{s} \sim 1.5 * 10^{10} \mathrm{~cm}^{-3}\right]$. Die Ausgleichsgerade der Steigung der Teilchenzahldichte nach Rayleigh liegt beim Ethylen bei $\mathrm{s} \sim 2.4 * 10^{10} \mathrm{~cm}^{-3}$ und die der Aggregatenzahldichte bei $\mathrm{s} \sim 1.7 * 10^{10} \mathrm{~cm}^{-3}$ [47]. Damit steigt die Aggregatenzahldichte in Abhängigkeit vom C/O-Verhältnis der untersuchten Benzol-LuftFlammen wesentlich deutlicher als das bei den Ethylen-Luft-Flammen der Fall ist. Die Ausgleichsgerade der Teilchenzahldichte hat einen Schnittpunkt mit der x-Achsen bei C/O = 0.565, der etwas unterhalb des kritischen C/O-Verhältnisses liegt. Die Ausgleichsgerade der Aggregatenzahldichte hat ihren Schnittpunkt mir der x-Achsen bei $\mathrm{C} / \mathrm{O}=0.62$, welcher etwas über dem kritischen $\mathrm{C} / \mathrm{O}-$ Verhältnis liegt und damit etwas $\mathrm{zu}$ hoch scheint. Auch diese Achsenschnittpunkte sind ein Indiz dafür, daß die gemessene Aggregatenzahldichte einen zu steilen und die gemessene Teilchenzahldichte eher einen zu flachen Verlauf haben.

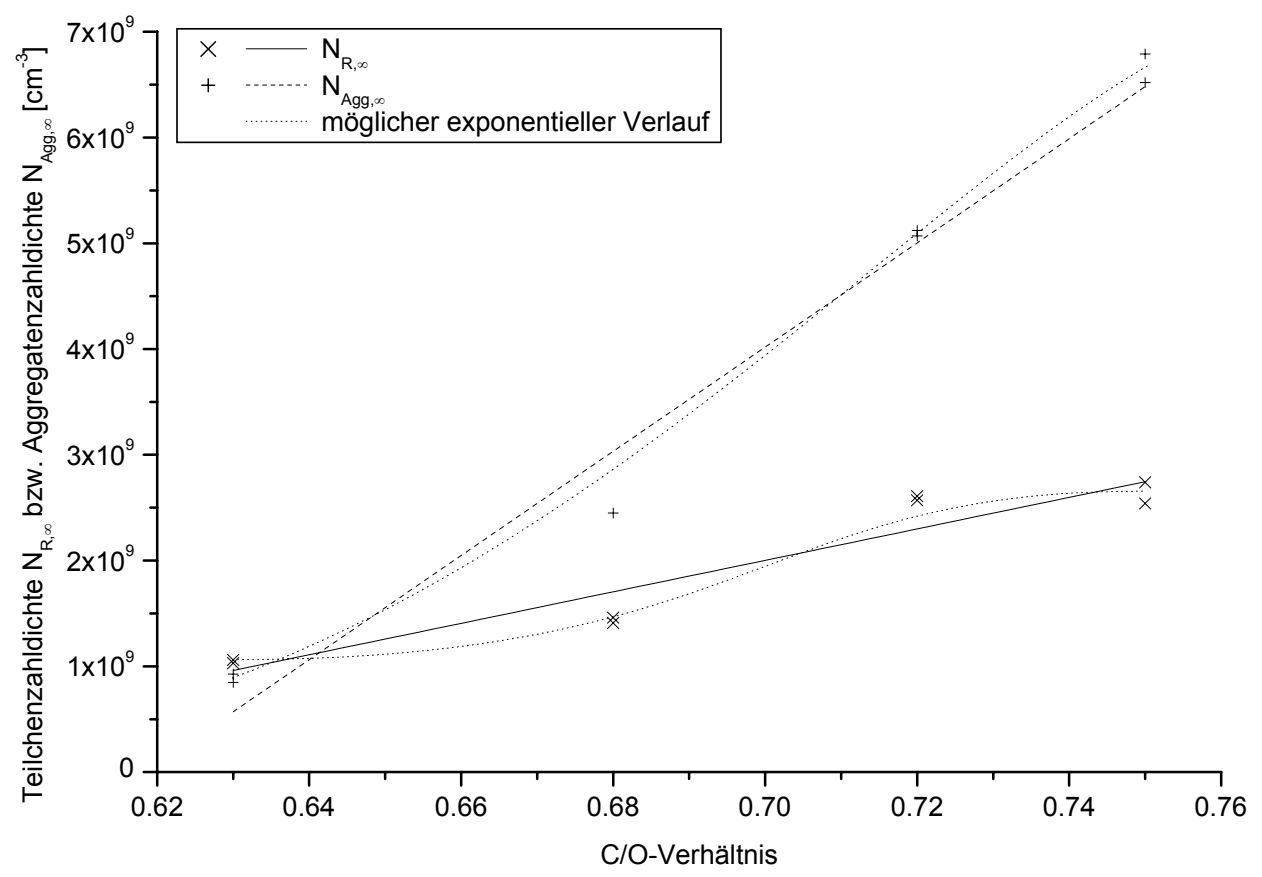

Abb. 4.24: Abhängigkeit der Teilchenzahldichte $\mathrm{N}_{\mathrm{R}, \infty}$ und der Aggregatenzahldichte $\mathrm{N}_{\mathrm{Agg}, \infty}$ vom C/O-Verhältnis für eine Höhe von $\mathrm{h}=17.7 \mathrm{~mm}$ und eine Frischgasgeschwindigkeit von $\mathrm{v}=5.1 \mathrm{~cm} / \mathrm{s}$

In der Arbeit [22] wurde für die Abhängigkeit der Teilchenzahldichte $\mathrm{N}_{\mathrm{R}, \infty}$ vom C/OVerhältnis im untersuchten Bereich an C/O-Verhältnissen ein exponentieller Anstieg der 
Teilchenzahldichte festegestellt. Zusätzlich wurde dort vermutet, daß eine Teilchenzahldichte von $10^{10}$ bei höheren $\mathrm{C} / \mathrm{O}$-Verhältnissen nicht überschritten wird. Ein möglicher exponentieller Anstiegt und ein Abflachen der Teilchenzahldichte sowie der Aggregatenzahldichte wurde deshalb mit gepunkteten Kurven in die Auftragung (Abb. 4.24) mit aufgenommen.

Die folgende Auftragung (Abb. 4.25) zeigt die Abhängigkeit der Teilchenzahldichte $\mathrm{N}_{\mathrm{R}, \infty}$ und der Aggregatenzahldichte $\mathrm{N}_{\mathrm{Agg}, \infty}$ von der Frischgasgeschwindigkeit:

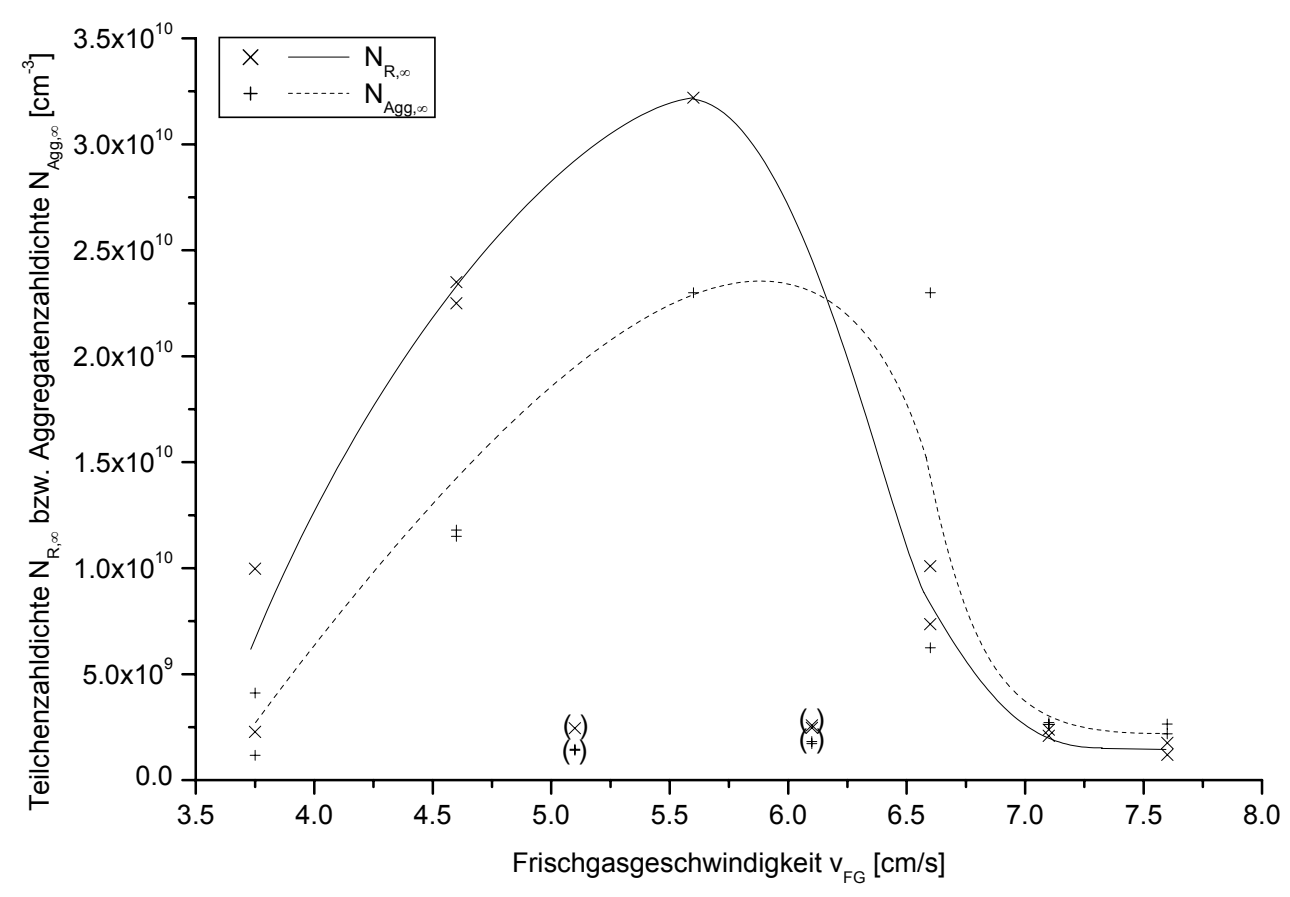

Abb. 4.25: Durchsatzabhängigkeit der Teilchenzahldichte für eine Höhe von $\mathrm{h}=17.7 \mathrm{~mm}$ und einem $\mathrm{C} / \mathrm{O}-$ Verhältnis von $\mathrm{C} / \mathrm{O}=0.68$

Sowohl die Teilchenzahldichte $\mathrm{N}_{\mathrm{R}, \infty}$ als auch die Aggregatenzahldichte $\mathrm{N}_{\mathrm{Agg}, \infty}$ durchlaufen ein Maximum bei einer Frischgasgeschwindigkeit von etwa $\mathrm{v}_{\mathrm{FG}}=5.5 \mathrm{~cm} / \mathrm{s}$. Es wird eine maximale Teilchenzahldichte von $\mathrm{N}_{\mathrm{R}, \infty}=3.2 * 10^{10} \mathrm{~cm}^{-3}$ und eine maximale Aggregatenzahldichte von $\mathrm{N}_{\mathrm{Agg}, \infty}=2.4 * 10^{10} \mathrm{~cm}^{-3}$ erhalten. Dabei nähern sich die Kurven der Teilchenzahldichte und der Aggregatenzahldichte mit zunehmender Frischgasgeschwindigkeit an. Bei Frischgasgeschwindigkeiten oberhalb von $\mathrm{V}_{\mathrm{FG}}=7 \mathrm{~cm} / \mathrm{s}$ beträgt die Teilchenzahldichte von $\mathrm{N}_{\mathrm{R}, \infty}=1.4 * 10^{9}$ und die Aggregatenzahldichte von $\mathrm{N}_{\mathrm{Agg}, \infty}=2.2 * 10^{9}$. Bei kleinen Frischgasgeschwindigkeiten werden ähnliche Werte erhalten. Der Verlauf von 
Teilchenzahldichte und Aggregatenzahldichte bei den untersuchten Benzol-Luft-Flammen widerspricht damit völlig dem Verhalten (exponentieller Anstieg mit der Frischgasgeschwindigkeit) der untersuchten Ethylen-Luft-Flammen [47]. Bei $p=10$ bar wurde in einer weiteren Arbeit auch ein exponentieller Abfall der Teilchenzahldichte mit der Frischgasgeschwindigkeit beobachtet [22]. Dies läßt die Vermutung zu, daß der Verlauf der Teilchenzahldichte sowie der Aggregatenzahldichte bei den untersuchten Benzol-LuftFlammen sich aus den beiden zitierten Ergebnissen der anderen Arbeiten zusammensetzt.

\subsubsection{Fraktale Dimension}

Die fraktale Dimension (vgl. Kap. 2.6) ist eine wichtige Größe der RDG/FA-Theorie. Die fraktale Dimension beschreibt die Struktur eines Aggregats bezüglich der drei Achsen. Eine fraktale Dimension von $\mathrm{D}_{\mathrm{f}}=1$ steht für eine lineare Struktur (Kette), Werte darüber bedeutet eine 2- oder 3-dimensionale Struktur der Aggregate. Ermittelt wurde dieser dimensionslose Parameter mit Hilfe einer doppeltlogarithmischen Auftragung des über den Absorptionskoeffizienten normierten Streufaktors $\mathrm{Q}_{\mathrm{vv}}$ gegen den Sinus des halben Streuwinkels $\Theta$ :

$$
\log \left(\frac{\mathrm{Q}_{\mathrm{VV}}}{\mathrm{K}_{\mathrm{abs}}}\right) \sim \log \frac{1}{[4 \pi \cdot \sin (\Theta / 2)]^{\mathrm{D}_{\mathrm{f}}}}
$$

Aus dieser Auftragung wird die fraktale Dimension durch Berechnung einer Ausgleichsgeraden im Power Law Abschnitt des Kurvenverlaufs erhalten. Ein Beispiel, wie die fraktale Dimension bestimmt wurde ist in den folgenden Abbildungen (Abb. 4.26 und Abb. 4.27) dargestellt: 


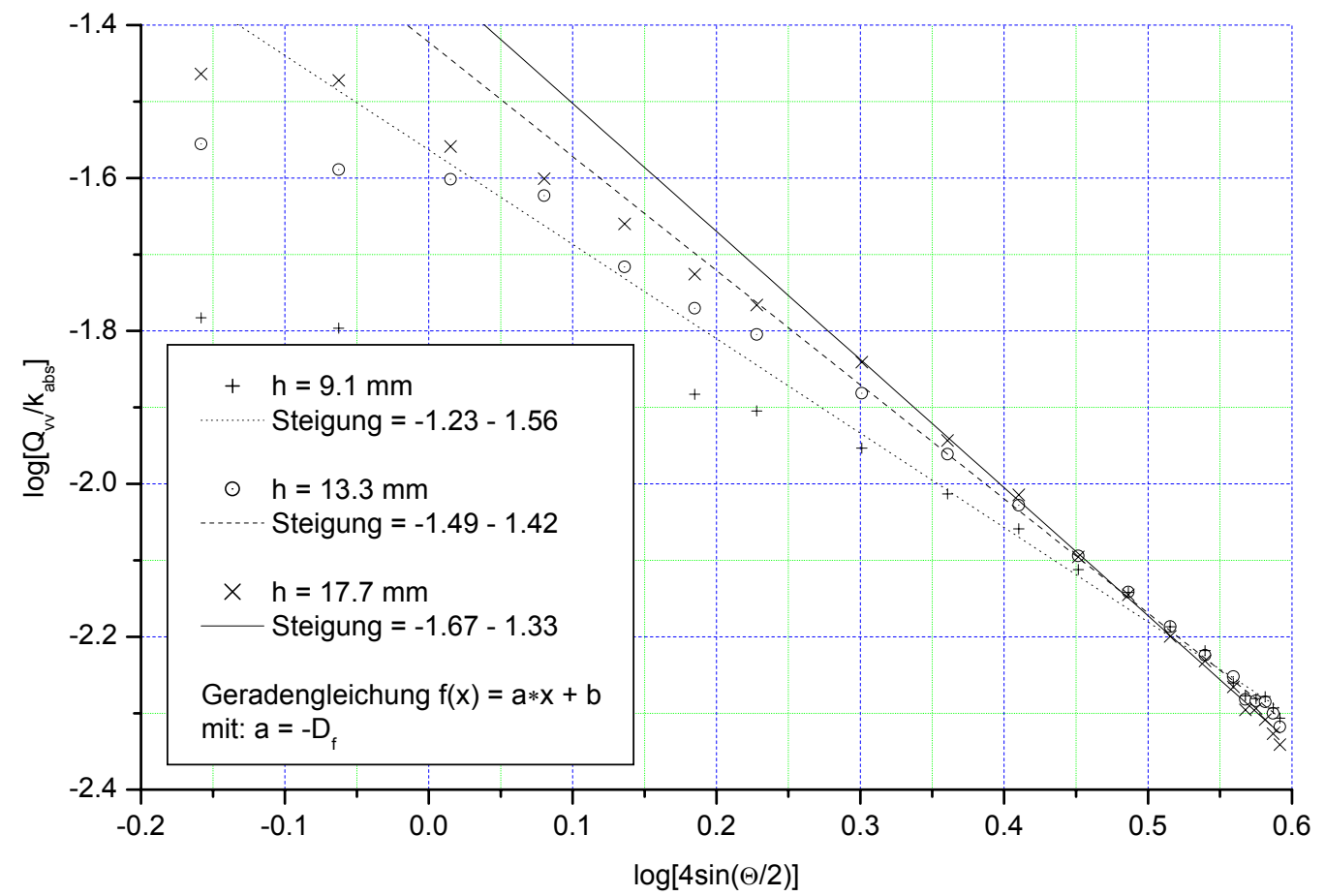

Abb. 4.26: Bestimmung des Parameters $\mathrm{D}_{\mathrm{f}}$ für verschiedene Höhen bei einem C/OVerhältnis von $\mathrm{C} / \mathrm{O}=0.72$ und einer Frischgasgeschwindigkeit von $\mathrm{v}=5.1$ $\mathrm{cm} / \mathrm{s}$

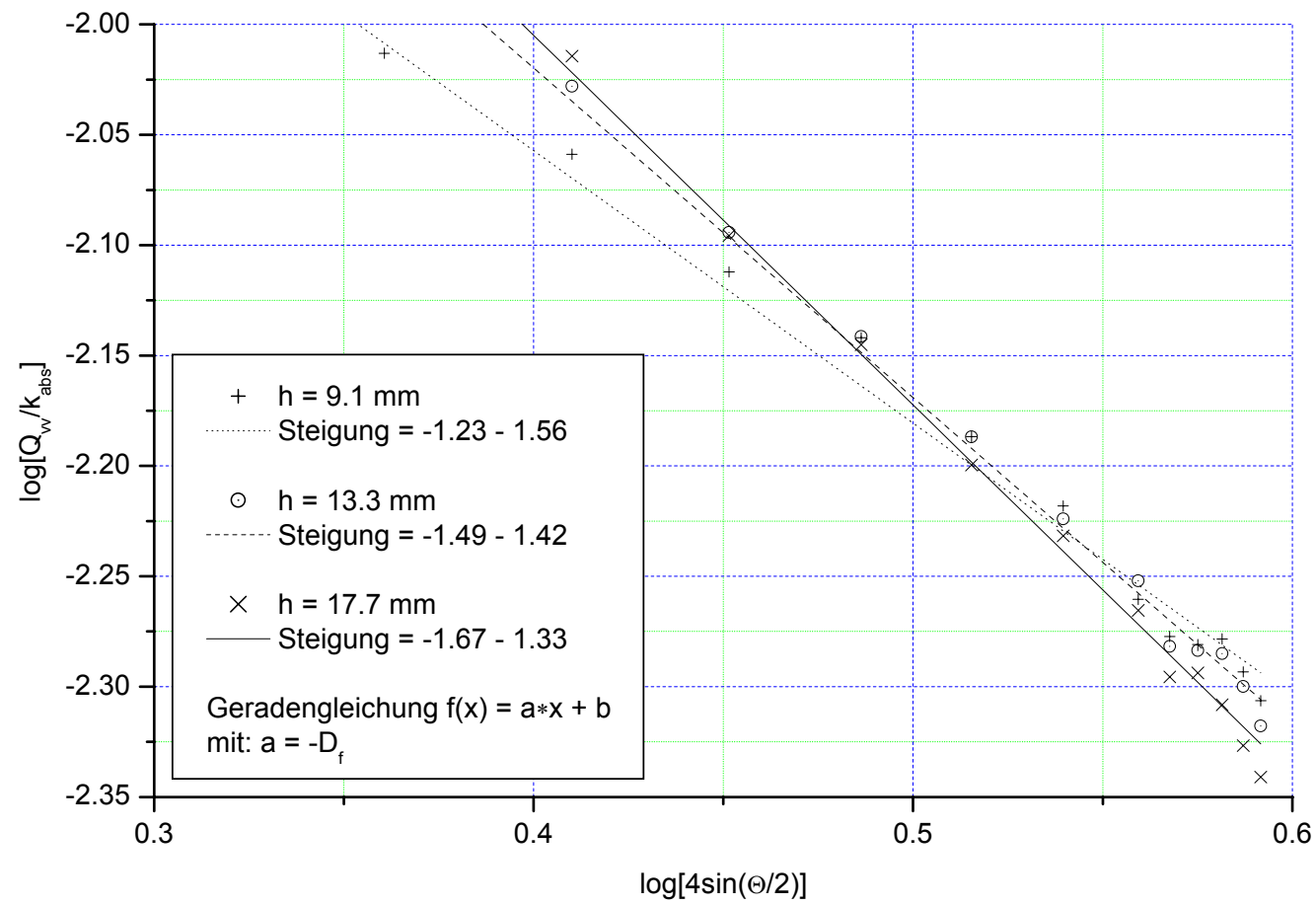

Abb. 4.27: Vergrößerung des relevanten Bereichs der Abb. 4.26 
Die fraktale Dimension $\mathrm{D}_{\mathrm{f}}$ nimmt bei Benzol-Luft-Flammen mit der Höhe fast linear zu (Abb. 4.28). Der Kurvenverlauf ist leicht abflachend. Im Vergleich zum Brennstoff Ethylen ist die Steigung geringer, da schon in der untersten Höhe die fraktale Dimension deutlich über eins liegt. Damit nimmt $\mathrm{D}_{\mathrm{f}}$ für alle Höhen physikalisch realistische Werte an, die tatsächlich mehrdimensionale Teilchen beschreiben.

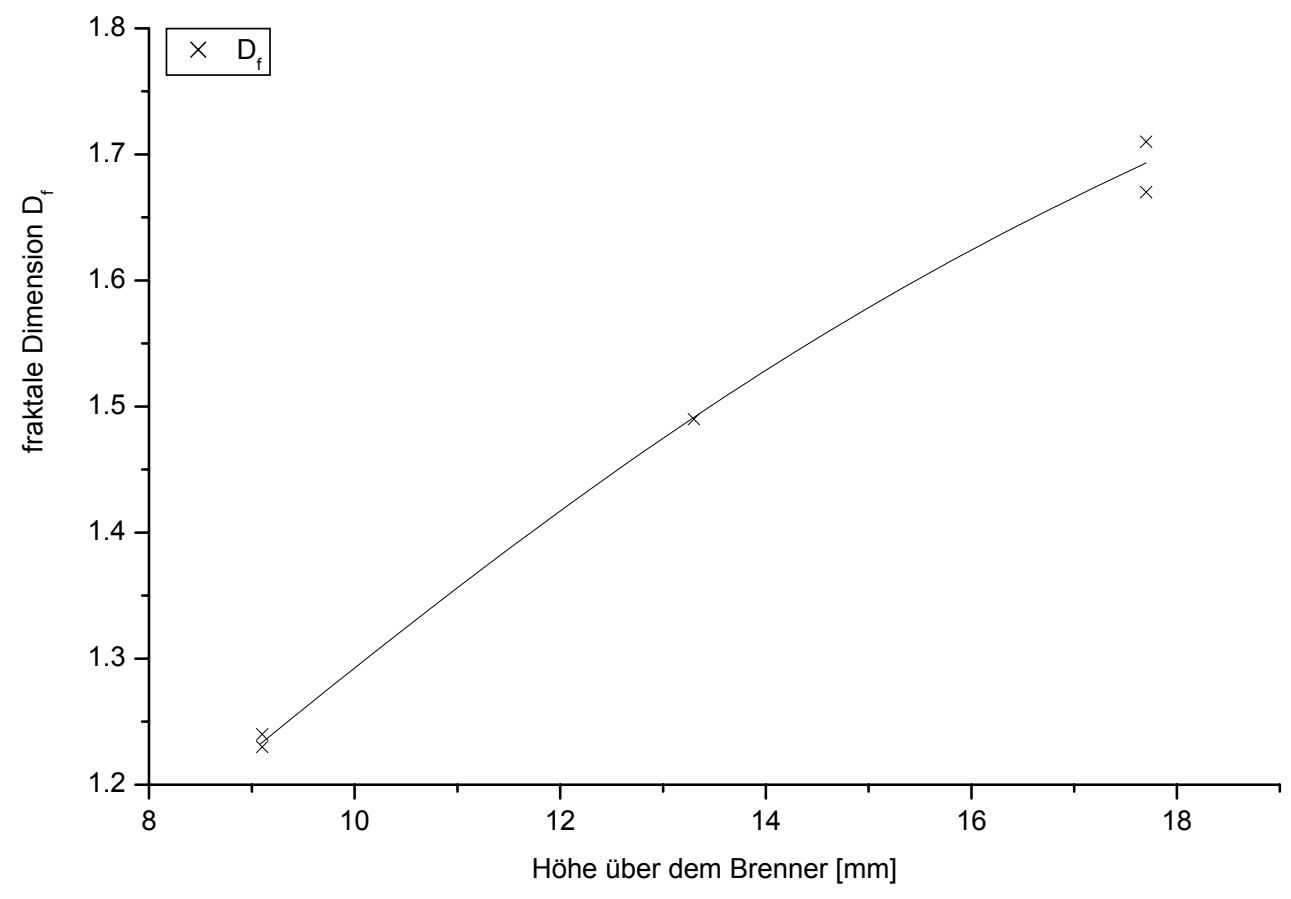

Abb. 4.28: Abhängigkeit der fraktalen Dimension von der Höhe für ein C/O-Verhältnis von $\mathrm{C} / \mathrm{O}=0.72$ und einer Frischgasgeschwindigkeit von $5.1 \mathrm{~cm} / \mathrm{s}$

Die Abhängigkeit der fraktalen Dimension vom C/O-Verhältnis (Abb. 4.29) wird deutlich geringer in Richtung höherer $\mathrm{C} / \mathrm{O}-$ Verhältnisse. Dies wurde auch bei den Ethylen-LuftFlammen beobachtet. Bei Benzol-Luft-Flammen nimmt die fraktale Dimension allerdings schon etwas über der Rußgrenze Werte von $\mathrm{D}_{\mathrm{f}} \sim 1$ an, d.h. es liegen dort kettenförmige Gebilde vor. Mit zunehmendem C/O-Verhältnis steigt die fraktale Dimension, wie beim Ethylen, zu Werten von $\mathrm{D}_{\mathrm{f}}=1.75$ (Ethylen: $\mathrm{D}_{\mathrm{f}}=1.78$ ). 


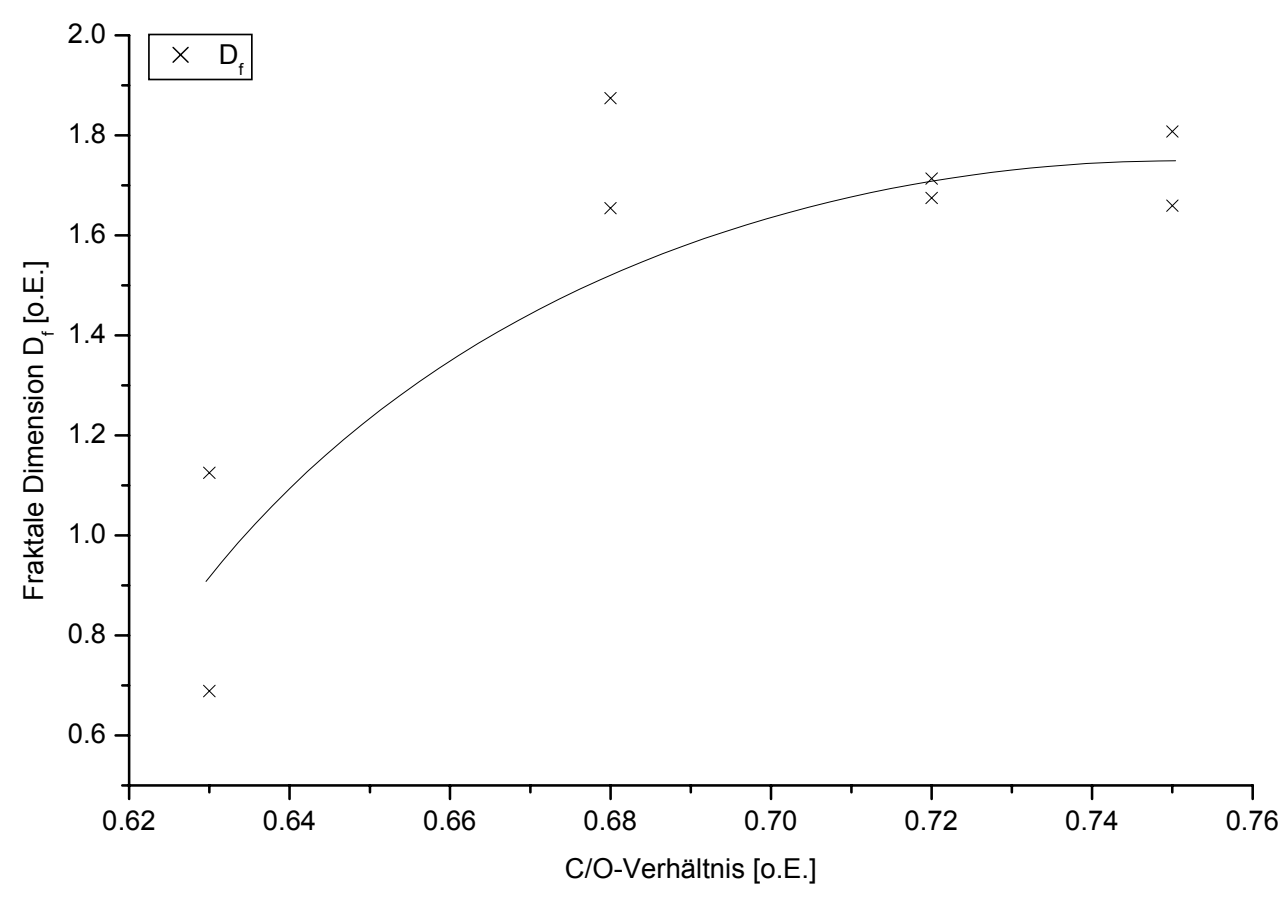

Abb. 4.29: Abhängigkeit der fraktalen Dimension vom C/O-Verhältnis für eine Höhe von $\mathrm{h}=17.7 \mathrm{~mm}$ und eine Frischgasgeschwindigkeit von $\mathrm{v}=5.1 \mathrm{~cm} / \mathrm{s}$

In ihrer Geschwindigkeitsabhängigkeit durchläuft die fraktale Dimension bei einem $\mathrm{C} / \mathrm{O}-$ Verhältnis von 0.68 und einer Höhe von $\mathrm{h}=17.7 \mathrm{~mm}$ analog zum Ethylen $(\mathrm{C} / \mathrm{O}=0.82 ; \mathrm{h}=$ $28 \mathrm{~mm}$ ) auch bei Benzol-Luft-Flammen ein Maximum. Auch der etwas flachere Anstieg und der steilere Abfall sind bei beiden Brennstoffen in etwa gleich ausgeprägt. Die fraktale Dimension nimmt hier in ihrem Maximum bei einer Frischgasgeschwindigkeit von $\mathrm{v} \approx 5.2$ $\mathrm{cm} / \mathrm{s}$ einen Wert von etwa $\mathrm{D}_{\mathrm{f}} \approx 1.72$ an.

Damit konnte gezeigt werden, daß die Rußpartikel aus Benzol-Luft-Flammen schon ab einer Höhe von etwa $\mathrm{h}=10 \mathrm{~mm}$, oder bei einem $\mathrm{C} / \mathrm{O}-\mathrm{Verhältnis} \mathrm{von} \mathrm{C} / \mathrm{O}=0.65$ und größer oder einer Frischgasgeschwindigkeit unter $v=7 \mathrm{~cm} / \mathrm{s}$ als Aggregate vorliegen müssen, da ihre fraktale Dimension dann deutlich über eins liegt. Dieses Phänomen wird durch das abweichende Verhalten des Rayleigh-Durchmessers vom scheinbaren Radius bzw. vom volumenäquivalenten Durchmesser (Kap. 4.1.2.7) in genau diesen Bereichen untermauert. 


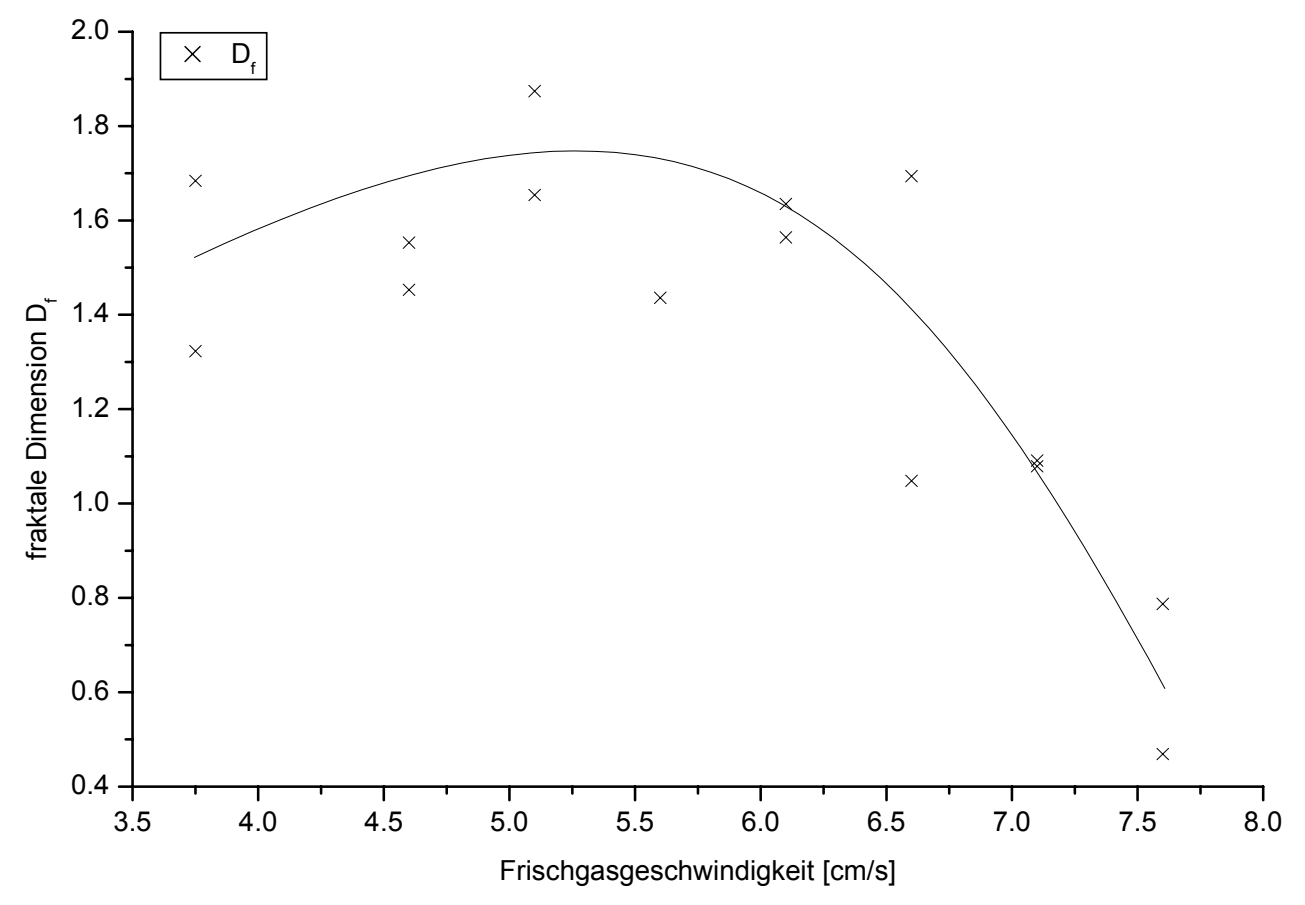

Abb. 4.30: Abhängigkeit der fraktalen Dimension von der Frischgasgeschwindigkeit für eine Höhe von $\mathrm{h}=17.7 \mathrm{~mm}$ und einem $\mathrm{C} / \mathrm{O}-$ Verhältnis von $\mathrm{C} / \mathrm{O}=0.68$

\subsubsection{Primärteilchen}

Die Primärteilchendurchmesser $d_{p}$ bzw. Primärteilchenzahldichten $n_{p}$ (s. Kap. 2.6) lassen sich aus dem optischen Größenparameter $x_{p}$ berechnen. Der optische Größenparameter kann mit Kenntnis der fraktalen Dimension aus Streufaktoren und Absorption berechnet werden. Durch die Abhängigkeit von vielen Größen sind der Primärteilchendurchmesser und die Primärteilchenzahldichte sehr fehlerbehaftete Größen. Im folgenden soll deshalb nur auf die Primärteilchendurchmesser kurz eingegangen werden.

Die Höhenabhängigkeit der Primärteilchendurchmesser verläuft genau entgegen den Erwartungen. Die Größenordnung des Primärteilchendurchmessers liegt bei $d_{p}=26 \mathrm{~nm}$ und damit im Bereich, der beim Ethylen festgestellten Werte, d.h. die Primärteilchen sind bereits unterhalb des untersuchten Höhenbereichs gebildet worden und auf ihre endgültige Größe angewachsen. Einen deutlich höhenabhängigeren Verlauf des Primärteilchendurchmessers ergaben die untersuchten n-Heptan-Luft-Flammen (s. Anhang, 7.1.1.2). 


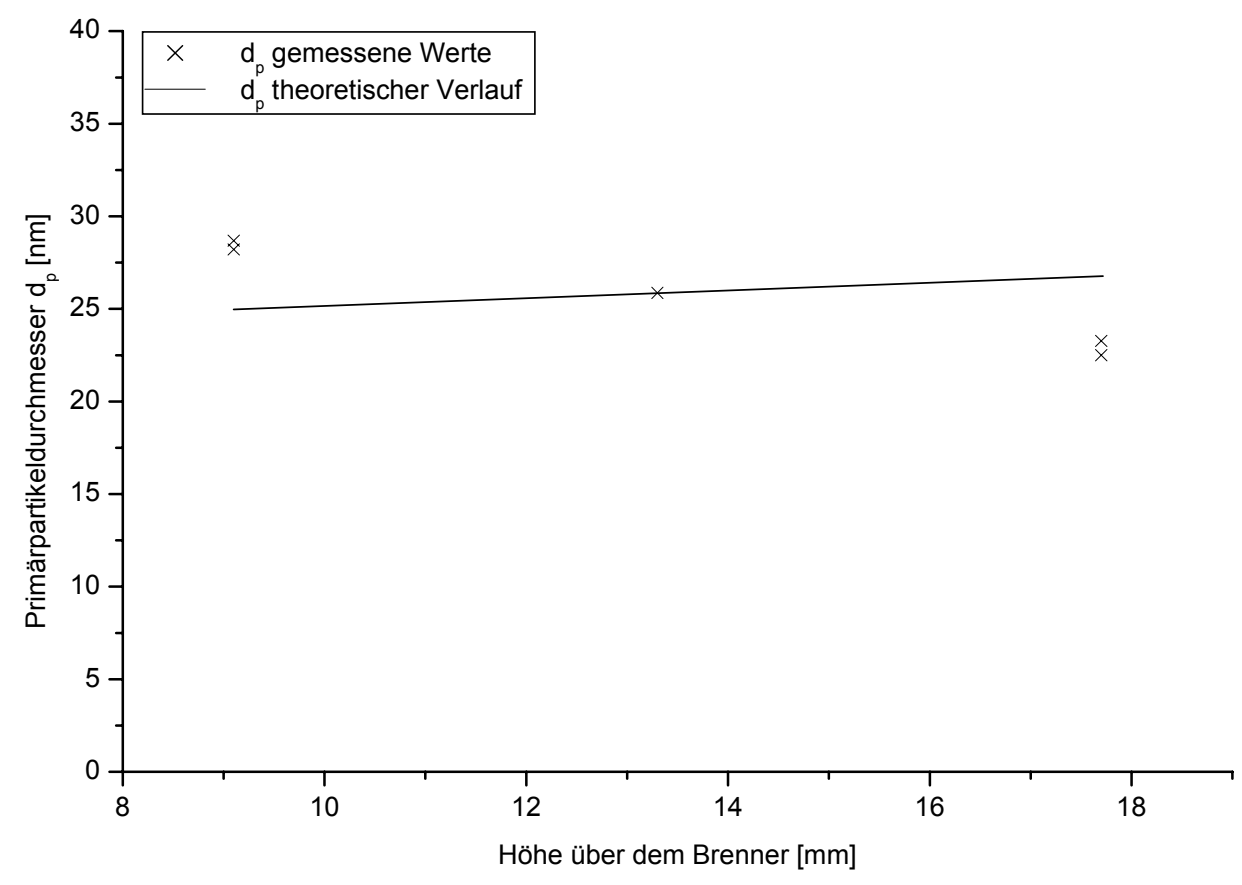

Abb. 4.31: Abhängigkeit des Primärteilchendurchmessers von der Höhe für ein C/OVerhältnis von $\mathrm{C} / \mathrm{O}=0.72$ und einer Frischgasgeschwindigkeit von $5.1 \mathrm{~cm} / \mathrm{s}$

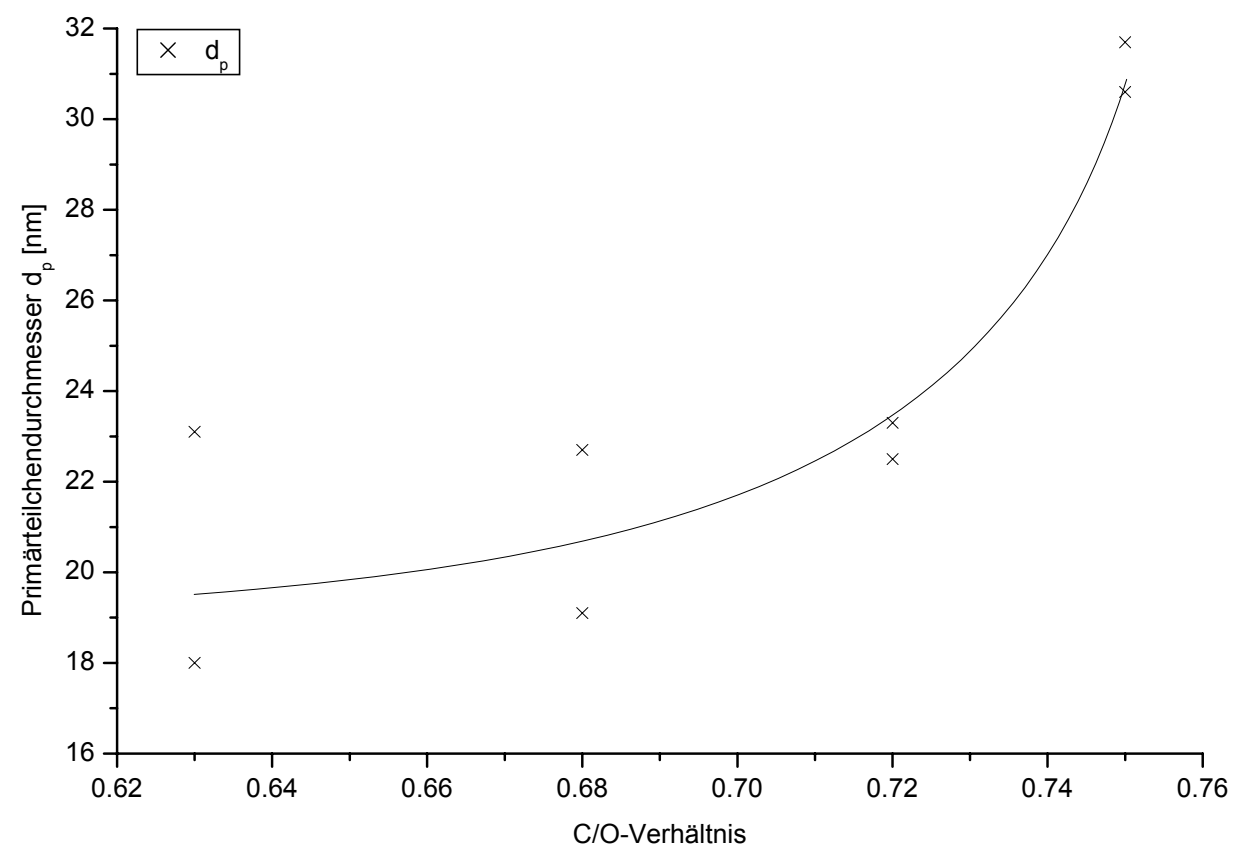

Abb. 4.32: Abhängigkeit des Primärteilchendurchmessers vom C/O-Verhältnis für eine Höhe von $\mathrm{h}=17.7 \mathrm{~mm}$ und eine Frischgasgeschwindigkeit von $\mathrm{v}=5.1 \mathrm{~cm} / \mathrm{s}$ 
Auch die Abhängigkeit des Primärteilchendurchmessers vom C/O-Verhältnis konnte bei Benzol-Luft-Flammen nur unzureichend ermittelt werden (Abb. 4.32). Zu erwarten wäre ein zunächst mit zunehmendem $\mathrm{C} / \mathrm{O}-$ Verhältnis steilerer Anstieg, der mit brennstoffreicheren Gemischzusammensetzungen zunehmend flacher wird. Auch hier liegt der Größenbereich der Primärteilchendurchmesser zwischen $d_{p}=20 \mathrm{~nm}$ und $d_{p}=30 \mathrm{~nm}$.

Eine Abhängigkeit des Primärteilchendurchmessers von der Frischgasgeschwindigkeit (Abb. 4.33 ) ist für das eingestellte $\mathrm{C} / \mathrm{O}-$ Verhältnis von $\mathrm{C} / \mathrm{O}=0.68$ festzustellen. Der Primärteilchendurchmesser durchläuft bei einer Frischgasgeschwindigkeit von $v_{\mathrm{FG}}=5.1 \mathrm{~cm} / \mathrm{s}$ mit Werten bis zu $d_{p}=23 \mathrm{~nm}$ ein Maximum. Sowohl mit geringeren als auch mit höheren Frischgasgeschwindigkeiten fällt der Primärteilchendurchmesser zunehmend. Bei hohen Frischgasgeschwindigkeiten mit $\mathrm{v}_{\mathrm{FG}} \geq 7.5 \mathrm{~cm} / \mathrm{s}$ beträgt der Primärteilchendurchmesser etwa $\mathrm{d}_{\mathrm{p}}=10-11 \mathrm{~nm}$. Bei Ethylen-Luft-Flammen (untersuchter $\mathrm{v}_{\mathrm{FG}}$-Bereich: $4.3 \mathrm{~cm} / \mathrm{s} \leq \mathrm{v}_{\mathrm{FG}} \leq 10.5$ $\mathrm{cm} / \mathrm{s}$ ) wurde nur der Bereich der mit der Frischgasgeschwindigkeit fallenden Primärteilchendurchmesser gefunden.

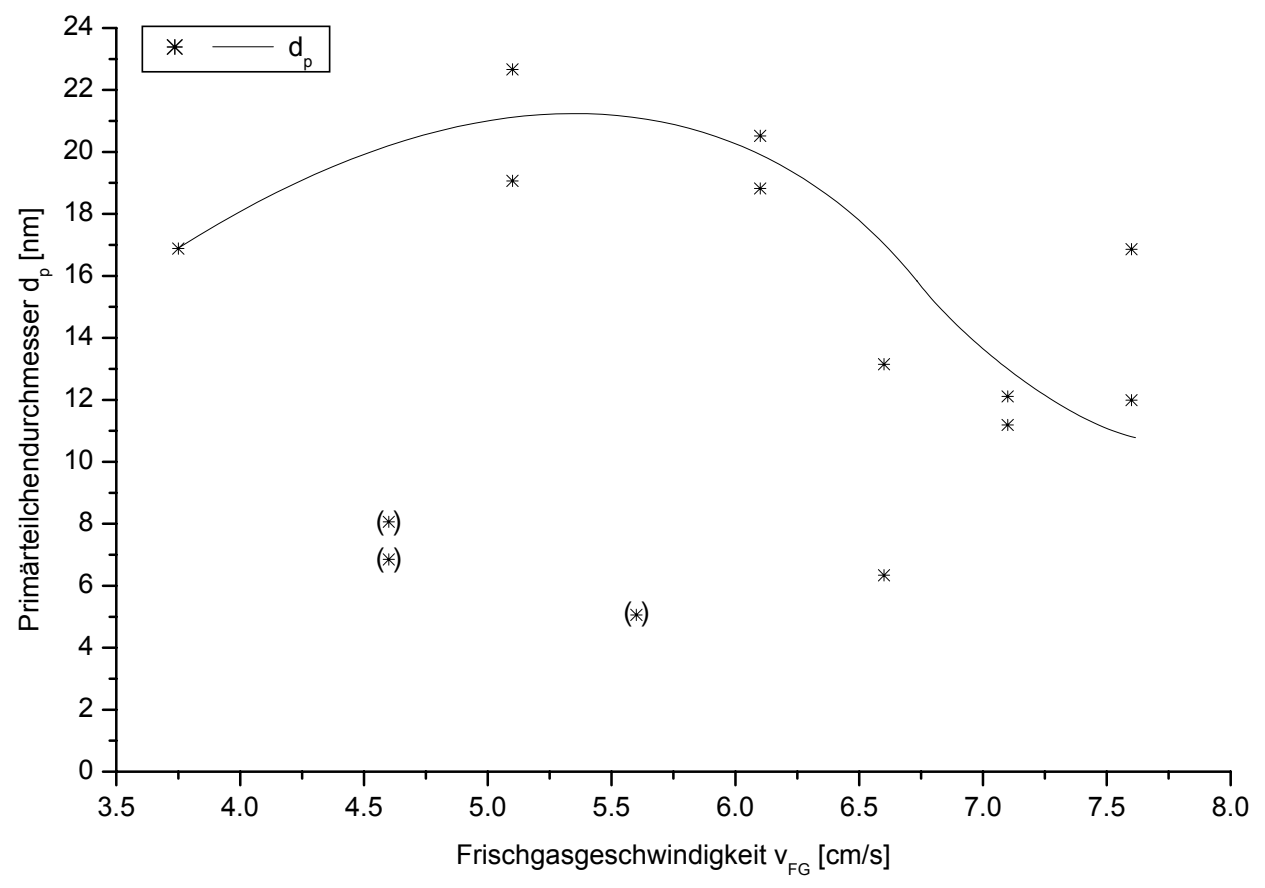

Abb. 4.33: Abhängigkeit des Primärteilchendurchmessers von der Frischgasgeschwindigkeit für eine Höhe von $\mathrm{h}=17.7 \mathrm{~mm}$ und einem $\mathrm{C} / \mathrm{O}$-Verhältnis von $\mathrm{C} / \mathrm{O}=0.68$ 
4.2 Ethylenflammen bei höherem Druck

\subsubsection{Zusammenstellung der Meßdaten}

Im folgenden sollen die bei höherem Druck gemessenen Ethylen-Luft-Flammen mit ihren unterschiedlich eingestellten Parametern tabellarisch (Tab. 4.6) zusammengestellt werden.

\begin{tabular}{|c|c|c|c|c|c|c|}
\hline $\begin{array}{l}\text { Druck } \\
\text { [bar] }\end{array}$ & $\begin{array}{l}\text { Frischgasgeschwin- } \\
\text { digkeit }[\mathrm{cm} / \mathrm{s}]\end{array}$ & $\begin{array}{l}\text { C/O- } \\
\text { Verhältnis }\end{array}$ & $\begin{array}{l}\text { Höhe } \\
{[\mathrm{mm}]}\end{array}$ & Datum & $\begin{array}{l}\text { Messung- } \\
\text { Nr.: }\end{array}$ & $\begin{array}{l}\text { Variierter } \\
\text { Parameter }\end{array}$ \\
\hline 3.10 & 5.8 & 0.75 & 7.5 & 030314 & $2 x$ & \\
\hline 3.27 & 5.5 & 0.66 & 7.5 & 030314 & $3 x$ & \multirow{4}{*}{$\begin{array}{l}\text { C/O- } \\
\text { Verhältnis }\end{array}$} \\
\hline 3.15 & 5.7 & 0.69 & 7.5 & 030414 & $11 x$ & \\
\hline 3.23 & 5.5 & 0.73 & 7.5 & 030414 & $12 x$ & \\
\hline 3.01 & 6.0 & 0.75 & 7.5 & 030414 & $13 x$ & \\
\hline 5.26 & 5.5 & 0.65 & 7.5 & 030414 & $14 x$ & \multirow{4}{*}{$\begin{array}{l}\text { C/O- } \\
\text { Verhältnis }\end{array}$} \\
\hline 5.10 & 5.8 & 0.65 & 7.5 & 030414 & $15 x$ & \\
\hline 5.20 & 5.5 & 0.68 & 7.5 & 030414 & $16 x$ & \\
\hline 4.99 & 6.1 & 0.71 & 7.5 & 030414 & $17 x$ & \\
\hline 5.14 & 3.8 & 0.68 & 7.5 & 030415 & $11 x$ & \multirow{4}{*}{$\begin{array}{l}\text { Frischgasge- } \\
\text { schwindigkeit }\end{array}$} \\
\hline 5.08 & 5.9 & 0.68 & 7.5 & 030415 & $12 x$ & \\
\hline 5.19 & 7.6 & 0.68 & 7.5 & 030415 & $13 x$ & \\
\hline 5.07 & 9.9 & 0.68 & 7.5 & 030415 & $14 x$ & \\
\hline 2.10 & 7.7 & 0.74 & 9.0 & 030430 & $11 x$ & \multirow[t]{4}{*}{ Druck } \\
\hline 3.03 & 7.9 & 0.75 & 9.0 & 030430 & $12 x$ & \\
\hline 3.95 & 8.1 & 0.75 & 9.0 & 030430 & $13 x$ & \\
\hline 5.13 & 7.8 & 0.75 & 9.0 & 030430 & $14 x$ & \\
\hline 5.13 & 7.8 & 0.75 & 2.5 & 030430 & $15 x$ & \\
\hline 1.99 & 8.1 & 0.75 & 3.0 & 030506 & $11 x$ & \multirow[t]{4}{*}{ Höhe } \\
\hline 2.00 & 8.1 & 0.74 & 6.0 & 030506 & $12 x$ & \\
\hline 2.02 & 8.0 & 0.74 & 9.0 & 030506 & $13 x$ & \\
\hline 2.03 & 7.9 & 0.74 & 12.0 & 030506 & $14 x$ & \\
\hline 3.92 & 8.2 & 0.75 & 12.0 & 030506 & $15 x$ & \multirow[t]{4}{*}{ Höhe } \\
\hline 3.96 & 8.1 & 0.75 & 9.0 & 030506 & $16 x$ & \\
\hline 3.97 & 8.1 & 0.75 & 6.0 & 030506 & $17 x$ & \\
\hline 3.95 & 8.1 & 0.75 & 3.0 & 030506 & $18 x$ & \\
\hline 1.18 & 9.0 & 0.69 & 9.0 & 030728 & 010 & \\
\hline 2.04 & 6.9 & 0.70 & 9.0 & 030728 & 020 & \multirow[t]{4}{*}{ Druck } \\
\hline 3.07 & 6.9 & 0.70 & 9.0 & 030728 & 030 & \\
\hline 4.10 & 6.8 & 0.70 & 9.0 & 030728 & 040 & \\
\hline 5.15 & 6.8 & 0.70 & 9.0 & 030728 & 050 & \\
\hline
\end{tabular}

Tab. 4.6: Zusammenstellung der Ethylen-Luft-Flammen bei höherem Druck

Eine Tabelle mit allen ausgewerteten Daten befindet sich im Anhang 7.1.2. 
In diesem Kapitel soll der Einfluß des Druckes auf die Meßgrößen Rußvolumenbruch $\left(f_{v}\right)$, die Teilchendurchmesser $\left(\mathrm{d}_{\mathrm{R}}, \mathrm{D}_{30}\right.$ und $\left.\mathrm{R}_{\mathrm{g}}\right)$, die Teilchenzahldichten $\left(\mathrm{N}_{\mathrm{R}}\right.$ und $\left.\mathrm{N}_{\mathrm{Agg}}\right)$, Primärteilchendurchmesser $\left(d_{p}\right)$, Primärteilchenzahldichte $\left(n_{p}\right)$ und fraktale Dimension $\left(D_{f}\right)$ dargestellt werden. Bei den hier behandelten Ethylen-Luft Hochdruckflammen handelte es sich um vorgemischte laminare Flammen in einem Druckbereich von 2 bar und 5 bar. Die Frischgasgeschwindigkeit wurde in einem Bereich von $\mathrm{v}_{\mathrm{FG}, \min }=3.85 \mathrm{~cm} / \mathrm{s}$ und $\mathrm{v}_{\mathrm{FG}, \max }=9.95$ $\mathrm{cm} / \mathrm{s}$ variiert. Die Gemischzusammensetzungen lagen in einem Bereich von $(\mathrm{C} / \mathrm{O})_{\min }=0.65$ und $(\mathrm{C} / \mathrm{O})_{\max }=0.75$. Die Flammen wurden durch eine nichtrußende Flamme des Außenbrenners stabilisiert. Ein Drahtnetz in einer Höhe zwischen $h=20 \mathrm{~mm}$ und $\mathrm{h}=30 \mathrm{~mm}$ diente zur weiteren Stabilisierung der Flammen.

Da in diesem Kapitel hauptsächlich auf die Druckabhängigkeit der Rußbildung eingegangen werden soll, werden in diesem Abschnitt Meßergebnisse, die bei unterschiedlichem Druck bei einem konstanten $\mathrm{C} / \mathrm{O}-$ Verhältnis von $\mathrm{C} / \mathrm{O}=0.70$ und einer konstanten Frischgasgeschwindigkeit von $\mathrm{v}_{\mathrm{FG}}=6.9 \mathrm{~cm} / \mathrm{s}$ erhalten wurden, herangezogen. Diese Flammen wurden in einer Höhe von $9 \mathrm{~mm}$ (die ersten Messungen mit $7.5 \mathrm{~mm}$ ) optisch untersucht. Weitere druckabhängige Auftragungen bei einem $\mathrm{C} / \mathrm{O}-$ Verhältnis von $\mathrm{C} / \mathrm{O}=0.75$ und einer Frischgasgeschwindigkeit von $\mathrm{V}_{\mathrm{FG}}=8.0 \mathrm{~cm} / \mathrm{s}$ befinden sich im Anhang 7.1.2 (Abb. 7.63 bis Abb. 7.71). Für jeweils eine Flamme bei $p=2$ bar und $p=4$ bar wurden die Messungen für 4 unterschiedliche Höhen (3, 6, 9 und $12 \mathrm{~mm}$ ) durchgeführt. Bei einem Druck von $\mathrm{p}=5$ bar wurde die Frischgasgeschwindigkeit in einem Bereich zwischen $\mathrm{v}_{\mathrm{FG}}$, $\min =3.85 \mathrm{~cm} / \mathrm{s}$ und $\mathrm{v}_{\mathrm{FG}}$, $\max =9.95 \mathrm{~cm} / \mathrm{s}$ variiert. Die Auftragungen hierzu befinden sich ebenfalls im Anhang 7.1.2 (Abb. 7.72 bis Abb. 7.75) im Anschluß an die Auftragungen der Druckabhängigkeit.

4.2.2 Erscheinungsbild der Ethylen-Luft-Flammen im Druckbereich von 2 bis 5 bar

Mit zunehmendem Flammendruck verändert sich eine rußende Flamme in zweierlei Weise:

1. Die Längenausdehnungen der Vorheizzone, der Hauptoxidationszone und der Beginn der Rußzone rücken immer näher zur Brenneroberkante hin. Das bedeutet, daß die Rußbildung mit steigendem Druck immer früher einsetzt.

2. Durch das frühe Einsetzen der Rußbildung kommt es in der Flamme nicht zu einer homogenen Rußverteilung. Die Flamme bekommt eine streifige Struktur. Diese Streifenbildung der vorgemischten Hochdruckflamme ist ein typisches 
Kennzeichen für Flammen im Hochdruck. Durch den in der Arbeitsgruppe Prof. Wagner entwickelten Folienflammenhalter konnte die Streifenbildung in der Flamme bis zu einem Druck von $p=5$ bar vollständig vermieden werden.

Die Photographien (Abb. 4.34 und Abb. 4.35) zeigen Ethylen-Luft-Flammen bei einem Druck von $p=2$ bzw. $p=5$ bar, bei denen keine streifige Struktur der Flamme zu beobachten ist.

Die Flammen wurden mit einer Digitalkamera durch das Beobachtungsfenster photographiert. Der helle Strich in den Flammen auf den Photographien ist ein Laserstrahl. Der Laserstrahl des $\mathrm{Ar}^{+}$-Ionenlasers ist über die ganze laterale Ausdehnung der Flamme von gleichmäßiger Intensität. Das Drahtnetz war bei der Flamme in der Abb. 4.35 in einer Höhe von etwa $20 \mathrm{~mm}$ montiert. Für die in der Abb. 4.34 dargestellte Flamme war das Drahtnetz in einer Höhe von etwa $30 \mathrm{~mm}$ angebracht worden. Auf dem Schauglas hat sich bei der 5 bar Flamme (Abb. 4.35) ein leichter Rußschleier gelegt, der das Bild etwas eintrübt.

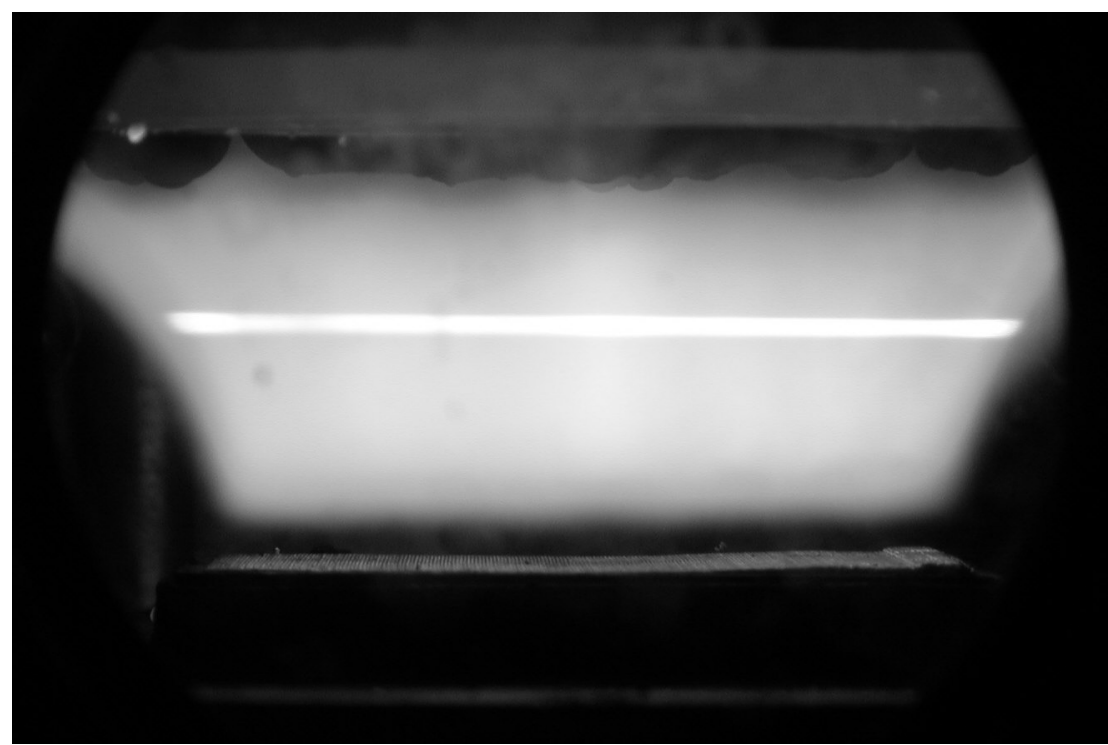

Abb. 4.34: Ethylen-Luft-Flamme bei einem Druck von $p=2$ bar, einem C/O-Verhältnis von $\mathrm{C} / \mathrm{O}=0.75$ und einer Frischgasgeschwindigkeit von $\mathrm{v}_{\mathrm{FG}}=8 \mathrm{~cm} / \mathrm{s}$

Die 2 bar Flamme hat einen deutlich größeren Abstand zwischen Brenneroberfläche und Untergrenze der Rußzone als das bei der 5 bar Flamme der Fall ist. Auch die geringere Höhe des Drahtnetzes bei der 2 bar Flamme ändert hieran nichts, obwohl diese Flamme durch die geringere Netzhöhe insgesamt etwas gestauchter erscheint als die 5 bar Flamme. So beginnt die Rußzone der 2 bar Flamme bei $2.2 \mathrm{~mm}$ (vgl. Laserstrahl bei $12 \mathrm{~mm}$ über dem Brenner), während sie in der 5 bar Flamme schon bei 1.2 mm anfängt (Abb. 4.35; vgl. Laserstrahl bei 9 
mm über dem Brenner). Damit setzt die Rußbildung in der 2 bar Flamme bei einer längeren Reaktionszeit, verglichen mit der 5 bar Flamme, ein.

Die deutlich bessere Ausleuchtung des Brenners in der Abbildung der 5 bar Flamme weist auf eine hellere Flamme hin. Dies wird durch die mit dem Druck steigende Flammentemperatur hervorgerufen. Diese Tendenz kann auch durch das niedrigere $\mathrm{C} / \mathrm{O}=$ Verhältnis nicht kompensiert werden.

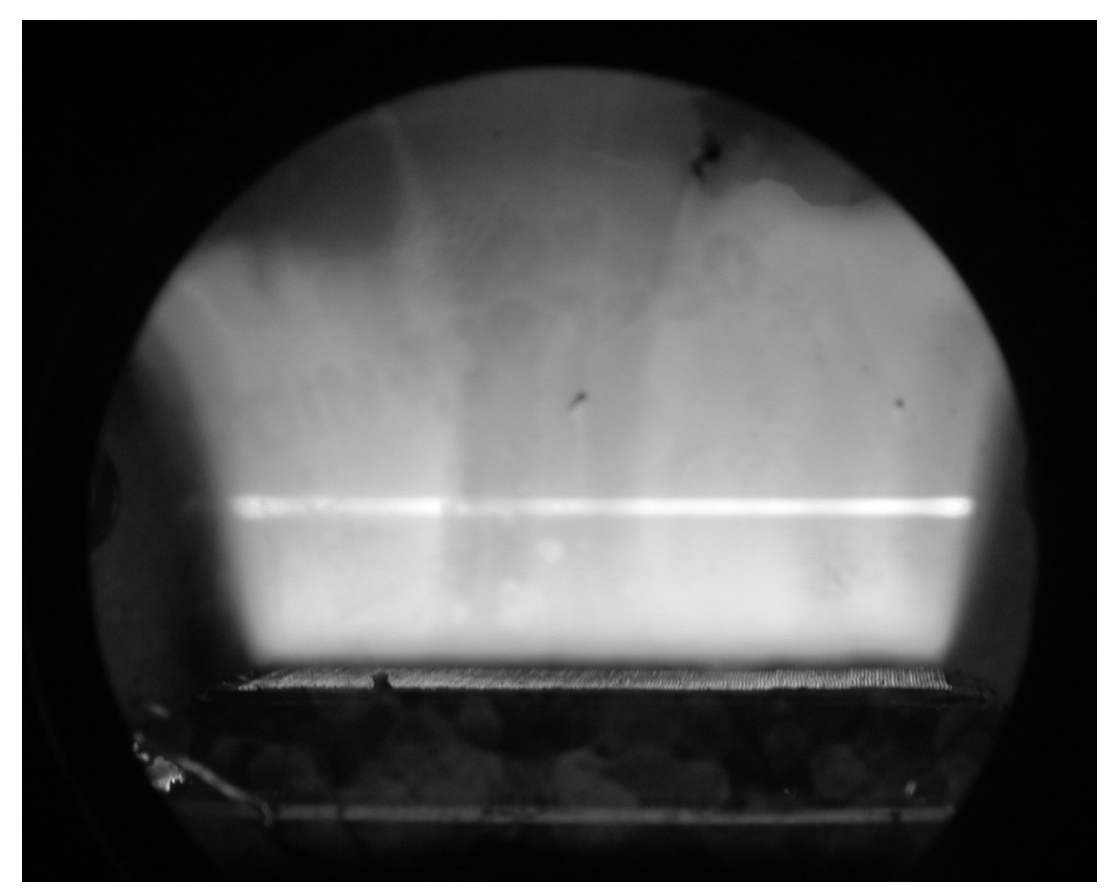

Abb. 4.35: Ethylen-Luft-Flamme bei einem Druck von $\mathrm{p}=5$ bar, einem C/O-Verhältnis von $\mathrm{C} / \mathrm{O}=0.70$ und einer Frischgasgeschwindigkeit von $\mathrm{v}_{\mathrm{FG}}=7 \mathrm{~cm} / \mathrm{s}$

\subsubsection{Rußgrenzen}

Der Einfluß des Flammendrucks auf das Rußgebiet einer Ethylen-Luft-Flamme wird aus der Abb. 4.36 deutlich. In Kap. 4.1.2.2 S. 43 wurde die visuelle Rußgrenze, und wie sie im Labor ermittelt wurde, erläutert. Die Abb. 4.36 zeigt bei 2 verschiedenen Drücken den Verlauf der

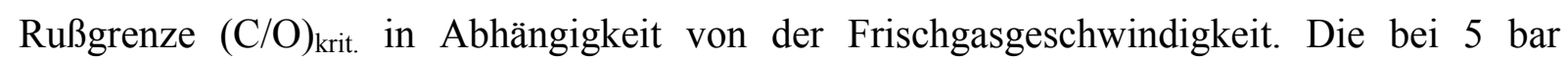
erhaltene Rußgrenze wurde der Diplomarbeit von Frau Bruhn [10] entnommen. 
Generell ist der Verlauf der Rußgrenze bei höherem Flammendruck dem der 1 bar Rußgrenze ähnlich. Für niedrige Frischgasgeschwindigkeiten (daher auch niedrige Flammentemperaturen) steigt die Rußgrenze sehr steil an und erreicht ein kritisches C/OVerhältnis von $(\mathrm{C} / \mathrm{O})_{\text {krit. }}>1$. Bei einer Frischgasgeschwindigkeit von $\mathrm{v}_{\mathrm{FG}}=2.2 \mathrm{~cm} / \mathrm{s}$ durchläuft die Rußgrenze ein Minimum mit $(\mathrm{C} / \mathrm{O})_{\text {krit. }} \sim 0.50$, und steigt dann mit zunehmender Frischgasgeschwindigkeit wieder an. Dabei ist besonders anzumerken, daß das Minimum der Rußgrenze der 5 bar Flamme bei einem kleineren kritischem C/O-Verhältnis liegt als die Rußgrenze der 1 bar Flamme $\left((\mathrm{C} / \mathrm{O})_{\text {krit. }} \sim 0.56\right.$ bei $\left.\mathrm{v}_{\mathrm{FG}}=5 \mathrm{~cm} / \mathrm{s}\right)$. Dies hat zur Folge, daß mit steigendem Flammendruck die Rußbildung immer eher einsetzt. Sie bewegt sich also mit steigendem Druck immer weiter in Richtung eines $\mathrm{C} / \mathrm{O}-$ Verhältnisses, welches einer stöchiometrischen Gemischzusammensetzung $(\mathrm{C} / \mathrm{O})_{\text {stöch. }}=0.33$ entspricht. Das hat zur Folge, daß bei höherem Druck und kleinen Frischgasgeschwindigkeiten bereits magere Gemischzusammensetzungen zu rußenden Flammen führen, die bei einem Druck von $p=1$ bar nicht rußen würden. Entsprechend ist auch die Rußmenge bei Flammen mit höherem Druck größer als es bei einem Druck von $\mathrm{p}=1$ bar der Fall ist.

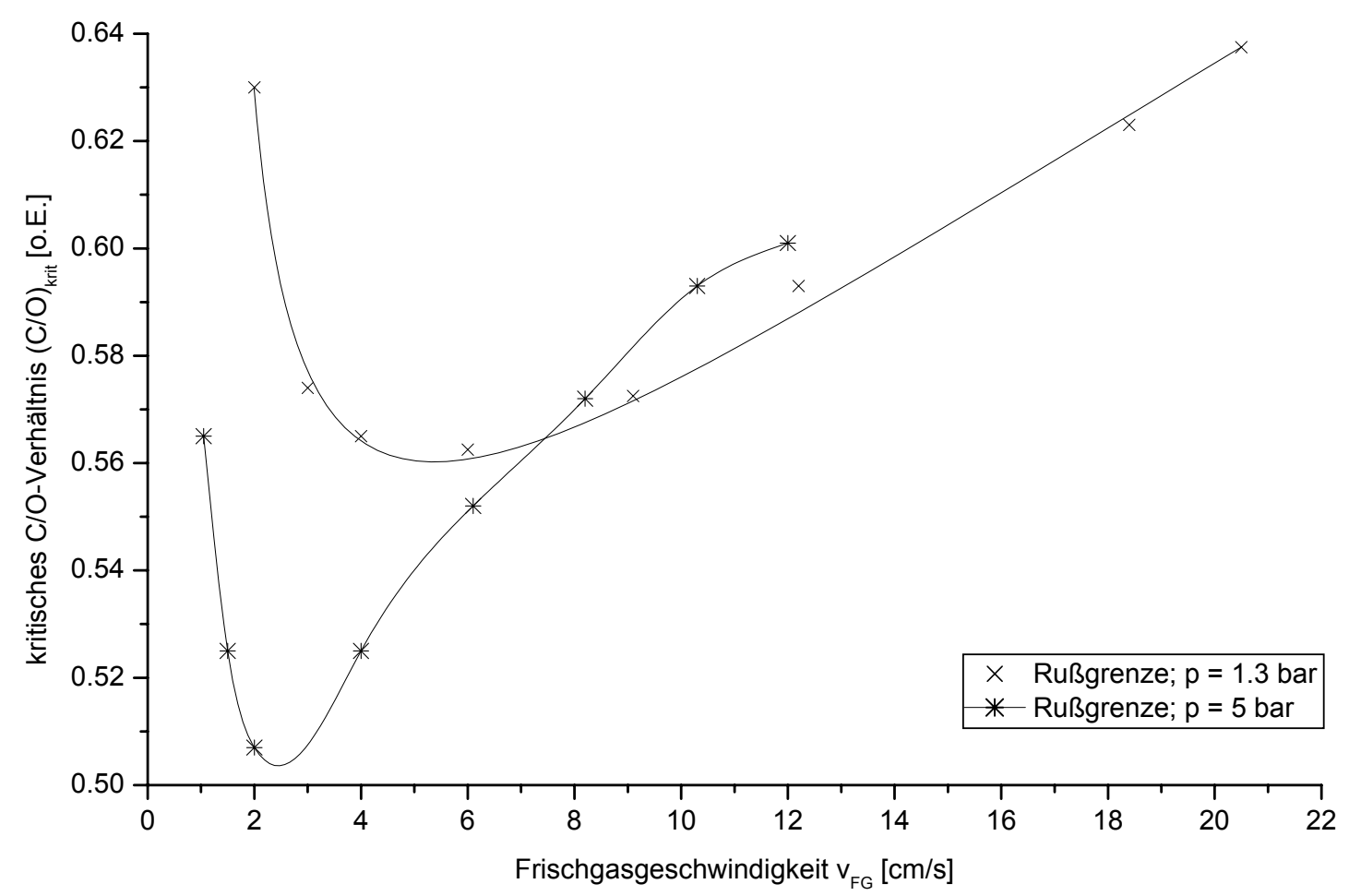

Abb. 4.36: Rußgrenzen einer Ethylen-Luft-Flamme bei 1.3 und 5 bar 


\subsubsection{Rußvolumenbruch}

Der Einfluß des Drucks auf die Rußbildung wird in der Abb. 4.37 anhand von 2 Drücken, 2 bar und 5 bar, gezeigt. Die kleinste Höhe, bei der stabile und reproduzierbare optische Messungen in der 2 bar Flamme möglich war ist $\mathrm{h}=3 \mathrm{~mm}$. Aus diesem Grund beginnen die höhenabhängigen Kurvenverläufe auch erst bei $\mathrm{h}=3 \mathrm{~mm}$. In dieser Höhe ist der Rußvolumenbruch bereits um mehrere Größenordnungen angewachsen.

$\mathrm{Zu}$ Beginn der Rußzone ist der Rußvolumenbruch mit einem Wert von $\mathrm{f}_{\mathrm{v}}=5 * 10^{-8} \mathrm{~cm}^{3}$ $(\mathrm{Ru} ß) / \mathrm{cm}^{3}$ (Flammenvolumen) zunächst sehr gering. Dies gilt auch für die Flammen bei höherem Druck. Mit steigender Höhe nimmt der Rußvolumenbruch für die untersuchten Drücke zwischen 2 bar und 5 bar aber einen unterschiedlichen Verlauf an. Je größer der eingestellte Druck der Flammen ist, desto stärker wächst der Rußvolumenbruch mit der Höhe an (s. Abb. 4.37).

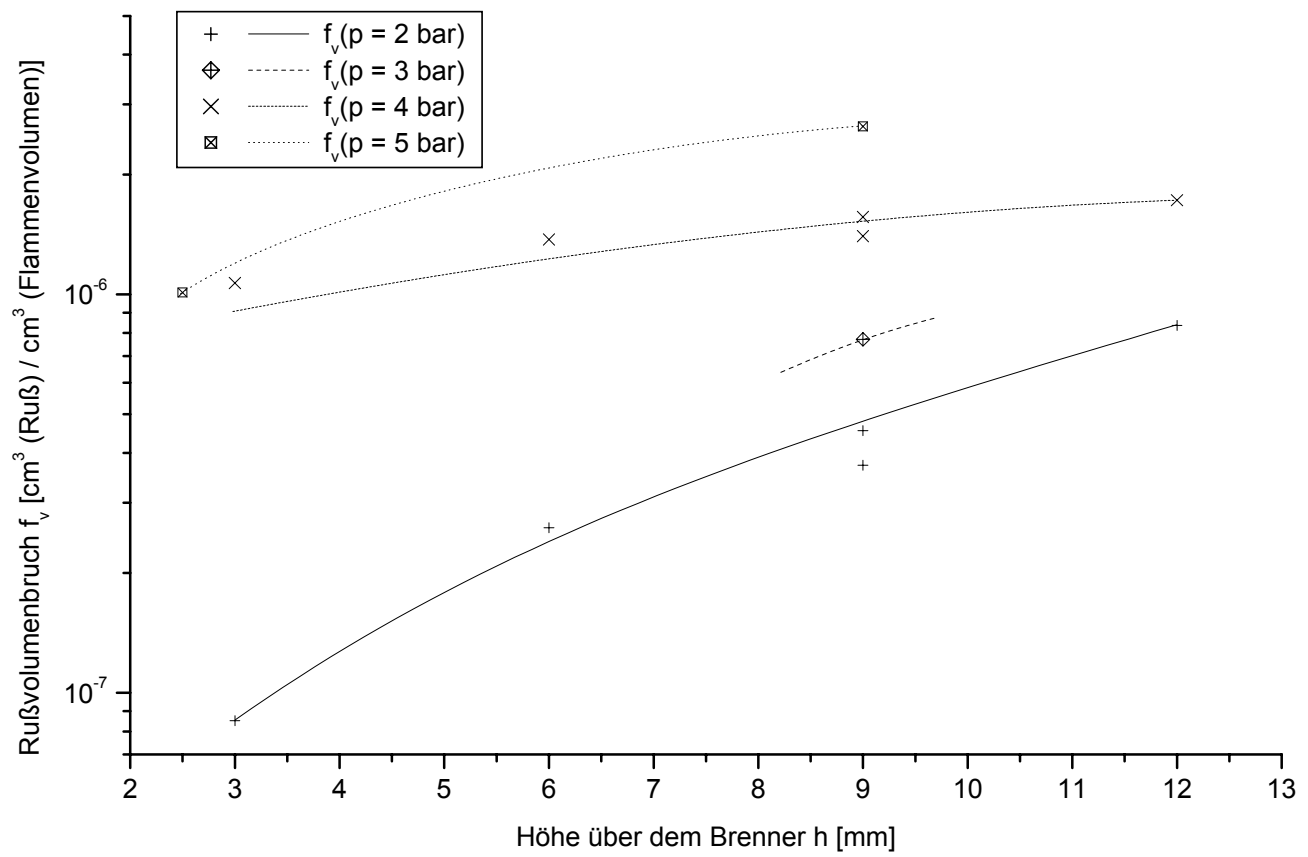

Abb. 4.37: Höhenabhängigkeit des Rußvolumenbruchs von Ethylen-Luft-Flammen bei einem Druck zwischen 2 bar und 5 bar mit einem $\mathrm{C} / \mathrm{O}-$ Verhältnis von $\mathrm{C} / \mathrm{O}=$ 0.75 und einer Frischgasgeschwindigkeit von $\mathrm{v}_{\mathrm{FG}}=8 \mathrm{~cm} / \mathrm{s}$ 


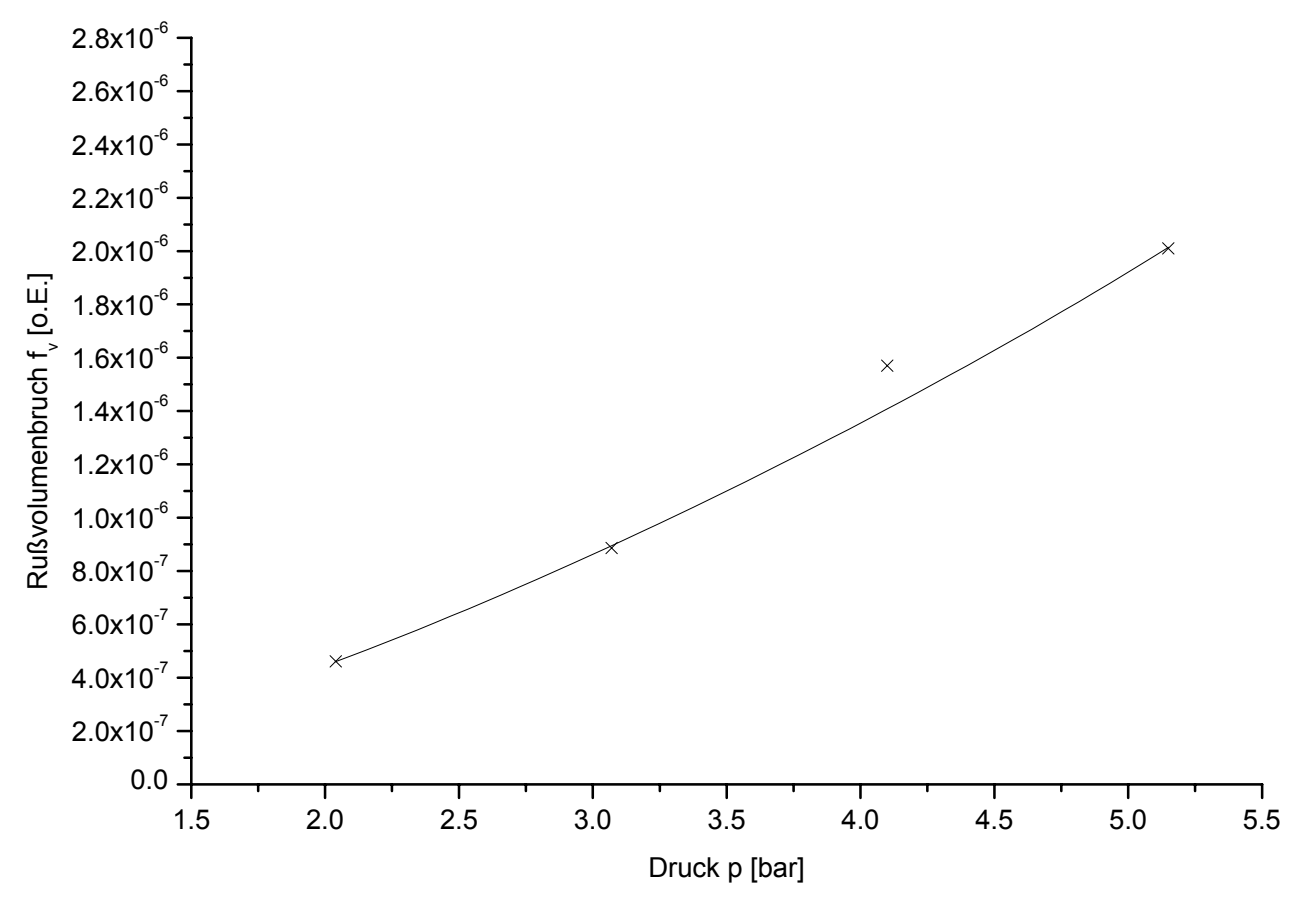

Abb. 4.38: Druckabhängigkeit des Rußvolumenbruchs von Ethylen-Luft-Flammen mit einem $\mathrm{C} / \mathrm{O}-$ Verhältnis von $\mathrm{C} / \mathrm{O}=0.70$ und einer Frischgasgeschwindigkeit von $\mathrm{v}=6.9 \mathrm{~cm} / \mathrm{s}$ und einer Höhe über dem Brenner von $\mathrm{h}=9 \mathrm{~mm}$

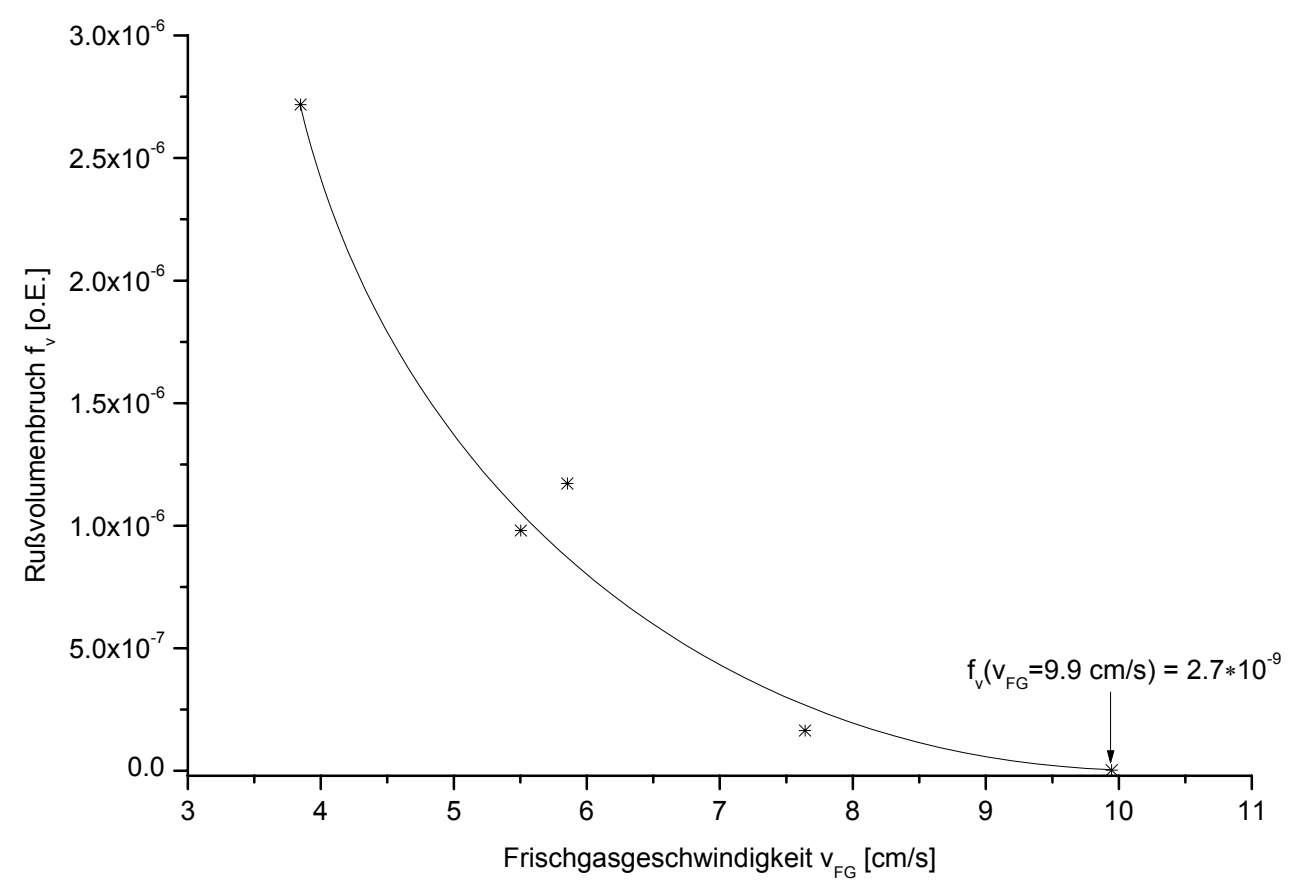

Abb. 4.39: Durchsatzabhängigkeit des Rußvolumenbruchs von Ethylen-Luft-Flammen mit $\mathrm{C} / \mathrm{O}=0.75$ und $\mathrm{h}=7.5 \mathrm{~mm}$ bei einem Druck von $5 \mathrm{bar}$ 
In Abb. 4.38 ist die Druckabhängigkeit des Rußvolumenbruchs dargestellt. Der Kurvenverlauf macht den starken Anstieg des Rußvolumenbruchs mit dem Druck im untersuchten Bereich von 2 bar $\leq \mathrm{p} \leq 5$ bar deutlich. Für eine Höhe von 9 mm (Abb. 4.38) und den Ergebnissen der Abb. 7.63 (s. Anhang) wurde eine quadratische Druckabhängigkeit des Rußvolumenbruchs $f_{v} \sim p^{2}$ ermittelt. Diese Ergebnisse stimmen mit den Resultaten aus vorangegangenen Arbeiten [40] überein.

Die Abhängigkeit des Rußvolumenbruchs von der Frischgasgeschwindigkeit (Abb. 4.39) ist bei einem Druck von $\mathrm{p}=5$ bar dem Verlauf bei einem bar sehr ähnlich, allerdings ist der Kurvenverlauf etwas steiler. Bei geringen Frischgasgeschwindigkeiten von 3 bis $4 \mathrm{~cm} / \mathrm{s}$ ist der Rußvolumenbruch der 5 bar Flamme deutlich höher als bei einem bar, mit steigender Frischgasgeschwindigkeit nähern sich die Kurvenverläufe etwas an.

\subsubsection{Teilchendurchmesser und Teilchenzahldichten}

\subsubsection{Teilchendurchmesser, volumenäquivalenter Durchmesser und scheinbarer Radius}

In der Abbildung (Abb. 4.40) ist die Höhenabhängigkeit der verschiedenen Teilchendurchmesser (Rayleigh-Teilchendurchmesser $d_{R}$, volumenäquivalenter Durchmesser $\mathrm{D}_{30}$ und scheinbarer Radius $\left.\mathrm{R}_{\mathrm{g}}\right)$ dargestellt. Alle Teilchendurchmesser $\left(\mathrm{d}_{\mathrm{R}}, \mathrm{D}_{30}\right.$ bzw. $\left.\mathrm{R}_{\mathrm{g}}\right)$ steigen bei geringer Höhe zunächst stark an. Die Steigung flacht aber mit zunehmender Höhe ab. Bei ungefähr $12 \mathrm{~mm}$ über dem Brenner sind die Endwerte aller Teilchendurchmesser $\left(\mathrm{d}_{\mathrm{R}}\right.$, $\mathrm{D}_{30}$ bzw. $\mathrm{R}_{\mathrm{g}}$ ) erreicht. Wie schon bei den 1 bar Ethylen-Luft-Flammen festgestellt wurde [47], sind die scheinbaren Radien größer als Rayleigh-Teilchendurchmesser und volumenäquivalenter Durchmesser. Der volumenäquivalente Durchmesser ist seinerseits noch geringfügig größer als der Rayleigh Teilchendurchmesser. Die Kurven der verschiedenen Teilchendurchmesser $\left(\mathrm{d}_{\mathrm{R}}, \mathrm{D}_{30}\right.$ bzw. $\left.\mathrm{R}_{\mathrm{g}}\right)$ bei unterschiedlichem Druck verlaufen annähernd parallel zueinander. Die Rayleigh-Teilchenradien und die volumenäquivalenten Durchmesser sind bei $\mathrm{p}_{2}=4$ etwa $15 \mathrm{~nm}$ größer als bei $\mathrm{p}_{1}=2$ bar. Die scheinbaren Radien für beide Drücke liegen $15 \mathrm{~nm}$ bis $30 \mathrm{~nm}$ auseinander und verlaufen in geringer Höhe noch nicht ganz parallel. 


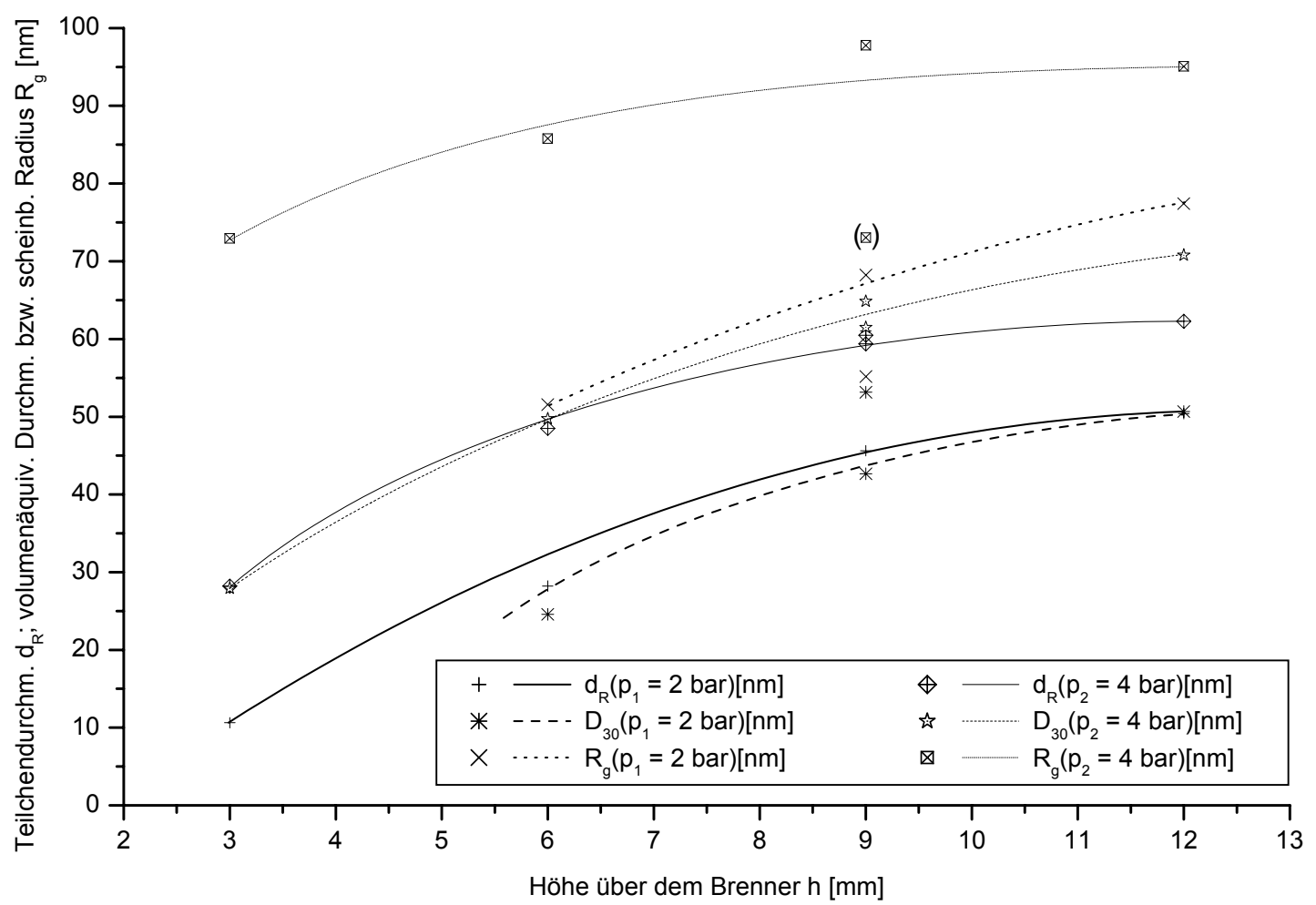

Abb. 4.40: Höhenabhängigkeit der Teilchendurchmesser $\mathrm{d}_{\mathrm{R}}$, der volumenäquivalenten Durchmesser $\mathrm{D}_{30}$ und der scheinbaren Radien $\mathrm{R}_{\mathrm{g}}$ von Ethylen-Luft-Flammen bei einem Druck von $\mathrm{p}_{1}=2$ bar bzw. $\mathrm{p}_{2}=4$ bar bei einem C/O-Verhältnis von $\mathrm{C} / \mathrm{O}=0.75$ und einer Frischgasgeschwindigkeit von $\mathrm{v}_{\mathrm{FG}}=8 \mathrm{~cm} / \mathrm{s}$

Die nächste Abbildung (Abb. 4.41) zeigt der Druckabhängigkeit des RayleighTeilchendurchmessers $d_{R}$, des volumenäquivalenten Durchmessers $D_{30}$ und des scheinbareren Radius $\mathrm{R}_{\mathrm{g}}$. Im untersuchten Druckbereich werden die Teilchendurchmesser nach Rayleigh mit zunehmendem Druck größer (Abb. 4.41). Diese Tendenz wird aber mit steigendem Druck immer geringer. Eine andere Arbeit [22] zeigt, daß bei etwa 5 bar eine Grenze des Teilchenwachstums bezüglich des Drucks erreicht ist. Die Abb. 7.64 bestätigt diese Tendenz. Bei den untersuchten Flammen steigt der Teilchendurchmesser auf bis $\mathrm{zu} \mathrm{d}_{\mathrm{R}}=65 \mathrm{~nm}$ bei einem Druck von 5 bar in Höhen von $\mathrm{h}=9 \mathrm{~mm}$ und höher. 


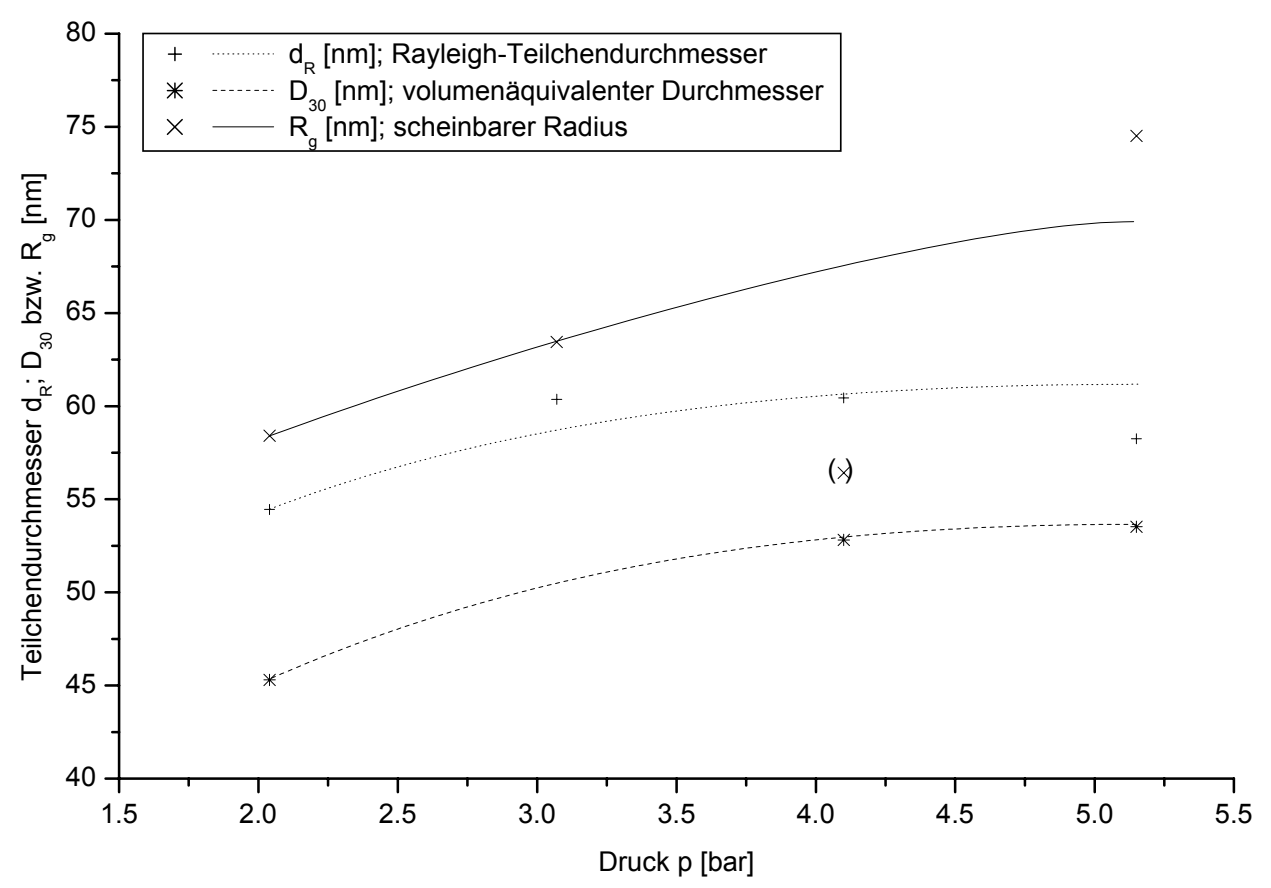

Abb. 4.41: Druckabhängigkeit der unterschiedlichen Teilchendurchmesser von EthylenLuft-Flammen mit einem $\mathrm{C} / \mathrm{O}-\mathrm{V}$ erhältnis von $\mathrm{C} / \mathrm{O}=0.70$ und einer Frischgasgeschwindigkeit von $\mathrm{v}=6.9 \mathrm{~cm} / \mathrm{s}$ und einer Höhe über dem Brenner von $\mathrm{h}=9 \mathrm{~mm}$

Im Vergleich zum Teilchendurchmesser nach Rayleigh liegen der volumenäquivalente Durchmesser um $10 \mathrm{~nm}$ und der scheinbare Radius um 15 bis $20 \mathrm{~nm}$ höher. Besonders der Anstieg des scheinbaren Radius mit dem Druck kann auf bei höherem Druck begünstigtes Aggregatenwachstum schließen lassen. Die Abb. 7.65 im Anhang zeigt allerdings, daß auch der scheinbare Radius mit steigendem Druck tendenziell geringer steigt.

\subsubsection{Teilchenzahldichte und Aggregatenzahldichte}

Die beiden folgenden Abbildungen (Abb. 4.42 und Abb. 4.43) zeigen die Höhenabhängigkeit der Teilchenzahldichte $\mathrm{N}_{\mathrm{R}}$ und der Aggregatenzahldichte $\mathrm{N}_{\text {Agg }}$ von Ethylen-Luft-Flammen bei Drücken zwischen 2 bar und 4 bar mit einer Gemischzusammensetzung von $\mathrm{C} / \mathrm{O}=0.75$ und einer Frischgasgeschwindigkeit von $\mathrm{v}_{\mathrm{FG}}=8 \mathrm{~cm} / \mathrm{s}$. 
Die höhenabhängigen Verläufe der Teilchenzahldichte $\mathrm{N}_{R}$ sowie der Aggregatenzahldichte $\mathrm{N}_{\text {Agg }}$ sind nahezu druckunabhängig wie die Abbildungen Abb. 4.42 und Abb. 4.43 zeigen. Wegen der Druckabhängigkeit der unteren Grenze der Rußzone in den Flammen, sind nur die Teilchenzahldichten $\mathrm{N}_{\mathrm{R}}$ bei einer Höhe von $\mathrm{h}=3 \mathrm{~mm}$ deutlich verschieden, sie erreichen aber in hinreichend großen Höhen annähernd identische Endwerte.

Die Teilchenzahldichte $N_{R}$ und die Aggregatenzahldichte $N_{\text {Agg }}$ haben auch im Vergleich miteinander einen fast identischen Verlauf. Nur die Werte für eine Höhe von $\mathrm{h}=3 \mathrm{~cm}$ differieren, da die Teilchenzahldichten $\mathrm{N}_{R}$ in dieser Höhe stark druckabhängig sind die Aggregatenzahldichte $\mathrm{N}_{\text {Agg }}$ jedoch nicht. Die Aggregatenbildung scheint damit nahezu druckunabhängig zu sein. Nur die Größe der Aggregate ist im unteren Druckbereich ( $p=2$ bis $p=5$ bar) leicht druckabhängig und geht erst bei einem Druck von $p=4$ bis $p=5$ bar ebenfalls in eine Druckunabhängigkeit über.

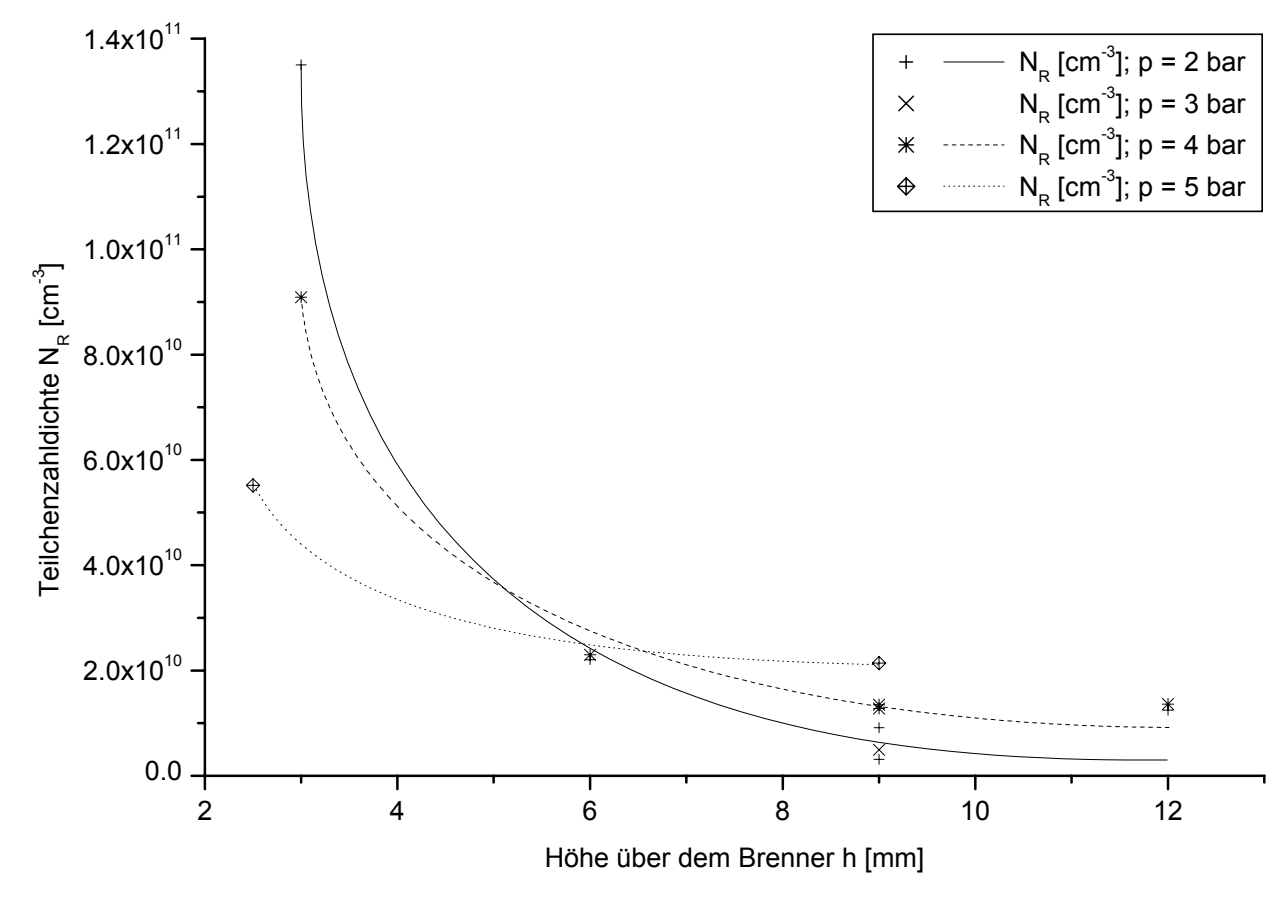

Abb. 4.42: Höhenabhängigkeit der Teilchenzahldichte $\mathrm{N}_{\mathrm{R}}$ von Ethylen-Luft-Flammen mit einem $\mathrm{C} / \mathrm{O}-$ Verhältnis von $\mathrm{C} / \mathrm{O}=0.75$ und einer Frischgasgeschwindigkeit von $\mathrm{v}=8.0 \mathrm{~cm} / \mathrm{s}$ bei verschiedenen Drücken von $\mathrm{p}=2$ bar bis $\mathrm{p}=5 \mathrm{bar}$ 


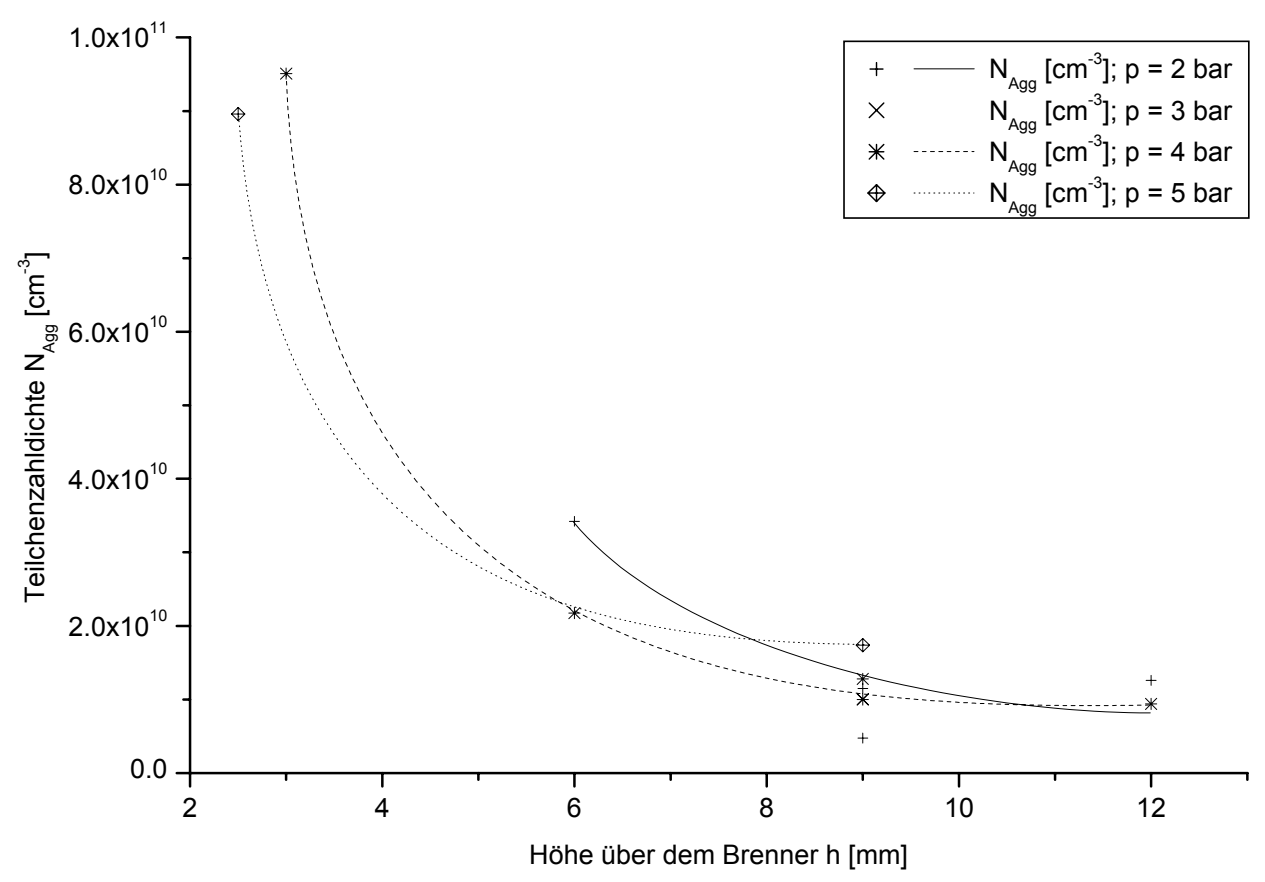

Abb. 4.43: Höhenabhängigkeit der Aggregatenzahldichte $\mathrm{N}_{\mathrm{Agg}}$ von Ethylen-Luft-Flammen mit einem $\mathrm{C} / \mathrm{O}-$ Verhältnis von $\mathrm{C} / \mathrm{O}=0.75$ und einer Frischgasgeschwindigkeit von $\mathrm{v}=8.0 \mathrm{~cm} / \mathrm{s}$ bei verschiedenen Drücken von $\mathrm{p}=2$ bar bis $\mathrm{p}=5 \mathrm{bar}$

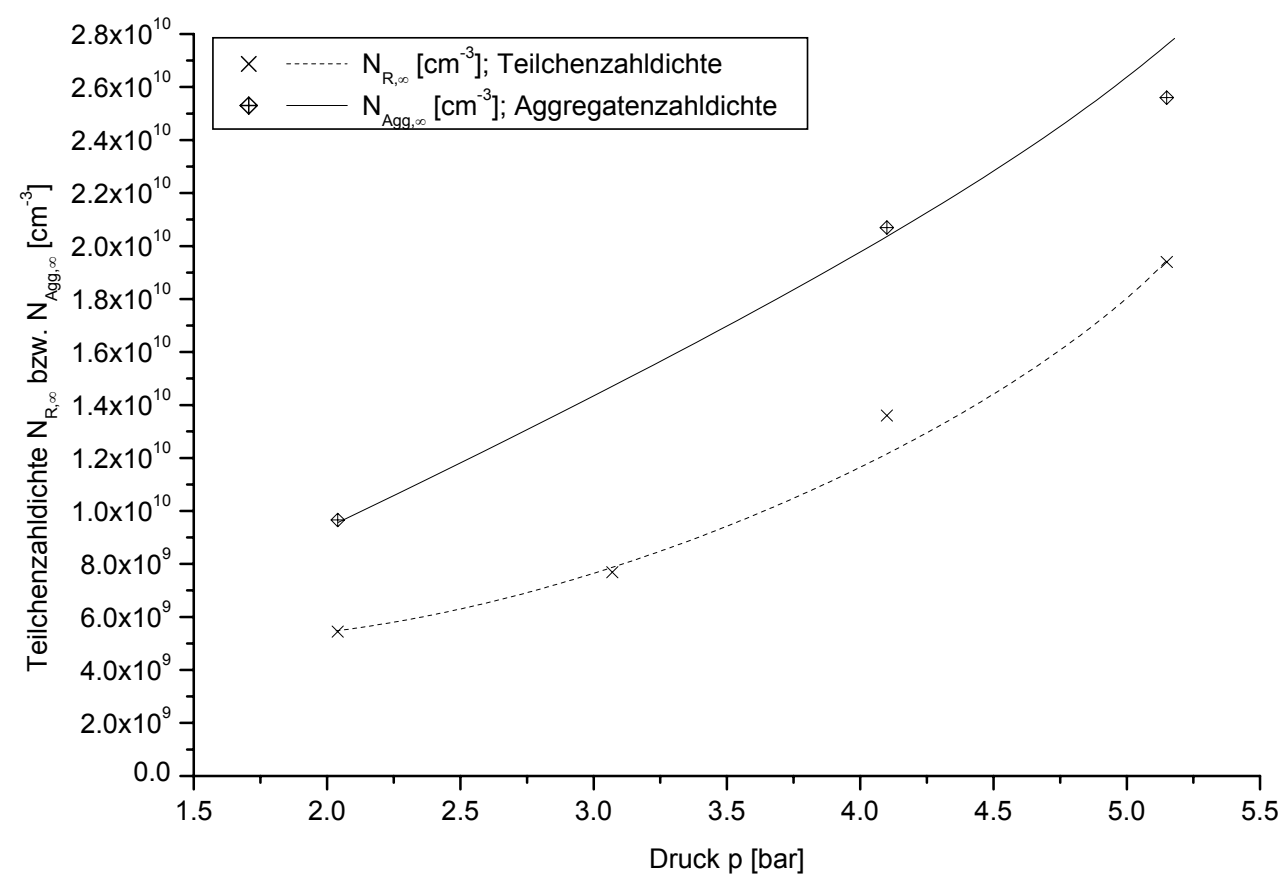

Abb. 4.44: Druckabhängigkeit der Teilchenzahldichte $\mathrm{N}_{\mathrm{R}, \infty}$ und der Aggregatenzahldichte $\mathrm{N}_{\mathrm{Agg}, \infty}$ von Ethylen-Luft-Flammen mit einem C/O-Verhältnis von $\mathrm{C} / \mathrm{O}=0.70$ und einer Frischgasgeschwindigkeit von v $=6.9 \mathrm{~cm} / \mathrm{s}$ und einer Höhe über dem Brenner von $\mathrm{h}=9 \mathrm{~mm}$ 
Die Abb. 4.44 zeigt die Druckabhängigkeit der Teilchenzahldichte und der Aggregatenzahldichte von einer Ethylen-Luft-Flamme mit einem $\mathrm{C} / \mathrm{O}-$ Verhältnis von $\mathrm{C} / \mathrm{O}=0.70$ und einer Frischgasgeschwindigkeit von $\mathrm{v}_{\mathrm{FG}}=6.9 \mathrm{~cm} / \mathrm{s}$. Untersucht wurde in einer Höhe von $\mathrm{h}=9 \mathrm{~mm}$, d.h. in einem Bereich der Flamme, in der die Koagulation der Teilchen nahezu aufgehört hat. Diese Teilchenzahldichte wird dann auch mit $\mathrm{N}_{\mathrm{R}, \infty}$ und die Aggregatenzahldichte mit $\mathrm{N}_{\text {Agg, }, \infty}$ bezeichnet. Die Teilchenzahldichte und die Aggregatenzahldichte steigen beide fast parallel zueinander mit dem Druck. Das Ausmaß der Steigung nimmt mit steigendem Druck noch zu. Erst mit Drücken oberhalb des untersuchten Bereichs mit $p \geq 10$ bar läßt die Steigungstendenz wieder nach [22].

Bemerkenswert ist die Tatsache, daß die Aggregatenzahldichte im untersuchten Druckbereich etwas höher ist als die Teilchenzahldichte $\mathrm{N}_{\mathrm{R}}$, obwohl der volumenäquivalente Durchmesser schon größer als der Teilchendurchmesser nach Rayleigh ist. Die Abb. 7.68 im Anhang relativiert dieses Verhalten etwa, da hier ab einem Druck von 4 bar die Teilchenzahldichte $N_{R}$ oberhalb der Aggregatenzahldichte $\mathrm{N}_{\text {Agg }}$ liegt.

Weder die Rayleigh-Theorie noch die Aggregatentheorie (RDG/FA) machen Aussagen, daß mit zunehmendem Flammendruck die Teilchenzahldichte $\mathrm{N}_{\mathrm{R}, \infty}$ bzw. Aggregatenzahldichte $\mathrm{N}_{\mathrm{Agg}, \infty}$ am Ende des Verbrennungsprozesses zunimmt. Das bedeutet, daß die Teilchen nicht mehr so gut koagulieren können, wie es in einer Flamme bei Normaldruck der Fall ist.

Abschließend soll der Vergleich der Druckabhängigkeiten von Teilchenzahldichte $\mathrm{d}_{\mathrm{R}}$ und Aggregatenzahldichte $\mathrm{N}_{\mathrm{R}}$ am Beispiel der Ethylen-Luft-Flamme mit einem C/O-Verhältnis von $\mathrm{C} / \mathrm{O}=0.70\left(\mathrm{v}_{\mathrm{FG}}=6.9 \mathrm{~cm} / \mathrm{h}\right.$ und $\left.\mathrm{h}=9 \mathrm{~mm}\right)$ gezeigt werden (Abb. 4.45). Im Gegensatz $\mathrm{zu}$ den anderen Parametern (Höhe, C/O-Verhältnis und Frischgasgeschwindigkeit) steigen in ihrer Druckabhängigkeit sowohl die Teilchendurchmesser als auch die Teilchenzahldichten. Allerdings nimmt die Steigungstendenz der Teilchendurchmesser mit dem Druck ab, während sie für die Teilchenzahldichten deutlich mit dem Druck zunimmt. 


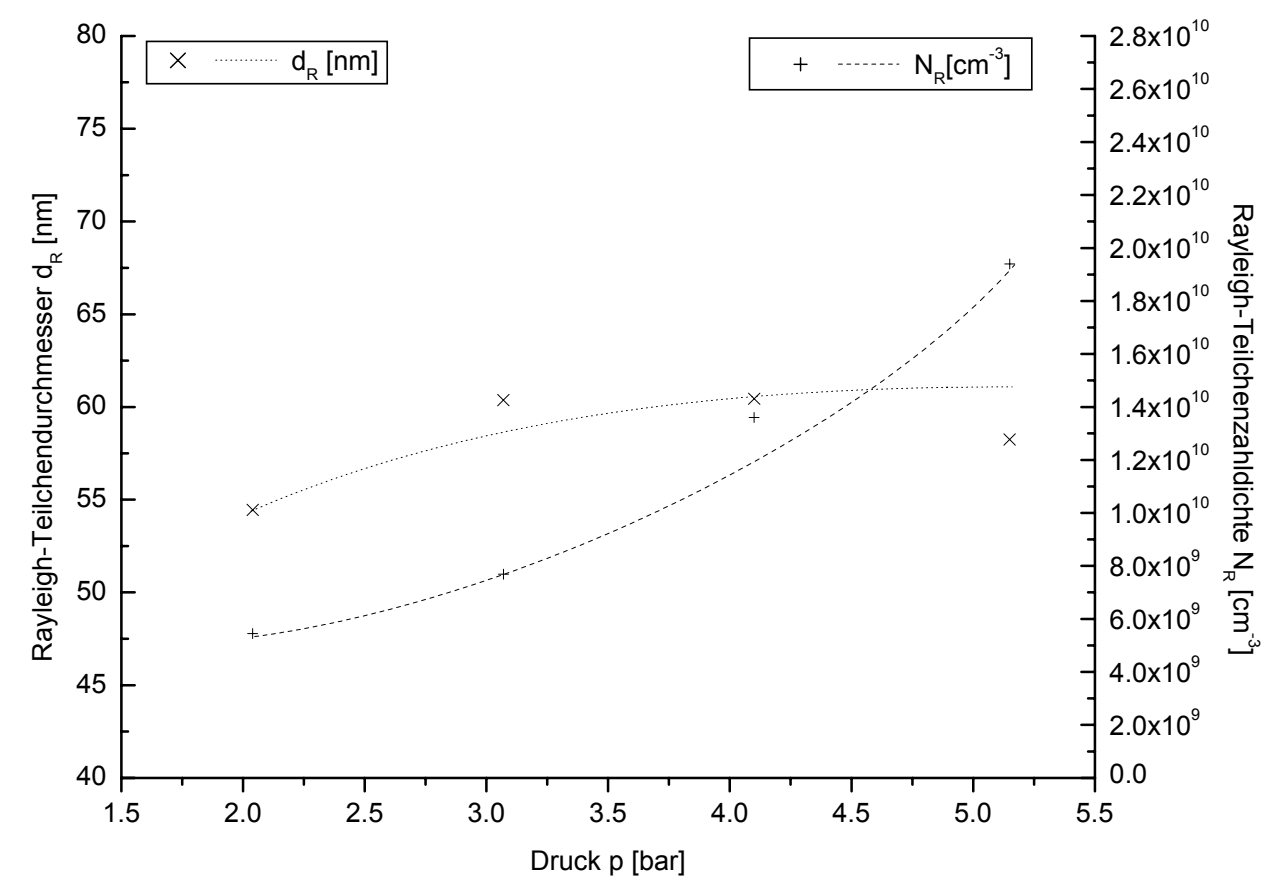

Abb. 4.45: Druckabhängigkeit des Teilchendurchmessers und der Teilchenzahldichte nach Rayleigh von Ethylen-Luft-Flammen mit einem $\mathrm{C} / \mathrm{O}-$ Verhältnis von $\mathrm{C} / \mathrm{O}=$ 0.70 und einer Frischgasgeschwindigkeit von v $=6.9 \mathrm{~cm} / \mathrm{s}$ und einer Höhe über dem Brenner von $\mathrm{h}=9 \mathrm{~mm}$

\subsubsection{Fraktale Dimension}

Der Begriff der fraktalen Dimension wurde bereits in Kap. 4.1.2.9 kurz erläutert. Die fraktale Dimension stellt den Zusammenhang zwischen Aggregaten einerseits und Primärteilchen andererseits her.

Die Abbildung zeigt den druckabhängigen Verlauf der fraktalen Dimension von einer Ethylen-Luft-Flamme mit $\mathrm{C} / \mathrm{O}=0.70\left(\mathrm{v}_{\mathrm{FG}}=6.9 \mathrm{~cm} / \mathrm{h}\right.$ und $\left.\mathrm{h}=9 \mathrm{~mm}\right)$ : 


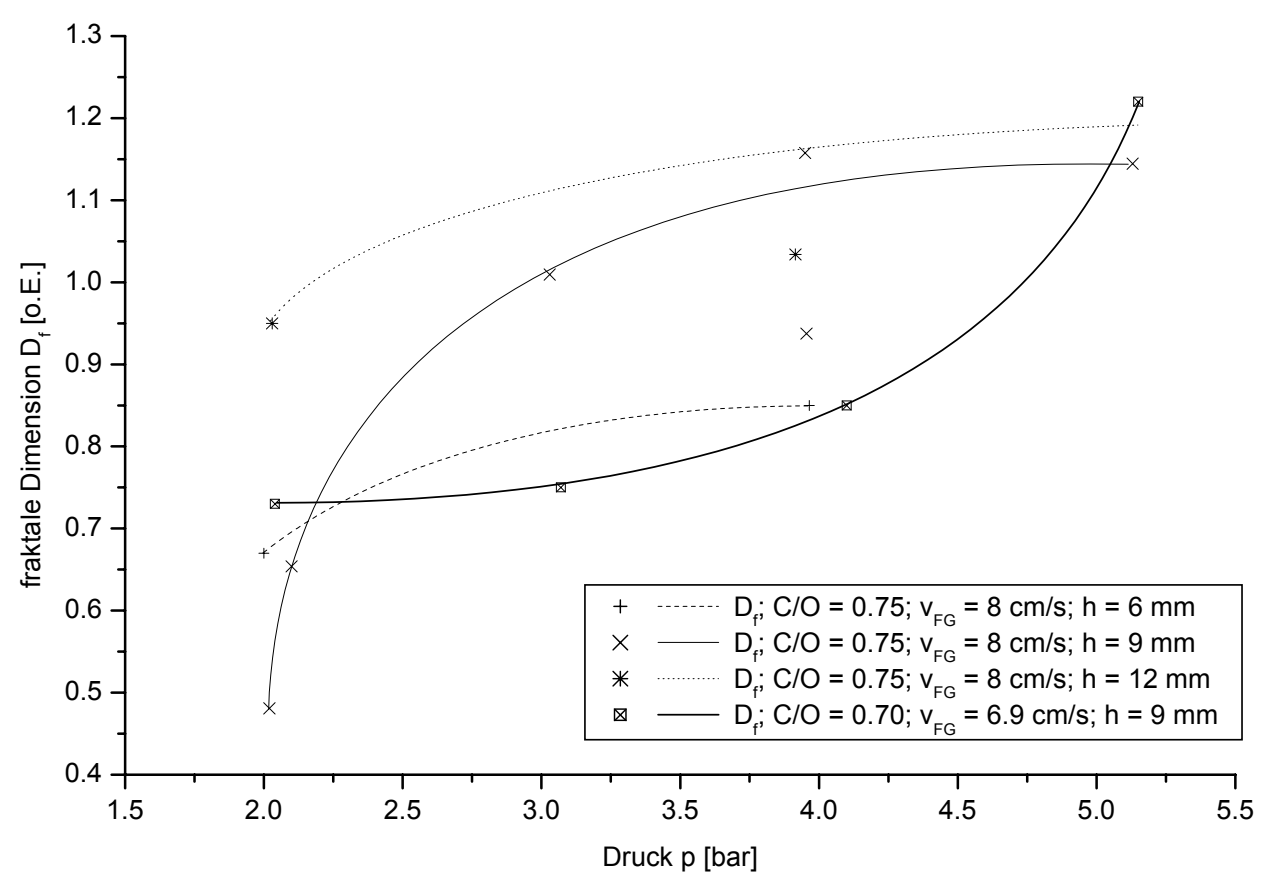

Abb. 4.46: Druckabhängigkeit der fraktalen Dimension von Ethylen-Luft-Flammen mit einem $\mathrm{C} / \mathrm{O}-$ Verhältnis von $\mathrm{C} / \mathrm{O}=0.70$ und einer Frischgasgeschwindigkeit von $\mathrm{v}=6.9 \mathrm{~cm} / \mathrm{s}$ und einer Höhe über dem Brenner von $\mathrm{h}=9 \mathrm{~mm}$

Bei dieser Flamme $(\mathrm{C} / \mathrm{O}=0.70)$ steigt die fraktale Dimension mit zunehmenden Druck mit steigender Tendenz an. Bei einem Druck von $p=5$ bar wird eine fraktale Dimension von $D_{f}=$ 1.2 erreicht.

Die Untersuchung einer weiteren Flamme mit einem $\mathrm{C} / \mathrm{O}-$ Verhältnis von $\mathrm{C} / \mathrm{O}=0.75$ und einer Frischgasgeschwindigkeit von $v=8 \mathrm{~cm} / \mathrm{s}$ ergab einen etwas anderen Verlauf (Abb. 7.69). Bei dieser Flamme steigt die fraktale Dimension zunächst stärker an, um dann ab einem Druck von $p=4$ bar in konstante Werte überzugehen.

Im Verglich mit den Ethylen-Luft-Flammen bei einem Druck von $p=1$ bar, sowie den Benzol-Luft-Flammen, konnte bei den Hochdruckflammen keine Werte für die fraktale Dimension von $\mathrm{D}_{\mathrm{f}}=1.7$ oder höher festgestellt werden. Allerdings wurden bei den Flammen mit höherem Druck nur C/O-Verhältnisse von höchstens $\mathrm{C} / \mathrm{O}=0.75$ gewählt, mit dem 1 bar Brenner wurden hingegen Flammen mit C/O-Verhältnissen von bis zu 1.2 untersucht. Mit einem Anstieg der fraktalen Dimension für noch höhere Drücke ist ebenfalls zu rechnen. 


\subsubsection{Primärteilchen}

Die Abbildungen Abb. 4.47 und Abb. 4.48 zeigen den höhenabhängigen Verlauf des

Primärteilchendurchmessers bzw. der Primärteilchenzahldichte von Ethylen-Luft-Flammen in einem Druckbereich von $\mathrm{p}=2$ bar bis $\mathrm{p}=5$ bar $\left(\mathrm{C} / \mathrm{O}=0.75\right.$ und $\left.\mathrm{v}_{\mathrm{FG}}=8 \mathrm{~cm} / \mathrm{s}\right)$. Der

Primärteilchendurchmesser steigt mit der Höhe mit geringer werdender Tendenz an. In einer Höhe von $\mathrm{h}=12 \mathrm{~mm}$ sind die Endwerte von etwa $d_{\mathrm{p}}=23 \mathrm{~nm}$ erreicht. Nur die Primärteilchendurchmesser bei einem Druck von $p=2$ bar erreichen einen geringeren Endwert in der Höhe von $\mathrm{h}=12 \mathrm{~mm}$, nämlich $\mathrm{d}_{\mathrm{p}}=18 \mathrm{~nm}$. Mit einem Sinterplattenbrenner [47] wurde bei Normaldruck am oberen Ende der Rußzone ein Primärteilchenradius von $d_{p}=$ $25 \mathrm{~nm}$ gefunden.

Die Primärteilchenzahldichte fällt mit der Höhe stark ab. Dabei flacht der höhenabhängige Kurvenverlauf der Primärteilchenzahldichte mit zunehmender Höhe ab und erreicht $\mathrm{ab} h=9$ mm seinen Endwert von etwa $2 * 10^{11} \mathrm{~cm}^{-3}$. Die Endwerte der Primärteilchenzahldichte sind nahezu druckunabhängig. Nur die Anfangswerte der Primärteilchenzahldichte in einer Höhe von $\mathrm{h}=3 \mathrm{~mm}$ variieren deutlich mit dem Druck.

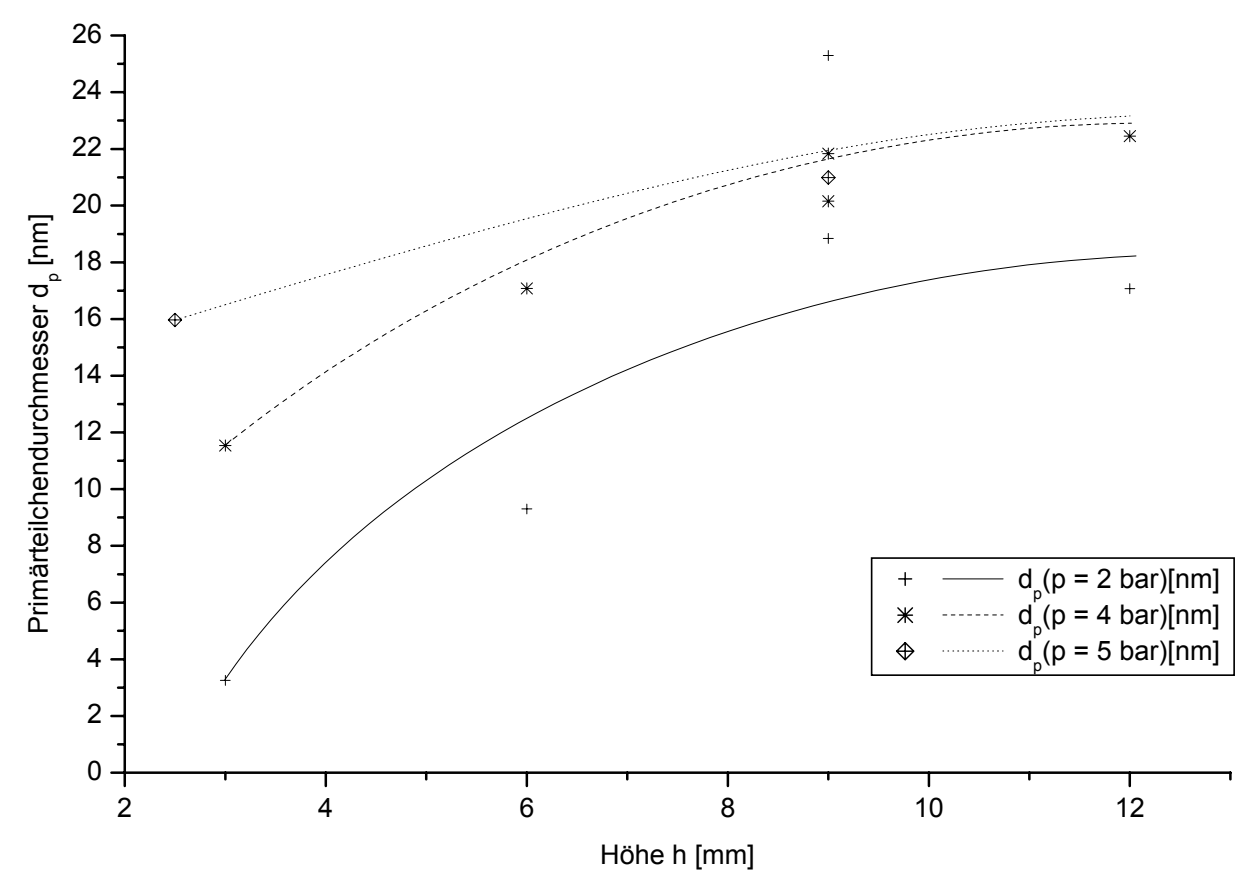

Abb. 4.47: Höhenabhängigkeit der Primärteilchendurchmesser $\mathrm{d}_{\mathrm{p}}$ von Ethylen-Luft-Flammen mit einem $\mathrm{C} / \mathrm{O}-$ Verhältnis von $\mathrm{C} / \mathrm{O}=0.75$ und einer Frischgasgeschwindigkeit von $\mathrm{v}=8.0 \mathrm{~cm} / \mathrm{s}$ bei verschiedenen Drücken von $\mathrm{p}=2$ bar bis $\mathrm{p}=5$ bar 


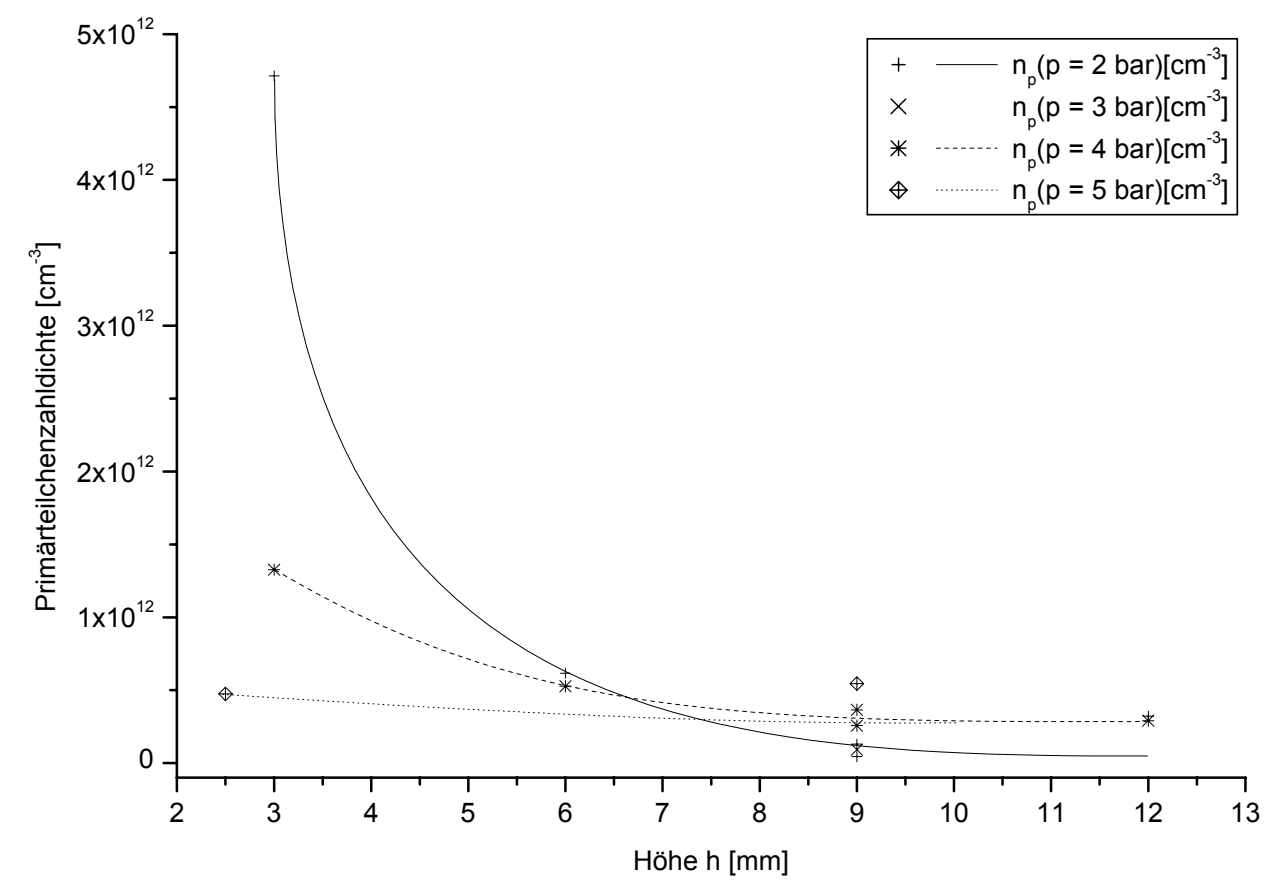

Abb. 4.48: Höhenabhängigkeit der Primärteilchenzahldichte $\mathrm{n}_{\mathrm{p}}$ von Ethylen-Luft-Flammen mit einem $\mathrm{C} / \mathrm{O}-$ Verhältnis von $\mathrm{C} / \mathrm{O}=0.75$ und einer Frischgasgeschwindigkeit von $\mathrm{v}=8.0 \mathrm{~cm} / \mathrm{s}$ bei verschiedenen Drücken von $\mathrm{p}=2$ bar bis $\mathrm{p}=5$ bar

Die letzte Auftragung Abb. 4.49) zeigt schließlich die Druckabhängigkeit der Primärteilchen bezüglich ihrer Durchmesser und Anzahldichte für Ethylen-Luft-Flammen in einem Druckintervall von $\mathrm{p}=2$ bar bis $\mathrm{p}=5$ bar für die Höhe $\mathrm{h}=9 \mathrm{~mm}\left(\mathrm{C} / \mathrm{O}=0.70\right.$ und $\mathrm{v}_{\mathrm{FG}}=6.9$ $\mathrm{cm} / \mathrm{s}$ ). Der Primärteilchendurchmesser (Abb. 4.49) steigt mit zunehmendem Druck immer schwächer an und erreicht schon bei einem Druck von etwa $p=3.5$ bis $p=4$ bar seinen Endwert von ungefähr $d_{p}=23 \mathrm{~nm}$. Dieses Verhalten wurde bei einer anderen Ethylen-LuftFlamme $\left(\mathrm{C} / \mathrm{O}=0.75\right.$ und $\left.\mathrm{v}_{\mathrm{FG}}=8 \mathrm{~cm} / \mathrm{s}\right)$ bestätigt (s. Anhang Abb. 7.70).

Die Primärteilchenzahldichte (Abb. 4.49) verläuft mit steigendem Druck stark ansteigend. Bei einem Druck von $\mathrm{p}=5$ bar wird eine Primärteilchendichte von $\mathrm{n}_{\mathrm{p}}=6 * 10^{11} \mathrm{~cm}^{-3}$ erhalten. Eine weitere Messung $\left(\mathrm{C} / \mathrm{O}=0.75 ; \mathrm{v}_{\mathrm{FG}}=8 \mathrm{~cm} / \mathrm{s}\right.$ und $\left.\mathrm{h}=9 \mathrm{~mm}\right)$ bestätigte dieses Ergebnis (s. Anhang Abb. 7.71). 


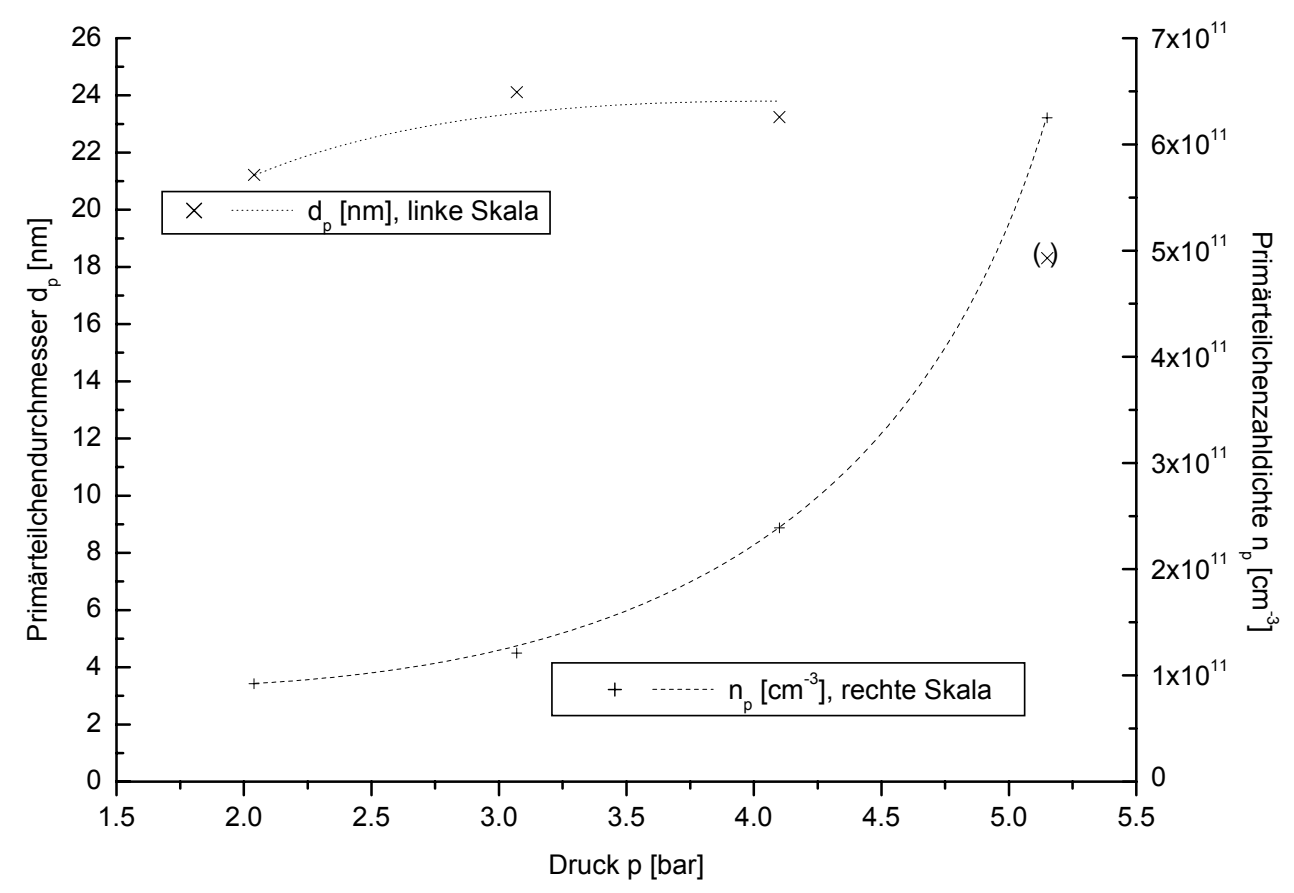

Abb. 4.49: Druckabhängigkeit des Primärteilchendurchmessers und der Primärteilchenzahldichte von Ethylen-Luft-Flammen mit einem C/OVerhältnis von $\mathrm{C} / \mathrm{O}=0.70$ und einer Frischgasgeschwindigkeit von $\mathrm{v}=6.9$ $\mathrm{cm} / \mathrm{s}$ und einer Höhe über dem Brenner von $\mathrm{h}=9 \mathrm{~mm}$ 


\subsection{Vergleich der Meßergebnisse bei Normaldruck}

\subsubsection{Rußgebiete und Stabilitätskriterien}

Die Ergebnisse, die mit Hilfe der Streulicht- und Absorptionsmessungen von den verschiedenen Kohlenwasserstoff-Luft-Flammen bei $\mathrm{p}=1$ bar gewonnen wurden, sollen in diesem Kapitel miteinander verglichen werden. Es werden hierzu nicht alle untersuchten Flammen herangezogen, sondern es soll vielmehr an einigen Beispielen mögliche vorhandene Gemeinsamkeiten, Unterschiede oder Trends des Teilchenverhaltens in den verschiedenen Kohlenwasserstoff-Luft-Flammen aufgezeigt werden.

Eine Vielfalt an Informationen über eine rußende Flamme erhält man z.B. aus der Lage des Rußgebietes und seiner Ausdehnung im Gemischzusammensetzungs-Temperaturfeld. An drei Beispielen, s. Abb. 5.1, werden die unterschiedlichen Positionen der Rußgebiete verschiedener KW-Luft-Flammen gezeigt. Begrenzt wird das Rußgebiet, das heißt das Gebiet stabiler rußender Flammen, durch eine untere Kurve, der Rußgrenze, und einer oberen Kurve, der Stabilitätsgrenze.

Die Rußgebiete einiger einzelner Flammen wie Benzol oder Ethylen, dieses bei unterschiedlichem Druck, sind im Kap. 4.1.2.2 (Benzol), Kap. 4.2.3 (Ethylen, Hochdruck) bzw. in den Anhängen Kap. 7.1.1.2 (n-Heptan), 7.1.1.3 (Isooktan) und 7.1.1.4 (Propen) dargestellt worden. In den jeweiligen Kapiteln wurde auch näher auf den Rußbeginn und der Meßmethode zu deren Ermittlung eingegangen.

Die Rußgebiete, die in Abb. 5.1 dargestellt sind, zeigen deutlich den Einfluß des Brennstoffmoleküls auf das Rußverhalten.

Je höher die C-Atomzahl in den Molekülen der aliphateischen Kohlenwasserstoffe ist, desto schmaler werden die Rußgebiete und desto eher fangen die Flammen an zu rußen. Außerdem tritt die Neigung zu gestörten Flammen mit kleineren C/O-Verhältnissen ein. Eine Ausnahme von diesem Verhalten macht das Benzol, dessen Rußgebiet vollständig im Rußgebiet der 
Ethylen-Luft-Flammen liegt. Seine Rußgrenze liegt z.B. bei wesentlich höheren C/OVerhältnissen als die des n-Heptans. Während die Ethylen-Luft-Flamme ein sehr ausgedehntes Rußgebiet besitzt, ist das Rußgebiet des Benzols sehr viel schmaler, was bedeutet, daß das Benzol nur in einem schmalen Bereich der Gemischzusammensetzungen stabile rußende Flammen unterhält und die Neigung, zu gestörten Flammen wesentlich ausgeprägter ist als bei den Ethylen-Luft-Flammen. Offensichtlich spielt hier neben der CAtomzahl auch die aromatische Struktur des Benzol-Moleküls bzw. der ungesättigte Charakter des Ethylen-Moleküls eine Rolle beim Rußbildungsprozeß.

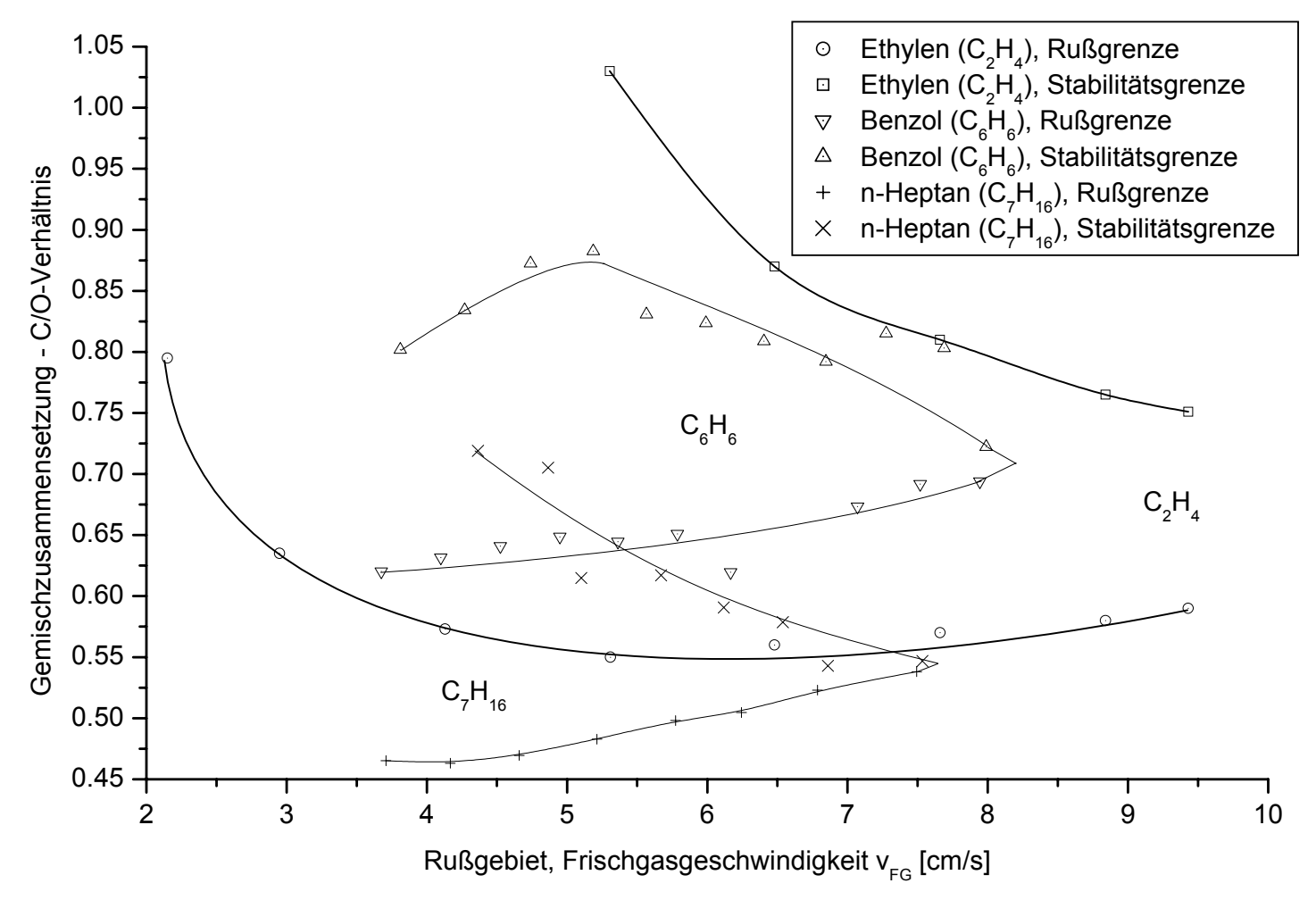

Abb. 5.1: Rußgebiete unterschiedlicher KW-Luft-Flammen.

\subsubsection{Teilchendurchmesser}

Der Einfluß des Brennstoffs auf die Aggregatenbildung soll anhand der Abb. 5.2 und Abb. 5.3 am Beispiel des Teilchendurchmessers in Abhängigkeit von der Flammenhöhe gezeigt werden. Aufgetragen sind die Teilchendurchmesser $d_{R}, D_{30}$ und $R_{g}$ einer Ethylen-LuftFlamme (Abb. 5.2) und $d_{R}$ und $D_{30}$ von n-Pentan-, n-Heptan- und Isooktan-Luft-Flammen (Abb. 5.3). 


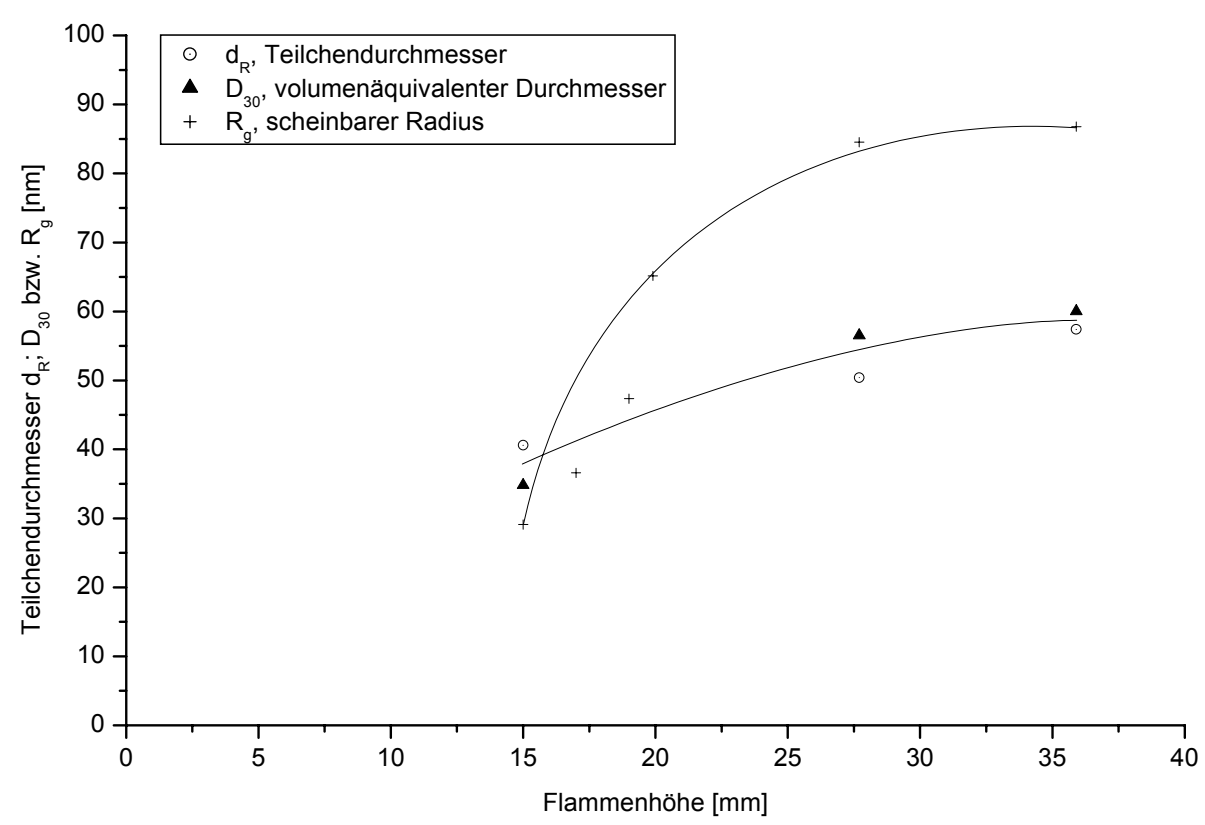

Abb. 5.2: Höhenabhängigkeit der Teilchendurchmesser von einer Ethylen-Luft-Flamme mit einem $\mathrm{C} / \mathrm{O}-$ Verhältnis von $\mathrm{C} / \mathrm{O}=0.84$ und einer Frischgasgeschwindigkeit von $\mathrm{v}_{\mathrm{FG}}=7.8$ $\mathrm{cm} / \mathrm{s},+$-Zeichen stehen für den scheinbaren Radius, Kreise für $\mathrm{d}_{\mathrm{R}}$ und geschlossene Dreiecke für $\mathrm{D}_{30}$

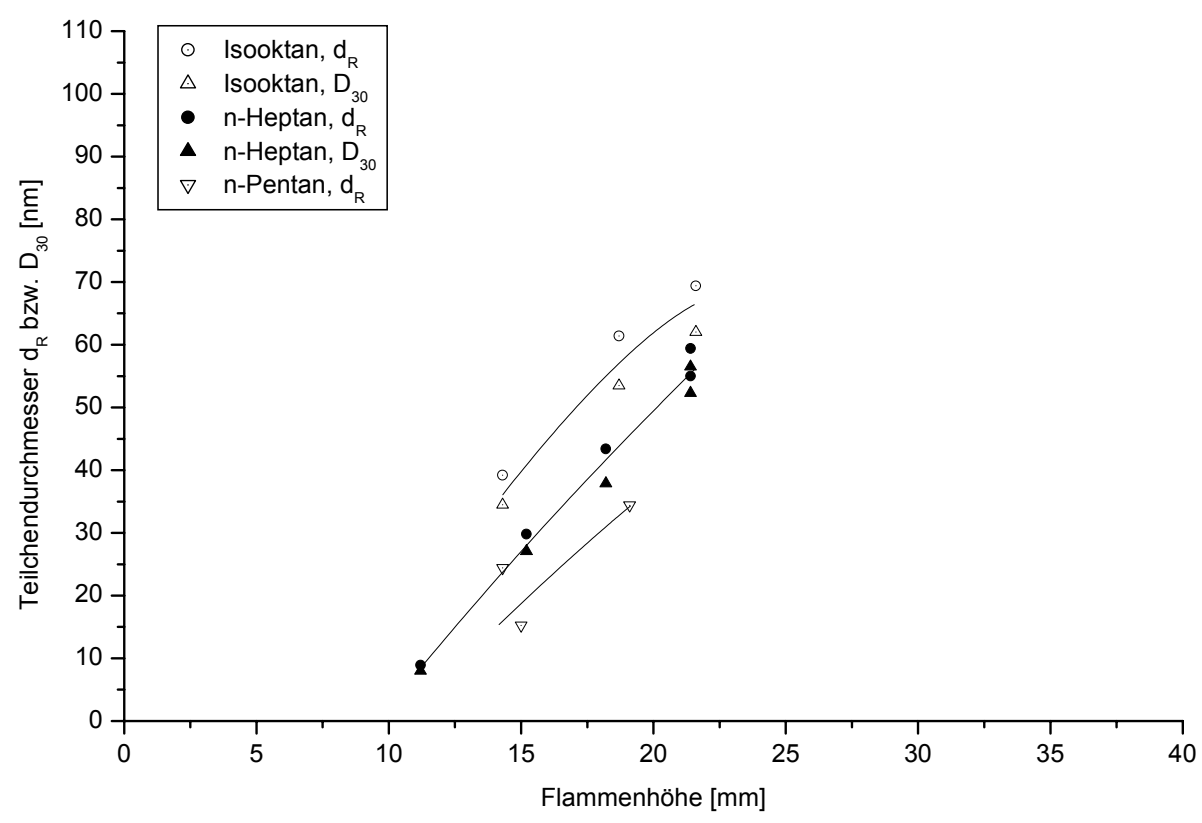

Abb. 5.3: Höhenabhängigkeit der Teilchendurchmesser von n-Pentan- $\left(\mathrm{C} / \mathrm{O}=0.68 ; \mathrm{v}_{\mathrm{FG}}=\right.$ $5.4 \mathrm{~cm} / \mathrm{s}), \mathrm{n}-$ Heptan- $\left(\mathrm{C} / \mathrm{O}=0.63 ; \mathrm{v}_{\mathrm{FG}}=4.9 \mathrm{~cm} / \mathrm{s}\right)$ und Isooktan- $(\mathrm{C} / \mathrm{O}=0.62$; $\mathrm{v}_{\mathrm{FG}}=4.6 \mathrm{~cm} / \mathrm{s}$ )- Luft-Flammen. Dargestellt sind $\mathrm{D}_{30}$ (Dreiecke) und $\mathrm{R}_{\mathrm{g}}$ (Kreise) 
Alle vier Flammen zeigen den typischen Anstieg, der Teilchendurchmesser mit der Flammenhöhe. In den Flammen mit größeren Kohlenstoffatomzahlen $\left(\mathrm{n}_{\mathrm{C}}>2\right)$ konnte aus Gründen der Flammenstabilität nur bis zu einer Höhe von $\mathrm{h}=25 \mathrm{~mm}$ gemessen werden. Es können deshalb keine Ergebnisse aus der späteren Phase des Verbrennungsprozesses angegeben werden. In Abb. 5.2, in der Ethylen-Luft-Flamme konnten jedoch beide Phasen des Wachstumsprozesses, sowohl der schmalen Anstieg, als auch der langsamere Teil gemessen werden. Die unterschiedlichen Teilchendurchmesser verhalten sich in allen Flammen ähnlich. In der schmalen Phase des Wachstumsprozesses der Rußbildung ist der Teilchendurchmesser in etwa gleich dem volumenäquivalenten Durchmesser $\mathrm{d}_{\mathrm{R}} \approx \mathrm{D}_{30}$, dies gilt für alle Flammen gleichermaßen. Es gilt weiterhin auch für die spätere Phase des Verbrennungsprozesses für schwach bis mittelstark rußende Flammen (Abb. 5.3). Nur für sehr stark rußende Flammen und für sehr hohe Flammenhöhen fallen der Teilchendurchmesser $d_{R}$ und der volumenäquivalente Durchmesser $\mathrm{D}_{30}$ nicht mehr zusammen $\left(d_{R} \neq D_{30}\right)$. Diese Ergebnisse zeigen, daß bis $\mathrm{zu}$ mittelstark rußende Flammen die Abweichungen der Rußteilchen von einer Kugel nicht sehr ausgeprägt sein können.

\subsubsection{Teilchenzahldichte und Aggregatenzahldichte}

Die Teilchenzahldichte ändert sich mit fortschreitender Reaktionszeit sehr stark. Zu Beginn der Rußzone ist die Teilchenzahl sehr hoch, wie in Abb. 5.4 zu erkennen ist, liegt dieser Wert bei etwa $\mathrm{N} \sim 10^{12} \mathrm{~cm}^{-3}$. Mit zunehmender Flammenhöhe nimmt sie, bedingt durch Koagulation, sehr stark ab und erreicht am Ende des Verbrennungsprozesses üblicherweise etwa $10^{9} \mathrm{~cm}^{-3}$.

Die Abb. 5.4 zeigt die Änderung der Teilchenzahldichte mit der Flammenhöhe von einer Ethylen-Luft-Flamme und einer n-Heptan-Luft-Flamme. Aufgetragen sind die Teilchenzahldichte $\mathrm{N}_{\mathrm{R}}$, die nach der Rayleigh-Theorie berechnet wurde sowie die Aggregatenzahldichte $\mathrm{N}_{\mathrm{Agg}}$, die nach der RDG/FA-Theorie ermittelt wurde. Die Daten für $\mathrm{N}_{\mathrm{R}}$ und $\mathrm{N}_{\mathrm{Agg}}$ beider Flammen zeigen keine wesentlichen Unterschiede. Sowohl in der EthylenLuft-Flamme als auch in der n-Heptan-Luft-Flamme ist die Abnahme der Teilchenzahldichten mit der Flammenhöhe $\frac{\Delta \mathrm{N}}{\Delta \mathrm{h}}$ gleich. Das zeigt, daß der Koagulationsprozeß in der ersten Phase des Wachstumsprozesses ein universeller Prozeß für die hier untersuchten KW-Luft-Flammen 
ist. Für sehr fette Gemischzusammensetzungen, d.h. für stark rußende Flammen sowie auch in der späteren Phase des Verbrennungsprozesses ändert sich das und der Prozeß der ClusterCluster-Aggregatbildung kann einsetzen.

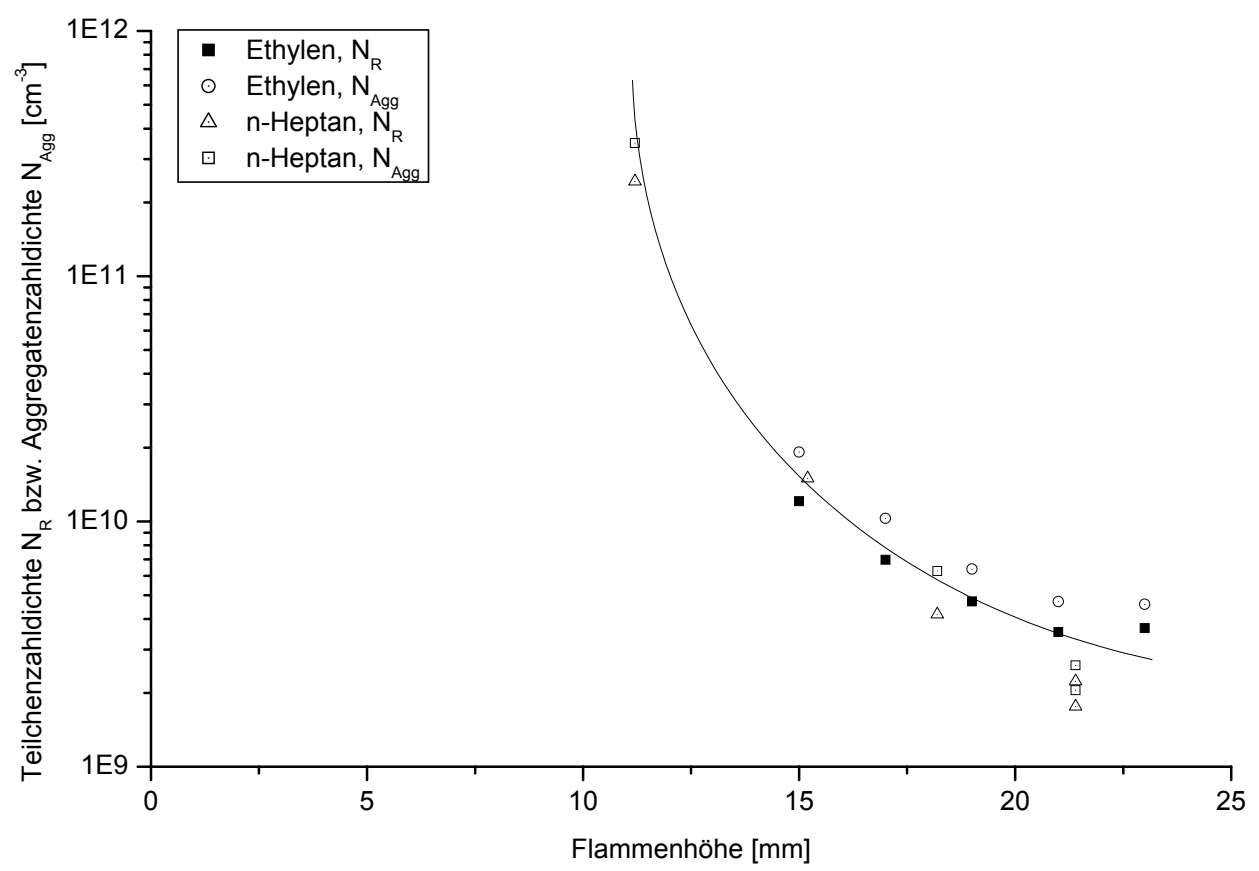

Abb. 5.4: Teilchenzahldichten in Abhängigkeit von der Flammenhöhe für zwei KWLuft-Flammen. Aufgetragen sind Ergebnisse, die nach der Rayleigh-Theorie $\mathrm{N}_{\mathrm{R}}$ bzw. nach der RDG/FA-Theorie $\mathrm{N}_{\text {Agg }}$ berechnet wurden. Geschlossene Quadrate geben $\mathrm{N}_{\mathrm{R}}$, offene Kreise $\mathrm{N}_{\text {Agg }}$ einer Ethylen-Luft-Flamme mit $\mathrm{C} / \mathrm{O}=0.88$ und $\mathrm{v}_{\mathrm{FG}}=7.9 \mathrm{~cm} / \mathrm{s}$ an. Offene Dreiecke sind $\mathrm{N}_{\mathrm{R}}$ und offene Quadrate $\mathrm{N}_{\mathrm{Agg}}$ einer n-Heptan-Luft-Flamme mit einem C/OVerhältnis von $\mathrm{C} / \mathrm{O}=0.65$ und $\mathrm{v}_{\mathrm{FG}}=4.9 \mathrm{~cm} / \mathrm{s}$

\subsubsection{Fraktale Dimension}

Die gesamte Information über die Struktur eines Teilchens liegt im Kurvenverlauf des Streufaktors $\mathrm{Q}_{\mathrm{vv}}(\Theta)$ gegen den Streuwinkel $\Theta$. Variiert z.B. der normierte Streufaktor mit $\frac{\mathrm{Q}_{\mathrm{VV}}(\Theta)}{\mathrm{k}_{\mathrm{abs}}} \approx \mathrm{F}^{-\mathrm{D}_{\mathrm{f}}}$, so liegt eine „fraktale“ Struktur vor [31]. Der Parameter $\mathrm{D}_{\mathrm{f}}$, die fraktale Dimension, ist ein Maß, welches darüber Auskunft gibt, wie kompakt ein Aggregat ist. Vergleiche von Computersimulationen, in denen verschiedene Wachstumsmodelle für die 
Teilchenaggregate angenommen wurden, und von experimentellen Messungen [31] ergaben, daß Aggregate sehr kompakt sind, wenn die fraktale Dimension einen Wert von etwa $D_{f}=3$ annimmt. Für $D_{f} \leq 2$ erhält man kettenartige Strukturen und für $D_{f} \geq 1$ ist ein Aggregat stäbchenartig.

Die Abb. 5.5 und Abb. 5.6 zeigen einige Ergebnisse von der fraktalen Dimension $\mathrm{D}_{\mathrm{f}}$ in Abhängigkeit von der Flammentemperatur (Abb. 5.5) und der Gemischzusammensetzung (Abb. 5.6) von verschiedenen KW-Luft-Flammen.

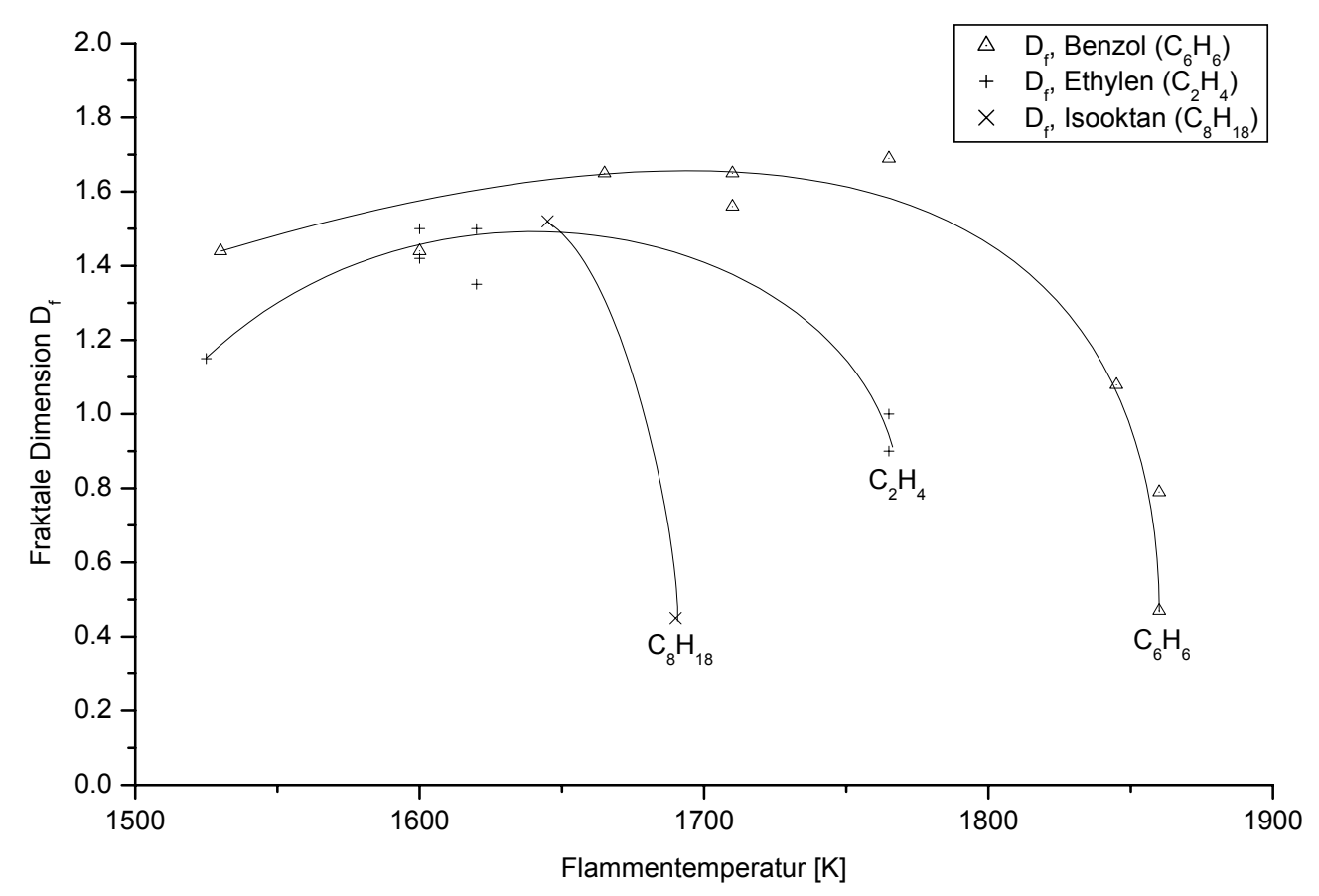

Abb. 5.5: Abhängigkeit der fraktalen Dimension $\mathrm{D}_{\mathrm{f}}$ von der Flammentemperatur von verschiedenen KW-Luft-Flammen:

$+\quad$ Ethylen-Luft-Flamme mit $\mathrm{C} / \mathrm{O}=0.83$ und $\mathrm{h}=17.5 \mathrm{~mm}$

$\Delta \quad$ Benzol-Luft-Flamme mit $\mathrm{C} / \mathrm{O}=0.65$ und $\mathrm{h}=17.7 \mathrm{~mm}$

$\mathrm{x} \quad$ Isooktan-Luft-Flamme mit $\mathrm{C} / \mathrm{O}=0.62$ und $\mathrm{h}=17.5 \mathrm{~mm}$

Die untersuchten Flammen zeigen bezüglich des Verlaufs der fraktalen Dimension mit der Flammentemperatur immer eine ähnliche Tendenz. Die fraktale Dimension durchläuft stets ein Maximum, das bei einer Flammentemperatur von $\mathrm{T}=1650-1700 \mathrm{~K}$ liegt und für die gemessenen Flammen einen maximalen Wert von $\mathrm{D}_{\mathrm{f}}=1.5$ annimmt. Für niedrigere und besonders für höhere Flammentemperaturen wird die fraktale Dimension sehr klein. Der 
Verlauf der fraktalen Dimension in Abhängigkeit von der Flammentemperatur zeigt, daß die Strukturen der Aggregate sich mit den Flammenbedingungen ändern und daß die Aggregate bei hohen Temperaturen wieder auseinanderfallen und zu kleineren Einheiten oder gar Einzelteilchen werden.

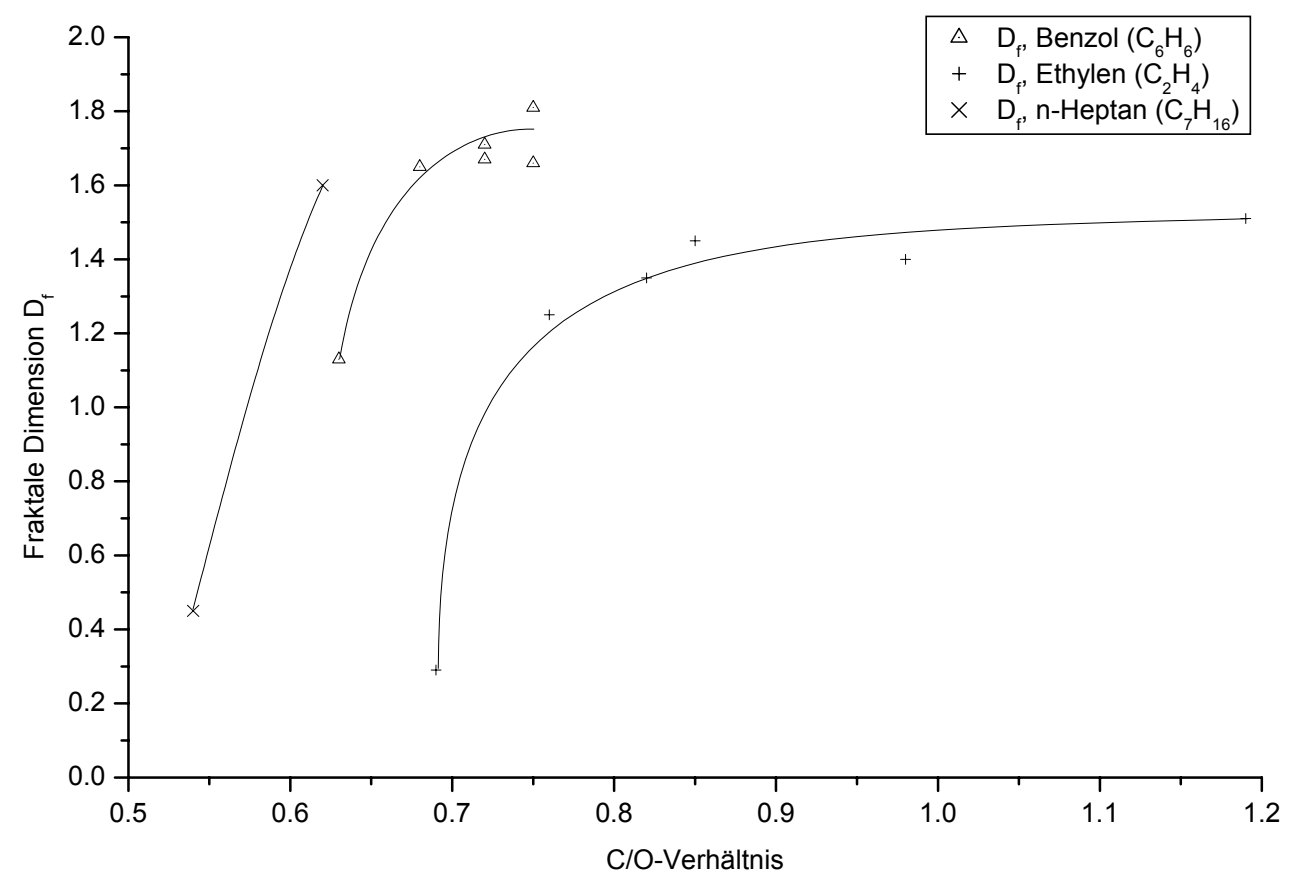

Abb. 5.6: Abhängigkeit der fraktalen Dimension $\mathrm{D}_{\mathrm{f}}$ von der Gemischzusammensetzung $\mathrm{C} / \mathrm{O}$ von verschiedenen $\mathrm{KW}-$ Luft-Flammen:

$+\quad$ Ethylen-Luft-Flamme mit $\mathrm{v}_{\mathrm{FG}}=8.3 \mathrm{~cm} / \mathrm{s}$ und $\mathrm{h}=17.5 \mathrm{~mm}$

$\Delta \quad$ Benzol-Luft-Flamme mit $\mathrm{V}_{\mathrm{FG}}=5.1 \mathrm{~cm} / \mathrm{s}$ und $\mathrm{h}=17.7 \mathrm{~mm}$

$\mathrm{x} \quad \mathrm{n}$-Heptan-Luft-Flamme mit $\mathrm{v}_{\mathrm{FG}}=3.8 \mathrm{~cm} / \mathrm{s}$ und $\mathrm{h}=17.5 \mathrm{~mm}$

Die Resultate der fraktalen Dimension in Abhängigkeit von der Gemischzusammensetzung ergaben auffallend ähnliche Zusammenhänge für alle untersuchten Flammen. Die fraktale Dimension $\mathrm{D}_{\mathrm{f}}$ nimmt für kleine $\mathrm{C} / \mathrm{O}-$ Verhältnisse geringe Werte an. Mit steigendem $\mathrm{C} / \mathrm{O}-$ Verhältnis steigt die fraktale Dimension bei den untersuchten Flammen rasch bis auf einen Wert von $\mathrm{D}_{\mathrm{f}}=1.5$ an. Für mittlere und hohe $\mathrm{C} / \mathrm{O}-$ Verhältnisse ändert sich der Betrag von $\mathrm{D}_{\mathrm{f}}$ nur noch langsam.

In der Ethylen-Luft-Flamme wurde ein maximaler Wert von $\mathrm{D}_{\mathrm{f}}=1.5$ berechnet. Für die nHeptan-Luft-Flammen und die Benzol-Luft-Flammen konnten aus Gründe der 
Flammenstabilität keine höheren $\mathrm{C} / \mathrm{O}-$ Verhältnisse als $\mathrm{C} / \mathrm{O}>0.75$ gemessen werden, so daß auch in diesen Flammen maximale Werte der fraktalen Dimension von nur $\mathrm{D}_{\mathrm{f}} \sim 1.6$ bestimmt wurden. Das bedeutet, daß die Aggregate, die in den Flammen vorliegen, aus bestenfalls kleinen Ketten bestehen und nicht sehr kompakt sein können.

In allen drei diskutierten Flammen liegt der Beginn des Kurvenverlaufs von $D_{f}$ bei unterschiedlichen $\mathrm{C} / \mathrm{O}-$ Verhältnissen, so daß sich für jede Flamme ein für sie typischer Verlauf von $\mathrm{D}_{\mathrm{f}}$ ergibt. Dieses Verhalten ist durch die unterschiedlichen Positionen der Rußgebiete im Gemischzusammensetzungs-Flammentemperatur-Feld begründet (s. Abb. 5.1). Dadurch wird auch verständlich, warum die Aggregatenbildung in der n-Heptan-Luft-Flamme schon bei so niedrigen C/O-Verhältnissen im Gegensatz zur Ethylen-Luft-Flamme einsetzt.

\subsection{Vergleich der Meßerbegnisse mit der Mie-Theorie}

Aus der Rayleigh-Näherung folgt, daß die vertikal polarisierte Komponente des Streulichts unabhängig vom Streuwinkel $\Theta$ ist. Diese Bedingung erfüllen kugelförmige Teilchen bei einer Wellenlänge von $488 \mathrm{~nm}$ bis zu einem Durchmesser von $\mathrm{d} \leq 50 \mathrm{~nm}$ nahezu exakt. Die Abb. 5.7 zeigt einige Beispiele von Teilchen mit verschiedenen Durchmessern. Verglichen wurden berechnete Teilchen anhand der Mie-Gleichungen und experimentell erhaltene Daten von Rußteilchen aus einer Isooktan.Luft-Flamme. Die Teilchendurchmesser sind für beide Teilchensorten etwa gleich. Mit zunehmendem Teilchendurchmesser weicht der winkelabhängige auf den $90^{\circ}$-Winkel normierte Intensitätsverlauf $\operatorname{IVv}_{\mathrm{VV}}(\Theta) / \mathrm{I}_{\mathrm{VV}}\left(90^{\circ}\right)$ immer mehr von der horizontalen Gerade mit $\operatorname{IVV}_{\mathrm{VV}}(\Theta) / \mathrm{IVV}_{\mathrm{VV}}\left(90^{\circ}\right)=1 \mathrm{ab}$. Für identische Durchmesser differieren der berechnete und der gemessene Kurvenverlauf ebenfalls. Je größer der Teilchendurchmesser wird, desto größer wird auch die Abweichung.

In der winkelabhängigen Streukurve sind zwei Informationen enthalten:

1. Der Teilchendurchmesser und

2. Die Teilchenstruktur

Aus den Resultaten der Abb. 5.7 wird deutlich, daß nur für kleine Flammenhöhen mit z.B. $\mathrm{h}<$ 15 mm für den verwendeten Röhrchenbrenner die Rußteilchen nahezu kugelförmig sind. Mit zunehmender Flammenhöhe weichen die Kurven immer deutlicher von den für sphärische Teilchen ab. 


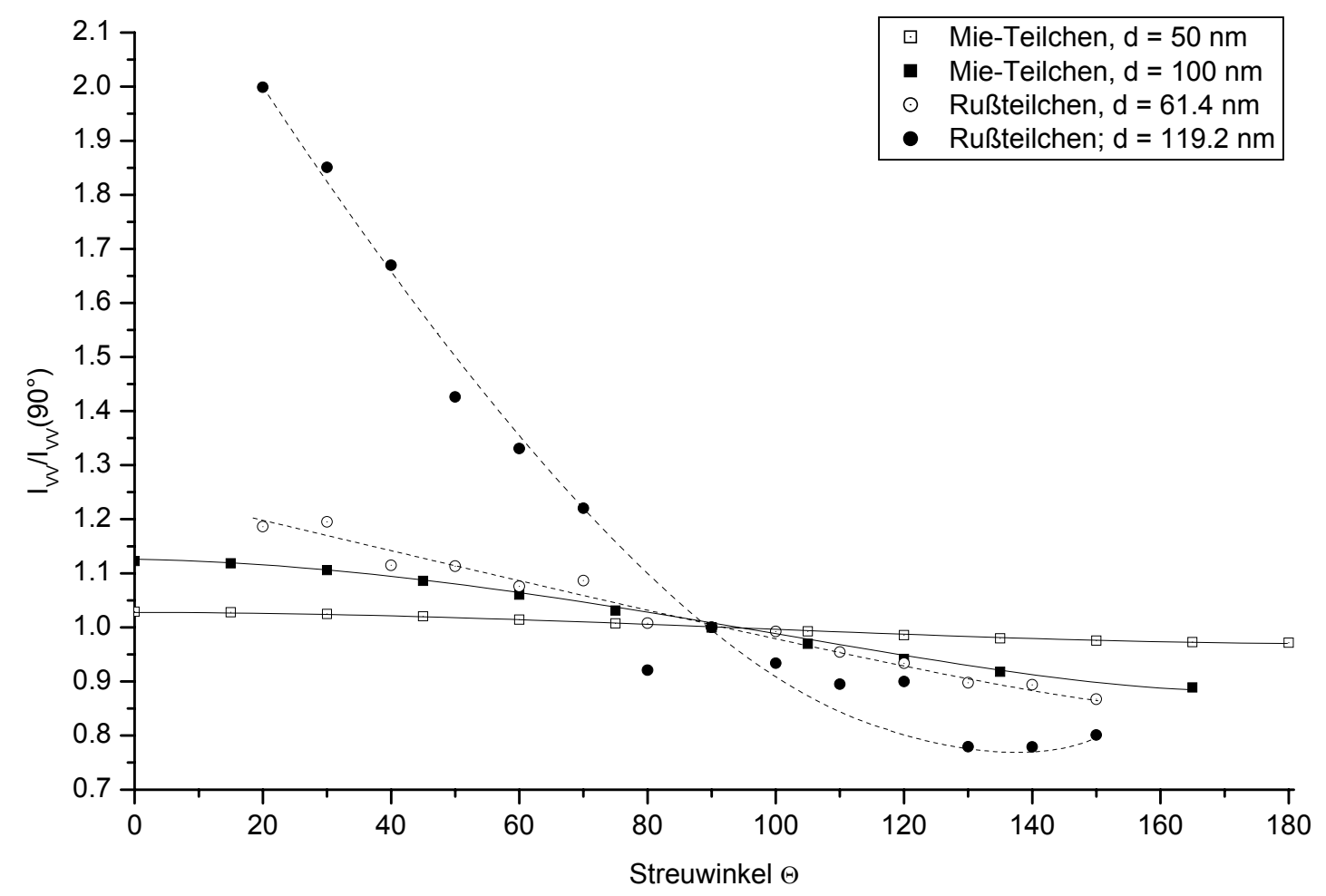

Abb. 5.7: Vergleich von experimentell bestimmten Streukurven (Rußteilchen) einer Isooktan-Luft-Flamme mit einem $\mathrm{C} / \mathrm{O}-$ Verhältnis von $\mathrm{C} / \mathrm{O}=0.62$ und einer Frischgasgeschwindigkeit von $\mathrm{V}_{\mathrm{FG}}=4.6 \mathrm{~cm} / \mathrm{s}$, gemessen in zwei Flammenhöhen von $\mathrm{h}_{1}=18.7 \mathrm{~mm}$ bzw. $\mathrm{h}_{2}=24.8 \mathrm{~mm}$ mit berechneten MieStreukurven (Mie-Teilchen) für zwei verschieden Teilchendurchmesser. Die Teilchendurchmesser betragen für die Rußteilchen $\mathrm{d}_{1}=50 \mathrm{~nm}$ bzw. $\mathrm{d}_{2}=100$ $\mathrm{nm}$ und für die Mie-Teilchen $\mathrm{d}_{1}=61.4 \mathrm{~nm}$ bzw. $\mathrm{d}_{2}=119.2 \mathrm{~nm}$. 
In Kohlenwasssersoff-Luft-Flammen kann eine Bildung von Rußaggregaten eintreten, diese differieren in unterschiedlichen Flammen in ihrer Größe und ihrer Gestalt. Die Zielsetzung dieser Arbeit war es diese Aggregatenbildung in vorgemischten Kohlenwasserstoff-LuftFlammen zu untersuchen. Dabei wurden KW-Luft-Flammen der Brennstoffe n-Pentan, nHeptan, Isooktan, Propen und Benzol unter atmosphärischen Bedingungen untersucht. Außerdem wurden Messungen bei höherem Druck für Ethylen-Luft-Flammen durchgeführt. Für alle Flammen wurden die untersuchte Flammenhöhe, die Gemischzusammensetzung und der Durchsatz innerhalb der jeweiligen Gebiete stabiler rußender Flammen variiert. Für die Ethylen-Luft-Flammen wurde der Druck in einem Bereich von $p=2$ bar bis $p=5$ bar variiert.

Es wurden winkelabhängige Streulichtmessungen sowie Extinktionsmessungen von alle Flammen ausgeführt. Bei den 1-bar-Flammen konnte der Streuwinkel zwischen $20^{\circ}$ und $150^{\circ}$ auf $1^{\circ}$ genau eingestellt werden. Im Hochdruck wurde das Streulicht in festen Winkeln von $25^{\circ}, 45^{\circ}, 70^{\circ}, 90^{\circ}, 110^{\circ}, 130^{\circ}$ und $150^{\circ}$ detektiert. Von den atmosphärischen Flammen wurden außerdem die Flammentemperaturen bestimmt.

Die aufgenommenen Streuintensitäten und Extinktionen wurden anhand der Rayleigh-Theorie und nach der RDG/FA-Theorie ausgewertet. Im Auswertungsteil dieser Arbeit wurden die Resultate der beiden Methoden vergleichend vorgestellt und diskutiert. Zusammenfassend können folgende Aussagen gemacht werden:

\section{$\underline{\text { Teilchendurchmesser }}$}

Es wurde der Rayleigh-Teilchendurchmesser $d_{R}$, sowie der volumenäquivalente Durchmesser $\mathrm{D}_{30}$ und der scheinbare Radius $\mathrm{R}_{\mathrm{g}}$ nach der RDG/FA-Theorie ermittelt. Alle Durchmesser der Teilchen weisen in ihrer Höhenabhängigkeit einen ähnlichen mit zunehmender Höhe in ihrer Steigung abflachenden Verlauf auf. Die Teilchendurchmesser verhalten sich auch in den unterschiedlichen Flammen ähnlich. Der Rayleigh-Durchmesser und der volumenäquivalente Durchmesser sind in geringen Höhen praktisch identisch und unterscheiden sich nur etwas in großen Höhen. Die Teilchendurchmesser der unterschiedlichen aliphatischen Brennstoffe liegen parallel zueinander verschoben vor, dabei hat das Isooktan in einer konstanten Höhe 
die größten Teilchendurchmesser und n-Pentan die kleinsten. Die scheinbaren Radien liegen in der Regel im gesamten untersuchen Höhenintervall etwas über den anderen Durchmessern $\mathrm{d}_{\mathrm{R}}$ und $\mathrm{D}_{30}$.

Nur bei sehr stark rußenden, d.h. brennstoffreichen, Flammen fallen der Teilchendurchmesser $\mathrm{d}_{\mathrm{R}}$ und der volumenäquivalente Durchmesser $\mathrm{D}_{30}$ nicht mehr zusammen. Daher kann man annehmen, daß die Rußteilchen erst bei sehr fetten Gemischzusammensetzungen von einer Kugelgestalt abweichen.

Mit größerem Durchsatz werden die Teilchendurchmesser bei allen Flamme geringer. Dabei fallen die unterschiedlichen Durchmesser bei hohen Frischgasgeschwindigkeiten zusammen. Mit geringer werdenden Frischgasgeschwindigkeiten trennen sich zunächst $d_{R}$ und $D_{30}$ von $R_{g}$. Für sehr kleine Durchsätze liegen die Teilchendurchmesser $d_{R}$ außerdem unter den volumenäquivalenten Durchmessern $\mathrm{D}_{30}$.

In ihrer Druckabhängigkeit verlaufen die unterschiedlichen Teilchendurchmesser weitgehend parallel zueinander, dabei ist der Rayleigh-Teilchendurchmesser $d_{R}$ im gesamten Druckbereich etwa um $10 \mathrm{~nm}$ kleiner als der volumenäquivalente Durchmesser $\mathrm{D}_{30}$, welcher wiederum um 5-10 nm kleiner als der scheinbare Radius $R_{\mathrm{g}}$ ist.

\section{Teilchenzahldichte und Aggregatenzahldichte}

Sowohl die Teilchenzahldichte $\mathrm{N}_{\mathrm{R}}$ als auch die Aggregatenzahldichte $\mathrm{N}_{\text {Agg }}$ nehmen mit der Flammenhöhe stark um drei Zehnerpotenzen von $10^{12}$ auf $10^{9}$ ab. Im untersuchten Höhenintervall verringern sich die Teilchenzahldichte und die Aggregatenzahldichte nur noch geringfügig. Die höhenabhängigen Verläufe der Teilchenzahldichte und der Aggregatenzahldichte fallen für die unterschiedlichen untersuchten Brennstoffe praktisch vollständig zusammen und sind damit nicht brennstoffspezifisch.

$\mathrm{Zu}$ brennstoffreicheren Gemischzusammensetzungen hin werden die am Ende des Verbrennungsprozesses gemessene Teilchenzahldichte $\mathrm{N}_{\mathrm{R}, \infty}$ und Aggregatenzahldichte $\mathrm{N}_{\mathrm{Agg}, \infty}$ leicht exponentiell ansteigend größer. Dabei steigt die Aggregatenzahldichte $\mathrm{N}_{\mathrm{Agg}, \infty}$ teilweise doppelt zu stark wie die Teilchenzahldichte $\mathrm{N}_{\mathrm{R}, \infty}$. 
Die Geschwindigkeitsabhängigkeit für die am Ende des Verbrennungsprozesses gemessenen Teilchenzahldichten $\mathrm{N}_{\mathrm{R}, \infty}$ und Aggregatenzahldichten $\mathrm{N}_{\mathrm{Agg}, \infty}$ verlaufen für die verschiedenen untersuchten Brennstoffe sehr unterschiedlich. Beim Propen wurde, wie es schon beim Ethylen gefunden wurde, ein exponentieller Anstieg mit dem Durchsatz sowohl von der Teilchenzahldichte $\mathrm{N}_{\mathrm{R}, \infty}$ als auch von der Aggregatenzahldichte $\mathrm{N}_{\mathrm{Agg}, \infty}$ beobachtet. Beim Benzol durchlaufen Teilchenzahldichte $\mathrm{N}_{\mathrm{R}, \infty}$ und Aggregatenzahldichte $\mathrm{N}_{\mathrm{Agg}, \infty}$ in ihrer Abhängigkeit von der Frischgasgeschwindigkeit ein Maximum und fallen von diesem Maximum mit kleineren und größeren Durchsätzen stark ab.

Mit dem Druck steigen die Teilchenzahldichte $\mathrm{N}_{\mathrm{R}, \infty}$ und die Aggregatenzahldichte $\mathrm{N}_{\text {Agg, }, \infty}$ fast parallel zueinander (leicht exponentiell) an. Die Aggregatenzahldichte $\mathrm{N}_{\mathrm{Agg}, \infty}$ liegt dabei um etwa $5 * 10^{9} \mathrm{~cm}^{-3}$ höher als die Teilchenzahldichte $\mathrm{N}_{\mathrm{R}, \infty}$.

\section{$\underline{\text { Fraktale Dimension }}$}

Die fraktale Dimension $\mathrm{D}_{\mathrm{f}}$ ist ein Parameter der RDG/FA-Theorie, der über die Form der Aggregate Auskunft gibt.

Die fraktale Dimension steigt (oft linear) in den untersuchten Flammen mit der Flammenhöhe auf Werte um $\mathrm{D}_{\mathrm{f}}=1.7$, diese Tendenz ist nahezu unabhängig vom Brennstoff.

Mit dem C/O-Verhältnis steigt die fraktale Dimension zunehmend abflachend und erreicht in brennstoffreichen Flammen ebenfalls Werte von bis zu $\mathrm{D}_{\mathrm{f}}=1.7$.

Die Geschwindigkeitsabhängigkeit der fraktalen Dimension weist in der Regel ein Maximum bei einer Frischgasgeschwindigkeit um die $5 \mathrm{~cm} / \mathrm{s}$ auf (brennstoffabhängig). Beim Benzol beträgt die fraktale Dimension in diesem Maximum etwa 1.8. Beim Propen beträgt $\mathrm{D}_{\mathrm{f}} \mathrm{im}$ Maximum aber nur etwa 0.8. Im Vergleich hierzu wurde beim Ethylen Wert bis unterhalb von 1.5 gefunden. Damit liegen die Aggregate in den untersuchen Flammen bestenfalls als kleiner Ketten vor, die wenig verzweigt sind und deshalb nicht sehr kompakt sein können Eine Druckabhängigkeit der fraktalen Dimension konnte ebenfalls gefunden werden. Zwischen 2 und 5 bar nimmt die fraktale Dimension von durchschnittlich 0.6 bis etwa $1.2 \mathrm{zu}$. 


\section{Primärteilchen}

Die Primärteilchen sind nach der RDG/FA-Theorie die kugelförmigen Grundbausteine der Aggregate. Sie liegen in den Aggregaten stets in etwa gleicher Größe vor, variieren aber mit unterschiedlichen Flammenparametern.

Eine deutliche Höhenabhängigkeit der Primärteilchen konnte nur für die Ethylen-LuftFlammen bei höherem Druck festgestellt werden. Der Primärteilchendurchmesser steigt z.B. in einer Ethylen-Flamme bei 4 bar von $3 \mathrm{~nm}$ in einer Flammenhöhe von $11 \mathrm{~mm}$ auf einen Wert von $23 \mathrm{~nm}$ in $12 \mathrm{~mm}$ Flammenhöhe an. Bei einer 2 bar Flamme liegen die Primärteilchendurchmesser $5 \mathrm{~nm}(\mathrm{~h}=12 \mathrm{~mm})$ bis $8 \mathrm{~nm}(\mathrm{~h}=3 \mathrm{~mm})$ tiefer als bei 4 bar. Die Primärteilchenzahldichten verlaufen bei unterschiedlichem Druck mit der Flammenhöhe fast identisch.

In den Benzol-Luft-Flammen konnte in einer Flammenhöhe von $17.7 \mathrm{~mm}$ und einer Frischgasgeschwindigkeit von $5.1 \mathrm{~cm} / \mathrm{s}$ ein Anstieg des Primärteilchendurchmessers mit dem $\mathrm{C} / \mathrm{O}-$ Verhältnis von $20 \mathrm{~nm}$ für $\mathrm{C} / \mathrm{O}=0.63$ ausgehend bis zu $30 \mathrm{~nm}$ bei einem $\mathrm{C} / \mathrm{O}-\mathrm{Verhältnis}$ von 0.75 festgestellt werden.

Wie die fraktale Dimension weisen auch die Primärteilchendurchmesser beim Benzol ein Maximum in ihrer Geschwindigkeitsabhängigkeit auf. Für die Propen-Luft-Flammen konnte innerhalb des Gebiets stabiler rußender Flammen nur eine Abnahme des Primärteilchendurchmessers $d_{p}$ mit der Frischgasgeschwindigkeit beobachtet werden.

\section{$\underline{\text { Schlußfolgerung }}$}

Aus den Meßergebnissen der vorliegenden Arbeit konnte gezeigt werden, daß sich mit Variation der Flammenbedingungen, wie Brennstoffart, Gemischzusammensetzung, Flammentemperatur und Druck, nicht nur die Rußmenge sowie die Teilchendurchmesser und Teilchenzahldichten ändern, es entstehen vielmehr auch Rußaggregate mit unterschiedlichen Strukturen. Die Bildung größerer Rußaggregate tritt hauptsächlich erst bei sehr stark rußenden Flammen in größeren Flammenhöhen und bei erhöhtem Brennkammerdruck ( $\mathrm{p} \geq 5$ bar) auf. 
7.1 Zusammenstellung aller Meßdaten

7.1.1 Flammen mit dem 1 bar Röhrchenbrenner 


\section{n-Pentan-Luft-Flammen}

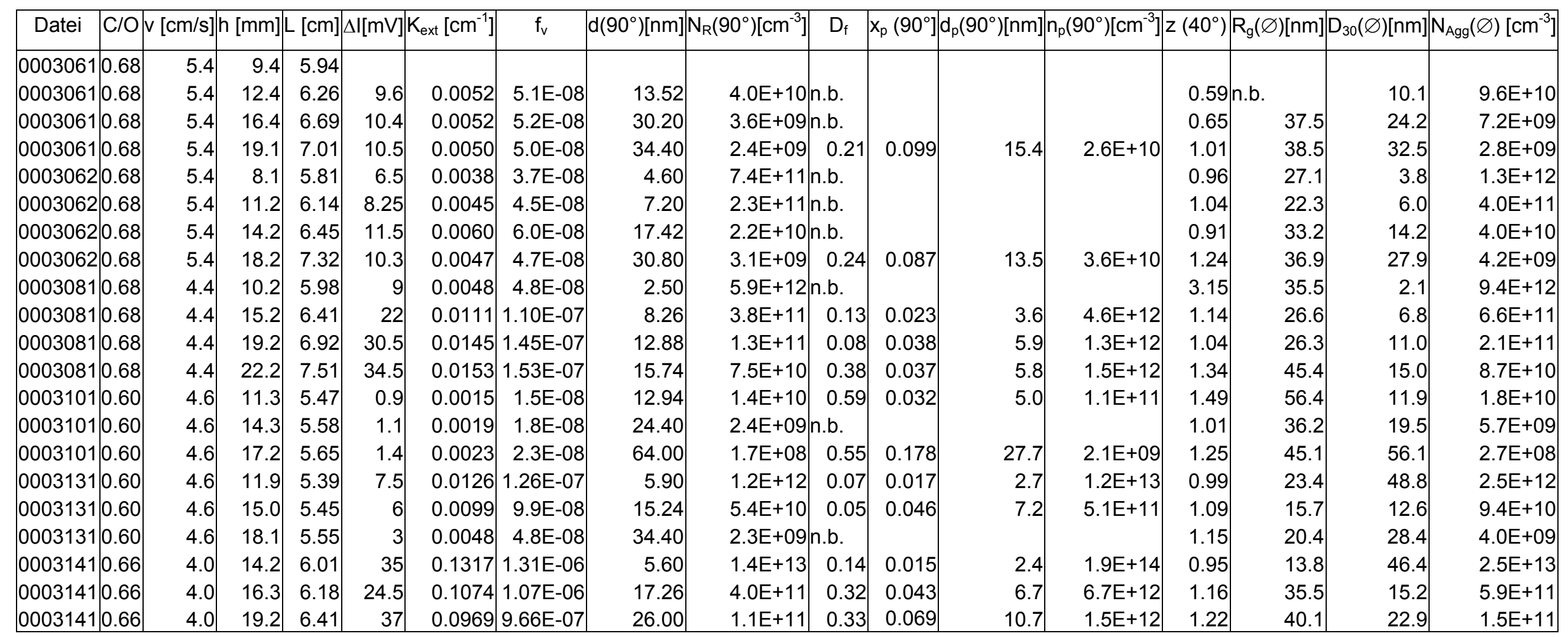

Tab. 7.1: Meßergebnisse der n-Pentan-Luft-Flammen 


\section{n-Heptan-Luft-Flammen}

\begin{tabular}{|c|c|c|c|c|c|c|c|c|c|c|c|c|c|c|c|c|c|}
\hline Datei & $\mathrm{C} / \mathrm{O}$ & $\mathrm{v}[\mathrm{cm} / \mathrm{s}]$ & h [mm] & $\mathrm{L}[\mathrm{cm}]$ & $\Delta \mathrm{I}[\mathrm{mV}]$ & $\mathrm{K}_{\mathrm{ext}}\left[\mathrm{cm}^{-1}\right]$ & $f_{v}$ & $\mathrm{~d}\left(90^{\circ}\right)[\mathrm{nm}]$ & $\mathrm{N}_{\mathrm{R}}\left(90^{\circ}\right)\left[\mathrm{cm}^{-3}\right]$ & $D_{f}$ & $x_{p}\left(90^{\circ}\right]$ & $\mathrm{d}_{\mathrm{p}}\left(90^{\circ}\right)[\mathrm{nm}]$ & $\mathrm{n}_{\mathrm{p}}\left(90^{\circ}\right)\left[\mathrm{cm}^{-3}\right]$ & $z\left(40^{\circ}\right) \mathrm{F}$ & $\mathrm{R}_{\mathrm{g}}(\varnothing)[\mathrm{nm}]$ & $\mathrm{D}_{30}(\varnothing)[\mathrm{nm}]$ & $\mathrm{N}_{\mathrm{Agg}}(\varnothing)\left[\mathrm{cm}^{-3}\right]$ \\
\hline 0003161 & 10.63 & 4.9 & 11.2 & 5.80 & 12 & 0.0090 & 9.0E-08 & 8.9 & $2.4 \mathrm{E}+11$ & 0.09 & 0.026 & 4.0 & $2.6 \mathrm{E}+12$ & 1.29 & 51.8 & 8.0 & $3.5 \mathrm{E}+11$ \\
\hline 0003161 & 0.63 & 4.9 & 15.2 & 6.15 & 24.3 & 0.0157 & 1.6E-07 & 29.8 & $1.1 \mathrm{E}+10$ & 0.19 & 0.086 & 13.3 & $1.3 \mathrm{E}+11$ & 1.15 & 39.4 & 27.1 & $1.5 \mathrm{E}+10$ \\
\hline 0003161 & 10.63 & 4.9 & 18.2 & 6.53 & 31.1 & 0.0178 & $1.8 \mathrm{E}-07$ & 43.4 & $4.2 \mathrm{E}+09$ & 0.20 & 0.128 & 19.8 & $4.3 E+10$ & 1.12 & 27.3 & 37.9 & $6.3 E+09$ \\
\hline 0003161 & 0.63 & 4.9 & 21.4 & 7.05 & 37.5 & 0.0194 & 1.9E-07 & 59.4 & $1.8 E+09$ & 0.81 & 0.149 & 23.1 & $3.0 \mathrm{E}+10$ & 1.70 & 58.7 & 56.5 & $2.0 \mathrm{E}+09$ \\
\hline 0003161 & 10.63 & 4.9 & 21.4 & 7.05 & 37.5 & 0.0194 & 1.9E-07 & 55.0 & $2.2 E+09$ & 0.82 & 0.134 & 20.8 & $4.0 \mathrm{E}+10$ & 1.60 & 57.3 & 52.3 & $2.6 E+09$ \\
\hline 0003171 & 0.65 & 3.8 & 12.0 & 5.71 & 13.5 & 0.0078 & 7.8E-08 & 9.2 & $1.9 \mathrm{E}+11$ & 0.27 & 0.023 & 3.5 & $3.4 \mathrm{E}+12$ & 0.75 & 39.9 & 7.6 & $3.4 \mathrm{E}+11$ \\
\hline 0003171 & 10.65 & 3.8 & 12.0 & 5.71 & 13.5 & 0.0078 & 7.8E-08 & 9.8 & $1.6 E+11$ & 0.00 & 0.031 & 4.8 & 1.3E+12 & 0.86 & 11.7 & 8.1 & $2.9 E+11$ \\
\hline 0003171 & 0.65 & 3.8 & 15.8 & 5.71 & 36 & 0.0219 & $2.2 \mathrm{E}-07$ & 31.4 & $1.3 E+10$ & 0.04 & 0.098 & 15.2 & $1.2 E+11$ & 0.99 & 21.2 & 26.3 & $2.3 E+10$ \\
\hline 0003171 & 0.65 & 3.8 & 19.2 & 5.68 & 51 & 0.0329 & 3.3E-07 & 50.2 & $5.0 E+09$ & 0.75 & 0.121 & 18.9 & $9.3 E+10$ & 1.52 & 56.4 & 46.8 & $6.1 \mathrm{E}+09$ \\
\hline 0003172 & 0.54 & 3.8 & 13.8 & 5.47 & 10.3 & 0.0061 & $6.1 \mathrm{E}-08$ & 9.4 & $1.4 \mathrm{E}+11$ & 0.00 & 0.030 & 4.6 & $1.2 \mathrm{E}+12$ & 0.97 & & 7.3 & $3.0 \mathrm{E}+11$ \\
\hline 0003172 & 0.54 & 3.8 & 17.6 & 5.64 & 4.8 & 0.0027 & $2.7 \mathrm{E}-08$ & 13.2 & $2.4 \mathrm{E}+10$ & 0.47 & 0.028 & 4.3 & $6.6 E+11$ & 1.18 & 41.1 & 11.9 & $3.1 \mathrm{E}+10$ \\
\hline 0003173 & 0.59 & 6.1 & 16.8 & 5.57 & 9 & 0.0067 & $6.6 \mathrm{E}-08$ & 8.4 & $2.2 E+11$ & 0.60 & 0.014 & 2.1 & 1.3E+13 & 1.33 & 44.9 & 7.0 & $4.0 \mathrm{E}+11$ \\
\hline 0003173 & 0.59 & 6.1 & 21.9 & 5.75 & 3 & 0.0021 & $2.1 \mathrm{E}-08$ & 30.0 & $1.5 E+09$ & 0.80 & 0.059 & 9.1 & $5.2 E+10$ & 0.74 & & 24.5 & $2.8 \mathrm{E}+09$ \\
\hline
\end{tabular}

Tab. 7.2: Meßergebnisse der n-Heptan-Luft-Flammen 


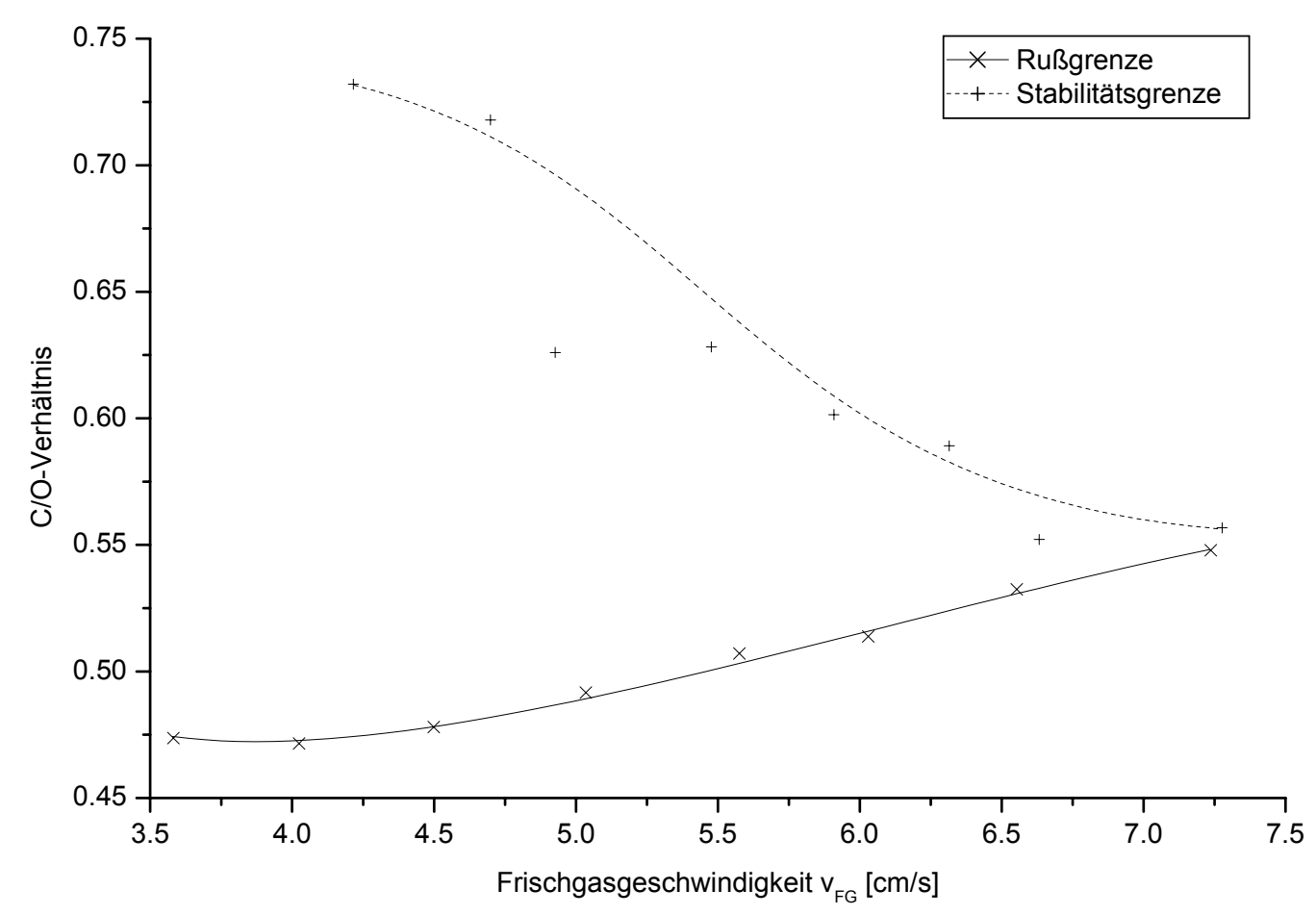

Abb. 7.1: $\quad$ Rußgrenze und Stabilitätsgrenze für n-Heptan-Luft-Flammen

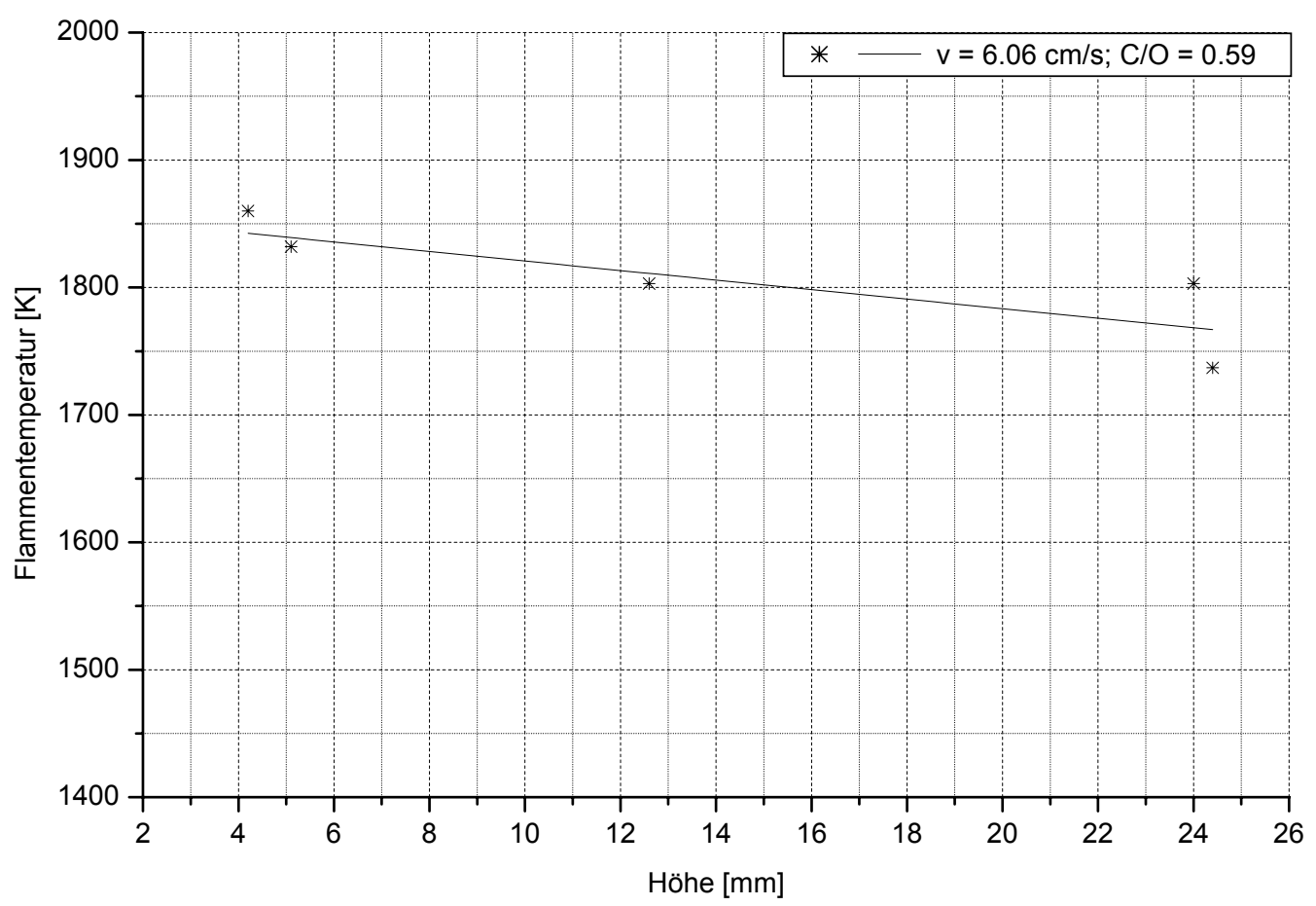

Abb. 7.2: $\quad$ Flammentemperaturen nach Kurlbaum einer n-Heptan-Luft-Flamme mit einem C/OVerhältnis von $\mathrm{C} / \mathrm{O}=0.59$ und einer Frischgasgeschwindigkeit von $\mathrm{v}=6.06 \mathrm{~cm} / \mathrm{s}$ 


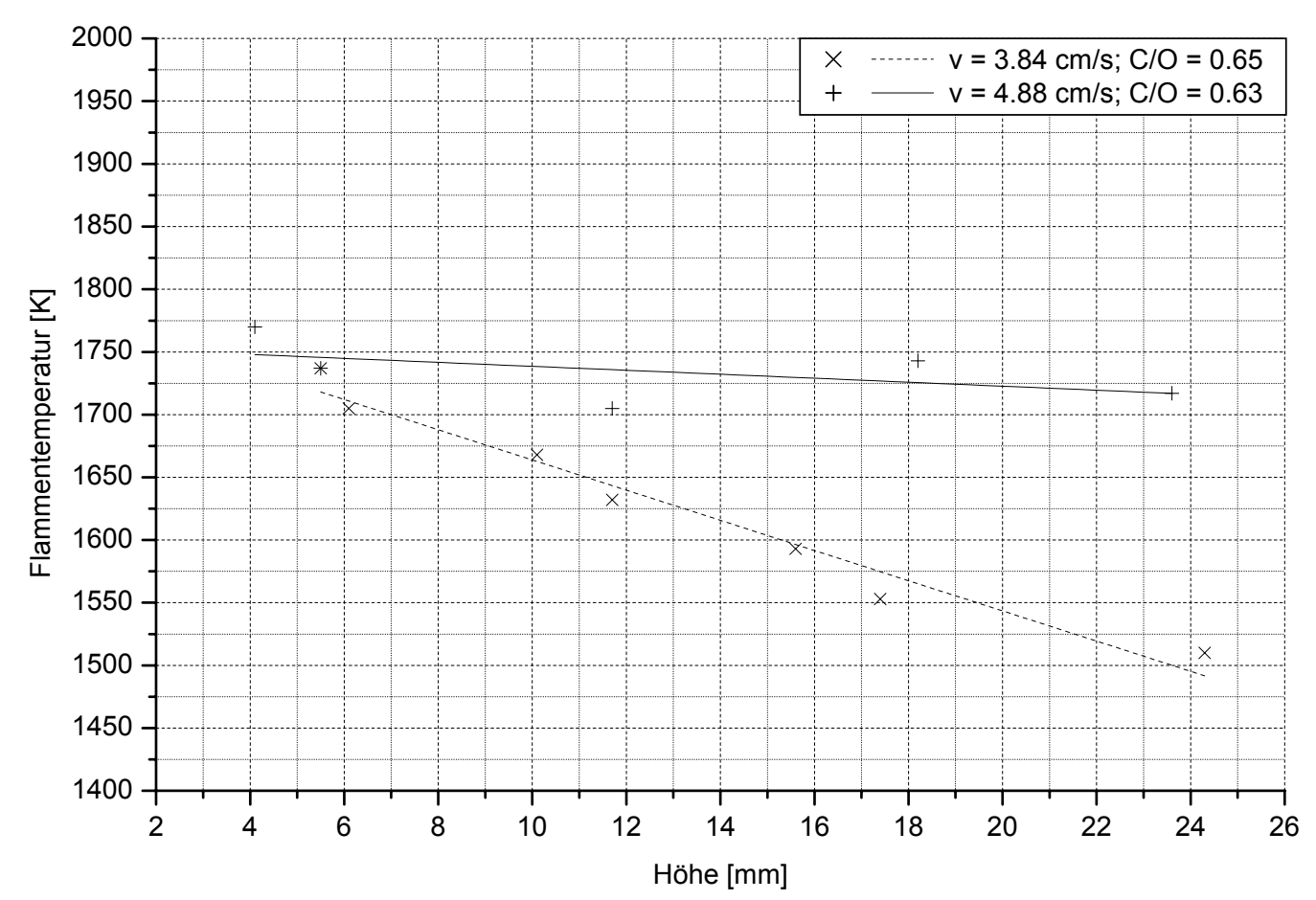

Abb. 7.3: Flammentemperaturen nach Kurlbaum von n-Heptan-Luft-Flammen mit einem C/OVerhältnis von $\mathrm{C} / \mathrm{O} \sim 0.64$ und verschiedenen Frischgasgeschwindigkeiten

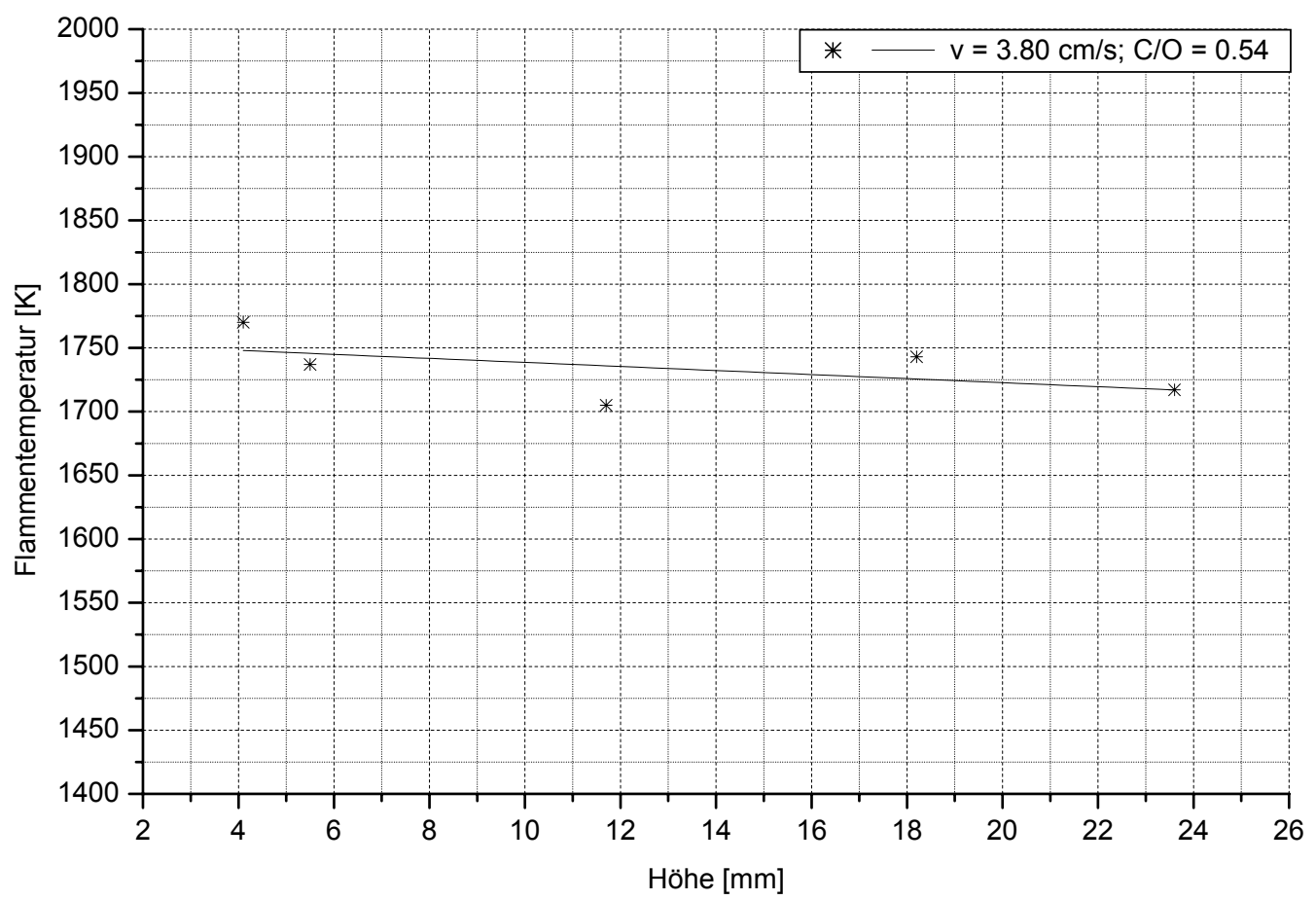

Abb. 7.4: Flammentemperaturen nach Kurlbaum einer n-Heptan-Luft-Flamme mit einem C/OVerhältnis von $\mathrm{C} / \mathrm{O}=0.54$ und einer Frischgasgeschwindigkeit von $\mathrm{v}=3.80 \mathrm{~cm} / \mathrm{s}$ 


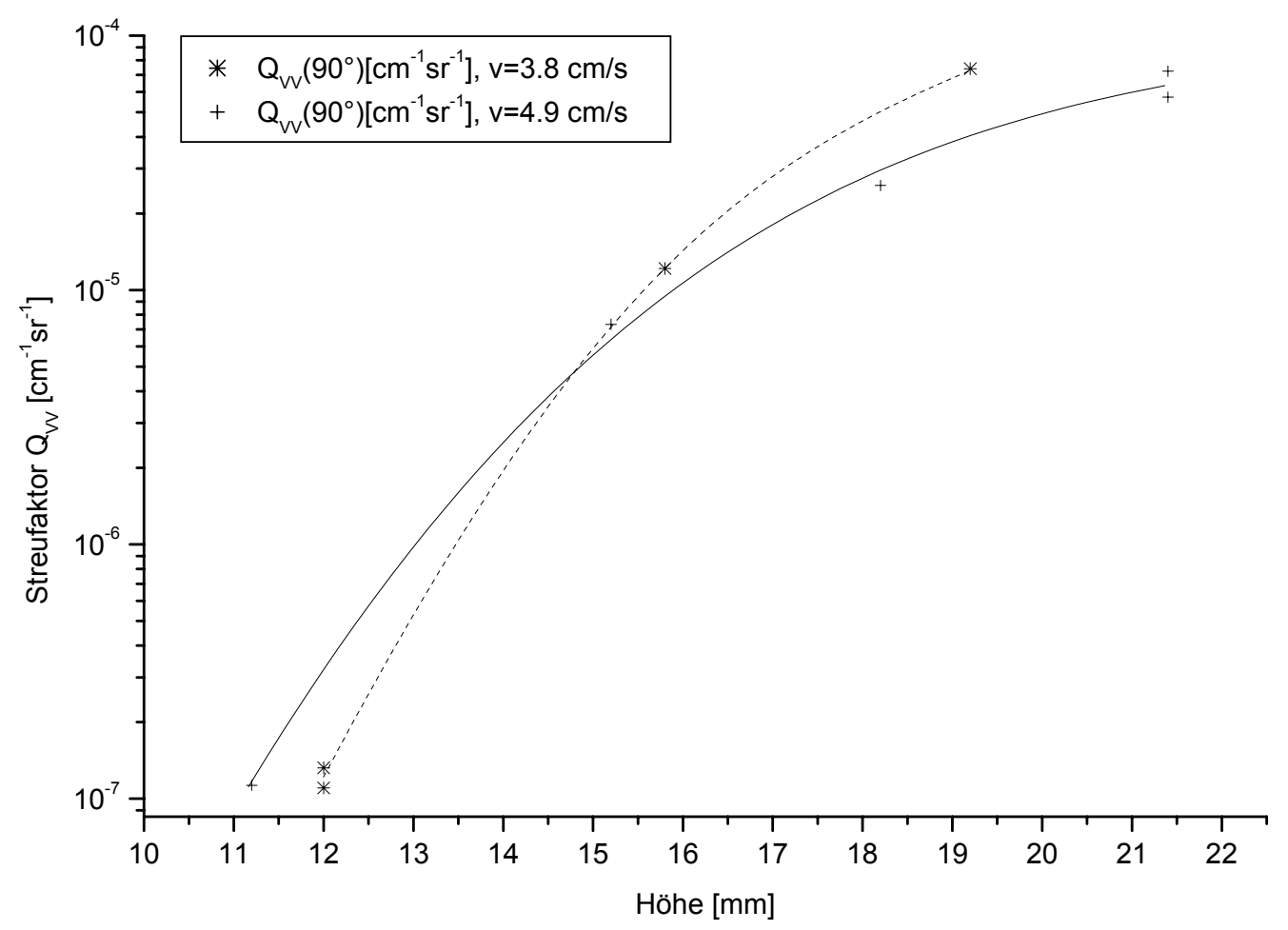

Abb. 7.5: Höhenabhängigkeit des Streufaktors einer n-Heptan-Luft-Flamme bei einem C/OVerhältnis von $\mathrm{C} / \mathrm{O} \sim 0.64$ und verschiedenen Frischgasgeschwindigkeiten

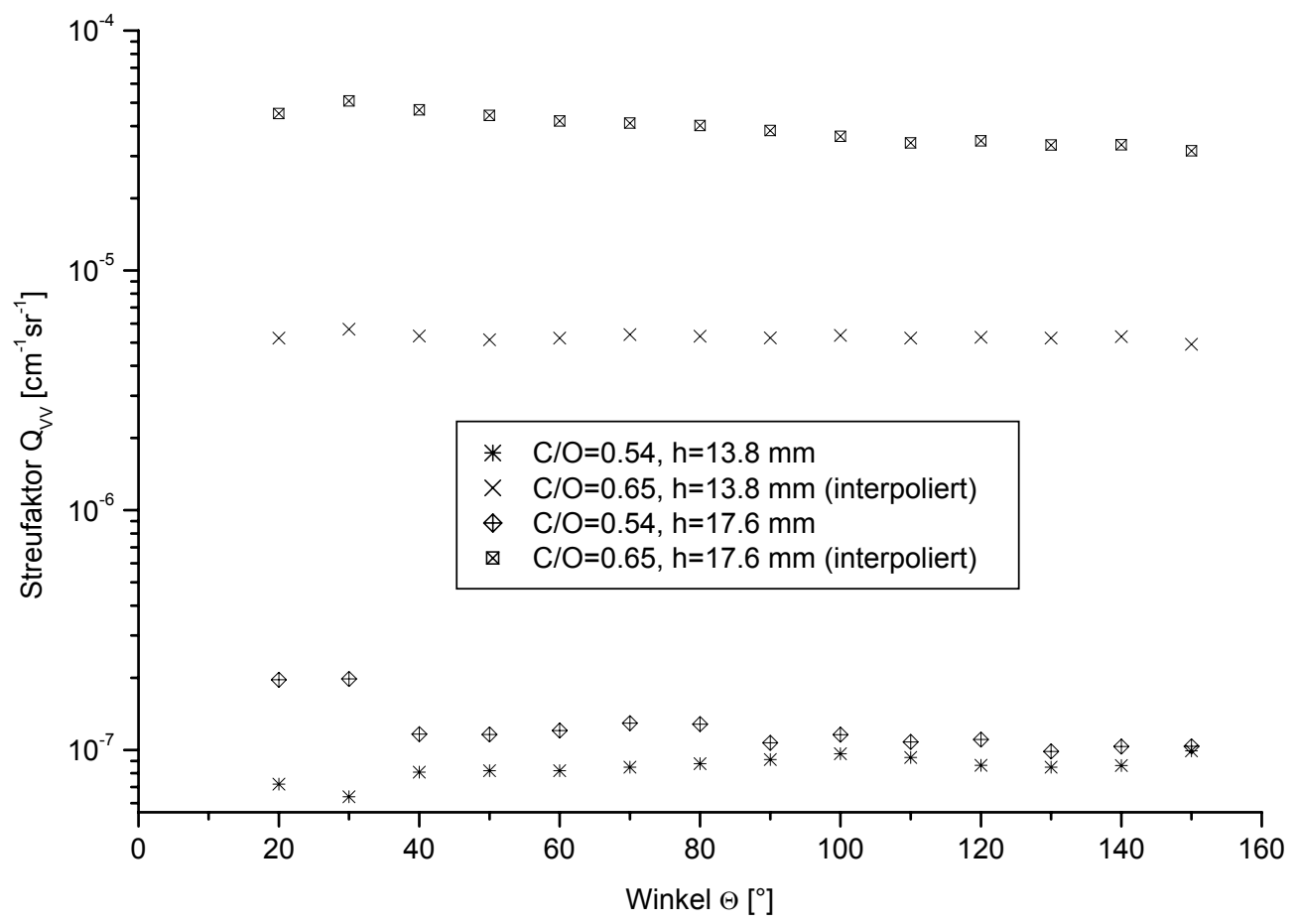

Abb. 7.6: Winkelabhängigkeit des Streufaktors von n-Heptan-Luft-Flammen bei verschiedenen C/OVerhältnissen und Höhen 


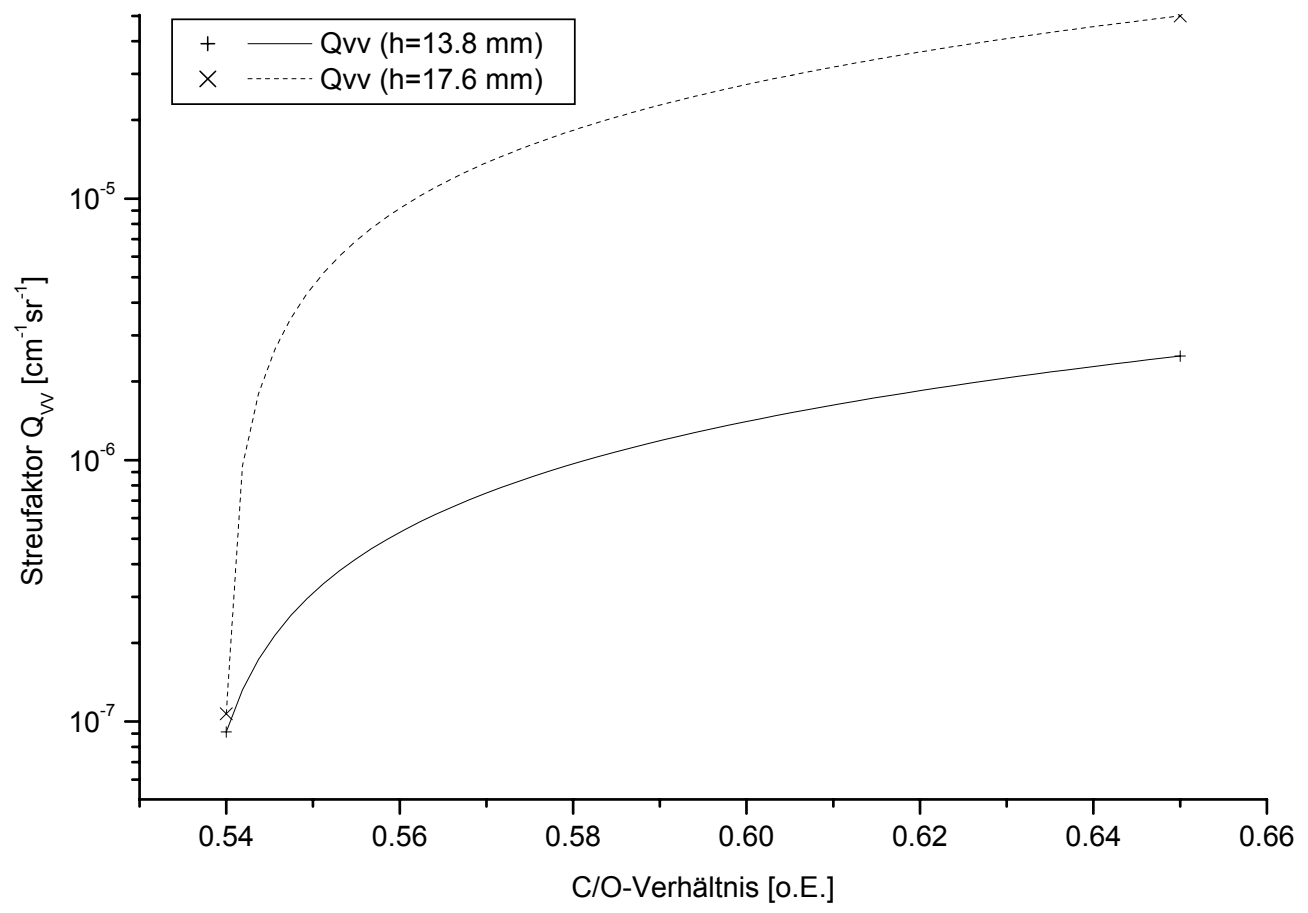

Abb. 7.7: Abhängigkeit des Streufaktors von n-Heptan-Luft-Flammen vom C/O-Verhältnis bei einer Frischgasgeschwindigkeit von $\mathrm{v}=3.8 \mathrm{~cm} / \mathrm{s}$

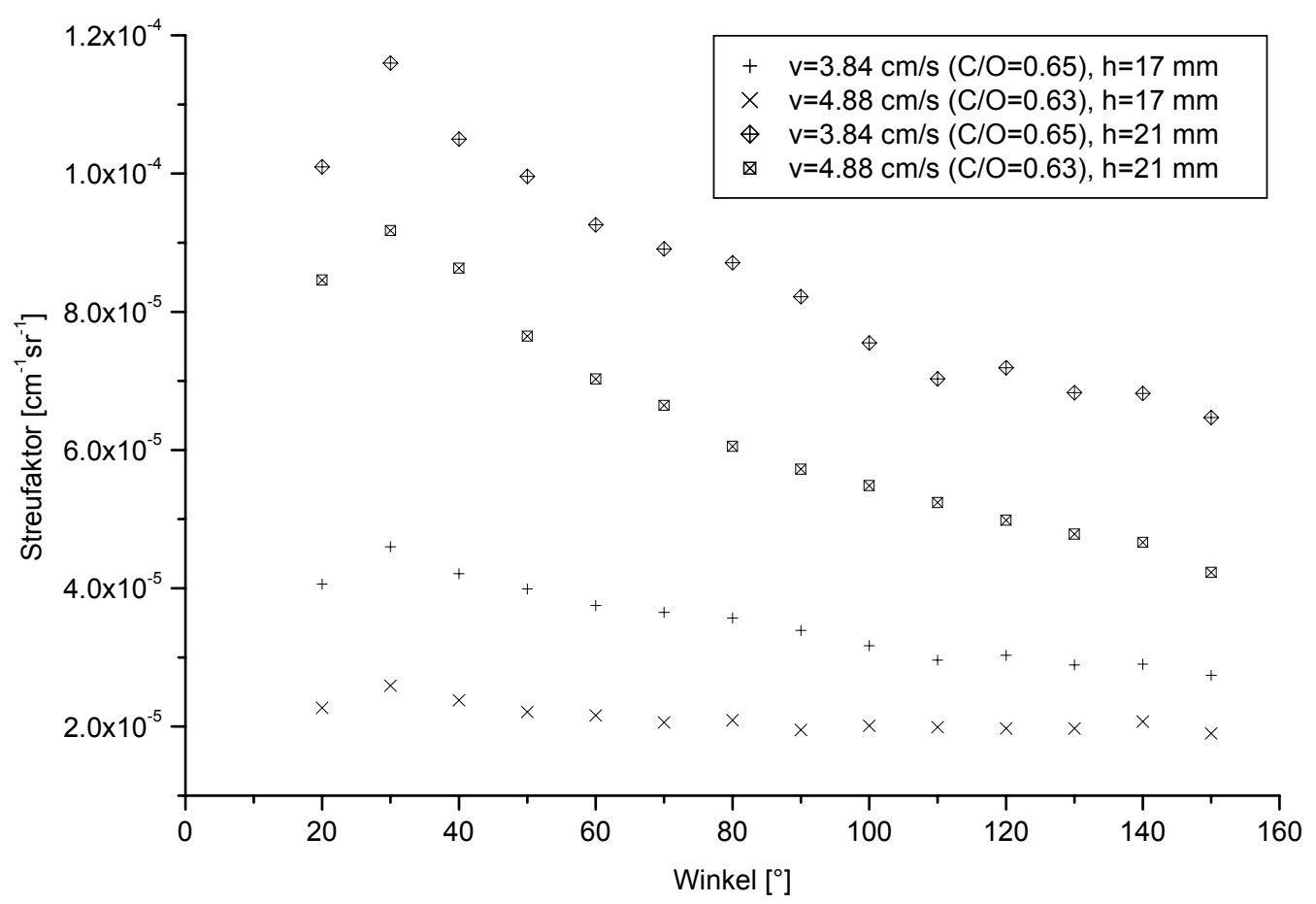

Abb. 7.8: Abhängigkeit des Streufaktors von n-Heptan-Luft-Flammen von der Frischgasgeschwindigkeit in verschiedenen Höhen für alle Streuwinkel 


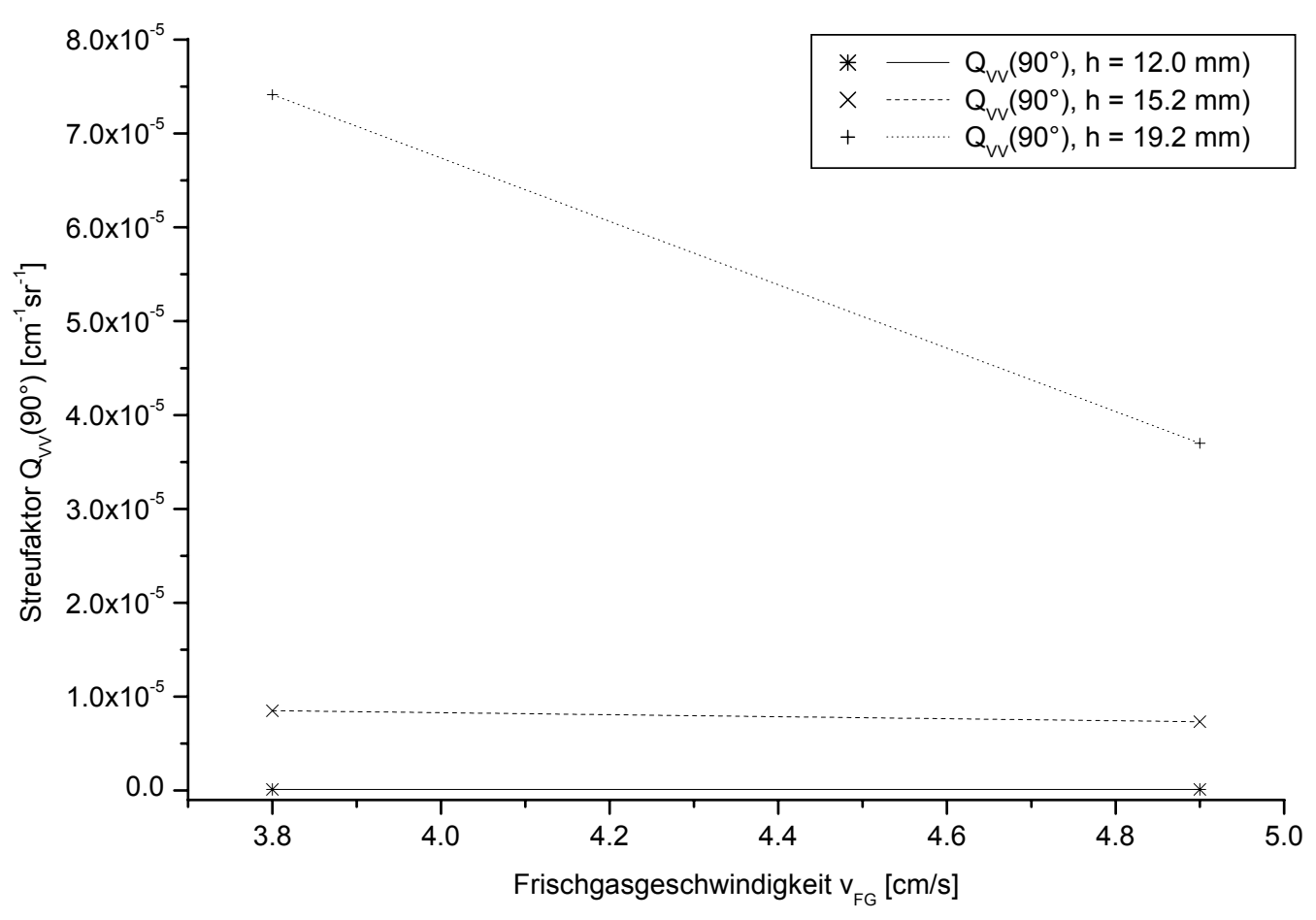

Abb. 7.9: Abhängigkeit des Streufaktors einer n-Heptan-Luft-Flamme von der Frischgasgeschwindigkeit in verschiedenen Höhen bei einem $\mathrm{C} / \mathrm{O}$-Verhältnis von $\mathrm{C} / \mathrm{O} \sim 0.65$

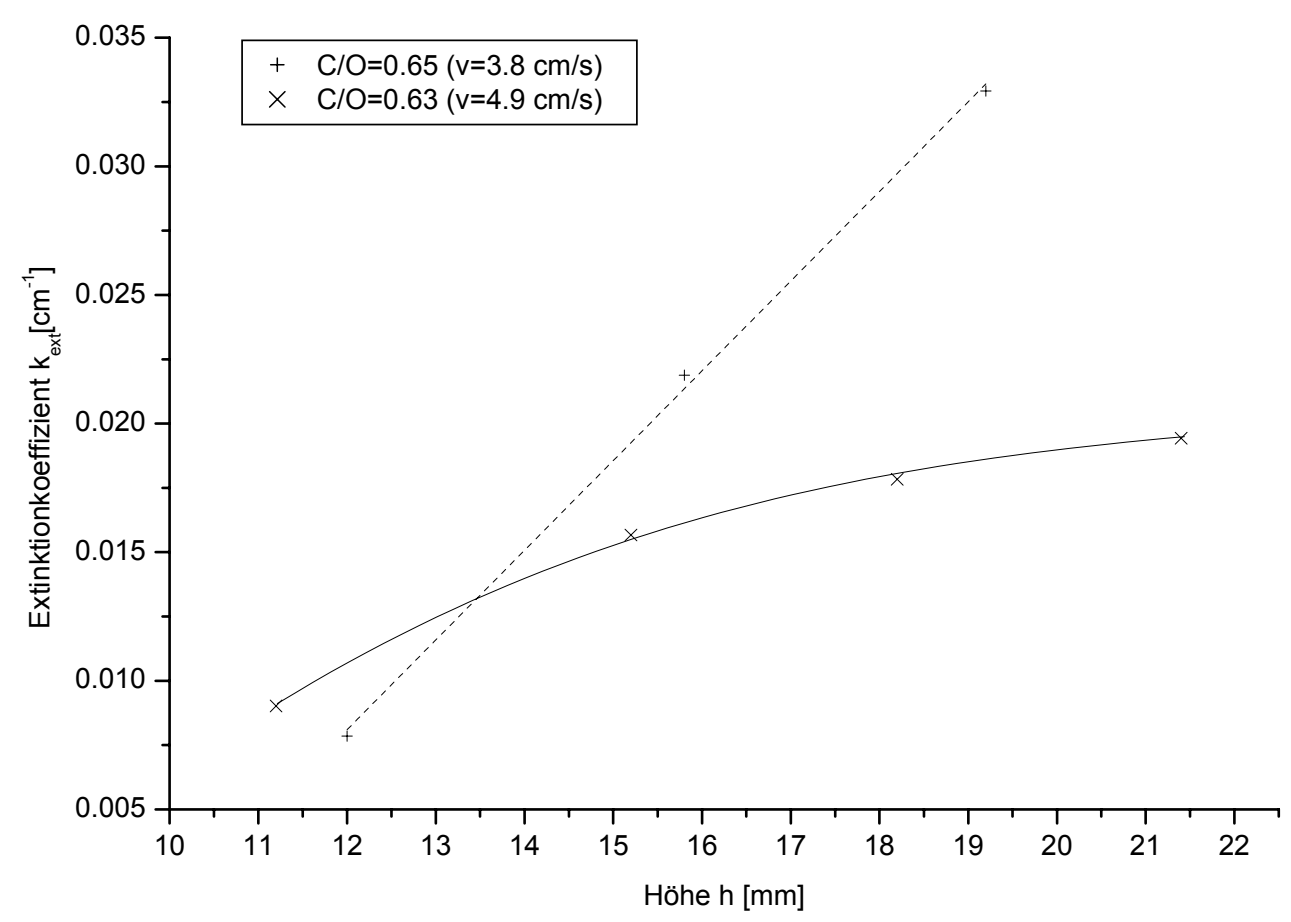

Abb. 7.10: Höhenabhängigkeit des Extinktionskoeffizienten von n-Heptan-Luft-Flammen bei einem $\mathrm{C} / \mathrm{O}$-Verhältnis von $\mathrm{C} / \mathrm{O} \sim 0.64$ und zwei Frischgasgeschwindigkeiten 


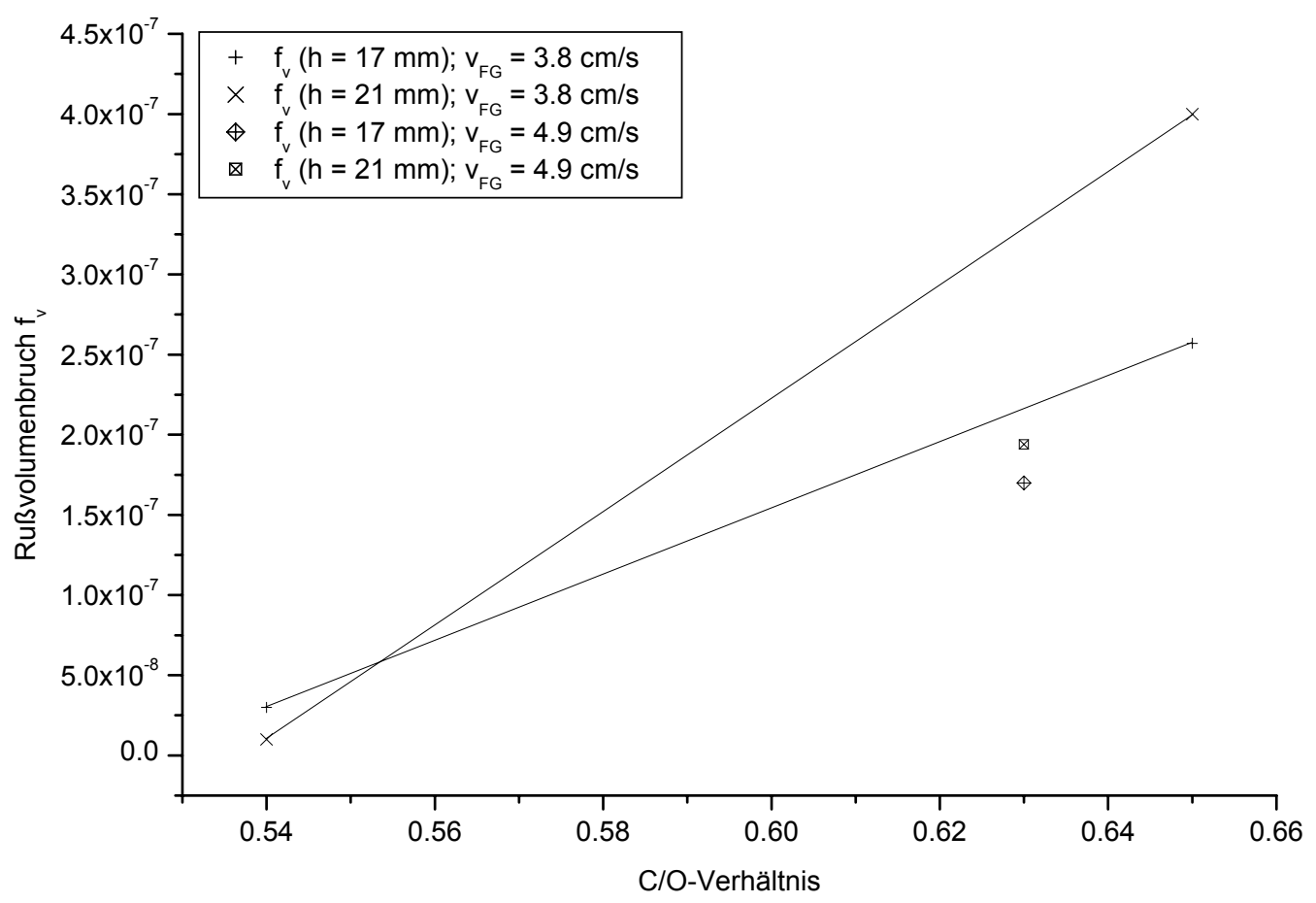

Abb. 7.11: Abhängigkeit des Rußvolumenbruchs $\mathrm{f}_{\mathrm{v}}$ von $\mathrm{n}$-Heptan-Luft-Flammen vom C/O-Verhältnis bei einer Frischgasgeschwindigkeit von $\mathrm{v}_{\mathrm{FG}}=3.8 \mathrm{~cm} / \mathrm{s}$ bzw. $4.9 \mathrm{~cm} / \mathrm{s}$ in zwei Höhen

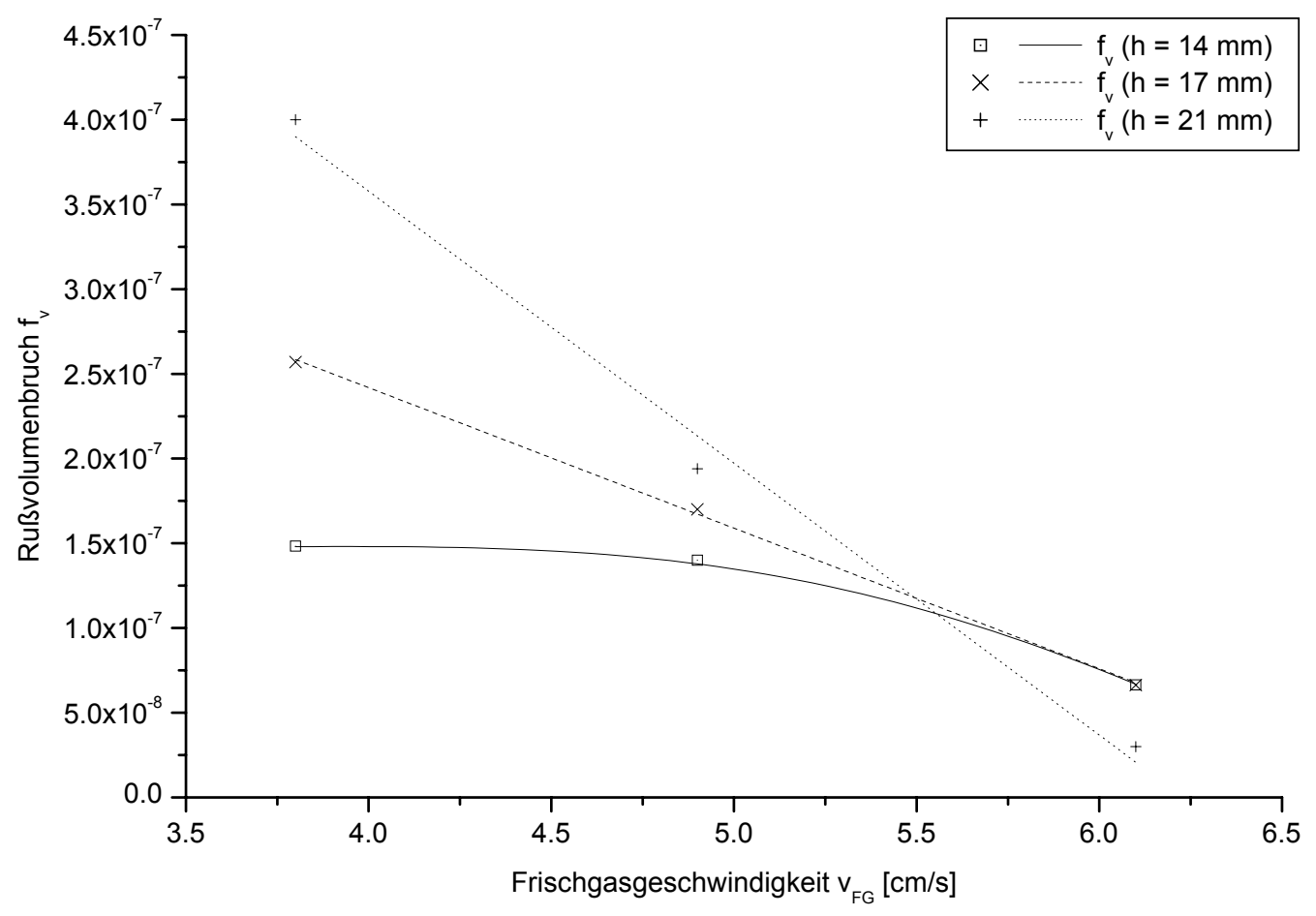

Abb. 7.12: Abhängigkeit des Rußvolumenbruchs $\mathrm{f}_{\mathrm{v}}$ von der Frischgasgeschwindigkeit in n-HeptanLuft-Flammen bei einem $\mathrm{C} / \mathrm{O}-$ Verhältnis von etwa $\mathrm{C} / \mathrm{O} \sim 0.6$ in verschiedenen Höhen 


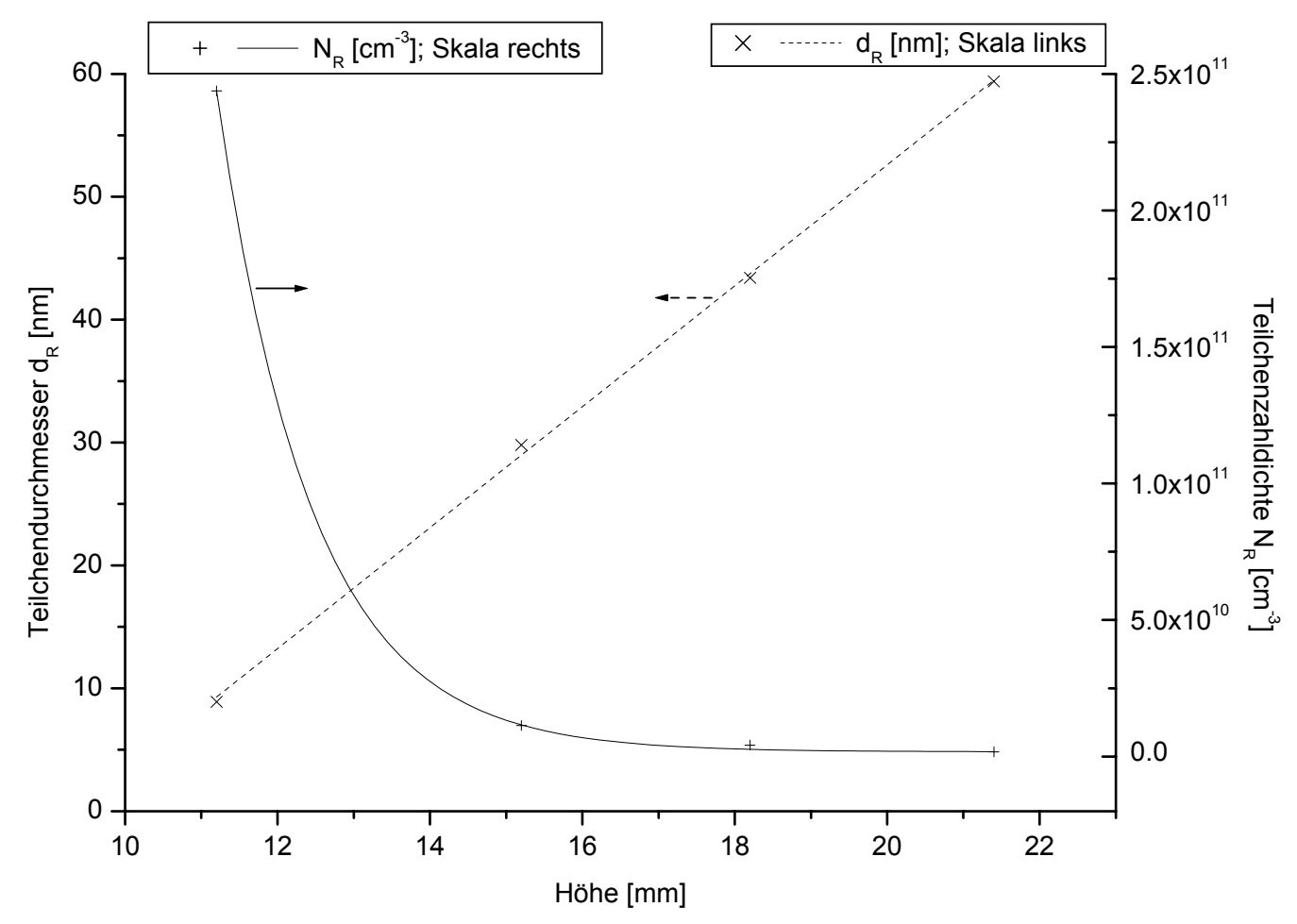

Abb. 7.13: Abhängigkeit des Teilchendurchmessers und der Teilchenzahldichte von der Höhe bei einem $\mathrm{C} / \mathrm{O}-$ Verhältnis von $\mathrm{C} / \mathrm{O}=0.63$ und einer Frischgasgeschwindigkeit $\mathrm{v}=4.9 \mathrm{~cm} / \mathrm{s}$

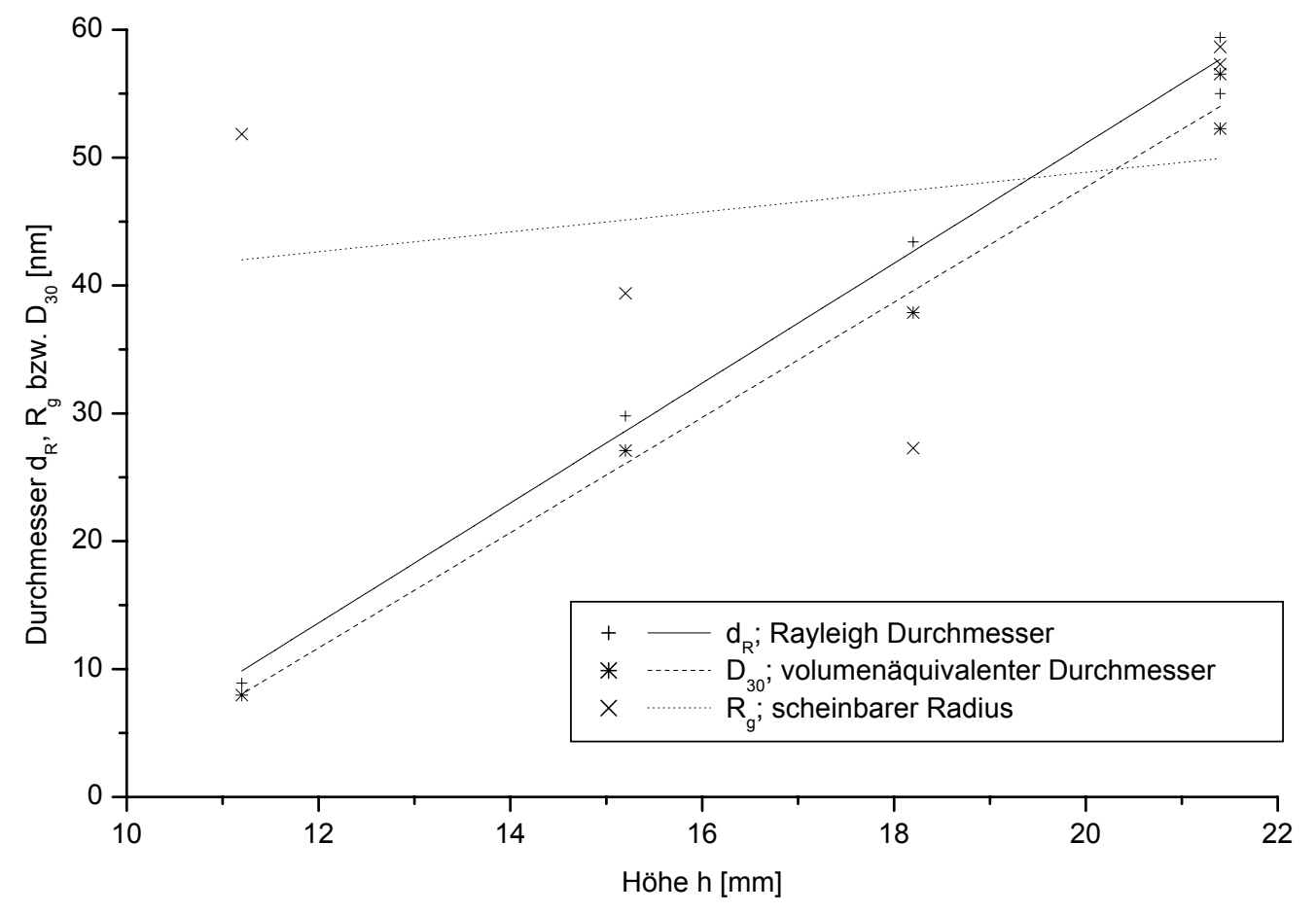

Abb. 7.14: Abhängigkeit der verschiedenen Rußteilchen-Durchmesser einer n-Heptan-Luft-Flamme von der Höhe für eine Frischgasgeschwindigkeit von v $=4.88 \mathrm{~cm} / \mathrm{s}$ 


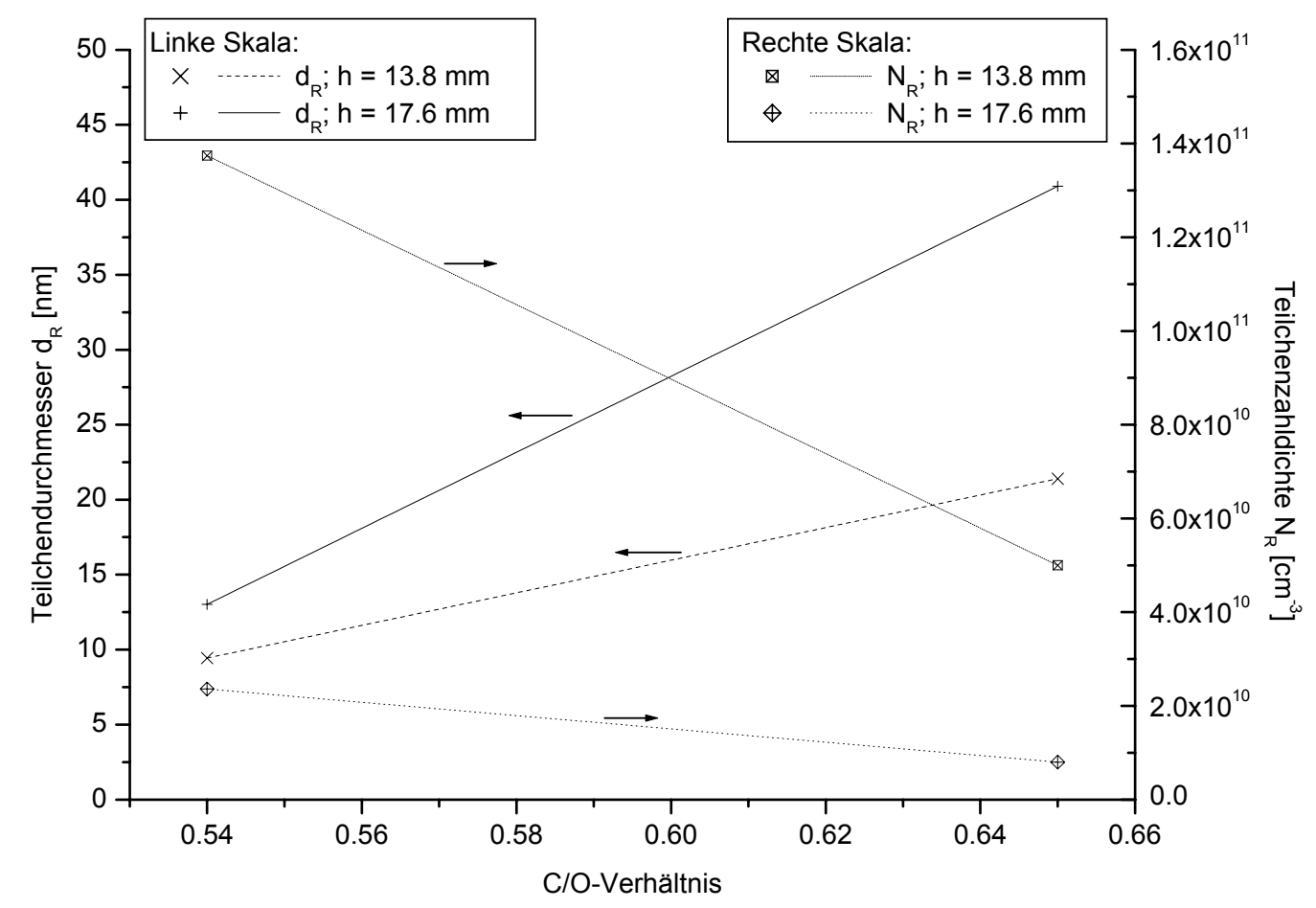

Abb. 7.15: Abhängigkeit des Teilchendurchmessers und der Teilchenzahldichte vom C/O-Verhältnis von n-Heptan-Luft-Flammen bei in einer Frischgasgeschwindigkeit von $\mathrm{v}=3.8 \mathrm{~cm} / \mathrm{s}$ in zwei verschiedenen Höhen

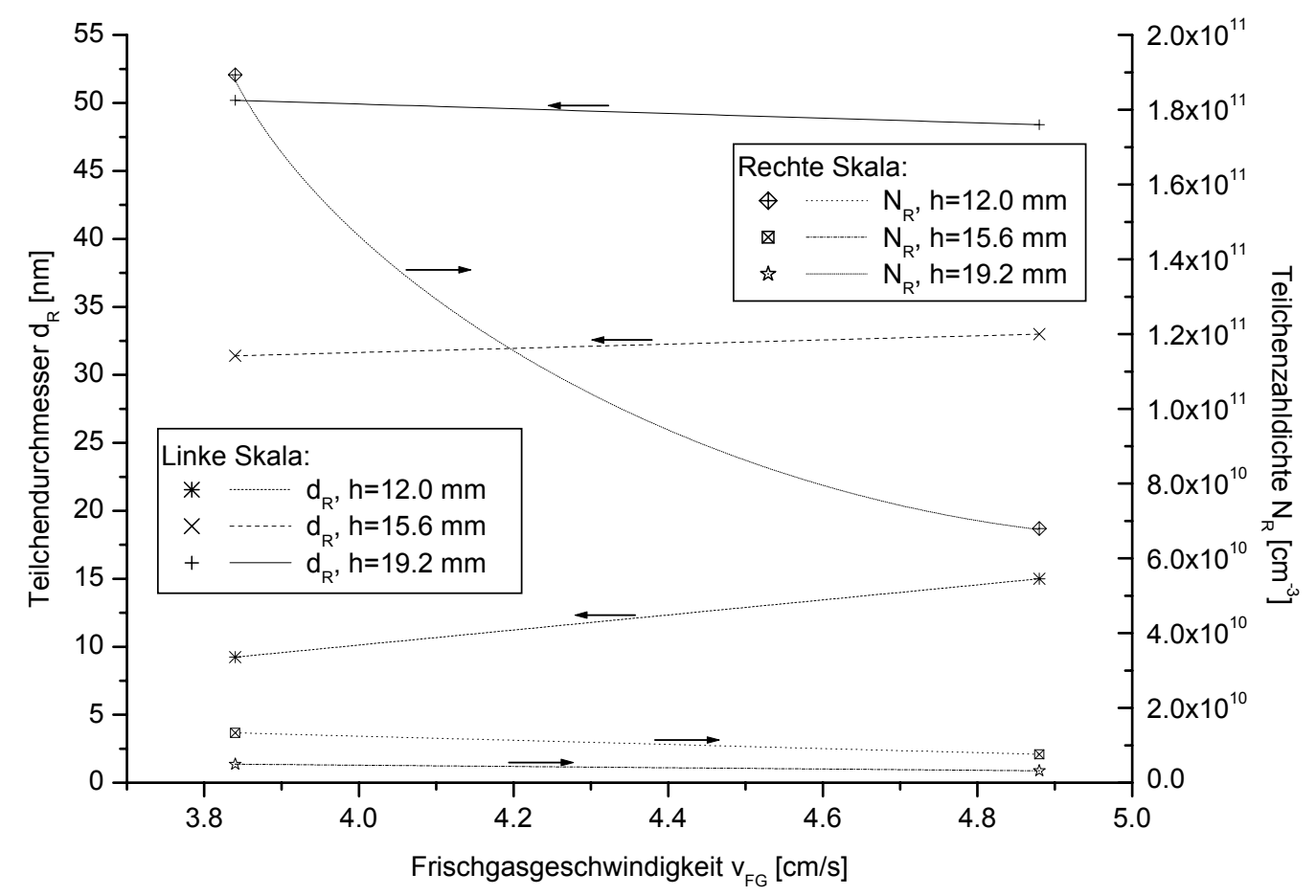

Abb. 7.16: Abhängigkeit des Teilchendurchmessers und der Teilchenzahldichte von der

Frischgasgeschwindigkeit von n-Heptan-Luft-Flammen für ein $\mathrm{C} / \mathrm{O}-$ Verhältnis von $\mathrm{C} / \mathrm{O}$ 0.64 in drei verschiedene Höhen 


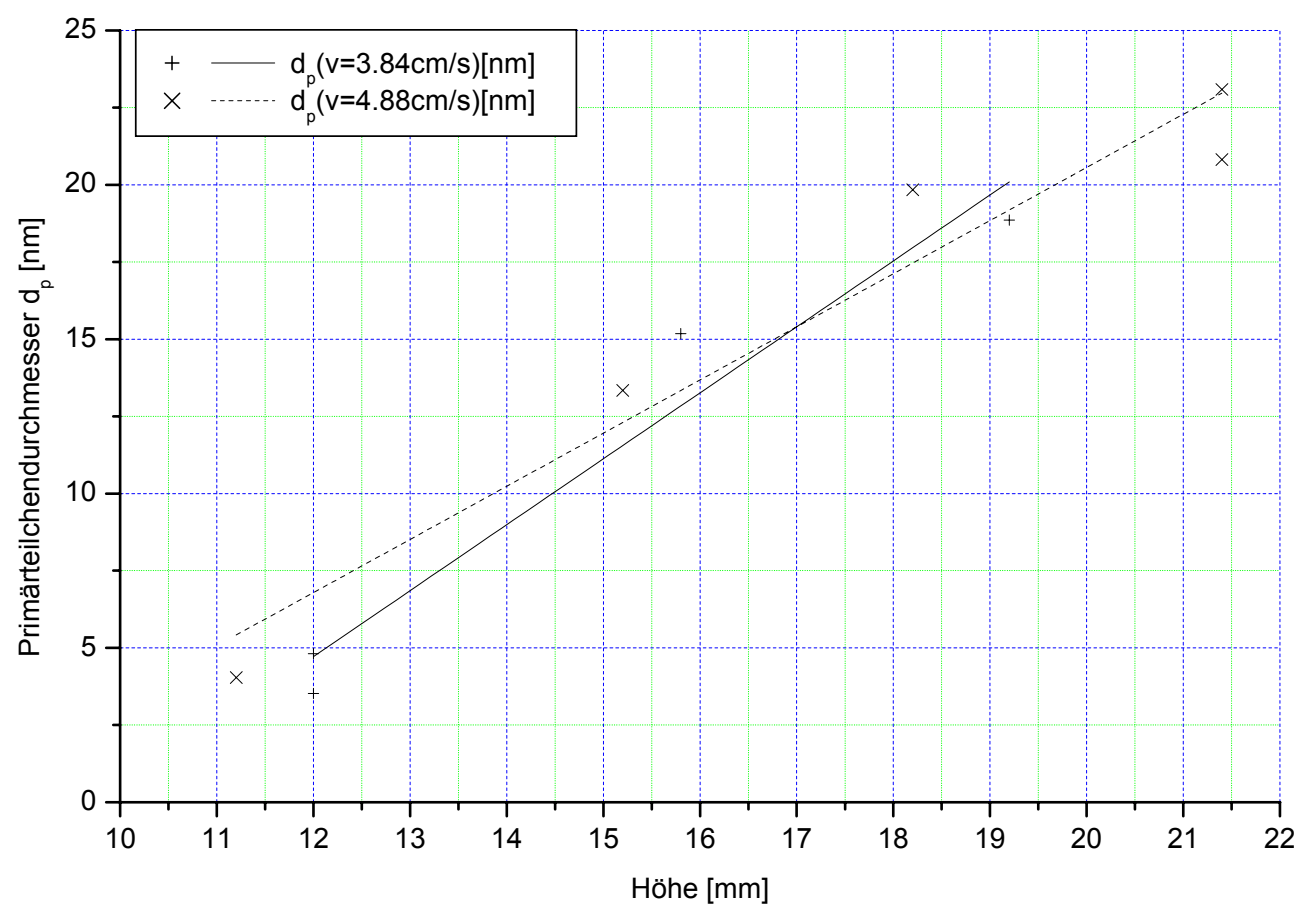

Abb. 7.17: Zusammenhang zwischen Primärteilchendurchmesser aus n-Heptan-Luft-Flammen und der Höhe über dem Brenner für zwei Frischgasgeschwindigkeiten

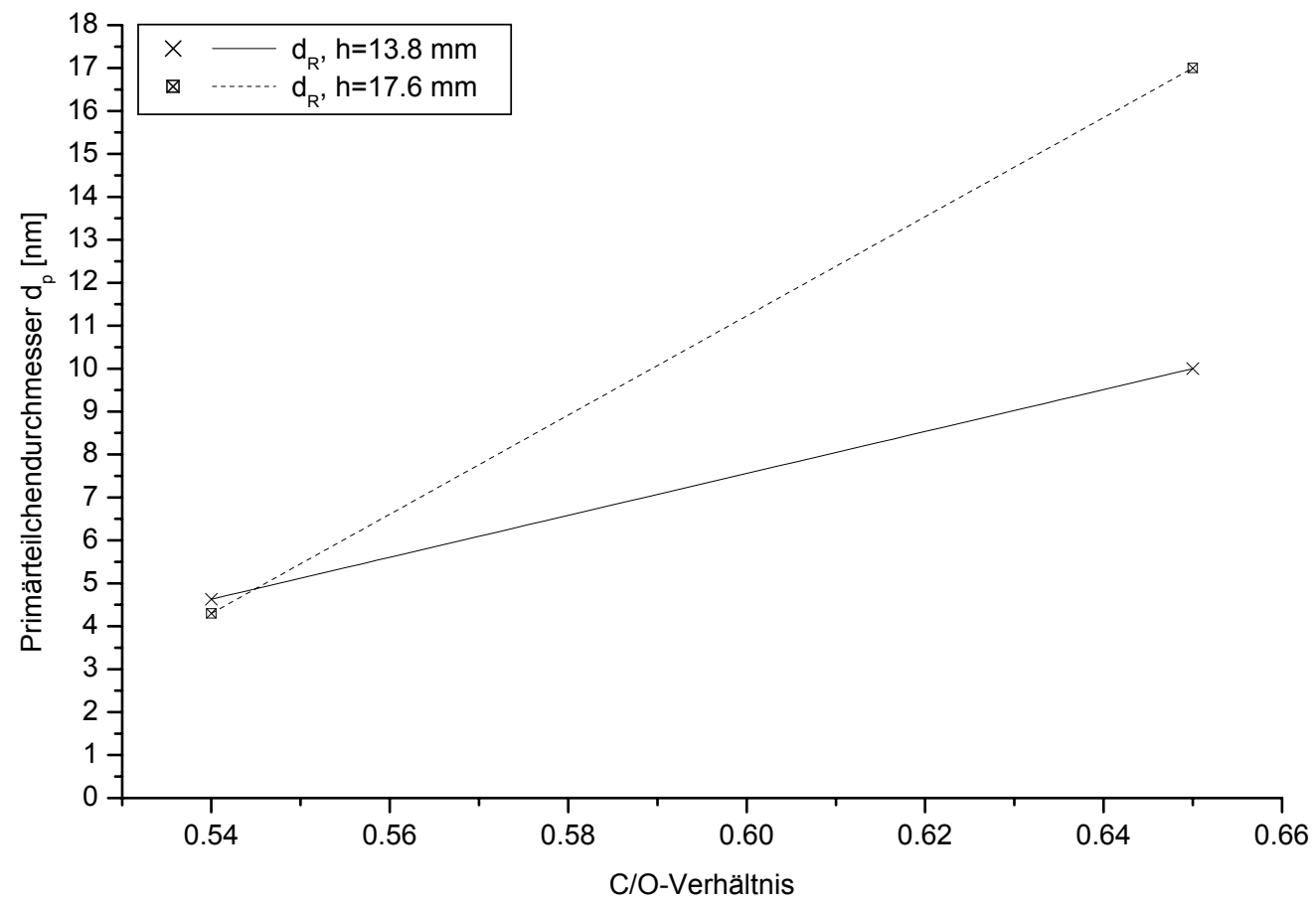

Abb. 7.18: Abhängigkeit des Primärteilchendurchmessers in n-Heptan-Luft-Flammen vom C/OVerhältnis bei einer Frischgasgeschwindigkeit von $v=3.8 \mathrm{~cm} / \mathrm{s}$ in zwei Höhen 


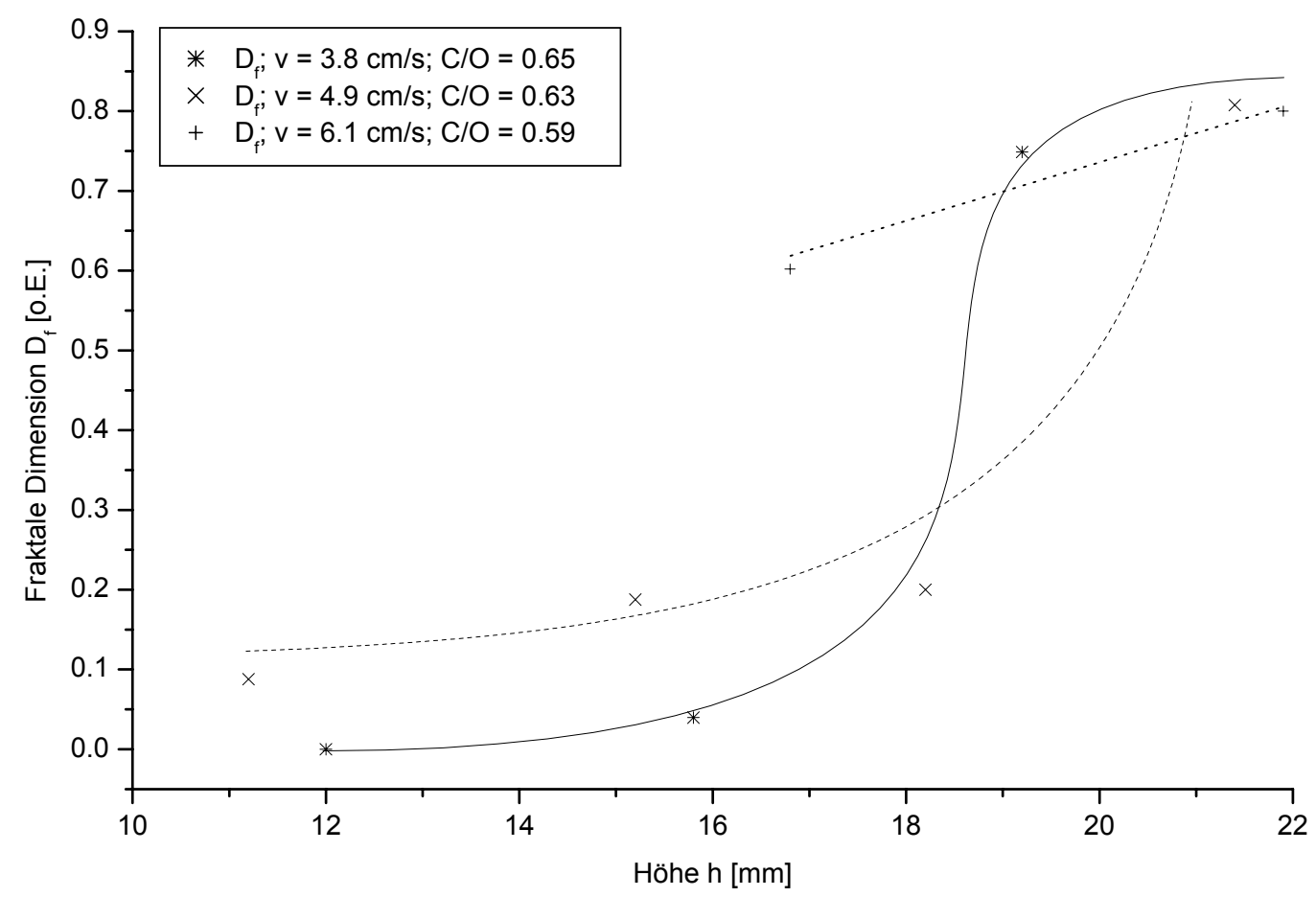

Abb. 7.19: Höhenabhängigkeit der fraktalen Dimension von n-Heptan-Luft-Flammen für ein C/OVerhältnis von etwa $\mathrm{C} / \mathrm{O} \sim 0.6$ und verschiedenen Frischgasgeschwindigkeiten 


\subsubsection{Isooktan}

\section{Isooktantan-Luft-Flammen}

\begin{tabular}{|c|c|c|c|c|c|c|c|c|c|c|c|c|c|c|c|c|c|c|}
\hline Datei & $|c / O|$ & $\mathrm{v}[\mathrm{cm} / \mathrm{s}] \mathrm{r}$ & $\mathrm{h}[\mathrm{mm}]$ & $\mathrm{L}[\mathrm{cm}]$ & $\Delta I[\mathrm{mV}]$ & $\mathrm{K}_{\mathrm{ext}}\left[\mathrm{cm}^{-1}\right]$ & $f_{v}$ & $\mathrm{~d}\left(90^{\circ}\right)[\mathrm{nm}]$ & $\mathrm{N}_{\mathrm{R}}\left(90^{\circ}\right)\left[\mathrm{cm}^{-3}\right]$ & $D_{f}$ & $x_{p}\left(90^{\circ}\right)$ & $\begin{array}{c}d_{p} \\
\left(90^{\circ}\right)[\mathrm{nm}]\end{array}$ & $d_{g}[n m]$ & $\begin{array}{c}\mathrm{n}_{\mathrm{p}} \\
\left(90^{\circ}\right)\left[\mathrm{cm}^{-3}\right]\end{array}$ & \begin{tabular}{|c|}
$z$ \\
$\left(40^{\circ}\right)$
\end{tabular} & $\mathrm{R}_{\mathrm{g}}(\varnothing)[\mathrm{nm}]$ & $\mathrm{D}_{30}(\varnothing)[\mathrm{nm}]$ & $\mathrm{N}_{\mathrm{Agg}}(\varnothing)\left[\mathrm{cm}^{-3}\right]$ \\
\hline 0003221 & 10.62 & 3.5 & 13.8 & 5.66 & 78 & 0.0328 & $3.3 \mathrm{E}-07$ & 39.0 & $1.1 \mathrm{E}+10$ & 0.27 & 0.111 & 17.2 & & $1.2 \mathrm{E}+11$ & 1.226 & 40.2 & 33.0 & $1.8 \mathrm{E}+10$ \\
\hline 0003221 & 10.62 & 3.5 & 16.8 & 5.79 & 119 & 0.0485 & $4.8 \mathrm{E}-07$ & 53.6 & $6.0 \mathrm{E}+09$ & 0.78 & 0.131 & 20.3 & 42.3 & $1.1 \mathrm{E}+11$ & 1.510 & 52.0 & 48.8 & $7.9 \mathrm{E}+09$ \\
\hline 0003221 & 10.62 & 3.5 & 19.8 & 5.85 & 155 & 0.0658 & $6.6 \mathrm{E}-07$ & 55.2 & $7.4 \mathrm{E}+09$ & 1.52 & 0.085 & 13.2 & 42.2 & $5.5 \mathrm{E}+11$ & 3.328 & 86.5 & 62.0 & $5.4 \mathrm{E}+09$ \\
\hline 0003221 & 10.62 & 3.5 & 22.6 & 5.98 & 176 & 0.0729 & 7.3E-07 & 77.2 & $3.0 \mathrm{E}+09$ & 0.99 & 0.202 & 24.6 & 41.7 & $4.6 \mathrm{E}+10$ & 1.915 & 62.7 & 77.5 & $2.8 \mathrm{E}+09$ \\
\hline 0003231 & 10.62 & 4.6 & 14.3 & 5.43 & 21 & 0.0104 & $1.0 \mathrm{E}-07$ & 39.2 & $3.3 \mathrm{E}+09$ & 0.3 & 0.11 & 17.1 & & $4.0 \mathrm{E}+10$ & 1.190 & 38.7 & 34.5 & $4.8 \mathrm{E}+09$ \\
\hline 0003231 & 10.62 & 4.6 & 18.7 & 5.59 & 37 & 0.0170 & 1.7E-07 & 61.4 & $1.4 \mathrm{E}+09$ & 0.45 & 0.174 & 27.1 & 35.8 & $1.6 \mathrm{E}+10$ & 1.273 & 37.8 & 53.5 & $2.1 \mathrm{E}+09$ \\
\hline 0003231 & 0.62 & 4.6 & 21.6 & 5.63 & 53 & 0.0243 & $2.4 \mathrm{E}-07$ & 69.4 & $1.4 \mathrm{E}+09$ & 0.48 & 0.2 & 31.1 & 41.7 & $1.5 \mathrm{E}+10$ & 1.356 & 48.0 & 62.0 & $1.9 \mathrm{E}+09$ \\
\hline 0003231 & 0.62 & 4.6 & 24.7 & 5.71 & 51 & 0.0228 & $2.3 \mathrm{E}-07$ & 119.2 & $2.6 \mathrm{E}+08$ & 0.87 & 0.391 & 60.8 & 34.1 & $1.9 \mathrm{E}+09$ & 1.830 & 65.7 & 116.8 & $2.8 \mathrm{E}+08$ \\
\hline 0003232 & 0.55 & 3.9 & 10.5 & 4.89 & 0.6 & 0.0005 & $5.4 \mathrm{E}-09$ & 8.0 & $2.0 \mathrm{E}+10$ & n.b. & n.b. & 3.9 & 30.5 & n.b. & 0.978 & 24.2 & 6.7 & $3.5 \mathrm{E}+10$ \\
\hline 0003232 & 0.55 & 3.9 & 14.9 & 5.14 & 1.35 & 0.0012 & $1.2 \mathrm{E}-08$ & 1.7 & $4.6 \mathrm{E}+12$ & n.b. & n.b. & 0.8 & 40.0 & n.b. & 0.727 & n.b. & 1.3 & $1.1 \mathrm{E}+13$ \\
\hline 0003233 & 30.67 & 4.8 & 18.9 & & 34 & 0.0253 & $2.5 \mathrm{E}-07$ & 21.4 & $4.9 \mathrm{E}+10$ & n.b. & n.b. & 10.5 & & n.b. & 1.146 & 37.6 & 19.6 & $6.4 \mathrm{E}+10$ \\
\hline 0003233 & 30.67 & 4.8 & 22.9 & 5.99 & 51.2 & 0.0363 & $3.6 \mathrm{E}-07$ & 32.2 & $2.1 \mathrm{E}+10$ & 0.44 & 0.082 & 12.7 & & $3.4 \mathrm{E}+11$ & 1.321 & 42.5 & 29.5 & $2.8 \mathrm{E}+10$ \\
\hline 0003233 & 0.67 & 4.8 & 26.9 & 6.30 & 68.5 & 0.0481 & $4.8 \mathrm{E}-07$ & 41.0 & $1.3 \mathrm{E}+10$ & 0.77 & 0.091 & 14.1 & 47.7 & $3.2 \mathrm{E}+11$ & 2.095 & 65.4 & 46.1 & $9.7 \mathrm{E}+09$ \\
\hline
\end{tabular}

Tab. 7.3: Meßergebnisse der Isooktan-Luft-Flammen 


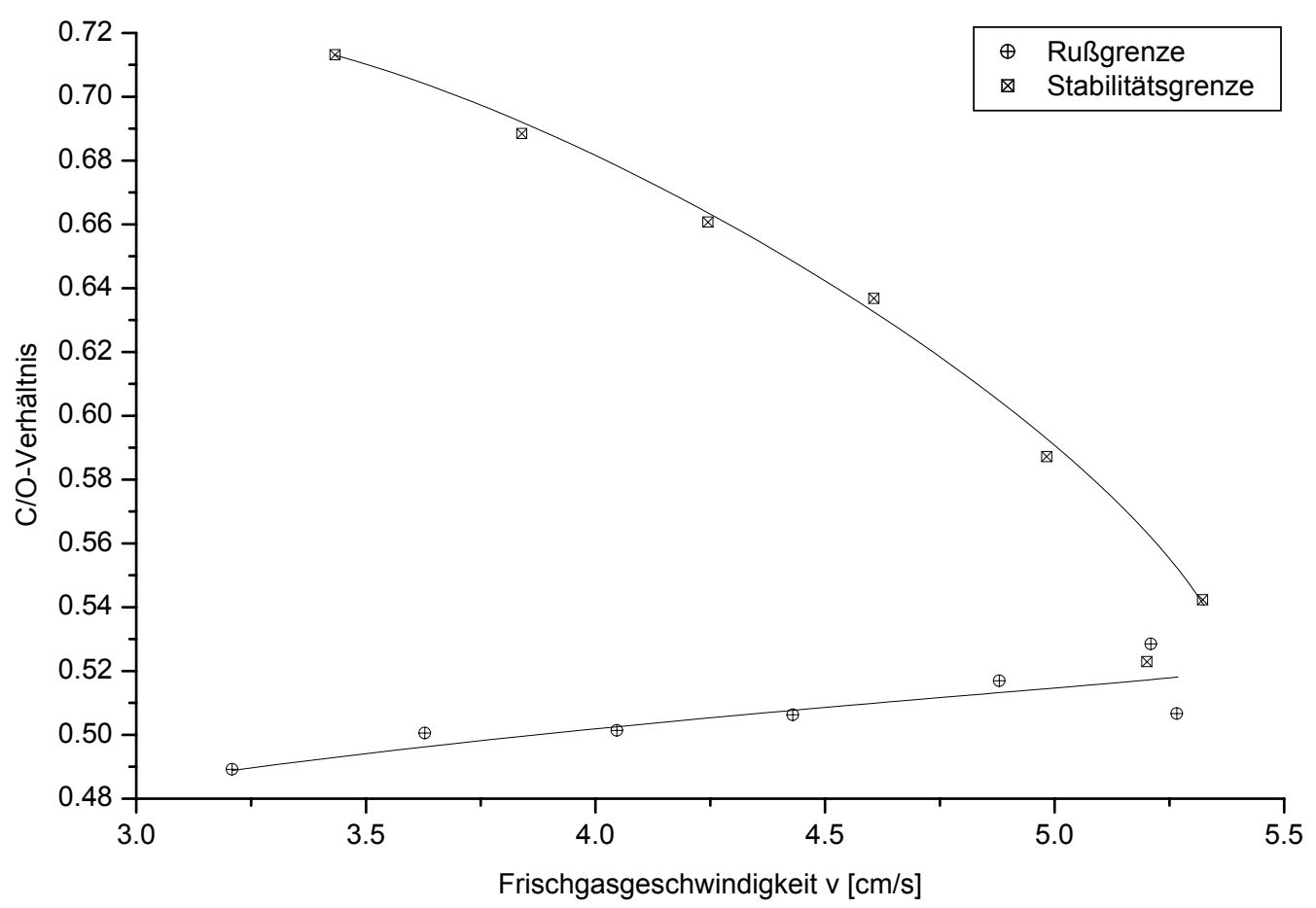

Abb. 7.20: Rußgrenze und Stabilitätsgrenze für Isooktan-Luft-Flammen

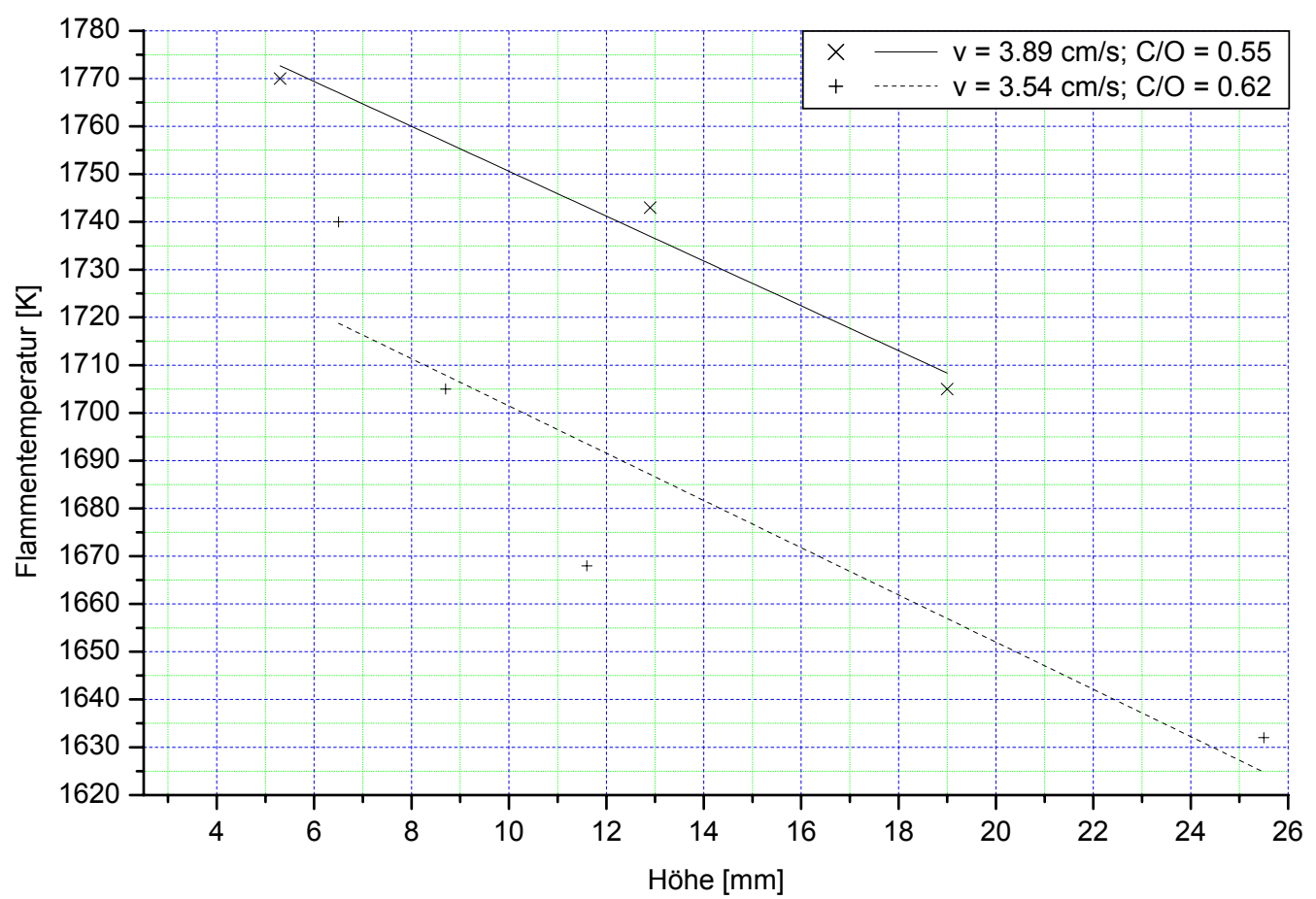

Abb. 7.21: Flammentemperaturen nach Kurlbaum von Isooktan-Luft-Flammen mit einer Frischgasgeschwindigkeit von etwa $3.7 \mathrm{~cm} / \mathrm{s}$ und unterschiedlichen C/O-Verhältnissen 


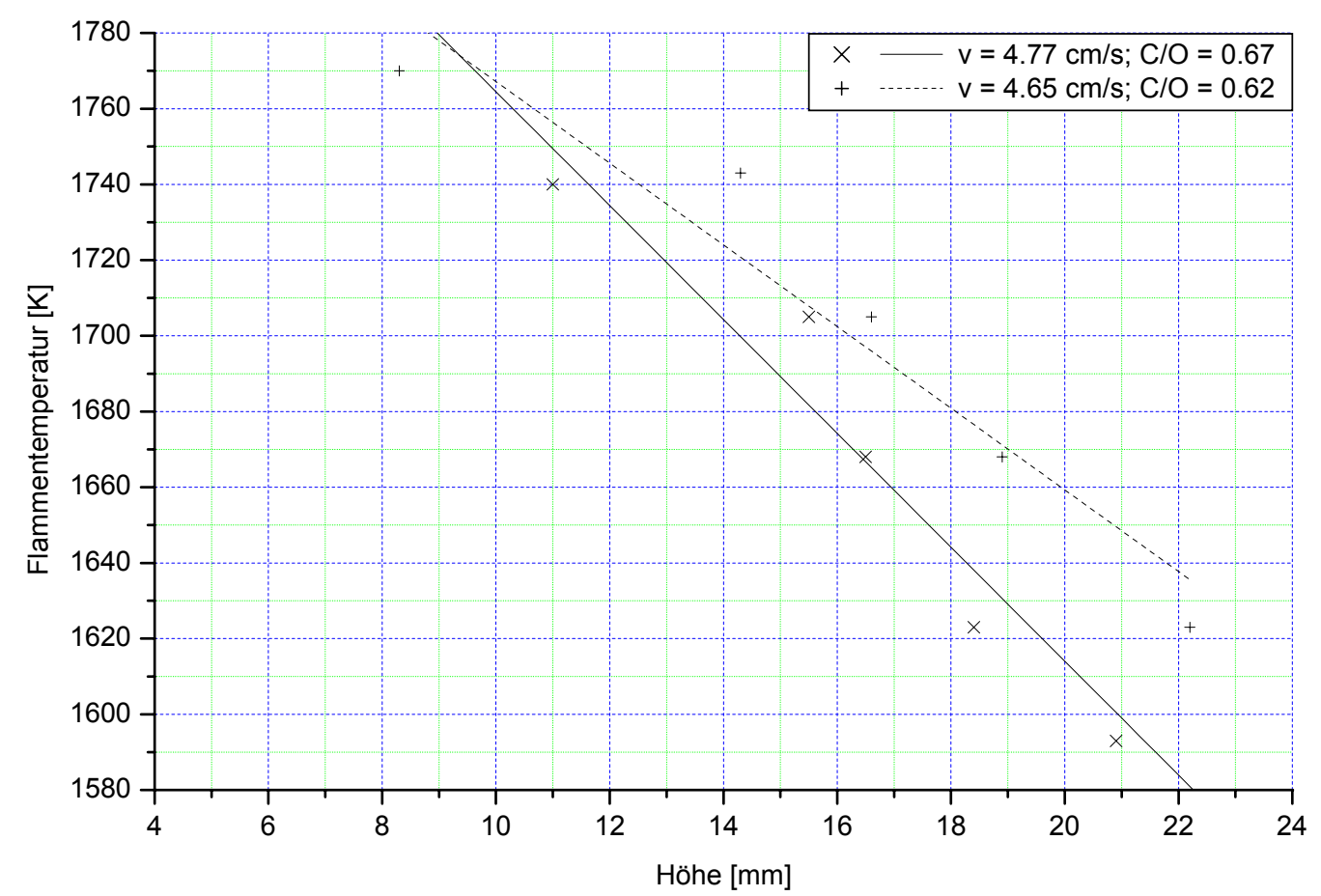

Abb. 7.22: Flammentemperaturen nach Kurlbaum von Isooktan-Luft-Flammen mit einer Frischgasgeschwindigkeit von etwa $4.7 \mathrm{~cm} / \mathrm{s}$ und unterschiedlichen $\mathrm{C} / \mathrm{O}-$ Verhältnissen

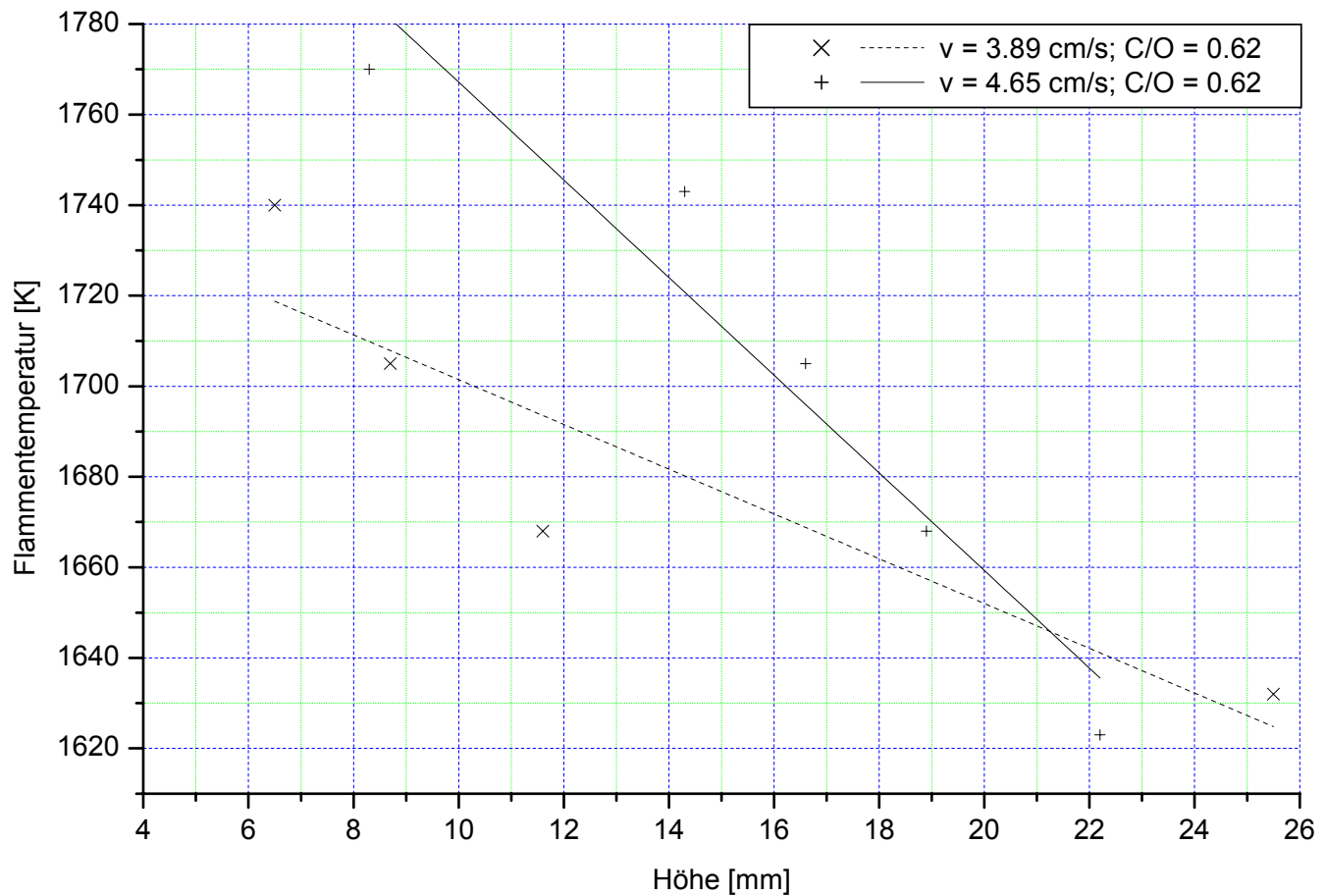

Abb. 7.23: Flammentemperaturen nach Kurlbaum von Isooktan-Luft-Flammen mit einem C/OVerhältnis von $\mathrm{C} / \mathrm{O}=0.62$ und unterschiedlichen Frischgasgeschwindigkeiten 


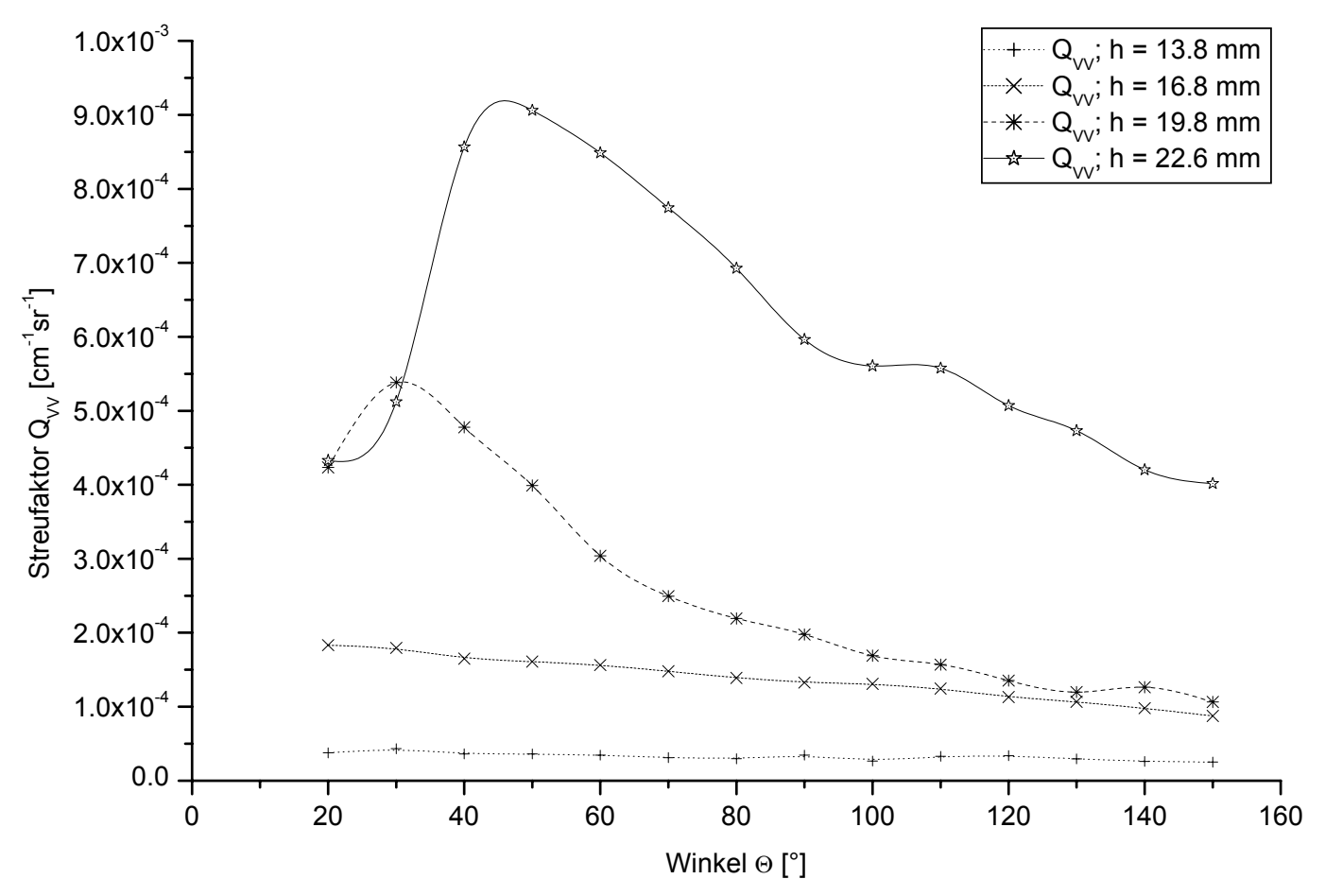

Abb. 7.24: Winkelabhängige Streufaktoren einer Isooktan-Luft-Flamme mit einem C/O-Verhältnis von $\mathrm{C} / \mathrm{O}=0.62$ und einer Frischgasgeschwindigkeit von $\mathrm{v}_{\mathrm{FG}}=3.5 \mathrm{~cm} / \mathrm{s}$ in mehreren Höhen $\mathrm{h}$ gemessen

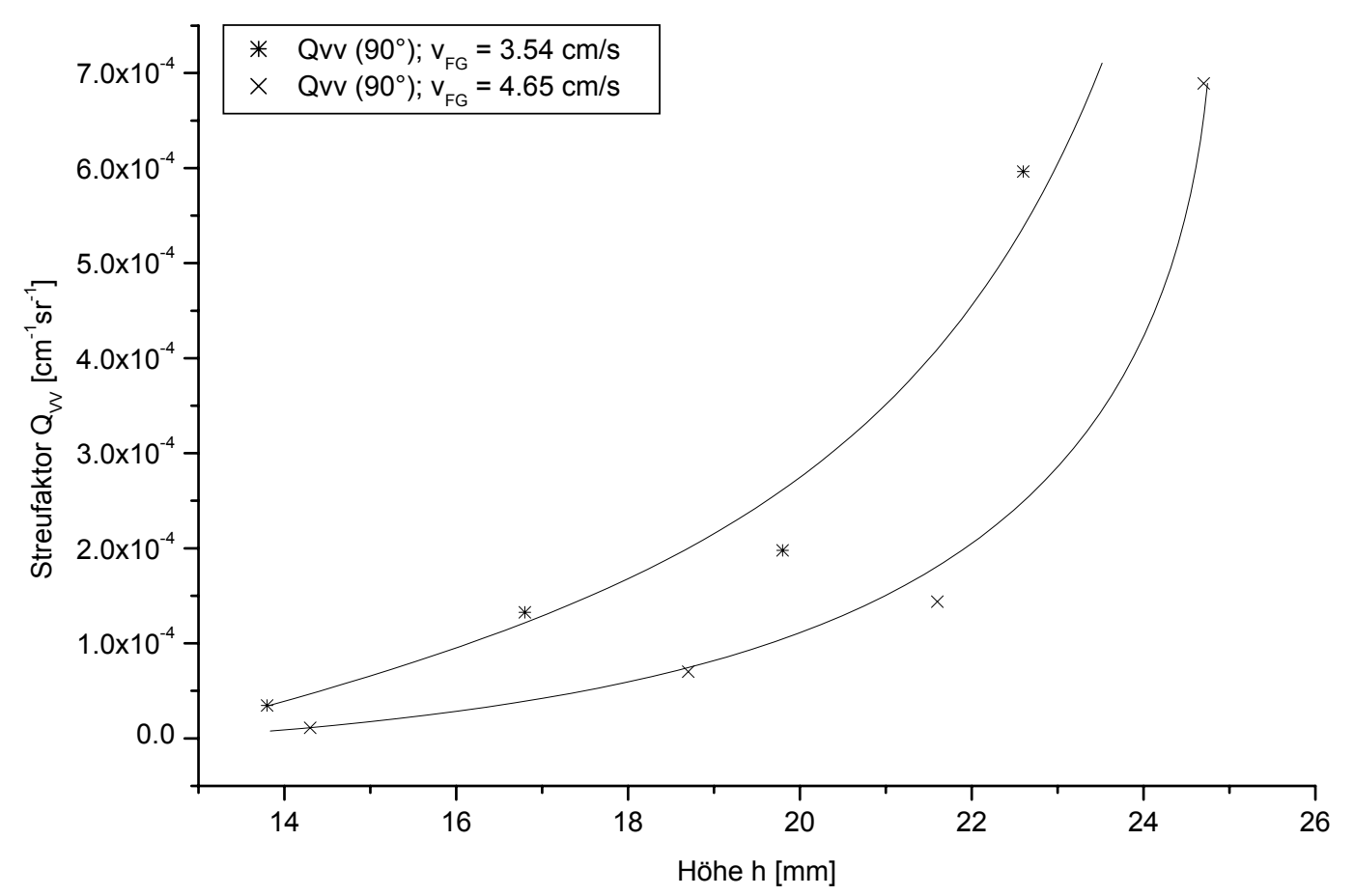

Abb. 7.25: Höhenabhängigkeit des Streufaktors von Isooktan-Luft-Flammen mit einem C/OVerhältnis von $\mathrm{C} / \mathrm{O}=0.62$ 


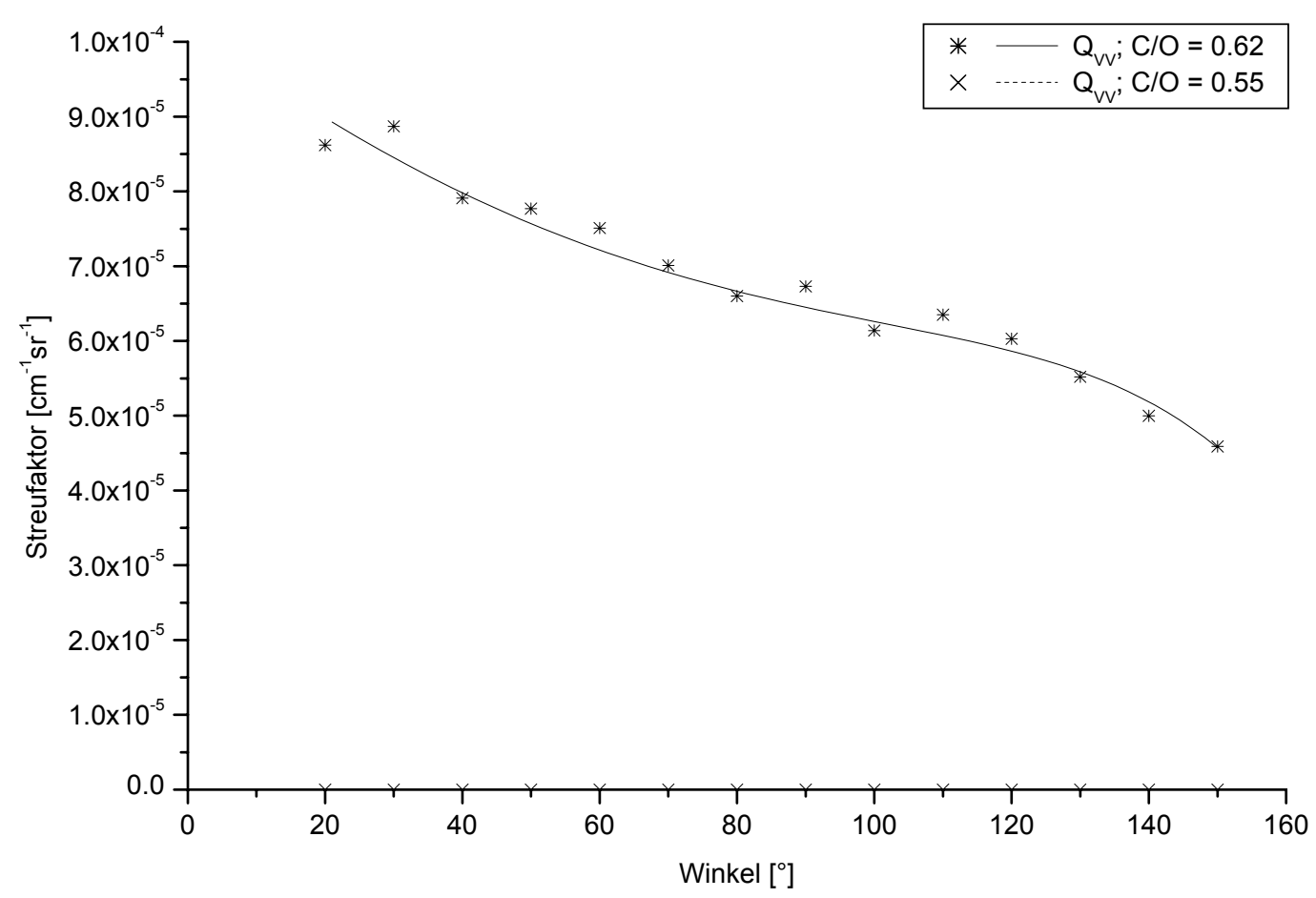

Abb. 7.26: Winkelabhängige Streufaktoren einer Isooktan-Luft-Flamme mit einer Frischgasgeschwindigkeit von $\mathrm{v}_{\mathrm{FG}}=3.5 \mathrm{~cm} / \mathrm{s}$ in einer Höhe $\mathrm{h}=14.9 \mathrm{~mm}$

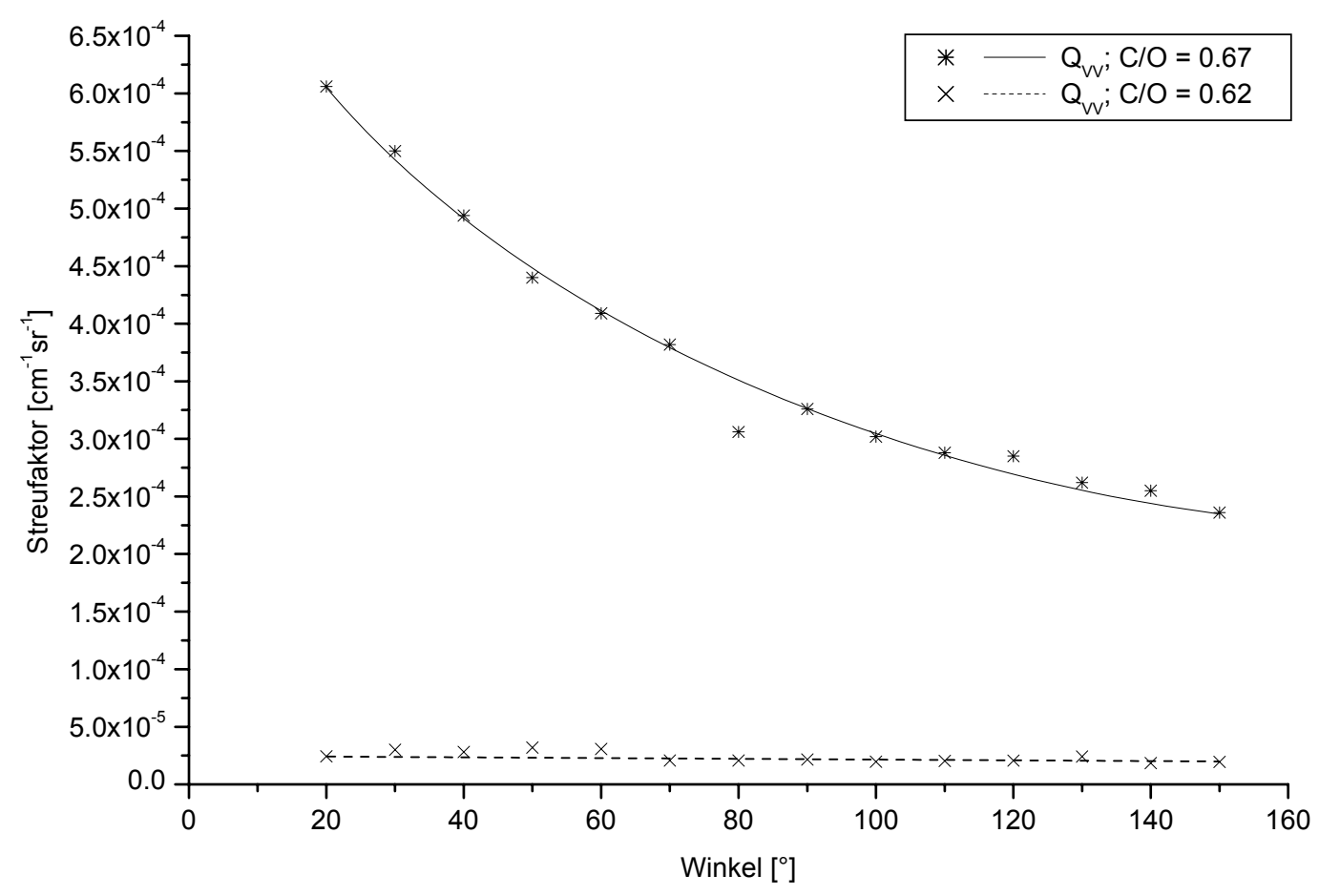

Abb. 7.27: Winkelabhängige Streufaktoren einer Isooktan-Luft-Flamme mit einer Frischgasgeschwindigkeit von $\mathrm{v}_{\mathrm{FG}} \sim 4.7 \mathrm{~cm} / \mathrm{s}$ in einer Höhe $\mathrm{h}=22.9 \mathrm{~mm}$ 


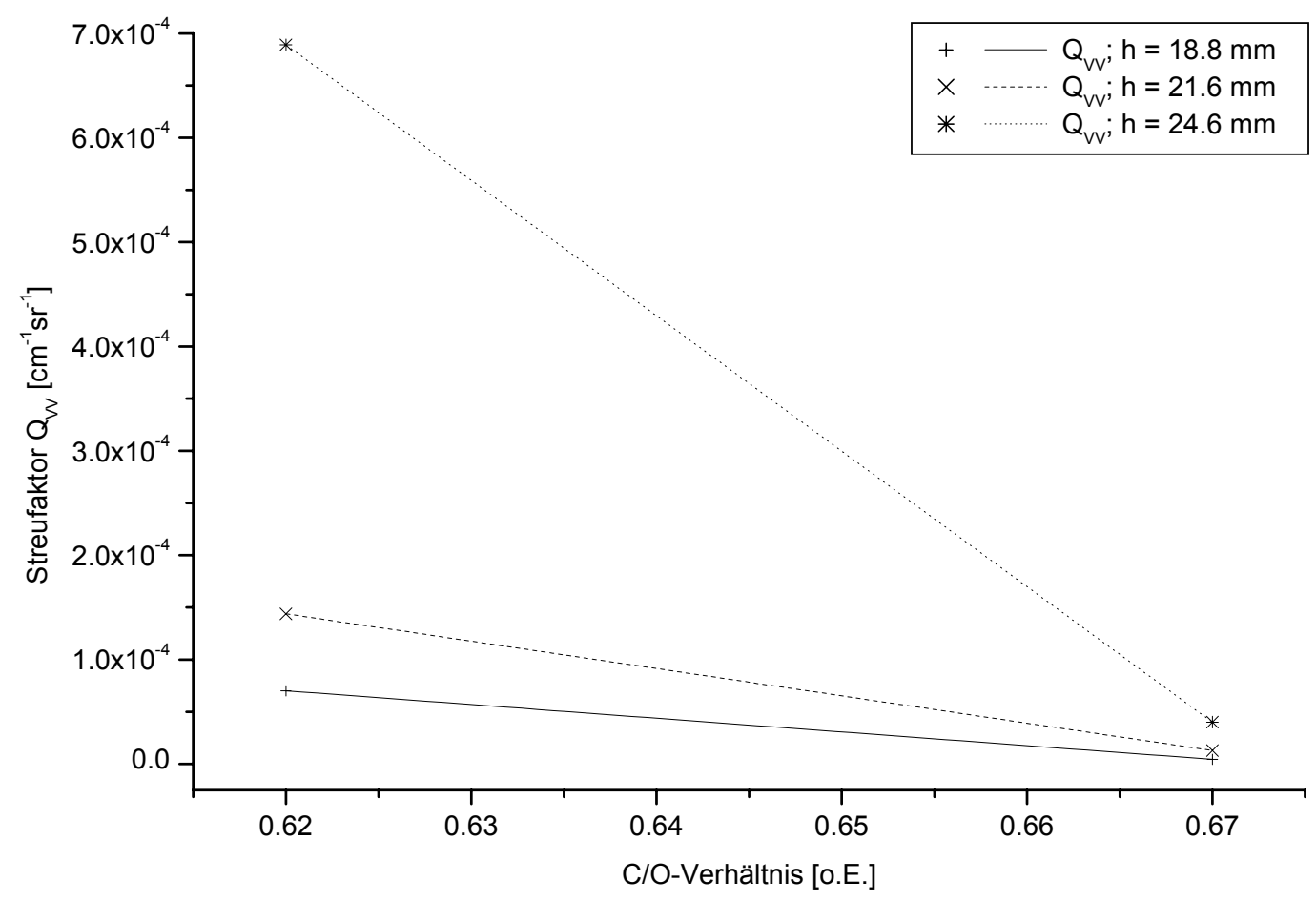

Abb. 7.28: Abhängigkeit des Streufakors vom C/O-Verhältnis von Isooktan-Luft-Flammen bei einer Frischgasgeschwindigkeit von $\mathrm{V}_{\mathrm{FG}} \sim 4.7 \mathrm{~cm} / \mathrm{s}$ in mehreren Höhen $\mathrm{h}$

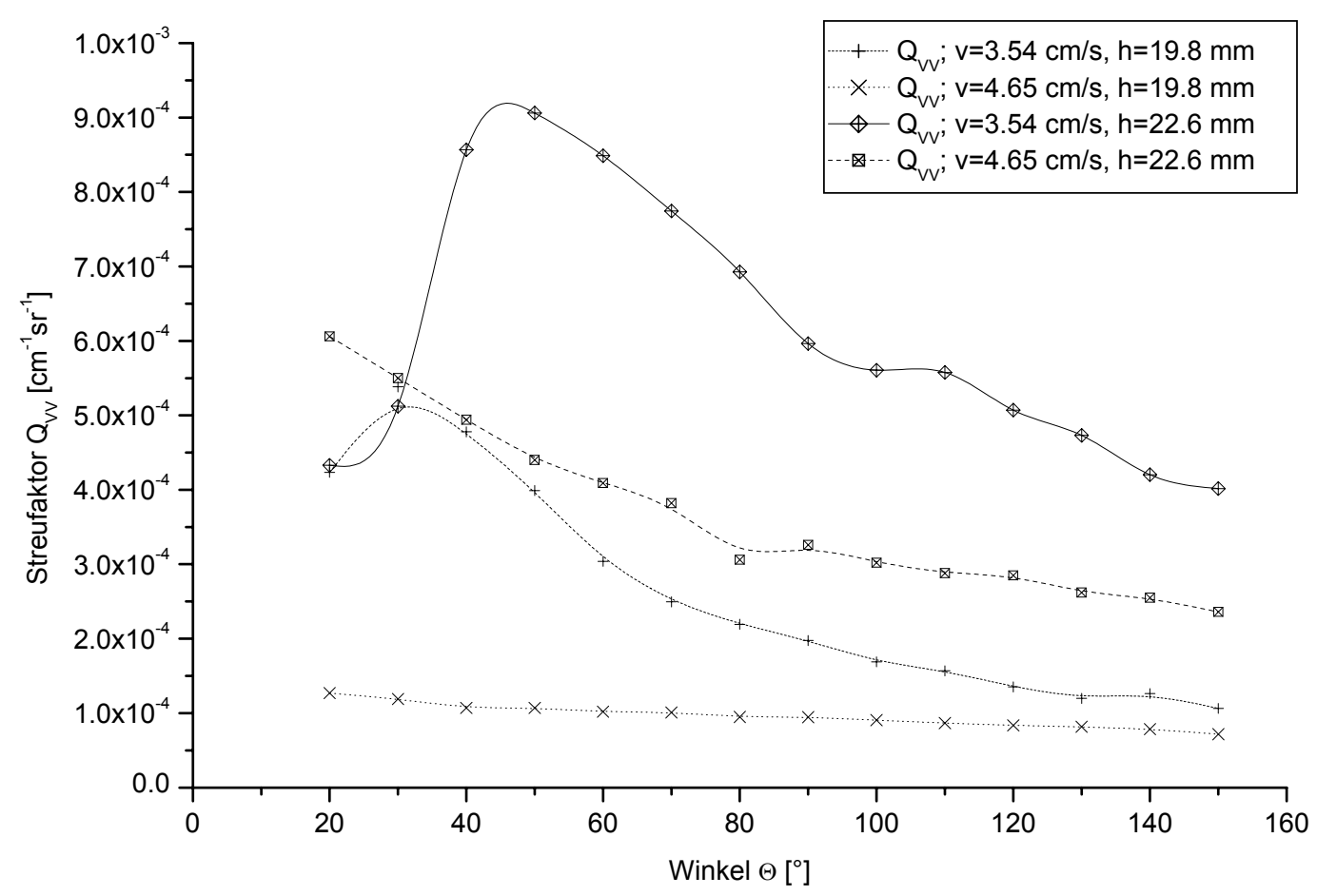

Abb. 7.29: Winkelabhängiger Verlauf des Streufaktors von Isooktan-Luft-Flammen bei einer Gemischzusammensetzung von $\mathrm{C} / \mathrm{O}=0.62$ 


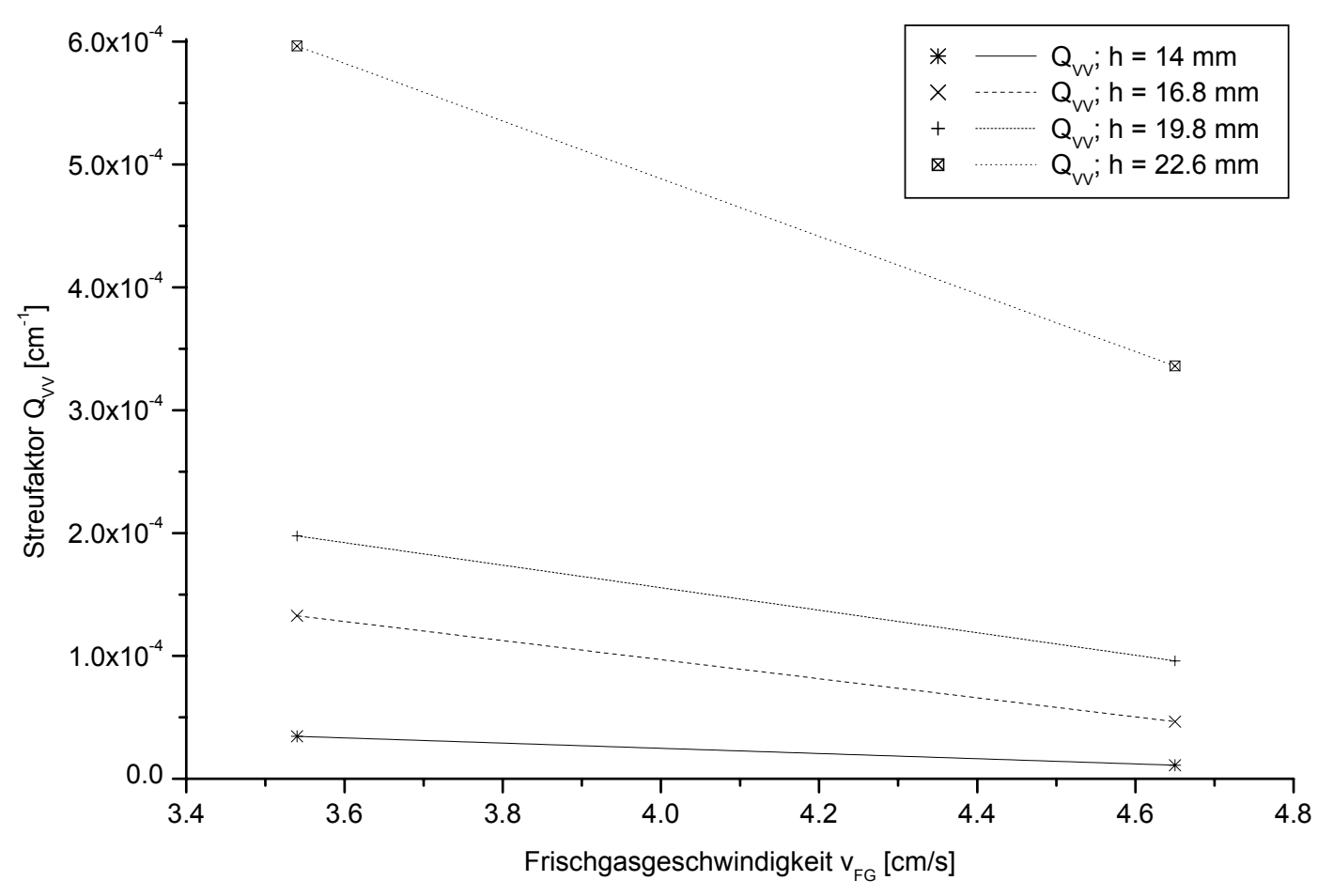

Abb. 7.30: Abhängigkeit des Streufaktors von der Frischgasgeschwindigkeit für Isooktan-LuftFlammen mit einem $\mathrm{C} / \mathrm{O}-$ Verhältnis von $\mathrm{C} / \mathrm{O}=0.62$ in mehreren Höhen $\mathrm{h}$

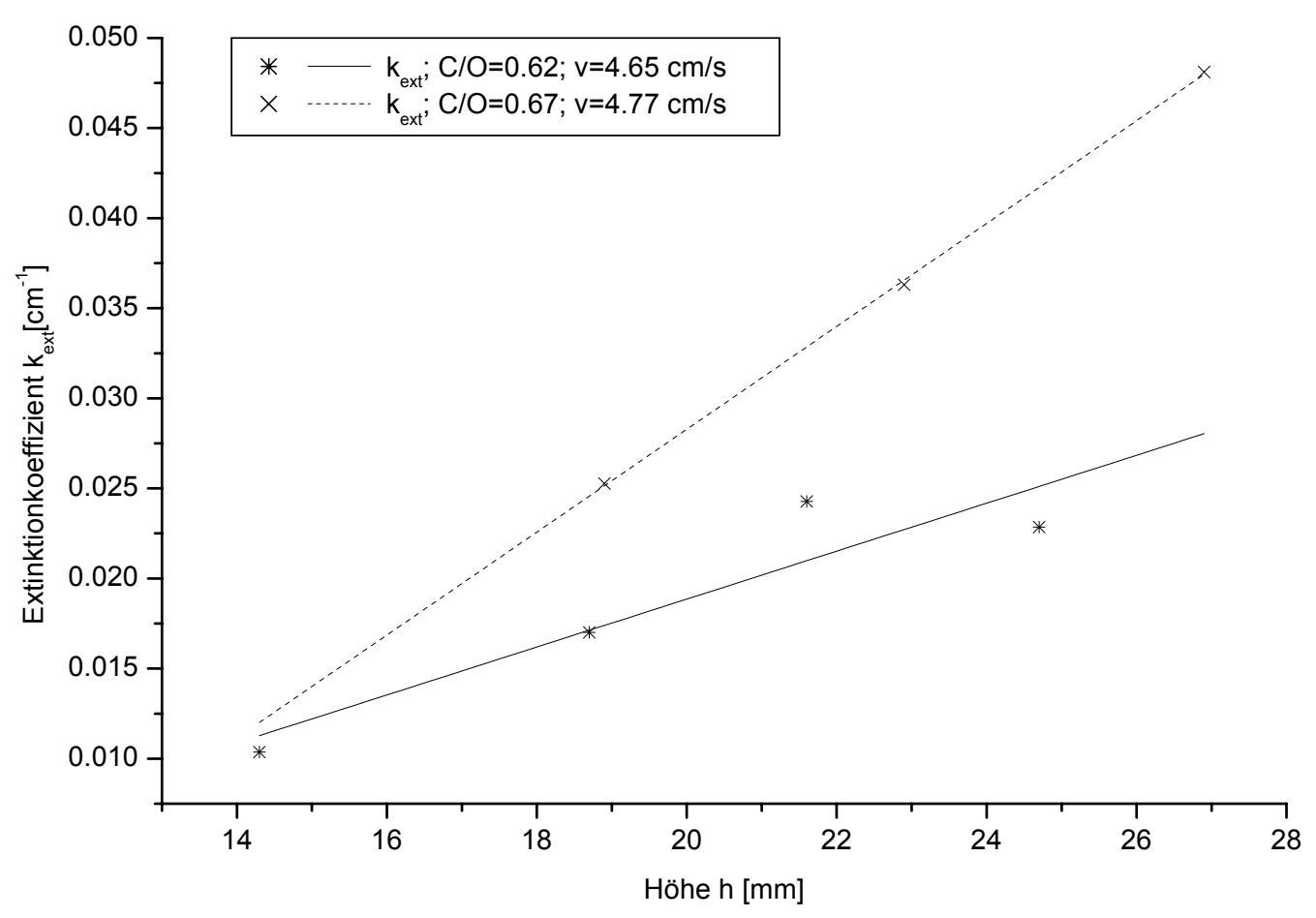

Abb. 7.31: Höhenabhängigkeit des Extinktionskoeffizienten von Isooktan-Luft-Flammen bei einer Frischgasgeschwindigkeit von $\mathrm{v}_{\mathrm{FG}} \sim 4.7 \mathrm{~cm} / \mathrm{s}$ 


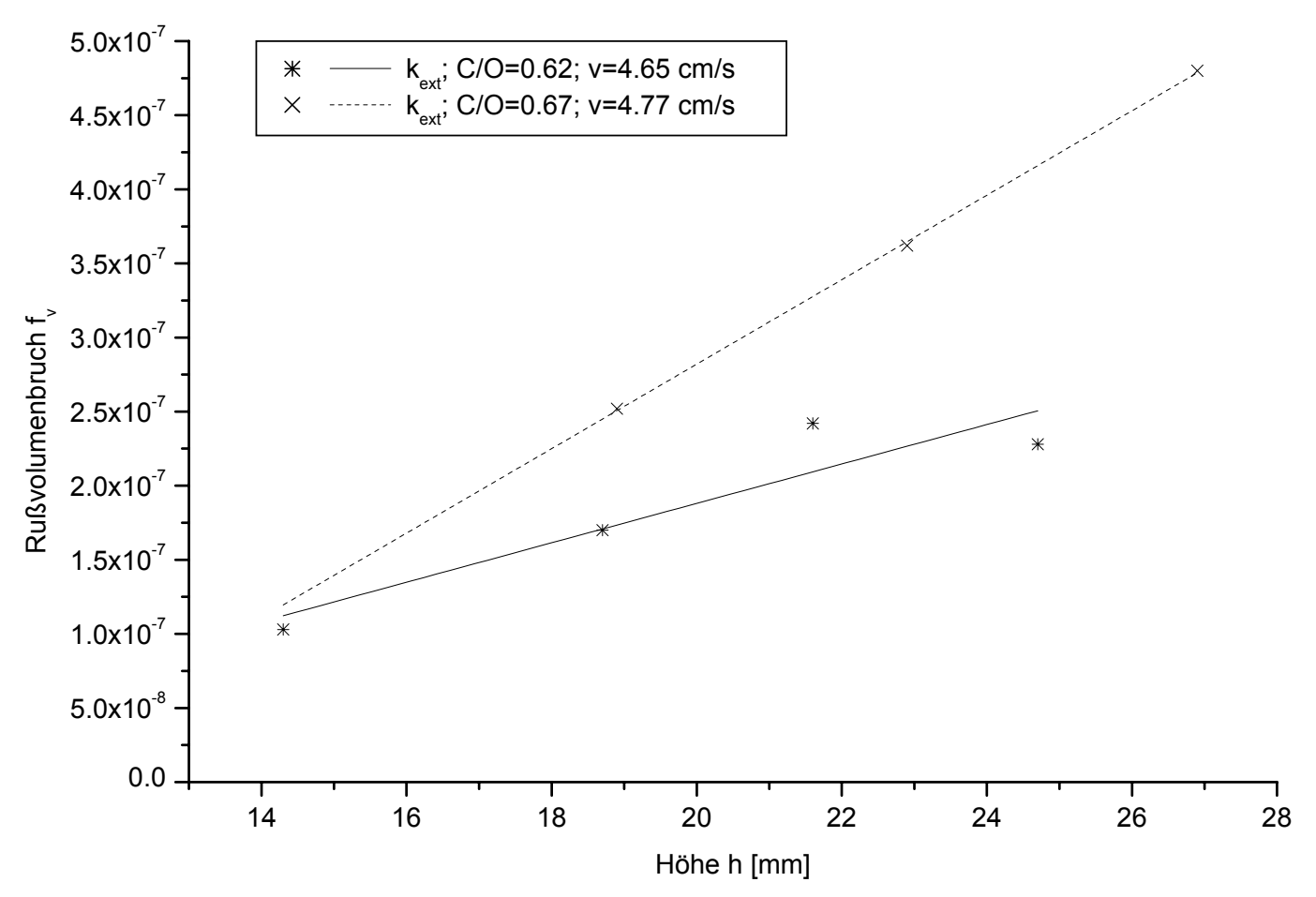

Abb. 7.32: Höhenabhängigkeit des Rußvolumenbruchs von Isooktan-Luft-Flammen bei einer Frischgasgeschwindigkeit von $\mathrm{v}_{\mathrm{FG}} \sim 4.7 \mathrm{~cm} / \mathrm{s}$

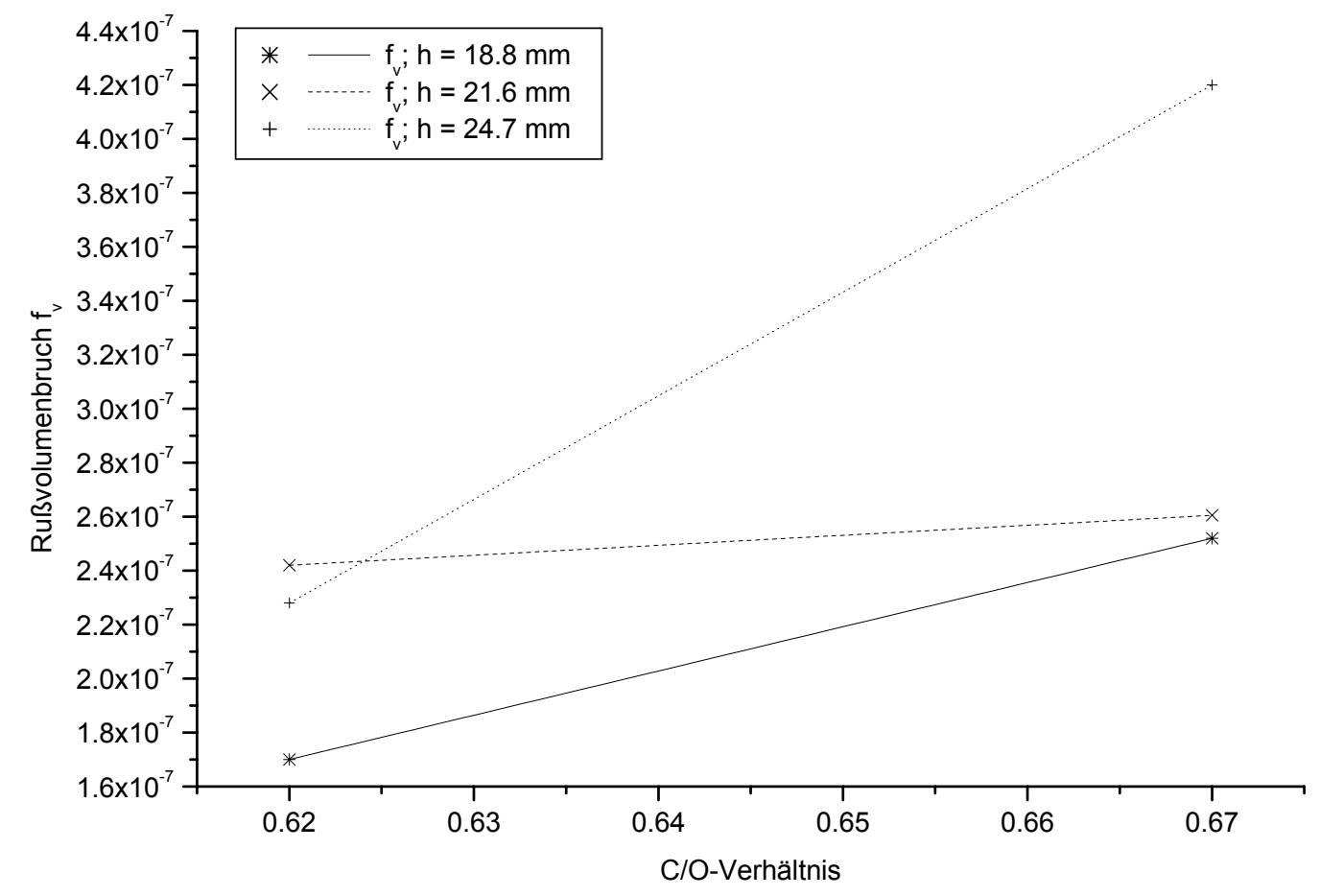

Abb. 7.33: Abhängigkeit des Rußvolumenbruchs vom C/O-Verhältnis bei Isooktan-Luft-Flammen mit einer Frischgasgeschwindigkeit von $\mathrm{v}_{\mathrm{FG}}=4.7 \mathrm{~cm} / \mathrm{s}$ in verschiedenen Höhen 


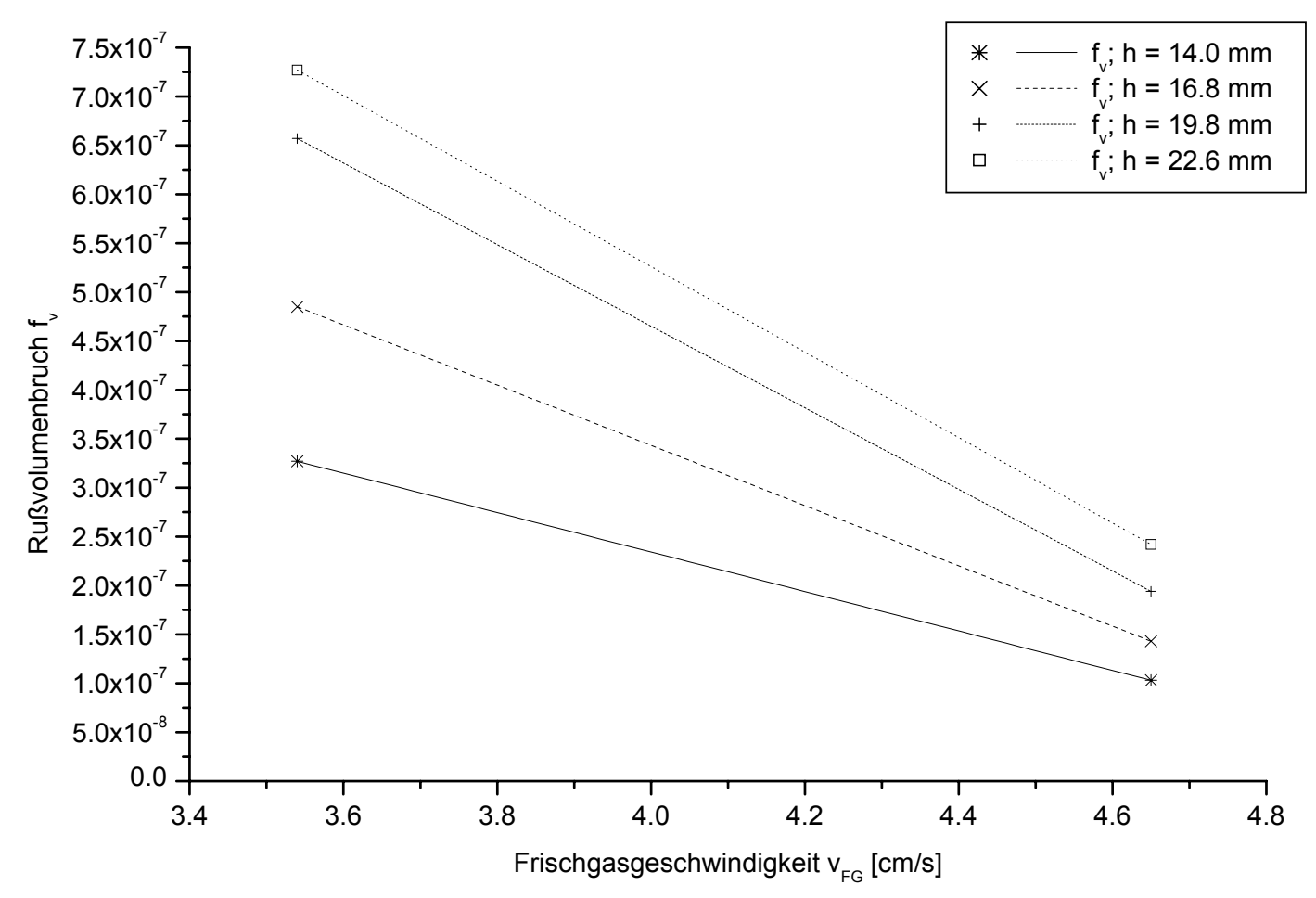

Abb. 7.34: Abhängigkeit des Rußvolumenbruchs von der Frischgasgeschwindigkeit bei Isooktan-LuftFlammen mit einem $\mathrm{C} / \mathrm{O}-$ Verhältnis von $\mathrm{C} / \mathrm{O}=0.62$ in verschiedenen Höhen

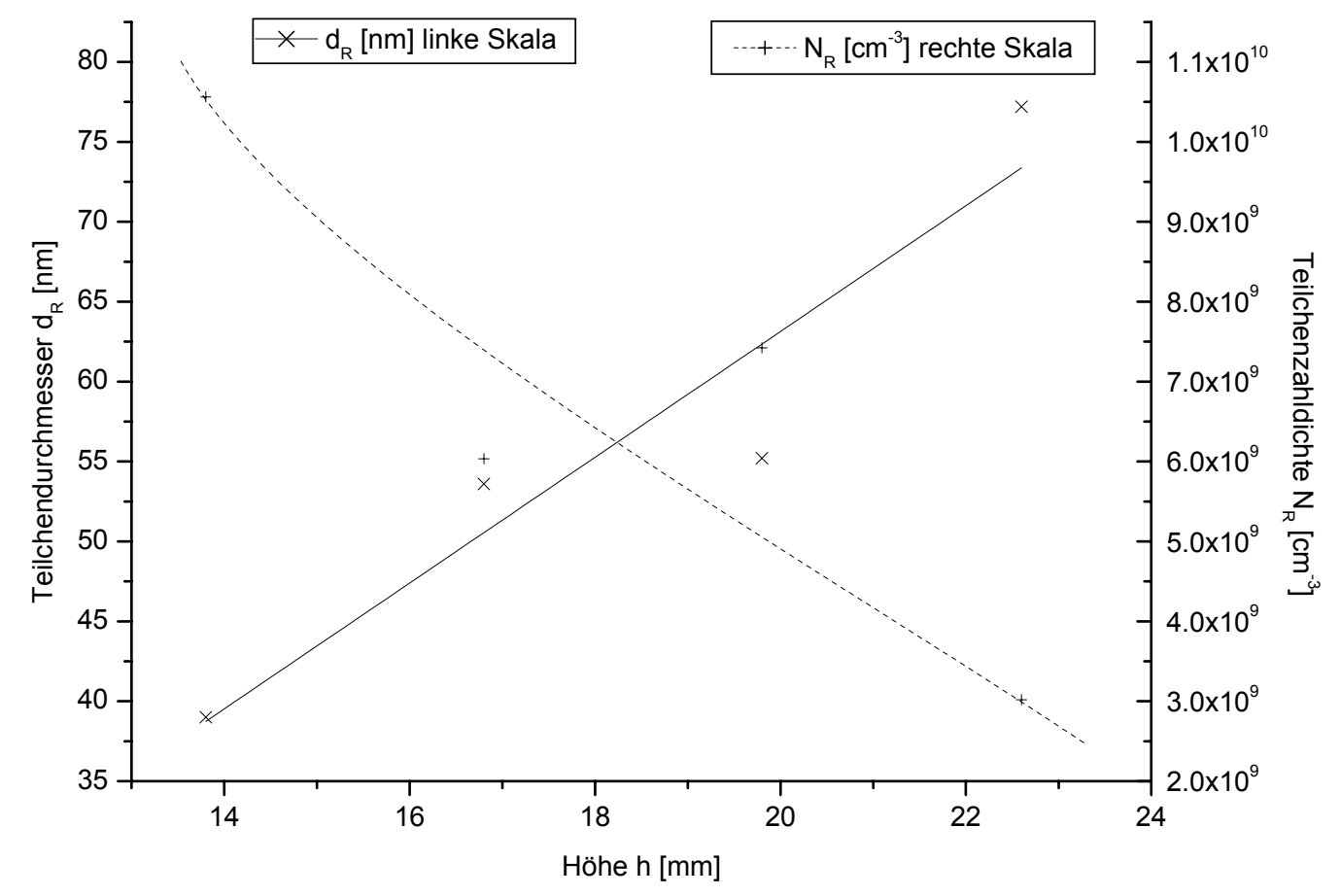

Abb. 7.35: Abhängigkeit des Teilchendurchmessers $d_{R}$ und der Teilchenzahldichte $\mathrm{N}_{R}$ von der Höhe in einer Isooktan-Luft-Flamme mit einem $\mathrm{C} / \mathrm{O}-$ Verhältnis von $\mathrm{C} / \mathrm{O}=0.62$ und einer Frischgasgeschwindigkeit $\mathrm{V}_{\mathrm{FG}}=3.5 \mathrm{~cm} / \mathrm{s}$ 


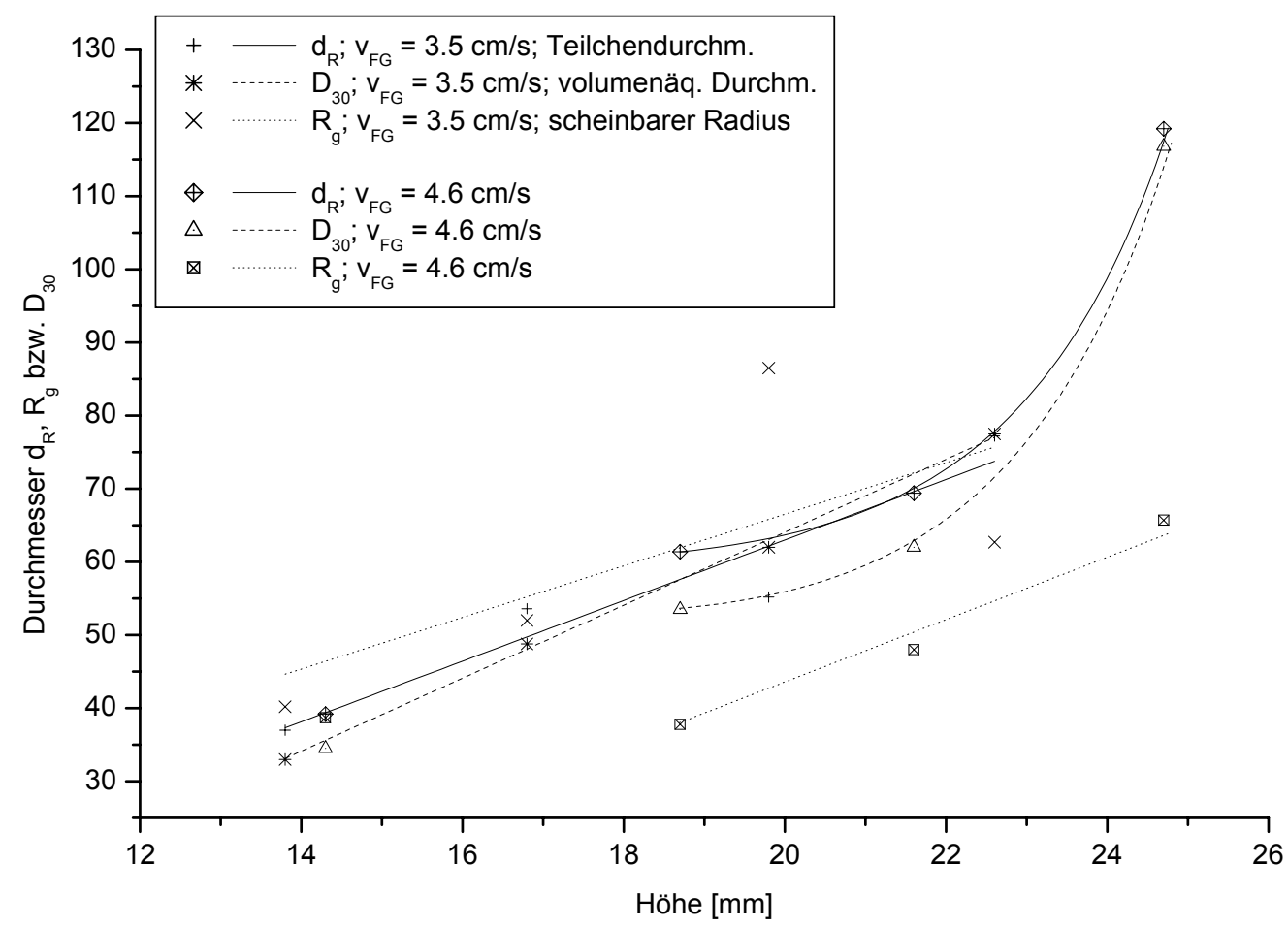

Abb. 7.36: Höhenabhängigkeit der verschiedenen Durchmesser der Rußteilchens in Isooktan-LuftFlammen mit einem $\mathrm{C} / \mathrm{O}-$ Verhältnis von $\mathrm{C} / \mathrm{O}=0.62$ und einer Frischgasgeschwindigkeit von $\mathrm{v}_{\mathrm{FG}}=3.5 \mathrm{~cm} / \mathrm{s}$ bZW. $\mathrm{v}_{\mathrm{FG}}=4.6 \mathrm{~cm} / \mathrm{s}$

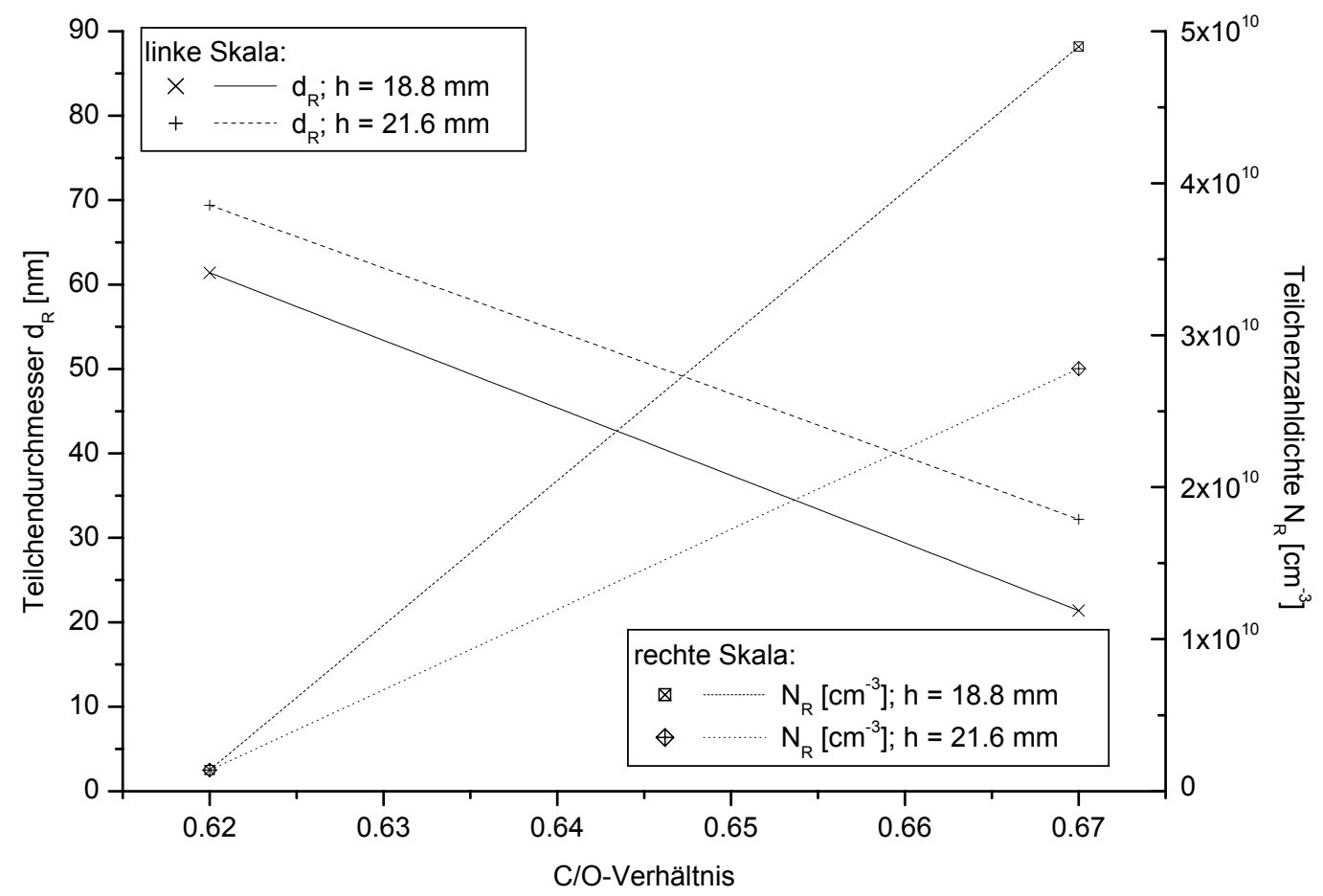

Abb. 7.37: Abhängigkeit des Teilchendurchmessers und der Teilchenzahldichte vom C/O-Verhältnis bei einer Isooktan-Luft-Flamme mit einer Frischgasgeschwindigkeit von $\mathrm{v}_{\mathrm{FG}}=4.7 \mathrm{~cm} / \mathrm{s}$ 


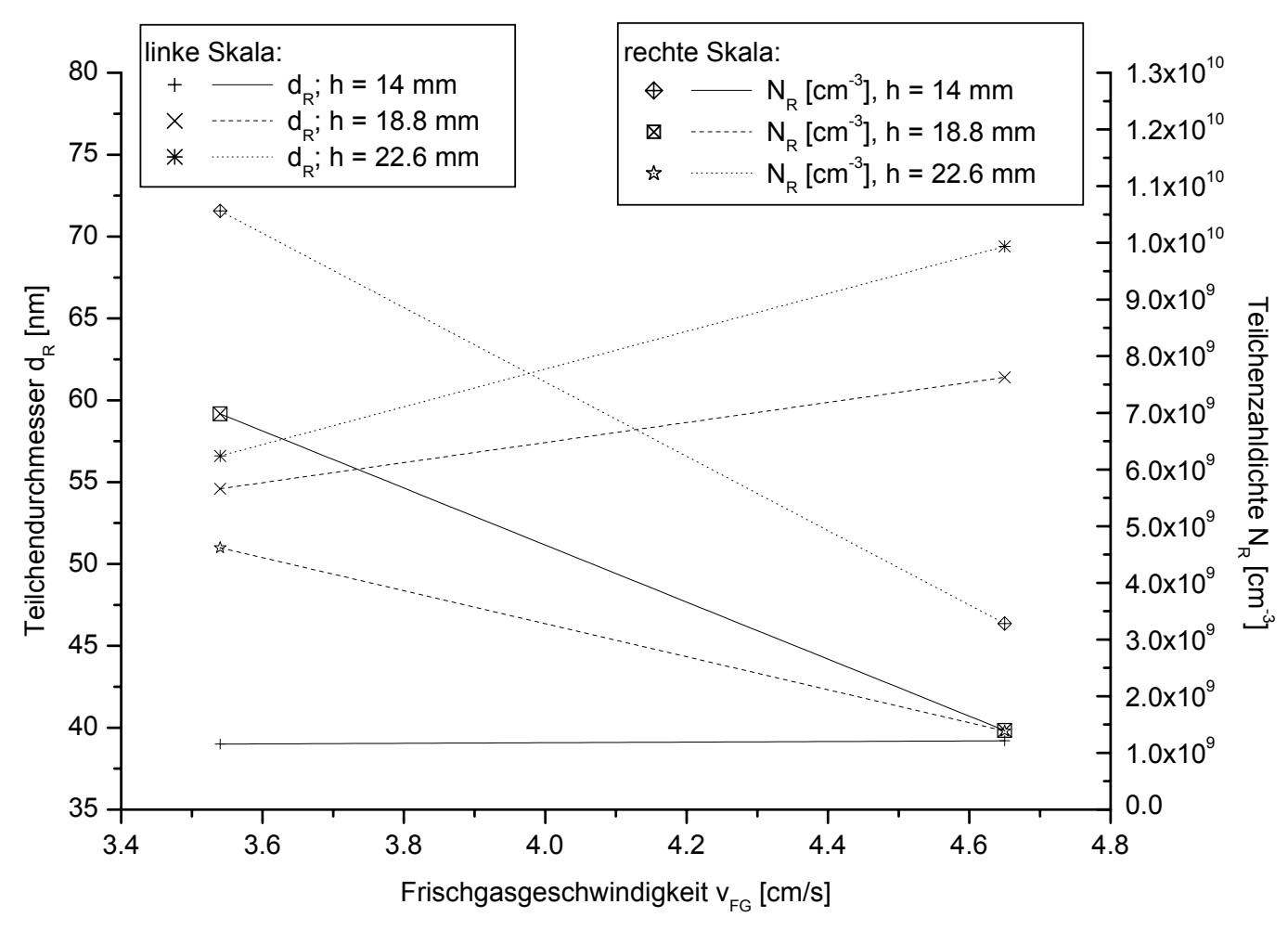

Abb. 7.38: Abhängigkeit der Teilchendurchmesser und Teilchenzahldichten von der Frischgasgeschwindigkeit für Isooktan-Luft-Flammen bei einem $\mathrm{C} / \mathrm{O}$-Verhältnis von $\mathrm{C} / \mathrm{O}=0.62$

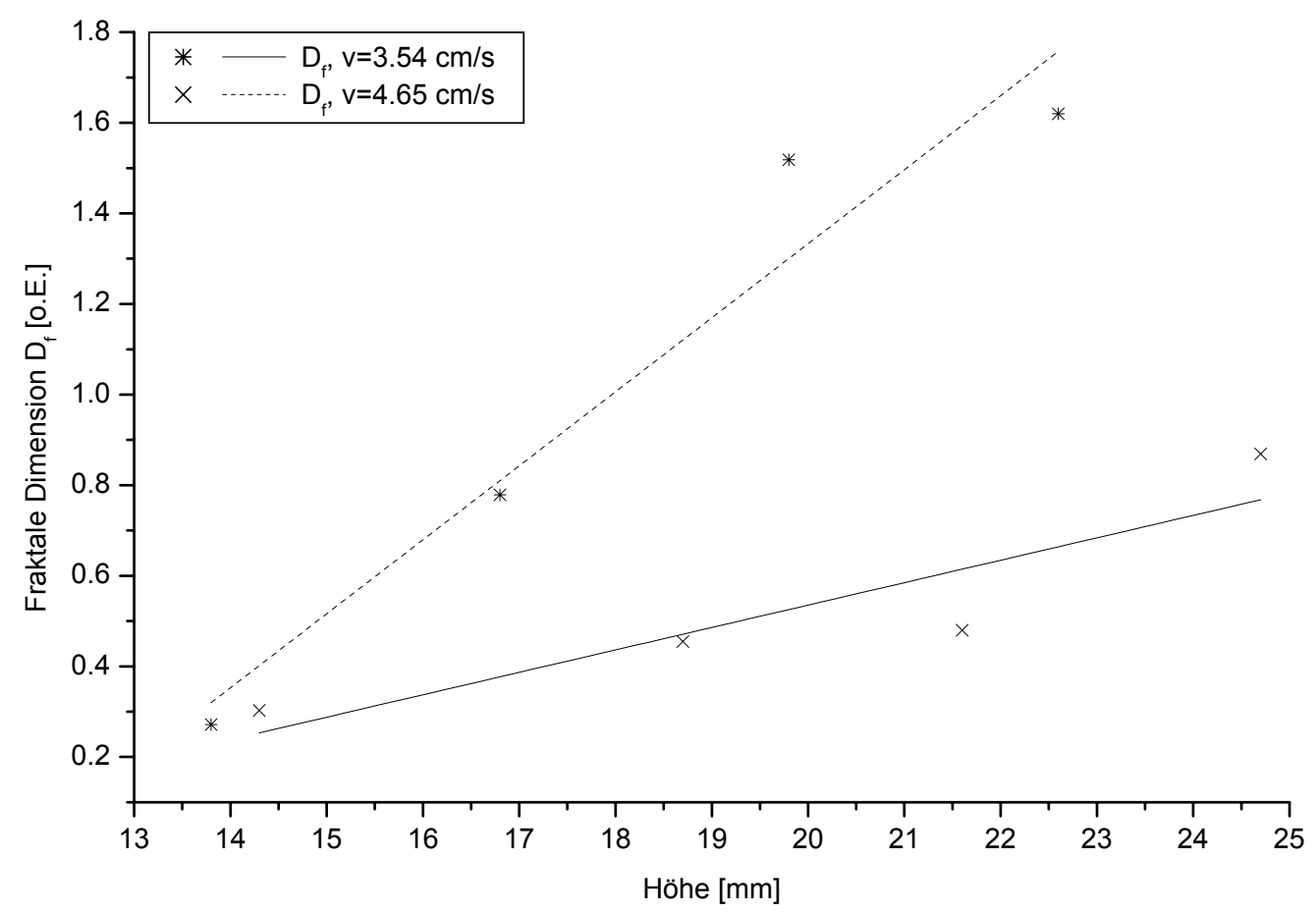

Abb. 7.39: Höhenabhängigkeit der fraktalen Dimension für Isooktan-Luft-Flammen bei einem C/OVerhältnis von $\mathrm{C} / \mathrm{O}=0.62$ und zwei verschiedenen Frischgasgeschwindigkeiten 


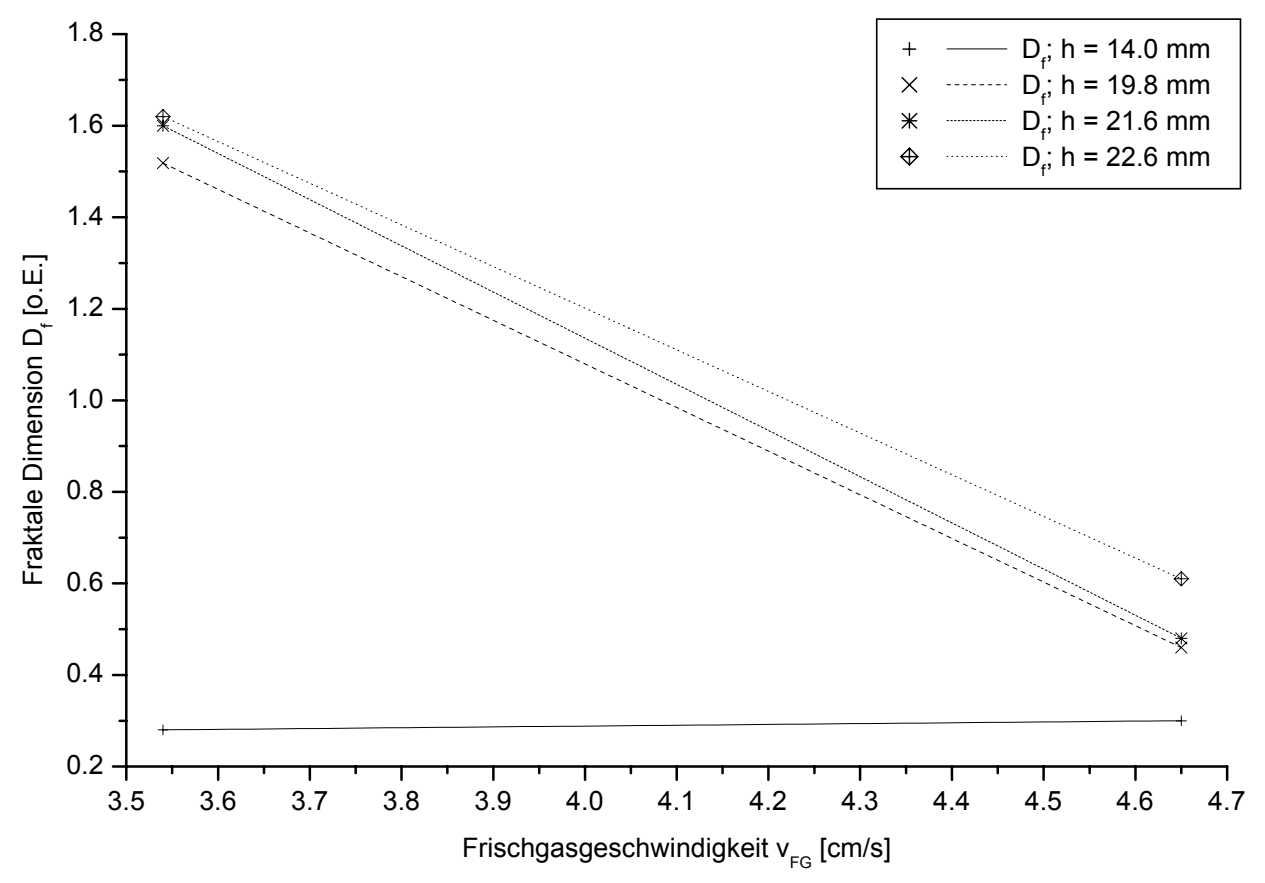

Abb. 7.40: Abhängigkeit der fraktalen Dimension von der Frischgasgeschwindigkeit bei einem C/OVerhältnis von $\mathrm{C} / \mathrm{O}=0.62$ in verschiedenen Höhen 


\section{Propen-Luft-Flammen}

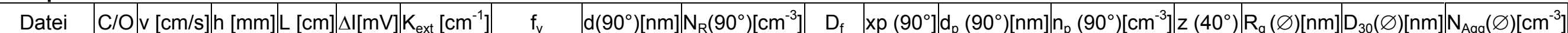

\begin{tabular}{|c|c|c|c|c|c|c|c|c|c|c|c|c|c|c|c|c|c|}
\hline prop001 & 0.71 & 6.2 & 16.7 & 5.23 & 24 & 0.0054 & $5.4 \mathrm{E}-08$ & 38.9 & 1.7E+09 & 0.64 & 0.092 & 14.3 & $3.5 \mathrm{E}+10$ & 1.980 & 63.5 & 38.0 & $1.9 \mathrm{E}+09$ \\
\hline prop002 & 0.71 & 5.2 & 16.7 & 5.21 & 35 & 0.0186 & $1.9 \mathrm{E}-07$ & 55.6 & $2.1 \mathrm{E}+09$ & 0.36 & 0.159 & 24.7 & $2.3 \mathrm{E}+10$ & 1.093 & 22.9 & 50.0 & $2.8 \mathrm{E}+09$ \\
\hline orop003 & 0.71 & 4.3 & 16.7 & 4.95 & 57.3 & 0.0390 & $3.9 \mathrm{E}-07$ & 60.1 & $3.4 \mathrm{E}+09$ & 1.52 & 0.100 & 15.5 & $2.0 \mathrm{E}+11$ & 2.222 & 68.7 & 58.8 & $3.6 \mathrm{E}+$ \\
\hline orop000 & 0.71 & 3.7 & 16.7 & 4.63 & 54.4 & 0.0334 & 4.0E-07 & 68.5 & $2.4 \mathrm{E}+09$ & 0.71 & 0.186 & 28.9 & $3.2 E+10$ & 1.771 & 58.1 & 65.0 & $2.8 \mathrm{E}+\mathrm{C}$ \\
\hline rop005 & 0.69 & 8.4 & 16.7 & 5.11 & 7.6 & 0.0045 & $4.5 \mathrm{E}-08$ & 5.6 & $4.6 \mathrm{E}+11 \mathrm{n}$ & b. & & & & $0.781 \mathrm{n}$ & & 4.8 & $4.8 \mathrm{E}+\mathrm{C}$ \\
\hline rop005a & 0.67 & 8.3 & 16.7 & 4.95 & 1.9 & 0.0017 & $1.7 \mathrm{E}-08$ & 11.0 & $2.4 \mathrm{E}+10$ & 0.12 & 0.032 & 4.9 & $2.7 E+11$ & 1.019 & 10.5 & 9.6 & 3.7E+ \\
\hline rop004 & 0.72 & 7.3 & 16.7 & 5.35 & 3.2 & 0.0028 & $2.7 \mathrm{E}-08$ & 10.7 & $4.3 E+10 n$ & b. & & & & $0.720 n$ & & 8.0 & $1.0 \mathrm{E}+$ \\
\hline prop004a & 0.70 & 7.4 & 16.7 & 5.27 & 3.5 & 0.0020 & 2.0E-08 & 31.0 & $1.3 \mathrm{E}+09$ & 0.65 & 0.068 & 10.6 & $3.2 E+10$ & 3.932 & 89.9 & 32.7 & $1.1 \mathrm{E}+$ \\
\hline prop004b & 0.70 & 7.4 & 16.7 & 5.27 & 3.5 & 0.0020 & 2.0E-08 & 12.5 & $2.0 \mathrm{E}+10$ & 0.41 & 0.028 & 4.3 & $4.7 E+11$ & 1.464 & 47.4 & 11.6 & $2.5 \mathrm{E}+$ \\
\hline rop006 & 0.65 & 6.2 & 16.7 & 4.63 & 0.9 & 0.0007 & $6.6 \mathrm{E}-09$ & 18.8 & $1.9 \mathrm{E}+09$ & 0.40 & 0.045 & 7.0 & $3.7 E+10$ & 1.672 & 55.1 & 17.0 & $2.5 \mathrm{E}+$ \\
\hline rop007 & 0.75 & 6.3 & 16.7 & 5.18 & 21 & 0.0155 & $1.5 \mathrm{E}-07$ & 49.5 & $2.4 \mathrm{E}+09$ & 0.50 & 0.133 & 20.6 & $3.4 \mathrm{E}+10$ & 1.554 & 51.0 & 45.0 & $3.2 \mathrm{E}+$ \\
\hline op008 & 0.71 & 4.3 & 16.7 & 5.07 & 42.2 & 0.0256 & 2.6E-07 & 76.2 & $1.1 \mathrm{E}+09$ & 0.55 & 0.221 & 34.3 & $1.2 \mathrm{E}+10$ & 1.850 & 60.3 & 72.3 & 1.3E+ \\
\hline op008a & 0.71 & 4.3 & 24.4 & 4.91 & 57.4 & 0.0400 & 4.0E-07 & 72.0 & $2.0 \mathrm{E}+09$ & 1.62 & 0.136 & 21.1 & $8.1 \mathrm{E}+10$ & 5.482 & 100.2 & 97.0 & $8.2 \mathrm{E}+$ \\
\hline рp008a & 0.71 & 4.3 & 24.4 & 4.91 & 57.4 & 0.0400 & 4.0E-07 & 72.9 & $2.0 \mathrm{E}+09$ & 1.38 & 0.160 & 24.9 & $5.0 \mathrm{E}+10$ & 4.099 & 91.3 & 90.0 & $1.0 \mathrm{E}+$ \\
\hline pp009 & 0.71 & 4.3 & 16.7 & 5.08 & 27.33 & 0.0209 & 2.1E-07 & 70.2 & $1.2 \mathrm{E}+09$ & 0.78 & 0.189 & 29.3 & 1.6E+10 & 1.785 & 58.5 & 65.2 & 1.4E+ \\
\hline p009a & 0.71 & 4.3 & 11.5 & 5.22 & 17.33 & 0.0138 & $1.4 \mathrm{E}-07$ & 47.0 & $2.5 E+09$ & 0.21 & 0.139 & 21.6 & $2.6 \mathrm{E}+10$ & 1.154 & 29.1 & 40.2 & $4.0 \mathrm{E}+$ \\
\hline p010_1 & 0.71 & 5.2 & 16.7 & 5.01 & 47.5 & 0.0249 & $2.5 \mathrm{E}-07$ & 60.8 & $2.1 \mathrm{E}+09$ & 0.90 & 0.148 & 23.0 & $3.9 E+10$ & 1.982 & 63.6 & 59.4 & $2.3 \mathrm{E}+$ \\
\hline p010_2 & 0.71 & 5.2 & 16.7 & 5.01 & 47.5 & 0.0249 & $2.5 \mathrm{E}-07$ & 56.9 & $2.6 \mathrm{E}+09$ & 1.00 & 0.128 & 19.8 & $6.1 \mathrm{E}+10$ & 2.423 & 72.3 & 57.4 & $2.5 \mathrm{E}+$ \\
\hline p010_3 & 0.71 & 5.2 & 16.7 & 5.01 & 47.5 & 0.0249 & $2.5 \mathrm{E}-07$ & 52.1 & $3.4 \mathrm{E}+09$ & 0.75 & 0.128 & 19.9 & $6.1 \mathrm{E}+10$ & 1.985 & 63.6 & 50.0 & $3.8 \mathrm{E}+$ \\
\hline op010a & 0.71 & 5.2 & 24.3 & 4.95 & 52 & 0.0267 & 2.7E-07 & 55.8 & $2.9 \mathrm{E}+09$ & 1.05 & 0.121 & 18.8 & $7.7 \mathrm{E}+10$ & 2.971 & 80.2 & 61.4 & $2.2 \mathrm{E}+$ \\
\hline op011_1 & 0.71 & 6.2 & 16.7 & 5.69 & 27.6 & 0.0110 & $1.1 \mathrm{E}-07$ & 51.4 & $1.5 \mathrm{E}+09$ & 0.63 & 0.132 & 20.5 & $2.4 \mathrm{E}+10$ & 1.781 & 58.4 & 48.7 & $1.8 \mathrm{E}+$ \\
\hline rop011_2 & 0.71 & 6.2 & 16.7 & 5.69 & 27.6 & 0.0110 & 1.1E-07 & 35.6 & $4.6 \mathrm{E}+09$ & 0.67 & 0.081 & 12.6 & $1.1 \mathrm{E}+11$ & 1.703 & 56.1 & 33.6 & $5.5 \mathrm{E}+$ \\
\hline rop012 & 0.70 & 7.4 & 16.7 & 5.85 & 13.5 & 0.0048 & $4.8 \mathrm{E}-08$ & 25.0 & $5.9 \mathrm{E}+09$ & 0.23 & 0.070 & 10.8 & $7.3 E+10$ & 1.164 & 30.0 & 22.0 & 8.7E+ \\
\hline rop013 & 0.71 & 4.8 & 16.7 & 5.05 & 34.8 & 0.0229 & 2.3E-07 & 59.9 & $2.0 \mathrm{E}+09$ & 0.90 & 0.145 & 22.5 & $3.8 E+10$ & 2.158 & 67.4 & 59.4 & $2.1 \mathrm{E}+$ \\
\hline op014_1 & 0.71 & 5.7 & 16.7 & 5.14 & 9.075 & 0.0066 & $6.5 \mathrm{E}-08$ & 36.7 & $2.5 E+09$ & 0.53 & 0.091 & 14.1 & $4.4 \mathrm{E}+10$ & 1.591 & 52.4 & 33.4 & $3.3 \mathrm{E}+$ \\
\hline p014 & 0.71 & 5.7 & 16.7 & 5.14 & 9.075 & 0.0066 & $6.5 \mathrm{E}-08$ & 34.9 & $2.9 \mathrm{E}+09$ & 0.53 & 0.086 & 13.3 & $5.3 \mathrm{E}+10$ & 1.431 & 46.0 & 30.8 & 4.2E \\
\hline
\end{tabular}

Tab. 7.4: $\quad$ Meßergebnisse der Propen-Luft-Flammen 


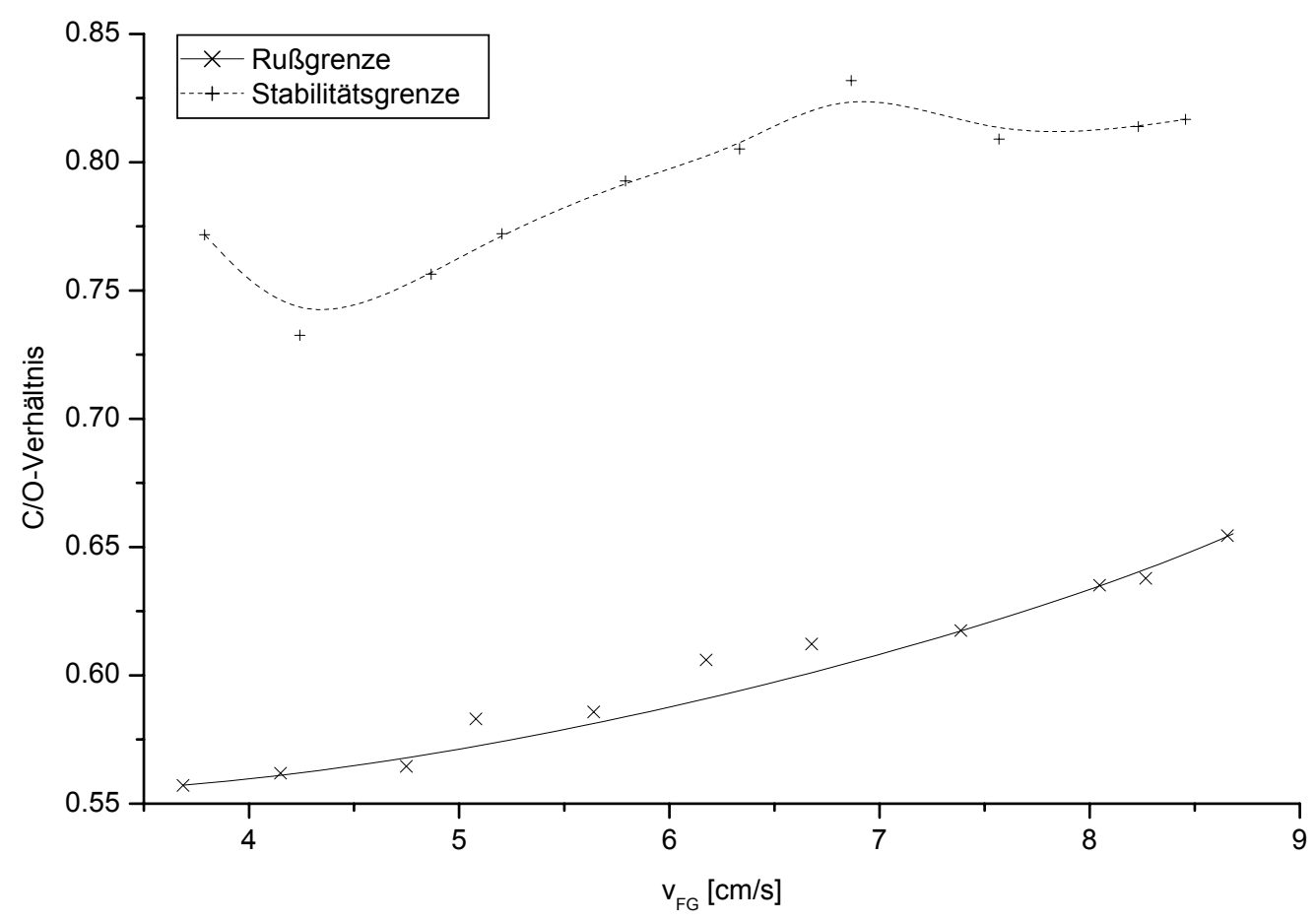

Abb. 7.41: Rußgrenze und Stabilitätsgrenze von Propen-Luft-Flammen

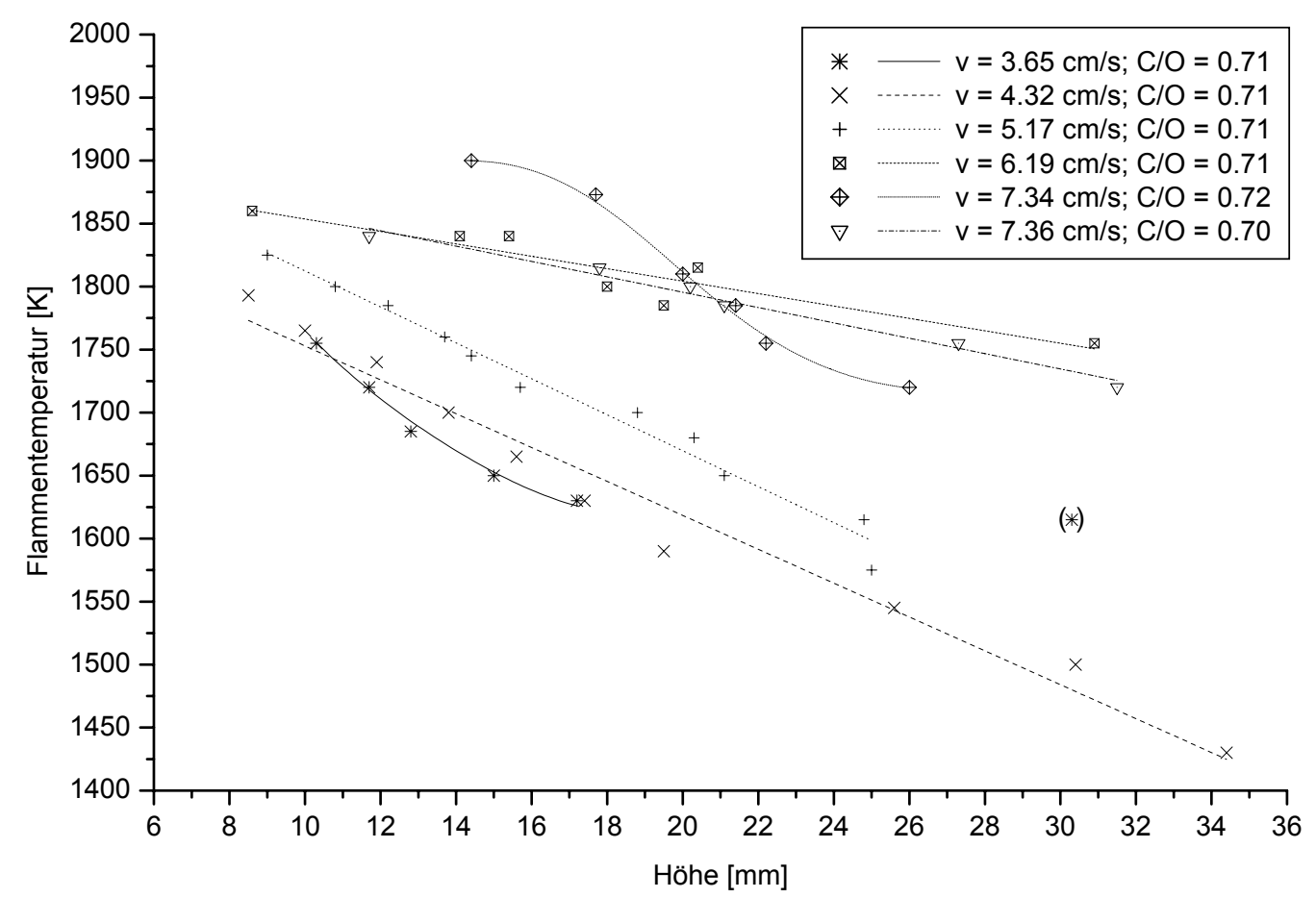

Abb. 7.42: $\quad$ Flammentemperaturen nach Kurlbaum von Propen-Luft-Flammen mit einem C/OVerhältnis von $\mathrm{C} / \mathrm{O}=0.71$ und Frischgasgeschwindigkeiten zwischen $\mathrm{v}=3.65 \mathrm{~cm} / \mathrm{s}$ und $\mathrm{v}$ $=7.36 \mathrm{~cm} / \mathrm{s}$ 


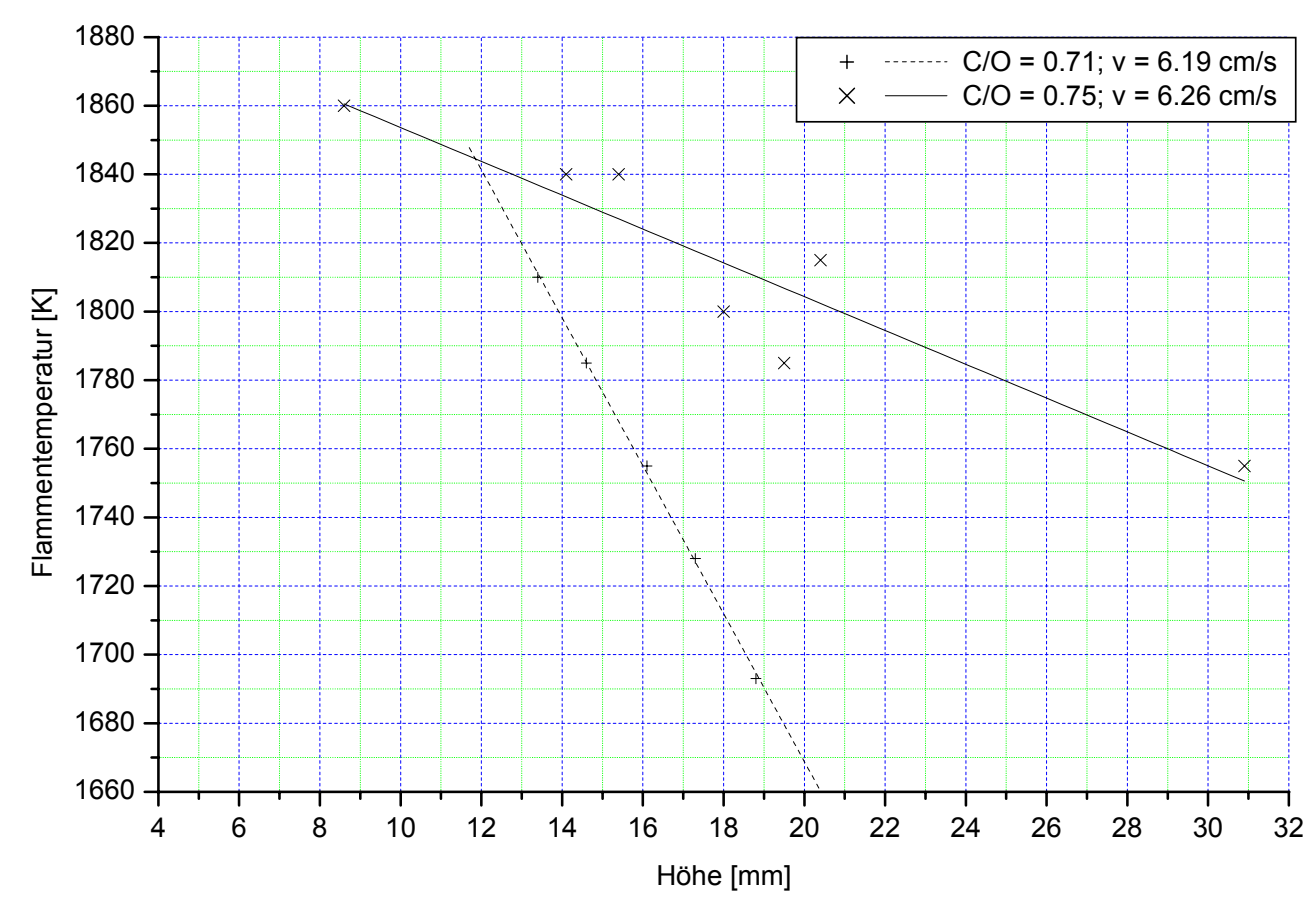

Abb. 7.43: Flammentemperaturen nach Kurlbaum von Propen-Luft-Flammen mit einer Frischgasgeschwindigkeit von etwa $\mathrm{v}=6.2 \mathrm{~cm} / \mathrm{s}$ und verschiedenen $\mathrm{C} / \mathrm{O}-$ Verhältnissen

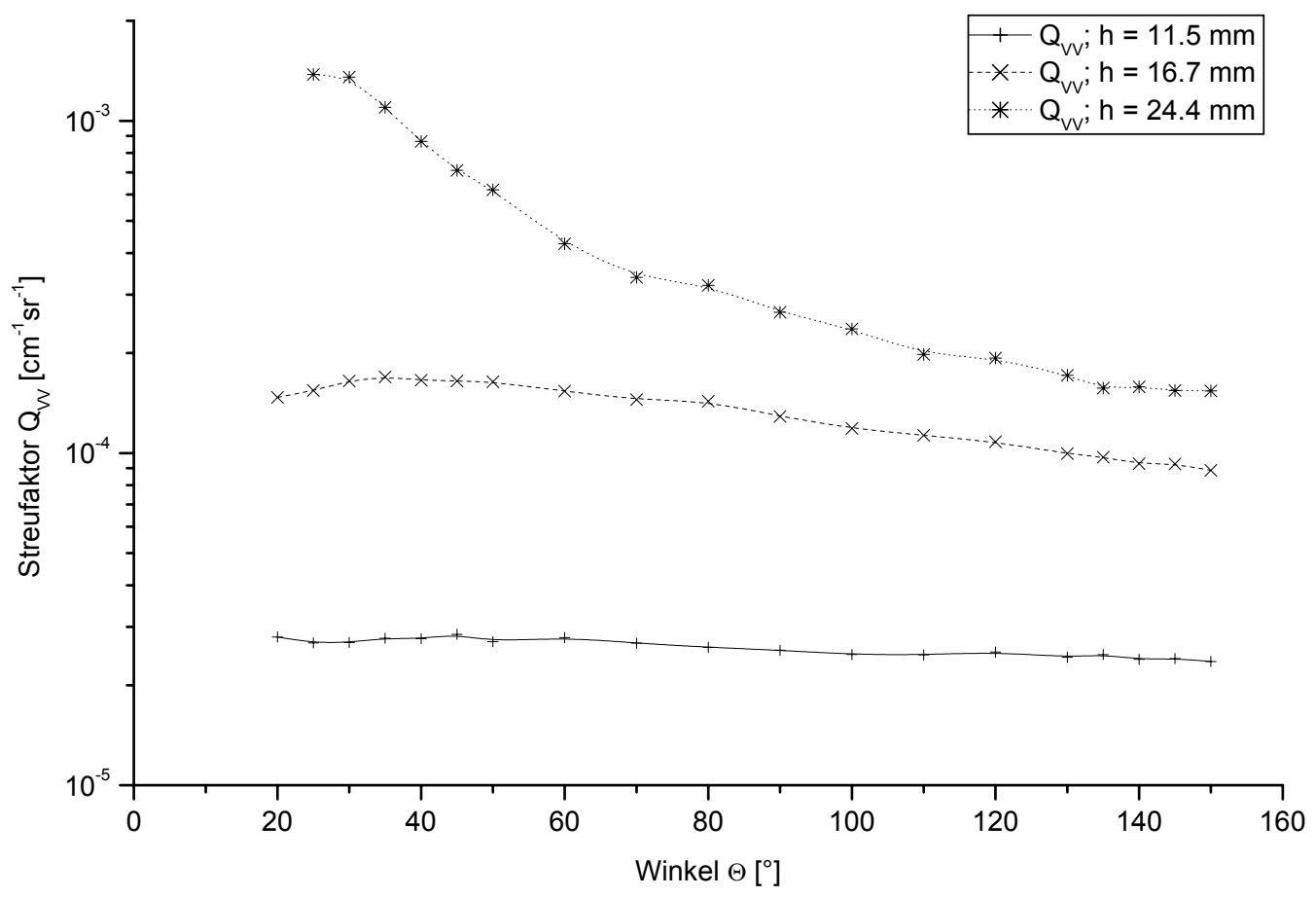

Abb. 7.44: Winkelabhängige Streufaktoren von Propen-Luft-Flammen mit einem C/O-Verhältnis von $\mathrm{C} / \mathrm{O}=0.71$ und einer Frischgasgeschwindigkeit von $\mathrm{v}_{\mathrm{FG}}=4.3 \mathrm{~cm} / \mathrm{s}$ für drei Höhen 


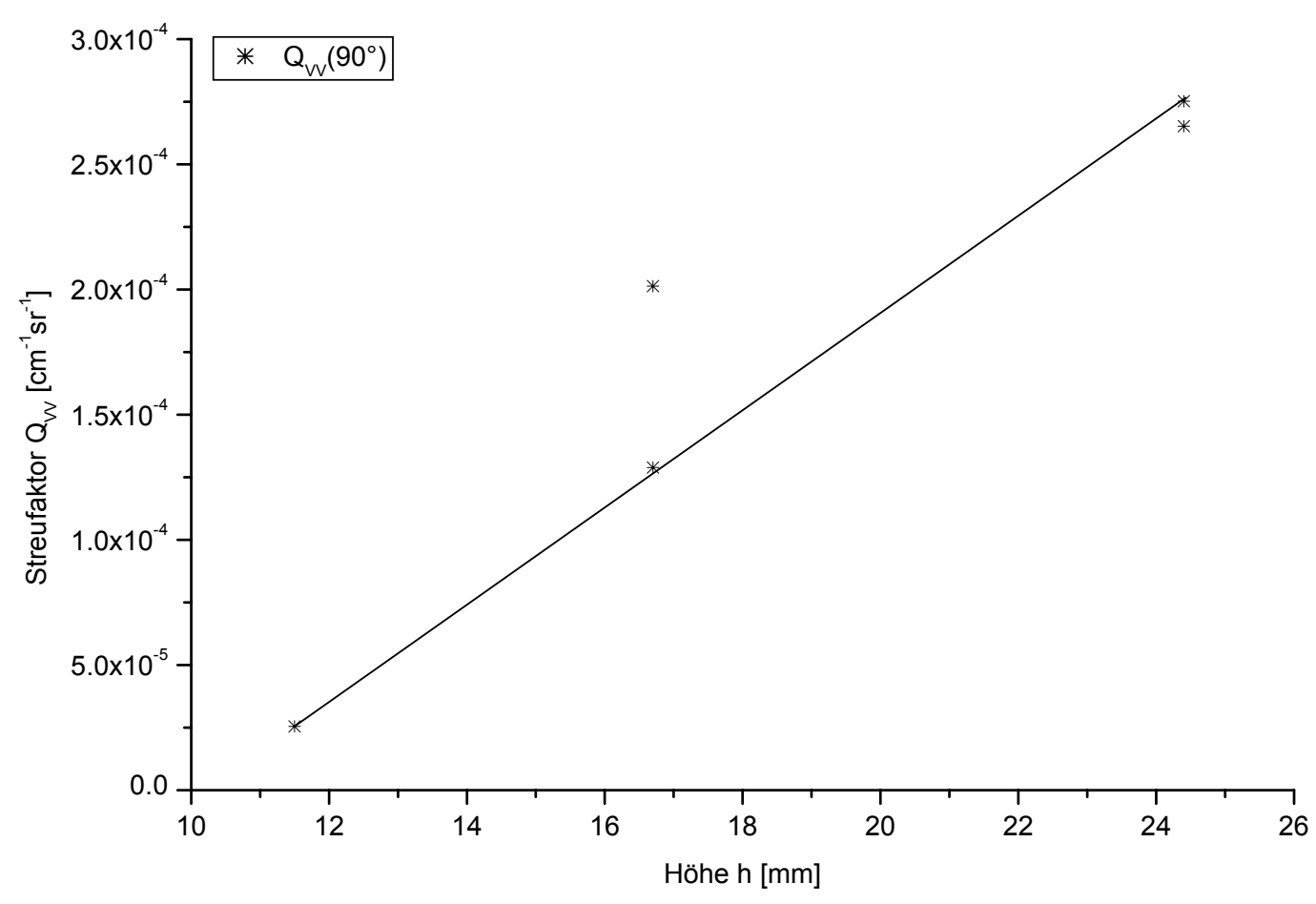

Abb. 7.45: Höhenabhängigkeit des Streufaktors einer Propen-Luft-Flamme bei einem C/O-Verhältnis von $\mathrm{C} / \mathrm{O}=0.71$ und einer Frischgasgeschwindigkeit von $\mathrm{v}_{\mathrm{FG}}=4.3 \mathrm{~cm} / \mathrm{s}$

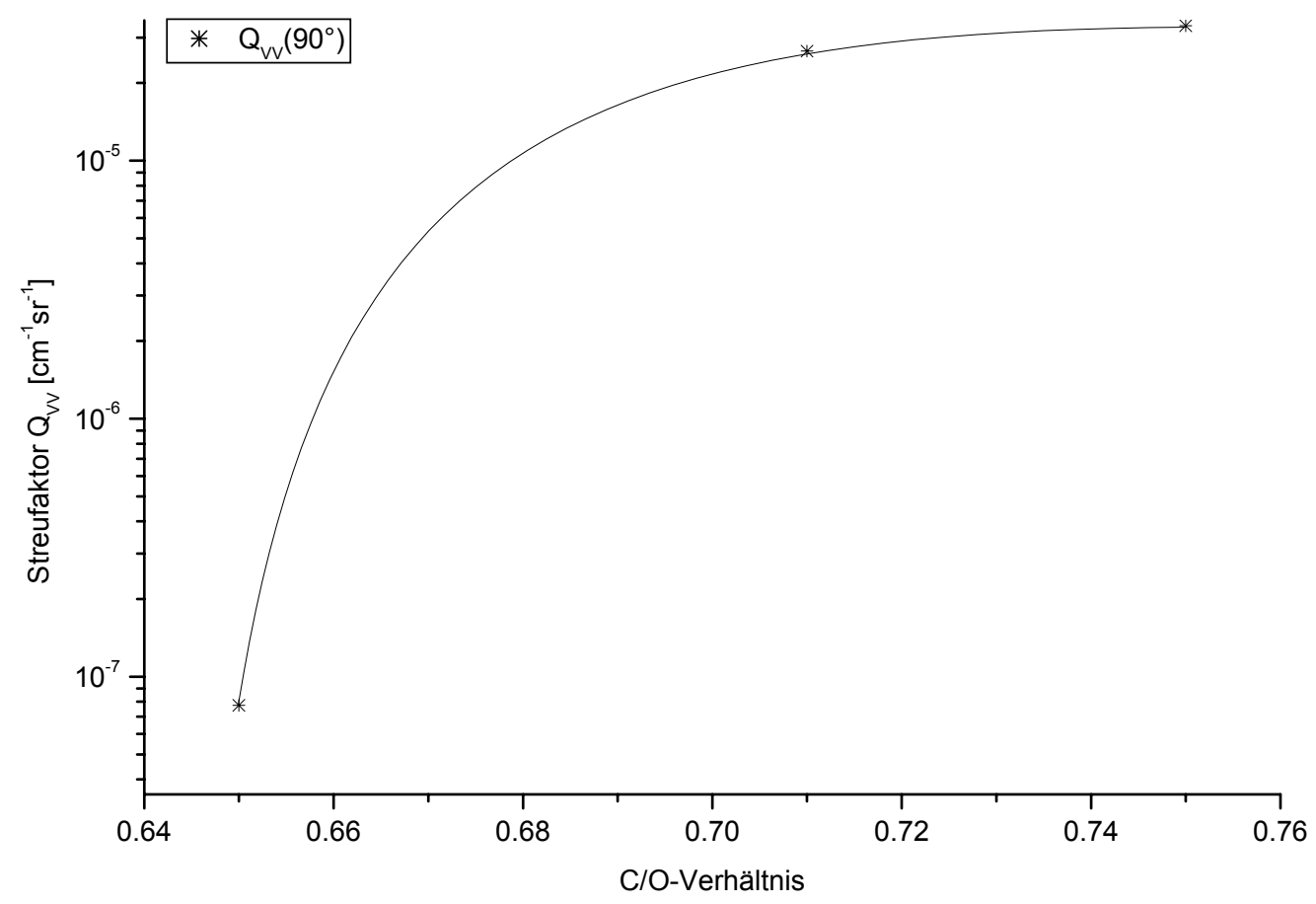

Abb. 7.46: Abhängigkeit des Streufaktors von Propen-Luft-Flammen vom C/O-Verhältnis bei einer Frischgasgeschwindigkeit von $\mathrm{v}_{\mathrm{FG}}=6.2 \mathrm{~cm} / \mathrm{s}$ in einer Höhe von $\mathrm{h}=16.7 \mathrm{~mm}$ 


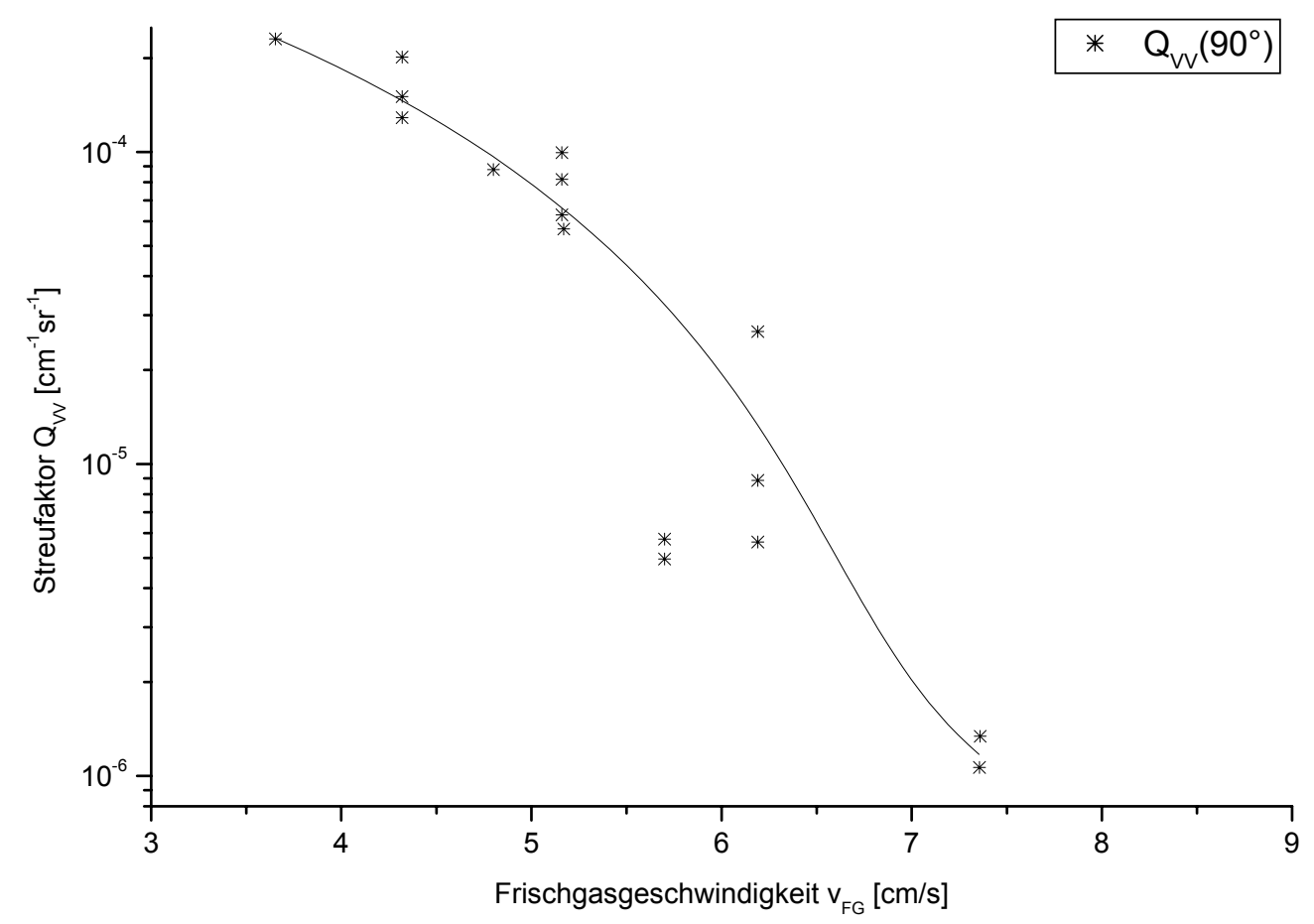

Abb. 7.47: Abhängigkeit des Streufaktors von der Frischgasgeschwindigkeit für Propen-LuftFlammen mit einem $\mathrm{C} / \mathrm{O}-$ Verhältnis von $\mathrm{C} / \mathrm{O}=0.71$ und einer Höhe von $\mathrm{h}=16.7 \mathrm{~mm}$

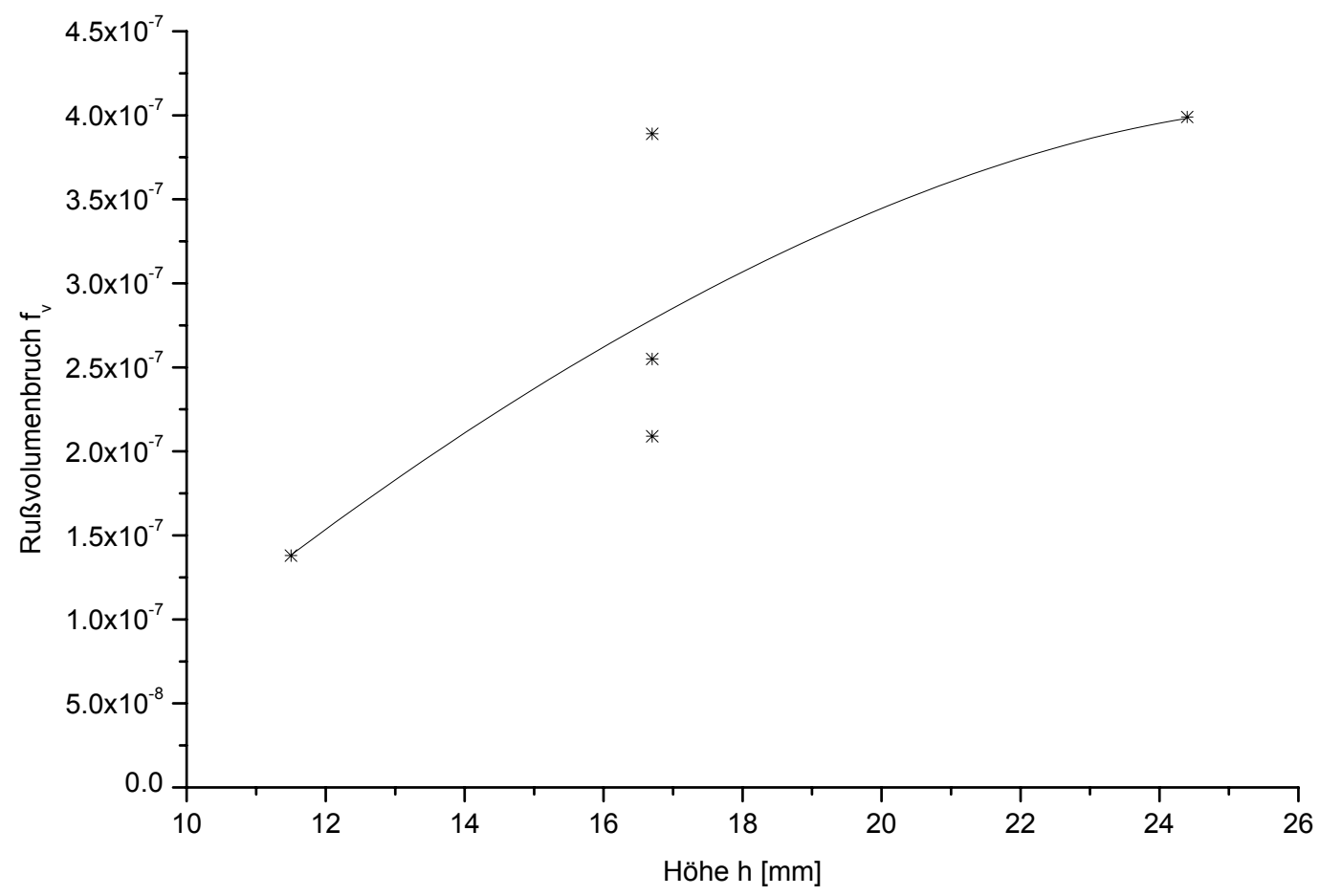

Abb. 7.48: Höhenabhängigkeit des Rußvolumenbruchs einer Propen-Luft-Flamme mit einem C/OVerhältnis von $\mathrm{C} / \mathrm{O}=0.71$ und einer Frischgasgeschwindigkeit von $\mathrm{v}_{\mathrm{FG}}=4.3 \mathrm{~cm} / \mathrm{s}$ 


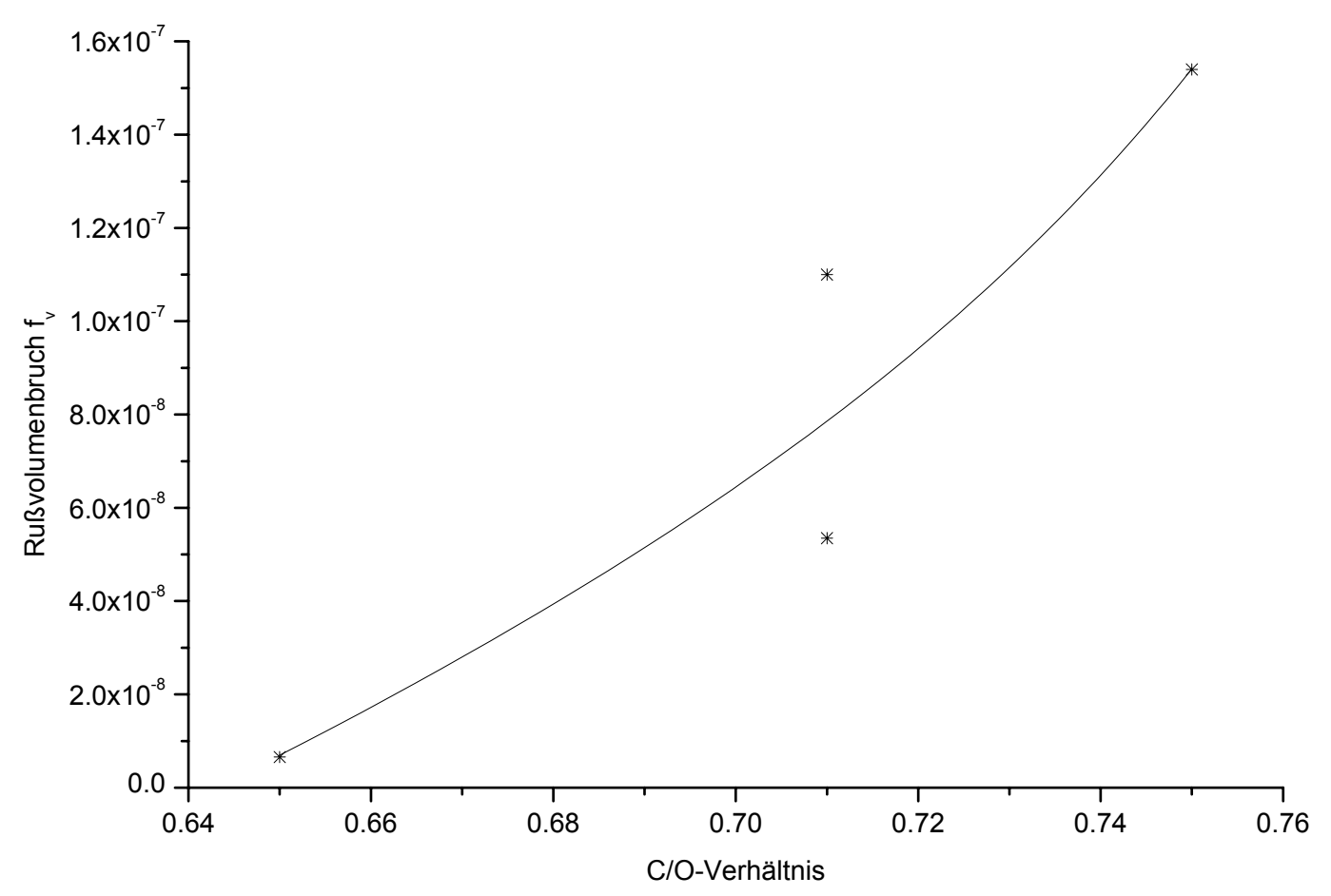

Abb. 7.49: Abhängigkeit des Rußvolumenbruchs vom C/O-Verhältnis für Propen-Luft-Flammen mit einer der Frischgasgeschwindigkeit von $\mathrm{V}_{\mathrm{FG}}=6.2 \mathrm{~cm} / \mathrm{s}$ in einer Höhe $\mathrm{h}=16.7 \mathrm{~mm}$

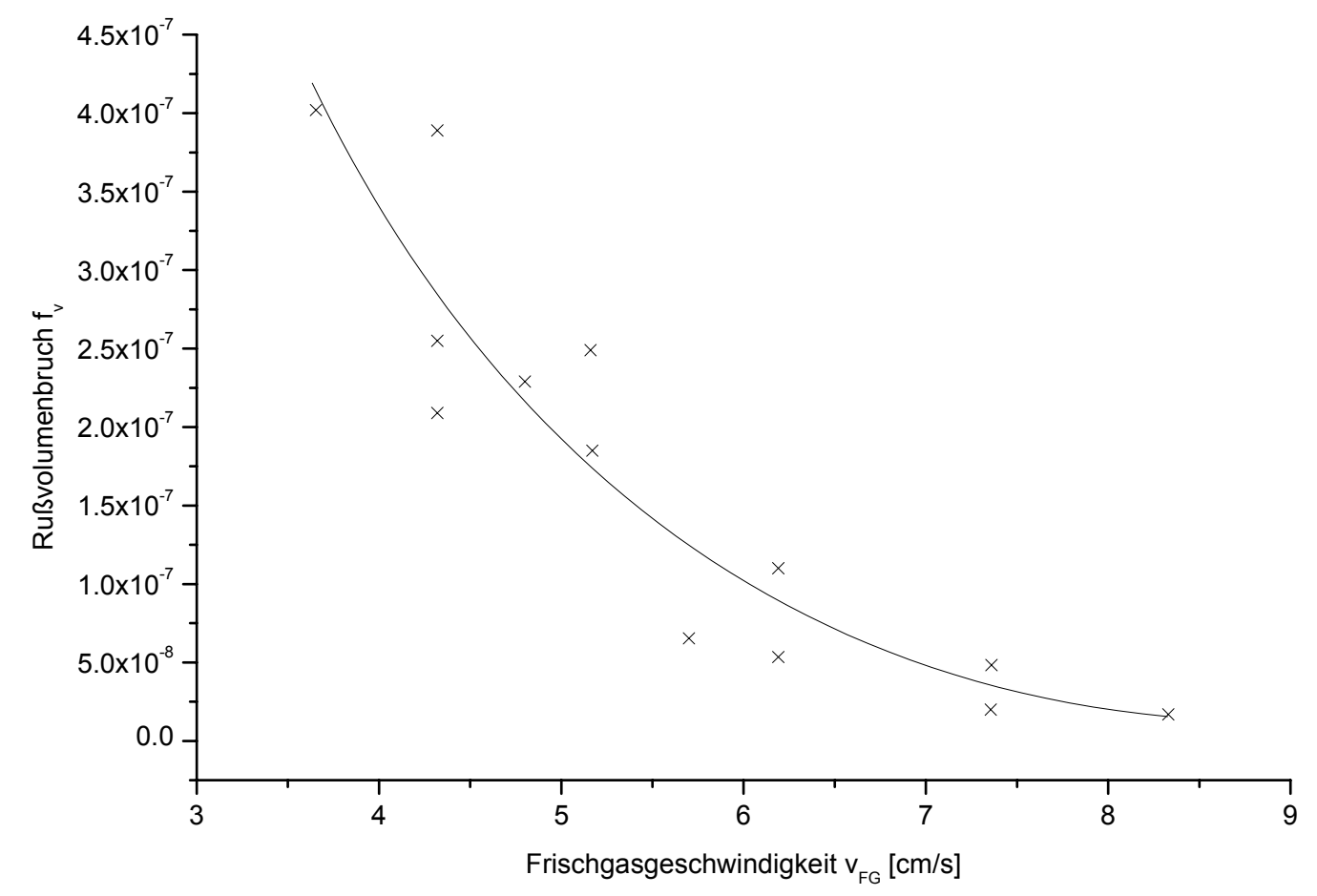

Abb. 7.50: Abhängigkeit des Rußvolumenbruchs von der Frischgasgeschwindigkeit für Propen-LuftFlammen mit einem $\mathrm{C} / \mathrm{O}-$ Verhältnis von $\mathrm{C} / \mathrm{O}=0.71$ in einer Höhe $\mathrm{h}=16.7 \mathrm{~mm}$ 


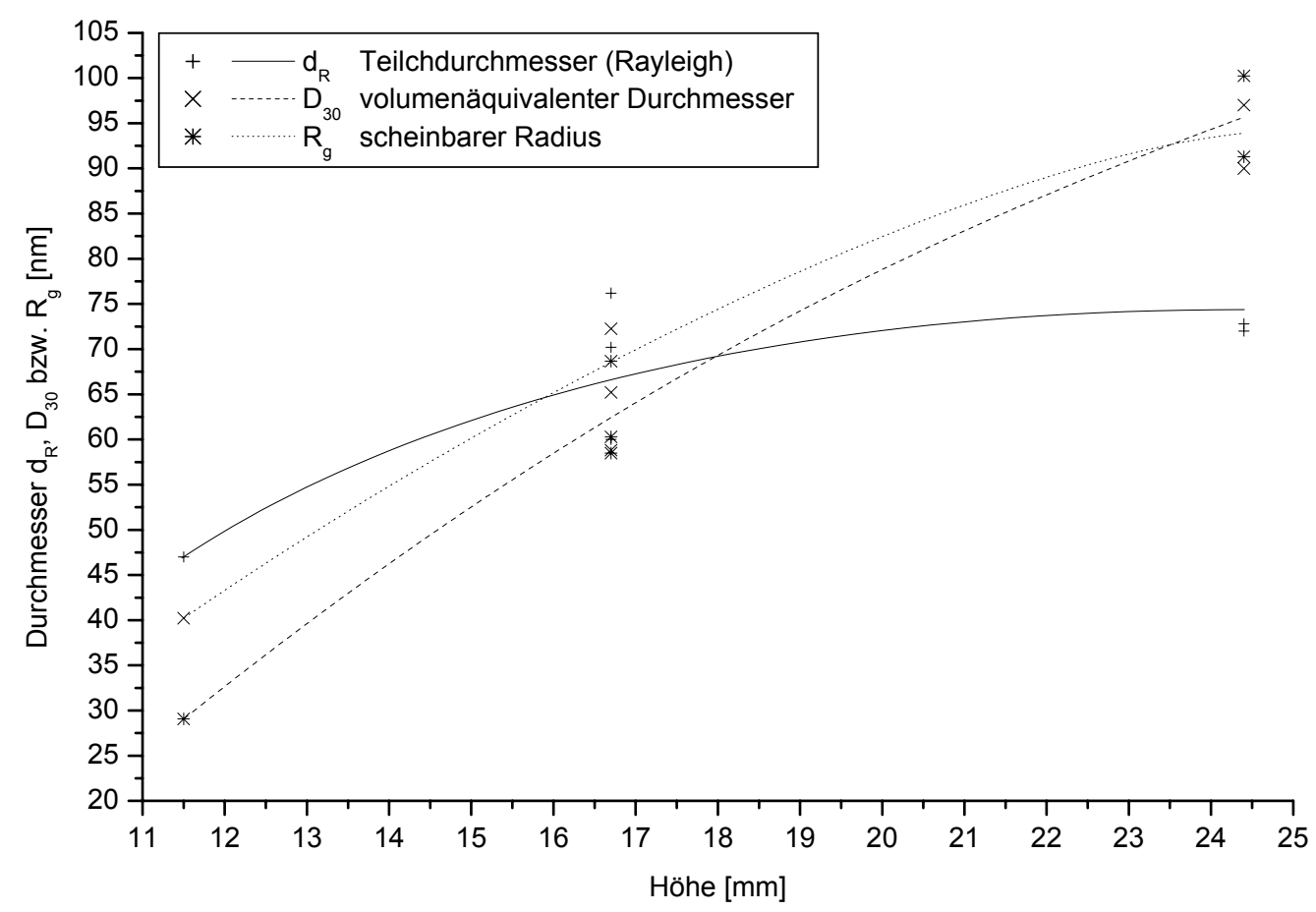

Abb. 7.51: Höhenabhängigkeit der verschiedenen Durchmesser der Rußteilchen einer Propen-LuftFlamme mit einem $\mathrm{C} / \mathrm{O}-$ Verhältnis von $\mathrm{C} / \mathrm{O}=0.71$ und einer Frischgasgeschwindigkeit $\mathrm{v}_{\mathrm{FG}}=4.3 \mathrm{~cm} / \mathrm{s}$

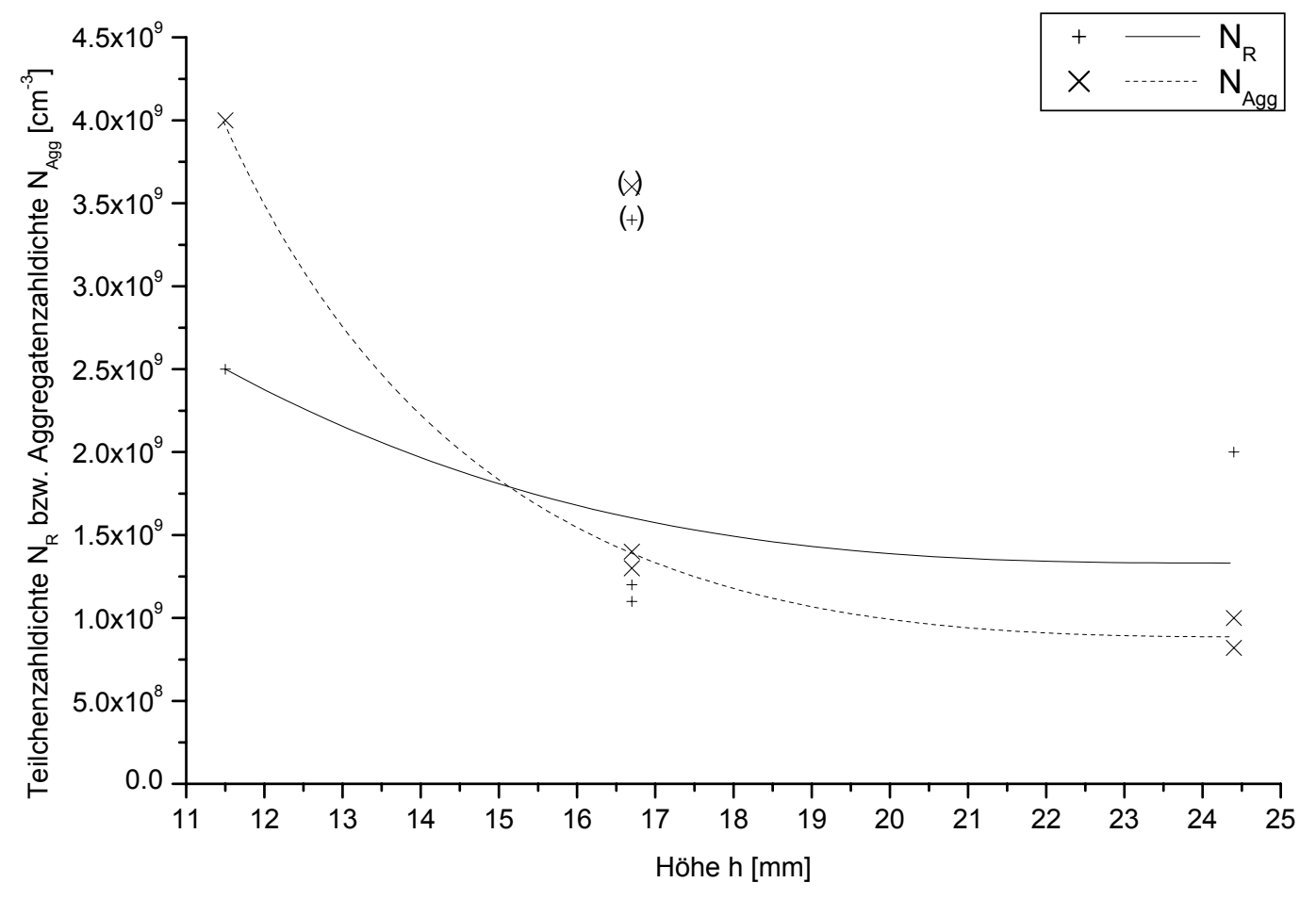

Abb. 7.52: Höhenabhängigkeit der Teilchenzahldichte $\mathrm{N}_{R}$ und der Aggregatenzahldichte $\mathrm{N}_{\text {Agg }}$ einer Propen-Luft-Flamme mit einem $\mathrm{C} / \mathrm{O}-$ Verhältnis von $\mathrm{C} / \mathrm{O}=0.71$ und einer Frischgasgeschwindigkeit $\mathrm{v}_{\mathrm{FG}}=4.3 \mathrm{~cm} / \mathrm{s}$ 


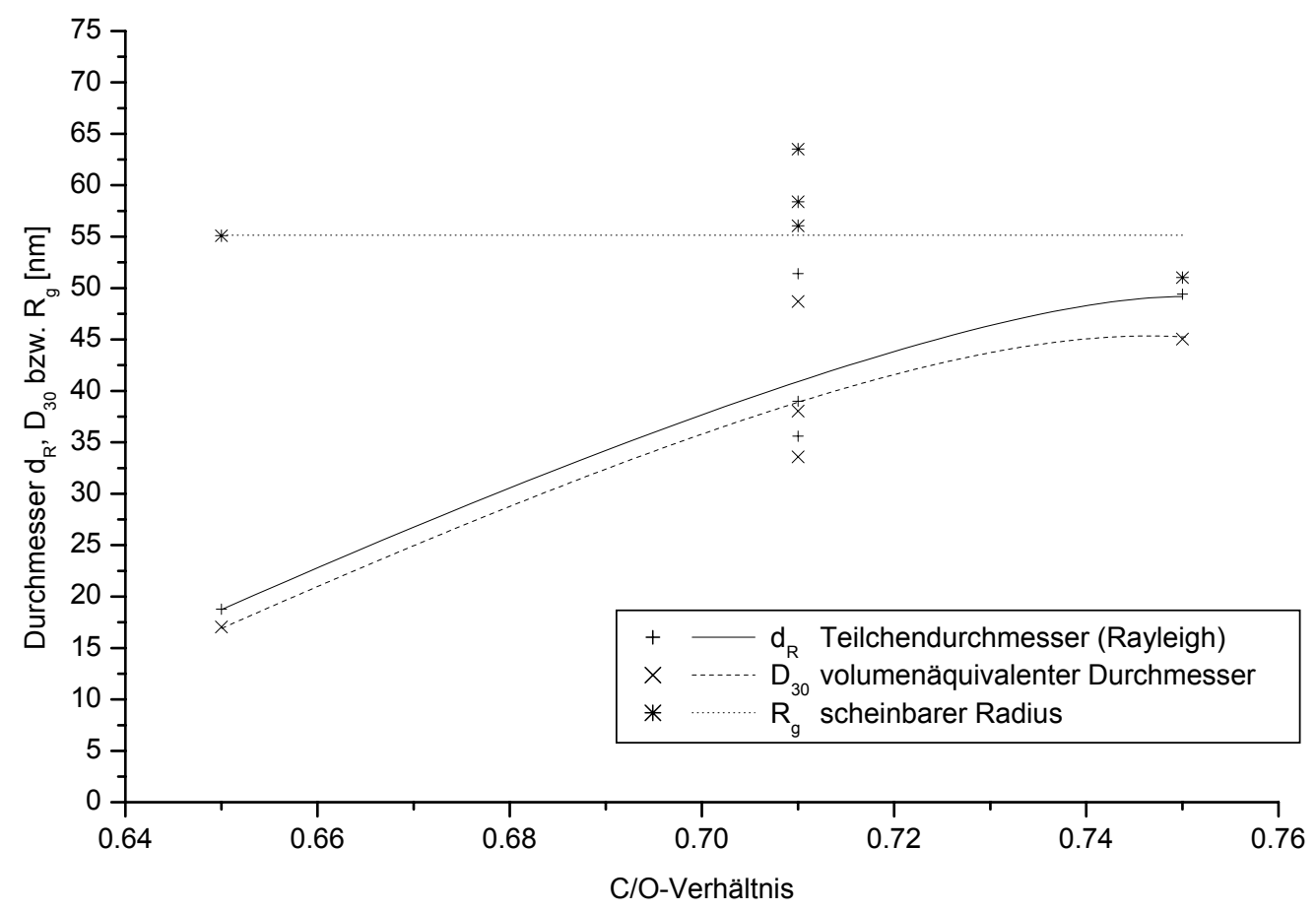

Abb. 7.53: Abhängigkeit der Teilchendurchmesser vom C/O-Verhältnis für Propen-Luft-Flammen mit einer Frischgasgeschwindigkeit $\mathrm{v}_{\mathrm{FG}}=6.2 \mathrm{~cm} / \mathrm{s}$ in einer Höhe $\mathrm{h}=16.7 \mathrm{~mm}$

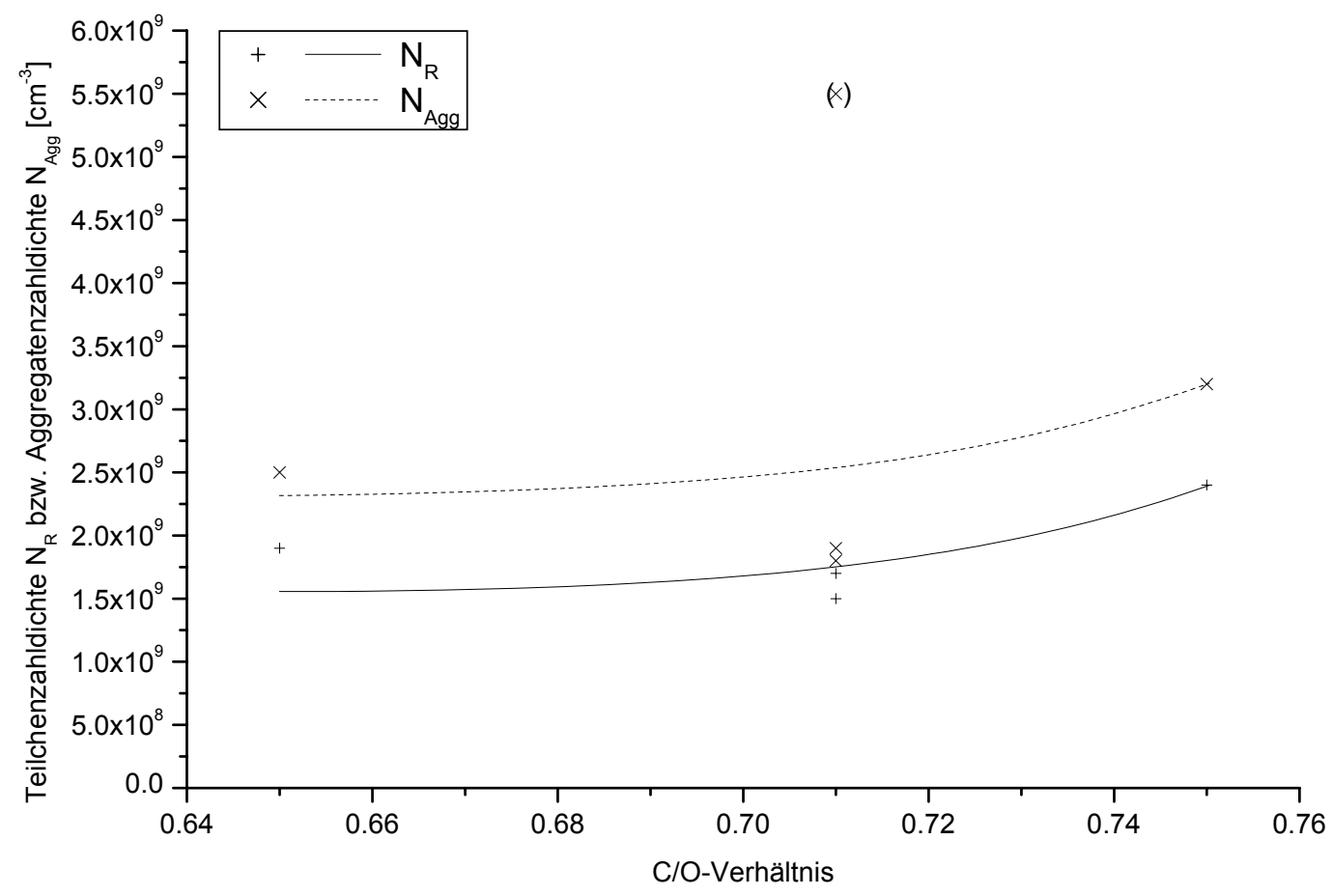

Abb. 7.54: Abhängigkeit der Teilchenzahldichte $\mathrm{N}_{\mathrm{R}}$ bzw. der Aggregatenzahldichte $\mathrm{N}_{\text {Agg vom C/O- }}$ Verhältnis für Propen-Luft-Flammen mit einer Frischgasgeschwindigkeit $\mathrm{V}_{\mathrm{FG}}=6.2 \mathrm{~cm} / \mathrm{s}$ in einer Höhe $\mathrm{h}=16.7 \mathrm{~mm}$ 


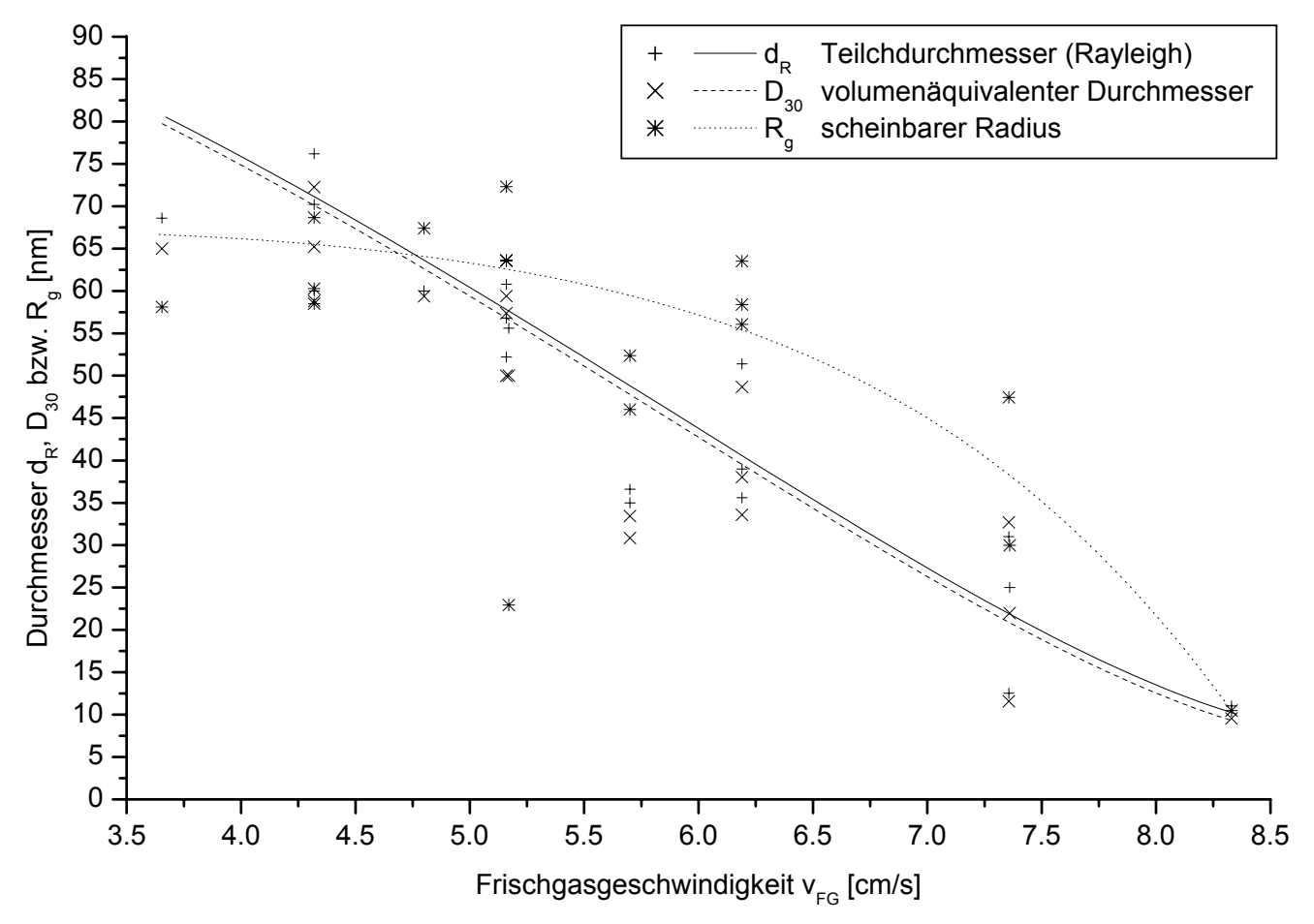

Abb. 7.55: Abhängigkeit der Teilchendurchmesser von der Frischgasgeschwindigkeit für Propen-LuftFlammen mit einem C/O-Verhältnis von 0.71 in einer Höhe $\mathrm{h}=16.7 \mathrm{~mm}$

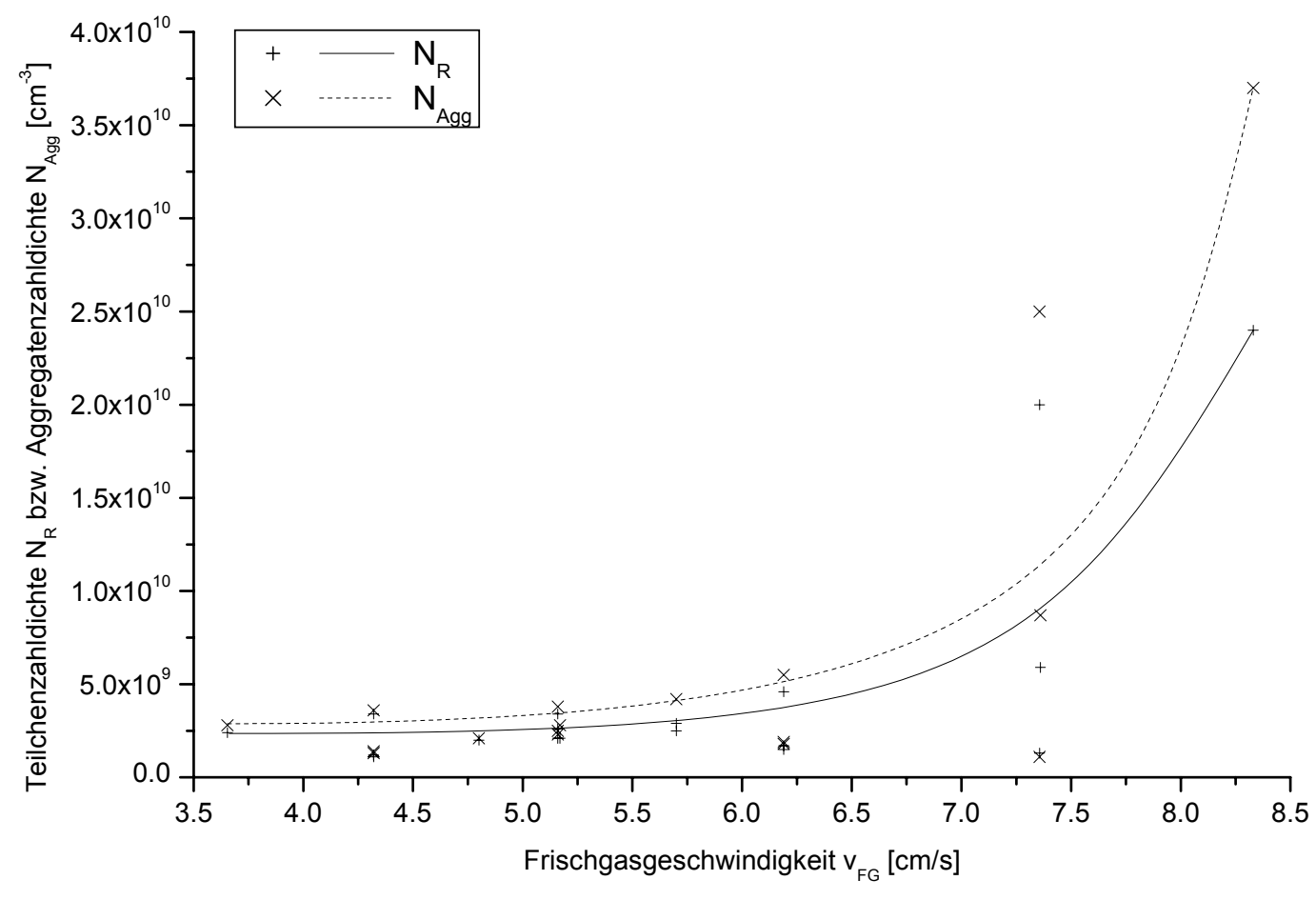

Abb. 7.56: Abhängigkeit der Teilchenzahldichte $\mathrm{N}_{\mathrm{R}}$ und der Aggregatenzahldichte $\mathrm{N}_{\text {Agg }}$ von der Frischgasgeschwindigkeit für Propen-Luft-Flammen mit einem C/O-Verhältnis von 0.71 in einer Höhe $\mathrm{h}=16.7 \mathrm{~mm}$ 


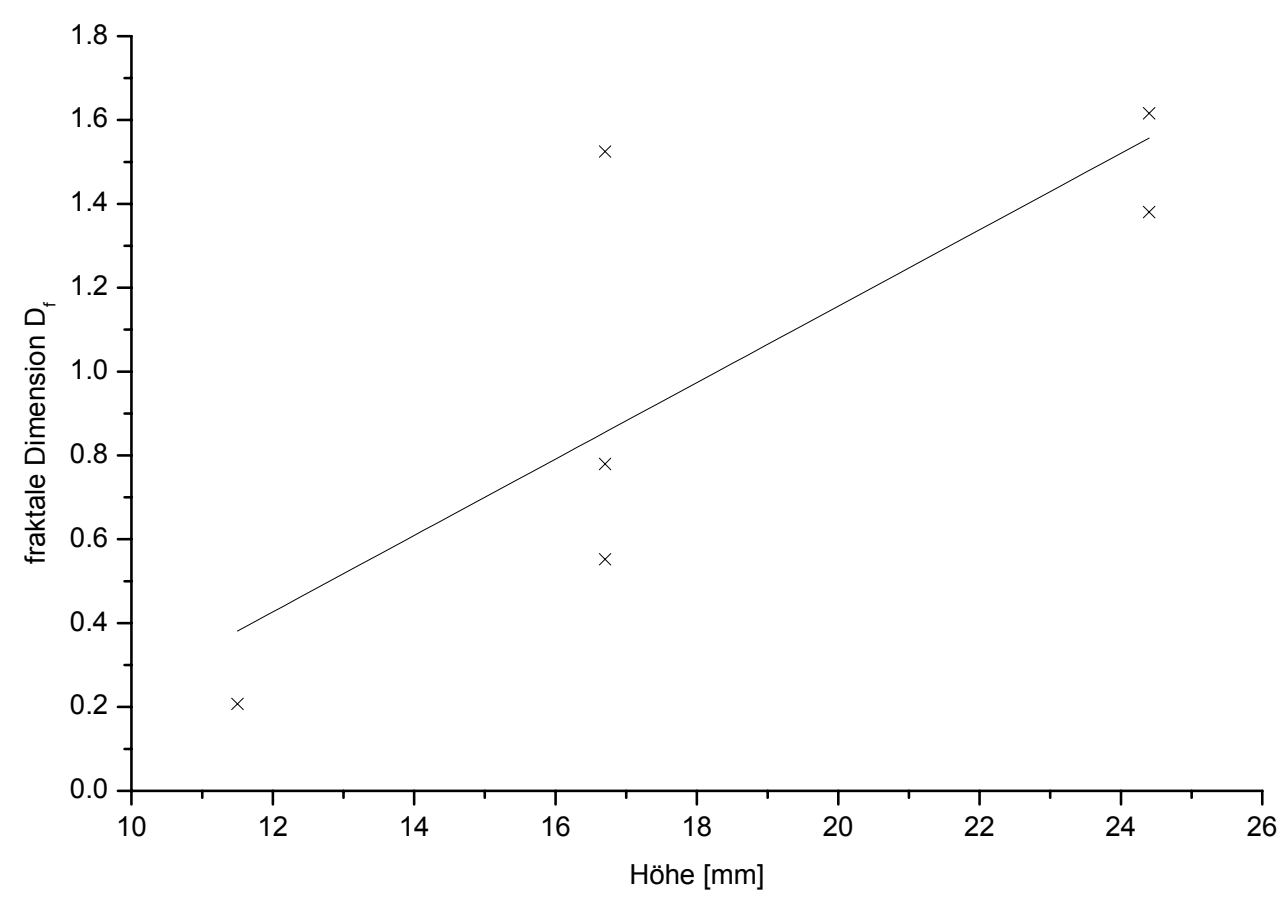

Abb. 7.57: Höhenabhängigkeit der fraktalen Dimension beim Propen für ein C/O-Verhältnis von C/O $=0.71$ und einer Frischgasgeschwindigkeit von $\mathrm{v}=4.32 \mathrm{~cm} / \mathrm{s}$

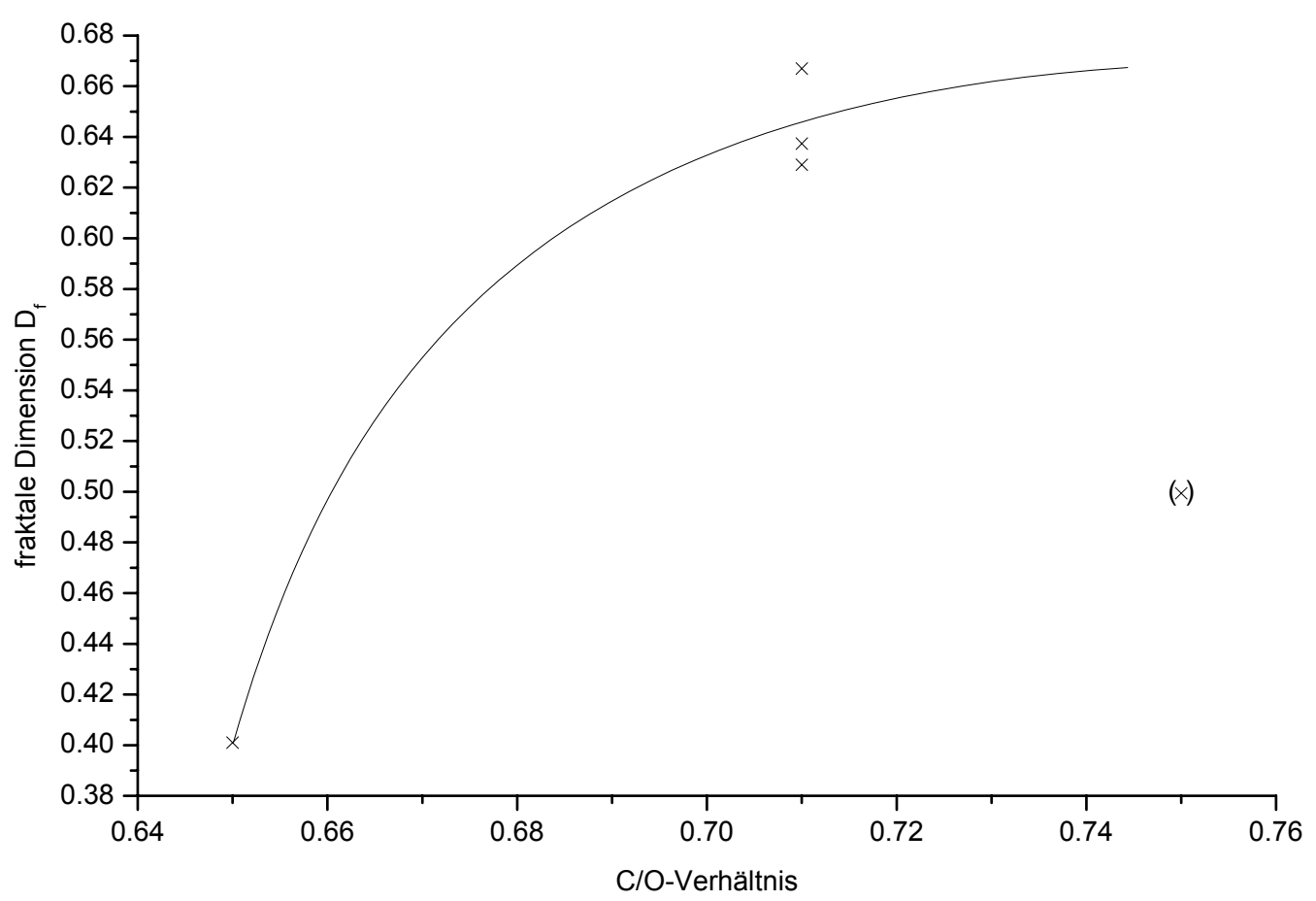

Abb. 7.58: Abhängigkeit der fraktalen Dimension beim Propen vom C/O-Verhältnis bei einer Frischgasgeschwindigkeit von $\mathrm{v}=6.2 \mathrm{~cm} / \mathrm{s}$ in einer Höhe von $16.7 \mathrm{~mm}$ 


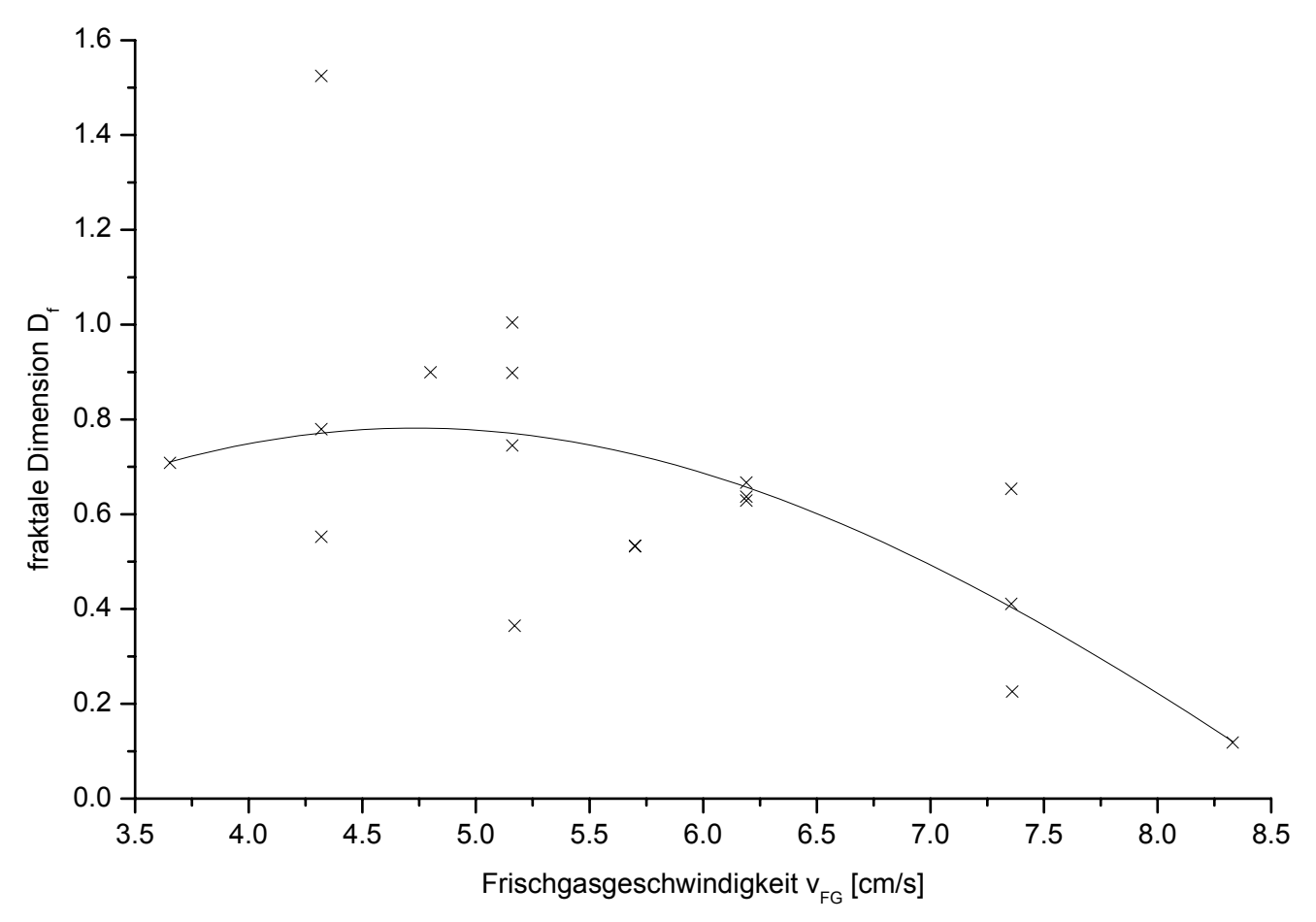

Abb. 7.59: Abhängigkeit der fraktalen Dimension beim Propen von der Frischgasgeschwindigkeit bei einem C/O-Verhältnis von 0.72 in einer Höhe von $16.7 \mathrm{~mm}$

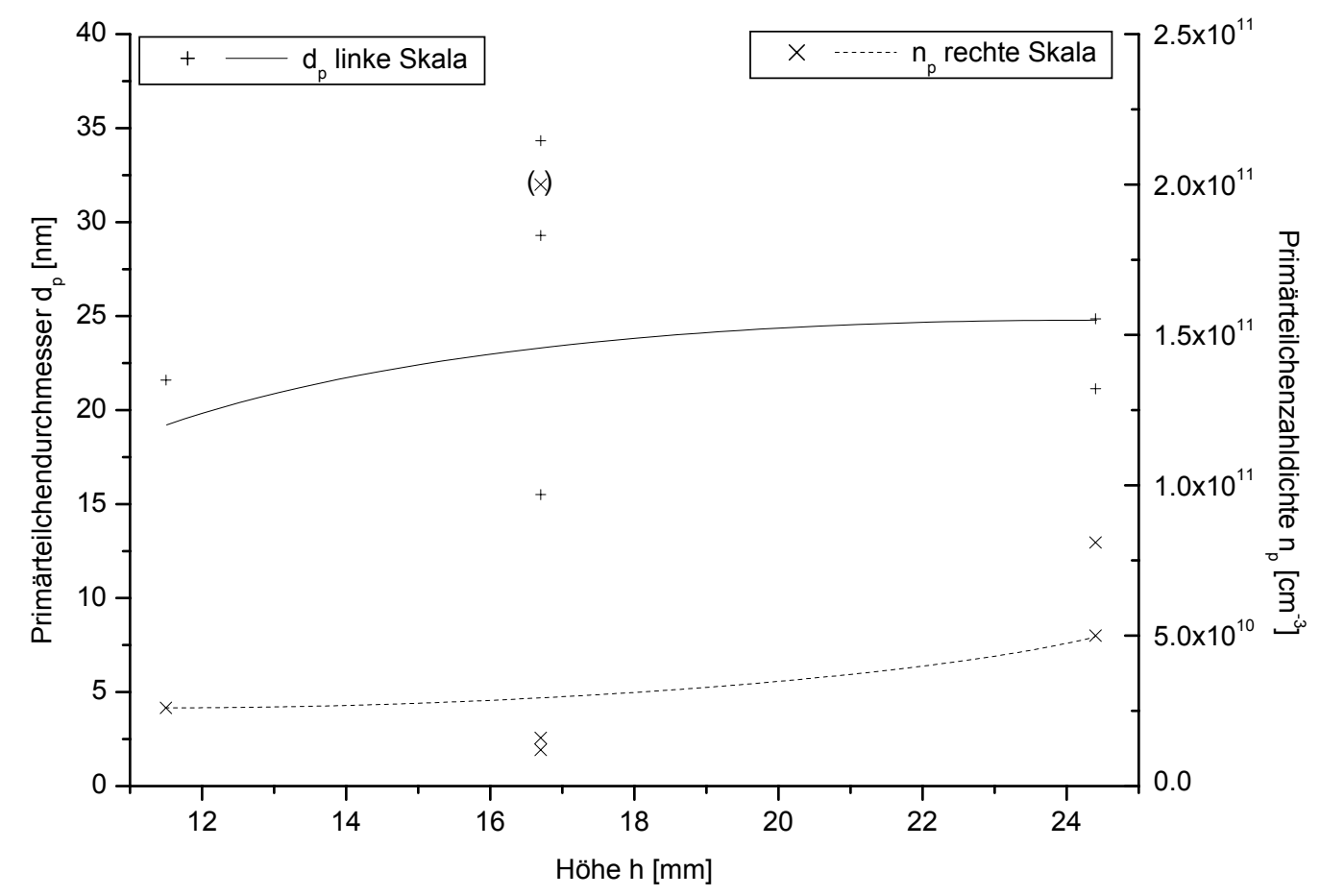

Abb. 7.60: Höhenabhängigkeit des Primärteilchendurchmessers $d_{p}$ und der Primärteilchenzahldichte $\mathrm{n}_{\mathrm{p}}$ von einer Propen-Luft-Flamme mit einem C/O-Verhältnis von $\mathrm{C} / \mathrm{O}=0.71$ und einer Frischgasgeschwindigkeit $\mathrm{v}_{\mathrm{FG}}=4.3 \mathrm{~cm} / \mathrm{s}$ 


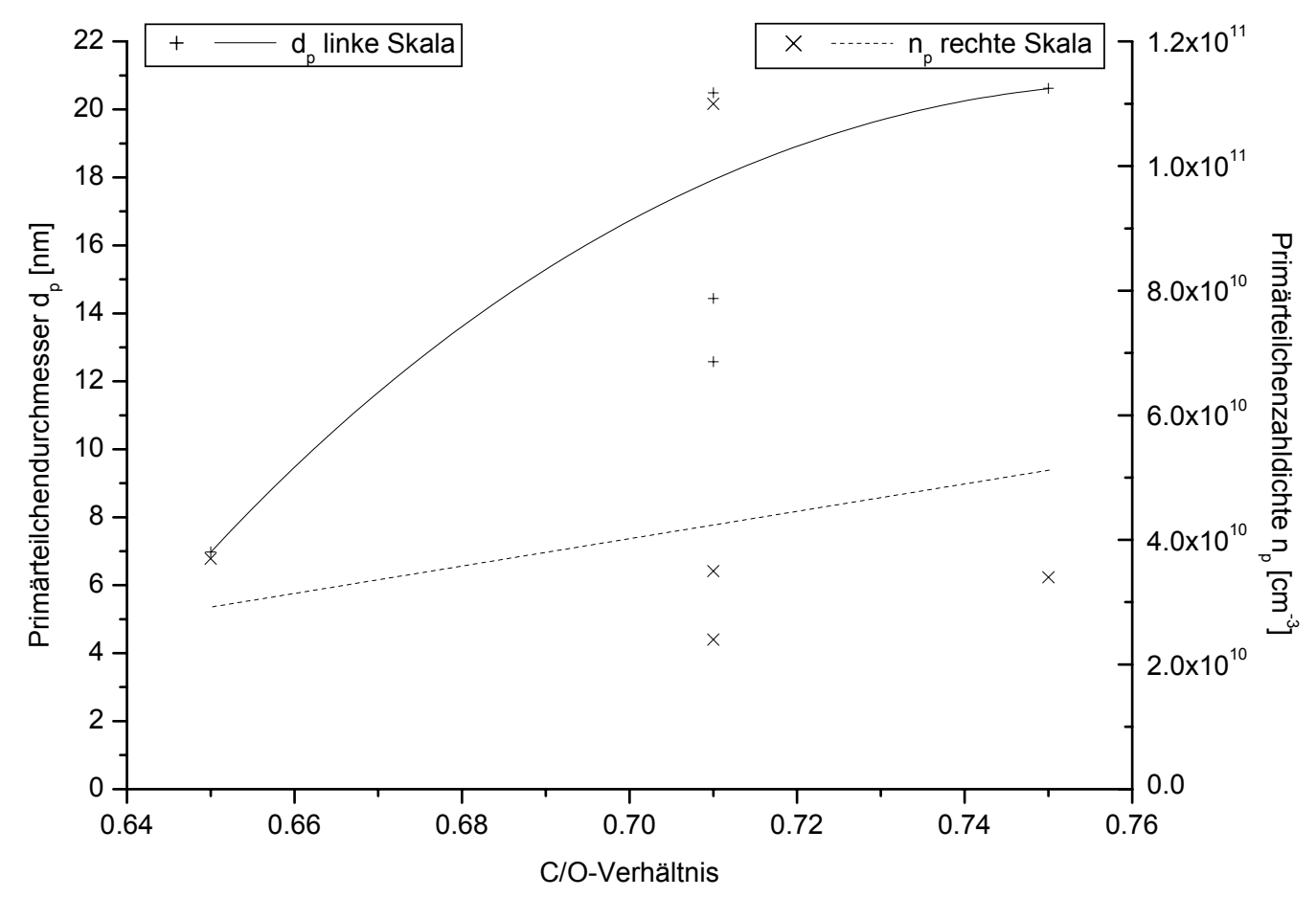

Abb. 7.61: Abhängigkeit des Primärteilchendurchmessers $d_{p}$ und der Primärteilchenzahldichte $n_{p}$ vom $\mathrm{C} / \mathrm{O}-$ Verhältnis für Propen-Luft-Flammen mit einer Frischgasgeschwindigkeit $\mathrm{V}_{\mathrm{FG}}=6.2$ $\mathrm{cm} / \mathrm{s}$ in einer Höhe $\mathrm{h}=16.7 \mathrm{~mm}$

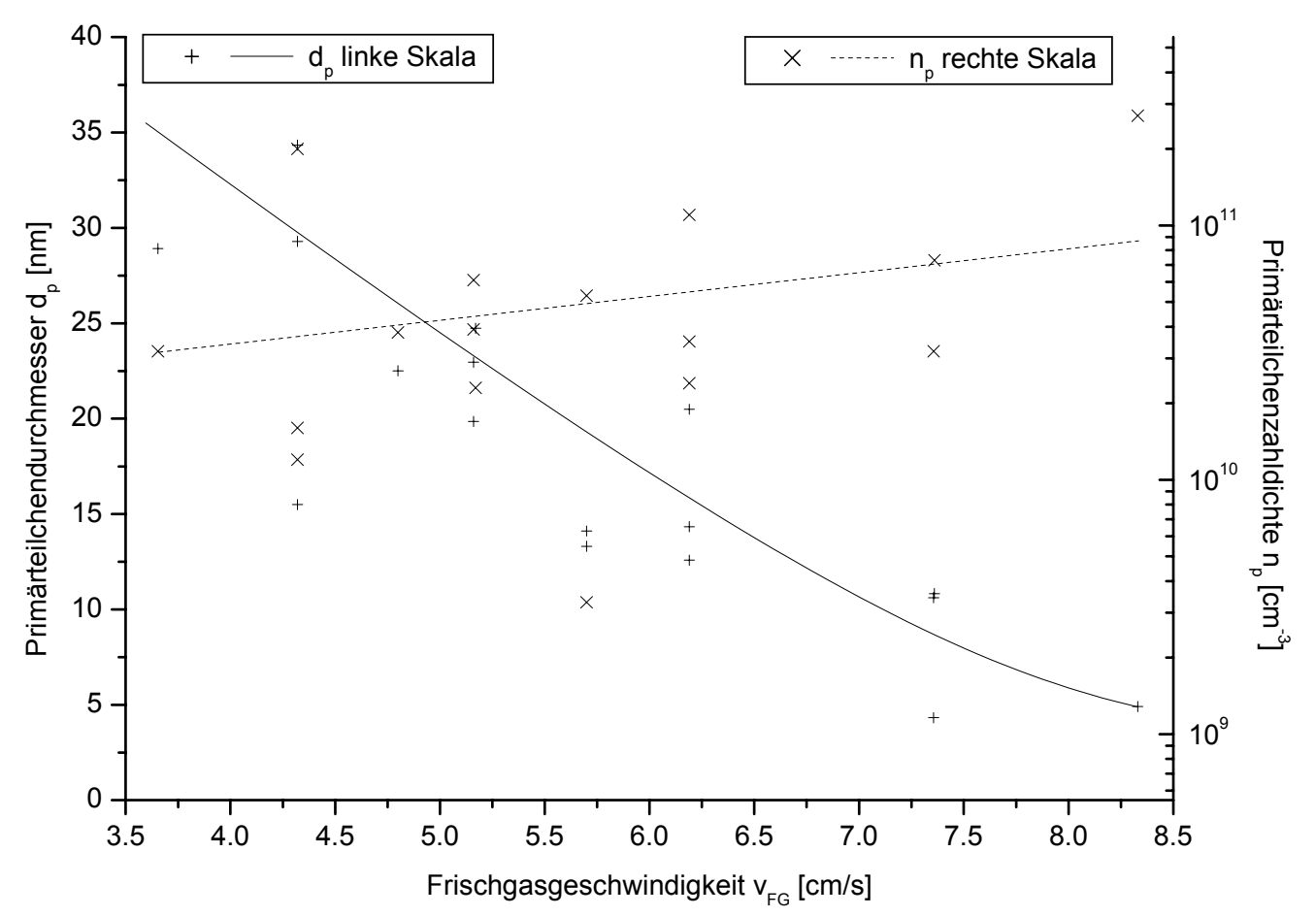

Abb. 7.62: Abhängigkeit des Primärteilchendurchmessers $d_{p}$ und der Primärteilchenzahldichte $n_{p}$ von der Frischgasgeschwindigkeit für Propen-Luft-Flammen mit einem C/O-Verhältnis von $\mathrm{C} / \mathrm{O}=0.71$ in einer Höhe $\mathrm{h}=16.7 \mathrm{~mm}$ 
Benzol-Luft-Flammen

\begin{tabular}{|c|c|c|c|c|c|c|c|c|c|c|c|c|c|c|c|c|c|}
\hline Datei & $\mathrm{C} / \mathrm{O}$ & $\mathrm{m} / \mathrm{s}] \mid \mathrm{r}$ & {$[\mathrm{mm}]$} & $\mathrm{L}[\mathrm{cm}]$ & $\Delta|[\mathrm{mV}]|$ & {$\left[\mathrm{cm}^{-1}\right]$} & $f_{v}$ & $\mathrm{~d}\left(90^{\circ}\right)[\mathrm{nm}]$ & $\left(90^{\circ}\right)\left[\mathrm{cm}^{-3}\right]$ & $D_{f}$ & $x_{p}\left(90^{\circ}\right) d$ & $d_{p}\left(90^{\circ}\right)[n m] r$ & $\mathrm{n}_{\mathrm{p}}\left(90^{\circ}\right)\left[\mathrm{cm}^{-3}\right]$ & $z\left(40^{\circ}\right)$ & $\mathrm{R}_{\mathrm{g}}(\varnothing)[\mathrm{nm}]$ & $\mathrm{D}_{30}(\varnothing)[\mathrm{nm}]$ & $\mathrm{N}_{\mathrm{Agg}}(\varnothing)\left[\mathrm{cm}^{-3}\right]$ \\
\hline 011130 & 0.71 & 6.2 & & 5.30 & 6 & 0.0014 & 1.4E-08 & 105.4 & $2.3 \mathrm{E}+07$ & 1.56 & 0.315 & 49.0 & $2.2 \mathrm{E}-13$ & & & & \\
\hline 011214 & 0.68 & 6.6 & 13.1 & 5.30 & 127 & 0.0459 & 4.6E-07 & 44.0 & $1.0 \mathrm{E}+10$ & 1.05 & 0.085 & 13.1 & $3.8 E+11$ & 2.44 & 72.6 & 46.6 & $8.6 \mathrm{E}+09$ \\
\hline 011217 & 0.68 & 6.6 & 17.7 & 5.30 & 107.5 & 0.0324 & 3.2E-07 & 43.8 & $7.4 \mathrm{E}+09$ & 1.69 & 0.041 & 6.3 & $2.4 \mathrm{E}+12$ & 2.80 & 77.9 & 46.2 & $6.2 \mathrm{E}+09$ \\
\hline 011221 & 0.68 & 5.6 & 17.7 & 5.30 & 155.7 & 0.0571 & $5.7 \mathrm{E}-07$ & 32.4 & $3.2 E+10$ & 1.44 & 0.033 & 5.1 & $8.4 \mathrm{E}+12$ & 3.26 & 83.5 & 36.2 & $2.3 \mathrm{E}+10$ \\
\hline 020103 & 0.68 & 7.6 & 17.7 & 5.30 & 21.5 & 0.0045 & 4.5E-08 & 41.4 & $1.2 \mathrm{E}+09$ & 0.47 & 0.109 & 16.9 & $1.8 \mathrm{E}+10$ & 1.07 & 21.0 & 33.9 & $2.2 \mathrm{E}+09$ \\
\hline 0201032 & 0.68 & 7.6 & 17.7 & 5.30 & 21.5 & 0.0045 & $4.5 \mathrm{E}-08$ & 36.4 & $1.8 \mathrm{E}+09$ & 0.79 & 0.077 & 12.0 & $4.9 \mathrm{E}+10$ & 1.22 & 34.4 & 31.8 & $2.6 \mathrm{E}+09$ \\
\hline $20103 \bar{a}$ & 0.68 & 7.1 & 17.7 & 5.30 & 35 & 0.0084 & 8.4E-08 & 42.6 & $2.1 \mathrm{E}+09$ & 1.08 & 0.078 & 12.1 & $9.1 \mathrm{E}+10$ & 1.69 & 55.7 & 39.4 & $2.6 \mathrm{E}+09$ \\
\hline 020103a_2 & 0.68 & 7.1 & 17.7 & 5.30 & 35 & 0.0084 & 8.4E-08 & 40.6 & $2.4 \mathrm{E}+09$ & 1.09 & 0.072 & 11.2 & $1.1 \mathrm{E}+11$ & 1.83 & 59.7 & 38.9 & $2.7 \mathrm{E}+09$ \\
\hline 020108 & 0.68 & 4.6 & 17.7 & 4.29 & 280 & 0.0853 & 8.5E-07 & 41.0 & $2.4 \mathrm{E}+10$ & 1.55 & 0.044 & 6.9 & $5.1 \mathrm{E}+12$ & 4.47 & 94.0 & 52.1 & $1.1 \mathrm{E}+10$ \\
\hline 020108_2 & 0.68 & 4.6 & 17.7 & 4.29 & 280 & 0.0853 & 8.5E-07 & 41.6 & $2.3 E+10$ & 1.45 & 0.052 & 8.1 & $3.1 \mathrm{E}+12$ & 4.04 & 90.8 & 51.6 & $1.2 \mathrm{E}+10$ \\
\hline $020108 \bar{a}$ & 0.68 & 3.8 & 17.7 & 4.39 & 440 & 0.1555 & 1.6E-06 & 66.6 & $1.0 \mathrm{E}+10$ & 1.68 & 0.109 & 16.9 & $6.2 \mathrm{E}+11$ & 5.05 & 97.8 & 89.1 & $4.1 \mathrm{E}+09$ \\
\hline 020108a_2 & 0.68 & 3.8 & 17.7 & 4.19 & 313 & 0.0939 & $9.4 \mathrm{E}-07$ & 92.2 & $2.3 E+09$ & 1.32 & 0.251 & 39.0 & $3.0 \mathrm{E}-11$ & 3.92 & 89.8 & 113.7 & $1.2 \mathrm{E}+09$ \\
\hline 20115 & 0.68 & 5.1 & 17.7 & 5.19 & 211 & 0.0546 & 5.4E-07 & 75.2 & $2.5 \mathrm{E}+09$ & 1.87 & 0.123 & 19.1 & $1.5 E+11$ & 4.11 & 91.3 & 89.7 & $1.4 \mathrm{E}+09$ \\
\hline $20115 a$ & 0.68 & 5.1 & 17.7 & 5.19 & 211 & 0.0546 & 5.4E-07 & 75.2 & $2.5 \mathrm{E}+09$ & 1.65 & 0.146 & 22.7 & $8.9 E+10$ & 3.71 & 88.0 & 88.7 & $1.5 \mathrm{E}+09$ \\
\hline 20115_2 & 0.68 & 6.1 & 17.7 & 5.35 & 179 & 0.0441 & 4.4E-07 & 68.8 & $2.6 \mathrm{E}+09$ & 1.63 & 0.121 & 18.8 & $1.3 E+11$ & 3.28 & 83.7 & 76.9 & $1.8 \mathrm{E}+09$ \\
\hline 20115 2a & 0.68 & 6.1 & 17.7 & 5.35 & 179 & 0.0441 & 4.4E-07 & 69.8 & $2.5 \mathrm{E}+09$ & 1.56 & 0.132 & 20.5 & $9.7 E+10$ & 3.35 & 84.5 & 78.4 & $1.7 \mathrm{E}+09$ \\
\hline 20115 3 & 0.68 & 6.1 & 8.0 & 5.41 & 159 & 0.0322 & 3.2E-07 & 60.2 & $2.8 \mathrm{E}+09$ & 1.1 & 0.133 & 20.6 & $7.0 \mathrm{E}+10$ & 1.70 & 56.0 & 55.3 & $3.6 \mathrm{E}+09$ \\
\hline 20118 & 0.75 & 5.1 & 17.7 & 5.65 & 751.5 & 0.2372 & 2.4E-06 & 87.4 & $6.8 \mathrm{E}+09$ & 1.66 & 0.204 & 31.7 & $1.4 \mathrm{E}+11$ & 5.20 & 98.7 & 116.7 & $2.7 \mathrm{E}+09$ \\
\hline $020118 a$ & 0.75 & 5.1 & 17.7 & 5.65 & 751.5 & 0.2372 & 2.4E-06 & 88.4 & $6.5 E+09$ & 1.81 & 0.197 & 30.6 & $1.6 \mathrm{E}+11$ & 5.36 & 99.6 & 119.5 & $2.5 \mathrm{E}+09$ \\
\hline 020118_2 & 0.72 & 5.1 & 17.7 & 5.29 & 425 & 0.1192 & $1.2 \mathrm{E}-06$ & 76.4 & $5.1 \mathrm{E}+09$ & 1.67 & 0.150 & 23.3 & $1.8 \mathrm{E}+11$ & 4.23 & 92.3 & 95.3 & $2.6 \mathrm{E}+09$ \\
\hline $020118 \_2 a$ & 0.72 & 5.1 & 17.7 & 5.29 & 425 & 0.1192 & $1.2 \mathrm{E}-06$ & 76.2 & $5.1 \mathrm{E}+09$ & 1.71 & 0.145 & 22.5 & $2.0 \mathrm{E}+11$ & 4.17 & 91.9 & 94.8 & $2.6 \mathrm{E}+09$ \\
\hline 020118_3 & 0.63 & 5.1 & 17.7 & 4.97 & 35.5 & 0.0085 & $8.5 \mathrm{E}-08$ & 55.8 & $9.3 E+08$ & 1.13 & 0.116 & 18.0 & $2.7 E+10$ & 1.88 & 60.9 & 53.4 & $1.1 \mathrm{E}+09$ \\
\hline 020118_3a & 0.63 & 5.1 & 17.7 & 4.97 & 35.5 & 0.0085 & $8.5 \mathrm{E}-08$ & 57.6 & $8.5 E+08$ & 0.69 & 0.149 & 23.1 & $1.3 \mathrm{E}+10$ & 1.79 & 58.5 & 53.8 & $1.0 \mathrm{E}+09$ \\
\hline 020118_4 & 0.72 & 5.1 & 9.1 & 5.41 & 345 & 0.0870 & 8.7E-07 & 75.6 & $3.8 \mathrm{E}+09$ & 1.23 & 0.182 & 28.2 & $7.4 \mathrm{E}+10$ & 2.63 & 75.5 & 80.0 & $3.2 \mathrm{E}+09$ \\
\hline 020118_4a & 0.72 & 5.1 & 9.1 & 5.41 & 345 & 0.0870 & 8.7E-07 & 76.4 & $3.7 E+09$ & 1.24 & 0.185 & 28.7 & $7.0 \mathrm{E}+10$ & 2.76 & 77.5 & 82.1 & $2.9 \mathrm{E}+09$ \\
\hline $020118 \_5$ & 0.72 & 5.1 & 13.3 & 5.26 & 387 & 0.1038 & 1.0E-06 & 76.6 & $4.4 \mathrm{E}+09$ & 1.49 & 0.167 & 25.9 & $1.1 \mathrm{E}+11$ & 3.64 & 87.4 & 90.6 & $2.6 \mathrm{E}+09$ \\
\hline
\end{tabular}

Tab. 7.5: Meßergebnisse der Benzol-Luft-Flammen 
7.1.2 Zusätzliche Meßdaten der Ethylen-Luft-Flammen im Hochdruck

\section{Ethylen-Luft-Flammen bei höherem Druck}

\begin{tabular}{|c|c|c|c|c|c|c|c|c|c|c|c|c|c|c|c|c|c|c|}
\hline Datei & Nr. & $\left.\begin{array}{|c|}\mathrm{p} \\
{[\mathrm{bar}]}\end{array}\right]$ & $\mathrm{C} / \mathrm{O}$ & $\left.\begin{array}{|c|}\mathrm{v} \\
{[\mathrm{cm} / \mathrm{s}]}\end{array}\right]$ & $\begin{array}{c}\mathrm{h} \\
{[\mathrm{mm}]}\end{array}$ & $\begin{array}{l}\mathrm{L} \\
{[\mathrm{cm}]}\end{array}$ & $\Delta[[\mathrm{mV}]$ & $\mathrm{K}_{\text {ext }}\left[\mathrm{cm}^{-1}\right]$ & $f_{v}$ & 0०) & $\mathrm{N}_{\mathrm{R}}\left(90^{\circ}\right)\left[\mathrm{cm}^{-3}\right]$ & $D_{f}$ & In & i & \begin{tabular}{|c|}
$z$ \\
$\left(45^{\circ}\right)$ \\
\end{tabular} & $\begin{array}{c}\mathrm{R}_{\mathrm{g}} \\
(\varnothing)[\mathrm{nm}] \\
\end{array}$ & \begin{tabular}{c|}
$\mathrm{D}_{30}$ \\
$(\varnothing)[\mathrm{nm}]$
\end{tabular} & \begin{tabular}{|c|}
$N_{\text {Agg }}$ \\
$(\varnothing)\left[\mathrm{cm}^{-3}\right]$ \\
\end{tabular} \\
\hline 30314 & $2 x$ & 3.10 & 0.75 & 5.8 & 7.5 & 4.31 & 2.19 & 0.1913 & $1.9 \mathrm{E}-06$ & 90.0 & $5.0 \mathrm{E}+09$ & \begin{tabular}{|l|}
1.00 \\
\end{tabular} & 39.8 & $5.8 \mathrm{E}+10$ & 1.778 & 70.0 & 86.6 & $5.6 \mathrm{E}+09$ \\
\hline 0314 & $3 x$ & $\mid 3.27$ & 66 & 5.5 & 7.5 & 31 & 0.22 & 0125 & $1.2 \mathrm{E}-07$ & 46.5 & $4 E+09$ & 0.75 & 17.0 & $4.9 \mathrm{E}+10$ & 1.134 & 48.1 & 38.2 & $4.4 \mathrm{E}+09$ \\
\hline 0414 & $11 x$ & 15 & 69 & 5.7 & 7.5 & .02 & 4.5 & 309 & $8.1 \mathrm{E}-07$ & 51.4 & 10 & 0.64 & 20.4 & $1.8 \mathrm{E}+11$ & 1.280 & 53.9 & 43.6 & $.9 \mathrm{E}+10$ \\
\hline 0414 & $12 x$ & 23 & .73 & 5.5 & 7.5 & 4.27 & 5.8 & & 1.1E-06 & 69.6 & 09 & 0.88 & 28.1 & 0 & 1.541 & 60.5 & 61.7 & $8.6 \mathrm{E}+09$ \\
\hline 0414 & $13 x$ & 01 & 0.75 & 6.0 & 7.5 & 4.31 & 7.5 & & 1.5E-06 & 77.4 & & 11 & 32.4 & 0 & & 62.0 & 8.9 & $E+09$ \\
\hline 0414 & $14 x$ & 26 & 0.65 & 5.5 & 7.5 & 4.13 & 1.8 & & -07 & 48.3 & & 0.50 & 20.0 & 0 & 22 & 47.3 & 42.0 & +0 \\
\hline 414 & $15 x$ & 10 & 64 & 5.8 & 7.5 & 14 & 0.9 & & 07 & .9 & & 0.49 & & & 1.266 & 46.6 & 48.2 & $E+0 s$ \\
\hline 4 & $16 x$ & 20 & 68 & 5.5 & 7.5 & 16 & 4.8 & & 9 & .2 & & 0.86 & .8 & & 515 & .4 & 54.6 & \\
\hline 414 & $17 x$ & 99 & .71 & 6.1 & 7.5 & 15 & 6 & 66 & 1.4 & 9.6 & +10 & 1.00 & 3 & +11 & 35 & 8.8 & 62.8 & +1 \\
\hline 415 & $11 x$ & 14 & 68 & 3.8 & 7.5 & 53 & 8.1 & 25 & 06 & .8 & +10 & 0.88 & 1 & $2.6 \mathrm{E}+11$ & 1.750 & 6.9 & 72.8 & +1 \\
\hline 415 & $12 x$ & 08 & .68 & 5.9 & 7.5 & 3.85 & 5.5 & & 06 & 2.4 & +09 & 0.7 & .2 & $1.4 \mathrm{E}+11$ & 1.475 & 0.6 & 59.9 & +1 \\
\hline 415 & $13 x$ & 19 & 0.68 & 7.6 & 7.5 & 4.04 & 0.9 & & 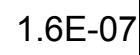 & 1.8 & +09 & 0.59 & .0 & $E+10$ & 1.525 & 2.6 & 47.4 & $+c$ \\
\hline 415 & $14 x$ & 07 & 0.68 & 9.9 & 7.5 & 4.24 & $E-04$ & 3 & 2.7 & 53.3 & +07 & 0.40 & .3 & $4.1 \mathrm{E}+08$ & 1.659 & 4.3 & 50.2 & $+c$ \\
\hline 430 & $11 x$ & 10 & 0.74 & 7.7 & 9.0 & .97 & 1.8 & 4 & 07 & .0 & +09 & 0.65 & 25.3 & $4.4 \mathrm{E}+10$ & 1.477 & 5.2 & 53.2 & $=0$ \\
\hline 430 & $12 x$ & 03 & 0.75 & 7.9 & 9.0 & .35 & 3.8 & 73 & 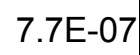 & 6.8 & +09 & 1.01 & 25.2 & $9.2 E+10$ & 1.979 & 67.9 & 57.7 & $1.0 \mathrm{E}+$ \\
\hline 430 & $13 x$ & 95 & 0.75 & 8.1 & 9.0 & .42 & 7 & 68 & 1.6 & 60.5 & +10 & 1.16 & 20.2 & $3.6 E+11$ & 2.211 & 73.0 & 61.5 & $1.3 \mathrm{E}+$ \\
\hline 0430 & $14 x$ & 13 & 0.75 & 7.8 & 9.0 & 4.44 & 9.4 & 46 & 2.6 & 1.7 & +10 & 1.14 & 21.0 & $5.4 \mathrm{E}+11$ & 2.460 & 78.1 & 66.3 & $1.7 \mathrm{E}+$ \\
\hline 0430 & $15 x$ & 13 & 0.75 & 7.8 & 2.5 & 3.75 & 4.3 & 14 & 1.0 & 7 & & 0.01 & 16.0 & $4.7 \mathrm{E}+11$ & $\mid 1.198$ & 42.9 & 28.0 & $9.0 \mathrm{E}+$ \\
\hline 506 & $11 x$ & 99 & 0.75 & 8.1 & 3.0 & 3.75 & 0.4 & 0085 & $8.5 \mathrm{E}-08$ & 10.6 & & 0.50 & 3.3 & $4.7 \mathrm{E}+12$ & 0.201 & & b. & $b$ \\
\hline 0506 & $12 x$ & 2.00 & 0.74 & 8.1 & 6.0 & 3.82 & 1.2 & 260 & $2.6 \mathrm{t}$ & 28.2 & -10 & $0.67 \mid$ & 9.3 & $6.2 E+11$ & $\mid 1.188$ & 51.6 & 24.6 & $3.4 \mathrm{E}+1$ \\
\hline 0506 & $13 x$ & .02 & 0.74 & 8.0 & 9.0 & 4.17 & 2.2 & 56 & 4.6 & 5.6 & & 0.48 & 8.8 & $1.3 E+11$ & 1.476 & 68.2 & 42.7 & $:+10$ \\
\hline 30506 & $14 x$ & .03 & 0.74 & 7.9 & 12.0 & 4.38 & 3.9 & 0838 & 8.4E-07 & .4 & +10 & 0.95 & 7.1 & $3.2 E+11$ & 1.901 & 77.4 & 50.7 & $1.3 \mathrm{E}+1$ \\
\hline 30506 & $15 x$ & 92 & 0.75 & 8.2 & 12.0 & 4.70 & 6.9 & 1728 & $1.7 \mathrm{E}-06$ & 62.3 & $E+10$ & 1.03 & 22.5 & $2.9 E+11$ & 2.660 & 95.1 & 70.8 & $9.4 \mathrm{E}+0$ \\
\hline 030506 & $16 x$ & 96 & 0.75 & 8.1 & 9.0 & 4.49 & 5.8 & 1404 & 1.4E-06 & 59.4 & $E+10$ & 0.94 & 21.8 & $2.6 \mathrm{E}+11$ & 2.410 & 97.8 & 64.9 & $1.0 \mathrm{E}+1$ \\
\hline 030506 & $17 x$ & 97 & 0.75 & 8.1 & 6.0 & 4.10 & 5.35 & 1377 & $1.4 \mathrm{E}-06$ & 48.5 & $E+10$ & 0.85 & 7.1 & $5.3 E+11$ & 2.325 & 85.8 & 49.7 & $2.2 \mathrm{E}+1$ \\
\hline 030506 & $18 x$ & 95 & 0.75 & 8.1 & 3.0 & 3.75 & 4.4 & 71 & $1.1 \mathrm{E}-06$ & 28.2 & +10 & 0.35 & 11.5 & $1.3 \mathrm{E}+12$ & 1.458 & 72.9 & 27.9 & $9.5 \mathrm{E}+1$ \\
\hline 0728 & 010 & 18 & 0.69 & 9.0 & 9.0 & 4.37 & 0.62 & 19 & $1.9 \mathrm{E}-08$ & 16.0 & +09 & 0.50 & 5.3 & $2.4 \mathrm{E}-10$ & 0.831 & 60.8 & 13.0 & $1.7 \mathrm{E}+1$ \\
\hline 0728 & 020 & 04 & 0.70 & 6.9 & 9.0 & 3.85 & 11 & 62 & 4.6 & 4.5 & & 0.73 & 21.2 & $9.2 \mathrm{E}+10$ & 1.324 & 58.4 & 45.3 & $9.7 \mathrm{E}+0$ \\
\hline & 030 & 07 & 0.70 & 6.9 & 9.0 & 3.92 & 20 & 88 & 8.9 & 1.4 & & 0.75 & 1 & $1.2 \mathrm{E}+11$ & 1.261 & 63.4 & 108.1 & $1.3 \mathrm{E}+0$ \\
\hline & 040 & 10 & 0.70 & 6.8 & 9.0 & 4.11 & 2.8 & & 1.6 & 0.4 & & 0.85 & 2 & & 1.050 & 56.4 & 52.8 & $2.1 \mathrm{E}+1$ \\
\hline 30728 & 050 & 15 & 0.70 & 6.8 & 9.0 & 4.17 & 37.5 & 2013 & $2.0 \mathrm{E}-06$ & 58.2 & $.9 \mathrm{E}+10$ & 1.22 & 18.3 & $6.2 E+11$ & $\mid 1.475$ & 74.5 & 53.5 & $2.6 \mathrm{E}+1$ \\
\hline
\end{tabular}

Tab. 7.6: Meßergebnisse der Ethylen-Luft-Flammen bei höherem Druck 


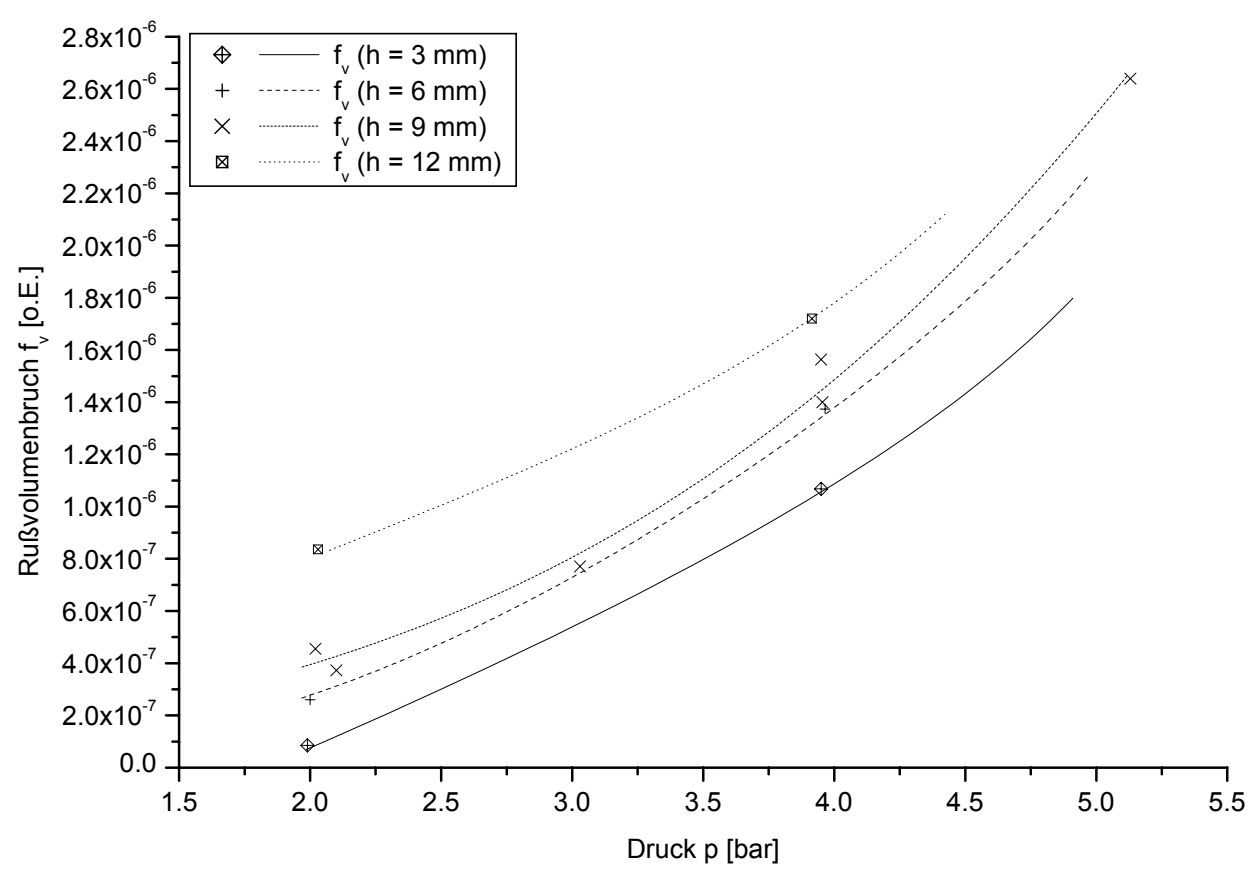

Abb. 7.63: Druckabhängigkeit des Rußvolumenbruchs von Ethylen-Luft-Flammen mit $\mathrm{C} / \mathrm{O}=0.75$ und $\mathrm{V}_{\mathrm{FG}}=8 \mathrm{~cm} / \mathrm{s}$ für verschieden Höhen $\mathrm{h}$

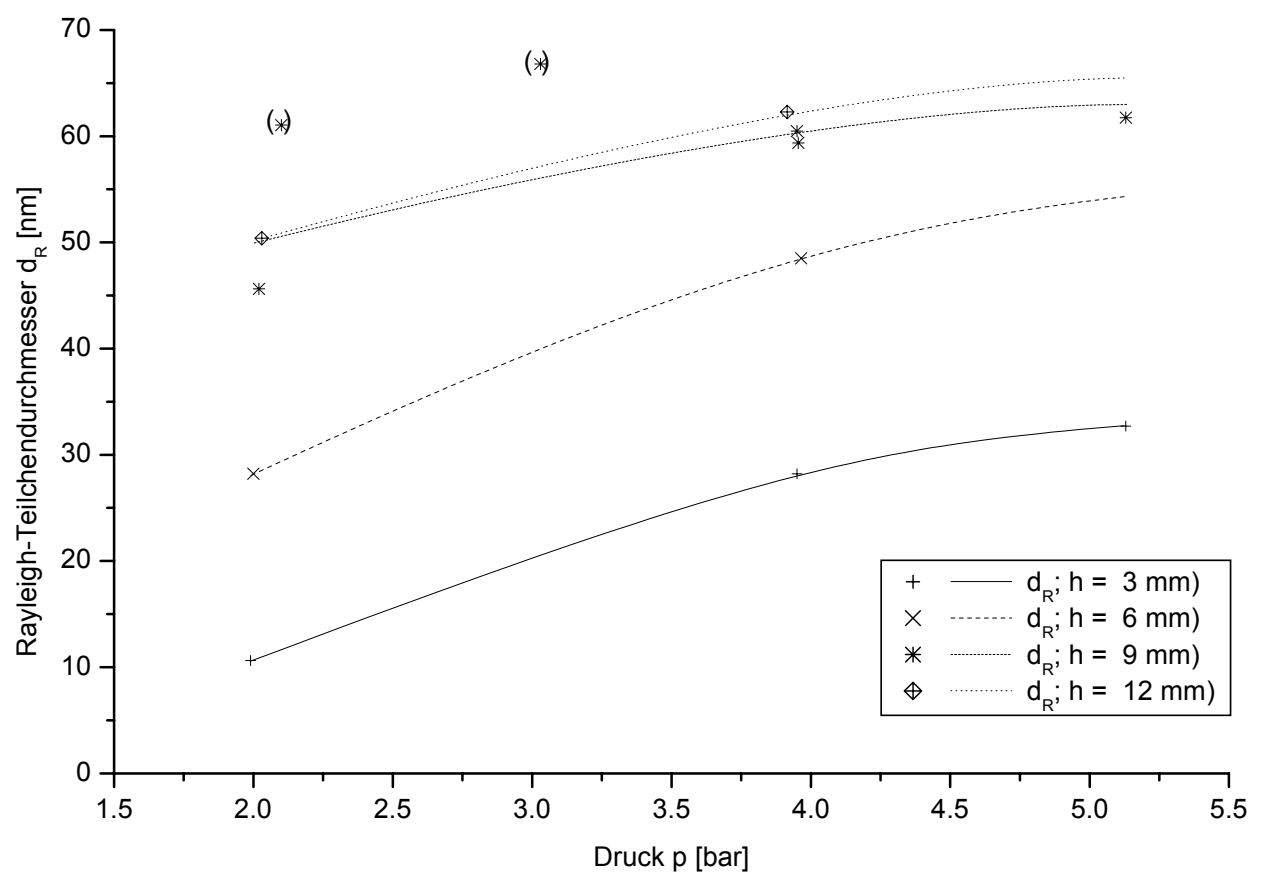

Abb. 7.64: Druckabhängigkeit des Teilchendurchmessers $d_{R}$ in Ethylen-Luft-Flammen mit $\mathrm{C} / \mathrm{O}=0.75$ und $\mathrm{v}_{\mathrm{FG}}=8 \mathrm{~cm} / \mathrm{s}$ für verschieden Höhen $\mathrm{h}$ 


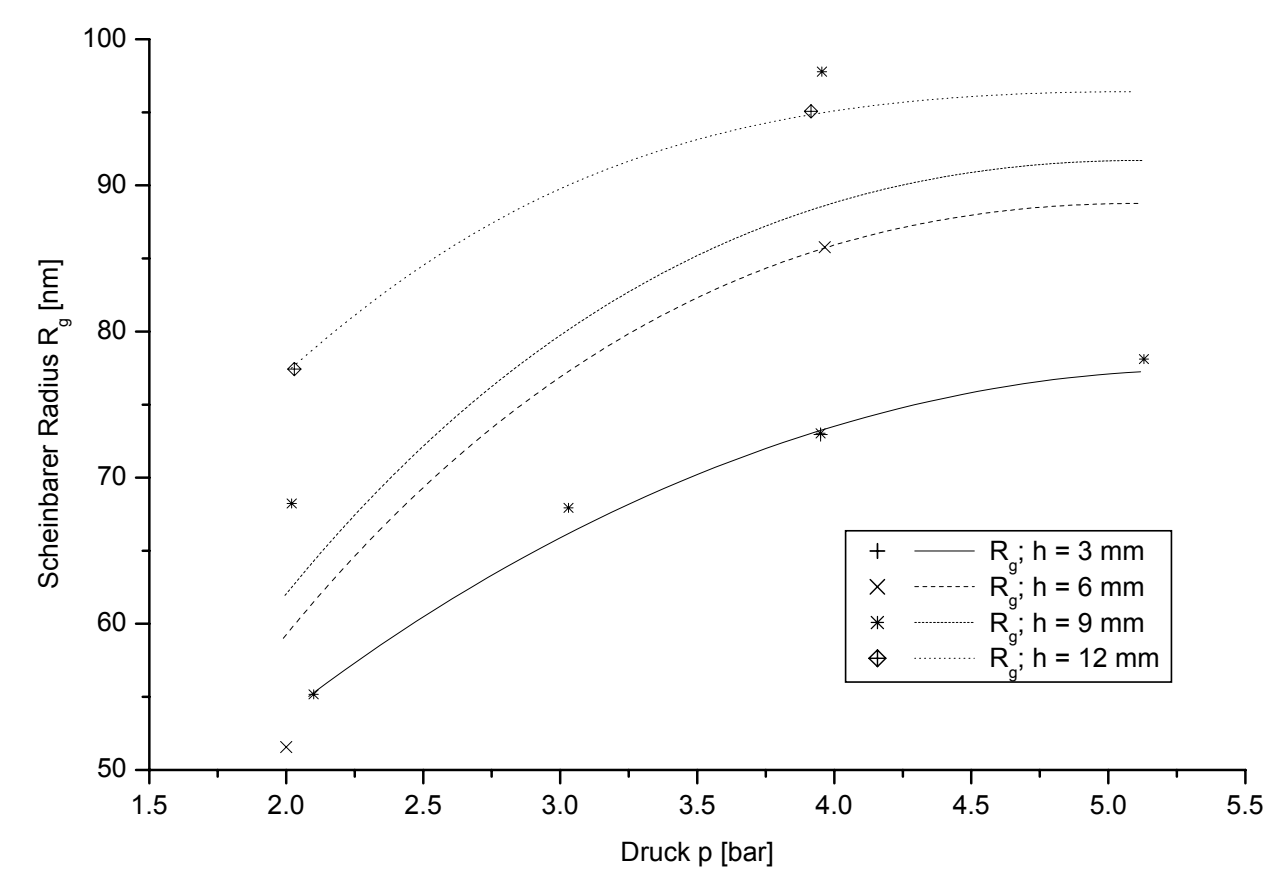

Abb. 7.65: Druckabhängigkeit des scheinbaren Radius $\mathrm{R}_{\mathrm{g}}$ in Ethylen-Luft-Flammen mit $\mathrm{C} / \mathrm{O}=0.75$ und $\mathrm{v}_{\mathrm{FG}}=8 \mathrm{~cm} / \mathrm{s}$ für verschieden Höhen $\mathrm{h}$

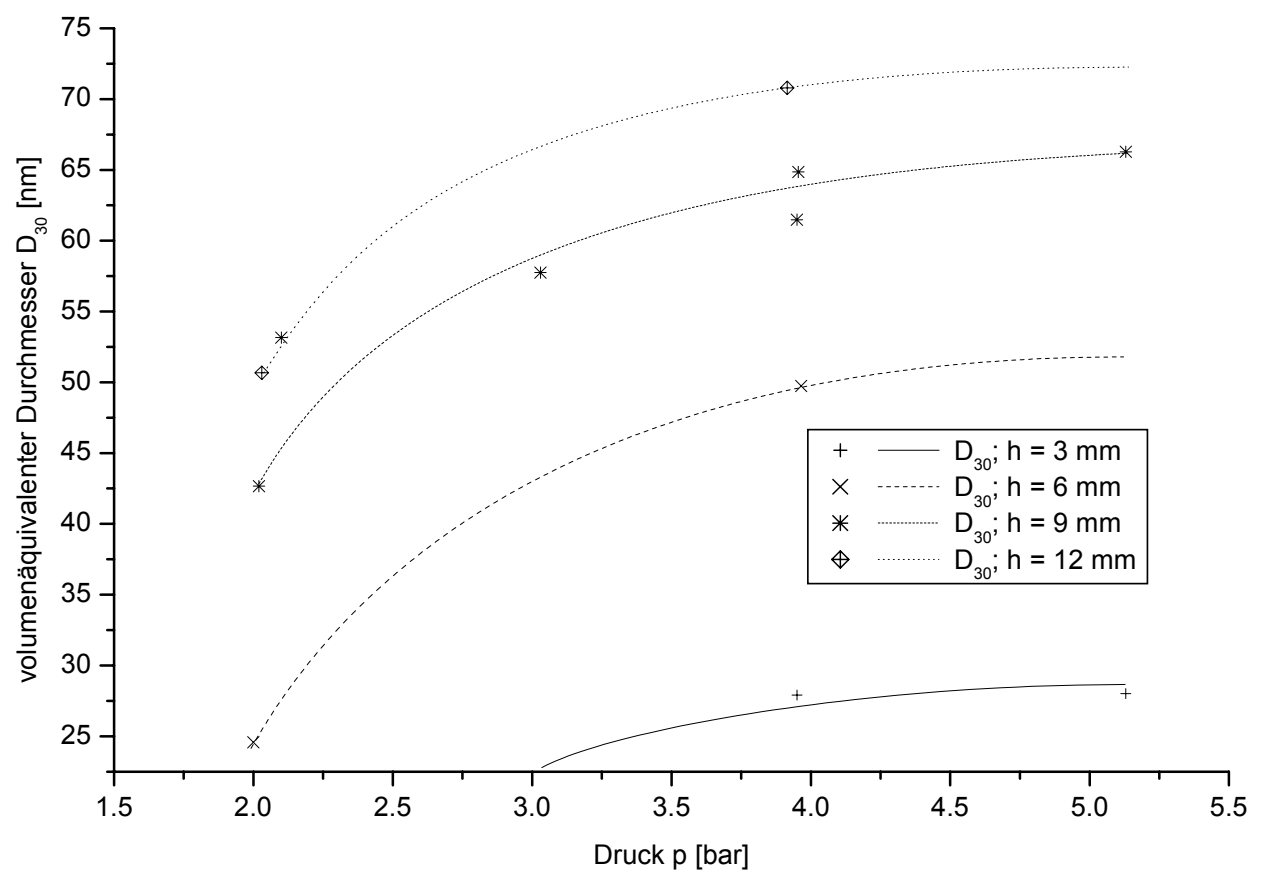

Abb. 7.66: Druckabhängigkeit des volumenäquivalenten Durchmessers $\mathrm{D}_{30}$ in EthylenLuft-Flammen mit $\mathrm{C} / \mathrm{O}=0.75$ und $\mathrm{v}_{\mathrm{FG}}=8 \mathrm{~cm} / \mathrm{s}$ für verschieden Höhen $\mathrm{h}$ 


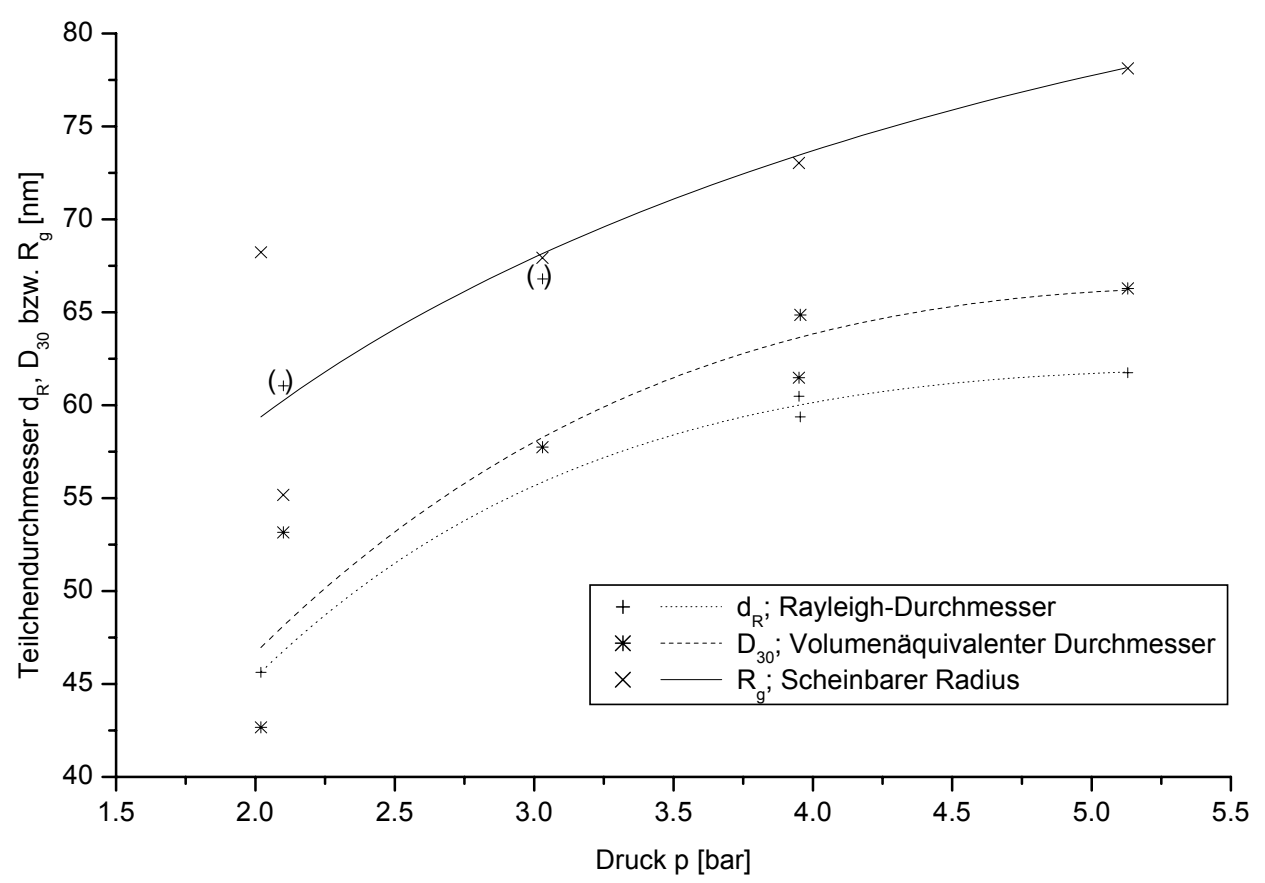

Abb. 7.67: Druckabhängigkeit der verschiedenen Teilchendurchmesser in Ethylen-LuftFlammen mit $\mathrm{C} / \mathrm{O}=0.75$ und $\mathrm{v}_{\mathrm{FG}}=8 \mathrm{~cm} / \mathrm{s}$ für verschieden Höhen $\mathrm{h}$

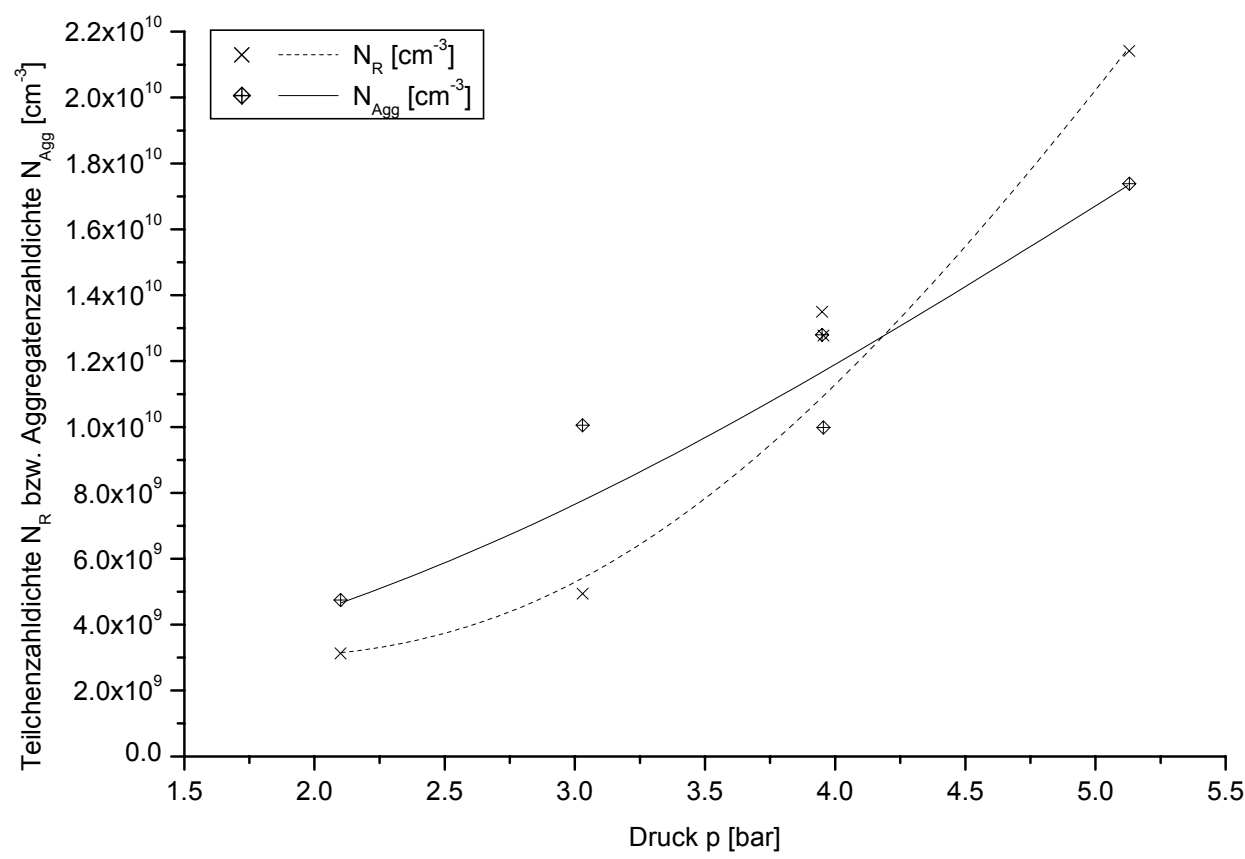

Abb. 7.68: Druckabhängigkeit der Teilchenzahldichte und der Aggregatenzahldichte in Ethylen-Luft-Flammen mit $\mathrm{C} / \mathrm{O}=0.75$ und $\mathrm{v}_{\mathrm{FG}}=8 \mathrm{~cm} / \mathrm{s}$ für verschieden Höhen $\mathrm{h}$ 


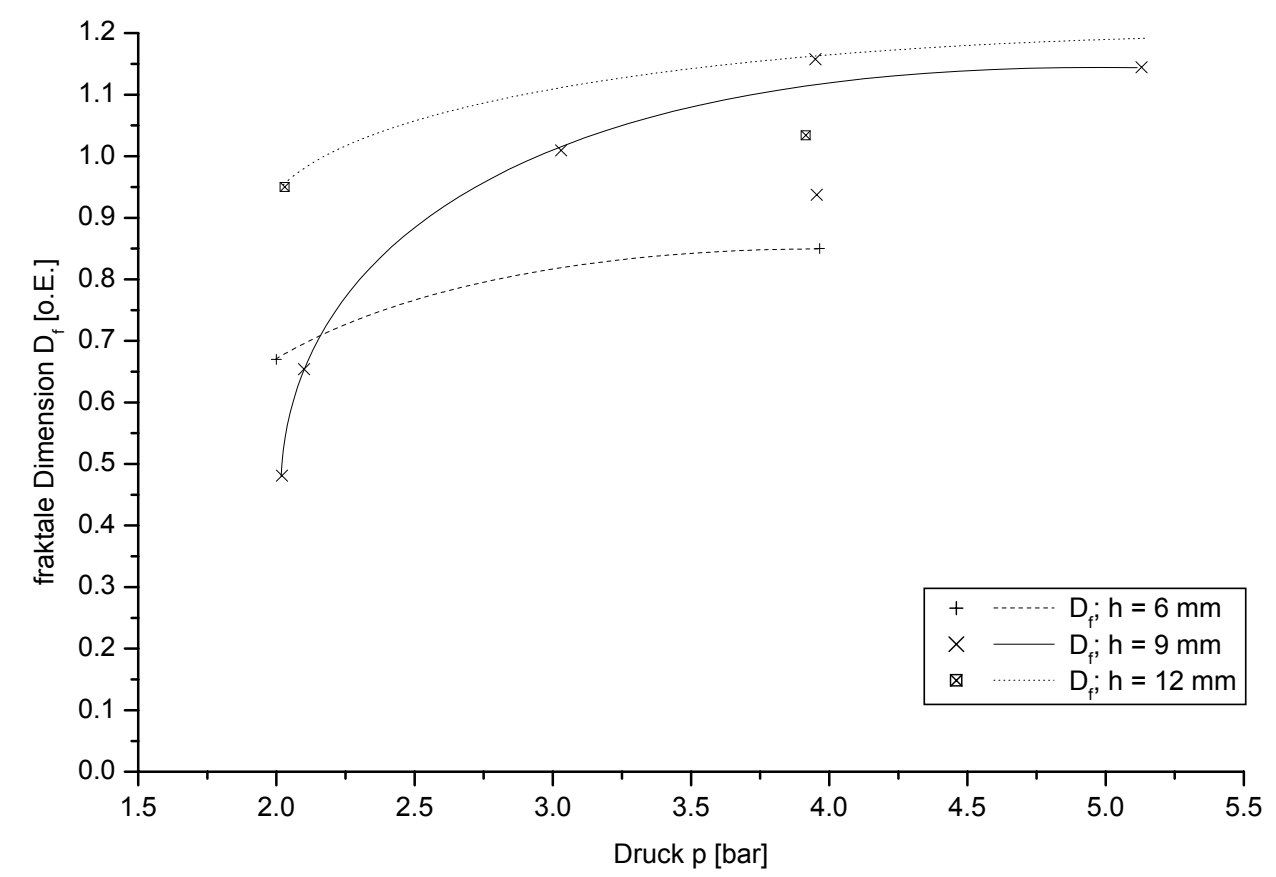

Abb. 7.69: Druckabhängigkeit der fraktalen Dimension in Ethylen-Luft-Flammen mit C/O $=0.75$ und $\mathrm{V}_{\mathrm{FG}}=8 \mathrm{~cm} / \mathrm{s}$ für verschieden Höhen $\mathrm{h}$

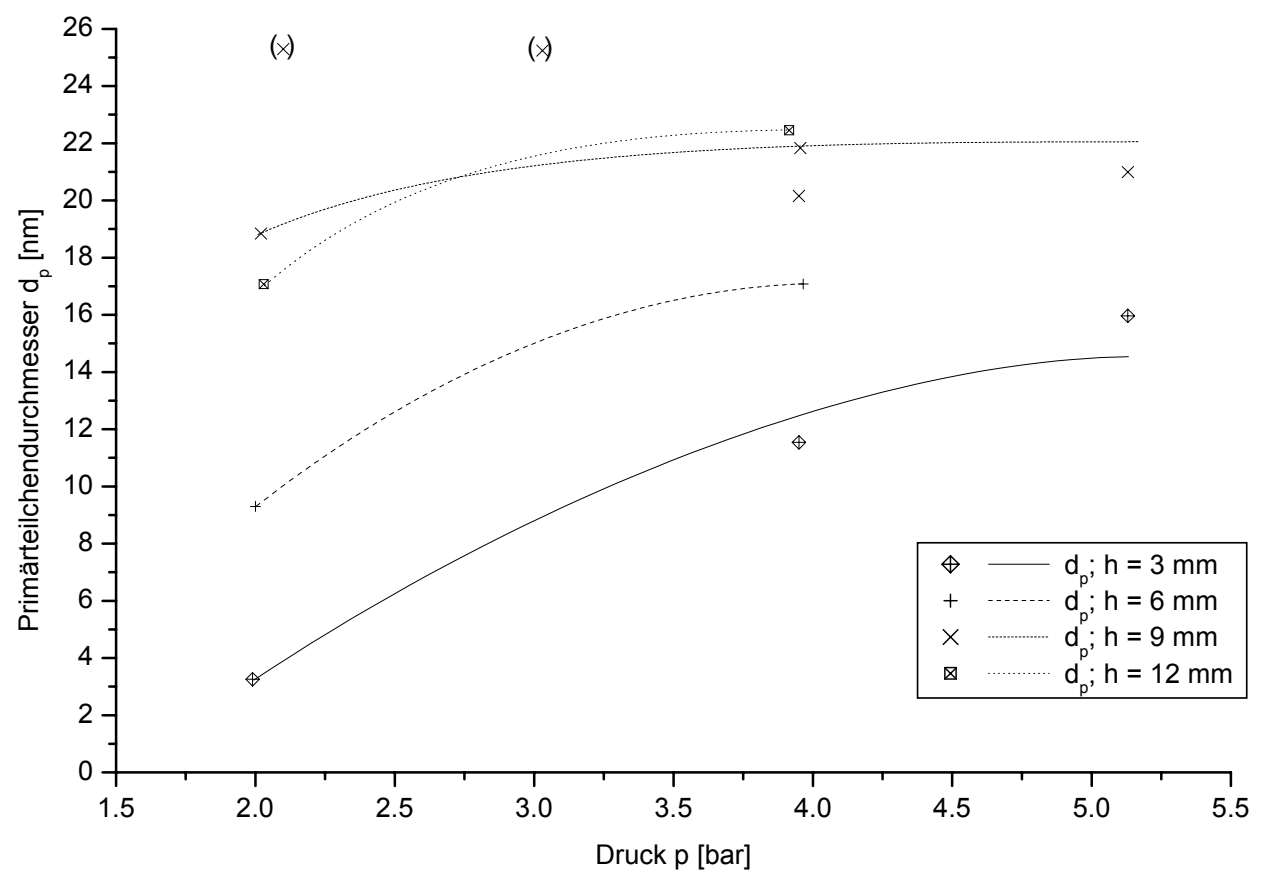

Abb. 7.70: Druckabhängigkeit des Primärteilchendurchmessers in Ethylen-Luft-Flammen mit $\mathrm{C} / \mathrm{O}=0.75$ und $\mathrm{v}_{\mathrm{FG}}=8 \mathrm{~cm} / \mathrm{s}$ für verschieden Höhen $\mathrm{h}$ 


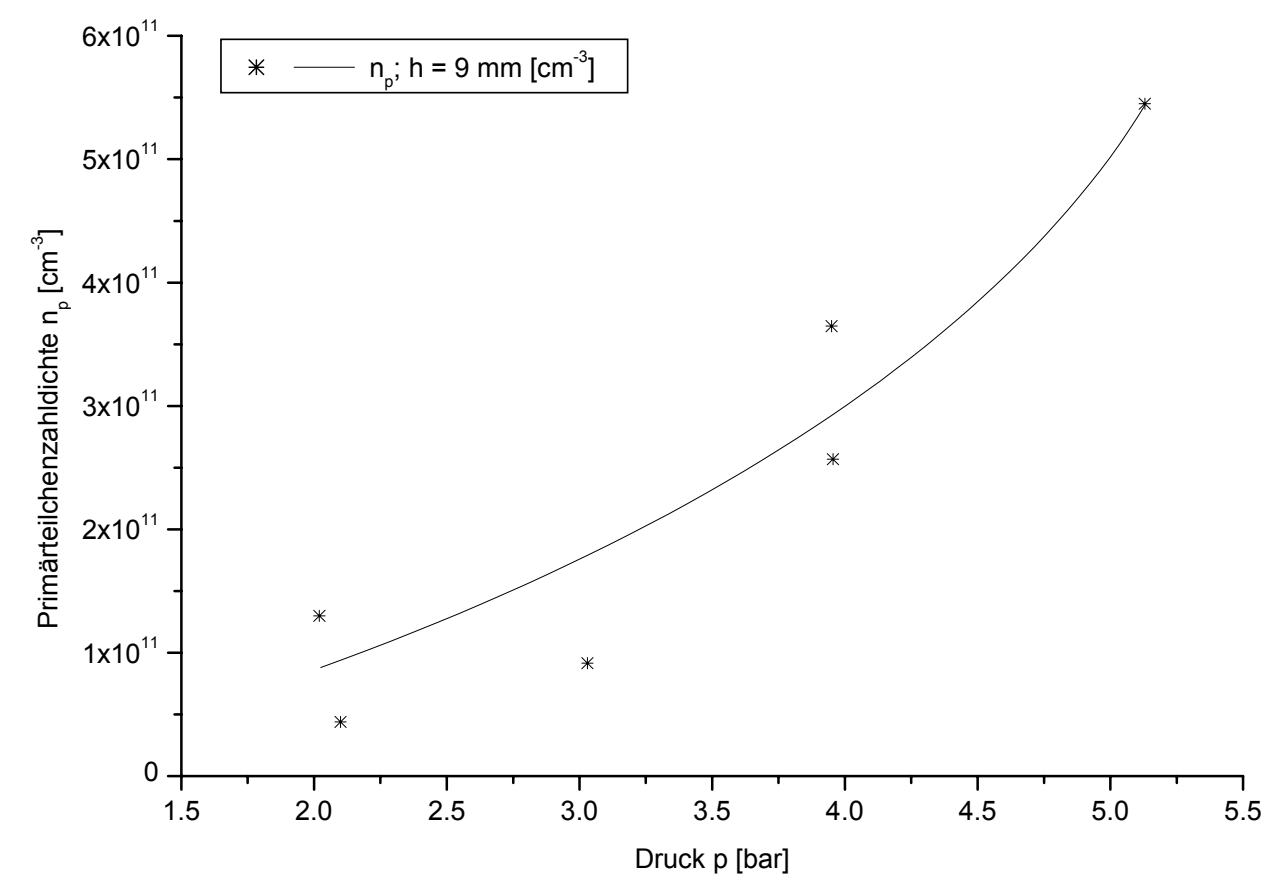

Abb. 7.71: Druckabhängigkeit der Primärteilchenzahldichte in Ethylen-Luft-Flammen mit $\mathrm{C} / \mathrm{O}=0.75$ und $\mathrm{v}_{\mathrm{FG}}=8 \mathrm{~cm} / \mathrm{s}$ in einer Höhe von $9 \mathrm{~mm}$

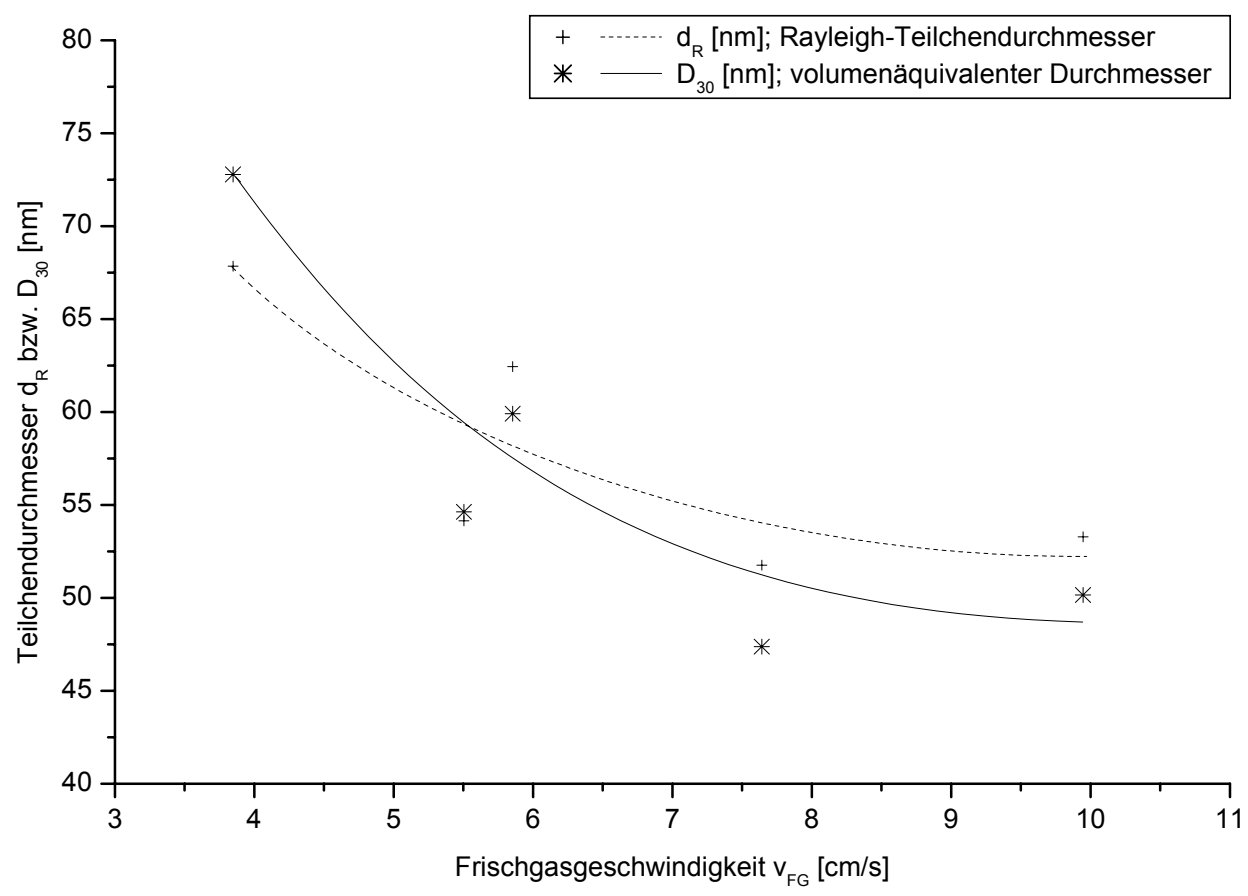

Abb. 7.72: Durchsatzabhängigkeit der verschiedenen Teilchendurchmesser $d_{R}$ bzw. $D_{30}$ in Ethylen-Luft-Flammen mit $\mathrm{C} / \mathrm{O}=0.75$ und $\mathrm{h}=7.5 \mathrm{~mm}$ bei einem Druck von 5 bar 


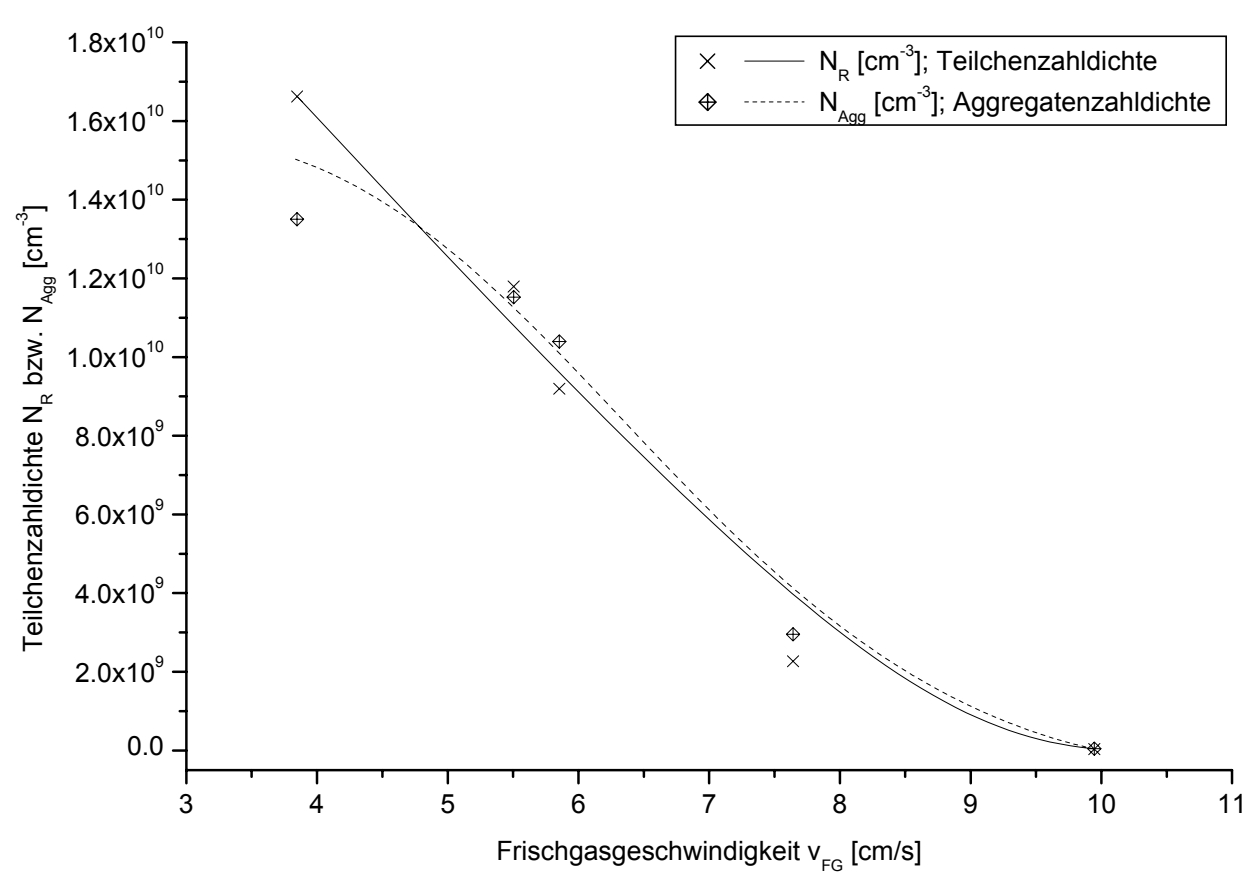

Abb. 7.73: Durchsatzabhängigkeit der Teilchenzahldichte $\mathrm{N}_{\mathrm{R}}$ bzw., Aggregatenzahldichte $\mathrm{N}_{\text {Agg }}$ in Ethylen-Luft-Flammen mit $\mathrm{C} / \mathrm{O}=0.75 ; \mathrm{h}=7.5 \mathrm{~mm}$ und $\mathrm{p}=5 \mathrm{bar}$

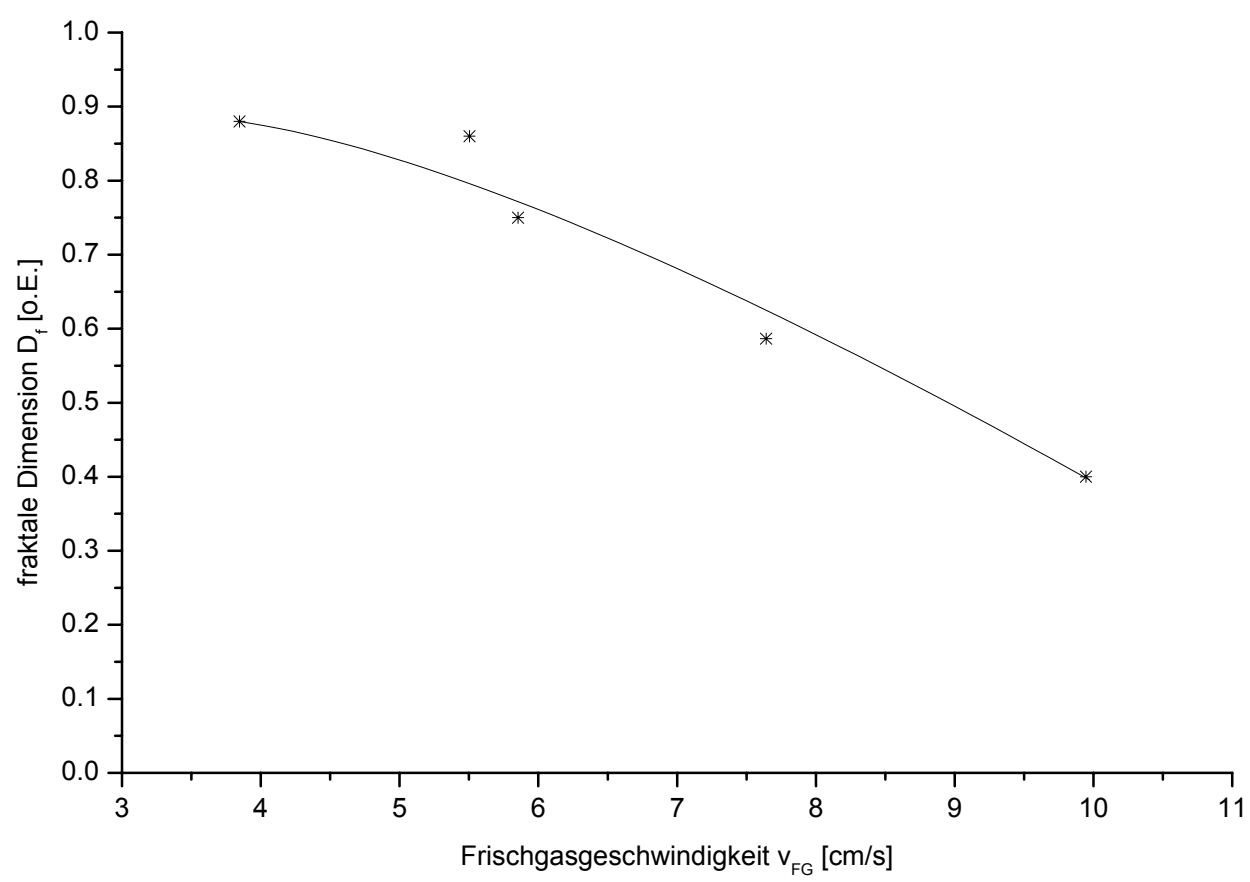

Abb. 7.74: Durchsatzabhängigkeit der fraktalen Dimension $\mathrm{D}_{\mathrm{f}}$ in Ethylen-Luft-Flammen mit $\mathrm{C} / \mathrm{O}=0.75$ und $\mathrm{h}=7.5 \mathrm{~mm}$ bei einem Druck von 5 bar 


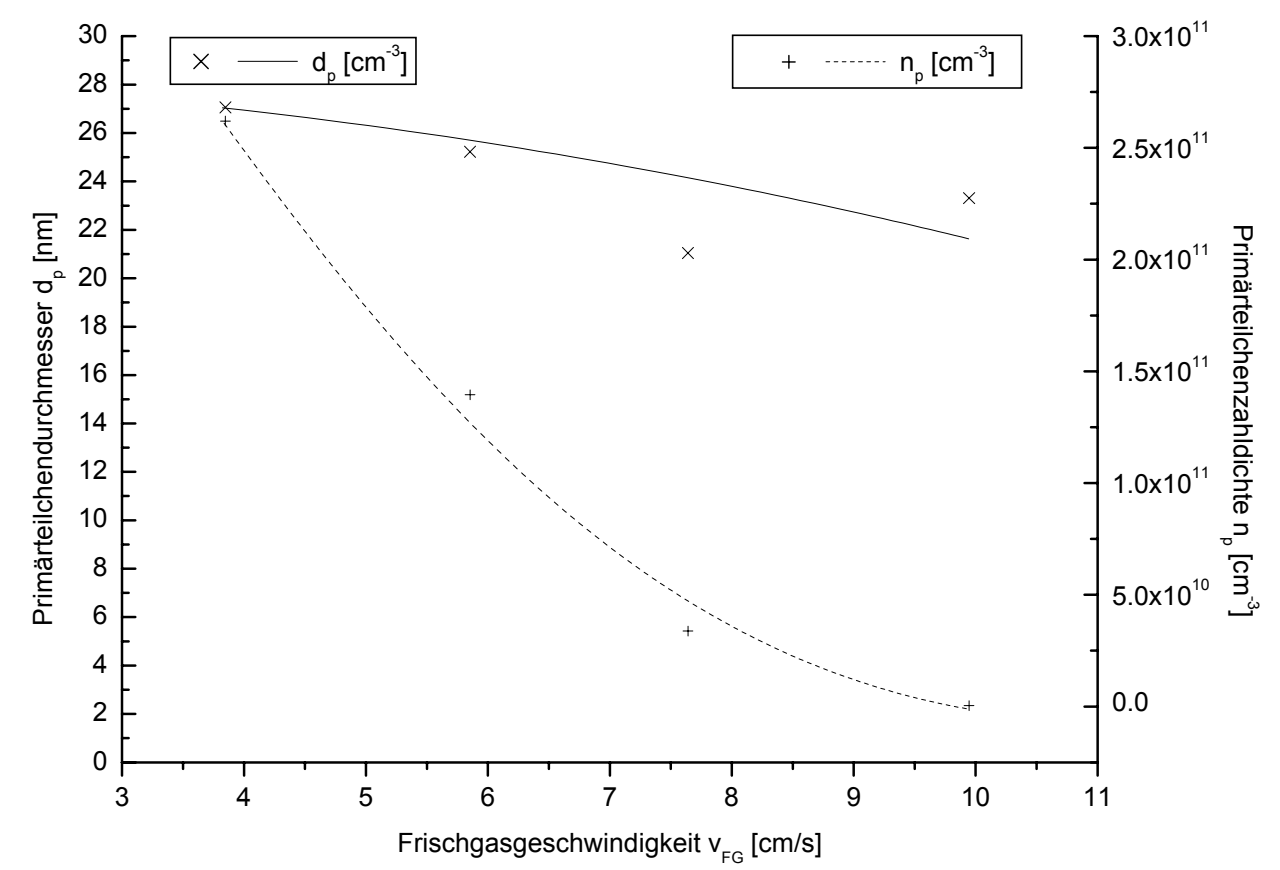

Abb. 7.75: Durchsatzabhängigkeit des Primärteilchendurchmessers $d_{p}$ und der Primärteilchenzahldichte $\mathrm{n}_{\mathrm{p}}$ in Ethylen-Luft-Flammen mit $\mathrm{C} / \mathrm{O}=0.75$ und $\mathrm{h}=$ $7.5 \mathrm{~mm}$ bei einem Druck von 5 bar

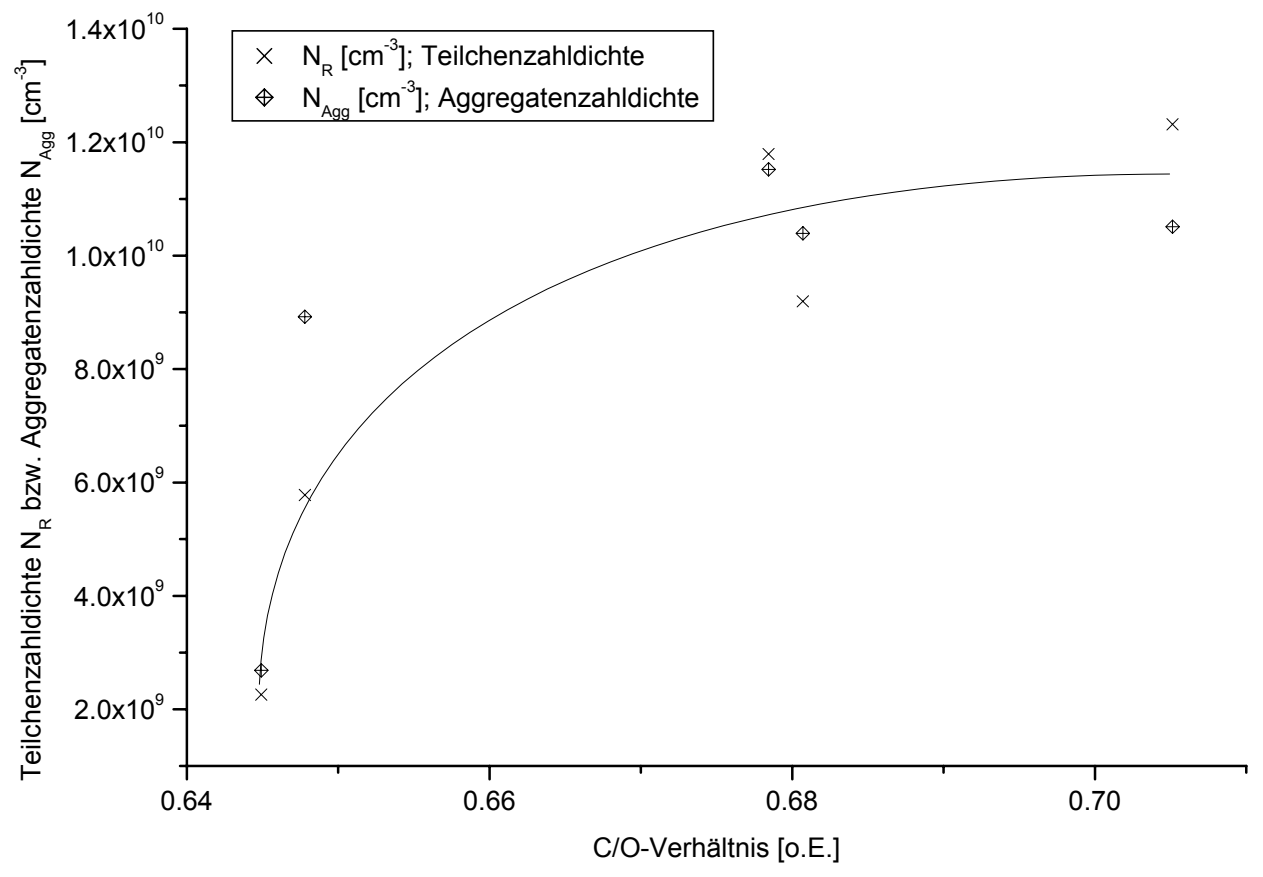

Abb. 7.76: Abhängigkeit der Teilchenzahldichte $\mathrm{N}_{\mathrm{R}}$ bzw. der Aggregatenzahldichte $\mathrm{N}_{\mathrm{Agg}}$ vom C/O-Verhältnis für Ethylen-Luft-Flammen mit $\mathrm{v}_{\mathrm{FG}}=6 \mathrm{~cm} / \mathrm{s}$ und $\mathrm{h}=7.5$ mm bei einem Druck von 5 bar 


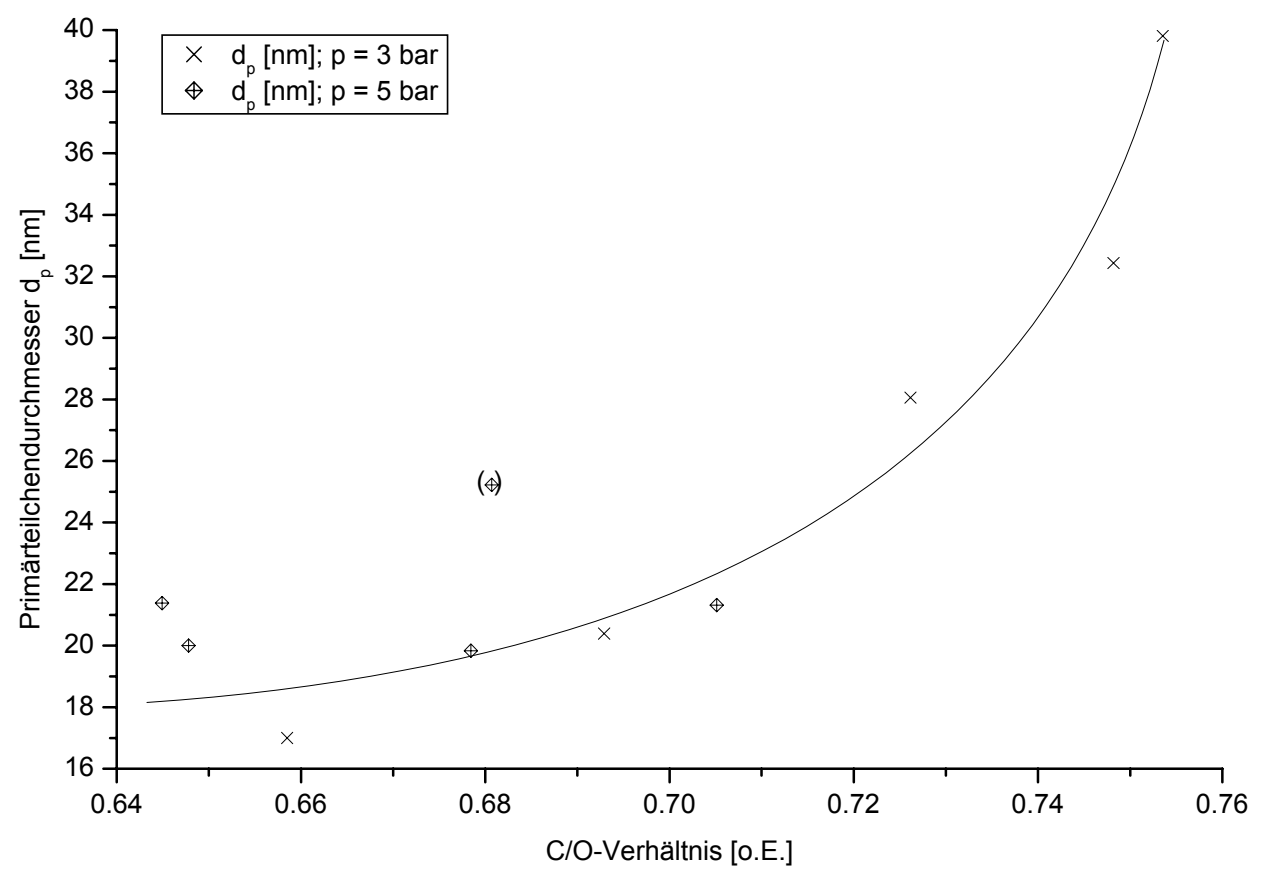

Abb. 7.77: Abhängigkeit des Primärteilchendurchmessers vom C/O-Verhältnis für Ethylen-Luft-Flammen mit $\mathrm{v}_{\mathrm{FG}}=6 \mathrm{~cm} / \mathrm{s}$ und $\mathrm{h}=7.5 \mathrm{~mm}$ bei einem Druck von 3 bzw. 5 bar 


\section{Literaturverzeichnis}

[1] BAUMGÄRTNER, L.; HESSE, D.; JANDER, H.; WAGNER, H. Gg.: Twentieth Symposium (International) on Combustion, S. 959, The Combustion Institute (1984), „Rate of Soot Growth in Atmospheric Premixed Laminar Flames".

BAUMGÄRTNER, LUTZ:

Dissertation; Göttingen 1983

] BAUMGÄRTNER,L.; JANDER,H. and WAGNER, H. Gg.:

Ber. Bunsenges. Phys. Chem. 87, 107, (1983): „Rußbildung in verschiedenen Brennstoff-Luft-Flammen“.

[4] BOCKHORN, H.; FETTING, F.; WANNEMACHER, G.; WENZ, H.W.:

Nineteenth Symposium (International) on Combustion, S. 1413, The Combustion Institute (1982), „Optical Studies of Soot Particle Growth in HydrocarbonOxygen Flames“.

[5] BOCKHORN, H.; FETTING, F.; WIESCHNOWSKY, U.:

Twenty-Second Symposium (International) on Combustion, S. 343, The Combustion Institute (1988), ,Some new Observations Concerning the Mass Growth of Soot in Premixed Hydrocarbon-Oxygen Flames“.

[6] BOCKHORN,H.; FETTING,F.; HEDDRICH,A.; WANNEMACHER, G.:

Twentieth Symposium (International) on Combustion, S. 979, The Combustion Insitute 1984, „Investigation of the Surface Growth of Soot in Flat low Pressure Hydrocarbon Oxygen Flames“.

[7] BÖHM, H.; FELDERMANN, Ch.; HEIDERMANN, Th.; JANDER, H.;LÜERS, B., WAGNER, H. Gg.:

Twenty-Fourth Symposium (Int.) on Combustion, S. 991. The Combustion Institute, 1992, „Soot Formation in a Premixed $\mathrm{C}_{2} \mathrm{H}_{4}$-Air Flame for Pressures up to 100 bar".

[8] BÖNIG, MATHIAS:

Dissertation Göttingen 1993

[9] BRIDGE, H. J.; BUCKINGHAM,A.D.:

Proc. Roy. Soc. A295, 334 (1966) 
[10] BRUHN, KATHRIN:

Diplomarbeit; Göttingen 1988

[11] CHAMBRION, Ph.; JANDER, H.; PETEREIT, N. and WAGNER, H.Gg.:

Z. Physikal. Chemie, 1996, „Soot Growth in Atmospheric $\mathrm{C}_{2} \mathrm{H}_{4} / \mathrm{Air} / \mathrm{O}_{2}$-Flames.

Influence of the Fuel Carbon Density“.

[12] CHARALAMPOPOULOS, T.T.; CHANG,H.:

Combust. Flame 87,S. 89-99, (1991). „Agglomerate Parameters and Fractal

Dimension of Soot Using Light Scattering-Effects on Surface Growth“.

[13] D'ALESSIO, A.

„Particulate Carbon: Formation During Combustion“, D. C. Siegla, G. W. Smith, eds., S. 207, Plenum Press, New York (1983), „Laser light Scattering and

Fluorescence

[14] D'AlESSIO, A.; BERETTA, F.; VENITOZZI, C.:

Combust. Sci. And Tech. 5, 263 (1972),“Optical Investigations on Soot Forming Methane-Oxygen Flames“.

[15] D'ALESSIO, A.; VAGLIECO, B. M.; BERETTA, F.:

„Aerosols: Formation and Reactivity“, 2nd Int. Aerosol Conference Berlin (1986),

„In Situ High Temperature Determination of the U.V. Optical Properties of Soot Particles from Light Scattering and Extinction Measurements“.

[16] DALZELL, W. H.; SAROFIM, A. F.:

J. Heat Transfer (Trans. ASME Sec. C) 91, 100 (1969)

[17] DASCH, C. J.:

Comb. Flame 61, 219 (1985), „The Decay of Soot Surface Growth Reactivity and its Importance in total Soot Formation“.

[18] di STASIO, S.; MASSOLI, P. and LAZZARO, M.:

J. Aerosol Sci. Vol. 27, Nr.6, S. 897-913,(1996), „Retrieval of Soot Aggregate Morphology from Light Scattering/Extinction Measurements in a high Pressure high Temperature Environment“.

[19] DOBBINS, R. A.; MEGARIDIS, C. M.:

Applied Optics, Vol.30, No. 33; S.4747, 1991. "Absorption and Scattering of

Light by Fractal Aggregates“،

[20] DOBBINS, R.A.; SANTORO, R.J.; SEMERJIAN,:

Twenty Third Symposium (International) on Combustion. The Combustion 
Institute, 1990. S. 1525-1532. Analysis of Light Scattering

„From Soot Using Optical Cross Sections For Aggregates“.

[21] FELDERMANN, CHRISTIAN:

Diplomarbeit; Göttingen 1988

[22] FELDERMANN, CHRISTIAN:

Dissertation; Göttingen 1992

[23] FELSKE,J.; HSU, P.-F. and KU, J.C.:

J. Quant. Spetrosc. Radiat. Transfer 35, S. 447-465, (1986), „The Effect of Soot

Particle Optical Inhomogenity and Agglomeration on the Analysis of Light

Scattering Measurements in Flames“.

[24] FRENKLACH, M., GARDINER, W. C.:

J. Phys. Chem. 88, 6263 (1984), „Representation of Multistage Mechanisms in

Detailed Computer Modelling of Polymerisation Kinetics“.

[25] FRENKLACH, M.; CLARY, D. W.; GARDINER, W. C.; STEIN, S. E.:

Twenty-First Symposium (International) on Combustion, S. 1067, The

Combustion Institute (1986), „Effect of Fuel Structure on Pathways to Soot““.

[26] GAYDON, A. G. UND WOLFHARD, H. G.: Flames: Their Structure, Radiation and Temperature; Chapman \& Hall; London 1979

[27] HARRIS, S. J.; WEINER, A. M.; GOLDSMITH, J. E. M.:

Twenty-First Symposium (International) on Combustion, S. 1033, The

Combustion Institute (1987), „Concentration Profiles in Rich and Sooting

Ethylene Flames“.

[28] HAYNES, B. S.; WAGNER, H. Gg.:

Prog. Energy Combust. Sci. 7, 229 (1981), „Soot Formation“.

[29] HAYNES, B.S.; WAGNER, H.Gg.:

Z. Phys. Chem. N.F. 133, 201 (1982), „The Surface Growth Phenomenon in Soot Formation".

[30] HESSE, DORIS:

Dissertation; Göttingen 1988

[31] HIEMENZ, P.; RAJAGOPALAN, R.:

"Principles of Colloid and Surface Chemistry"

Marcel Dekker; Inc., New York,1997.

[32]

JANDER,H.; PETEREIT, N.; RAZUS, D.M.:

Z. Phys. Chem. Bd.188, S.159 (1995). „The Influence of Carbon Density on Soot 
Growth in Atmospheric $\mathrm{C}_{2} \mathrm{H}_{4}\left(\mathrm{Air}, \mathrm{O}_{2}\right)$-Flames“.

[33] JULLIEN, R. and BOTET, R. 1987:

„Aggregation and Fractal Aggregation“"

World Scientific Publishing Co., Singapore S. 46-60.

[34] KERKER, M.:

„The Scattering of Light“", Academic Press, New York (1969).

[35] KÖYLÜ, Ü.Ö.:

Comb. Flame 106, S. 488-500 (1996), „Quantitative Analysis of In Situ Optical

Diagnostics for Inferring Particle / Aggregate Parameters in Flames: Implications for Soot Surface Growth and total Emissivity“.

[36] KÖYLÜ,Ü.Ö.; FAETH,G.M.; FARIAS, T.L. and CARVALHO, M.G.:

Journal of Heat Transfer, Vol. 117, S. 152-159, (1995), „Computational

Evaluation of Approximate Rayleigh-Debye- Gans/Fractal-Aggregate Theory for the Absorption and Scattering Properties of Soot".

[37] LAHAYE, J.; PRADO, G.:

"Particulate Carbon" (Edited by Siegla, D.C. and Smith, G.W.), S. 31-55.

Plenum, New York. „Morphology and Internal Structure of Soot and Carbon

Blacks“.

[38] LANDOLT; BÖRNSTEIN:

Wärmetechnik; Band IV, 4. Teil

Springer-Verlag, 1972

[39] LIDE, DAVID R.:

Handbook of Chemistry and Physics, $81^{\text {st }}$ Ed., CRC Press 2000

[40] MÄTZING, HARTMUT:

Dissertation; Göttingen 1986

[41] MEGARIDIS, CONSTANTINE M.; DOBBINS, RICHARD A.:

"Morphological Description of Flame-Generated Materials"

Combust. Sci. andTech., 1990, Vol. 71, S. 95-109

[42] MIE, G.:

Ann. Physik 25, 377-445 (1908) „Beiträge zur Optik trüber Medien, speziell

kolloidaler Lösungen"

[43] NIST, National Institute of Standard and Technology: www.nist.gov

[44] PAPE, THOMAS:

Diplomarbeit; Göttingen 1993 
[45] PAPE, THOMAS:

Dissertation; Göttingen 1997

[46] SANTORO, R. J.; SEMERJIAN, H. G.; DOBBINS, R. A.:

Comb. Flame 51, 203 (1983), „Soot Particle Measurements in Diffusion Flames“.

[47] STAHLBERG, WILHELM:

Diplomarbeit; Göttingen 1999

[48] UMWELTBUNDESAMT:

Umweltdaten Deutschland Online; http://www.umweltbundesamt.de; Stand 2003

Angaben sind für das Jahr 2001

[49] VAN DE HULST, H.C.:

"Light Scattering by Small Particles"; Wiley, New York, 1957

[50] WARNATZ, J.:

18th Symposium (International) on Combustion, The Combustion Institute,

Pittsburgh, S. 369. „The Structure of Laminar Alkane-, Alkene-, and Acetylene Flames".

[51] WARNATZ, J.; BOCKHORN, H.; MÖSER, A.; WENZ, H.W.:

Nineteenth Symposium (International) on Combustion, S.197, The Combustion Institute (1982), „Experimental Investigations and Computational Simulation of Acetylene-Oxygen Flames from near Stoichiometric to Sooting Conditions“. 


\section{Lebenslauf}

Am 23.12.1964 wurde ich als erstes von zwei Geschwistern des Rechtsanwalts Wilhelm Johann Heinrich und seiner Frau Eva-Elfriede Anne-Christine Stahlberg, geb. Bremer, in der Freien Hansestadt Bremen geboren.

In den Jahren 1972 bis 1976 besuchte ich in Bremen die Grundschule „Schule an der CarlSchurz-Straße". Anschließend wechselte ich auf das Kippenberg-Gymnasium in Bremen. Am 30.06.1986 habe ich das Zeugnis der allgemeinen Hochschulreife erhalten.

Von Juli 1986 bis Juni 1988 diente ich als Soldat bei der Marine.

Im Herbst 1988 immatrikulierte ich mich an der Universität Bayreuth im Diplomstudiengang Chemie. Während des Grundstudiums habe ich 1990 für zwei Monate bei der Firma Bayer im Pflanzenschutzzentrum Monheim als Werksstudent gearbeitet. Beschäftigt habe ich mich dabei hauptsächlich mit der Trennung und Identifizierung von Metaboliten unter anderem aus Bodenextrakten. Im Juni 1991 habe ich an der Universität Bayreuth die Vordiplomsprüfung bestanden.

Im Oktober 1993 wechselte ich an die Georg-August-Universität Göttingen. Im August 1998 begann ich am Institut für Physikalische Chemie unter der Anleitung von Herrn Prof. Dr. Wagner meine Diplomarbeit mit dem Thema „Aggregatenbildung von Rußteilchen in vorgemischten atmosphärischen laminaren Ethylen/Luft-Flammen“. Am 02.07.1999 habe ich die Diplomprüfung abgelegt.

Seit April 2000 bin ich als wissenschaftlicher Mitarbeiter am Institut für Physikalische Chemie beschäftigt und habe Übungen zu Vorlesungen und Praktika betreut.

Von Oktober 2000 bis August 2001 habe ich an einem viersemestrigen Hochschulkurs zum Grundlagenschwerpunkt Materialwissenschaften Göttingen mit Erfolg teilgenommen. Außerdem habe ich Vorlesungen mit Übungen und Praktika in Technischer und Makromolekularer Chemie sowie in Materialphysik erfolgreich belegt.

Meine Dissertation mit dem Thema „Aggregatenbildung der Rußteilchen in Kohlenwasserstoff/Luftflammen in einem Druckbereich von ein bis fünf bar" habe ich im September 1999 unter Anleitung von Prof. Dr. Wagner in der Flammenabteilung unter Leitung von Frau Dr. Jander begonnen. 